\title{
UWE WOLF
}

\section{NOTATION UND AUFFÜHRUNGSPRAXIS}

STUDIEN ZUM WANDEL VON NOTENSCHRIFT UND NOTENBILD IN ITALIENISCHEN MUSIKDRUCKEN

DER JAHRE 1571-1630

Band I: Darstellung

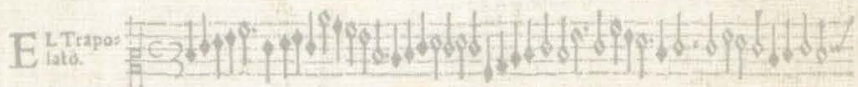

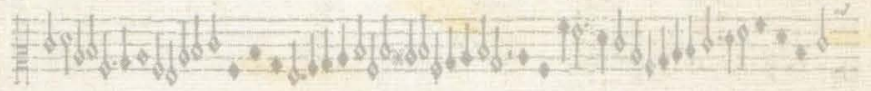

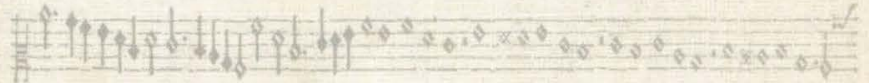

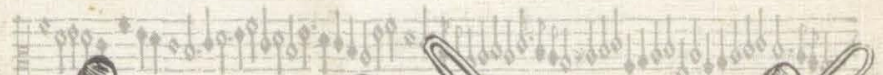

SLUB Dresden

LR

55800

W855

$-1$

zell 1

MERSEBURGER 
zell1 FHA N/LR 55800 W855-1 
Uwe Wolf

Notation und Auffuihrungspraxis

Band I 


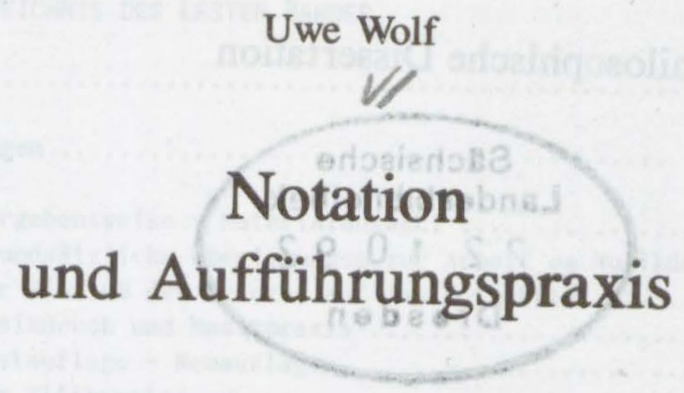

Studien zum Wandel von Notenschrift und Notenbild in italienischen Musikdrucken der Jahre 1571-1630

Band I

Darstellung

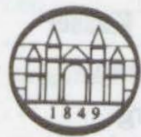

1992

Merseburger 


\section{7 Göttinger philosophische Dissertation}

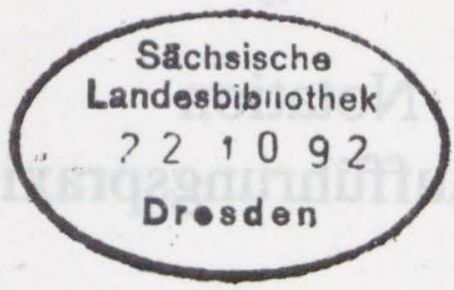

CIP-Titelaufnahme der Deutschen Bibliothek

Wolf, Uwe:

Notation und Auffuihrungspraxis : Studien zum Wandel von

Notenschrift und Notenbild in italienischen Musikdrucken der

Jahre 1571 - 1630 / Uwe Wolf. - Kassel : Merseburger

Zugl.: Göttingen, Univ., Diss. 1991

ISBN 3-87537-248-4

Bd. 1. Darstellung. -1992

Edition Merseburger 1542

(91992 Verlag Merseburger Berlin GmbH, Kassel

Alle Rechte vorbehalten · Printed in Germany

Umschlaggestaltung: Dorothea Lindenberg

Gesamtherstellung: Dissertations Druck Darmstadt GmbH

ISBN 3-87537-248-4 


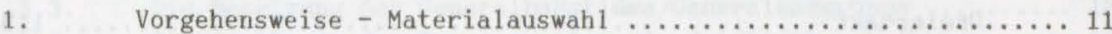

2. Grundsätzliche überlegungen zur Arbeit an Musikdrucken ...... 14

2.1. Der Einfluß der Drucker auf das Notenbild $\ldots \ldots \ldots \ldots \ldots \ldots \ldots 14$

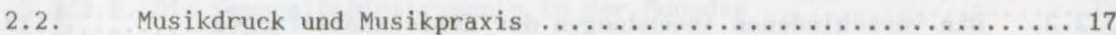

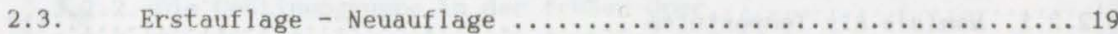

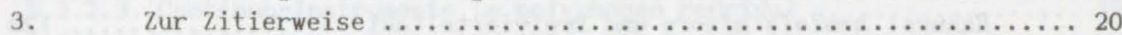

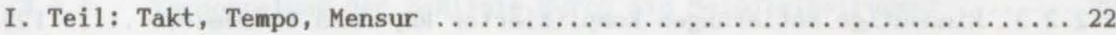

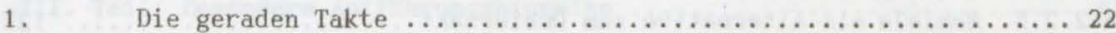

1.1. Die Gattungsmensuren des späten 16. Jahrhunderts $\ldots \ldots \ldots \ldots \ldots 22$

1.1.1. "madrigalische" und "motettische" Mensur ................ 23

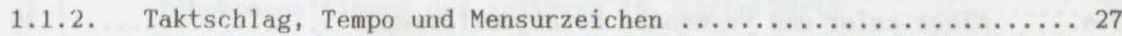

1.1.3. Die geraden Mensuren in den anderen Gattungen $\ldots \ldots \ldots \ldots \ldots \ldots . \ldots \ldots 2$

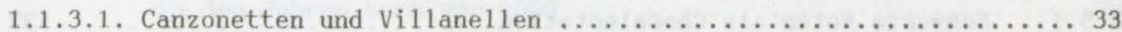

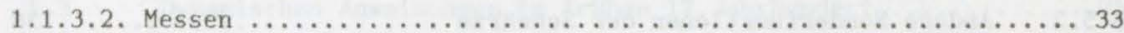

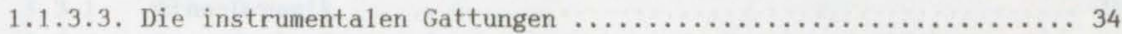

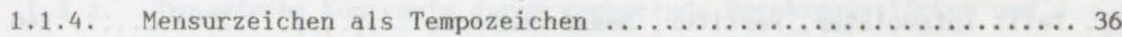

1.2. Auflösung des Systemes der Gattungsmensuren ab $1600 \ldots \ldots \ldots \ldots 40$

1.2.1. Angleichung der Motetten an die Madrigale zu Beginn des 17. Jahrhunderts .............................. 40

1.2.2. Der Bedeutungsverlust der Mensurzeichen .............. 42

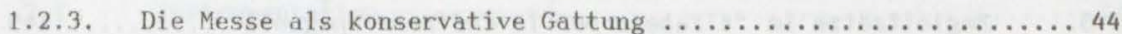

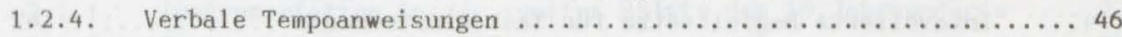

1.2.4.1. Tempoanweisungen in uberschriften $\ldots \ldots \ldots \ldots \ldots \ldots \ldots \ldots \ldots \ldots 4$

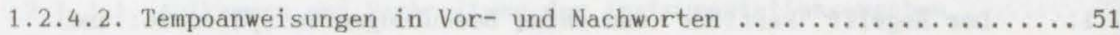

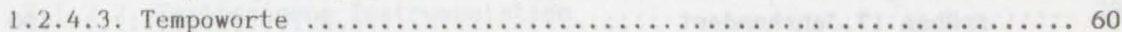

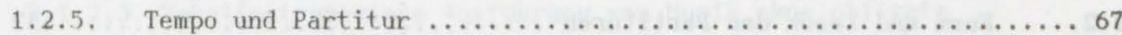

1.3. Taktstrich und Taktschlag .............................. 69

1.4. Andere Verfahren, das Notenbild zu untergliedern $\ldots \ldots \ldots \ldots \ldots 80$

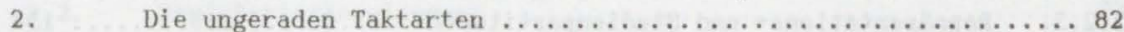

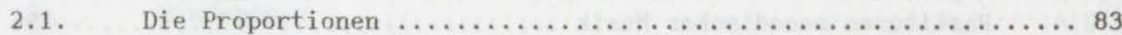

2.2. Die Abhängigkeit der ungeraden Takte von der Mensur des geraden Taktes ....................................... 87

2.3. Die Abhängigkeit der ungeraden Takte von der Gattung ....... 88

2.4. Das Tempo der erstarrten Dreiertakte ................... 89

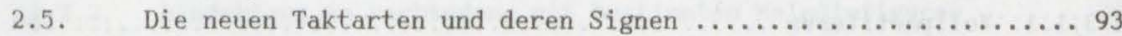

2.6. Drucke mit verschiedenen Dreiertakten $\ldots \ldots \ldots \ldots \ldots \ldots \ldots \ldots . \ldots 6$

2.6.1. "Verdopplungstheorie" nach Willi Apel . ................ 98

2.6.2. "Hemiolentheorie" nach Karin Paulsmeier .................. 98

2.6.3. "Mischtheorie" nach Helmut Hell ....................... 99

2.6.4. "Notenbildtheorie" nach Etienne Darbellay und Magaret Murata .. 99

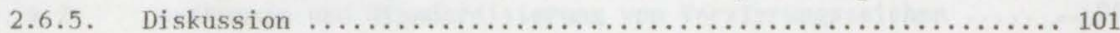


2.6.6. Versuch einer neuen unproportionalen Darstellung der Tempo-

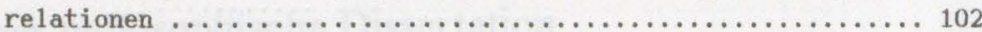

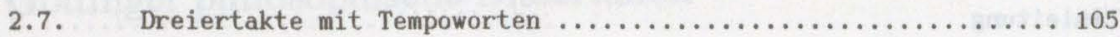

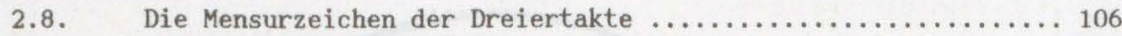

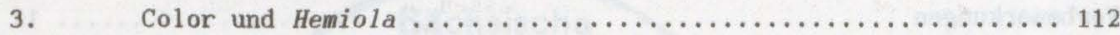

3.1. Color als Zeichen für Imperfektion sowie als Warnzeichen im

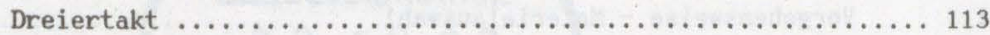

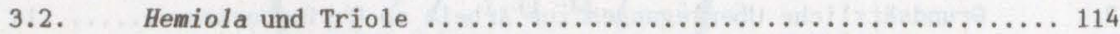

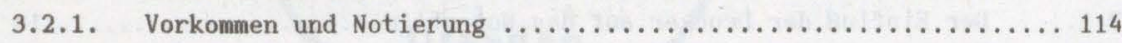

3.2.2. Die verschiedenen Anwendungen der Hemiolanotierungen $\ldots \ldots \ldots 117$

3.2.2.1. Hemiole als Tempostufen ............................ 117

Exkurs: hemiola minore und Dreivierteltakt ............... 120

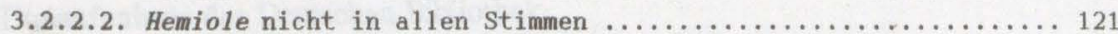

3.2.2.3. Eindeutige Notierungen komplizierter Rhythmen in hemiole .... 123

3.2.2.4 Hemiole zur Notation von Neunertakten ................. 126

3.2.2.5. Hemiola als Alternative zum Dreiertakt . . . . . . . . . . . 127

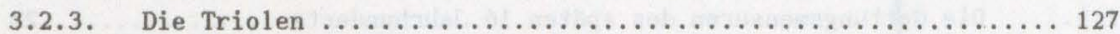

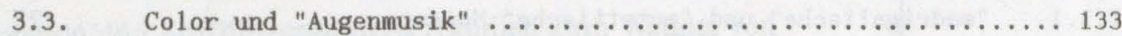

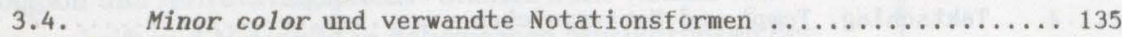

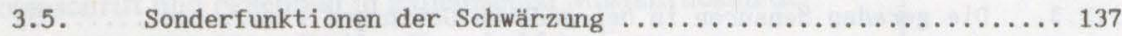

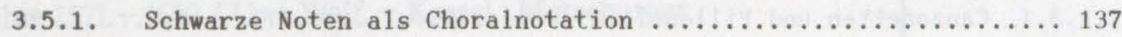

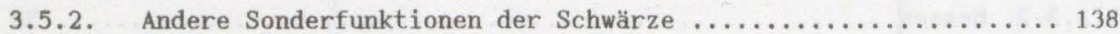

II. Tei1: Intavolatur, Partitur, Basso continuo $\ldots \ldots \ldots \ldots \ldots \ldots \ldots \ldots 140$

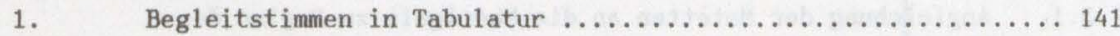

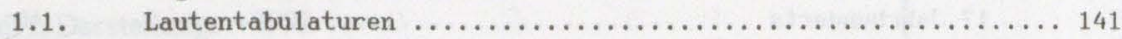

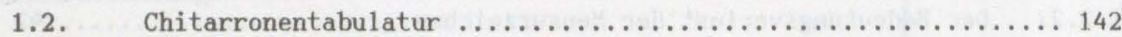

1.3. Begleitsätze im "Alfabeto per la Chitarra spagnola" ........ 145

1.4. Intavolierte Begleitsätze für Tasteninstrumente $\ldots \ldots \ldots \ldots \ldots 147$

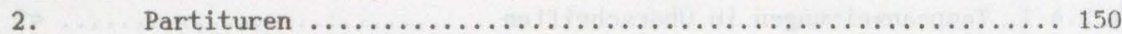

2.1. Der Begriff "Partitur" und seine Bedeutungen im späten 16. und

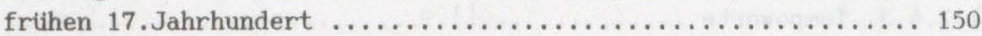

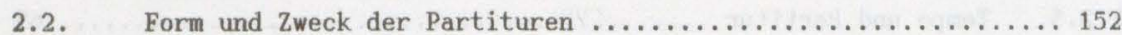

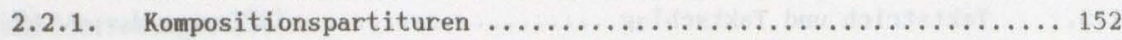

2.2.2. Zwischenpartituren beim Vorgang des Intavolierens ........ 152

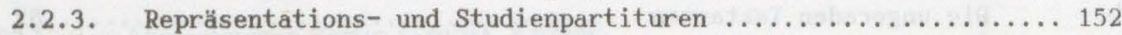

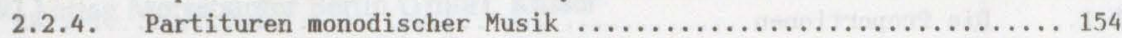

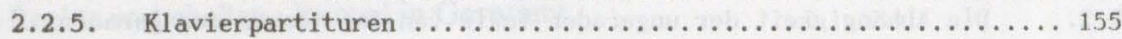

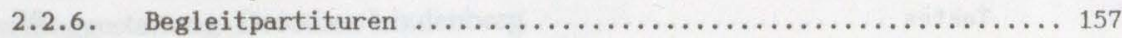

2.3. Die Begleitung anhand einer Begleitpartitur . . . . . . . . 159

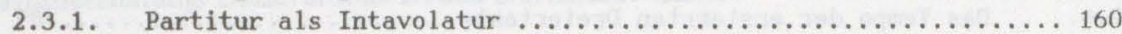

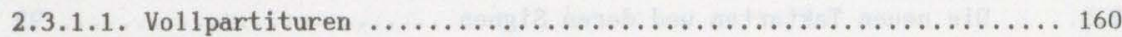

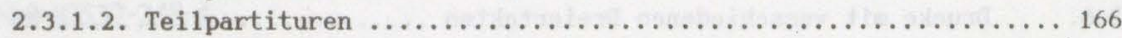

2.3.2. Partitur anstelle einer Bezifferung oder zu deren Ergänzung .. 169

2.3.3. Partituren zur Ermöglichung eines freien Vortrages ......... 170

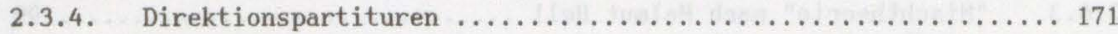




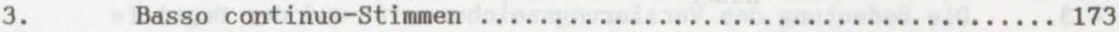

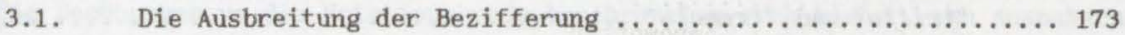

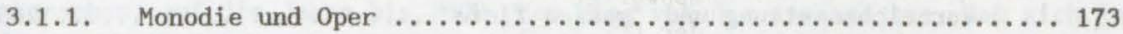

3.1.2. Die Bezifferung in den Generalbässen der geistlichen Musik ... 176

3.2. Die Direktionsfunktion der Continuo-Stimmen $\ldots \ldots \ldots \ldots \ldots \ldots \ldots 1$

3.3. Die Besetzung der Generalbaßstimme/Generalbaßgruppe ........ 183

3.3.1. Die Besetzung des Basso continuo in der Kirchenmusik ....... 183

3.3.2. Die Besetzung des Generalbasses in der weltlichen Musik ..... 192

3.3.2.1. Die Generalbaßinstrumente in der Monodie ............... 192

3.3.2.2. Die Continuogruppe in der fruhen oper $\ldots \ldots \ldots \ldots \ldots \ldots \ldots \ldots . \ldots \ldots$

3.3.2.3. Continuo-Instrumente im polyphonen Madrigal $\ldots \ldots \ldots \ldots \ldots \ldots 196$

3.3.3. Die Besetzung der Generalbässe in der Instrumentalmusik ..... 199

3.3.4. Verdoppelung der Baßlinie durch ein Melodieinstrument ...... 203

III. Teil: Besondere Auffuhrungshinweise $\ldots \ldots \ldots \ldots \ldots \ldots \ldots \ldots \ldots \ldots \ldots \ldots \ldots$

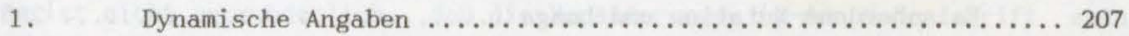

1.1. Echokompositionen des späteren 16.Jahrhunderts . . . . . . 207

1.2. Die Verwendung der dynamischen Angaben "piano" und "forte"

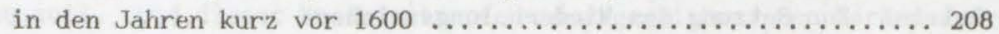

1.3. Dynamischen Anweisungen im frihen 17 .Jahrhundert $\ldots \ldots \ldots \ldots .210$

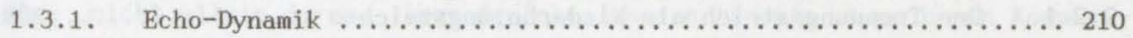

1.3.2. Dynamische Kontraste durch wechselnde Besetzungsstärken und deren Verdeutlichung durch dynamische Angaben ............ 214

1.3.3. Andere dynamische Effekte im Dienste des Textaffektes ...... 218

1.4. Crescendo, Diminuendo und "Gesangsdynamik" ............... 221

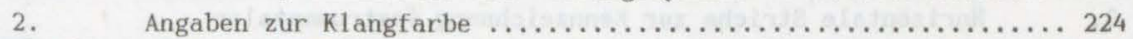

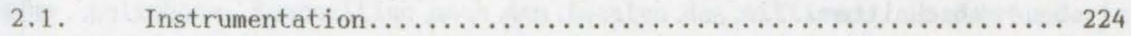

2.1.1. Instrumentation in der zweiten Hälfte des 16. Jahrhunderts $\ldots 224$

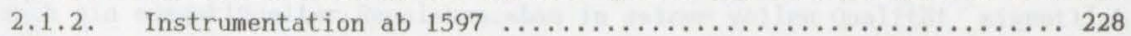

2.1.2.1. Aufkommen und Verbreitung der Instrumentationsangaben ...... 228

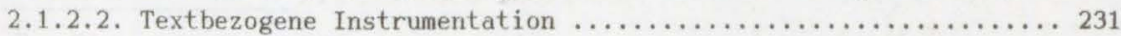

2.1.2.3. Vokalinstrumentale Ausfuihrung von Musik ohne obligate

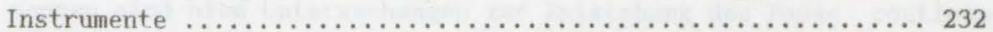

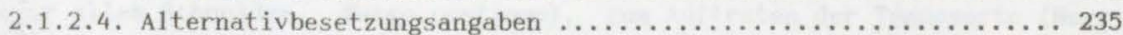

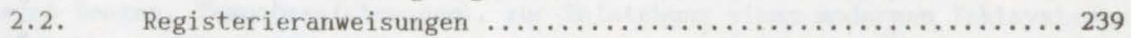

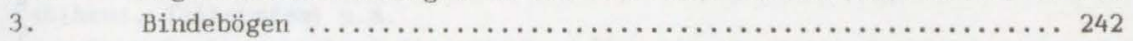

3.1. Bindebögen zwischen Noten derselben Tonhöhe $\ldots \ldots \ldots \ldots \ldots \ldots .243$

3.2. Bindebögen zwischen Noten unterschiedlicher Tonhöhe in den

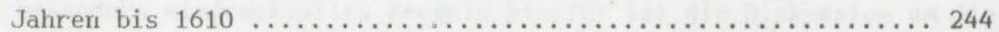

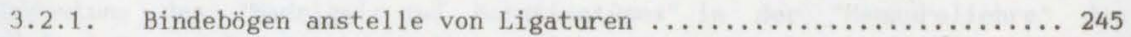

3.2.2. Bindebögen in Verbindung mit bestimmten Melodiefiguren ...... 245

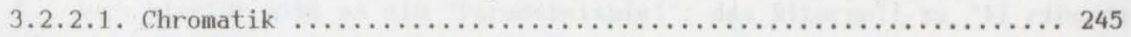

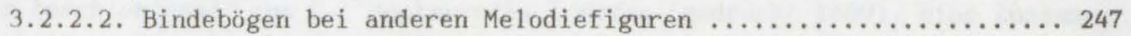

3.3. Die weitere Verbreitung der Bindebögen in den Jahren bis 1630. 250

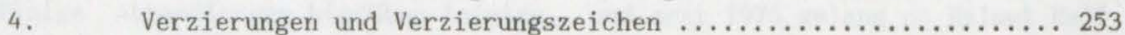

4.1. Die Verzierungspraxis im späten 16. und frühen 17.Jahrhundert. 253

4.2. Aufkommen und Standardisierung von Verzierungszeichen ...... 258 
4.3. Die Bedeutung des Verzierungszeichens " $t$ " und der Begriffe

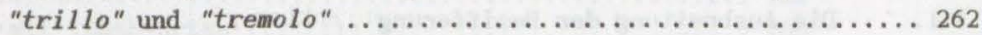

5. Vorzeichensetzung und "musica ficta" ................... 265

6. Transpositionsangaben - "Chiavettenfrage" ............... 270

6.1. "Chiavette" als Transpositionsanweisung? ................ 270

6.2. Die Erweiterung des Tonraumes im späten 16. und frihen

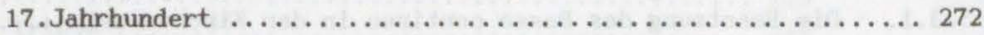

6.3. Vorgeschriebene oder bereits ausgefuhrte Transposition in

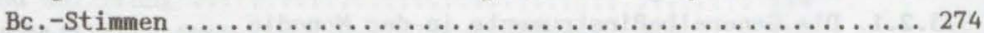

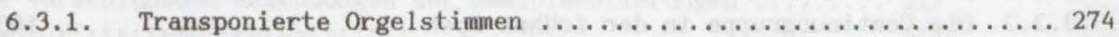

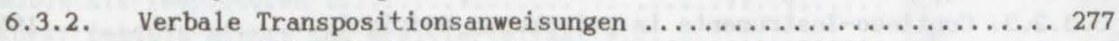

6.4. Transposition in Monteverdis "Marienvesper"? -

IV. Teil: Weitere Besonderheiten in der Notation dieser Zeit $\ldots \ldots \ldots 285$

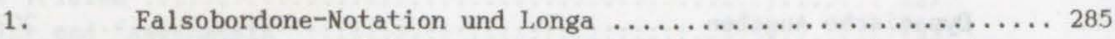

2. Wiederholungszeichen, Trennungsstrich, Fermate und Kongruenz-

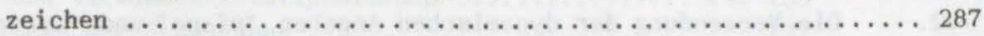

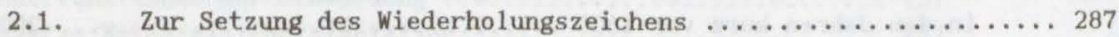

2.2. Die Anwendungen des Trennungsstriches $\ldots \ldots \ldots \ldots \ldots \ldots \ldots \ldots . \ldots \ldots$

2.2.1. Der Trennungsstrich als Wiederholungszeichen $\ldots \ldots \ldots \ldots \ldots \ldots 288$

2.2.2. Der Trennungstrich zur Abschnittsbildung als "Pausa generalis" - Trennungsstrich und Fermate ............... 289

2.2.3. Weitere Verwendungen des Trennungsstriches .............. 290

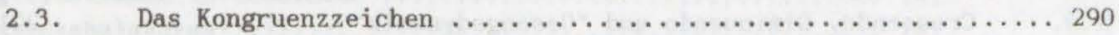

3. Horizontale Striche zur Kennzeichnung instrumentaler

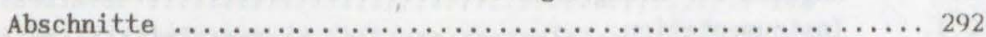

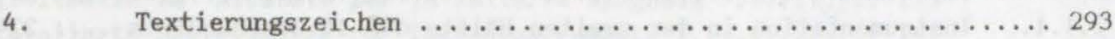

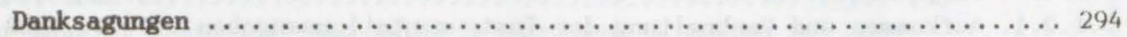




\section{EINLEITUNG}

Die Bemühungen um die Notationskunde beschränkten sich bisher, von Ausnahmen abgesehen, auf die Jahre bis 1600. Man ging von der Annahme aus, daß sich in dieser Zeit das heutige System der Notation etabliert habe ${ }^{1}$. Einige Spezialstudien zeigten, daß dies nicht ohne eine lange ubergangsperiode, in der sich Neues nur langsam durchsetzte und Altes fortbestand, geschah ${ }^{2}$. Angeregt von der eher konservativen Haltung vieler Theoretiker wurde gar das Notationssystem des mittleren 16.Jahrhunderts - und auch ein solches existiert nur in den Lehrbichern - auf das 17. Jahrhundert ibertragen ${ }^{3}$. Wurden Veränderungen in der Notation von der Forschung wahrgenommen, so geschah dies anhand der Musiktheorie ohne uberprüfung der Gegebenheiten in den musikpraktischen Quellen ${ }^{4}$. Ungewohntes in musikpraktischen Quellen hingegen wurde oft als Einzelfall behandelt und eine Lösung nur aus dem gegebenen Fal1 heraus angestrebt 5 . Eine eingehende Untersuchung des Wandels in der Notation un 1600 fehlt jedoch bislang.

Es ist nicht verwunderlich, daß die Veränderungen in der Notenschrift etwa gleichzeitig mit einem Wandel in der Musik iberhaupt vonstatten gingen. Im Gegenteil: eine Notation wird immer bestimmt durch die Musik, welche sie darstellen soll, und dieser Musik entsprechend angeglichen. Den Veränderungen in der Notenschrift um 1600 kommt jedoch eine besondere Bedeutung zu: Es ging nicht allein darum, irgendwelche inneren Aspekte einer neuen Kompositionsmanier, wie um 1400 neue, feinere rhythmische Nuancen, notierbar zu machen, sondern die Notation hatte hier auch die Aufgabe, einen bisher nicht in dieser Weise beachteten musikalischen Bereich, nämlich die Aufführung, in die notierte Komposition mit einzubeziehen.

Eine polyphone Komposition nach den Idealen des mittleren 16.Jahrhunderts etwa kann fast nicht besser bestaunt werden, als am Notenbild - ähnlich wie auch ein ausgeklügelter Renaissancebau in seiner vollen Qualität eigentlich

1. Hierzu sehr gut: Chew, Notation.

2. $\mathrm{Zu}$ nennen sind hier Untersuchungen zur Entstehung des Basso continuo (vor allem Schneider, Basso continuo), zum Auftreten der Tempoworte (Herrmann-Bengen, Tempobezeichnungen), zur Entstehung eines modernen Taktsystemes (Dahlhaus, Taktsystein) u.a.

3. So z.B. in dem Abschnitt "Musical Notation in Italy in the SevententhCentury" in Aldrich, Rhythm, S.22 ff.

4. Ein besonders eindruckvolles Zeugnis hierfür ist die Diskussion um die Bedeutung des "Madrigal- und Motettentypus" in der "Mensurallehre" des Michael Praetorius (siehe zu dieser Diskussion unten im Abschnitt I.1.1.4.). 5. Auch hierfür gibt es ein "Paradebeispiel": das Ritornell zu "Vi ricorda o bosch'ombrosi" aus C. Monteverdis L'Orfeo (gedruckt 1609). Eine Zusammenstellung der verschiedenen Lösungsvorschläge bis 1951 gibt Ape1, Ritornello. Einige Abhandlungen hieruber folgten, und erst 1975 gelang es Helmut Hell, Rhythmus, mittels Vergleichen das graphische Erscheinungsbild dieses Satzes einigermaßen zu erklären. 
nur am Bauplan erfaßbar ist. Ein moderner Komponist des frỉhen 17.Jahrhunderts will jedoch nicht mit der Faktur seiner Komposition Aufsehen erregen, sondern er will das Publikum verbluffen, bewegen, er will eine Wirkung hervorrufen, "meraviglia"6! Diese Intention entzieht sich dem theoretischen Studium - Giovanni Maria Artusi mußte Monteverdi falsch verstehen; auch viele Bauwerke dieser Zeit verbluffen den Betrachter, wenn er vor ihnen steht, nicht jedoch, wenn er ihren Bauplan betrachtet?. Werner Braun unterscheidet zwischen einem "Aufführungsgebot" für den neuen Stil und der "Aufführungsgelegenheit" für den alten ${ }^{8}$.

Mit der Auffuhrung kommt auch der Frage, wie musiziert wird, große Bedeutung zu. Bis ins 16. Jahrhundert hinein dient die Notation in erster Linie dazu, ein Stïck zu fixieren. Eine Sonderstellung kommt in dieser Beziehung den $\mathrm{Ta}-$ bulaturen zu. Sie sind immer Aufführungsvorschrift, während die Mensuralnotation mehr eine "allgemeingultige" Form darstellt. Diese "allgemeingültige" Form konnte entweder bearbeitet (intavoliert) oder aber direkt aufgefürt werden - beides oblag allein den Ausführenden; sie ibernahmen dies entsprechend ihrer Ausbildung und damit entsprechend einer Tradition. Die Mensuralnotation selbst enthält hierzu nur wenig uber die Noten hinausgehende Informationen.

Wollte nun ein Komponist der Jahre um 1600 die Wirkung seiner Musik durch eine bestimmte Auffuhrungsart unterstrichen wissen, so konnte er entweder, in Kenntnis der Tradition, herkömmliche Zeichen in einer veränderten Art und Weise verwenden und damit den Aufführenden seine Absicht zu erkennen geben oder aber ein neues Zeichen in einem Vorwort einfuhren und entsprechend einsetzen oder schlieblich verbale Anweisungen in die Noten hineinschreiben. Dies geschah freilich nicht immer auf dieselbe Art und Weise. Konnte man in friherer Zeit noch von einem in den Grundzïgen einheitlichen Notationssystem ausgehen, so wurde diese Einheitlichkeit nun zugunsten recht unterschiedlicher Experimente aufgegeben. Eine Erklärung eines neuen Zeichens in einem Vorwort z.B. hat zunächst nur Gültigkeit für den zu dem Vorwort gehörenden Musikdruck und ist nicht grundsätzlich ibertragbar.

Obwohl seit der Jahrhundertmitte erste Tendenzen in diese Richtung spirbar wurden", konzentrierte sich die Umdeutung und besonders die Neueinfuihrung von Zeichen auf die Jahre um 1600. Bereits im zweiten und dritten Jahrzehnt

6. Vg1. dazu z.B. den Abschnitt iber den "Stilwandel" bei Leopold, Monteverdi, $\mathrm{S} .47 \mathrm{ff}$.

7. Es sei hier auf die immer noch grundlegende Arbeit Renaissance und Barock von Heinrich Wölfflin verwiesen. Das ihm häufig vorgeworfene Ausgehen vom visuellen Eindruck trägt allerdings gerade zu der Nachvollziehbarkeit seiner Ausfuhrungen bei.

8. Stilwandel S.8.

9. Zu nennen ist zum Beispiel die neue Rolle, die den Mensurzeichen bei den "madrigali a note nere" (s.u. im Abschnitt I.1.1.1.) zukommt oder die frïhen Echo-Sätze, in denen eine gezielte Dynamik "entdeckt" wird. 
des 17.Jahrhunderts wird von den neuen Möglichkeiten der Notenschrift grofer Gebrauch gemacht - wahrscheinlich sind Auffürungszeichen in dieser Zeit sogar häufiger verwendet worden als im späteren 17.Jahrhundert, denn vieles, was un 1620 noch ungewöhnlich war - gegen die vorhandene Tradition verstieß - war in der zweiten Jahrhunderthälfte bereits seinerseits wieder zu Tradition geworden.

Diese Arbeit möchte die Entwicklungen in der Notenschrift auf dem Hintergrund der jeweiligen auffihrungspraktischen Tradition untersuchen. Sie möchte dabei sowoh1 Herausgebern als auch Musikern ein Nachschlagewerk für Edition und Aufführungspraxis bieten - aus diesem Grund wurde das Inhaltverzeichnis so ausfuhrlich gestaltet - als auch zur Vervollständigung des Bildes dieses für die Musikgeschichte so wichtigen Stilwandels beitragen.

\section{VORBEMERKUNGEN}

1. VORGEHENSWEISE - MATERIALAUSWAHL

Ausgewertet wurden für diese Arbeit neben den theoretischen Schriften der Zeit vor allem Musikeinzeldrucke italienischer Verleger. Ohr 3 Zweifel wurde die Entwicklung damals von Italien bestimmt - nicht selten wird etwa in deutschen Quellen dieser Zeit eine Neuerung mit dem bloßen Verweis auf "itzige italiänische Manier" eingefuhrt. Die Drucke bilden die wichtigsten Quellen fur diese Zeit. Im Gegensatz zur Zeit etwa ab der Mitte des 17.Jahrhunderts sind Handschriften aus dem späten 16. und frihen 17.Jahrhundert nur in vergleichsweise geringer Zahl erhalten, während man von einem "Boom" des italienischen Notendruckes sprechen kann 10.

Die Musikeinzeldrucke sind aber nicht nur die häufigsten Quellen dieser Zeit. Für diese Untersuchungen eignen sie sich auch, weil sie die einzigen Quellen sind, die sich sicher datieren lassen. Handschriften sind nur selten datiert. Dariberhinaus kann man oft nicht mit Sicherheit sagen, ob alles gleichzeitig eingetragen wurde, oder ob vielleicht gerade Aufführungsbezeichnungen später hinzugekommen sind. Gegenüber den Sammeldrucken haben die Einzeldrucke nicht nur den Vorteil der besseren Datierung (die verschiedenen Werke innerhalb eines Sammeldruckes können weit auseinanderliegen), sondern auch der größeren Nähe zum Komponisten.

Um die Entwicklung der Notation einigermaßen sicher dokumentieren zu können, mußte ein Zeitraum gefunden werden, innerhalb dessen sich nicht nur die wesentlichen Wandlungen vollzogen, sondern in dem auch die Zeit vor dem eigentlichen Umbruch deutlich dokumentiert ist, um zu sehen, auf welchem

10. Aus den Jahren 1571 - 1630 sind (ohne Neuauflagen) etwa 3000 Musikeinzeldrucke erhalten, hinzu kommen zahlreiche Verluste (Bianconi, Sussidi bibliografici, hat alle nachweisbaren (d.h. auch die heute verlorenen) Musikdrucke sizilianischer Komponisten des 16. und 17.Jahrhunderts zusammengestelit. Aus dieser Zusammenstellung ergibt sich, daß nur noch etwa $2 / 3$ dieser Drucke in mindestens einem Stimmbuch erhalten sind!). 
Hintergrund die Veränderungen stattfanden. Auch nach hinten muBte eine gewisse Zeit nach den eigentlichen Jahren des Wandels in diese Untersuchung mit einbezogen werden, denn erst allmählich entstand aus dem Experimentieren mit Neuem wieder eine annähernd einheitliche Notenschrift.

Eine Abgrenzung nach hinten wird durch äußere Unstände erleichtert. Nach dem schon in den 1620er Jahren eine Wirtschaftkrise ${ }^{11}$ die Drucktätikeit der italienischen Musikdrucker dämpfte, kam der italienische Notendruck - und sicher zu einem großen Teil auch das Musikleben - in den Jahren 1630/1631 durch eine große Pestwelle nahezu vollständig zum erliegen ${ }^{12}$. Nach 1631 erholten sich Musikdruck und Musikleben nur allmählich - die große Lust zum Probieren ist danach nicht mehr zu spüren - sowohl in der Notation als auch in der Komposition. Nicht umsonst gilt das Jahr 1630 als wichtiger Einschnitt innerhalb des italienischen Barock ${ }^{3}$.

Eine Abgrenzung nach vorne war weitaus schwieriger zu finden. Um die Entwicklung voll erfassen zu können, mußten die ältesten mit einbezogenen Quellen den Stand vor Beginn der hier zu untersuchenden Wandlungen repräsentieren. Nachdem zunächst das Jahr 1560 als Grenze angestrebt worden war, konnte diese auf 1570 (bzw. 1571, um einen statistisch gut auswertbaren Zeitraum von genau 60 Jahren zu bekommen) angehoben werden. Es stellte sich nämlich heraus, daß vor den 1580er Jahren kaum entscheidende Entwicklungen in Richtung eines neuen Notationsverständnisses spürbar werden - wenngleich Notation freilich immer irgendwie in einem Veränderungsprozeb steht.

Die große Zahl der Quellen legte von Anfang an fest, daß nur ein Teil der Musikeinzeldrucke dieser Zeit in diese Arbeit mit einbezogen werden konnte. Um aber das Ergebnis nicht schon von vornherein zu beinflussen, mußte ein Zufallssystem gefunden werden, nach dem die zu bearbeitenden Quellen ausgewählt wurden. Diese Zufälligkeit wurde durch eine Gesamterfassung der italienischen Einzeldrucke verschiedener Bibliotheken - z.T. für den vollständigen Zeitraum 1571 - $1630^{14}$, z.T. aber auch nur für den Kernzeitraum 1586 -

11. Vgl. hierzu vor allem Bianconi, $17^{\text {th }}$ Century, S.28.

12. Vgl. z.B. Denis Arnold, Art. Venedig in MGG, Bd.13, Sp.1380.

13. So z.B. bei Friedrich Blume, Art. Barock in MGG, Bd.1, Sp.1323. Einen Einschnitt in dieser Zeit hat bezogen auf die Architektur auch Wölfflin, Renaissance, S.13, festgestel1t (die Kunstgeschichte tendiert mittlerweile dazu, den Barock iberhaupt erst in dieser Zeit beginnen zu lassen - vgl. z.B. Blunt, Kunst und Kultur, S.9 ff oder Braunfels, Kunstgeschichte, S.408 ff. Für die fruhe Barockperiode Wölfflins wird heute der Begriff des Manierismus verwendet, mit dem sich die Musikforschung bisher jedoch nicht hat anfreunden können - vgl. die Diskussion in Band 3 der Studi musicali (1974)).

14. Augsburg, Staats- und Stadtbibliothek; Frankfurt a.M., Stadt- und Universitätsbibliothek; Kasse1, Deutsches Musikgeschichtliches Archiv; Kassel, Landesbibliothek und Murhardsche Bibliothek; Münster, Diözesan-Bibliothek: Santini-Sammlung; Wolfenbutte1, Herzog August Bibliothek. 
$1615^{15}$ gewährleistet. Freilich durfte dieses Prinzip nicht iberbewertet werden. Eine Anzahl von zusätzlichen Quellen, die dem Titel nach wichtig erschienen oder bereits fuir ihre notationstechnischen Besonderheiten bekannt waren, wurden zusätzlich mit einbezogen. Ausgewertet wurden freilich auch die im Faksimile greifbaren Musikeinzeldrucke aus dieser Zeit. Eine Verfälschung des statistischen Bildes ist hierdurch nicht zu erwarten: Zum einen ist die Zahl der bewußt hinzugezogenen Quellen verhältnismäßig gering und zum anderen sind fast alle Quellen nur in einem bestimmten Punkt interessant und in Bezug auf viele andere Fragestellungen eher durchschnittlich. Auf diese Weise wurde insgesamt mehr als ein Drittel der erhaltenen italienischen Produktion an Musikeinzeldrucken aus dem zu behandelnden Zeitraum bearbeitet (zusammen knapp 1000 verschiedene Drucke von 400 Komponisten ${ }^{16}$ ).

Das sich aus der Auswertung dieser Quellen ergebende Bild wurde durch die Miteinbeziehung von Neuauflagen ${ }^{7}$, Sammeldrucken, Drucken außerhalb der gesetzten zeitlichen Grenzen sowie Handschriften ergänzt. Diese Quellen werden immer dann Beachtung finden, wenn sie sich von den Musikeinzeldrucken in notationstechnischer Hinsicht unterscheiden; stimmen sie uberein, bleiben sie weitgehend unberỉcksichtigt.

Die Musiktheorie wurde in größerem Umfang in diese Arbeit miteinbezogen. Es galt jedoch der Grundsatz, daß die Musiktheorie dazu dienen sollte, daß in den praktischen Quellen Vorgefundene zu erklären. Probleme und Fragestellungen, die nur in der Musiktheorie behandelt werden, aber für die praktischen Quellen keine Bedeutung haben, wie etwa die oft sehr ausführlichen Abhandlungen uber Proportions- und Mensurzeichen ${ }^{18}$, bleiben auch in dieser Arbeit unberücksichtigt. Die Zahl der wirklich auf die veränderte Notation eingehenden Theoretikeräußerungen ist recht gering. In der Notationskunde herrscht seit dem späten 16. Jahrhundert etwa dasselbe Dilemma wie in der Kompositionslehre. Im 16.Jahrhundert ist ein gut lehrbarer Regelapparat zusammengetragen worden, der - mehr oder weniger - die Kompositionslehre bzw. Notationskunde dieser Zeit beinhaltete - freilich auch hier nur einen Ausschnitt aus einer tatsächlich viel bunteren Wirklichkeit. In Bezug auf die Kompositionslehre hat man sich angewöhnt, dies als den "Palestrinastil" zu bezeichnen. Die Entwicklungen nach 1600 aber entzogen sich einer Zusammenfassung zu Regeln. Dies hat dazu gefuhrt, daß der "Palestrinastil" iber Jahrhunderte Grundlage der Kompositionsausbildung war, ohne zugleich Spiegel der jeweils zeitgenössischen Komposition zu sein ${ }^{19}$.

15. London, The British Library; München, Bayerische Staatsbibliothek; Oxford, Christ Church Library; Wien, Osterreichsche Nationalbibliothek.

16. Alle für diese Arbeit ausgewerteten Drucke sind im Band II dieser Arbeit aufgelistet.

17. Zu der Bewertung der Neuauflagen siehe unten.

18. Ein Beispiel: S. Picerli gibt in Specchio primo di musica (1630) eine Tabelle uber die Bedeutung von insgesamt 71 Mensurzeichen ( $\mathrm{S} .28 \mathrm{ff}$ ). Immerhin 7 davon findet man auch in praktischen Quellen - häufig nur 4.

19. Zur Beziehung zwischen Kompositionspraxis und Musiktheorie um $1600 \mathrm{vgl}$. 
Die Notationskunde der Mensuralnotation hat freilich nicht eine ganz so lange Tradition aufzuweisen, dennoch finden sich auch durchaus im späteren 17. Jahrhundert noch Lehrbicher, die Dinge beschreiben, die seit Beginn des Jahrhunderts keine musikpraktische Bedeutung mehr hatten ${ }^{20}$. Darüber hinaus läst sich feststellen, daß große Teile insbesondere der italienischen Musiktheorie auch kaum Interesse an der Notenschrift zeigen. Wichtige Quellen hingegen sind die von der Musikwissenschaft oft nicht recht gewirdigten Fibeln für den Schulgebrauch, wie sie aus Deutschland in großer Zahl vorliegen. Ihre Autoren beschreiben oft "unverbildet" den tatsächlichen Gebrauch verschiedener Zeichen. So verbirgt sich auch hinter den von Roland Eberlein ${ }^{21}$ beschriebenen Unterschieden in der Bedeutung der Proportionszeichen bei deutschen und italienischen Theoretikern nicht etwa eine unterschiedliche Praxis in Deutschland und Italien, sondern nur eine größere Praxisnähe der deutschen Musiktheorie. Auch solche Werke wurden daher für diese Arbeit ausgewertet.

Sehr viel aussagekräftiger als Traktate sind Vorworte. Sie entstanden meist gerade aufgrund der Anderungen in der Notation und weisen den Benutzer in die neuen Bedeutungen ein; und die können zur gleichen zeit bei verschiedenen Komponisten recht unterschiedlich sein. Die Jahre um 1600 sind auch in Bezug auf die Notation die große Zeit des Experimentierens.

\section{GRUNDSÄTZLICHE UBERLEGUNGEN ZUR ARBEIT AN MUSIKDRUCKEN 2.1. DER EINFLUB DER DRUCKER AUF DAS NOTENBILD}

Eine weitgehend an Musikdrucken orientierte Arbeit mü sich die Frage ste1len, inwieweit das Notenbild des Druckes wirklich auf die Intention des Komponisten zurïkgeht, oder ob hier ein großer Einfluß des Druckers oder auch der Drucktechnik anzunehmen ist.

Immer wieder, insbesondere im Zusammenhang mit der Einführung des Genera 1basses, ist ein großer Einfluß der Drucker bzw. Verleger auf das Notenbild angenommen worden 22 . Als Quelle hierfü diente in erster Linie eine kurze Bemerkung Giacomo Vincentis unter dem Inhaltsverzeichnis zur Spartitura der achtstimmigen Motetten von Giovanni Croce (1594)23. Dort weist Vincenti auf seine zahlreichen, den Organisten dienenden Publikationen hin. Dieses Eigenlob Vincentis scheint seine Bestätigung darin zu finden, daß tatsăchlich in den Jahren bis 1600 nur bei wenigen Verlegern Continuo-Stimmen und Parti-

Groth, Musiktheorie.

20. Vg1. hierzu Wolf, Handbuch, Bd.I, S.423 ff. So beschreibt nach Wolf noch A. Kircher (1650) den "tactus alla maxima" und iber 20 verschiedene Proportionen. Ahnliches findet sich auch noch 1673 bei G. M. Bononcini (Musico prattico - vgl. Wolf, S.425 f).

21. Proportionsangaben.

22. So zunächt von Kinkeldey, Orge1, S.196. Der Argumentation Kinkeldeys ist bis hin zu Horsley, Scores, S.467, unwidersprochen gefolgt worden.

23. Abgedruckt bei Kinkeldey, S.196 u.a. 
turen erschienen - neben den Mailänder Verlegern Pietro Tini (bzw. Tini \& Francesco Besozzi) und Agostino Tradate auch Giacomo Vincenti und, allerdings erst ab 1600, auch Riccardo Amadino. Diese wenigen Verleger druckten jedoch zusammen rund $50 \%$ der gesamten Musikproduktion dieser Jahre ${ }^{24}$; nur ein großer Verleger - und zugleich der größte zu dieser Zeit -, nämlich Angelo Gardano brachte noch keine Partituren oder Generalba日stimmen heraus. Bei Gardano ist in den Jahren um 1600 wenig richtungsweisende Musik erschienen. Der Anteil Gardanos an der italienischen Musikdruckproduktion sank in den Jahren um 1600 von tiber 40\% (1581-1590) auf gerade 20\% (1601-1610). Seine marktführende Position verlor er bereits kurz nach 1600 an Riccardo Amadino. Das Fehlen von Generalbaßstimmen bei Gardano ist tatsächlich verwunderlich, nicht aber, daß zwei Großverleger wie Amadino und Vincenti in ihrem Programm neben vielem anderen auch Partituren und Generalbaßstimmen haben. Interessant ist jedoch die Vorliebe der Mailänder Drucker für Partituren und Generalbaßstimmen. Sieben von sechzehn Generalbaßstimmen25 und vier von füf Begleitpartituren der Jahre 1591-1600 sind in Mailand erschienen und zwar bei Verlegern, deren Anteil an der gesamten italienischen Musikdruckproduktion dieser Zeit zusammen bei knapp 5\% lag.

Da aber der bei weitem größte Teil der Musikdrucke bei wenigen, im großen Stil arbeitenden venezianischen Verlegern erschien ${ }^{26}$, wird man einen großen Einfluß der Drucker auf den Inhalt der Drucke kaum feststellen können. Eher das Gegenteil ist zu beobachten: Wechselt ein Komponist den Verleger, so gehen die Eigenheiten seiner Drucke mit ihm zum neuen Verleger uber; und auch hierfür liefern die frühen Partituren und Generalbaßstimmen Belege:

24. Die Prozentangaben basieren auf einer statistischen Auswertung aller in RISM verzeichneten Musikeinzeldrucken der entsprechenden Jahre, deren Komponisten mit den Buchstaben $\mathrm{A}-\mathrm{F}$ beginnen.

25. Dazu sind auch die BaBpartituren zu Drucken doppelchöriger Musik gerecht. Sie stehen in ihrer Funktion einer Continuo-Stimme näher als einer Partitur (vgl. im Abschnitt II.2.2. sowie II.3. dieser Arbeit).

26. Die venezianischen Verleger hatten in den Jahren 1571-1630 zusammen einen Anteil zwischen etwa $96 \%$ und etwa $72 \%$ an der gesamten italienischen Musikdruckproduktion. Der ständige Rückgang des venezianischen Anteiles ging vor allem zugunsten der römischen Verleger (1621-1630 fast 19\% der italienischen Musikdruckproduktion). Dies ist sicherlich auch im Zusammenhang mit der steigenden Bedeutung geistlicher Musik an der Gesamtproduktion zu sehen. Lediglich im ersten Jahrzehnt des 17. Jahrhunderts kommt auch den Druckorten Mailand und Neapel eine gewisse Bedeutung zu (jeweils etwas uber 6\%). Auch innerhalb Venedigs ist die Aufteilung in diesem Blitezeitraum des Musikdruckes am größten: Vier verschiedene Verleger haben in diesem Jahrzehnt einen nennenswerten Anteil an der Produktion: Riccardo Amadino, Angelo Gardano, Giacomo Vincenti und Alessandro Raverij (ein Neffe Gardanos). Am Anfang und am Ende des behandelten Zeitraumes dominieren je zwei Verleger (zu Anfang Gardano und Scotto, zu Ende der Gardano-Erbe Magni und Vincenti), sonst sind jeweils drei Verlage führend (Gardano bzw. Magni, Amadino und Vincenti). 
Orfeo Vecchi gibt Drucke mit solchen Orgelstimmen 1597 bei Tradate, 1598 bei Tini \& Besozzi, 1601 wieder bei Tini \& Besozzi, 1602 und 1603 wiederum bei Tradate heraus ${ }^{27}$. Antonio Mortaro (Tini \& Besozzi und Amadino), Ottavio Vernizzi (Vincenti und Amadino) u.a. brachten ebenfalls ihre fruhen Genera1baßstimmen bei verschiedenen Verlegern heraus. Auch Beispiele aus anderen Bereichen lassen sich anfüren. So erschienen Adriano Banchieris frühe Drucke mit Tempoworten 1611 - 1613 bei Amadino in Venedig und bei Rossi in Bologna28. Besonders eindrucksvolle Beispiele hierfür finden sich im Werk Giovanni Giacomo Kapsbergers. Sein erstes und drittes Buch der Villanellen (1610 und 1619) sowie sein erstes Buch der Arie passeggiate (1612) erschienen als Stiche (ohne Angabe des Stechers) mit dem in Stichen ublichen, an Handschriften erinnernden Notenbild. Das zweite Buch der Arie und das vierte Buch der Villanellen erschienen 1623 in Rom bei Luca Antonio Soldi im Typendruck. Bei beiden Drucken wurde versucht, das Notenbild der Stiche im Typendruck zu imitieren. Dies bedeutete vor allem, daß die kurzen Notenwerte (ab der Croma!), sofern sie nicht syllabisch textiert waren, nicht mit Fähnchen, sondern mit Balken notiert wurden. Da dies im italienischen Typendruck nicht möglich war, mubten die Balken in die fertigen Drucke von Hand eingetragen werden ${ }^{29}$.

Selbst bei Musiksammeldrucken haben sich die Verleger offenbar mit der Zusammenstellung des Inhaltes begnügt, sofern nicht auch dies von Herausgebern libernommen wurde. Eine notationsmä日ige Angleichung fand nicht statt 30 .

Auch die Unterschiede zwischen Handschriften und Drucken sind in dieser Zeit kleiner als man vermuten möchte. Sie beschränken sich im wesentlichen auf die im Typendruck nur schwer mögliche Balkung. Es sind dem Verfasser kaum Beispiele bekannt, bei denen eine der Neuerungen in der Notation dieser Zeit in den erhaltenen Handschriften frïher belegt ist als in den Drucken - auch dies liegt sicher in der insgesamt nur spärlichen handschriftlichen Uberlie-

27. Vg1. die Listen der Partituren und der frihen Generalbaßstimmen in Band II dieser Arbeit.

28. Vgl. die Liste der Drucke mit Tempoworten in Band II dieser Arbeit. Alle genannten Drucke mit Ausnahme des Terzo libro di nuovi pensieri (1613) erschienen bei Amadino.

29. Der Vollständigkeit halber soll auch angemerkt werden, daß sich diese handgemalten Balken in dem in Rom bei Giovanni Battista Robletti 1619 im Typendruck erschienen zweiten Buch der Villanellen nicht finden. Auf weitere Beispiele solcher Balkungen wird unten im Abschnitt I.1.4. hingewiesen.

30. So hat man den Eindruck, daß die Mensurzeichen in dem beruhmten Samme1druck Canzoni per sonare ... libro primo, erschienen 1608 bei Alessandro Raverij, völlig wahllos verwendet worden seien. Ein Grund für die Verwendung der verschieden Zeichen $(C$ und $\ell$ ) ist am Notenbild nicht zu erkennen. Wie Bartolomew, Raverij, S.257, nachgewiesen hat, besteht jedoch ein enger Zusammenhang zwischen der Generation des jeweiligen Komponisten und dem Mensurzeichengebrauch - vgl. hierzu weiter unten im Abschnitt 1.1.1., bes. I.1.1.3.3. 
ferung begriindet. Die große Bedeutung des Musikdruckes in dieser Zeit machte es offenbar nötig, den Typendruck sogleich den jeweiligen Gegenbenheiten anzupassen.

\subsection{MUSIKDRUCK UND MUSIKPRAXIS}

Wenn iber den Musikdruck EinfluB auf die Auffuhrungsweise genommen werden sol1, so setzt dies voraus, daß die Musikdrucke auch tatsächlich der praktischen Musikausubung dienten. Das ist in der Tat nicht ganz so eindeutig der Fall, wie man vermuten könnte. Es gibt durchaus Musikdrucke, aus denen so nicht musiziert werden kann, etwa weil in einem Chorbuchdruck nicht alle Stimmen gleichzeitig zu blättern haben. Hier mußte man, sofern man nicht mehrere Exemplare des Chorbuches hatte - und davon ist kaum auszugehen -, einzelne Stimmen von Hand herausschreiben.

Im italienischen Musikdruck ist jedoch eine große Sorgfalt in Bezug auf die praktische Verwendbarkeit der Musikdrucke spirbar. Sind mehrere Stimmen aus einem Chorbuch oder auch aus einem Stimmbuch (etwa bei einem achtstimmigen Stück in einem uberwiegend fünstimmigen, und daher nur in fünf Stimmbuichern gedruckten Madrigalbuch) zu musizieren, so sind die Blätterstellen, soweit dem Verfasser bekannt, immer an denselben Stellen. Oft werden halbe Seiten oder noch mehr freigelassen, um ein Blättern im Stück auch bei normaler Einzelstimmennotation nach Möglichkeit zu vermeiden. Bei Drucken mit Musik unterschiedlicher Besetzungen ist häufig bei Stücken, bei denen eine Stimme pausiert, in deren Stimmbuch eine leere Seite gedruckt oder aber die Seitenzahl ubersprungen worden, so daß in allen Stimmbüchern dieselben Stucke auf den gleichen Seitenzahlen stehen. Ein Stimmbuch, dem einige Seitenzahlen fehlen, muß also nicht immer unvollständig sein ${ }^{31}$.

Ein Beispiel besonderer verlegerischer Sorgfalt sind zwei Madrigalbuicher des neapolitanischen Komponisten und Lautenisten Giovan Domenico Montel1a ${ }^{32}$. Wohl um die Probenarbeit zu erleichtern, sind hier nicht nur die Seitenwechsel, sondern auch die Zeilenwechsel in allen Stimmbiichern synchron; die einzelnen Zeilen sind durchnumeriert ${ }^{33}$. Dieses Verfahren beansprucht freilich erheblich mehr Platz als das ubliche Druckverfahren, da stets die

31. Vgl. z.B. RISM C 893: Der Baß des - einzig erhaltenen - Muinsteraner Exemplares ist nicht unvollständig, wie in RISM angezeigt. Die "fehlenden" Seiten 1-7 haben nie existiert, da - in den anderen Stimmbichern - auf diesen Seiten Musik ohne Baß notiert ist. Auf Seite 8 steht dann das erste dreistimmige Stiick, und zwar in allen Stimmbiichern.

32. Ottavo libro de madrigali a 5 (1607) und Secondo libro de madrigali a 4 (1607); beide erschienen in Neapel bei Sottile.

33. Taktzahlen sind in dieser Zeit noch nicht iblich. Der einzige dem Verfasser aus dieser Zeit bekannte Druck mit Taktzahlen ist G. M. Trabacis secondo libro de ricercare (1615). Die Taktzahlen (z.T. mit "Cas." oder auch "Case1." bezeichnet) dürften hier jedoch kaum einen probentechnischen Zweck erfuillen, da es sich bei diesem Druck um eine Klavierpartitur handelt. 
kürzesten Notenwerte die Ausdehnung auch der langen Noten in den anderen Stimmen bestimmen ${ }^{34}$.

Vor diesem Hintergrund ist auch der Druck Sanctissimae Virgini Missa von Claudio Monteverdi (1610) zu sehen; der Druck mit der berihmten "Marienvesper". Sollte diese Vesper tatsächlich als aufführungspraktisches Gesamtwerk gedacht worden sein, so wäre dies ein ganz außergewöhnliches Beispiel von "Schlamperei", wie man es in dem zu dieser Zeit marktfürenden Verlag, Riccardo Amadino, eigentlich nicht erwarten düfte. Als Gesamtwerk nämlich ist die "Marienvesper" nach diesen Stimmbüchern nur auffuhrbar, wenn man entweder im großen Umfang Stimmen herausschreibt oder aber während der Aufführung Stimmbìcher untereinander austauscht35. Während nämlich innerhalb der einzelnen geschlossenen Teile dieses Druckes auf Auffihrbarkeit groBer Wert gelegt wurde ${ }^{36}$, stehen selbst bei direkt aufeinanderfolgenden Sätzen die verschiedenen Instrumentalstimmen in ganz unterschiedlichen Stimmbüchern ${ }^{37}$. Sicher kann man davon ausgehen, daß in dieser Zeit auch in großem Umfang abgeschrieben wurde, nur hat es eher den Anschein, daß die Drucke auch gleich das Muster für dieses Abschreiben bieten sollten, und nicht erst der Einrichtung bedurften. Ganz ohne Zweifel ist die "Marienvesper" kompositorisch dennoch ein geschlossener Zyklus ${ }^{38}$, dessen Gesamtauffuhrung durchaus

34. Dieses Verfahren ist aber offenbar nicht in allen Madrigalbuichern Montellas angewandt worden. Für diese Arbeit wurde auch sein siebtes Madrigalbuch zu füf Stimmen (1605, ebenfalls bei Sottile) ausgewertet. Dies ist. jedoch in der herkömmlichen Art gedruckt.

35. Parrotts Behauptung, die "Marienvesper" sei nach den Originalstimmen gut auffuihrbar ist entweder unzutreffend oder' setzt die Vertauschung von Stimmbïchern während der Auffuhrung stillschweigend vorraus (Transposing, S.505).

36. Als geschlossene Teile gelten hier die Messe, die beiden Magnificat sowie die einzelnen Psalmen, Concerti und die Sonata. Die Verteilung der Musik in den Stimmbuichern ist teilweise mit viel Bedacht vorgenommen worden. So sind in der Sonata (die ja zwei Stimmen mehr hat als Stimmbücher vorhanden sind) die notenreichen Diskantstimmen einzeln in je ein Stimmbuch gedruckt - Blättern bleibt hier trotzdem ein Problem -, die getrageneren Baßund Tenorstimmen je zwei in einem Stimmbuch; der Cantus erhält wegen der vielen Pausen und der Tempowechsel einen durchgehenden Basso continuo in Partitur unterlegt.

37. Sa gibt es in der Verteilung der Instrumentalstimmen auf die Stimmbiicher zwischen Sonata und Hymnus (im Druck direkt hintereinander) immerhin noch drei mögliche ubereinstimmungen: Im Altus steht in der Sonata die Violine 2, im Ritornel1 des Hymnus die dritte Stimme (auf Violine möglich), im Sextus steht in der Sonata die Violine 1, im Hymnus-Ritornell die zweite Stimme, im Bassus jeweils eine Baßstimme. Zwischen dem Hymnus und dem sich daran anschließenden Magnificat gibt es aber keine ubereinstimmung mehr, wenn man auch die Sonata-Besetzung hinzuzieht: Im Altus steht nun der zweite Zink, im Bassus die zweite Violine und im Sextus der dritte Zink. Die anderen Stimmen sind von den Wechseln z.T. noch stärker betroffen. 
lohnend sein kann; liturgische Ergänzungen aller Art sind jedoch kaum angebracht, da eine liturgische Einheit nur bei einer als liturgisches Gesamtwerk gemeinten Vesper sinnvo11 erscheint - und das ist die "Marienvesper" sicher nicht ${ }^{39}$.

\subsection{ERstauflage - Neuauflage}

Durch die Art der Quellenauswahl (s.o.) ergab es sich, daß nicht immer die Erstauflagen 40 der jeweiligen Drucke vorlagen; von einigen Drucken gar sind die Erstauflagen heute iberhaupt nicht mehr nachzuweisen. Es stellte sich die Frage, unter welchem Jahr die Neuauflagen einzuordnen sind: Repräsentiert eine Neuauflage den notationstechnischen Standard des Jahres der Erstauflage oder aber des Jahres der vorliegenden Auflage? Alfred Einstein hat sich anhand von Claudio Merulos Ausgabe der Madrigale Verdelots sowie der zahlreichen Auflagen der Ricercari Gramatico Metallos mit diesem Problem beschäftigt ${ }^{41}$. Bei der Ausgabe der Verdelot-Madrigale durch Merulo stellt Einstein neben Verbesserungen an der Musik selbst die Auflösung von Ligaturen, die Einfügung weiterer Vorzeichen sowie insgesamt eine peinlich genaue Vorzeichensetzung fest; zu den Neuauflagen von Metallos Ricercari schreibt er gar "Man kann die Fortschritte der Notation, die ja immer auch Wandlungen in den jeweiligen musikalischen Anschauungen anzeigen, an oft und spät aufgelegten Werken wie z.B. G.Metalli's zweistimmigen Ricercari leicht beobachten, mit innigem Vergnügen an der beherzten ZeitgemäBheit der Drucker und Herausgeber" 42 .

Von den 16 Auflagen der Ricercari besitzt The British Libray in London sieben aus den Jahren 1605 (friheste erhaltene Auflage) bis 1674 (vorletzte Auflage), so daß man dort sehr gute Vergleichsmöglichkeiten hat ${ }^{3}{ }^{\text {. So gro }}$

38. Vg1. Leopold, Monteverdi, S.188 ff, bes. 202.

39. Es erubrigt sich daher auch, auf die Fulle der Literatur zur liturgischen Einheit oder Nicht-Einheit der "Marienvesper" einzugehen.

40. Es sei an dieser Stelle darauf hingewiesen, daß der Begriff "Auflage" in dieser Zeit eine andere Bedeutung hatte als heute. Verschiedene Exemplare derselben Auflage sind keineswegs zwingend identisch. Wie unten im Abschnitt I.1.4. noch gezeigt werden wird, unterlagen die fertigen Drucke vor der Auslieferung einer Durchsicht, bei der z.B. nicht druckbare Zeichen von Hand ergänzt wurden. Dabei wurden auch Fehler verbessert. Nun kommt es vor, daB in einem Exemplar einer Auflage ein Fehler von Hand verbessert ist, in einem anderen Exemplar derselben Auflage aber bereits richtig gedruckt wurde (so an einigen Stellen beobachtet an drei Exemplaren der Partiturausgabe von Frescobaldis Primo libro delle canzoni (1628)). Daraus ergibt sich, daß eine Auflage nicht in allen Exemplaren hintereinander gedruckt wurden, sondern je nach Bedarf eine kleinere Anzahl von Exemplaren hergestellt wurde.

41. Merulo.

42. S. 221.

43. Vgl. die Auflistung der Auflagen in der Liste der ausgewerteten Quellen in Bd.II dieser Arbeit. Die Auflage von 1614 wurde nicht in London, sondern 
wie Einsteins überschwänglicher Text vermuten lä日t, sind die Unterschiede jedoch nicht. Die venetianischen Ausgaben (1605 - 1665) gleichen einander fast genau, lediglich eine Kanon-Anweisung ist 1665 etwas ausfuhrlicher formuliert, und ein Satz am Schluß ist hinzugekommen. Die römischen Verleger haben etwas stärker eingegriffen. Taktstriche, die noch in der Auflage von 1643 an manchen Stellen von Hand eingetragen sind, erscheinen in den römischen Auflagen von 1654, 1665 und 1674 vereinzelt an unibersichtlichen Ste1len gedruckt. Auch Ergänzungen finden sich in den römischen Auflagen häufiger. 1654 lassen gerade die Ergänzungen den Anachronismus der Metallo-Ricercare erkennen: Sie sind deutlich virtuoser. In der römischen Auflage von 1665 kommt im Anhang ein neuer Anachronismus hinzu: Ein pseudo-komplizierter Proportionen-Kanon von Francesco Foggia44. Die römische Auflage von 1674 entspricht etwa der von 1654; die Taktstriche sind ein wenig häufiger.

Die Unterschiede sind also, bedenkt man den Zeitraum, doch sehr gering. Bei den meisten Neuauflagen liegen zwischen der Erstausgabe und der Neuauflage nur wenige Jahre, oft erschienen sie bei demselben Verleger. Für diese Arbeit wurden von 25 Drucken zwei und von drei Drucken mehr als zwei Auflagen miteinander verglichen ${ }^{45}$. In Bezug auf die Notation ergaben sich nur in den seltensten Fällen Abweichungen. Die von Einstein zitierte Neuauflage der Verdelot-Madrigale darf als Sonderfall gelten: Der Komponist war zur Zeit ihrer Erstellung bereits mindestens 14 Jahre tot, der Drucker selbst war Komponist und es war die erste, also sicher Aufsehen erregen wollende Ausgabe eines neuen Verlages46. All dies ist im Normalfall nicht gegeben. Neuauflagen unterscheiden sich von den Erstausgaben oft nur im Titelblatt, von gleichzeitigen Erstausgaben desselben Komponisten hingegen deutlich. Bei statistischen Fragestellungen wurden Neuauflagen aus diesen Grínden unter dem Jahr der Ersterscheinung geführt, bei anderen Darstellungen werden sie nur mit Vorbehalt miteinbezogen. Auf Unterschiede zwischen einzelnen Auflagen wird ggf. verwiesen. Insgesamt haben dem Verfasser nur etwa 10\% der ausgewerteten Musikeinzeldrucke nur in einer Neuauflage vorgelegen; von der uberwiegenden Zah1 der Quellen konnte also die Erstauflage bearbeitet werden.

\section{ZUR ZITIERWEISE}

Werden im folgenden musikpraktische Quellen als Nachweise angegeben, so wird in aller Regel nur auf den Druck bzw. auf die Handschrift als Ganzes verwiesen. Dies liegt zum einen darin begründet, daß notationstechnische Besonderheiten in der Regel nicht auf eine Stelle innerhalb der Quellen beschränkt sind, sondern die Quelle insgesamt betreffen oder zumindest an mehreren

im Musikgeschichlichen Archiv in Kassel eingesehen.

44. Foggia lebte von 1604 bis 1688 und gilt als der letzte Vertreter der römischen Schule; vgl. Howard E. Smither, Art. Foggia, Francesco, NG, Bd.6, S. $686 \mathrm{f}$.

45. Vgl. die Quellenliste in Bd.II.

46. Vg1. Einstein, Merulo, S.220. 
Stellen auftreten. Zum anderen wäre eine genauere Zitierweise in vielen Fä1len entweder gar nicht oder nur sehr umständlich durchfuhrbar: Nicht immer decken sich die Seitenzählungen in den einzelnen Stimmbüchern, Taktzahlen sind den Quellen fremd und auch nicht ohne weiteres zu ergänzen, und auch die Verteilung des Notentextes auf die Systeme ist von Stimmbuch zu Stimmbuch unterschiedlich. In Fällen, in denen das beschriebene Phänomen tatsächlich nur auf ein Stuick innerhalb der Quelle beschränkt ist, wird der Tite1 dieses Stiickes genannt, um ein Auffinden der entprechenden Stelle zu erleichtern.

Die Titel der Quellen werden in den Anmerkungen verkürzt zitiert. Die Nennung der Stimmenzahl - auf den Drucktiteln dieser Zeit ublich - wird in den Anmerkungen nur angegeben, wenn dies an der gegebenen Stelle sachlich relevant oder aber zur Identifizierung des genannten Druckes nötig ist. Sie erfolgt in verkürzter Form (z.B. "à 5 " statt "à cinque voci"). Lateinische Titel zeigen häufig grammatische Fehler. Diese wurden selbstverständich nicht verbessert, sondern in der vorgefundenen Gestalt zitiert. 
I. TEIL: TAKT, TEMPO, MENSUR

1. DIE GERADEN TAKTE

Der Gebrauch der Taktzeichen gehört zu den Teilen der Notation, in denen die tiefgreifenden Wandlungen der Jahre um 1600 besonders gut zu beobachten sind. Es wurden althergebrachte Zeichen in neuer Art und Weise verwendet und die bisherigen Möglichkeiten durch neue Zeichen und verbale Anweisungen ergänzt. Schon ein Blick auf den Gebrauch der Zeichen für den geraden Takt, also das "tempus imperfectum diminutum" und das "tempus imperfectum non diminutum", in der italienischen Nomenklatur der Zeit "semicircolo tagliato" (q) und "semicircolo semplice" (C), in den Jahren zwischen 1571 und 1630 zeigt, wie sehr sich das Bild in dieser Zeit veränderte: Während in dem ersten Jahrzehnt dieses Zeitabschnittes (1571-1580) nur in etwa 5\% der Musikdrucke alleinig der semicircolo semplice Verwendung fand, war dies im letzten Jahrzehnt (1621-1630) bereits in $80 \%$ der Musikeinzeldrucke der Fall. Diese Entwicklung vollzog sich jedoch nicht gleichmäßig durch alle Musikgattungen hindurch. Es kam vielmehr im letzten Drittel des 16. Jahrhunderts zu gattungseigenen Notationsweisen in den beiden Hauptgattungen Madrigal und Motette. In einer zweiten Phase, beginnend um 1600, ist eine neuerliche Angleichung und Vermischung dieser Gattungen zu beobachten.

\subsection{Die GatTUngSMENSUREN DES SPÄten 16. JAHRHUNDERTS}

Im frühen 16. Jahrhundert war der semicircolo tagliato zur Standardmensur geworden. In Madrigalen und Motetten werden etwa dieselben Notenwerte eingesetat und auch der Standardnotenwert der Textdeklamation ist der gleiche. Diese Einheitlichkeit wurde von den ab 15401' erscheinenden "madrigali a note nere" 2 verlassen. Sie unterscheiden sich von den herkömmlichen Madrigalen (und damit auch von den Motetten) durch die häufig auftretenden kurzen (schwarzen) Notenwerte und in der Regel durch den semicircolo semplice als Mensurzeichen ${ }^{3}$. Um 1570 sind die "note nere" und bald auch der semicircolo semplice eine Selbstverständlichkeit im Madrigal4. In den Motetten sind in diesem Zeitraum keine vergleichbaren Veränderungen zu beobachten. Es bestanden zwei unterschiedliche Notationsarten für diese beiden Gattungen, die ihren Ursprung in unterschiedlichen Bewegungstypen haben, nebeneinander (mit "Bewegung" ist der von den verwendeten Notenwerten, dem taktgebenden Notenwert und dem Tempo des Taktschlages abhängige Tempoeindruck gemeint) ${ }^{5}$.

1. Schmalzried, Schütz, S.63, sowie Hans Enge1, Art. Madrigal in MGG, Bd.8, Sp. $1426 \mathrm{f}$.

2. Vgl. hierzu Kroyer, Chromatik, S. 51 ff und, mit Vorbehalten, Bank, Tactus, S. 237-241.

3. Auf die von Kroyer aufgezeigte Austauschbarkeit des Titels "a note nere" mit "a misura breve" sowie auf die Bedeutung des letzteren Begriffes wird weiter unten eingegangen werden.

4. Vgl. dazu weiter unten. Die Entwicklung von den ersten Madrigali "à note nere" bis zum Jahr 1570 kann nicht Gegenstand dieser Arbeit sein. Untersuchungen hieruber fehlen bisher. 
Parallel zu den Haupttypen Madrigal und Motette lassen sich gattungseigene Notationsweisen auch in den anderen Gattungen erkennen. Aufgrund der sehr viel geringeren Materialdichte ${ }^{6}$ in diesen Gattungen sind die Unterschiede allerdings schlechter dokumentiert. Das Phänomen der gattungseigenen Notation so11 nun zunächst anhand der geraden Takte in den Hauptgattungen Madrigal und Motette vorgestellt werden. Es darf dabei nicht aus den Augen verloren werden, daß diese gattungseigene Notation nur eine Außerung einer verschiedenen Kompositionsweise und auch Aufführungspraxis in den einzelnen Gattungen ist?. Sie ist äußeres Kennzeichen des für die Musik des späteren 16. Jahrhunderts bestimmenden Stilgegensatzes Madrigal/Motette ${ }^{8}$.

\subsection{1. "MAdrigalische" und "MOtettische" Mensur}

Mit den "madrigali a note nere" trat der semicircolo semplice als Hauptmensurzeichen für die Madrigale auf, während die Motetten weiter unter dem semicircolo tagliato notiert wurden. Trotz der Bindung der beiden Mensurzeichen (semicircolo semplice und tagliato) an den jeweiligen Gattungstyp sind sie nicht das eigentliche Kennzeichen der madrigalischen bzw. motettischen Bewegung. Der jeweilige Typ ist auch an den verwendeten Notenwerten zu erkennen ${ }^{9}$, und zwar an dem jeweils kürzesten benutzten Notenwert, am Wert der Textdeklamation und an den Notenwerten der Kadenzforme110.

5. Auf gattungseigene Bewegungstypen wurde erstmals in Machatius, Tempi (1956, gedruckt 1977) hingewiesen. Machatius unterschied zwischen einem madrigalischen, einem motettischen sowie einem Canzonettentypus (S.90). Wieder aufgegriffen wurde die Idee der unterschiedlichen Bewegungstypen im Zusammenhang mit der "Tactlehre" des Michael Praetorius (Hiekel, Motettentypus; Brainard, Diminution; Dahlhaus, Taktlehre). Seitdem ist immer wieder - insbesondere unter Berufung auf Praetorius - darauf verwiesen worden. Im Mittelpunkt all dieser uberlegungen stand die Frage nach dem Tempounterschied zwischen den beiden Gattungen (Machatius) bzw. zwischen den beiden Taktvorzeichnungen (Hiekel, Dahlhaus, Brainard).

6. In den letzten 30 Jahren des 16. Jahrhunderts entfallen allein iber $72 \%$ aller Drucke auf die beiden Hauptgattungen Madrigal und Motette; in den ersten 30 Jahren des 17. Jahrhunderts gar fast $80 \%$. (Im einzelnen ergeben sich folgende Prozentverteilungen: 1571-1630: Madrigale 37,9\%, Motetten 34,6\%, Canzonetten (u.ä.) 13,4\%, Messen 11,3\% und Instrumentalmusik 2,8\%; 1601-1630: Madrigale 25,8\%, Motetten 52,9\% (!), Canzonetten (u.ä) 4,8\%, Messen 9,6\% und Instrumentalmusik 7,0\% (statistische Werte, gewonnen durch Auswertung der Buchstaben A-F nach RISM. Drucke mit mehreren Gattungen wurden nur einmal bericksichtigt (Hauptgattung)).

7. Vg1. hierzu u.a. Kolneder, Dynamik, S.343 ff.

8. Vgl. Dahlhaus, Proportionenlehre, S.352 ff.

9. Das Mensurzeichen konnte daher eigentlich auch entfallen. Dies erklärt nicht nur das oft innerhalb der Drucke bei einzelnen Stücken ein Mensurzeichen fehlt - es mangelte hier wohl an Sorgfalt - sondern auch das gänzliche Fehlen des Hauptmensurzeichen in einze1nen Quellen ist nur so zu erklären. Während das Hauptmensurzeichen in Individualdrucken soweit dem Ver- 
Eine theoretische Behandlung erfuhren diese Gattungsunterschiede in Pietro Pontios Ragionamenti di musica (1588), jedoch ohne Hinweise auf Taktschlag oder Mensurzeichen. In seinem recht kurzen Kapitel iber die Mensurzeichen ${ }^{11}$ werden der semicircolo tagliato und der semicircolo semplice nur als zwei (unter mehreren) Mensurzeichen genannt, die zueinander in der "dupla proportione"12 stehen.

Am Ende des Ragionamento quarto steht in kurzen Abschnitten, worauf beim Komponieren der einzelnen Gattungen zu achten ist ${ }^{13}$. Darunter finden sich auch Anweisungen, welche Notenwerte zu verwenden, sowie welche Werte zu synkopieren sind. Dort heibt es, die Motetten seien langsam und still ("grave, e quieto"), die Stimmen sollen sich "con gravita" bewegen. Wenn einige Komponisten dennoch schnelle Bewegungen schrieben, wie in Madrigalen oder Canzonen, so beachteten diese nicht die Gravität der Motetten ${ }^{14}$. Den Madrigalen

fasser bekannt nie gänzlich fehlt, sind durchaus Sammeldrucke oder Handschriften ohne Hauptmensurzeichen anzutreffen, so z.B. in den Sacrae cantiones quinque vocum (1583) und den Cantici beatae Mariae Virginis (1586).

10. Vg1. dazu Braun, 17.Jahrhundert, S.131 sowie ders., Stilwande1, s.15. Die Unterschiede des madrigalischen und des motettischen Bewegungstypes können z.B. hervorragend an G. Aichingers Sacrae cantiones ... cum quibusdam aliis que vocantus madrigali (1590) studiert werden. Hier treffen die beiden Typen unmittelbar aufeinander.

11. S.123-136. Pontio beschreibt (wie auch andere) zwei verschiedene Arten der Mensurzeichen. Eine der "antichi", bei der Perfektion bzw Imperfektion des "modo minore" sowie des "tempo" durch Ziffern angezeigt werden, sowie die ubliche Art durch Initialpausen (beide wurden 1588 schon lange so gut wie nicht mehr benutzt). Als Gewährsmann dafür, "come gli moderni fogliano fare $^{\prime \prime}$ fuhrt er Lanfranco an - Lanfrancos Scintille, von Pontio als Quelle genannt (S.126), war 1588 immerhin 55 Jahre alt!

12. $\mathrm{S} .133 \mathrm{ff}$.

13. Laut Tavola des Ragionamento quarto: "Del Modo che si tiene in fare un Motetto" (S.154); "Del Modo, che si tiene in far vna Messa" (S.155); "Del Modo, che si tiene in far Salmi" (S.156); "Del modo, che si tiene in far Magnificat" (S.157); "Del Modo, che si tiene in far le Lettioni della Settimana Santa" (S.158); "Del Modo, che si tiene in far vn Recercare" (S.159); "Del Modo, che si tiene in fare un Madrigale" (S.160).

14. "Il Modo, o stile, che dir vogliamo, volendo far vn Motetto, è graue, \& quieto; doue si vede le parti mouersi cō grauità ... ancora c'hoggi di in alcuni cōpositori frà suoi Motetti, \& cose ecclesiastiche non seruano tal ordine; ma talmēte pógono le parti insieme cö moto veloce, \& velocissimo, che paiono Madrigali, e Cazoni; \& valersi in luogo della Semibreue sincopata, della Minima sincopata, qual nō couiene alla grauità del Motetto (...). Io intedo; che nelle parti vi siano assai Breui, \& Semibreui, \& Minime col püto, quali vadano insieme, \& facciano grave la cōpositione, $p$ esser il moto tardo" (S.154). 
hingegen seien die Semiminime und die Minime in der Synkopation eigen. Die Stimmen gingen gewöhnlich "zu gleich" in einer schnellen Bewegung ${ }^{15}$.

Ein Bezug zu Taktschlag und Mensurzeichen fehlt bei Pontio. Gerade zur Zeit des Erscheinens von Pontios Ragionamento, und damit zur Zeit der vollen Blute des italienischen Madrigals16, war aber die Verwendung der beiden unterschiedlichen Taktzeichen besonders ausgeprägt. In den Jahren 1581-1600 stehen gut $80 \%$ der Madrigale unter dem semicircolo semplice als Hauptmensurzeichen und gut $80 \%$ der Motetten unter dem semicircolo tagliato 17.

Erst kurz nach 1600 wird auch in der Musiktheorie ausdrücklich der semicircolo semplice für Madrigale und Canzonen verlangt, und erklärt, daß der semicircolo tagliato nur für "Cantilene"18, die ein "caminare, \& proceder graue" (es sei an Pontios Forderungen für die Motette erinnert) aufweisen ${ }^{19}$, zu verwenden sei.

Eine Anzahl von musikpraktischen Quellen beweist, daß Pontios Beobachtungen zu den unerschiedlichen Bewegungstypen, unabhängig vom Mensurzeichen, in der Tat zutreffen. Auch ein Teil der - freilich wenigen - Drucke, in denen das in der jeweiligen Gattung unibliche Taktzeichen verwendet wurde, kann nämlich trotzdem dem Gattungstyp voll zugerechnet werden. So weisen auch alle der untersuchten Madrigaldrucke unter dem semicircolo tagliato mit nur einer Ausnahme20, ebenso wie diejenigen, unter dem semicircolo semplice, die Semicroma als kürzesten Notenwert auf ${ }^{21}$. Die erwähnte Ausnahme wird nicht so sehr verwundern, wenn man bedenkt, daß der Komponist dieser Stuicke, Andrea Gabrieli, zu ihrem Erscheinungsjahr bereits 79 Jahre alt war und damit noch

15. "Vi so anco sapere, che il suo proprio è di fargli delle Semiminime assai, \& anco delle Minime fatte in sincopa. Sappiate ancora, che spesse volte le parti debbono andare vgualmente insieme, con moto perd veloce di Minime, ouero Semiminime" (S.160).

Natiurlich entsprechen die von Pontio genannten Notenwerte nur denen der konservativen Komponisten seiner Zeit, wie auch iberhaupt Pontios Ragionamento als konservativ gelten kann (vg1. Sachs, Pontio, S.142). Dennoch sind die Gattungsbeschreibungen Pontios noch 1613 in P. Cerones El melopeo y maestro eingeflossen (vg1. die bei Strunk, Readings, S.263-273 abgedruckten Ausschnitte).

16. Vg1. die Tabellen uiber die Anzah1 der Madrigaldrucke bei Engel, Madrigal, S.41 und Bianconi, $17^{\text {th }}$ Century, S.2.

17. Vgl. die Tabellen 1 und 2 im zweiten Band dieser Arbeit.

18. "Cantilene" meint hier sicherlich keine spezielle Gattung (vgl. zu Cantilena als Gattungsbezeichnung Arnold, Croce, S.45 und Günther, Grandi, S.52 ff).

19. So im letzten Avvertimento der insgesamt als sehr konservativ zu bezeichnenden Prattica musica S. Cerretos (1601), \$.263 f (Abdruck im zweiten Band dieser Arbeit auf S.22 f).

20. Es handelt sich um die Madrigali et ricercari (1589) von A. Gabrieli. 21. Maximal zwei Semicromen in Folge. 
in einer älteren Tradition steht. Warum die anderen Komponisten sich des semicircolo tagliato bedienten, wird sich oft nicht klären lassen. Bei einem Teil der Komponisten zeigt es sich, daß Bewegung und Mensurzeichen nicht unbedingt parallel dem in der Zeit ublichen angepaßt werden. Diese Entwicklung läßt sich sehr gut in dem ausgesprochen umfangreichen madrigalischen OEuvre Philippo de Montes beobachten. Beginnend mit seinem zweiten Madrigalbuch zu füf Stimmen (1567) benutzt er in seinen Madrigalen Semicromen2z. Während alle seine Madrigalbücher bis 1580 unter dem semicircolo tagliato notiert sind, schreibt er ab seinem vierten Buch zu vier Stimmen bzw. seinem zehnten Buch zu 5 Stimmen (beide 1581) nur noch den semicircolo semplice $23-$ an den Notenwerten ändert sich jedoch nichts. Eine vergleichbare Entwicklung läbt sich auch bei Benedetto Pallavincino und Tiburtio Massaino beobach$\operatorname{ten}^{24}$. Gründe für eine Abweichung von der Norm können auch in einem außeritalienischen Wirkungsort eines Komponisten 25 oder schlicht in einer Eigenwilligkeit zu suchen sein.

Anders als bei den Madrigaldrucken, entsprechen viele der Motettendrucke unter dem gattungsunublichen semicircolo semplice auch sonst nicht dem ublichen. So haben die Motettendrucke Gregorio Turinis26 und Asprilo Pacellis27 fast schon madrigalisches Aussehen. An Madrigale erinnern auch die unter dem semicircolo semplice notierten Sätze aus den Magnificat quatuor vocum von Theodoro Leonardo (1594). Dieser interessante Druck enthält insgesamt 16 Magnificatvertonungen - zweimal auf jedem der acht Kirchentöne; je einmal unter dem semicircolo semplice und unter dem semicircolo tagliato. Die Notenwerte unter dem semicircolo tagliato entsprechen dem in Motetten iblichen, die unter dem semicircolo semplice denen der Madri$g_{a l e}^{28}$. Einige andere Komponisten verwendeten auch bei nur tendenziell

22. Ebenfalls maximal zwei in Folge.

23. Vgl, auch Hans Engel, Art. Madrigal in MGG, Bd.8, Sp.1434.

24. Pallavincino verwendet den semicircolo tagliato nur noch in seinem ersten Madrigalbuch zu funf Stimmen (1581); Massaino benutzt ihn als Hauptmensurzeichen bis 1587 (drittes Buch der Madrigale zu fünf Stimmen).

25. Schmalzried, Schütz, S. 64, sieht am Ende dieser Entwicklung die ausschliebliche Verwendung des semicircolo tagliato. Wie aus der Tabelle 1 in Bd.II dieser Arbeit zu ersehen ist, ist in Italien aber genau das Gegenteil der Fall. Es deckt sich jedoch mit den Erfahrungen des Verfassers, daß in Deutschland im frïhen 17.Jahrhundert tatsächlich fast ausschließlich der semicircolo tagliato verwendet wurde. Vermutlich stüzt sich Schmalzried allein auf die Entwicklung dort (vgl. hierzu Wolf, Barbarino, S.51). So könnte auch die Verwendung des semicircolo tagliato als Hauptmensur in C. Ardesis Primo 1ibro de madrigali (1597 gedruckt in Venedig) mit dem Wirkungsort des Komponisten (Prag) zu erk1ären sein.

26. Cantiones (1589).

27. Motectorum et psalmorum ... liber primus (1597).

28. Von den Magnificat der ersten Hälfte des Druckes (unter semicircolo tagliato) besitzt die Murhardsche und Landesbibliothek Kassel (sie besitzt auch das einzige erhaltene Exemplar des Druckes) dariberhinaus noch eine 
madrigalischer (man könnte auch sagen: moderner) Kompositionsart ihrer Motetten bereits in den letzten Jahren des 16. Jahrhunderts den semicircolo semplice 29 .

Die Tatsache, daß Madrigale unter dem semicircalo tagliato sich kaum von denen unter dem semicircolo semplice unterscheiden, Motetten unter dem semicircolo semplice in aller Regel jedoch deutlich von denen unter dem semicircolo tagliato, ist sicherlich auf die Entwicklung des Mensurzeichengebrauches uberhaupt zuriuckzuführen. Es erscheint verständlich, daß ein althergebrachtes Mensurzeichen (der semicircolo tagliato) auch bei geändertem Bewegungsablauf "mitgeschleppt" wird, während die Verwendung eines noch unublichen Mensurzeichens wohl erst geschieht, wenn eine Notwendigkeit dafür empfunden wird.

\section{1,2, TAKTSCHLAG, TEMPO UND MENSURZEICHEN}

Ehe nun auf die anderen Gattungen eingegangen werden so11, ist es notwendig, etwas über die Temporelationen der beiden Bewegungstypen zu sagen. Schon die aufgezeigte Entwicklung läßt eine strenge "proportio dupla', wie sie die Theorie für die beiden Zeichen verlangt, unwahrscheinlich erscheinen. Ein Taktschlag "alla Breve" wurde offenbar schon im 16.Jahrhundert immer seltener. 1533 beschrieb Stefano Vanneo ${ }^{30}$ noch beide Takttypen - "alla Breve" 31 und "alla Semibreve"32, hier "Mensura maior" und "Mensura minor" - und deren Zuordnung zu den beiden Mensurzeichen, dem (semi)circolo tagliato sowie dern (semi)circolo semplice ${ }^{33}$. Ganz selbstverständlich ist eine solche Zuordnung auch noch für Zarlino ${ }^{34}$.

1585 erwähnt Giovarni Battista Rossi35 soweit bekannt zum ersten Mal den Zusammenhang zwischen den Mensurzeichen und den Gattungen, wenn auch auf recht indirektem Wege. Er berichtet, daß man nur noch in "Capelle ben ordinate" $3_{6}$ "alla Breve" singt; Rossi räumt aber auch ein, daß das Singen "alla Breve" selbst dort nicht mehr immer beachtet wuirde. Alle Messen seien

Abschrift in einem Chorbuch (Ms. Mus fol. 3), datiert auf 1598.

29. Zu nennen sind Giulio Belli, Giovanni Croce und Antonio Mortaro.

30. Recanetum de musica aurea (1533).

31. "alla Breve" meint einen zweiteiligen Taktschlag, der insgesamt eine Brevis-Note dauert, d.h. er entspricht unserem Zwei-Ganze-Takt.

32. "alla Semibreve" meint einen zweiteiligen Taktschlag, der insgesamt eine Semibrevis-Note dauert, d.h. er entspricht unserem Zwei-Halbe-Takt.

33. fol. 54.

34. Institutiones harmoniche (1558) fol.209 f.

35. Organo de cantori, gedruckt Venedig 1618. Aus dem Schlußvermerk des ersten Teiles geht jedoch hervor, daß das Werk bereits 1585 beendet wurde: "...è qui finisse questo nostr'organo alli cinque di Maggio 1585." (S.79).

36. "Capelle" ist sicher hier im Gegensatz zu "Concerto" - also nur bezogen auf geistliche Musik zu sehen (vgl. ähnliche Zitate bei Vincentino, Bovicelli, Zacconi und Cerreto weiter unten). 
aber unter dem semicircolo tagliato geschrieben und dürften auch nur "alla Breve" gesungen werden ${ }^{37}$.

Auch Lodovico Zacconi erwähnt $1596^{38}$ nicht nur in seinem Kapitel uber die Synkope die Tatsache, daß nicht mehr alle Capellen den Takt "alla Breve" gebrauchen ${ }^{39}$, sondern er gibt an anderer Stelle sogar eine Alternativmög1ichkeit: Das Singen "alla Breve" werde nicht mehr gebraucht, da man mit einem langsameren oder schnelleren Taktschlag dasselbe erreichen könne 40 . Auch im weiteren Verlauf seines Buches ist der Semibreventakt unabhängig vom Mensurzeichen ein unausgesprochener ${ }^{41}$ oder ausgesprochener Standard ${ }^{42}$.

Den Ausfihrungen Antonio Brunellis von 1606 ist zu entnehmen, daß der Breventakt so gut wie ausgestorben ist, wenngleich die Unrichtigkeit des Singens "alla Semibreve" unter dem semicircolo tagliato noch bewußt bleibt43. Bei Bernardo Bottazzi, Choro et Organo, libro primo (1614)

37. "... come sarebbe dire il semicircolo semplice valere la breue dua tatti, il traverso vno solo, cantandosi però alla Breve come si vsa nelle capelle ben ordinate, soto il qual segno habbiamo composte tutte le nostre Messe, \& da cantarsi in questa maniera \& non altrimente: se bene veramente hoggidi non è osseruato da' Musici cotal stile \& ordine" (S.11).

38. Prattica di musica.

39. "Questa maniera di syncope uà per le mani solamente a quei cantori che cantano nelle Cappelle, \& in quelle particularmente in che il tatto delle Breve si suole usare" ( fol. 40').

40. "... il che è stato causa che hora il cantar le cantilene al tatto della Breue da molti cantori non siano ben cantate, non havendone l'uso: \& molti altri che lo fanno, \& l'intendano, per l'uso grande che hanno del cantar col tatto della Semibreue, cantano al tatto della Breue come per prattica, \& come in foggia nuova. (...) \& il cantore di cantarle comunque $1 i$ torna commodo, \& li pare o piace: perche con l'attenzione, \& sumministartione del tatto largo e stretto si può hauer l'istesso, \& cauarne il medemo effetto" (fol.24' f).

41. Unausgesprochen z.B. auf fo1. 23: Auf einem Notensystem stehen alle Notenwerte Massima bis Semicroma; ein Mensurzeichen ist nicht vorgesetzt. Uber den Noten stehen die Zahlen $8,4,2,1,1 / 2,1 / 4,1 / 8,1 / 16$. Wie aus dem Text hervorgeht, beziehen die Zahlen sich auf den Werte der jeweiligen Note in Bezug zum "tatto" ("che sempre il numero superiore dimostra il tatto"). Die Bezugseinheit ist also die Semibrevis.

42. Ausgesprochen auf fol 41': Ein Beispiel unter dem semicircolo tagliato ist bezeichnet als "Essempio della Breue", ein anderes unter dem semicircolo semplice als "Essempio ordinario \& commune". Auch der Begriff "tempo ordinario" wird hier in Bezug auf den "tatto della Semibreue" gebraucht.

43. Regole utilissime per il scolari (1606), S.17: " $E$ ben vero si puó cantare come sopra mandano vna Semibreve a battuta: ma questo si fa per leuare 1a difficultà al cantare, non già che sia suo proprio". Für den deutschsprachigen Raum hat Ruhnke, Burmeister, $\mathrm{S} .77 \mathrm{ff}$, bes. S.82, bereits ein Aussterben des Breventaktes im letzten Drittel des 16. Jahrhunderts nach- 
schließlich wird der Semibreven-Takt unter beiden "semicircoli" ohne Erklärung als selbstverständlich vorausgesetzt 44 .

Adriano Banchieri erschuf in verschiedenen Traktaten für sich ein neues System der Mensuren 45. Dabei führt er für die beiden Mensuren, die bisher in der Regel als "tempo imperfetto minore" und "maggiore" bezeichnet wurden, neue Begriffe ein. In seinen Conclusioni nel suono dell'organo (1609) unterscheidet er zwischen "tempo perfetto" (semicircolo tagliato) und "tempo imperfetta" (semicircolo semplice) "6 und fürt interessante Beispiele für den Gebrauch der beiden tempi an: Das "tempo perfetto" $(\varphi=$ zwei Semibreven "alla battuta") gebraucht man in den "compositioni gravi \& facili", wie in den Messen, Vespern und Motetten von Giovanni Matteo Asola47. Diese Art zu Singen werde aber von der Mehrheit der Sänger nicht mehr gebraucht. Sie sängen ohne Unterschied alles "alla Semibreve". Das "tempo imperfetto" (C = zwei Minimen "alla battuta") wird in den Kompositionen "di note nere, \& allegre" verwendet, wie in den Messen, Vespern und Motetten von Giulio Belli ${ }^{48}$ und anderen ${ }^{49}$. An die Stelle eines Gattungsunterschiedes ist hier bereits ein Generationsunterschied getreten.

In der Cartella musicale von 1614 greift Banchieri wieder auf die minore/maggiore-Terminologie zurück, benennt jedoch jetzt alle ungeraden Takte mit "tempo perfetto"50. Die Unterschiede werden auch hier zunächst in ihrer klassischen Definition (semicircolo tagliato = "alla Breve", semicircolo semplice = "alla Semibreve") erläutert, dann aber unter Hinweis auf den Mißbrauch "Einiger" wieder in Frage gestellt. In Wahrheit nämlich würden einige Praktiker unter beiden Zeichen "alla Semibreve" singen und pausieren indem sie das "tempo perfetto maggiore" $(q)$ wegen der weißen Noten "presto" und das "tempo perfetto minore" (C) wegen der schwarzen Noten "adagio"

gewiesen.

44. Dies geht aus der Behandlung der Ligaturen (S.10) hervor.

45. Soweit dem Verfasser bekannt, hat sich aus dem, auch weiterhin Veränderungen unterworfenen (s.u.), System Banchieris keine Tradition entwickelt. Michael Praetorius folgt Banchieris Cartella (s.u.) jedoch in einigen Punkten.

46. Banchieri kennt und beschreibt zunächst auf S.34 die alte Terminologie, hält sie jedoch für uberholt und fährt fort (S.34): "Tutta via essendo le cantilene cöposte sotto tanti, \& variati segni difficili à gli Cantori, \& ancora per gli stirachiamente aspiri all'vdito, da gli Musici moderni sono sdimesi, \& ridotti sotto dui tempi simili, più facili à gli Cantori, \& più soaui al concento, \& questi ancor loro vengono nominati tempo perfetto, \& tépo imperfetto".

47. 1532-1609.

48. $1560-1621$.

49. S.34.

50. In dieser Terminologie folgt M. Praetorius, Syntagmatis musici ... tomus tertius (1619), S.45 ff, Banchieri. Vgl. dazu auch Dahlhaus, Taktsystem, S.232 sowie Tactlehre, S.163. 
schlagen ${ }^{51}$. Daß der Begriff "Einige" hier kaum wörtlich zu nehmen ist, ergibt sich aus der Tatsache, daß schon 5 Jahre vorher (Conclusioni) Banchieri diese Art des Singens als "von der Mehrheit der Sänger nicht mehr gebraucht" beschrieb (s.o.).

Auf Gattungsunterschiede in der Verwendung der Zeichen geht Banchieri in der Cartella nicht ein. Er erwähnt jedoch - ähnlich wie auch schon in den Conclusioni - den unterschiedlichen Taktzeichengebrauch einiger Komponisten in deren "Concerti"52: "Gio. Felice Anerio della Romana Schola"53 sowie Enrico Radesca di Foggia ${ }^{54}$ benutzen nach Banchieri den semicircolo semplice, Giacomo Finettis5 hingegen den semicircolo tagliato.

Besonders einige Notenbeispiele der Theoretiker zu den beiden Signen, legen - oft im Gegensatz zu den Texten - die Vermutung nahe, es bestehe kein Tempounterschied zwischen dem "tempa minor" und dem "tempo maior"; lediglich der optische Eindruck sei ein anderer ${ }^{56}$. Zu Recht ist eine solche Theorie der "poportio dupla" zwischem dem "tempo minor" und dem "tempo maior" in Bezug auf die geraden Takte in der Forschung immer wieder abgelehnt worden $^{57}$. Schon die oben gezeigte Typisierung der Gattungen - die auch bei abweichendem Mensurzeichen erhalten blieb - spricht dagegen. Ein unterschied-

51. "Vero è che al giorno d'oggi, per modo d'abuso convertito in vso, vengono amendui praticati l'istesso cantando, \& pausando sott'il valore della Semibreue, \& battendo il perfetto maggiore presto (per essere di note bianche) \& il minor perfetto adagio essendo di note negre ..." (S.29).

Ein "tempo imperfetto" erwähnt Banchieri nicht. Das alte "tempo perfetto" bezeichnet Banchieri als "tempo chiuso" - offenbar hergeleitet vom geschlossenen Kreis (S.168).

52. S. $167 / 168$.

53. $1567-1630$.

54. Zweite Hälfte 16. Jahrhunderts bis nach 1620 .

55. Lebensdaten unbekannt.

56. Es würde hier zu weit führen, alle diese Notenbeispiele aufzuführen sie finden sich in fast jedem Traktat der Zeit. Verwiesen sei aber auf die Diskussion iber solche Notenbeispiele aus M. Praetorius Syntagmatis musici ... tomus tertius (1619), insbesondere S.49, durch Hieke1, Motettentypus und Brainard, Diminution. Ein Notenbeispiel dieses Types findet sich im zweiten Band dieser Arbeit auf S.23.

57. Sachs, Rhythm, S.369, Brainard, Diminution, sowie in allen Aufsätzen von Dahlhaus zu diesem Thema. Die Kritik Werner Brauns an den sich mit. dieser Frage befassenden Aufsätzen ist grundsätzlich berechtigt. Braun, Stilwandel, S.16, weist darauf hin, daß die gattungsdifferenzierenden Mensurzeichen in erster Linie nicht als Anweisung für die Aufführungspraxis, sondern zur Unterscheidung der Stile benutzt wurden. Braun ibersieht jedoch, daß die verschiedenen Stile wiederum eine unterschiedliche Auffuhrungspraxis zur Folge hatten, und somit den Mensurzeichen auch auffuhrungspraktische Bedeutung zukommt (vgl. auch weiter unten im Abschnitt 1.1.4. dieser Arbeit). 
licher Tempoeindruck bzw. eine unterschiedliche Bewegung zwischen den verschiedenen Gattungen war bereits Vincentino gegenwärtig - zu einer Zeit also, da noch fast alle Musik unter dem semicircolo tagliato notiert wurde58. Ebenso kann auch die von Pontio verlangte "gravità" oder der "moto tardo" der Motette nicht allein vom Notenbild herruhren. Es muB davon ausgegangen werden, daß unterschiedliche Gattungen schon frih nach unterschiedlichen Tempi verlangten. Auch die Bezeichnung, mit der differenzierende Mensurzeichen eingeftihrt wurden, "a missura breve", meint solche unterschiedlichen Tempi. Dieser Zusatz findet sich auf einigen Drucken mit "madrigali a note nere" und war mit "a note nere" austauschbar59. Es ist unwahrscheinlich, daß damit "alla Breve" gemeint wurde, denn "alla Breve" war in dieser Zeit der normale Taktschlag. Die Formulierung "a missura breve" ("mit kurzem Takt") kann nur folgenden Sinn ergeben: Geschlagen wird unter dem semicircolo semplice nun die Semibrevis (im Einklang mit allen Theoretikern). Ein solcher Semibreven-Takt soll jedoch kürzer (= schneller) sein als ein ublicher (Breven-) Takt - die Verlangsamung (durch die Wahl der anderen Bezugsnote) wird durch diese Beschleunigung z.T. wieder aufgehoben es ergibt sich eine beschleunigte Bewegung 60 . Dieser leichte Tempounterschied veranlaßte auch Giovanni Battista Bovicelli, in den Vorbemerkungen zu seinen Regole passaggi di musica (1594) vor bestimmten Verzierungen beim "cantare nel concerto" (als Gegensatz zur Capella) zu warnen, da diese wegen des langsamen Tempos wie ubungen wirken wïrden ${ }^{\circ 1}$.

Infolge der gegen Ende des 16.Jahrhunderts zu beobachtenden Verkürzung der Notenwerte und der damit einhergehenden Verlangsamung des Tempo (s.u.) wurde der Taktschlag "alla Breve" in den meisten Gattungen sicherlich schon im 16. Jahrhundert aufgegeben ${ }^{2}$. Ebenso wie die kurzen Notenwerte dringt der Taktschlag "alla Semibreve" und schlieBlich auch das Mensurzeichen des Madrigals in die motettischen Gattungen ein. Die im 17.Jahrhundert aufkommende Praxis, längere Pausen zusätzlich mit Zahlen zu bezeichnen, bestätigt die - mit wenig Ausnahmen - Alleingultigkeit des Taktschlages "alla Semibreve": Diese Zahlen geben immer die Zahl der Semibreven wieder ${ }^{63}$. War tat-

58. L'antiqua musica (1555), bes. fol.88 f.

59. Kroyer, Chromatik, S.51 ff.

60. Vg1. zur Verwirrung, die dieser Terminus schon in 16.Jahrhundert ausgelöst hat Dahlhaus, Taktsystem, S.227 f. Dahlhaus gelangt zu einer ähnlichen Interpretation des Terminus (S.228).

61. "Perche cantando non da Capella, ma da Concerto, doue la battuta deue esser graue, $i$ uolere far crome, che non uadino per grado, pare che sia quasi lo studiare una lettione". (S. 14). Zur Benutzung des Terminus Capella Vgl. Rossi (s.o.) und Zacconi (s.u.).

62. Vgl. oben die Zitate von Rossi, Zacconi und Cerreto. Ein später Beleg für den Taktschlag alla Breve findet sich im Titel von F. Spongia Uspers Salmi Vespertini (1627). Er lautet vollständig: Salmi Vespertini per tutto l'anno, parte a doi chori, parte concertati all'uso moderno, \& parte alla breve, come si cantano nelle capelle de principi, a 4.5. \& otto voci, con il basso continuo ... opera quinta. 
sächlich ein Taktschlag "alla Breve" oder eine "proportio dupla" gemeint, so wurde häufig nicht der semicircolo tagliato sondern eines der anderen Zeichen hierfür verwendet ("C 2 " oder umgekehrtes "C") 64 .

\subsubsection{Die GERADEN MENSUREN In DEN ANDEREN GATTUNGEN}

Ein Großteil der verbleibenden Gattungen ist mit den beiden Hauptgattungen, Motette und Madrigal, eng verwandt. Zu nennen sind auf der einen Seite die dem Madrigal verwandten Gattungen Canzonette und Villanelle 65 und auf der anderen die der Motette satztechnisch fast gleichzusetzende Messe 66.

63. So bei L. Viadana, Cento concerti $(1602,2 / 1604)$, B. Tomasi, I1 primo libro de sacri fiori (1611), G. Finetti, Concerti (2/1612, 1. Auf1. nicht nachweisbar), A. Borsara, Odorati fiori (1615), G. Gabrieli, Sacrae symphoniae ... libro secondo (1615), T. Merula, Il primo libro delle canzoni (1615), I. Donati, Motetti concertati (1618), S. Bernardi, I1 terzo libro de madrigali (1624), A. Grandi, Salmi a 8 (1629) und A. Gualtieri, Motetti ... libro terzo (1630). Nur in einigen der Drucke ist bereits die bis heute ublich gebliebene Kennzeichnung längerer Pausen mit Zahlen und Pausenzeichen anzutreffen. Viadana benutzte lediglich Zahlen im System (die Notenlinien sind unterbrochen, in der Lïcke steht die Zah1). Finetti und Gabrieli fügen das Wort "Pause" hinzu, Tomasi schreibt hinter die Zahl "battute". Immer ist die Zahl der Semibreven gemeint, sofern es sich um einen geraden Takt handelt. Bei Dreiertakten bezieht sich die Zahl in der Regel auch auf einen Takt (also z.B. perfekte Brevis im Dreiganzetakt). Lediglich Tomasi und Merula zählen auch im Dreiganzetakt die Semibreven. Dies kann jedoch bei Merula kaum zu Verwirrungen führen, da er außer der Zahl auch Pausenzeichen drucken 1ieb. Die Zahl ist hier also lediglich eine Lesehilfe. Ganz im modernen Sinn, also mit Pausenzeichen und darüber einer Zahl, sind Pausen bereits in dem Manuskript A. Striggios Ecce beatam lucem (Ratsschulbibliothek Zwickau, datiert auf 1587) notiert. Auch hier ist die Semibrevis die Einheit.

Interessant sind in diesem Zusammenhang auch handschriftliche Ergänzungen in Musikdrucken, die altertüliche Notationsgewohnheiten "ubersetzen". Natürlich sind diese handschriftlichen Ergänzungen nicht genau zu datieren. Sofern es sich jedoch um Buchstaben oder Zahlen handelt, ist, im Gegensatz etwa zu nachträglich eingefügten Taktstrichen, wenigstens eine annähernde Datierung möglich. So stammen die Zahlen uber den ligaturen und Longen im Münsteraner Exemplar von Palestrinas Missae ... liber septimus (1594, 3/1609) sicher aus dem 17.Jahrhundert (Santini Sammlung, Dr.588). Sie bezeichnen jeweils den Wert der Ligaturen und Longen in Semibreven.

64. Vg1. David Fellows, Art Tempo and Expression Marks in NG, Bd.18, S. 680 .

65. Vg1. Hans Engel, Art. Madrigal in MGG, Bd.9, Sp.1424 ff.

66. Vg1. weiter oben zum Quarto Ragionament aus P. Pontios Raginamenti (1588). 
Auch in der Instrumentalmusik sind diese beiden Grundtypen - hier in Canzone und Ricercar - vertreten ${ }^{7}$, wenngleich die Ausprägung dieses Kontrastpaares erst im friben 17.Jahrhundert in aller Deutlichkeit hervortritt.

\section{1 .3 .1 . CANZONETTEN UND VILLANELLEN}

Die Canzonetten sowie die anderen meist dreistimmigen Gattungen 68 zeigen sich auch in Hinblick auf die Verwendung der Mensurzeichen dem Madrigal verwandt. Diese Gattungen wurden fast ausschließlich unter dem semicircolo semplice notiert - auch schon zu einer Zeit, in der sich diese Schreibweise im Madrigal erst durchzusetzen begann 69 .

Franz-Jochen Machatius sah in der Canzonette einen dritten, schnelleren Bewegungstypus ${ }^{70}$. In der Tat sind die kürzesten Notenwerte in der Canzonette seltener als im Madrigal. Es erscheint dennoch fraglich, ob hierin tatsächlich ein Indiz für ein doppeltes Tempo (!) - so Machatius gesehen werden kann ${ }^{71}$. Vielmehr hat es den Anschein, daß hier der allgemeinen Tendenz zum Einfachen in diesen Gattungen auch der Verzicht auf die kleinen Notenwerte zuzuschreiben ist.

\section{$1,1,3,2$, MESSEN}

Wie die Canzonetten mit den Madrigalen, so sind die Messen satztechnisch mit den Motetten verwandt. Auch hier 1 äßt sich in der Verwendung der Mensurzeichen eine Entsprechung zur Satztechnik erkennen: Der eher konservativen Kompositionsart der Messen entspricht die Verwendung des semicircolo tagliato bis weit in das 17. Jahrhundert hinein 72 . Im späten 16. Jahrhundert stehen alle Messen init einer Ausnahme ${ }^{73}$ unter dem semicircolo tagliato und zeichnen

67. Vg1. Braun, Handbuch, S.260 f.

68. In erster Linie ist hier die Villanelle gemeint. Zu dieser Gattungsgruppe gehören jedoch auch einige geistliche Gattungen, wie die Canzonette spirituali und die Laude.

69. Schon in den ausgewerteten Canzonettendrucken der Jahre $1561-1580$ war $\mathrm{zu}$ uber $80 \%$ der semicircolo semplice als Hauptmensurzeichen zu finden. Danach sind es annähernd $100 \%$. (Die Prozentzahlen sind bei allen Gattungen außer Madrigal und Motette mit Vorsicht zu betrachten, da das Vergleichsmaterial ungleich geringer ist).

70. Tempi, S.90 sowie S.120 ff.

71. Machatius gibt für seine Tempi eine objektiv kaum überprüfbare Begrüdung. Im Zusammenhang mit der Canzonette fuhrt er eine Stelle bei Vincentino an, in der dieser das "cantare in chiese" als "grave", das der "Canzona francese" als mit "velocită" bezeichnet (Machatius S.120. Vgl. ähnliche Zitate - insbesondere bei Bovicelli weiter oben). Hierin ist jedoch nichts anderes, als eine Differenzierung der beiden Hauptbewegungstypen zu sehen. Die Wurzel des schnellen "Canzonettenpulses" sieht Machatius in der Frottola; den Bewegungstyp selbst als eine Art anthropologische Konstante.

72. S.u. 
sich oft durch ausgesprochen wenig schwarze Notenwerte aus. Die Croma wird zwar verwendet 74 , aber nur äußerst selten.

\section{$1,1,3,3$. Die instrumentalen GatTungen}

Bei der Instrumentalmusik des späten 16.Jahrhunderts ist es besonders schwierig, zu klaren Aussagen über den Zusammenhang von Gattung und Mensurzeichen zu kommen. Die Instrumentalmusik entwickelte erst gegen Ende des 16.Jahrhunderts eigene Gattungen und die Zahl der reinen Instrumentaldrucke ist in diesem Zeitraum noch gering ${ }^{5}$. Weitere Instrumentalmusik ist in Drucken vokaler Musik iberliefert76. In Bezug auf Notation und Taktzeichen bereitet ein Vergleich der selbständig gedruckten Instrumentalmusik mit solcher aus Vokaldrucken Schwierigkeiten. Nicht selten wurde die Notation in den Drucken mit vokaler und instrumentaler Musik dazu benutzt, ein instrumentales Stïck von den vokalen in Bezug auf das Tempo zu unterscheiden ${ }^{77}$. In einem reinen Instrumentaldruck hingegen wurde die gleiche Methode verwendet, um zwischen zwei instrumentalen Gattungen zu differenzieren ${ }^{78}$. Es versteht

73. G. Croce, Messe a 8 (1596). Zunächst ist man geneigt, die Verwendung des semicircolo semplice in Croces Motetten (s.o.) und Messen schon im 16. Jahrhundert einer modernen Haltung des Komponisten zu den Mensurzeichen zuzuschreiben (Croce nimmt auch in Bezug auf den Generalbaß eine moderne Haltung ein (s.u.), die ihm jedoch im Hinblick auf seine Kompositionen nicht. unbedingt zuzusprechen ist - vgl. Arnold, Croce, S.38). Erstaunlich wäre dann jedoch, daß er in den Messe a 5 e 6 (1599), sowie in den Sacrae cantiones (1601) und Magnificat omnium tonorum (1605) den semicircolo tagliato verwendet. Unter Umständen gab für Croce die Stimmenzahl den Anlaß, seine achstimmigen Werke unter dem semicircolo semplice erscheinen zu lassen, um bei diesen das Tempo zu bremsen ("Monumentalstil"?). Ahnliches ist auch in den Missae sacrae von Giulio Belli (1608) zu beobachten. Hier steht lediglich die erste der beiden achtstimmigen Messen unter dem semicircolo semplice.

74. Natürlich nur maximal zwei in Folge.

75. Vgl. Sartoris Bibliografia.

76. Vgl. Sartoris Bibliografia.

77. In A. Banchieri, Concerti ecclesiastici (1595), J. Gallus, Totius libri ... partitio (1598) und P. Vinci, Motetti e ricercari (1591), wurden nur die Instrumentalwerke unter dem semicircolo semplice, die Vokalwerke hingegen unter dem semicircolo tagliato notiert. Es ist also auch möglich, dak Ricercare, die meist - auch noch im 17. Jahrhundert - unter dem semicircolo tagliato stehen (oft auch in Abgrenzung zu Canzonen), unter den semicircolo semplice gedruckt werden, um sie wiederum von (noch langsameren?) Motetten abzuheben (so bei Vinci). Zur schnelleren Bewegung instrumentaler Stücke gegeniber vokalen s.u.

Während bei diesen Beispielen jeweils Instrumentalstuicke von Motetten durch den "madrigalischen" semicircolo semplice unterschieden wurden, differenzierte A. Gabrieli, Madrigali et ricercare (1589) die Instrumentalstiucke (Ricercare) mit dem "motettischen" semicircolo tagliato von den Madrigalen. 
sich von selbst, daß diese unterschiedlichen Absichten nicht miteinander vergleichbar sind.

Ein weiteres Problem liegt in der Uneindeutigkeit der instrumentalen Gattungsnamen. Zwar lä日t sich im Groben eine Differenzierung zwischen Ricercar, Fantasie, Toccata, Canzone und Sonata erkennen; diese ist jedoch keineswegs verbindlich ${ }^{79}$. So stehen die Ricercare Giovanni Bassanos nicht unter dem semicircolo tagliato, da es sich hier nicht um das klassische polyphone Ricercar, sondern vielmehr um einstimmige Instrumentaletuden von virtuosem Charakter mit entsprechend kurzen Notenwerten handelt 80.

Das instrumentale Gegenstiuck zu dem Kontrastpaar Madrigal-Motette, das Paar Canzone-Ricercare ${ }^{81}$ ist, wie bereits erwähnt, im späten 16.Jahrhundert noch nicht ausgeprägt82. Insgesamt herrscht bei den alleinigen Instrumentaldrucken des späten 16.Jahrhunderts der semicircolo tagliato vor; sowohl bei Ricercaren als auch bei Canzonen und Toccaten ${ }^{83}$. Der semicircolo semplice hingegen begegnet uns häufig bei Instrumentalstücken in iberwiegend vokalen Drucken ${ }^{84}$. Die von Leland Earl Bartolomew85 beobachtete Generationsabhängigkeit in der Verwendung der Mensurzeichen innerhalb des Sammeldruckes Canzoni per sonare (1608) bestätigt diese Entwicklung.

78. F. Spongia Usper, Ricercari et arie francese (1595).

79. Es kann nicht Aufgabe dieser Arbeit sein, die Probleme der Definition instrumentaler Gattungsnamen im 16. und frihen 17.Jahrhundert erneut zu erläutern. Im großen und ganzen seien die Gattungsdefinitionen hier vorausgesetzt. Lediglich wo diese für die Notation von Wichtigkeit sind, wird im Folgenden darauf eingegangen werden.

80. G. Bassano, Ricercate, passaggi (1585). Vergleichbare Ricercare finden sich in D. Ortiz' Tratado de glosas (1553) sowie in II dolcimelo von A. Virgilliano (ms um 1600; Vg1. hierzu auch: Smith, Renaissancequerflöte, S.34 ff. Dort ist eines der Ricercare Virgilianos abgedruckt). Auch Ortiz verwendet für die Ricercare den semicircolo semplice - sicher ebenfalls im Hinblick auf die kurzen Notenwerte. Virgiliano schreibt allerdings unter dem semicircolo tagliato.

81. Braun, Handbuch, S.260 f.

82. Nur bei F. Spongia Usper, Ricercari et arie francese (1595) ist dieser Kontrast bereits anzutreffen. Drei der insgesamt vier Arie francese (= (anzoni francese) stehen unter dem semicircolo semplice, alle Ricercare hingegen unter dem semicircolo tagliato. Es ist verständlich, daß dieser Kontrast dort am deutlichsten hervortritt, wo die beiden Gattungen unmittelbar aufeinander treffen; ähnlich dann zu Anfang des 17.Jahrhunderts bei A. Bonelli, Ricercari e canzoni (1602), G. M. Trabaci, Ricercari, canzoni francese (1603) und G. P. Cima, Partito de Ricercari (1606).

83. Auf eine Ausnahme ist oben schon hingewiesen worden (Bassano). Weitere Ausnahmen: G. Bassano, Fantasie a 3 (1585), F. Guami, Ricercari a 2 (1588) sowie C. Merulo, Toccate d'intavolatura (1598).

84. Siehe die Beispiele weiter oben.

85. Raverij, S.257. ij 


\subsubsection{MENSURZEICHEN ALS TEMPOZEICHEN}

In dem bisher Gesagten wurde zwischen Drucken, in denen nur ein Mensurzeichen anzutreffen war und solchen, in denen ein Mensurzeichen überwiegend Anwendung fand - von Ausnahmen abgesehen - nicht unterschieden. Dies erscheint gerechtfertigt, da die Tatsache, daß ein Komponist innerhalb eines Druckes einzelne Stücke von den anderen unterschieden wissen wil1, nicht in einem grundsätzlichen Gegensatz zu den Gattungsmensuren steht.

Es wurde gezeigt, daß verschiedene Mensurzeichen für den geraden Takt innerhalb eines Druckes ihre Ursache in einer Gattungsverschiedenheit der einze1nen Stücke haben können86. Die Mehrzah1 der Drucke aber, in denen außer einem Hauptmensurzeichen für den geraden Takt zusätzlich für eine geringe Zahl der Stücke das jeweils andere Verwendung fand, beinhaltet nur Musik einer Gattung. Für diese Drucke, in denen - oft ohne ersichtlichen Grund beide Mensurzeichen auftreten, wurde behauptet, es wäre willkürlich mal dieses und mal jenes Zeichen verwendet worden ${ }^{87}$. Es fäl1t jedoch auf, daß bei fast allen Drucken in allen Stimmbiichern dieselben Stücke dasselbe Mensurzeichen tragen und bei mehrteiligen Kompositionen in der Rege188 alle Teile unter dem gleichen Zeichen stehen. Bei einer Anzahl von Drucken kommen weitere deutliche Anzeichen für eine durchaus bewubte Verwendung beider Zeichen hinzu. So kann man bei einigen Sätzen etwa von "madrigalischen

86. Vgl. die oben bereits angefuhrten, instrumental-vokalen Drucke. Darliberhinaus wurde in folgenden Drucken eine solche Gattungsdifferenzierung vorgenommen: P. de Monte, Madrigali a 5, libro quarto (1571, benutzte Auflage von 1581; alle Madrigale stehen unter dem semicircolo tagliato (vgl. oben), eine Canzone unter dem semicircolo semplice), G. Costa, I1 primo libro de motetti e madrigali spirituali (1581; Motetten: semicircolo tagliato, Madrigali spirituali: semicircolo semplice), A. Banchieri, Messa solenne a 8 (1599; enthält eine Fantasie unter dem semicircolo semplice), V. Bona, Psalmi omnes (1600; Psalmi: semicircolo tagliato, Magnificat: semicircolo semplice (!!)).

87. Z.B. Bellermann, Mensuralnotation, S.64.

88. Natürlich gibt es auch Fälle, wo verschiedene Teile einer mehrteiligen Komposition unter unterschiedlichen Mensurzeichen stehen. Folgende Kompositionen sind zu nennen: R. del Mel, "Et dignum ducis" aus Motectorum liber tertius (1585). Bei dieser dreiteiligen Komposition steht nur der Mittelteil unter dem semicircolo tagliato. Es ist interessant, daß die beiden äußeren Teile dieses Stückes die einzigen Kompositionen dieses Druckes unter dem semicircolo semplice sind; A. Striggio, "O messagi del cor" aus $I 1$ primo libro de madrigali a $6(1560,6 / 1578)$, P. Vinci, "Altiera donna" aus $I 1$ terzo libro de madrigali a 5 (1571, 2/1579) und "Le braccia aprendo" aus Quattordeci sonetti spirituali (1580), T. Massaino, "Amorosetti augel1i" sowie "La ver l'aurora", beide aus Il terzo libro de madrigali (1587), J. Peetrinus, "Sincerae animalae" aus Liber primus motectorum (1591), G. Croce, "Cruda 1a fune" aus Il secondo libro de madrigali (1592) sowie das Madrigal in "genere chromatico Il cantar nuovo" aus H. Bottrigaris I1 Melone (1603). 
Motetten"89 oder umgekehrt sprechen. Hier ist für einige Stiucke ein eigentlich "gattungsfremder" Bewegungstypus gewählt worden. Dies ist natülich umso häufiger zu beobachten, je näher die Drucke dem 17.Jahrhundert stehen, in dem es ohnehin zu einer Vermischung dieser Typen kommt90. Einige Komponisten unterschieden mittels der Mensurzeichen moderne von eher konservativen Sätzen'1. Auch die Verwendung der Dreiertakte beweist, daß hier keine Willkür geherrscht hat: In den meisten Drucken wurde konsequent unter dem semicircolo tagliato nur der groBe Dreier.92, unter dem semicircolo semplice nur der kleine Dreier ${ }^{9}$ verwendet.

Es kann daher davon ausgegangen werden, daß die Mensurzeichen semicircolo semplice und semicircolo tagliato in aller Rege194 bewußt gesetzt wurden. Bei der iberwiegenden Anzahl der Drucke mit beiden Mensurzeichen (beide Men-

89. $\mathrm{Zu}$ den wechselseitigen Einfluissen der verschiedenen Gattungen aufeinander am Ende des 16. Jahrhunderts vgl. auch Friedrich Blume: Art. Renaissance in MGG, Bd.11, Sp. 267.

90. Es kann nicht verwundern, daß auch die Differenzierungen mittels beider Mensurzeichen zuerst im Madrigal Arwendung fanden und erst zu einer Zeit, da sie im Madrigal schon wieder weniger benutzt wurden, in den Motetten zu vermehrter Anwendung kamen. Auch hier zeigt sich die insbesondere in der zweiten Hälfte des 16.Jahrhunderts zu beobachtetende Modernität des Madrigals.

91. Schmalzried, Schütz, S.63.

92. Gemeint ist der Dreiganzetakt.

93. Gemeint ist der Dreihalbetakt.

94. Selbstverständlich kann nicht grundsätzlich immer von bewußtem Setzen der Zeichen ausgegangen werden. Oft steht in einem Druck, der sonst nur den semicircolo semplice enthält an, einer stelle einmal ein semicircolo tagliato. Da dieser dann nur in einem Stimmbuch steht, kann solch ein Fall meist leicht von einer bewußten Setzung der Zeichen unterschieden werden. Es gibt durchaus aber auch den Fall, daß man zu spüren glaubt, daß hinter der Zeicherisetzung eine Absicht gestanden hat, diese jedoch durch Fehler im Druck nicht mehr recht zu erkennen ist (eine solche Absicht läbt sich zum Beispiel an einer Konzentration der Zeichenvermischung auf wenige Stiucke erkennen). Eine solche Vermischung der Zeichen beim Druck veranlaßte Praetorius zu den aufschlußreichen Sätzen in der Ordinatz zur Polyhymnia cadutrix (1619): "Daß die Signa Tactus Aequalis \&\& $C$ untereinander / ohne mein verursachen vermengt seyn / wolle sich niemand jrren lassen: Besonders jedes nach seinem Tact / nach deme jhme gut deuchtet / dirigieren." (Praetorius-GA, Bd. 17, S.XV. Vg1. Machatius, Tempi, S.217, Grï, Notation, S.75). Die in der genannten Literatur daraus gezogen Folgerungen, der Affekt (Machatius) bzw. die Notenwerte (Griiß) seien wichter für das Tempo denn die Signa, mag im Grunde stimnen. Dennoch ist insbesondere in Italien auf die Setzung dieser Zeichen einige Sorgfalt verwendet worden. Auch Praetorius hatte sich offenbar die Mühe gemacht, in seinem Manuskript die Zeichen genau einzutragen. Daß der Drucker sie durcheinandergebracht hat, ist sicher noch kein Beleg für die uberflüssigkeit der Unterscheidung zwischen beiden Signa. 
surzeichen wurden immerhin in rund 40\% der Drucke aus den Jahren 1581 - 1600 benutzt) läBt sich kein äußerer oder formaler Grund hierfür feststellen. In den Traktaten wird schon seit der Mitte des 16.Jahrhunderts immer wieder darauf hingewiesen, daß sich auch das Tempo nach dem Text zu richten habe95. Die Vermutung liegt nahe, auch diese abweichenden Mensurzeichen mit dem Text bzw. dem erzielten Affekt in Verbindung zu bringen. Der Zusammenhang zwischen Mensurzeichen und Geschwindigkeit (und nicht nur Bezugswert) des Taktschlages ist in Italien 96 durch Lodovico Zacconi ausdrücklich festgestellt worden (s.o.) - zahlreiche AuBerungen anderer Theoretiker lassen sich kaum anders verstehen.

Drei Typen der Verwendung tempomodifizierender Mensurzeichen lassen sich feststellen:

1. Die Notenwerte laufen der Beschleunigung/Verlangsamung durch die Taktzeichen entgegen. Hier nähert sich das Notenbild dem des jeweils anderen Gattungstypes. Ein Madrigal unter dem semicircolo tagliato ähnelt dann von den Notenwerten her einer Motette. Trotz beschleunigendem Mensurzeichen ist also die Bewegung im Effekt langsamer.

2. Die Notenwerte der Stücke mit dem abweichenden Mensurzeichen gleichen den anderen des Druckes. Hier wirkt nur das Mensurzeichen - ein semicircolo tagliato beschleunigend, ein semicircolo semplice verlangsamend.

3. Die Notenwerte und das Mensurzeichen bewegen sich in dieselbe Richtung. In einem Druck, im dem überwiegend der semicircolo tagliato verwendet wurde, hat ein einzelnes Stück unter dem semicircolo semplice zusätzlich langsamere Notenwerte. Das Mensurzeichen verstärkt hier die Wirkung der langsamen Notenwerte 97 .

Bei der Beurteilung dieser Fälle muß jedoch bedacht werden, daß die Spanne der verwendeten Notenwerte insbesondere im Madrigal des späten 16.Jahrhunderts sehr groB ist - Brevis bis Semicroma98. Nicht immer kommen alle werte innerhalb eines Madrigals vor, sondern es gibt sowohl Stücke mit eher kurzen als auch solche mit eher langen Notenwerten. Diese sicher mit dem Text in Verbindung stehenden Differenzierungen sollen natülich nicht durch einen wechselnden Taktschlag wieder aufgehoben werden. Wo ein abweichendes Mensur-

95. Am bekanntesten N. Vincentino, L' antica musica ridotta (1555), fol.94. 96. Im deutschen Sprachraum sind viele Probleme in der Musiktheorie praxisnäher behandelt worden als in Italien. Die Möglichkeit der Tempobeschleunigung mittels Mensurzeichen wurde dort schon von Glarean erwähnt (Dahlhaus, Rhythmik, S.122). Ebenfalls in der Mitte des 16.Jahrhunderts beschrieb in Spanien A. Mudarra, Tres libros de musica (Sevilla 1546) die Tempowirkung verschiedener von ihm benutzter Mensurzeichen (John M.Ward, Art. Mudarra, Alfonso, in MGG, Bd.9, Sp.844).

97. Dieser Fall ist recht selten. Ein Beispiel hierfür bietet G. Gabrielis "Canzona 7. \& 8. toni" aus den Sacrae symphoniae (1597).

98. Vg1. auch Braun, Handbuch, S.143. 
zeichen auftritt, kann jedoch von einer Modifikation des Tempos ausgegangen werden. Die Differenzierungsmöglichkeiten werden durch wechselndes Tempo vergrößert.

Die durch das abweichende Taktzeichen hervorgerufene Tempomodifikation ist nicht unbedingt mit dem Tempounterschied der beiden Gattungstypen gleichzusetzen. Es kann sein, daß ein Komponist tatsächlich an das Tempo der anderen Gattung gedacht hat, dies ist aber kaum zwingend der Fall.

Es muß nicht besonders betont zu werden, daß diese Tempomodofikationen nichts mit dem Proportionssystem (oder besser Proportionssystemen) des 15. und 16.Jahrhunderts gemein haben. Hier sind Beschleunigungen bzw. Verlangsamungen gemeint, die auch als solche empfunden werden sollen - dies ist bei einer dupla-Proportion nicht unbedingt der Fal199.

Ein besonderer Fall eines tempomodifizierenden Mensurzeichens findet sich im ersten Buch der Madrigali a cinque voci von Mogens Peders $\phi n$ (1608). Dem letzten Teil des Madrigals "Se nel partir" ist in allen Stimmen ein semicircolo semplice und direkt darunter ein semicircolo tagliato vorgezeichnet 100 . Wahrscheinlich sol1 die Wiederholung dieses Abschnittes beschleunigt vorgetragen werden. Ähnliche Anweisungen sind auch in verbaler Form aus etwas späterer Zeit bekannt101.

Nur schwer zu beurteilen ist eine weitere Art der Verwendung der beiden semicircoli: In einer ganzen Reihe von Musikdrucken des späten 16. und frihen 17. Jahrhunderts steht in Sätzen, die unter dem semicircolo semplice notiert sind, nach einem Dreiertakt nicht wieder ein semicircolo semplice sondern nun ein semicircolo tagliato102. Zunächst wird man hier an Ungenauigkeiten des Druckes denken. Die Häufigkeit dieser Erscheinung und vor allem die Konsequenz, mit der sie auftritt103, deuten jedoch auf eine $\mathrm{Ab}$ sicht hin ${ }^{04}$. Denkbar ist sowohl, daß man einer möglichen Verlangsanung beim

99. Es ist kaum möglich, zwischen einer Dupla und der Verwendung kürzerer bzw. längerer Notenwerte vom Höreindruck her zu unterscheiden.

100. Ahnliche Vorzeichnungen sind in Proportionstenören häufig. Hier sollen dieses Stimmen nacheinander unter jedem der gesetzten Zeichen mensuriert werden.

101. S.u. in den Abschnitt 1.2.4.1. - 1.2.4.3. dieser Arbeit.

102. Besonders häufig ist dies in Musikdrucken S. Rossis der Fall, nämlich in $I 1$ primo libro delle sinfonie (1607), Il secondo libro delle sinfonie (1608), Il terzo libro de varie sonate (1613?, 2/1622), Il quarto libro de varie sonate (1622), Madrigaletti a 2(1628), aber auch bei G. Croce, Motetti a 8 (1594), L. Bellanda, Canzonette spirituali (1599), D. M. Megli, Musiche (1602), M. A. Negri, Affetti amorosi libro secondo (1611), E. Radesca di Foggia, Madrigali a 5 e 8 (1615), G. Mussi, Il primo libro delle canzoni (1620) und L. Valvasensi, Letanie della B.V. (1622).

103. So findet sich die Setzung der semicircoli fast immer in allen Stimmbiichern parallel. 
Wechsel vom Dreier zurick in den geraden Takt entgegenwirken wollte, als auch, daß tatsächlich eine Beschleunigung des zweiten Teiles im geraden Takt gegenuber dem ersten gemeint ist105. Beides ist freilich nur denkbar, nachdem ein proportionales Verhältnis von Dreier und geradem Takt aufgehoben war106.

\subsection{Auflösung DES SYSTEMES DER GATTUNGSMENSUREN AB 1600}

Schon in den Quellen aus den letzten Jahren des 16.Jahrhunderts konnten die Anfänge der Auflösung des Gattungsmensursystemes beobachtet werden ${ }^{10}$ ?. Der Bruch in den Jahren um 1600 tritt dennoch in aller Deutlichkeit hervor: Wurde im letzten Jahrzehnt des 16. Jahrhunderts lediglich in etwas mehr als 11\% der Motettendrucke der semicircolo semplice als Hauptmensurzeichen notiert, so geschah dies in den ersten zehn Jahren des 17.Jahrhunderts bereits in fast 60\%108. Der Grund für diese Entwicklung ist sicher auch in einer Angleichung eines Teiles der Motettenproduktion an die Madrigale zu suchen.

\subsubsection{Angleichung der Motetten an die Madrigale zU Beginn des 17. JAHRHUNDERTS}

An die Ste1le des Gattungsgegensatzes Madrigal-Motette trat im 17.Jahrhundert in beiden Gattungen eine Anzahl unterschiedlicher Stilrichtungen, die auch zahlreiche uberschneidungen von Stil und Gattung hervorbrachten 109 . Dies wird in den für Madrigal wie Motette neuen Stilrichtungen - und hierzu

104. Ein möglicher Gedanke wäre auch, daß in den Setzereien ein uberfluß an semicircolc tagliato-Lettern vorhanden war (brauchte man doch un die Jahrhundertwende immer mehr den semicircalo semplice und immer weniger den tagliato). Da aber nur das Mensurzeichen am Satzbeginn Bedeutung gehabt habe, hätte man so die alten Letter verwenden können. Dagegen spricht aber schon die Tatsache, daß die Werke Rossis zunächst bei Amadino, und dann bei Vincenti erschienen, beides Verleger die erst in den letzten Jahren des 16. Jahrhunderts ihre Tätigkeit begannen (1583), also zur "Blitezeit" des semicircolo tagliato noch gar nicht verlegten. Zum anderen muß man sich wohl aufgrund der enormen Produktion gerade dieser Verleger fragen, warum diese Erscheinung nicht gleichmäßig verteilt auf die gesamte Produktion auftritt. Es kann davon ausgegangen werden, daß nicht nur die Zahl der vorhandenen Letter sehr groß gewesen ist, sondern daß diese auch nach Belieben zu beschaffen waren.

105. Kommen in einem Stuick mehrere Abschnitte im Dreiertakt vor, so steht der erste Abschnitt im geraden Takt unter dem semicircolo semplice, alle weiteren unter dem semicircolo tagliato.

106. Vg1. dazu weiter unten.

107. Vg1. Tabelle $1+2$ in Bd.II dieser Arbeit.

108. Vgl. ebenda.

109. Vgl. hierzu Bianconi, 17th century, S.45 ff sowie Haack, Generalbaß, S.72 f. 
zäh1t die Monodie wie auch andere geringstimmige Formen - am besten deutlich. Von den geringstimmigen Madrigal- und Motettendrucken110, die für diese Arbeit ausgewertet wurden ${ }^{11}$, ist ein großer Teil im Erscheinungsbild sehr ähnlich. Rund 50\% der geringstimmigen Madrigal- und Motettendrucke der Jahre 1601- 1620 enthalten Musik von mittlerer Virtuosität (Semicromen in Läufen, jedoch keine Biscromen). Die ubrigen 50\% der Motetten stehen zum großen Teil in der Nachfolge von Lodovico Viadanas Cento concerti ecclesiastici (1602)112. Es sind geringstimmige Werke mit Basso continuo, die in Anlehnung an den klassischen Motettenstil komponiert wurden, ohne direkt von der Monodie beeinflußt zu sein113. Der Großteil der verbleibenden Madrigaldrucke hingegen enthält die "wilden" hochvirtuosen Monodien des fruher 17.Jahrhumderts114. Bereits in der geringstimmigen Vokalmusik des dritten Jahrzehntes des 17. Jahrhunderts kann eine weitere Annäherung verzeichnet werden: Sowoh1 die Zahl der hochvirtuosen Monodiedrucke als auch die der konservativen Generalbaßmotetten ist zurückgegangen - rund $70 \%$ der geringstimmigen Madrigal- und Motettendrucke gleichen nun einander.

Ahnliche Entwicklungen sind - wenn auch nicht ganz so deutlich - in den Drucken mit polyphonen Motetten zu beobachten: auch sie gleichen sich (auch) in der Bewegung immer mehr den Madrigalen an. Dies ermöglicht nicht zuletzt das erneute Auftreten von geistlichen Kontrafakturen polyphoner Madrigale115 und sogar von Monodien116. Es gibt allerdings daneben auch bis zum Ende des hier zu betrachtenden Zeitraumes ausgesprochen konservative Werke einiger Vertreter der römischen Schule117. Die klaren Gattungsgrenzen zwischen Madrigal und Motette, wie sie im späten 16. Jahrhundert bestanden, ja für die Musik der Zeit bestimmend waren, sind nicht mehr zu erkennen. Die Vermischung der Stile hat nicht zuletzt auch zur Entstehung des ConcertatoSatzes beigetragen: Hier sind der polyphone und der solistisch-virtuose Stil

110. Als Drucke mit geringstimniger Musik sollen hier diejenigen gelten, die mehrheitlich Sätze mit max. zwei Singstimmen und Bc. enthalten.

111. Insgesammt rund $200-v g 1$. Quellenliste.

112. Im ersten Jahrzehnt sind es rund 44\% dieser Motettendrucke; nur 6 enthalten hochvirtuose Solomotetten.

113. Vg1. Arnold, Croce, S. 37 ff sowie Braun, Handbuch, S.154 ff und Haack, Generalbaß, S. 208 f.

114. Hierzu zählen im ersten Jahrzehnt des Jahrhunderts rund 47\% im zweiten rund $42 \%$ der geringbesetzten Madrigaldrucke.

115. Z.B. die Musica tolta da $i$ madrigali di Claudio Monteverdi, e d'altri autori ... fatta spirituale da Aquilino Coppini (1607, 2/1611), die geistliche Fassungen zu Monteverdis fünftem Madrigalbuch enthält (vgl. Horsley, Scores, S.466) oder die Concerti sacri scielti, \& trasportati dal secondo, \& terzo libro de madrigali von S. Bernardi (1621).

116. So enthält Monteverdis Selva morale (1641) eine geistliche Kontrafaktur der monodischen Fassung des Lamento d'Arianna (vgl. Bianconi, 17th century, S.22).

117. Neben verschiedenen Motettendrucken der ersten Jahre des 17.Jahrhunderts sind besonders G. Brunettis Motecta (1625) zu nennen. 
in einem Satz verbunden; sowoh1 in der Concertato-Motette als auch im Concertato-Madrigal118.

\subsubsection{Der Bedeutungsverlust Der Mensurze ichen}

Schon zu Beginn des 17.Jahrhunderts haben die Taktzeichen an Bedeutung viel verloren. Besonders deutlich wird dies in den nun aufkommenden verbalen Tempoangaben. Nicht nur Tempoworte im modernen Sinn - auch diese kommen im beginnenden 17.Jahrhundert auf (s.u.) - sondern auch Vorschriften, die - wie zur Sicherheit - die Bedeutung eines Mensurzeichen verbal wiederholen, fanden Verwendung 119 .

$\mathrm{Zu}$ beobachten ist dieser Bedeutungsverlust auch in der Setzung der Hauptmensurzeichen. So erfolgt die Verwendung der Taktzeichen in den geringstimmigen Werken (s.o.) - solange beide Zeichen dort noch zu beobachten sind - nun weitgehend unabhängig von Stil und Notenwerten. Innerhalb dieser Werkgruppe sind dem Verfasser noch sechs Motetten- und vier Madrigaldrucke unter dem semicircolo tagliato als Hauptmensurzeichen bekannt120. Nur zwei dieser sechs Motettendrucke sind mehr oder weniger dem konservativen "Viadanastil" zuzurechnen $^{121}$, unter den Madrigaldrucken unter dem semicircolo tagliato befinden sich auch zwei der virtuosesten Monodiedrucke122. Vereinzelt ist nun auch im 17. Jahrhundert wieder zu beobachten, daß ein Hauptmensurzeichen ganz fehlt, also für nicht notwendig gehalten wurde123.

Auch in polyphonen Motettendrucken der ersten Jahre nach 1600 wurde nun ungeachtet des Stils oder der Notenwerte iberwiegend der semicircolo semplice verwendet, sogar in den Drucken einiger ausgesprochen konservativer Vertreter der römischen Schule124. Andererseits verwendteen einige Komponisten

118. Vg1. auch Haack, Generalbaß, S.72 f. Zum Begriff Concerto vg1. Bianconi, $17^{t h}$ century, S.33 ff.

119. G. M. Trabaci hebt in seinem zweiten Buch der Ricercare (1615) einige Stuicke von den anderen durch den semicircolo tagliato ab und schreibt hinzu "Battuta stretta" (Vgl. unten).

120. L. Luzzaschi, Madrigali (1601), G. Caccini, Le nuove musiche (1601/1602), L. Viadana, Cento concerti ecclesiastici (1602), S. Bonini, Madrigali, e canzonette spirituali (1607), T. Massaino, Musica per cantare (1607), S. Bernardi, Motecta (1610), G. B. Biondi, Il quarto libro delli concerti (1611), G. M. Cesare, Concerti ecclesiastici (1614), B. Corsi, Motecta (1615), G. Fornaci, Amorosi respiri musicali (1617).

121. Viadana (1602) und Corsi (1615).

122. Luzzaschi (1601) und Caccini (1602).

123. Z.B. in M. da Gaglianos Dafne (1608) oder den Handschriften Verona, Biblioteca dell'Accademia filarmonica 228 und Florenz, Biblioteca Nazionale Centrale Mag. XIX 138 (beide mit Instrumentalmusik des frühen 17.Jahrhunderts).

124. Besonders zu nennen sind G. F. Anerio, G. Metallo, und P. Nenna. Recht konservativ sind auch die motettischen Werke A. Agazzaris. Deutlich ist der 
für alle ihre Motetten weiterhin auch noch im zweiten Jarhzehnt des 17.Jahrhunderts den semicircolo tagliato, wie z.B. Pietro Pace125 - oder schließen sich erst relativ spät dem neuen Brauch an, wie z.B. Giacomo Finetti126.

Schon in den ersten Jahren des 17. Jahrhunderts gibt es also unter einem Mensurzeichen, nämlich dem semicircolo semplice, sowohl motettische Werke auch polyphone - mit Biscromen in Ketten, als auch solche, in denen nur maximal zwei Cromen in Folge verwendet werden. An ein einheitliches Tempo dieser Stiicke wird man kaum glauben körnen. Offenbar spielt es für das Tempo keine große Rolle mehr, welches Zeichen einem Satz vorgesetzt ist.

Eine tempoangebende Bedeutung bleibt den Mensurzeichen jedoch noch in zumindest einigen der nun rasch weniger werdenden Drucke erhalten in denen auch nach 1600 noch beide Mensurzeichen verwendet wurden. Auch wenn das Hauptmensurzeichen seine tempoangebende Bedeutung verloren hat, kann das abweichende Zeichen noch immer für eine Tempomodifikation stehen. Um auch wirklich sicher sein zu können, daß er richtig verstanden wurde, hob Giovanni Maria Trabaci in seinem secondo libro de ricercari (1615) nicht nur einige Ricercare und Versi127 von den anderen Säzen128 mittels eines semicircolo tagliato ab, sondern uberschrieb diese zusätzlich mit "Battuta stretta"129.

Gegensatz Mensurzeichen / Musik z.B. in den Messe B. Strozzis (1626). Unter den ausgesprochen konservativen Messen dieses Druckes befindet sich auch eine "Missa A 5 Ad imitatione di quella di Papa Marcello", also eine Nachahmung von Palestrinas beriuhmter "Missa Papae Marce11i" aus dem zweiten Messbuch von 1567. Tatsächlich gleicht Strozzis Messe diesem Werk u.a. auch in der Bewegung. Dennoch ist der semicircolo semplice vorgezeichnet.

125. Alle Motettendrucke Paces, die für diese Arbeit eingesehen wurden (insgesamt füf aus den Jahren 1614 bis 1619, vgl. Quellenliste) stehen unter dem semicircolo tagliato.

126. Finetti benutzt in seinen motettischen Werken bis 1612 den sernicircolo tagliato als llauplmensurzeichen, ab 1613 den semicircolo semplice, olne dab eine Zuwendung zu einem virtuoseren Stil zu sehen wäre. Die kurzen Notenwerte sind im Gegenteil in seinen späteren Motetten sogar seltener.

127. Unter dem semicircolo tagliato stehen 11 von 14 Ricercaren (unter dern semicircolo semplice nur das Ricercar "Nono Tono con tre fughe", die verbleibenden sind im Dreiertakt notiert) und 10 der 100 Versi.

128. Außerdem enthält dieses Buch Gagliarden (auch im geraden Takt!), Partiten, Toccaten und eine Canzone (alles unter dem semicircolo semplice).

129. S.46, 51, 56, (2x) $60,66,77,79$ und damit bei fast allen Versi unter dem semicircolo tagliato. Zusätzlich tragen diese auch in der Tavola den Vermerk "in battuta stretta". Bei all diesen Sätzen handelt es sich um Stücke mit sehr langsamen Notenwerten, bei denen die "battuta stretta" den Kontrast zu den anderen Stuicken mildern - nicht jedoch aufheben - soll (vgl. oben). Auch in Trabacis Ricercare canzoni francese (1603) werden beide Taktzeichen (in erster Linie zur Gattungsdifferenzierung, aber auch innerhalb der Gattungen) verwendet, jedoch ohne eine verbale Erklärung. Wahrscheinlich erschien Trabaci 1603 eine solche Erklärung, im Gegensatz zu 1615, noch 
Bei den Versi steht zusätzlich die Anweisung "Et tutti quelli versi che stanno con lo Circolo tagliato si suoneranno in battuta stretta, gia che dove bisogna ritrovare 1'aviso"130. Die Nähe der Taktzeichenverwendung zum Tempowort ist hier nicht zu ubersehen.

Der Bedeutungsverlust der Mensurzeichen ist auf ganz andere Weise in dem zweiten Buch der Ricercari e canzoni francese von Antonio Cifra (1619) zu beobachten. Dieser Druck enthält acht Ricercare und acht Canzonen. Die Canzonen tragen im Titel den Zusatz "sopra il primo (secondo, terzo... ottavo) Ricercare". Gemeint ist, daß die Themen der Canzonen denen der Ricercare entlehnt wurden - allerdings sind die Notenwerte in den Canzonen um die Hälfte verkürzt. Es ist hier kaum anzunehmen, daß beide im selben Tempo zu spielen sind; dennoch stehen die Canzonen und die Ricercare unter dem semicircolo semplice. Die unterschiedlichen Gattungstempi wurden hier offenbar vorausgesetzt131.

\section{$1,2,3$. DiE MESSE ALS KONSERVATIVE GATTUNG}

Werner Braun hat bereits darauf hingewiesen, daß die Messe bis weit in das 17. Jahrhundert hinein die Verkörperung eines "alla breve-Ideals" darstel1$\operatorname{te}^{132}$. Während die Grenzen zwischen Motette und Madrigal immer undeutlicher wurden ${ }^{133}$, blieb das Erscheinungsbild der Messen auch im 17.Jahrhundert weitgehend unverändert. Dieser Gegensatz konservative Messe / moderne Motette könnte nicht deutlicher sein als in Angelo Antonellis Missa ac sacrarum cantionum (1629). Dieser Druck enthä1t neben einer Anzahl hochvirtuoser geringbesetzter Motetten unter dem semicircolo semplice eine Messe, notiert unter dem semicircolo tagliato mit maximal zwei Cromen in Folge. Hier treffen die Welt des monodisch-virtuosen und die der "alla breve-Polyphonie" unmittelbar aufeinander. Ganz ähnlich - wenn auch vielschichtiger - ist die Situation in Claudio Monteverdis berühmtem Druck Sanctissimae Virgini missa ... ac vesperae (1610). Der Cantus enthält in der "Missa In illo tempore"134 nur insgesamt vierma1 zwei Cromen, in den Concerti hingegen fehlt es nicht an Biscromen. Zusätzlich zu der konservativen Messe und den virtuosen Concerti enthält dieser Druck jedoch auch eine Anzah1 von motettischen Werken in denen eine Synthese zwischen den traditionellen Motetten und den modernen konzertanten Gattungen gesehen werden

nicht nötig.

130. S.41. Gemeint ist mit "Circo10", wie aus den Noten ersichtlich, der semicircolo.

131. Vg1. weiter unten zu einer Anweisung aus dem Vorwort zu A. Banchieris Partitura moderna armonica (1612). Banchieri spricht hier von einem "adagio in der Art der Ricercare". Die langsame Bewegung in der "Art der Ricercare" kann durch ein langsames Tempo oder - wie sicherlich bei Cifra gemeint durch doppelte Notenwerte bei nur etwas schnellerem Tempo erreicht werden.

132. Braun, Stilwandel, S.16.

133. Vg1. Braun, Handbuch, S.186 ff.

134. Parodiemesse nach Gombert. 
darf. Auch in diesen Druck steht lediglich die Messe unter dem semicircolo tagliato.

Ebenso deutlich hat sich das optische Erscheinungsbild der "alla breve-Polyphonie" in den reinen Messdrucken bis weit in das 17 .Jahrhundert hinein erhalten135. $\mathrm{Zu}$ nemen sind hier insbesondere die Partiturdrucke mit Messen von Paolo Agostini136 (erschienen ab 1627). Trotz der modernen Erscheinungsform in Partitur sind diese Messen (und Motetten ${ }^{137}$ ) nicht nur im Hinblick auf die Taktzeichen deutliche Beispiele für die mit Palestrina geschaffene und von da ab von der allgemeinen Entwicklung der Musik losgelöste Tradition der römischen Schule138. Hier gibt es zahlreiche Ligaturen und sogar einige Stuicke mit Proportionen - freilich einfachster Art139.

Einige Komponisten schrieben Messen sowohl im konservativen "Palestrinastil" als auch in der moderneren Form der "Concertato Messe"140. In Ansätzen ist dies in den Messe a quattro et cinque voci, parte sono per capella, e parte per concerto (1615) von Steffano Bernardi zu beobachten. Die mit "in concerto" iberschriebenen Messen beschränken sich zwar ebenso auf lange Notenwerte wie diejenigen "per capella", verfugen jedoch uber solistische Passagen141. Ganz gegensätzliche Messen haben sich von Leandro Gallerano142, Giovanni Priuli und Giovanni Valentini erhalten. Bei Valentini wird die stilistische Stellung der beiden Messdrucke schon durch den Titel angezeigt: Die Missae quatuor partim octonis partim duodenis vocibus (1621) gehören trotz des Generalbasses 143 - zu den traditionellen Messen; die Missae concertatae (1617) zu den modernen. Nichts jedoch deutet in den Titeln von Priulis Messdrucken des Jahres 1624 auf eine stilistische Verschiedenheit

135. Braun nennt als ein Beispiel Messen von Scachi, 1646 (Stilwandel, S.16). An anderer Stelle (Handbuch, S.309) bietet Braun ein Faksimile der "Missa Clementina $I I$ " von Alessandro Scarlatti (1716) in einem handgeschriebenen Chorbuch. Das Notenbild gleicht, abgesehen von dem Fehlen von Ligaturen, ganz dem einer Messe aus dem 16. Jahrhundert.

136. RISM verzeichnet sechs Messpartiturdrucke aus dem Jahr 1627 (fünf von diesen wurden fïr diese Arbeit ausgewertet) sowie einen posthumen Messdruck in Partitur aus dem Jahr 1630 - vgl. dazu weiter unten im Abschnitt II.2.2. sowie II.2.2.6.).

137. Drei der Drucke enthalten auch Motetten (vgl. die Titel in RISM). Sie sind unter dem semicircolo semplice notiert, unterscheiden sich jedoch sonst nicht wesentlich von den Messen.

138. Vgl. Fellerer, Kirchenmusik.

139. In Spartitura delle messe e motetti steht einmal der Tenor in einer Proportio dupla.

140. Braun sieht in den "Concertato Messen" ab 1610 sogar eine eigenständige Gattung (Handbuch, S.201).

141. Vgl. hierzu auch Arnold, Croce. Arnold beschreibt hier einen Motettendruck Croces mit Stücken ganz ähnlicher Faktur.

142. Jerome Roche, Art. Gallerano, Leandro, in NG, Bd. 7, S. 102.

143. Allerdings "ad placitum". 
des Inhaltes. Dennoch enthält der Druck Missae quatuor, sex, \& octo vocibus concinendae 144 ausgesprochen konservative Messen mit nur sehr wenig Cromen, während die Missae octo novemq. vocibus atque etiam instrumentis musicis concinendae konzertierende und z.T. hochvirtuose Kompositionen darstellen. Die Formulierung "atque etiam instrumentis musicis concinendae" meint hier ganz anders als im 16.Jahrhundert - selbständige, virtuose Instrumenta1stimmen ${ }^{45}$. Bei allen drei Komponisten stehen die konservativen Messen unter dem semicircolo tagliato, die modernen unter dem semicircolo semplice. Die Zahl der Messen mit einem modernen Erscheinungsbild ist jedoch insgesamt. recht gering 146 .

\section{$1.2,4$, VERBALE TEMPOANWE ISUNGEN}

Bereits in den Quellen aus dem 16.Jahrhundert 1ieß sich der Wunsch nach Tempodifferenzierungen deutlich erkennen. Die beiden Zeichen für den geraden Takt, der semicircolo tagliato und der semicircolo semplice, wurden benutzt, um einzelne Sätze in Bezug auf das Tempo von anderen zu unterscheiden. Trotz des Bedeutungsverlustes dieser Zeichen zu Beginn des 17.Jahrhunderts wurden sie weiterhin in dieser Weise verwendet, wenn auch mit stark abnehmender Häufigkeit. Seit dem Anfang des 17. Jahrhunderts erschienen nun auch verbale Tempoangaben in den Musikdrucken ${ }^{147}$. Im dritten Jahrzehnt des 17.Jahrhumderts wurden verbale Tempovorschriften bereits häufiger verwendet als unterschiedliche Mensurzeichen148, denen ohnehin zu dieser Zeit kaum noch Bedeutung beigemessen werden kann. Die verbalen Tempoangaben erschienen zunächst in Form von uberschriften uber einzelnen Musikstücken oder in Voroder Nachworten zu einzelnen Musikdrucken. Das Vokabular für solche Tempoanweisungen war bereits im 16.Jahrhundert ausgeprägt. Seit der Mitte dieses Jahrhunderts wurden in den Musiktraktaten regelmäBig verschiedene Arten der Tempomodifikation beschrieben ${ }^{149}$; ausgedrückt mit den bis heute ublichen Tempoworten.

144. Auch hier trägt die Bc.-Stimme den Zusatz "si placet".

145. In den Instrumentalstimmen kommen auch Ketten mit Biscromen vor (in den Vokalstimmen nur Semicromen).

146. Zu nennen sind hierfür u.a. Kompositionenen von Pietro Lappi (ab) 1608), Adriano Banchieri (1619), Marco da Gagliano (1614) sowie Tomaso Cecchino (1618).

147. Diesen Wandel beschreibt 1626 sehr schön Nicolaus Gengenbach. An die Erklärung des Tempoverhältnisse zwischen den beiden semicircoli fügt er in seiner Musica nova den Hinweis an: "Heutiges Tages aber werden sie so genaw nicht in acht genommen / Sondern / wenn es langsam und gravitetisch gehen sol / setzen sie darzu Adagio, Presto aber / wenn es geschwinde darvon gehen sol." (S.78 f, zitiert nach Bartels, Aspekte, S.181). Ahnliche Zusammenhänge beschreibt auch M. Praetorius. Für ihn sind die Tempoworte ein Ersatz der beiden semicircoli. Sie verhindern die bei häufigem Wechsel zwischen den semicircoli mögliche Konfussion (Syntagmatis musici ... tomus tertius, 1619, S. 51).

148. s.u. 


\section{$1,2,4,1$, TEMPOANWEISUNGEN IN UBBERSCHRIFTEN}

Die verbalen Tempoanweisungen treten zunächst nicht innerhalb einzelner Sätze, sondern in der Form von überschriften auf. Diese uberschriften weisen einzelnen Sätzen ein von dem Standardtempo abweichendes Tempo zu. Sie treten damit an die Stelle tempomodifizierender Mensurzeichen, die ebenfalls einzelne Sätze von der Mehrheit unterschieden. Irngard Herrmann-Bengen fuhrt einige Beispiele solcher Tempouiberschriften schon aus dem zweiten Drittel des 16.Jahrhunderts an ${ }^{150}$. Es handelt sich hierbei um Anweisungen aus spanischen und deutschen Lautentabulaturen ${ }^{151}$.

In den italienischen Quellen erschienen solche Tempoüberschriften zunächst in der Form von Titeln, wie "Fantasia Allegra"152 oder "Canzona La Grave"159. Es ist bei diesen Titeln in der Regel nicht mit Sicherheit zu erkennen, ob sie als Anweisung oder als Beschreibung zu verstehen sind. In den meisten Fällen ist hier eine beschreibende Funktion wahrscheinlicher. Ganz deutlich ist dies bei der "Sonata Terza Fuga Grave" aus der ersten Auflage von Adriano Banchieris $L^{\prime}$ organo suonarino (1605)154. Im Vergleich mit den anderen Titeln in diesem Druck wird deutlich, daß "L uga Grave" die besondere Art des der Sonata zugrundeliegenden Themas beschreibt155. Ein Bezug zum Taktschlag ist hingegen bei Biagio Marinis "La Bocca Sinfonia Allegra" aus Affetti musicali (1617)156 wahrscheinlich. Hier ist nicht nur

149. Auf zahlreiche solche Äußerungen wurde oben im Zusammenhang mit den Tempodifferenzen zwischen den Gattungen bzw. den Mensurzeichen hingewiesen. Beachtung verdienen in diesem Zusammenhang auch die äußerungen in dem Kapitel "Chi, \& quale debba essere il maestro di Capella" in L. Zacconis Prattica di musica (1596), fol.76.

150. Tempobezeichnungen, S. $30 \mathrm{ff}$.

151. Herrmann-Bengen nennt El Meastro von L. Milan (1536), Seys libros del Delphin von L. de Narvàres (1538) sowie Newgeordenet künstlich Lautenbuch von H. Newsidler $(1536 / 1544)$.

152. A. Gabrieli, Il terzo libro de ricercari (1596; vgl. hierzu auch: Bonicatti, Fantasia). Schon hier muß auf die Sonderstellung des Wortes "allegro" innerhalb der Tempoworte hingewiesen werden. Ahnlich wie auch "grave" und "largo" hat es im außermusikalischen Italienisch keine Tempobedeutung (im Gegensatz etwa zu "presto" oder "adagio"). Vgl. hierzu Robert Donington: Art. Tempo, in NG, Bd.18, S.677, sowie weiter unten in den beiden folgenden Abschnitten.

153. G. Guami, Partitura per sonare (1601).

154. S.26.

155. Andere Untertitel lauten z.B. "Fuga Plagale" (S.22), "Fuga Triplicata" (S.24, S.32), "Fuga Chromatica" (S.28) etc. Die "Sonata Terza Fuga Grave" entspricht in den durchschnittlichen Notenwerten den anderen Sonaten. Außergewöhnlich ist lediglich das Thema mit einer punktierten Brevis zu Anfang.

156. Herrmann-Bengen, Tempobezeichnungen, S.43, ging hier von einer Charakterbezeichnung aus, allerdings ohne das Stiick selbst zu kennen. 
die Sinfonia in verhältnismäßig kurzen Notenwerten geschrieben, sondern ihr ist auch ein semicircolo tagliato (der einzige dieses Druckes) vorgesetzt. Allerdings sind 1617 auch Tempoworte im modernen Sinn bereits bekannt157, so dab eine solche Bedeutung hier nicht verwundert 158.

Eindeutige verbale Tempovorschriften in Form von Uberschriften sind dem Verfasser aus der Zeit ab 1606159 bekannt: Das einzige Madrigal des sonst nur Canzonetten enthaltenden Druckes Della ricreatione di Posilipo von Gioseffo Biffi trägt die Uberschrift "Madrigale artificioso. Et si canta à missura larga". Auch diese Tempoanweisung trifft mit den einzigen semicircolo tagliato dieses Druckes zusammen 160.

Eine Anzahl solcher Tempoanweisungen findet sich in Claudio Monteverdis Sanctissimae Virgini missa (1610)161. Alle Tempoanweisungen stehen nur in der Bassus Generalis-Stimme; auch in zahlreichen späteren Drucken finden sich Tempoanweisungen überwiegend oder nur in den Continuo-Stimmen, die zugleich die Funktion von Direktionsstimmen ibernahmen ${ }^{162}$. Monteverdis Bassus Generalis-Stimme enthält nicht nur Anweisungen zum Tempo, sondern

157. Vg1. weiter unten. Außer der "Sinfonia Allegra" enthält Marinis Affetti musicali in dieser Art noch "La Albana Symphonia Breve" und "La Zorzi Symphonia Grave". Der Zusatz "Breve" bei "La Albana" scheint in der Tat nur die Kürze des Stückes zu meinen (Vg1. Herrmann-Bengen, S.43). Das Wort "Breve" ist auch sonst als Tempowort nicht bekannt. Bei "La Zorzi Symphonia Graue" könnte auch eine Beschreibung und nicht eine Tempovorschrift gemeint sein. In der Tat fällt dieses Stiick durch lange Notenwerte insbesondere zu Anfang - auf. In Angleichung an die "Symphonia Allegra" ist hier jedoch auch eine unterstützende Tempomodifikation zu vermuten.

158. Weitere Beispiele für ähnliche Titel: S. Rossi, "Sinfonia graue, a 5" aus I1 primo libro delle sinfonie (1607) mit ausgesprochen langen Notenwerten sowie A. Brunelli "Ballo Graue per sonare" in Scherzi, arie (1616; Vg1. Sartori, Bibliografia I, S.221, Herrmann-Bengen, S. 43, He11, Rhythmus, S.139 f). Brunellis "Ballo Graue" liegt in einer Neuausgabe in der Sammlung Baletto delle ninfe, hrsg.v. Silke Leopold, vor. Zum Rhythmus, wie zur besonderen Notierung dieses Stückes vgl. weiter unten. Nach Herrmann-Bengen finden sich ähnliche Titel noch 1639 bei Domenico Mazzocchi (S.43). Hell, S.139 f, vermutet für Tanzsätze mit solchen Angaben (deren Verbreitung er wohl uberschätzt) keine Bedeutung für das Tempo der Musik, sondern vielmehr für den Tanzschritt.

159. Das bei Herrmann-Bengen, Tempobezeichnungen, S.34, abgebildete Beispiel aus G. Caccinis Le nuove musiche gehört nicht hierher, da es sich dabei weniger um eine Tempovorschrift, als vielmehr um eine Auffiuhrungsbeschreibung handelt.

160. Es ist denkbar, daß dieses Mensurzeichen hier das "artificioso" unterstreichen sol1 - in Anlehnung an die kunstvollen klassischen Motetten. Zur Bedeutung des Begriffes "missura larga" vg1. weiter unten.

161. Sie stehen in den beiden Magnificat.

162. Dazu weiter unten im Abschnitt II.3.2. 
auch zur Dynamik und zur Registration 163. Die auch das Tempo betreffenden Angaben sind besonders interessant, da sie nicht nur die Anweisungen geben, sondern sie auch begrinden: Fast immer werden die kurzen Notenwerte der anderen Stimmen angefuhrt, um ein langsames Tempos zu rechtfertigen 164 . Solche Tempoanweisungen sind in dem umfangreichen Druck nur deshalb relativ selten vertreten, da fast alle virtuosen Sätze in der Generalbaß-Stimme in Partitur oder doch zumindest in Teilpartitur gegeben werden 165 . Die in den Tempoanweisungen zum Ausdruck gebrachte Abhängigkeit des Tempos von den Notenwerten kann auch dort angenommen werden, wo eine entsprechende Anweisung fehlt, weil eine Partitur sie uberflissig macht.

Einige recht ähnliche Anweisungen finden sich in verschiedenen Musikdrucken Adriano Banchieris aus den Jahren 1613-1619 - wenn auch hier ohne solche Begriundungen 160 . Neben diesen uberschriften verwendete Banchieri z.T. in denselben Drucken auch schon reine Tempoworte im modernen Sinn ${ }^{167}$. Auch Banchieris uberschriften gehen nicht nur auf das Tempo ein.

163. Vg1, im Abschnitt III.1.2. sowie III.2.2. dieser Arbeit.

164. Diese Anweisungen lauten: "Et exultavit, Principale solo, va sonata tardo perchè li doi tenori cantano di semicroma" (S.41), "Quia fecit, principale solo, \& si suona adaggio perche le parti cantano \& sonano di Croma \& Semicroma" (S.41), "Suscepit Israel a 3. voci, Principale solo, si suona adaggio perchè $1 i$ duoi soprani cantano di echo" (S.46), "Et misericordia, à 3. voci \& si suona adaggio perche li soprani cantano di Croma" (S.50). Vgl. dazu auch Herrmann-Bengen, Tempobezeichnungen, S.42. Alle diese Angaben sind in der Neuausgabe innerhalb der Monteverdi-GA abgedruckt.

Diese erläuterten Tempovorschriften belegen zugleich, daß diese Worte auch zu dieser Zeit tatsächlich das Tempo, und nicht den Charakter der Stücke meinten (so z.B. Adler, Stil, S.133 oder Robert Donington, Art. Tempo, in NG, Bd.18, S. 677. Witzerimann, Mazzocchi, S.210, sieht die Worte zumindest auch als Affektbezeichnung, wenn auch zugleich als Tempoworte). Eine Verlangsamung des Tempos aufgrund vieler kurzer Notenwerte erwähnte bereits Bardi (um 1580; vg1. Irmgard Bengen, Art. Tempo in MGG, Bd.16, Sp.1826).

165. Vg1. hierzu weiter unter im Abschnitt II.2.2.3.

166. Terzo libro di nuovi pensieri (1613): "Sinfonia piena, \& allegra" (S.18), "Vna \& due Voci spirituoso pieno, \& allegra" (S.50); Salmi festivi (1613), in der Orgelstimme zum "Magnificat à versi \& intiero": "A 4. Allegro Quia fecit, \& pieno" (S.28), "Et misericordia Tre Tenori che sono Basso Tenore \& Alto, suonarsi graue" (S.28), "A 4 Fecis potentiam Allegro \& pieno" (S.28), "Sicut erat graue \& larga" (S.29); Sacra armonica a 4 (1619), "Missa vittoria": "Gloria in Excelsis concertata graue" sowie, nur in der Orgelstimme: "Ultimo Kyrie tutti graue". Interessanterweise stehen diese uberschriften bei Banchieri wie auch bei Monteverdi besonders bei Magnificatsätzen. Vielleicht sollen auch hiermit die i.d.R. sehr kurzen Magnificatverse gegeneinander kontrastiert werden - ein ähnliches Bemühen ist auch in anderen Magnificatvertonungen zu spüren (z.B. in dem deutschen Magnificat aus den Symphoniae Sacrae II von Heinrich Schütz (SWV 344)).

167. Terzo libro di nuovi pensieri ecclesiastici (1613). Die beiden anderen 
Ähnlich wie Biffi verlangt Giovanni Maria Trabaci eine "battuta larga" in seinem zweiten Buch der Ricercare (1615) für eine Intavolatur des Madrigales "Ancidetemi pur" von Arcadelt168.

In die Gruppe dieser Tempoanweisungen gehört auch eine iberschrift in Monteverdis Concerto ( $=7$. Madrigalbuch) von 1619. Sie lautet: "Sequita il ballo a 5 con istromenti e voci, Concertato e adagio"169.

Solche Tempoüberschriften wurden schlieblich auch noch zu einer Zeit verwendet, in der auch die bis heute iblichen Tempoworte schon vermelart Anwendung fanden. Späte Beispiele für Tempoüberschriften finden sich bei Pellegrino Possenti170, Adriano Banchieri171, Martino Pesenti172, Pietro Francesco Garzi173, und Guiseppe Scarani174.

Drucke enthalten ebenfalls uber die uberschriften hinaus Tempoanweisungen (im Titel bei den Salmi festivi (1613) sowie in der Vorrede bei der Sacra armonica (1619) - s.u.).

168. S. 126: "Ancidetemi pur, Per l'Arpa" mit der Anweisung: "Questo Madrigale particolarmente si sonerà in Battuta larga, ma in principio del. Tenor' e Contralto per non scriuere quella sorte di Trillo sempre disteio, doue se ritrouerà questo segno + vi seruirete di detto Trillo". (Ähnliche Anweisungen in Bezug auf den Trillo finden sich auch an anderen Stellen in Trabacis Werk (s.u.im Abschnitt III.4.).

169. Vgl. Monteverdi-GA, Bd. 7, S.196.

170. Accenti pietosi (1625), S.30: "Questa Stanza si dice più presto delle altre".

171. Il virtuoso ritrovo (1626), "Lamento Di Leonardo Pastore" (in der Bc.Stimme): "Violone o Trombone, sonandosi stromento da pienezza s'arpeggia con attenzione e battuta larga" (in diesem Druck finden sich dariberhinaus einzelne Tempowörter wie auch eine "Avertenza" zum Tempo - vgl, unten).

172. Il terzo libro de madrigali (1628), "Per l'acque al fonte": "Si canti questo Madrigale adagio adagio fuor che la Canzonetta che canta il Soprano solo la quale và cantata mediocremente", "Più non $t$ 'amera": "Si canta la proportione maggiore adagio, \& la minor presto" (Vgl. dazu auch weiter unten), "Dietro al poggio": "Si canti adagio adagio"; Il primo libro delle correnti (2/1635, erste Auflage 1630 oder fruher), S.5: "Corrente detta la Querina Va sonata presto", S.12: "Volta delta la Liona Va sonata presto", S.25: "Balletto a Tre Si douerà sonar a battuta longhissima". Auch in diesem Druck findet sich eine weitere Temoanweisung im Nachwort (s.u.). Eine Anweisung "va sonata presta" will Herrmann-Bengen, Tempobezeichnungen, S.42, auch in Trabacis Secondo libro de ricercari (1615) gesehen haben. Dies beruht offensichtlich auf einem Irrtum. In der dem Verfasser vorliegenden Faksimileausgabe (siehe Quellenverzeichnis) ist diese Angabe ebensowenig zu finden, wie bei Sartori, Bibliografia. Wahrscheinlich verwechsell sie dies mit dem Pesenti-Druck (der auch von Sartori mit Angabe der Tempoiberschriften genannt wird (S.348 f)). Auch die Anzahl der Angabe "va sonata presto", die Herrmann-Bengen bei Trabaci gesehen haben will, stimmt mit der bei Pesenti uberein. 
Verwandt mit diesen uberschriften sind Forderungen, die Wiederholungen in schnellerem Tempo zu spielen bei Salomone Rossi175. Rossi verwendet neben diesen Tempovorschriften in Satzform auch Tempowörter in denselben Drucken 176 .

\section{$1,2,4,2$, TEMPOANWEISUNGEN IN VOR- UND NACHWORTEN}

Die Tempoanweisungen in Vor- bzw. Nachworten sind den Tempouiberschriften in den Formulierungen eng verwandt, gelten jedoch in der Regel nicht für ein bestimmtes Stück, sondern für eine ganze Sammlung. Hierbei kann zwischen Vorworten, die sich allgemein auf den gesamten Druck oder aber nur auf bestimmte Situationen beziehen, unterschieden werden.

Am Anfang der Reihe der allgemeineren Vorworte mit Tempoanweisungen steht das Vorwort zur Bc.-Stimme zu Antonio Agazzaris Sacrae Laudes... liber secundus (1603)177. Agazzari verlangt in diesem Vorwort eine "missura molto larga"178, die der (neue) Stil erfordere, besonders bei "esclamationi" und "parole affetuose". Er weist jedoch darauf hin, daß diese "missura molto larga" auch stellenweise aufgehoben werden kann, so bei einem Dreiertakt ("proportione") oder einer "fuga ribattuta". Das wiirde dem Gesang mehr "affetto" verleihen, ohne dabei der in der Kirche geforderten "gravità" entgegenzuwirken.

Fünf Jahre später erschien das umfangreiche Vorwort zu Ottavio Durantes Arie devote, le quale in se contengono la maniera di cantar con gratia l'imitatione delle parale (1608)179. Dieses Vorwort handelt im wesentlichen von der richtigen Art, Passaggien anzubringen, geht jedoch auch auf andere Fragen des Vortrages, wie Generalbaßspiel und Ausfuhrung von Bindebögen etc.

173. Madrigali e canzonette (1629): "Fili mia": "Canzonetta allegra per passa tempo" (Canto primo), "Cantata allegra" (Canto secondo).

174. Sonate concertate (1630): Sonata 18: "Và portata tutta larghissima". Dieser Druck enthält auch zahlreiche Tempoworte. Die Sonata 18 ist zusätzlich mit "adasio" iberschrieben.

175. "Si replica l'ultima parte più presto" steht mehrfach im dritten und vierten Buch seiner Varie sonate $(1613 \mathrm{bzw}$. 1622). Vgl. auch die bereits erwähnte, ähnliche Anweisung in dem Vorwort zu Banchieris Partitura moderna armonica (1612). Auf den Versuch, eine solche Beschleunigung der Wiederholung mittels Mensurzeichen anzuzeigen, wurde bereits hingewiesen ( $M$. Peters $\phi$, Madrigali a 5, libro primo (1608)).

176. So im vierten Buch der Varie sonate (s.u.).

177. Dieses Vorwort ist im zweiten Band dieser Arbeit auf S. 6 abgedruckt.

178. Auf die Deutung des Begriffes "missura larga" wird weiter unten ausfuihrlich eingegangen.

179. Das Vorwort ist vollständig abgedruckt und ubersetzt bei Goldschmidt, Gesangsmethode, S.29 ff. 
ein ${ }^{180}$. Zu den Arie schrieb Durante, daß man sich hier aus Rïcksicht auf den Affekt die größte Freiheit im Kontrapunkt nimmt. Auch die Sänger miißten danach trachten, den Text zu erfassen und dem Hörer zu vermitteln. Sie sollten darauf achten, gut zu intonieren und "adagio, cioè con la battuta larga" zu singen ${ }^{181}$.

Ganz ähnlich sind die Forderungen in den Vor- bzw. Nachworten zu Adriano Banchieris Sacra armonica (1619)182, Dialoghi, concerti, sinfonie e canzoni (1625)183 sowie Il virtuoso ritrovo (1626)184 und Constanzo Portas Sacro convitio musicale (1620)185. Auch hier wird eine "missura larga", "missura larghissima" bzw. "battuta larga" gefordert. Auf eine vergleichbare Forderung im Titel von Banchieris Salmi festivi (1613)186 sei ebenfalls in diesem Zusammenhang verwiesen .

Diese Forderungen nach einer "missura" oder "batutta larga" verlangen nach einer eingehenderen Betrachtung. Die Angabe "largo", "missura larga" oder "battuta larga" ist im fruhen 17. Jahrhundert fast nur in Vor- bzw. Nachworten zu finden; Ausnahmen bilden lediglich eine der Tempoüberschriften in Banchieris Salmi festivi - dort jedoch zusammen mit dem Tempowort "Grave"187 -, der Titel dieser Sammlung (s.o.), sowie eine Tempoüberschrift Giuseppe Scaranis (s.o.) - dort zusammen mit dem Tempowort "adasio". Zunächst wird man auch hier an das aus späterer Zeit bekannte Largo, also an eine, dem Adagio verwandte Tempoangabe denken.

Schon bei den allgemeineren Vorworten erscheint aber die Forderung nach einen langsamen Tempo recht unverständlich, da die Musik der genannten Drucke selbst bereits in überwiegend langen Notenwerten geschrieben ist. Recht aufschlußreich ist in diesem Zusammenhang der die "prima messa

180. Hierauf wird unten in den entsprechenden Kapiteln einzugehen sein.

181. Die Stelle lautet im Original: "Alle Arie si permette qualche licenza nel contrapunto per causa degli affetti. I Cantori devono procurar di capir bene in se stessi, quel che hanno da cantare, massime quando cantano soli, accio intendolo e possendendolo bene, 10 possino far intender all altri, che li stanno a sentire, che questo è il loro scopo principale, e devono avertire di intonar bene, e di cantar adagio, ciè con la battuta larga, porgendo la voce con gratia e pronnuntiando le parole distintamente ...".

182. Abgedruckt im zweiten Band dieser Arbeit auf S.7 f. Zu den Tempoanweisungen dieses Druckes vgl. auch weiter oben.

183. Unter der Tavola: "AVERTIMENTO. Tutti li Tenori, si cantano in Soprani, o li Soprani in Tenori, vi si ricerca battuta larga, \& attenzione nelle pause."

184. Unter der Tavola: "AVERTENZA. Dar una scorsa alle parole, e battuta larga reuscirà di gusto."

185. Siehe Anhang S.14.

186. Der Titel lautet: "Salmi festivi intieri, cortesi, allegri, et moderni da recitarsi à battuta larga in concerto di quatro voci, \& organo".

187. "Sicut erat Grave \& largo", s.o. 
Vittoria" betreffende Abschnitt aus dem Vorwort zu Banchieris Sacra armonica (1619). Während er für die "terza messa" ganz allgemein eine "missura larghissima" fordert (allerdings mit der interessanten Begriundung "per esser musica recitate"), beschreibt seine Angabe zur "prima messa Vittoria" eine recht kuriose Art der Tempodifferenzierung: Banchieri gebraucht hier die Schreibweise des Textes, un unterschiedliche Tempi zu kennzeichnen. Dazu heißt es im Vorwort188: Dort wo man Majuskeln findet, handelt es sich um ein "concerto solo o bino", und deshalb bediene man sich hier einer "missura largha"189.

Diese Sola- oder Duettabschnitte sind ebenfalls nicht besonders virtuos gestaltet190, und es ist nicht einzusehen, daß hier ein besonders langsames Tempo erforderlich sei. Das gleichzeitige Auftreten des Wortes "larga" mit dem Tempowort "grave" oder "adagio" bestätigt", daß "larga" nicht - oder zumindest nicht nur - "langsam" meinen kann191.

Tatsächlich legen die Wortlaute und Zusammenhänge einiger der Vorworte, in denen das Wort "larga" Verwendung fand, auch eine andere Wortbedeutung nahe. Agazzari schreibt 1603, daß der Stil der Stücke die "missura molto larga" erfordere, und zwar besonders bei den "esclamationi" und "parole affettuose"192. Banchieri erklärt 1619, daß die "missura larghissima" sich bei seiner "terza messa corrente" anbiete, da es sich um ein "musica recitate" handelt193. Frescobaldi fordert ein "tempo largo" in dem Vorwort zur zweiten Ausgabe seines ersten Buches der "Toccate e partite" (1615)194 nicht nur für

188. Vgl. den originalen und vollständigen Wortlaut in Band II, S.7 f.

189. Majuskeln im Text wurden auch von anderen Komponisten gebraucht. In den meisten Fällen zeigen sie jedoch die Instrumentation an; dazu ausfihrlicher weiter unten.

190. Vg1. hierzu das Notenbeispiel I,1 im zweiten Band dieser Arbeit: das "Gloria in Excelsis concertata graue" aus der "Messa Vittoria". Da die AltStime dieses Druckes verloren ist, wurde der von Johannes Donfried herausgegebene Sammeldruck Corolla musica missarum XXXVII (Straßburg: Zetzner 1628, RISM 16282) zur Vervollständigung herangezogen (vgl. zur uberlieferung der Messen Adriano Banchieris: Wilbert Banchieri, S.18-25). Vorwort und Terapoangabe in Titel fehlen bei Donfried ebenso wie die Großschreibung der "concerto"-Stellen. Nur gelegentlich ist noch ein Wort - wohl aus Versehen in Majuskeln stehengeblieben. In dem Notenbeispiel wurde die Großschreibung in der Alt-St imme rekonstruiert.

191. S.o.

192. Abdruck in Bd.II, S.6. Auch Pier Francesco Valentini (ca. 1570-1654) sagt, daß "passi affetuosi" in Motetten und Madrigalen nach einer "battuta larga" verlangen (Murata, Valentini, S.334, leider ohne Angabe des Originalwortlautes und auch ohne genaue Quellenangabe).

193. Aludruck des Vorwortes in Bd.II, S.7 f. Die "terza messa" ist fast durchgängig homophon mit recht kurzen Deklamationsnotenwerten komponiert.

194. Unter Punkt. 9. - vgl. den Abdruck des Vorwortes in zweiten Band dieser Arbeit auf S.13 f. 
Verzierungen in den Partiten, sondern auch für die Toccaten insgesamt. Zu Anfang dieses Vorwortes vergleicht Frescobaldi die "battuta" in den Toccaten mit der der "Madrigali moderni": "hor languida, hor veloce, è sostenendola etiandio in aria secondo $i$ loro affetti, ó senso delle parole"195. Das Wort "largo" trägt hier ganz offensichtlich - zumindest auch - den Sinn von "frei", eine Bedeutung, die auch in Einklang mit dem außermusikalischen Italienisch steht196. Sehr deutlich ist dieser Sinn des wortes "largo" in einer Bemerkung Ignatio Donatis197, in der die "misura Larghissima" als Gegensatz zu "Batter la battuta" verwendet wird198.

Aus den Wortlauten einiger Vorworte ergibt sich aber auch, daß "largo" darüber hinaus " "verzögernd" bedeutet bzw. bedeuten kann. So kann laut Agazzari (1603) die "misura molto larga" durch ein "ristrengere"190 - also eine "Wiederbeschleunigung" - aufgehoben werden; Francesco Lambardi200 nennt "stretta", also "drängend" als Gegensatz zu "larga"201. Auch bei Paolo Quagliati (1608)202 erscheint die ubersetzung des Wortes "larga" mit "verzögernd" passend, obwohl hier nicht "stretta", sondern "presta" den Gegensatz bildet, da "verzögernd" auch den Sinn von "langsam" mit ubernehmen kann. In ähnlicher Weise ergibt die ubersetzung des Wortes "larga" auch an den anderen Stellen mit "frei" und/oder "verzögernd" eirien Sinn203. Es ist

195. Unter Punkt 1.

196. Das Dizionario delle lingue italiana e tedesca von Vladimiro Macchi, Rom, Wiesbaden, ohue Jahr (I Grandi Dizionari Sansoni), S.752, kennt fiir "largo" die Bedeutungen "freigebig" und "großzigig". In Neues italienischdeutsches und deutsch-italienisches Wörterbuch von Oskar Bulle und Giuseppe Rigutini, Leipzig, Mailand 1902, wird dariber hinaus "largo interpretatione" als "freie Interpretazion", "freie ibersetzung" angefuihrt (Bd.I, S.427). Auch Goldschmidt, Gesangsmethode, s.31 uibersetzte Durantes "di cantar adagio, cioè con la battuta larga" mit "mit Sorgfalt" (??) "zu singen, d.h. mit gröBter Freiheit im Tact".

197. Aus Il secondo libro de motetti a voce sola, Venedig: Vincenti 1636, zitiert nach Goldschmidt, Gesangsmethode, S.77.

198. "In questa sorte di Cantilena di voce sola non si deve mai Batter la battuta, ma solo attendere a Cantare con misura Larghissima posatamente, con far quelli affetti".

199. Vg1. Wortlaut in Bd.II, S.6.

200. Il secondo libro de villanelle (1614) sowie Canzonette a tre ... libro terzo (1616); siehe hierzu weiter unten. Bei D. Mazzocchi, Dialoghi e sonetti (1634) findet sich die Angabe "Largo, e poi stretto" (Witzemmann, Mazzocchi, S.210).

201. Vg1. zu diesem Gegensatzpaar auch die Ausfiihrungen zun wechselnden Tempo bei A. Pisa, Battuta della musica (1611): "Ma non essendo necessario, che questa misura vadi tanto veloce, quello che regge il canto la gouerna à sua volontà \& la fà larga, e stretta quando gli piace." (S.95; vgl. hierzu: Dür, Auftakt, S.29).

202. Vorwort zu Il primo libro de madrigali; vgl. weiter unten.

203. Die Bedeutung "frei" erscheint so auch in den Beispielen aus G. 
durchaus wahrscheinlich, daß die Bedeutung "verzögernd" schließlich zu der Bedeutung "langsam" gefiihrt hat; aber auch im späteren 17.Jahrhundert hat "largo" noch nicht immer seine - dem Begriff "adagio" verwandte - Bedeutung als selbständiges Tempowort erlangt 204.

Statt der von Agazzari, Banchieri, Donati, Durante und Porta geforderten "battuta" oder "misura larga", wird in den Vorworten Francesco Lambardis, Marcello Albanos und Martino Pesentis ein eher schnelles Tempo verlangt. Dabei werden wiederum Gattungsdifferenzierungen sichtbar.

Besonders deutlich treten diese Gattungsdifferenzierungen bei Francesco Lambardi auf. Zu seinem secondo libro de villanelle (1614) schrieb er, man solle die Villanellen - besonders diejenigen in der "proportione menori" (= Dreihalbetakt) nicht zu treibend singen - die Canzona (hier = Villanella) verlange nach "autoritä" - aber auch nicht zu schleppend, denn sie verlange nach "spirito". Insgesamt empfielt Lambardi ein mittelmäßiges Tempo, aber eher treibend als schleppend, damit die Canzonen sich noch von den "arie graui" unterscheiden205. Mit den "arie graui" meint Lambardi die beiden Arien für Tenor und Generalbaß, die in dem Buch von 1614 eben falls enthalten sind206. In seinem dritten Buch (1616) beruft Lambardi sich auf die Tempoanweisung des zweiten Buches und faßt zusammen, die "battuta" sei weder zu treibend, noch zu schleppend 207 .

Caccinis Le nuove musiche (1601/02) durchaus sinnvo11. Der Unterschied zwischen der "escla senza misura" und der "escla con misura più larga" (beide auf der vorletzten Seite des Vorwortes) ist trotzdem gegeben. Der Ausruf "senza misura" besteht aus einer Folge von Noten, die "quas $i$ favellando in armorica con la suddetta sprezzatura" gesungen werden sollen. "senza misura" meint hier also "dem Sprachrhythmus folgend". Die "escla con misura più larga" hingegen bezieht sich auf eine lange Note, die nicht genau im Takt gehalten werden muß, also init einem "eher freiem Maß" gesungen werden soll (vgl. eine andere Deutung bei Herrmann-Bengen, Tempobezeichnungen, S.34).

204. So findet sich eine Nennung von zwei Tempoworten auch noch 60 Jahre später in der "Sonala La Pelicana" von Maurizio Cazzati (1670). Der erste Satz dieser Sonate ist iberschrieben mit "Presto \& Largo" (vgl. die Edition der Sonate in Giegling, Solosonate, S.33 ff). Wenige Jahre später setzte W. C. Printz in seinem Compendium musicae (1689) die Worte "adagio", "lento" und "largo" gleich (S.22).

205. "Avertimento à Lettori: II modo con che si haveranno d̀ cantare queste Villanelle, e particolarmente quelle in proportione menori sard che non si cantino tanto strette, che la Canzona perda l'autorità ne anco tanto larghe, che perda lo spirito, peró mediocremente, e più tosto cantate strette, che larghe, poiche le canzoni sono differente delle arie gravi. Et anco riusceranno assai meglio, s'elle verrano cantate con istrumenti perfetti, come Gravicimbali, Leuti, Arpe doppie \&c. ", zitiert nach Vogel, Bibliothek, Bd.I, S.341.

206. In Tenorstimmbuch in Partitur. 
Ein schnelles Tempo verlangen ausdrücklich Marcello Albano und Martino Pesenti. Pesenti gibt im Nachwort zu seinem primo libro delle correnti (1630?, 2/1635) eine recht interessante Begrundung hierfü: Man solle sich nicht wundern, wenn man einige Nonen, Septimen, Tritoni, verminderte Quinten und andere Dissonanzen fände, denn, wenn man diese nicht mit Mittelstimnen versehe 208 und mit einer "battuta presta" spiele, wiirden diese, entgegen ihrer Natur, Lieblichkeit und "affetto" ergeben209. Albano fordert in seinem primo libro di canzoni e madrigaletti (1616) nicht nur ein schnelles Tempo sondern, fügt noch hinzu, daß die Binnenkadenzen "à battuta giusta" gesungen werden sollen, die Schlußkadenzen jedoch möge man etwas länger als die anderen halten 210 .

Nicht ein schnelles oder langsames, sondern vielmehr ein wechselndes Tempo verlangen schlieBlich Paolo Quagliati und Horatio Modiana. Quagliati schreibt im Vorwort zu seinem primo libro de madrigali (1608), er winsche sich eine "missura alternata", die mal "larga" und mal "presto" sei211. Modiana fordert im Vorwort zu seiner Primitie di sacri concerti a voce sola (1623) nicht nur eine "battuta hor lento hor veloce", um dem Sim der Worte gerecht zu werden, sondern er erlaubt auch, bei schnellen Passaggien den Taktschlag außer acht zu lassen und sie ganz entsprechend der eigenen Fähigkeit ("dispositione") zu singen. Am Ende eines Passaggios möge man jedoch immer ein wenig langsamer werden212.

207. "... dette Canzonette si havramno da cantare dell'istesso modo, come mi dechiaro nel 2.libro mio, ciò è che la battuta non sia troppo larga, ne troppo stretta, \& anco sonate con istrumenti perfetti.", zitiert nach Vogel, Bibliothek, Bd. I, S.341.

208. Pesentis Tänze sind, wie auch eine Anzahl von Klavierwerken Banchieris, nur in Randstimmenpartitur notiert. Die Mittelstimnen hatte der Ausfuhrende in der Regel selbst zu ergänzen.

209. "Alli signori sonatori. Non vi apporterà meraviglia ritrovare in alcune di queste mie Correnti, None, Settime, Tritoni, Semiquinte, e simili dissonanze, poiche non accompagnandole con le parti di mezzo, e sonandoli d̀ battuta presta, rendono vaghezza, et affetto contrario alla natura. Et vivete felici".

210. Dieses Vorwort ist im zweiten Band dieser Arbeit auf S.6 abgedruckt. 211. "AI LETTORI. ... molti de quali (mostrandone non mediocre diletto) si degnano all'occasione cantarli con vn certo giuditio, col quale esorto ciasuno, che voglia dar di essi, à cantarli; cioè con vna missura alternata, tal'hora larga, e tal'hora presta, per dar tempo, e commodita a $i$ gratiosi cantanti ..." ".

212. "Avertiscano $1 i$ Signori Cantori, che non sono molto prattichi del Cantare moderno, e con dispositione a non sgomentarsi nelle Passaggi espressi con Semicrome e talvolta con duplicate piu veloci per schivare la multiplicità delle note; suspendosi la Batutta si cantano conforme alla dispositione del Cantante tanto l'une quanto l'altre, rallentandosi sempre aliquanto la voce nel fine del Passaggio. Rimentendosi poi l'Autore al discreto Cantore circa l'imitatione delle parole, che ricercano la battuta 
Mit ihren Anweisungen gehören die zitierten Vorreden Albanos und Modianas auch schon zu den Vorworten des zweiten Types, die nicht nur allgemeine, sondern spezielle Tempoanweisungen für bestimmte Situationen enthalten. Zu den Vorworten dieser Gruppe gehört die "Quinta avertenza" aus dem Vorwort. zu Banchieris Partitura moderna armonica (1612). Dort empfiehlt Banchieri die Canzonen zunächst "adagio" in der Art der Ricercare 213 zu spielen und dann, in der Wiederholung, schneller, da diese Abwechslung zu neuer Freude führe214. Daß diese Idee der beschleunigten Wiederholung hier kein Einze1fall ist, wurde oben bereits gezeigt.

Geradezu beriihmt fuir die Vielzahl ihrer Tempoanweisungen sind einige Vorworte von Girolamo Frescobaldi215, Das erste dieser Vorworte erschien 1615 zur ersten Ausgabe der Toccate e partite ... libro primo. Dort gibt Frescobaldi folgende Tempoangaben: Die Anfänge der Toccaten sollen langsam gespielt werden. Im weiteren Verlauf achte man auf die Unterscheidung der einzelnen Passagen. Sie sollen, je nach ihrem Affekt, mehr oder weniger schnell gespielt werden. "Passi doppui"216 sind der Deutlichkeit halber langsam zu spielen. Auf der letzten Note eines Trillers oder anderen Effektes 217 halte man inne. In den Partiten nehme man ein "tempo giusto" und "proportionato" 218 und, da in einigen auch schnelle Passaggien zu finden sind, bediene man sich eines bequemen Tempos, um nicht schnell anzufangen und dann stockend fortzufahren, denn sie sollen ganz in ein und demselben Tempo gespie1t werden. Und es bestehe kein Zweifel: Die Perfektion beim Spielen besteht hauptsächlich in der Fähigkeit, das richtige Tempo zu erfassen.

hor lento hor veloce...", zitiert nach Goldschmidt, Gesangsmethode, S.77f.

213. Auch hier wird auf eine Gattungsdifferenzierung Bezug genommen. Die "Art der Ricercare" kann hier nur die langsamere Bewegung meinen. Um die Canzonen dieser anzupassen, muß ein langsamerer Taktschlag erfolgen - bei Cifra (vgl. oben) war für die Ricercare ein schnellerer Taktschlag nötig, damit sie nicht ganz. so langsam wurden. Es zeigt sich auch hier wieder, daß entscheidend das hörbare Resultat war.

214. Das Vorwort ist vollständig abgedruckt bei Sartori, Bibliografia I, S.184 (1612 a). Die "Quinta avertenza" lautet: "Quinta, 1a prima fiata deuesi suonare adagio in guisa di ricercare, \& nella replica stretamente, rendendo tal varietà nuouo diletto, e per ció vengono signate le Repliche".

215. Alle Vorworte zu Frescobaldis Instrumentaldrucken sind zugänglich uber Sartoris Bibliografia. Dort sind sie vollständig abgedruckt. In Abhandlungen iber das Tempo oder iber Frescobaldi wurden sie bereits wiederholt herangezogen. Aufgrund der enormen Wichtigkeit gerade der beiden Toccaten-Vorworte sind diese in zweiten Band dieser Arbeit nochmals vollständig wiedergegeben (S.12 ff).

216. Herrmann-Bengen, Tempobezeichnungen, S.37, Anm.19, ibersetzt "Passi doppui" ıit "Passagen, in denen beide Hände parallel laufen".

217. Gemeint sind wohl Verzierungen.

218. "proportionato" hier wohl: angemessen. 
Frescobaldis erstes Toccatenbuch erfuhr noch im Jahr seiner Erstveröffentlichung eine zweite Auflage. Dieser zweiten Auflage gab Frescobaldi ein neues Vorwort bei, das zwar auf dem ersten basiert, jedoch so weitgehend umgearbeitet und erweitert wurde, daß man von zwei verschiedenen Vorworten sprechen muß. Zusätzlich zu dem Vorwort der ersten Ausgabe enthält das erweiterte Vorwort folgende Aussagen zum Tempo219: Diese Art. zu spielen (bei Toccaten) soll nicht einem gleichen Takt unterworfen sein, sondern eher wie bei den "madrigali moderni", wo der Takt mal schleppend, mal schnell geschlagen wird oder sogar innehält, je nach dem Affekt und dem Sinn der Worte. Die Kadenzen sollen, auch wenn sie schnel1 (= mit schnellen Notenwerten) geschrieben sind, ausgehalten werden, und wenn man sich dem Ende des Laufes oder der Kadenz nähert, solle man in ein "tempo più adagio" gehen. Wo gleichzeitig in einer Hand ein Triller, und in der anderen ein Lauf steht, solle man den Triller nicht Note für Note aufteilen, sonder nur versuchen den Triller schnel1, und den Lauf langsamer und "affetuoso" zu nehmen. Findet man in den Partiten "passaggi" oder andere Verzierungen ( "affetti"), so wird es gut sein, ein "tempo larga" zu nehmen, und dieses wird auch bei den Toccaten beachtet. Die anderen kann man mit irgendeinem schnelleren Takt spielen ("alqunto allegre di battuta").

Einige weitere interessante Tempohinweise gibt Frescobaldi in der Vorrede zu dem Primo libro dei capricci (1624)220. Auch dort fordert er ein langsames Tempo für die Anfänge. So gäbe man den darauffolgenden Passagen mehr Geist ("spirito") und Lieblichkeit. Bei den Kadenzen halte man inne, ehe man mit der nächsten Passage fortfahre. Frescobaldi gibt in diesem Vorwort dariberhinaus eine Tempodifferenzierung der Dreier- bzw. Sechsertakte auf die auch weiter unten noch eingegangen werden wird. Dort heißt es, die großen Dreier (= Dreiganzetakte) seien "adagio", die kleinen Dreier (= Dreihalbetakte) etwas schneller und wenn sie aus drei Semiminimen bestehen (= Dreivierte1takt221) (noch) schneller, und wenn es ein Sechsvierteltakt ist222, so gibt

219. Vg1. den Abdruck dieses Vorwortes in Bd.II dieser Arbeit auf S.13 f. 220. Dieses Vorwort ist vollständig abgedruckt bei Sartori, Bibliografia I, S.295 f (1624 b). Der sich auf das Tempo beziehende Abschnitt lautet: "Si deuono $i$ principij cominciarli adagio a dar maggior spirito e vaghezza al seguente passo \& nelle Cadenze sostenerle assai prima che si incominci l'altro passo, e nelle trippole, o sesquialtere, se saranno maggiori, si portino adagio, se minori alquāto piu allegre, se di tre semiminime, piu allegre se saranno sei per quattro si dia illor tempo con far caminare la battuta allegra".

221. Entgegen der Behauptung von Machatius, Tempi, S. 132 ist der Dreivierteltakt in dieser Zeit wenn auch nicht sehr verbreitet, so doch bekannt.

222. Die ubersetzung dieser Stelle bei Hermann-Bengen, Tempobezeichnungen, (S.52, Anm.25) ist irrefuhrend. Sie Ubersetzt "sesquialtere" mit "Sechsertakte", die "sesquialtera" ist jedoch ein Dreiertakt mit der Vorzeichnung $3 / 2$. Daß Frescobaldi diesen Abschnitt so pauschal mit "nelle trippole, $\delta$ sesquialtere" beginnt, zeigt wie unwichtig ihm die Taktvorzeichnung of fenbar war, und daß die Notenwerte fur ihn das eigentlich Bedeutsame waren. Auch 
man ihm sein Tempo, indem man mit der "battuta allegra" fortfährt223. Die "battuta allegra" ist offensichtlich für Frescobaldi ein "tempo ordinario"224. Ein Tempowechsel kann gar nicht gemeint sein, da in diesem Druck an mehreren Stellen der Sechsviertel-Takt nicht in allen Stimmen gleichzeitig verlangt wird. Der Sechsvierte1-Takt - wie auch der Zwölfachtel-Takt - wird von Frescobaldi zur Triolierung auch in einzelnen Stimmen verwendet 225

Auf ganz bestimmte Situationen zugeschnitten sind auch die Tempoangaben im Vorwort zu Francesco Severos Salmi passeggiati (1615)226. Dort heibt es, die Intonationen seien "adagio" zu singen. Wenn in den Versetti eine Stelle erscheint, bei der man viele Wörter auf einer Note rezitieren muß (= Falsobordone227), so halte man stets auf der ersten Silbe mit Anmut inne und nehme die zweite "presto", und so fort, immer zwei und zwei Silben. Semicromen singe man immer so schnell wie man könne ("con vivacità et presto il più che sarà possibile").

Eine sehr spezielle, aber im Zusammenhang mit den Dreiertakten (s.u.) höchst interessante Tempoanweisung gibt schließlich Sigismondo d'India im Vorwort zu seinen Musiche e balli (1621)228. Er schreibt dort, daß man die "sestupla" (Sechsvierteltakt) wegen ihrer "velocità" und der sich daraus ergebenden Schwierigkeit auch als Dreiertakt mit je drei Semiminime "à battuta presta" singen kann229.

der letzte Satz dieser Stelle: "se saranno sei per quattro si dia illor tempo cou far caminare la battuta allegra" ist von Herrmann-Bengen mißverständlich ibersetzt wurden. Die ubbersetzung muß lauten: "Wenn sechs gegen vier gehen (6/4) gibt man ihnen (den Noten) das ihnen eigene Tempo indem man die "battuta allegra" (weiter)laufen läBt".

223. Siehe dazu auch weiter unten im nächsten Abschnitt.

224. In dern Vorwort Bartolomeo Grassis zu der Partiturausgabe von Frescobaldis Ensemble-Canzonen (1628) wird darn auch das Wort "tempo ordinario" im gleichen Sachverhalt gebraucht: "Se trouaranno qualche tempo non solito, cioè essere ad'vna parte, segnato il tempo di proportione, \& all'altra seguire il tempo ordinario, non perció credano, che sia per errore, ma considerino bene la partitura, che troveranno non esser molto aggiustata, \& facile, tanto più che come vedranno quest' opera è stata stampata con si esquisita diligentia in questa parte, che ogni vna delle note porta il suo valore al debita luogo ...". Der beschriebene Fall ("tempo ordinario" in einer Stimme, "proportione" in einer anderen) tritt nur beim 6/4-Takt auf.

225. Näheres hierzu weiter unten im Abschnitt 1.2.5. und 2.6.

226. Vg1. den Abdruck des Vorwortes in Band II dieser Arbeit, S.16 f.

227. Zur Verbreitung des Falsobordone im frühen 17. Jahrhundert vgl. im Abschnitt IV.1. dieser Arbeit.

228. Auch dieses Vorwort ist im zweiten Band dieser Arbeit, S.14, abgedruckt.

229. Mehr dazu weiter unten im Abschnitt I.2.6. 
Alle diese Vor- und Nachworte belegen zum einen erneut, daß das Tempo in den ersten Jahren des 17. Jahrhunderts keinesfalls festgelegt und unveränderlich war und zum anderen, daß das Vokabular der Tempoworte breits bekannt und auch deutlich im Bezug zum Tempo verstanden wurde, und nicht etwa als Charakterbezeichnungen - denn eine "heitere" oder "schwermütige battuta" macht keinen Sinn. Dariber hinaus wird deutlich, daß die Tempodifferenzierungen weiterhin auch von den Gattungen abhängig sind; besonders ausgeprägt ist dies bei Lambardi und Frescobaldi.

\section{$1,2,4,3$, TEMPOWORTE}

1611 erschienen in der zweiten Auflage von Adriano Banchieris L'argano suonarino erstmals - soweit bekannt - Tempoworte im "eigentlichen Sinn"230, d.h. herausgelöst aus einem Satzzusammenhang und innerhalb eines Stückes. Fast alle Tempoworte dieses Druckes stehen in der "Battaglia"; dort allerdings in einer sobald nicht wieder zu beobachtenden Häufung (10 Anweisungen auf zwei Seiten zweistimmiger Partitur ${ }^{31}$ ). Die Verwandtschaft zu den ungefähr gleichzeitig auftretenden Tempoanweisungen in Satzform bei Claudio Monteverdi und Banchieri selbst (s.o.) ist deutlich erkennbar: Auch hier werden die Tempoworte zu einem großen Teil mit Registrationsanweisungen gekoppelt. Diese Tempoworte Banchieris haben verschiedene Deutungen erfahren. HerrmannBengen 232 sieht in ihnen zusätzliche Erläuterungen des bereits im Notenbild Ausgedrückten oder Alternativen zu den Möglichkeiten des Notenbildes. Lediglich der ersten Tempoangabe (Takt 9) gesteht sie Notwendigkeit zu; das darauf folgende "Presto" (Takt 21) erkläre nur die schwarze Notation. Aus dem zweiten "Adagio" (Takt 29) meint sie erkennen zu können, daß der semicircolo alleine offenbar seine Eindeutigkeit (Rückkehr zum langsameren Ausgangstempo) verloren habe. Für das folgende "allegro" (Takt 37) bietet sie gleich zwei Interpretationsmöglichkeiten: Entweder ändere sich am Tempo gar nichts und das "allegro" beschreibe lediglich die schnelleren Notenwerte, oder aber derselbe Taktschlag beziehe sich nun auf den nächst größeren Notenwert. Die Temposteigerung bei gleichen Notenwerten durch Tempoworte zunächst "Presto" (Takt 57) und dann darauf "Veloce" (Takt 64) - hätte Banchieri nach Herrmann-Bengen auch durch Halbierung der Notenwerte erreichen können²33.

Nur auf den Adagio-Allegro-Wechsel von Takt 37 geht Carl Dahlhaus ein234. Für ihn gehört in dieser Zeit ein Wechsel von Adagio zu Allegro untrennbar

230. Herrmann-Bengen, Tempobezeichnungen, S.44.

231. Die Battaglia ist im zweiten Band dieser Arbeit als Notenbeispiel I,2 abgedruckt.

232. Tempobezeichnungen, S.44.

233. Die Berufung auf Vincentino - er versah die unterschiedlichen Notenwerte mit je einem Tempowort (z.B. Semiminima = presto, Croma = veloce; $v$ gl . Herrmann-Bengen, S.44 sowie S.24) - ist doch etwas weit hergeholt.

234. Taktsystem, S.236 ff, besonders 239, wiederholt in Proportionenlehre, S. 360 , 
zusammen mit einem Wechsel von einem 2/2- zu einem 2/4-Takt; auch diesen Adagio-Allegro-Wechsel erklärt er mit einem solchen Schlagwechsel. Dabei wird der Taktschlag zwar beschleunigt, geht aber gleichzeitig von der Semibrevis auf die Mirima über, womit die Semiminima des "allegro" also langsamer wäre als die des "Adagio235. Ein solcher Minimentakt ist aber zumindest in der Theorie des späten 16. und fruhen 17. Jahrhunderts in Italien nicht nachweishar und auch kaum zu vermuten236.

Warum aber sollen die Tempoworte, wie Banchieri sie hier gebraucht, nicht schon im modernen Sinne verstanden werden? Tempomodifikationen waren - wenn auch nicht in dieser Fulle - bereits im 16. Jahrhundert bekannt und verbreitet; Vorworte verlangen für bestimmte Gattungen geradezu ein wechselndes Tempo. Auch das Notenbild selbst erklärt manches. So könnte der Grund für das erste "adagio" (Takt 9) durchaus auch in den, fiir einen Dreihalbetakt?37 in dieser Zeit ungewöhnlich kurzen Notenwerten liegen ${ }^{33}$. Freilich erklärt das darauf folgende "Presto" (Takt 21) die schwarze Notation239, fordert darüber hinaus aber zu einem nicht triolisch sondern wahrscheinlich stärker beschleunigtem Tempo auf240. Wenn nämlich das "Presto" nur die schwarze Notation erklärte, wäre das "Adagio" in Takt 29 nicht nötig, un wieder in das Anfangstempo zuríckzukommen, denn die Schwärzung endet dort ganz offensichtlich. Daß das oben mehrfach angesprochene "Allegro" in Takt 37 nicht einen Taktwechsel fordert und auch keine reine Beschreibung darstellt, ergibt sich aus der Parallelstelle in Takt 72. Hier steht bei denselben Noten dieselbe Tempobezeichmung, obwohl hier kein "Adagio" sondern ein "Veloce" vorangeht. Das kann nur einen Sinn ergeben, wenn "Allegro" in diesem Fall eine bestimmte, unabhängige Tempostufe meint. Wahrscheinlich bezeichnet "Allegro" für Banchieri - ebenso wie auch fuir Frescobaldi241, das - oder vielmehr ein - Grundtempo, ein "tempo ordinario". Insgesamt vier Abschnitte von Banchieris Battaglia sind ganz offensichtlich verwandt. Die Abschnitte Takt 1-8, 37-44, 72-79 und $89 \mathrm{~b}-$ Sch1uß weisen nicht nur dieselben Notenwerte auf, sondern sind auch die einzigen als "melodisch" zu bezeichnenden Teile dieses Werkes; sie werden dariber hinaus durch dieselbe Registration ("Ottava \& Flauto") verklammert. Daß bei allen diesen Teilen auch "Allegro"

235. Dahlhaus, Taktsystem, S.239.

236. Vg1. dazu weiter oben im Abschnitt I.1.3.

237. Unter Umständen soll die weiße Notation - sonst ublich bei der Prolatio perfecta (s.u.) - hier zusätzlich als "optische Bremse" wirken.

238. Die Dreiertakte dieser Zeit haben in der Regel Ritorne11-Funktionen. Cromen beim 3/2-Takt bzw. Semiminimen beim 3/1-Takt sind nur sehr selten anzutreffen - fast nie mehr als zwei in Folge. Vg1. dazu auch Arnold, Crace, S. 43 und 46 f sowie unten im Abschnitt I.2.4.

239. Weiter unten im Abschnitt I.3.2.2. Dort werden einige vergleichbare Stellen mit einem Umschwung von weißer in schwarze Notation zu nennen und besprechen sein.

240. Theoretisch wäre ein schwarzer Takt so lang wie zwei Drittel eines weißen Taktes (Triolierung der nicht perfekten Semibrevis).

241. S.o. 
vorgeschrieben ist, mit Ausnahne des ersten - hier versteht sich das Grundtempo von selbst - bestätigt diese These.

Ganz sicher handelt es sich bei der Folge "adagio" (Takt 45) - "Presto" (Takt 57) - "Veloce" (Takt 64) um eine Art Accelerando242. Jeweils eine Verdoppelung des Tempos, wie Herrmann-Bengen annimmt, wird man jedoch nicht vermuten, da die dynamische Wirkung einer Beschleunigung dadurch verloren geht. Die Tatsache, daß "Presto" und "Veloce" in diesem Accelerardo benutzt werden, nicht jedoch "Allegro", bestärkt erneut die These, daß es sich bei "Allegro" um ein Grundtempo handelt. Die anderen Tempoworte sind darm als Modifikationen dieses Grundtempos zu verstehen 243 .

AuBer in der Battaglia verwendet Banchieri in der zweiten Auflage des L'organo suonarino (1611) nur noch zweimal ein Tempowort, un eine besoridere Schlußwirkung zu erzielen: Sowohl beim "Primo" als auch beim "Secondo Dialogo"244 steht in den letzten Takten "Pieno \& graue". Der "Secondo Dialogo" enthält daruber hinaus auch Registrationsangaben ${ }^{245}$. Interessanterweise wird im "Secondo Dialogo" das Wort "grave" auch als Lagenbezeichnung verwendet: Der vollständige Titel lautet "Secondo Dialogo: Acuto \& Graue". Der Titel bezieht sich auf die beiden dialogisierenden Parteien, deren erste in Sopran- bis Tenorlage, die zweite aber in Alt- bis Baßlage notiert ist. "Pieno \& graue" kann jedoch nicht mit den Lagen zusammenhängen, denn diese werden jeweils mit einem Schlüsselwechsel angezeigt.

Insgesamt ist die Anzahl der Drucke mit Tempoworten im zweiten Jahrzehnt des 17.Jahrhunderts noch relativ gering. Der zweite dem Verfasser bekannte italienische Musikdruck246 mit Tempoworten ist das schon weiter oben im Zusammenharg mit den Tempoubberschriften zitierte Terzo libro di nuovi pensieri (1613), ebenfalls von Adriano Banchieri. Auch hier treten die Tempoworte nicht einzeln, sondern gemeinsam mit Lautstärkeangaben auf, die in vokaler Musik den Registerangaben der Orgelmusik vergleichbar sind. Für

242. So auch Braun, Stilwande1, S.84. Ein durch Tempoworte ausgedricktes Accelerando verwendete Adriano Banchieri auch in der "Bizaria del Primo Tuono al Graduale col Flauto all'ottaua" aus der dritten Auflage des L'organo suonarino" (1622), S.135; dort allerdings mit den Tempoworten "Più presto" und "Prestissimo" (die "Bizaria" ist auch enthalten in der Teilfaksimileedition der Auflage von 1638, S.57).

243. Der Gebrauch von Tempoworten zur Modifikation eines Grundtempos entspricht der historischen Entwicklung aus dem Gebrauch der Mensurzeichen. Diese Deutung der Tempoworte ermöglicht es auch, das Auftreten von Tempowortpaaren in dieser Zeit - s.u. - zu verstehen.

244. Auch die beiden Dialoghi sind in der Teilfaksimileausgabe enthalten (S. $31 \mathrm{ff}$ ).

245. Vg1. hierzu weiter unten im Abschnitt III.2.2.

246. Es ist anzunehmen, daß Tempoworte in anderen Ländern erst später Verwendung fanden. Schon die Tatsache, daß die italienischen Worte allgenein üblich geworden sind, bestätigt dies. 
den Organisten enthält der Titel des entsprechenden Stückes eine Anweisung zur Umsetzung dieser Angaben: "Vndecimo Concerto. Cantarsi piano con vn Registro, \& forte con dua"247. Die auftretenden Tempoangaben lauten "PIANO \& graue", "FORTE \& spirituoso" (!) sowie "PIANO, \& allegro"248. Zusätzlich enthält dieser Druck noch ein einzeln auftretendes "A11egro"249.

Bei diesen beiden Drucken Banchieris handelt es sich urn Partiturdrucke. Der früheste dem Verfasser bekannte Stimmbuchdruck mit Tempoworten im engeren Sinn ist Giovanni Valentinis Secondo libro de madrigali... concertati (1616). In diesem Druck finden sich vereinzelt die Tempoworte "Presto" und "Lento" - allerdings - wie auch schon die Tempouiberschriften - nur in der Continuo-Stimme.

Gattungsdifferenzierende Tempoworte finden sich in Giovanni Priulis Sacrorum concentuum ... pars prima und altra (1618/19). Zu Anfang jeder Canzone ist "Presto" notiert, während die Vokalkompositionen und auch die Sonaten keine Tempoworte tragen250. In einer Anzah1 dieser Canzonen finden sich darüber hinaus einige mit "Tardo" bezeichnete Teile - besonders an den Kadenzen. Wie auch in späteren Beispielen (s.u.) sind die Tardo-Abschnitte zusätzlich in langen Notenwerten geschrieben 251 und fallen durch ihre blockhafte Setzweise auf252. Das Ende eines solchen Tardo-Abschnittes markiert ein erneutes "Presto". Die Tempoworte stehen in allen Stimmen. Das Tempowortpaar "Presto" - "Adagio" benutzte nach Irngard Herrmann-Bengen ebenfalls 1619 Francesco Spongia Usper ${ }^{25}$.

247. S. 26. Zur direkten Parallelität von Lautstärkebezeichnungen und Registerangaben und vergleichbaren Angaben auch bei anderen Komponisten siehe unten im Abschnitt III.1.3.2.

248. Alle diese Angaben stehen im "Undecima concerto" (S.26-27). Es ist im zweiten Band dieser Arbeit als Notenbeispiel III, 3 wiedergegeben.

249. S. 68 .

250. Auch hierin ist wiederun eine Bestätigung der Theorie der Gattungstempi zu sehen. Während in vielen Drucken mit instrumentaler und vokaler Musik die Notenwerte in den instrumentalen Werken kürzer bzw. bedeutend kiirzer sind, entsprechen die Notenwerte vokaler und instrumentaler Musik in den Drucken Priul is einander. Für die Canzonen ist jedoch ein schnellerer Taktschlag vorgeschrieben. Daß diese Vorschrift bei den Sonaten fehlt, entspricht einer allgemeinen Tendenz bei Ensemble-Sonaten zur Gravität (vgl. แ. a. Giovanni Gabrielis "Sonata pian e forte" (1597)).

251. Auch solche das Notenbild verstärkenden Tempomodifikationen konnten bei der Verwendung der Mensurzeichen bereits beobachtet werden. Erst im dritten Jahrzehnt des 17. Jahrhunderts beginnt es sich durchzusetzen, daß Tempoworte in der Regel dem Notenbild entgegenstehen, wie dies aus späterer Zeit allgemein bekannt ist. Dazu weiter unten mehr.

252. Vgl. die Neuausgabe von Priulis Sacrarum concentuum ... pars prima, hrsg. von Albert Biales.

253. Tempobezeichnungen, Anhang, Tabelle 1. 
Auch eine Ritardando-Vorschrift findet sich bereits in einem Musikdruck des zweiten Jahrzehntes des 17.Jahrhunderts. In Giovanni Maria Trabacis secondo libro de ricercare (1615) steht am Ende einiger Versi254, einer Toccata255, einer Gagliarde256 und einer Partita257 "Allarga la Battuta". Einmal findet sich dieser Eintrag auch vor einem ubergang von einem Dreiertakt zu einem geraden Takt258. Nie hingegen tritt diese Ritardando-Vorschrift in den polyphonen Stücken (z.B. Ricercaren) dieses Druckes auf.

War die Anzah1 der Drucke mit Tempoworten aus dem zweiten Jahrzehnt des 17. Jahrhunderts noch recht uberschaubar, so ist im dritten Jahrzehnt eine starke Zunahme in der Benutzung der Tempoworte zu verzeichnen. Knapp $20 \%$ der ausgewerteten Drucke der Jahre 1621-1630 sind bereits mit Tempoworten versehen259. Aufgrund dieser großen Zahl soll im folgenden nicht melir jeder Druck einzeln behandelt werden, sondern es sollen vielmehr die Hauptanwendungsarten der Tempoworte zur Sprache kommen und nur einige Beispiele näher betrachtet werden.

Auch im dritten Jahrzehnt des 17. Jahrhunderts werden Tempoworte oft verwendet, um einzelne, langsamere Abschnitte einzuschieben, wie dies berejts bei Priuli (5.o.) zu beobachten war260. Die Bezeichnung für diese Einschiibe: lautet "Adagio" (mit den Nebenformen "Adasio", "Adaggio" etc.), seltener auch "Tardo". Nicht immer ist das Ende des Adagio- bzw. Tardo-Teiles ausdricklich durch ein zweites Tempowort angezeigt261. Teilweise fehlt der Hinweis auf einen solchen Einschub auch ganz262. Da sich jedoch ein ganz

254. S.45, 47, 50, 54, 57, 61, 71, 74, 78 .

255. "Toccata Quarta, \& v1tima, d̀ Cinque" (S.95).

256. "Gagliarda Terza a 5 sopra 1a Montoana" (S.110).

257. "Partita Terza" (S.119).

258. "Gagliarda Terza a 5 sopra la Montoana" (S.110).

259. Vg1. Liste aller Drucke mit Tempoanweisungen im zweiten Band dieser Arbeit.

260. $\mathrm{Zu}$ nennen sind folgende Drucke: N. Corradini, Motetti (1624), G. Frescobaldi, In partitura il primo libro delle canzoni (1628), 0. M. Grandi, Sonate (1628), D. Castello, Sonate concertate... libro primo und libro secondo (beide 1629), A. Grandi, Motetti a 1, et 2 (1629), B. Marini, Sonate (1629), B. Montalbano, Sinfonie (1629) und G. Rovetta, Madrigali concertati (1629).

261. So bei B. Marini und 0. M. Grandi. Parallelen zu einer solchen Praxis finden sich auch bei den dynamischen Bezeichnungen (s.u.).

262. Die als Adagio-Einschub gekennzeichneten Stellen sind nicht in allen Ausgaben zu Frescobladis primo libro delle canzoni gleichermaßen verzeichnet. Während in der ersten Ausgabe als Stimmbuchdruck ( 1628 - bei der gelegentlich zitierten Ausgabe von 1623 handelt es sich um einen Lesefehler) Tempoworte ganz fehlen, finden sich in der von Frescobaldis Schüler Bartolomeo Grassi herausgegebenen Partiturausgabe (ebenfalls 1628) neun AdagioAllegro Paare - acht davon als Einschub und eins am Anfang einer Sonate. In der Basso per l'organo-Stimme (nur sie enthält alle Canzonen) der Stimm- 
typischer Stil für diese Einschube herausgebildet hat, sind sie in der Regel gut zu erkennen. In der uberwiegenden Zahl werden diese Einschibe am Ende eines Abschnittes verwendet. Frescobaldi bevorzugt die ubergänge von einem Dreier zu einem geraden Takt, Priuli benutzt Tardo-Stellen an jeder Art von Kadenz. In der geringstimmigen Musik zeichnen sie sich durch eine große Zahl von Vorhalten und besonders afféktreichen Figuren aus. In Einzelfällen, etwa bei einigen Sonaten Biagio Marinis, ist es jedoch kaum möglich, ein fehlendes Tempowort, z.B. zwischen zwei Tardo-Teilen zu ergänzen263. Unter Umständen rechnet Marini hier sogar mit einer allmählichen Beschleunigung.

Mit den langsanen Eirschiben ist in der geringstimmigen Musik wahrscheinlich auch ein freieres Spiel verbunden. In auffälliger Weise nimmt die Zahl der Tempoworte in der Partiturausgabe von Frescobaldis primo libro delle canzoni (1628) mit steigender Stimmenzahl $\mathrm{ab}^{264}$. In diesen Adagio-Teilen kommt es nur äußerst selten vor, daß zwei Stimmen sich gleichzeitig bewegen. In der Regel wechselt eine Stimne die Tonhöhe während die andere(n) eine Note aushält/halten. Nur in einer einzigen Canzone sind im Adagio zwei. Stimmen außer dem $B c$, besetzt265.

Es sei daran erinnert, daß die Bedeutung "frei" auch fiir "Largo" festgestellt wurde, dort allerdings als Hauptbedeutung. Der Grund fur diese Parallele wird weniger in den Wortbedeutungen, als vielmehr in den mit diesen Worten bezeichncten Stellen zu suchen sein. Die Adagio-Einschibe Frescobaldis erinnern sehr an die "esclamationi" und an die Vertonungen von "parole affettuose", die nach Agazzari die "missura molta larga" erfordern. "Largo" als Tempowort gab es in dieser Zeit jedoch nicht (s.o.).

Eng verwandt mit diesen Einschüben sind Tardo-bzw. Adagio-Schlußteile 266 oder -Satzanfänge267. Sie entsprechen diesen in der Kompositionsart.

buchausgabe von 1634 tritt das Paar Adagio-Allegro nun 33 mal auf; hinzu komut drei mal Adagio-Presto sowie zahlreiche einzelne Tempoworte. Die Canzonen sind jedoch weitgehend dieselben (vgl. Harper, Canzonas, Tabellen auf S.271 f).

In anderen Drucken sind solche Einschibe grundsätzlich nicht gekennzeichnet, soudern nur an ilurer Faktur zu erkennen, so z.B. in G. B. Riccios Terzo libro delle divine lodi (1620; besonders deutlich in der Canzon la Savoldi vg1. die Neuausgabe, hrsg. von Rudolf Ewerhart, Heft. II, S.13 ff, Takt 13, 27 und 40 ).

263. Vg1. die "Sonata quarta" aus Sonate (1629), abgedruckt bei Iselin, Marini, Notenheispiele, S.11 ff.

264. In sechs Canzonen "a uno", zwei Canzonen " $a$ due", keiner "a tre" und keiner "a quattro" finden sich Tempoworte.

265. "Canzona nona" (S.23).

266. Adagio-Schliisse finden sich in folgenden Drucken aus dem zweiten Jahrzelint des 17. Jahrliunderts: A. Grandi, Motetti a $t$ e 2 (1621), Motetti a voce sola (1621 zitiert nach Seelkopf, Grandi, Bd.II, S.155) sowie Motteti a 1 e 
Ebenfalls recht häufig werden Tempoworte zur zusätzlichen Erläuterung bei Taktwechseln verwendet. Hier zeigt sich deutlich, daß auch gleich notierte Dreiertakte verschiedene Tempostufen repräsentieren können. Ein und dieselbe Taktart kann innerhalb eines Satzes einmal mit "Presto" und ein anderes mal mit "Adagio" überschrieben sein268. Auf verschiedene Tempostufen deutet es aber auch hin, wenn eine Taktart innerhalb eines Druckes sowohl mit als auch ohne Tempowort vorkummt269.

Am Ende des dritten Jahrzehntes des 17.Jahrhunderts beginnt die Verwendung der Tempoworte bereits die zunächst engen Schranken zu verlassen und der Gebrauch der Worte wird zunehmend flexibler. Stellvertretend auch für andere Drucke mit ausgedehntem Gebrauch der Tempoworte, sol1 num ihre Verwendung in dem ersten Buch der Sonate concertate von Dario Castello (1629)270 dargestellt werden 271 .

Nicht nur die Zahl der Drucke mit Tempoworten, sondern auch die Zahl der Tempoworte innerhalb eines Druckes selbst nahn in dritten Jahrzehnt des 17. Jahrhunderts zu. Dario Castellos primo libro enthält (in der Generalbaßstimme 272) 87 Tempoworte in insgesamt 12 Sonaten - also im Durchschnitt

2 ... libro terzo (1629), S. Rossi, Il quarto libro de varie sonate (1622), G. B. Buonamente, Il quarto libro de varie sonate (1626), D. Mazzocchi, La catena d'Adone (1626), P. Possenti, Concentus armonici (1628) und B. Marini, Sonate (1629). Tardo-Schlüsse verwendete auch Priuli in Sacrarum concentuum ... pars prima/altera $(1618+1619)$. Vgl. dazu auch Apel, Klaviermusik, S. 469 .

267. Adagio/Tardo-Anfänge sind dem Verfasser aus dieser Zeit aus einer Sonate aus S. Rossis quarto libro de varie sonate (1622), einer Canzone aus Frescobaldis In partitura il primo libro delle canzoni (1628) sowie einer Sonate aus B. Marinis Sonate (1629) bekannt.

268. Z.B. in den Sonate concertate von G. Scarani (1630), vgl. auch unten im Abschnitt I.2.7.

269. Es gilt immer noch, daß auch ein Mensurzeichen alleine - in Abhängigkeit von der Gattung - eine Tempostufe beinhaltet.

270. Eitner, Quellen-Lexikon, Bd.II, S.361 verzeichnet von Castellus primo libro eine Auflage von 1621. Da kein weiterer IIinweis auf diese Auflage bekannt ist und sich auch im Titel der Auflage von 1629 kein Hinweis auf ein friihere Auflage findet, wird im folgeuden davon ausgegangen, daß die Auflage von 1629 die fruheste war. Diese Ansicht vertritt auch Marcello Castellani im Vorwort zur Faksimileausgabe der Sonate concertate (vgl. (2uellenliste). Winkler, Instrumentalwerke, S.300, ordnet jedoch das primo libro in seiner chronologischen ubersicht unter 1621 ein.

271. Das "primo 1ibro" ist in einer Faksimileausgabe nach der Ausgabe von 1658 mit einer verkleinerten Wiedergabe der Stimmbiicher der Ausgabe von 1629 als Anhang des Canto primo-Stimmbuch (vg1. Quellenliste im Anhang) zugänglich. Die Sonaten 3, 5, 9 und 12 sind auch in Neuausgabe, hrsg. von Eleanor Selfridge-Field erschienen.

272. Fast alle Tempoworte stehen genauso in den anderen Stimmen. 
etwas mehr als sieben Tempoworte pro Sonate. Keine Sonate hat weniger als drei $273 \mathrm{bzw}$. mehr als zehr274 Tempoworte.

Häufigste Anwendung der Tempoworte sind hier wieder Adagio-Einschübe. Das Ende eines Adagio-Teiles kann auch ein beliebiger Taktwechsel anzeigen275. offenbar genügt sogar eine Fermate, um die Gultigkeit eines Tempowortes zu beenden, denn gelegentlich wird es nach einer Fermate wiederholt 276 .

Fast alle Sonaten tragen ein Tempowort gleich zu Anfang in der Art einer Satziuberschrift277. Dieses lautet 9 mal "Alegra" und einmal "Adasio"278.

"Presto" und "alegra" bzw. "alegro" können - wahrscheinlich ohne Unterschied - die Funktion der Beendigung eines Adagio-Abschnittes ubernehmen. Sie werden jedoch auch als verschiedene Tempostufen in Folge eingesetzt. Dies ist am auffälligsten in der 6.Sonate. Hier wird der Anfangsteil ("Alegra") ohne Veränderung in der musikalischen Faktur sechs Takte vor dem ersten "Adagio" durch ein "Presto" nochmals beschleunigt279.

Castello verwendete in Adagio-Abschnitten bereits ausgesprochen häufig viele kurze Notenwerte, wie es in späterer Zeit iblich wird280.

\subsubsection{TEMPO UND PARTITUR}

Auf eine Wechselbeziehung zwischen verbaler Tempoanweisung und Partitur wurde weiter oben im Zusammenhang mit den Tempoanweisungen in Monteverdis Druck Sanctissimae Virgini messa (1610) bereits hingewiesen. Hier erschienen verbale Tempoangaben nur in der Continuo-Stimme einiger geringstimmiger Sätze, die - im Gegensatz zu vielen anderen - in der Generalbaßstimme nicht in Partitur gedruckt sind. Die Tempoanweisungen Monteverdis begründen das geforderte Tempo mit einer Beschreibung der zu singenden Partien: "va sonato tardo perchè li doi tenori cantano di semicroma" etc.281. Es ist offensichtlich, daß diese Anweisung hätte entfallen können, wenn diese Stiicke in

273. Nur drei Tempoworte stehen in der Sonate $\mathrm{Nr} .3$.

274. 10 Tempoworte sind in den Sonaten Nr. 4, 8 und 9 eingetragen.

275. Dies ist ganz offensichtlich in der 2. Sonate der Fall: Das drittletzte Tempowort ("adasio") steht in einem Abschnitt im geraden Takt. Beim folgenden Taktwechsel zun Dreiertakt steht erneut "adasio". Der letzte Abschnitt im geraden Takt beginnt mit zwei Takten ohne Tempowort und einer Kadenz, nach der ein letztes "adasio" steht.

276. So z.B. in der 1 . Sonate.

277. Lediglich die Sonaten fünf und sieben beginnen ohne Tempowort.

278. Sonate $\mathrm{Nr} .2$.

279. Ähnlich eine Stelle in der 6. Sonata. Der vorletzte Abschnitt im geraden Takt beginnt "adasio", dann folgt ein "alegro" und darauf wiederum ein mit "presto" überschriebener Dreier.

280. Adler, Stil, S.139.

281. Vol1ständig weiter oben. 
Partitur gedruckt wären, denn dann hätte der Organist sehen kömnen, welche Notenwerte gesungen werden. Wahrscheinlich geht auch Monteverdi davon aus, daß der Organist in solch einem Fall selbständig ein angemessenes Tempo gewählt hätte, denn bei keinem der Sätze in Partitur findet sich ein vergleichbarer Tempohinweis.

Es stellt sich die Frage, warum Monteverdi die mit Tempoanweisungen versehenen Sätze nicht in Partitur gedruckt und welche Konsequenzen dies für die in Partitur gedruckten Sätze hat. Das Partiturproblem in seiner Gesamtheit wird an späterer Stelle - im Zusammenhang mit der Generalbaßproblematik - noch zur Sprache kommen. Für die Tempofrage mag zunächst ein Blick auf alle in Vol1partitur282 wiedergegebenen Stücke dieses Druckes genügen. In vollständiger Partitur enthält die Generalbaßstimme des Druckes von 1610:

1. das "Crucifixus quatuor vocibus" der "Missa in illo tempore"

2. die Concerti "Pulchra es" und "Duo Seraphim"

3. aus dem Magnificat I das "Deposuit" und das "Gloria Patri" sowie das "Deposuit" aus dem Magnificat II.

Das erste Stiick in Vollpartitur, das Crucifixus der Missa, ist in einem, von den anderen Stücken verschiedenen Zusammenhang zu sehen. Auf diese Tradition wird bei der Behandlung der Partituren noch näher einzugehen sein. Rein äußerlich ist der Unterschied schon daran zu erkennen, daß die Partitur des Crucifixus nur die vier Chorstimmen enthält, ohne eine zusätzliche Generalbaßstimme, wie sie in den anderen Partituren zu finden ist.

Die beiden Concerti "Pulchra es" und "Duo seraphim" gehören dem Bereich des von der Monodie stark beeinflußten geistlichen Konzertes an. Zahlreiche Äußerungen uber die Freiheit des Tempos in solchen Kompositionsformen - und auch iber die Notwendigkeit der Partitur bei solcher Musik - sind bekannt283. Es war in dieser Zeit ublich, solche solistischen sticke in der Generalbaßstimme in Partitur zu drucken 284.

Auch die Partitur des "Gloria Patri" (Magnificat I) ist im Zusammenhang mit dem freieren Musizieren monodischer Musik zu sehen. Die hochvirtuosen Tenorstimmen können unmöglich ständig genau im Takt gesungen werden285. Weitere Temposchwankungen bringt das Echo mit sich - insbesondere bei entfernt plaziertem Echo-Sänger. Dies ist besonders deutlich an Stellen, wo innitten eines Laufes die Harmonie wechselt. Ebenfalls in Echo haben die Partituren der beiden "Deposuit"-Sätze ihre Berechtigung. Auch hier sind die Harmoniewechsel problematisch.

282. Eine ganze Reihe von Sätzen sind in der Generalbaßstimme in Teilpartitur wiedergegeben. Eine ausfuhrliche Beschreibung derselben wird wejter unten folgen. Auch die Teilpartituren können freilich eine Bedeutung fiir das Tempo haben.

283. Vgl, weiter unten im Abschnitt II.2.3.3.

284. Vg1. ebenda.

285. Vg1. Monteverdi-GA, Bd.XIV, 2.Halbband, S.315 ff. 
Ganz anders aber die Sätze mit den Tempoanweisungen. Hier verlangen die in verhältnismäßig kurzen Notenwerten gehaltenen canti fermi nach einer genauen Rhythmisierung; nur darf das Tempo nicht zu schnell sein, um diese auch durchhalten zu können.

Es mag an dieser Stelle dies eine Beispiel genuigen, un auf den Zusammenhang von Partiturveröffentlichung - Partituren sind in dieser Zeit entgegen der verbreiteten Meinung bereits recht häufig (s.u.) -, verbalen Tempoanweisungen und Tempo hiruzuweisen. Genauer wird hierauf im Kapitel uber die Partituren einzugehen sein.

\subsection{TAKTSTRICH UND TAKTSCHLAG}

Bei der Betrachtung der Entwicklung des Takstriches ist zunächst zwischen Takstrichen in partiturartigen Quellen und solchen in Einzelstimmen zu unterscheiden. Takt- oder besser Gliederungsstriche in Partituren oder partiturähnlichen Quellen gibt es fast seit den Anfängen der mehrstimmigen Musik. Senkrechte Striche in in Partituranordnung geschriebener Musik sollen die Orientierung erleichtern. Solche Orientierungstriche finden sich bereits in friihen Organum-Partituren286 und in fast allen Arten der mehrstimmigen Tabulaturen287. In Verbindung mit der weißen Mensuralnotation findet man solche Orientierungsstriche in den seit Beginn des 16. Jahrhunderts erscheinenden Drucken mit Musik für Laute und Gesang. Die in den Lautentabulaturen iblichen orientierungsstriche werden hier auch in die Gesangsstimme übernommen288. Die Abstände dieser Striche entsprechen in der Regel schon denen der späteren Taktstriche: Die Norm ist der Abstand von einer Brevis zwischen zwei Strichen. Ganz selbstverständlich ist es dann auch, daß solche Orientierungsstriche in den aus dem 16.Jahrhundert erhaltenen Spartierungen meist vokaler Musik ebenso enthalten sind, wie in allen frijhen Partiturdrucken289.

In der zweiten Hälfte des 16. und vermehrt zu Beginn des 17. Jahrhunderts erschienen nun auch senkrechte Striche in Notierungen nur einer Stimme. Auch diese Striche dienen zunächst nur der Gliederung und zur Orientierung für den Musiker. Eine solche Untergliederung des Notenbildes wurde nötig durch eine steigende Zahl von Noten, die, bei gleichzeitiger Verlangsamung des Tempos, innerhalb einer Battuta gesungen oder gespielt werden sollten. Ein "tactus alla minima", wie er um die Mitte des 16 . Jahrhunderts in verschie-

286. Vgl. etwá Besseler Schriftbild, Abb. 6 ff.

287. Vgl, daselbst, Abb.74-82. Fehlen die Orientierungsstriche, so sind sie in der Regel durch Zwischenräume ersetzt.

288. Vgl. daselbst, Abb. 82 sowie die Abbildung aus Bossinensis Tenori e contrabassi intabulati col soprano in canto figurato, gedruckt 1509 bei Petrucci bei Gianluigi Dardo, Art. Bossinensis, Franciscus in MGG, Bd.15, Tafel 35 .

289. Vg1. außer den in Quellenverzeichnis genannten Partiturdrucken die Abbildungen bei Lowinsky, Early Scores sowie weiter unten im Abschnitt II.2. 
denen, meist deutschen Traktaten beschrieben wird, ist in den italienischen Quellen nicht nachzuweisen290.

Eine Aufteilung der taktgebenden Semibrevis in vier Teile ist zwar schon aus dem 16.Jahrhundert belegt, jedoch kann die damals praktizierte Vierteiligkeit nicht mit einem wirklichen Minimentakt verglichen werden: Zusätzlich zu Aufschlag und Niederschlag beschreiben einige Autoren Ruhepunkte in den beiden Extrempositionen als eigene Schlagzeit. Die frïheste dem Verfasser bekannte Beschreibung eines solchen vierteiligen Taktschlag findet sich im Organo de cantori Giovarni Battista Rossis (geschrieben 1585). Nach Rossi besteht der Takt aus vier Semiminimen, deren jede ihr eigenes "tempo" bekomnt: Zuerst eine Abwärtsbewegung, dann ein Ruhighalten, anschließend wieder eine Aufwärtsbewegung und wiederum ein Ruhighalten; entsprechend besteht auch ein Dreier aus zwei Bewegungen und einem Ruhighalten291. Eine ähnliche Aussage von Francesco Piovesana (1627)292 1 ieß Carl Dahlhaus schon

290. Einzig Agostino Pisa erwähnt den Taktschlag "alla minima", lehnt ihm jedoch ab (vgl. Dirr, Auftakt, S.30). Dennoch ist ein solcher Minimentactus von Machatius, Tempi, S.90 ff, Herrmann-Bengen, Tempoanweisungen, $\$ .42$ und $53 \mathrm{ff}$, sowie von Dahlhaus, Taktschlag u.a., immer wieder zur Deutung vieler Probleme herangezogen worden - einen Beleg für diesen Taktschlag bleiben jedoch alle schuldig.

291. Vollständig lautet diese Stelle bei Rossi: "Questo tatto si fà con dua mouimèti cotrarij cō due quieti, vn'all in giù, \& l'altro all'in su: perche com'habbiamo visto nella fisica, (libro ottauo) mostrassimo insieme col filosofo, che è impossibile far questi motti retti in su \& in giù sēza quiete, il che no occorre nell'circolare che puote il suo moto esser senza quiete. Di qui soluerai vn dubio da'Teorici non più mosso, ò per dir meglio saprai partire, che diuidendo vna semibreue in quattro semiminime ad ogn'una darai il suo tempo, \& vno non eccederà l'altro: il primo sarà, il primo moto all'andar in giù, nel posarti per tonar all'indietro, sarà il secondo tempo che porterà la secondo (semi-)minima, il terzo tempo sarà l'altro mouimento che porterà la terza semiminima, l'altra quiete porterà l'ultima nota nera, è cosi due quieti, \& dua moti saranno quattro tempi equali, \& ogn'vna di queste nere hauerá il suo tempo equale, come battendo \& cantando si pud esperimentare da qual si voglia perito cantore. Vedete nell'ottauo della nostra fisica d̀ carte 244. Lettione 17. colonna terza, dove dice, impossibile est esse continuum motum qui est rectitudine, \&c. Questo s'intende del tatto ma l'inequale si divide in tre tempi, \& vno non eccede 1'altro: cioè in dua moti \& vna quiete, è tutti alterati, \& la quiete non eccedi $i$ moti. Vna delle tre figure, va al prima moto, la seconda, alla quiete, la terza all'altro moto, perche in questo moto retto, Tanta, est quies quantus est motus" (S.10).

292. Misure harmoniche, Venedig 1627, S.60: "La Compositione di poi della battuta è di due parti, la prima delle quale è il battere, e la seconda l'elevar della mano: di più in cadauna di queste parte sono doui Tempi, di modo que in tutto sono quattro: et questi si distribuiscono in questo modo: cioè, nell'isteso tempo dell'abbassat'uno, e nel fermar la mano à basso, un' 
fiir das frihe 17. Jahrhundert einen Viervierteltakt annehmen293. Ferner glaubt er, den verschiedenen Stilen verschiedene Taktierarten zuordnen zu können: Für Messen und Motetten in der Tradition der prima prattica ein Fortleben der iberlieferten Taktierart der "continua motio", für die moderneren Werke hingegen den Viervierteltakt, der bereits den "Akzentstufentakt" repräsentiere294. Eine solche Differenzierung der Taktierarten wird jedoch in keiner der dem Verfasser bekannten Quellen erwähnt. Sofern man von einem anderen Taktbewustsein sprechen kann - und in der Tat spricht einiges hierfuir - wird man dies jedoch auf alle Musik anwenden muissen. Gerade der fruihste bekannte Zeuge fiir das vierteilige Verständnis des Taktschlages kommt aus dem Bereich der prima prattica: Rossis Organo de cantori behandelt ausschlie日lich traditionelle Musik für den gottesdienstlichen Gebrauch295. Bedenkt man die Bedeutung des Wortes Battuta (Schlag), so erscheint die "continua motio" eher als Idealvorstellung denn als Beschreibung der Realität. Die Tatsache, daß nun beginnend mit dem späteren 16. Jahrhundert diese Abweichungen der Praxis von der Theorie wahrgenommen werden, bestätigt, daß die Akzentpunkte an Bedeutung gewinnen296. Hinzu kommt sicher die Tatsache, daß der verlangsamte Taktschlag, den man aufgrund der kurzen Noten wird annehmen müssen, den tatsächlichen Verlauf der Taktierbewegung deutlicher sichtbar macht 297 .

Zwar bedeutet die von Rossi und Piovesana beschriebene Vierteiligkeit des Taktschlages sicher eine Betonung der ersten Zählzeit298 (kein gleichmäßig

altro vien distribuito: nell' elevar poi similmente si applica il terzo, e nel fermar la mano in alto, il quarto: il qual modo di distribuir questi tempi è il verso, e reale." (Zitiert nach Dahlhaus, Geschichte des Taktschlagens, S.117).

293. Dahlhaus, Taktschlag, S.116 ff. Dahlhaus kennt die entsprechende Ste1le bei Rossi nicht (vgl. auch Dahlhaus, Proportionenlehre, S.360 f).

294. Ebenda, S.119 f.

295. Vgl. die Beispiele in der Seconda parte dell'organo de cantori. Dieser zweite Teil enthält in erster Linie motettische Werke sowie einige Instrumentalkanzonen - der gottestdienst1iche Gebrauch solcher Werke ist vielfältig belegt (s.u. im Abschnitt II.3.3.2.4) - und Falsobordoni. Den Abschluß des Beispiel-Teiles bilden Proportionsbeispiele von Palestrina und Josquin interessanterweise ohne Text.

296. Die Lehren von der Dissonanzbehandlung zeigen, daß auch dem 16.Jahrhundert ein BewuBtsein für Taktakzente nicht völlig fehlte (vgl. u.a. Dahlhaus, Taktschlag, S.120).

297. Es ist kaum anzunehmen, daß sich die Bewegung des Dirigierenden im gleichen Maße verlangsant hat, wie der Takt.

298. Interessanterweise hat sich die Lehre von den Ruhepunkten anscheinend in Bezug auf die Dreiertakte am schnellsten verbreitet. So erklärt D. Hizler, Newe Musica, 2/1628 (von der ersten Auflage von 1615 ist kein Exemplar nachweisbar), den geraden Takt noch in herkömmlicher Art und Weise: "Was ist mensura temporis binaria oder Communis? Es ist ein Tact oder Schlag / welcher in zwey gleiche theil der zeit / darinnen er geschicht / kan abge- 
Hinauf und Hinab sondern ein Hinabschlagen), es ergibt sich jedoch daraus noch kein wirklicher Vierertakt. Bei einem Vierertakt bekommt jeder Taktteil eine eigene Bewegung, die dem Musiker genau dessen Länge anzeigt; ein Innehalten allein hilft wenig. Der zweiteilige Schlag (den ja auch Rossi und Piovesana ausdrick1ich beschreiben) wird durch die Unterteilung in Bewegung und Ruhe nur deutlicher, nicht aber aufgehoben 299 .

Der Zusammenhang der erwähnten Ruhepunkte mit den Betonungen (Schlagen!) und nicht mit einer vierteiligen Aufteilung des Taktes, wird sehr schön deutlich in der Beschreibung des Taktschlages durch Pier Francesco Valentini (15861657). Um 1643300 beschreibt er ebenfalls einen vierteiligen Taktschlag, doch beim ihm handelt es sich nicht um vier gleiche Teile, sondern die beiden Bewegungen dauern je ein Achtel des Taktes, die Ruhezeiten je drei Achte1301. Hier ist also das Schlagen noch sehr viel ausgeprägter. Von einem Vierertakt liegt dies jedoch weit entfernt ${ }^{302}$.

theilet werden: Nämlich / ein theil darinn man niderschlägt / unnd ein theil / darinn man auffhebet: heisset auch Tactus vulgaris." (S.56 f). Der Dreier ist fur Hizler jedoch bereits dreiteilig: "Was ist mensura temporis proportionata oder tripla? Es ist ein Tact oder Schlag / welcher in drey gleiche theil der zeit / darinnen er geschicht / kan abgetheilet werden: Nämlich in eine zeit / darinnen man niderschlägt / die ander zeit / da man unten hält / und die dritte zeit / darinnen man wider auffhebt: heisset auch mensura ternaria, oder Tactus perfectus." (S.57). Einen dreiteiligen Taktschlag kennt auch Agostino Pisa (1611, vg1. Dürr, Auftakt, S.27).

299. Ein wirklicher vierteiliger Taktschlag - mit vier Bewegungen - kommt wohl erst Ende des 17. Jahrhunderts auf. Noch L. Penna, Li primi albori musicali (2/1679, erste Auflage 1672) beschreibt nach Schünemann, Dirigieren, S.122 f, einen vierteiligen Takt ganz ähnlich dem von Rossi beschriebenen. D. Speer, Grundrichtiger Unterricht (2/1697), beschreibt zwar fur den "schlechten" sowie für den "Trippel" den alt überkommenen Taktschlag (besonders prägnant in der "TABELLA GENERALIS" (S.19): "Das XIV. Capite1. Zeigt den Tact. Dieser ist eine gleiche Nieder= und Aufhebung mit der Hand im schlechten Gesang. Im Trippel aber wird die Hand langsam nieder / und geschwinder aufgehoben.", erwähnt aber für einen Ausnahmefall bereits den Viererschlag, wie er bis heute benutzt wird: "6. Ein Zwölffachtel TrippelGesangs=Tact" (hier ist in der Auflage von $1597 \mathrm{fälschlicherweise} \mathrm{8/12} \mathrm{statt}$ $12 / 8$ gedruckt) "dieser hat eine besondere Mannier / theils tractieren ihn nach eines schlechten Gesangs langsamen Tact, welches nicht gar unrecht , aber am gewiesesten und besten ists / wann er Viertelweiß tactiert wird / als das erste Viertel mit der Hand unter sich / das andere auf die lincke Seit / das dritte auf die rechte Hand / das vierte uber sich oder in die Höhe / diß ist seine rechte Manier." (S.17).

300. Nach Murata, Valentini, S.327.

301. S.328 ff.

302. Es mag dahingestellt bleiben, ob die Beobachtungen Rossis und Piovesanas oder die Valentinis näher an den tatsächlichen Taktierbewegungen waren. 
Die verwendeten Notenwerte wurden im späten 16. und frihen 17. Jahrhundert immer kiirzer, woraus auf eine Verlangsamung des Tempos zu schließen ist ${ }^{303}$. Da weiterhin der "tactus alla semibreve" die Norm war, mußten immer mehr Noten auf einen immer länger andauernden Taktschlag verteilt werden. Die Orientierung wurde zweifelsohne komplizierter. Das Einfügen von Orientierungsstrichen konnte hier die ubersicht erleichtern.

Es ist sicher kein Zufall, daß es sich bei dem friihsten bekannten Musikdruck mit Orientierungstrichen in einzelstimmenähnlicher Notation ${ }^{304}$ um Diego Ortiz' Trattado de glosas von 1553 handelt ${ }^{305}$. Ohne daß sich ein Vorbild in einstimmiger Notierung firden läßt, versah Ortiz einen Großteil der Stücke seines Trattado mit solchen Orientierungs- oder Taktstrichen ${ }^{306}$. In diesen Stiicken unterscheidet sich seine Taktstrichsetzung nicht von der im 17 . Jahrhundert iblich gewordenen. Die Taktstriche stehen im wesent1ichen rege1mäßig im Abstand einer Brevis. Ein Taktstrich steht jedoch nicht:

1. zwischen zwei langen Nuten (etwa Semibreven oder Breven) oder

2. wenn nach einer längeren Note Pausen folgen.

Ein Grund, warum gerade in diesem Druck Taktstriche stehen, kann nur in dem besonderen Inhalt. des Druckes gesehen werden. Nachdem im ersten Buch des Trattado kurze Verzierurgen zu einzelnen Intervallen und Klauseln behandelt. wurden - voneinander mit Trennungstrichen ${ }^{307}$ getrennt - folgen im zweiten Buch Bearbeitungen von verschiedenen Chansons und Madrigalen sowie Improvisationen iber bestimmten Akkordfolgen. Während die Chansons und Madrigale in der Regel aus weiken Noten bestehen und Cromen nur sehr selten in Zweier-

303. Auf die ungleichmäßige Zunahme der kurzen Notenwerte in den verschiedenen Gattungen und auf die daraus resultierenden ungleichen Gattungstempi wurde bereits hingewiesen.

304. Mit einzelstimmenähnlicher Notation sind hier Notierungen nur einer Stimme außerhalb eines Stimmensatzes gemeint. Dies ist z.B. in den Diminutionstraktaten der Fal1.

305. Von Ortiz Tratado erschienen zugleich zwei verschiedene Ausgaben. Diese unterscheiden sich jedoch nur im Titelblatt sowie in den längeren Texten; in der einen Ausgabe sind sie italienisch, in der anderen spanisch (beide erschienen in Rom). Die Noten sind jedoch dieselben. Auch kürzere Texte zwischen den Nuten sind in beiden Ausgaben spanisch geblieben. Die Taktslrichsetzung kann nach dem Faksimile der italienischen Ausgabe (vgl. Quellenliste) oder aber auch nach der recht genauen Neuausgabe Max Schneiders studiert werden. Vgl. hierzu auch Wolf, Handbuch, Bd. I, S.427 f.

306. So besagt auch der Name "Taktstrich" zunächst nur, daß er nach jedem Takt oder "tactus", "tatto", "battuta" steht - er war ja sowohl als Begriff als auch als Maß durchaus bekannt. Die akzentgebende Bedeutung des Taktstriches ist nachträglich hinzugekommen; vgl. wejter unten.

307. Diese Trennungsstriche unterscheiden sich im Äußeren - zunindest in den an dieser Stelle zu besprechenden Quellen - nicht von den Takt- oder Orientierungsstrichen. Sie haben hier die Aufgabe, das Ende eines Beispieles anzuzeigen. Vgl. hierzu weiter unten im Abschnitt IV.2.2.2 und 2.2.3. 
gruppen als Kadenzverzierungen auftreten, bestehen die Bearbeitungen überwiegend aus Cromen; gelegentlich finden sich sogar Gruppen von bis zu vier Semicromen. Diese Gruppen von Semicromen sind in der Mitte des 16. Jahrhumderts noch etwas Besonderes, eine Tatsache die sich auch in Spielanweisungen Ortiz' niederschlägt308. Die Taktstriche hatten hier, wie auch in den Partituren und Tabulaturen, die Funktion von Orientierungsstrichen; sie halfen dem Musiker, die Vielzahl der Noten auf den Takt zu verteilen. Ortiz hat sie vermutlich von den Tabulaturen übernommen.

In ganz ähnlichem Zusammenhang benutzte Girolamo Dalla Casa im zweiten Buch seines Vero modo di diminuir (1584) und Giovanni Bassano in den Ricercari (1585) Orientierungs- bzw. Taktstriche. Der Auftau Dalla Casas Vero morlo gleicht im wesentlichen dem des Trattado Ortiz': Den Verzierungen einzelner Intervalle und Klauseln schließen sich im zweiten Buch längere Bearbeitungen meist weltlicher Vokalwerke an. Auch hier sind diese Bearbeitungen zum Teil mit Taktstrichen versehen, und wie Ortiz verwendete auch Dalla Casa für seine Zeit ungewöhnlich kurze Notenwerte. In einem Vorwort Dalla Casas werden, wie auch ein Jahr später bei Bassano ${ }^{309}$, die "quadruplicate che sono 32. per battuta" also unsere Zweiundreißigstelnote (bei Bassano heißt sie "Bissicroma"), sowie noch ein weiterer Notenwert, nämlich die "treplicale. che sono 24. per battuta", also ein Notenwert, der einer Sechzehulelnote aus einer Sextolengruppe entspricht, erklärt310. Die Ricercari Bassanos sind ähnlich virtuos wie die Diminutionen Da1la Casas und ebenfalls init Taktstrichen versehen.

Neben dem Taktstrich verwendet Dalla Casa auch Punkte zur Untergliederung des Notenbildes. Diese Punkte wurden z.B. auf Seite 2 und 4 des zweiten Buches dazu benutzt, eine Reihe gleicher Noten zu unterteilen ${ }^{311}$. Die Ver-

308. Gemeint ist die Anweisung Ortiz', Gruppen von Semicromen auf einem Bogenstrich zu spielen, also zu binden, vg1. S.XXIX der Neuausgabe.

309. Das Vorwort zu Bassanos Ricercari ist bei Sartori, Bibliografia I, S.52 (1585 g) abgedruckt.

310. Diesen Notenwert verwendete nur Giovanni Dalla Casa. Aurelio Virgiliano hat in dem ersten, die passaggi behandelnden Buch seines Dolcimelo (ms um 1600) eine Spalte für "Triplicate" vorgesehen (Es gibt dort die Spalten "Crome", "Semicrome", "Perfidie" (Diminutionen mit verschicdenen Notenwerten), "Sestuple", "Triplicate" und "Quadruplicate"). Die Spalten "Triplicate" und "Quadruplicate" sind in diesem unvollständig gebliebenen Werk leider immer leer geblieben. "Sestuple" sind an einigen Stellen eingetragen (S.14, 16 und 18 der Faksimileausgabe). Es handelt sich hierbei um Triolierungen auf der Ebene der Croma, notiert mittels der Taktvorzeichnung "6/4". Ob die "Triplicate" als eigener Notenwert (wie bei Dalla Casa) geplant waren, oder auch mittels Taktvorzeichnung erreicht werden sollten, bleibt ungeklärt.

311. Natürlich sind alle diese Anwendungen nicht konsequent gehandhabt worden. So stehen auf Seite 318 Semiminimen d" ohne irgendwelche Gliederungen. 
wendung dieser Punkte geschah sicherlich in Anlehnung an den Divisionspunkt der Mensuralnotation, der, wenn auch mit abnehmender Häufigkeit, immer noch verwendet wurde.

Diese Takt- oder Orientierungspunkte erschienen wenige Jahre später in den Concerti di Andrea, et di Giovanni Gabrieli (1587) sowie in dem Sammeldruck Dialoghi musicali von 1590. In beiden Drucken sind Battaglien (1587 eine Battaglia von Andrea Gabrieli, 1590 abermals die Battaglia von Andrea Gabrieli sowie eine weitere von Annibale Padoano312) mit diesen Punkten ausgestattet. Bei diesen beiden Sätzen werden fast regelmäßig Gruppen von der Dauer einer Semibrevis im geraden Takt bzw. eines Taktes im Dreier von solchen Punkten abgegrenzt. Anlaß fir die Verwendung dieser Punkte mögen die vielen unübersichtlichen Tonrepetitionen (Cromen) gewesen sein. Die Punkte treten erstmals bei der ersten stelle mit Tonrepetitionen in der ersten Battaglia in Erscheinung. Im weiteren Verlauf der Stücke werden sie jedoch auch an ibersicht lichen Stellen verwendet ${ }^{313}$.

Die bisher genannten Grüde für den Einsatz von Orientierungsstrichen bzw. -punkten bleiben auch im frühen 17. Jahrhundert bestehen. Die meisten der mit Taktstrichen versehenen Drucke verwenden diese nicht durchgängig, sondern nur in einzelnen Stimmen an einzelnen Stellen. Ein Taktstrich wurde notiert bei: 1. Pausen auf betonter Taktzeit oder Auftaktigkeit 314

2. außergewöhnlichen Rhythmen in Dreiertakten ${ }^{315}$

3. Stellen besonders hoher Virtuosität316

4. Tonrepetetionen ${ }^{317}$.

312. Vg1. das Vorwort zur Notenausgabe Musiche strumentali von Giacomo Benvenuti, S.XCII ff.

313. Die Setzung dieser Orientierungspunkte kann auch in der Neuausgabe dieser Sticke in Benvenutis Musiche strumentali, S.93 ff beobachtet werden, da Benvenuti diese mit abdruckt. Unabhängig von kurzen Notenwerten verwendele G. M. Asola einmal einen solchen orientierungspunkt in seinen Madrigali a 2 (1587, 4/1604). Hier findet der Orientierungspunkt an einer Stelle Verwendung, bei der ein komplizierter Rhythmus mit einem Zeilenwechsel zusammenfällt.

314. R. Micheli, Musica vaga (1615), C. Monteverdi, Concerto (1619), G. Priuli, Sacrorum concentuum... pars altera (1619, vgl. das Notenbeispiel I,3 im zweiten Band dieser Arbeit), A. Grandi, Motetti a 1 et 2 (1621), P. Lappi, Concerti sacri ... libro secondo (1623), F. Turini, Motetti a voce sola (1629). An Stellen anderer rhythmischer Unibersichtlichkeit im geraden Takt stchen Trktstriche in folgenden Drucken: L. Leoni, Sacri fiori (1612), S. Bernardi, Concerti sacri (1621) und Concerti accademici ... libro primo (1616).

315. S. Bernardi, Il terzo libro de Madrigali (1624), G. Bruschi, Missa et psalmi (1627), D. Bellante, Concerti accademici (1629), A. Signoretti, Vespertinae omnium solemnitatum psalmodiae (1629, vgl. Notenbeispiel I,4 im zweiten Band dieser Arbeit).

316. T. Massaino, Musica per cantare (1607), Sammeldruck Canzoni da sonare 
In einigen Musikdrucken des frühen 17.Jahrhunderts ist eine Bevorzugung des Orientierungs- oder Taktstriches bei textlosen Stelleu bzw. textloser Musik $\mathrm{zu}$ beobachten. Hier seien in erster Linie zwei Drucke Giulio Bruschis genannt318, in denen Orientierungsstriche nur bei längeren Melismen in den Vokalstimmen verwendet werden. Offensichtlich sollten hier die Orientierungsstriche die fehlende textliche Untergliederung ersetzen. Es erscheint durchaus wahrscheinlich, daß das Fehlen eines gliedernden Textes auch die Veranlassung bot, bevorzugt Instrumentalmusik mit Orientierungsstrichen zu versehen. So sind in I. Vivarinos Primo libro de motetti ... con otte sonate (1620) nur die acht Sonaten in der Solostimme mit. Strichen versehen - hier allerdings überaus regelmäßig ${ }^{319}$.

Die Vorliebe für Taktstriche in textloser Musik wird bestätigt durch die Tatsache, daß bis zum Ende des zweiten Jahrzehntes des 17. Jahrhunderts alle dem Verfasser bekannten regelmäßig in den Stimmbuichern mit Taktstrichen versehenen Drucke entweder nur instrumentale oder zumindest instrumental-vokale Musik enthalten ${ }^{320}$. Erst aus dem Jahr 1630 sind dem Verfasser rein vokale Musikdrucke mit durchgängiger Taktstrichsetzung in den Stimmbüchern be-

(1608), P. Lappi, Concerti sacri ... libro secondo (1623), f. Priuli, Missae ... octo, novenq. (1624), F. Turini, Madrigali ... Iiloro primo (1624) sowie Motetti a voce sola (1629) und D. Bellante, Concerti accademici (1629).

317. A. Banchieri, Metarmorfosi musicale (1601), T. Merula, Canzoni (1615). Vgl. zu dieser Aufstellung auch Wolf, Handbuch, Bd.I, S.429. Es sei an dieser Stelle bereits darauf hingewiesen, daß es auch einzelne Striche optisch dem Taktstrich gleich - in einer großen Zahl von Quellen gibt, die mit den Orientierungsstrichen nichts gemein haben. Es handelt sich dabei um Trennungsstriche, vergleichbar den weiter oben zu ortiz' bereits erwähnten. Diese trennen nun nicht einzelne ubungen voneinander, sondern unterteilen Kompositionen in Abschnitte, vergleichbar einer Generalpause. Eine besonders beliebte Stelle hierfür ist vor dem Schlußamen in motettischen Kompositionen. Diese Striche werden weiter unten im Abschnitt IV.2.2.2. und 2.2.3. gesondert behandelt.

318. Liber secundus sacrarum modulationum (1625) sowie Il terzo libro delli concerti ecclesiastici (1629).

319. Schünemann, Dirigieren, S.72, berichtet von einer deutschen Tradition des 17.Jahrhunderts, Vokalstimmen ohne Taktstriche, die dazugehörigen Instrumentalstimmen aber mit Taktstrichen zu notieren; vgl. auch Tappolet, Notenschrift, S.33.

320. Es sind dies N. Corradini, Motetti (1624), 0. M. Grandi, Sonate (1628), D. Castello, Sonate concertate ... libro primo (secondo) (1629), B. Marini, Sonate (1629), G. Rovetta, Madrigali concertati ... libro primo (1629). Bei den hier genannten vokal-instrumentalen Drucken erfolgte die Taktstrichsetzung jedoch in der Regel sowohl in den Vokal-als auch den Instrumentalstimmen. 
kannt321. Selbstverständich ist auch eine hier als durchgängig bezeichnete Taktstrichsetzung nicht durchgängig im heutigen Sinne. Durchgängig meint, daß in den meisten Stiicken die meisten Stimmen mit arıähernd regelmäßigen Taktstrichen versehen sind, ohne daß für die Taktstrichsetzung jeweils ein besonderer Grund vorliegen muß.

Aufgrund der in den Instrumentalwerken üblichen kurzen Notenwerte ${ }^{322}$ ist nicht zu entscheiden, ob die Textlosigkeit oder die kurzen Notenwerte den Komponisten oder den Drucker dazu veranlaßten, Taktstriche zu verwenden. Wahrscheinlich wird beides hier zusammengewirkt haben.

Die in Hardschriften und auch in deutschen Drucken anzutreffenden kleinen Orientierungsstriche nur durch eine Linie - vergleichbar dem heutigen Zäsurstrich (Atemzeichen) - sind in italienischen Musikdrucken nicht anzutreffen ${ }^{32.3}$. Dennoch interessant ist das Kapitel "Von den Virgulis und Strichlin"

321. M. Delipari, I baci sowie A. Gualtieri, Motetti ... libro terzo. Ebenfalls mit durchgängiger Taktstrichsetzung versehen sind aus dem Jahr 1630 die Sanate concertate von G. Scarani.

322. Auf dieses Phänomen wurde bereits kurz hingewiesen. Abgesehen von den sowohl instrunentalen als auch vokalen Diminutionstraktaten sind es in erster linie Instrumentaldrucke für ein bestimmtes Instrument, die zuerst extrem kurze Notenwerte verwenden. Die wichtige Rolle, die dabei der Festlegung des zur Ausfihrung verwendeten Instrumentes zukommt, kann sehr gut anhand des ersten Ricercarbuches von Jacobus Buus beobachtet werden. Es gibt vor diesem ersten Buch sowohl eine Stimmbuchausgabe (1547) als auch eine Intavalatura (1549) - allerdings haben beide Ausgaben nur ein Stuick gemeinsam. Während die Notenwerte des Stimmbuchdruckes nur selten einmal zwei Semicromen in Eolge aufweisen, sind die intavolierten Ricercare mit langen Semicromenläufen reich diminuiert. Man hat hier eine allgemeine Formulierung ("da cantare et sonare"), die sicherlich nach den jeweiligen Möglichkeiten noch diminuiert werden kann/soll, sowie eine spezielle Formulierung fiir ein Tasteninstrument, dessen virtuose Möglichkeiten von Buus hereits mit einbezogen wurden. Interessant in diesen Zusammenhang ist auch, daß in der Stimbuchausgabe jedem Stiack der semicircolo tagliato vorgezeichnet ist, während in der Intavolatura ein Mensurzeichen zu Beginn der Ricercare tehlt.

Auch in der Folgezeit fallen Intavolaturen durch kurze Notenwerte auf, so z.B. C. Merulos Canzoni d'intavolatura (1592) mit langen Biscramen-Läufen. Auch die nächste Stufe - unsere Vierundsechzigstelnote - wird in der Tastenmusik erstmals erreicht, nämlich im ersten Ricercarebuch G. M. Trabacis (1603) (dort allerdings in Partiturnotierung). Wie auch in der Intavolatura Merulos mußte der Drucker hier von Hand nachhelfen, da ihm entsprechende Typen fehlten. Vg1. dazu auch L. Zacconi, Prattica di musica (1596), fol. 22 ff. Zacconi beschreibt den Wert der Biscroma als unsingbar und nur fiir Instrumentalisten zu gebrauchen.

323. Vgl. zu diesen Strichen Wolf, Handbuch, Bd.I, S.428 f, sowie Schiinemann, Dirigieren, s.72 f. 
aus Syntagmatis musici ... tomus tertius von Michael Praetorius ${ }^{324}$. Er erwähnt als Grund für die Setzung dieser Striche die vielen "Fusen" (in Deutschland üblicher Name der italienischen Croma), die "in etlichen Cantionibus vnd Gesängen / sonderlich aber in den Symphonien ohne Text" zu finden seien325. Praetorius rechnet dariber hinaus mit Schwierigkeiten "sonderlich auch in den Proportionibus", da "primo intuitu, wegen des tacts gar leichtlich irrungen vorfallen" können. Oft sei nicht zu erkennen, ob es sich um eine "Tripla" (Dreiganzetakt) oder "Sesquialtera" (Dreihalbetakt) handele. Er zieht "kleine Strichlin vnd Virgulae" den Punkten vor, da diese auch leicht mit Augmentationspunkten verwechselt. werden könnten.

Eine Sonderstellung in Bezug auf die Taktstriche nimmt das Stimmbuch des Basso continuo ein. Fast seit es Basso continuo-stimmen gibt, sind diese oft mit Taktstrichen versehen ${ }^{326}$. Nach dem bisher Gesagten liegt es nahe, den Grund für die Taktstrichsetzung in den Continuostimmen in deren Textlosigkeit zu sehen. Wichtig ist in diesem Zusammenhang jedoch auch die Tatsache, daß der Continuo-Spieler stets wissen muß, auf welchem Taktteil er sich befindet, denn danach richtet sich u.a. der auszusetzende Tonsatz. Er muß eindeutig Hauptnoten von Durchgangsnoten unterscheiden können. Selbstverständlich muß man annehmen, daß auch alle anderen Stimmen stärdig wissen sollten, auf welchem Taktteil sie sich gerade befinden. Dies ist für den Organisten (bzw. anderen Continuo-Spieler ${ }^{327}$ ) jedoch durch die Vielzahl der

324. S.34 f.

325. Die Deutung Schünemanns, Dirigieren, S.73, "durch diese Orientierungsstriche sollte auch äußerlich die freie Rhythmik der vokalen Literatur von der Taktmetrik der Instrumentalkunst unterschieden werden" ist sicher verfehlt.

326. Vg1. hierzu Kinkeldey, Orgel, S. 198, Schneider, Basso Contimo, S.66 ff, sowie weiter unten im Abschnitt II.2.1. Schneider wies darauf hin, daß die fruihste bekannte Continuo-Stimme - es handelt sich um die "Bassone"Stimme zu Striggios "Ecce Beatam Lucem" im Manuskript der Ratschulbib]iothek Zwickau, datiert auf 1587 (vgl. Schneider, S.66 ff) - bereits mit einer taktstrichähnlichen Abteilung versehen ist. In unregelmäßigen Abständen sind hier die oben erwähnten kleinen Striche in die Stirme eingetragen. Bei eingehender Betrachtung der Continuo-Stimme fällt jedoch auf, daß es sich hierbei nicht um Vorläufer des Taktstriches, sondern vielmehr um Orientierungshilfen anderer Art handelt. Hier werden Textzusamnenhänge und die Stellen mit Textwiederholungen gekennzeichnet. Daruber hinaus kommen diese Striche nicht nur in der Continuo-Stimme, sondern auch in den anderen Stimmen vor.

Schinemann, Dirigieren, S.71, zitiert eine Stelle aus Erasmus Sartorius' Institutionum musicarum tractatio (1635), in der die Taktstriche der Continuo-Stimmen mit denen der Tabulaturen und Partituren in Zusammenhang gebracht werden: "Tempus bedeutet in der Musica zween tactus, als wenn ein Organist seine tabulatur oder ein Componist seine partitur in tempora eintheilet / so machet er allezeit nach 2 Schlägen ein strich durch die Linien: Wie der Bassus continuus heutiges tages in tempora distribuirt wird". 
ihm zukommenden Aufgaben - außer der Aussetzung in der Regel wohl auch die Direktion 328 - schwierig und wichtig zugleich ${ }^{329}$.

Eine besondere Art des Taktstrichgebrauches fällt in einigen Continuo-Stimmen zu Werken Antonio Agazzaris auf. Diese Continuo-Stimmen sind nicht durchweg mit einer Taktstrichsetzung versehen, sondern nur die ersten drei oder vier Takte, und zwar manchmal im Abstand von einer Semibrevis, manchmal aber auch im Abstand einer Brevis330. Vielleicht sollte hier der Versuch unternommen werden, auf anderem Wege als durch die ihrer Bedeutung entledigten Mensurzeichen, anzuzeigen, ob "alla semibreve" oder "alla breve" zu schlagen ist 331 .

Eine Behandlung der frihen Taktstriche wäre unvollständig, wiirde nicht auch nach dem Zusammenhang von Taktstrich und Akzentstufentakt gefragt. Nach heutigem Verständnis gehört es zu den Hauptaufgaben des Taktstriches, iber die Plazierung der Betonungen zu informieren. Dies war im frihen 17.Jahrhundert sicher nicht der Eall. Die Entwicklung zum Akzentstufentakt fand im wesent1ichen vor der Einfuhrung der Taktstriche stat.t. Schon in 16.Jahrhundert wurde die Battuta als gliedernde Einheit in der Musik empfunden (s.o.). Taktstriche wurden gesetzt, wenn die Deutlichkeit dieser Gliederung - z.B. durch viele kleine Notenwerte - gefährdet war. Die Setzung der Taktstriche hätte ohne die auch sonst vorhandene Gliederung der Musik keinen Sim gehabt: Sie gliederten die Musik nicht neu, sondern sie halfen, die vorhandene Gliederung der Musik zu erkennen. Erst nachdem Taktstriche allgemein iblich geworden waren, konnte sich dieses Verhältnis umkehren, und die Taktstriche selhst zur Gliederung werden.

327. Zur Besetzung des Generalbasses siehe weiter unten im Abschnitt II.3.3.

328. In zahlreichen Continuo-Stimmen finden sich eindeutige Hinweise auf ihre Bedeutumng als Direktionsstimnen - vgl. dazu weiter unten im Abschnitt II. 3.2.

329. Vg1 hierzu A. Banchieris Bevorzugung der "Bassi continui spartiti" da sie dem Organisten helfen das Ensemble "in battuta" zu führen (Cartella musicale (1614), S.215, vgl. den Abdruck dieser Stelle im zweiten Band dieser Arbeit auf S.21 f sowie die Ausfihrungen hierzu weiter unten im Abschnitt II. 3,2,).

330. Sacrae landes ... liber secundus (1603), Sacrae cantionum ... liber tertius $(1603,2 / 1608)$ und Missae quattuor $(1614,2 / 1617+3 / 1625)$. Ähnliche Taktstriche nur zu Anfang einiger Sticke in der Continuo-Stimme finden sich auch in G. Metallos Messe a 5 (1610), dort allerdings im Abstand von einer Longa.

331. Ein "tactus alla breve" ist bei einigen Werken Agazzaris - insbesondere bei den Missae quattuor - gut denkbar (sehr lange Notenwerte). 


\subsection{ANDERE VERFAHREN, DAS NOTENBILD ZU UNTERGLIEDERN}

Das mit Abstand am weitesten verbreitete Druckverfahren fuir Noten im 16. und 17. Jahrhundert war der Typendruck, also der Notendruck mit beweglichen Lettern. Für jede Note, zusammen mit einem Stiick Notensystem, war eine Meta11type vorhanden. Diese Typen wurden zum Druck aneinandergereiht. Um eine gleiche Lănge der Systeme zu erreichen, fügte man Typen mit Notenlinien, aber ohne Noten ein. Ein Nachteil dieses Druckverfahrens war es, daß keine Gruppierungen von kurzen Noten mittels Balkung, wie in Handschriften auch damals ublich, möglich waren. Es sind wiederum die Diminutionstraktate mit ihren extrem kurzen Notenwerten, bei denen dieser Mangel besonders deutlich spiurbar wurde.

Eine mögliche Abhilfe boten die oben erwähnten Typen ohne Noten: Nach je vier oder acht Semicromen bzw. Biscromen konnte eine solche Lücke eingefügte werden, wodurch das Notenbild erheblich ubersichtlicher wurde ${ }^{332}$. Fast konsequent fand dies Verfahren in Dalla Casas vero modo di diminuirs3a und darüber hinaus in zahllosen späteren Typendrucken Verwendung.

Auf verschiedenen Wegen fanden aber auch die Balken Einzug in die Musikdrucke. So ermöglichte der im späten 16.Jahrhundert aufkommende und zunehmend beliebter werdende Notenstich ohne Probleme auch die Balkung. Für die Entwicklung dieses Verfahrens steht in erster Linie der römische Verleger Simone Verovio334. Dieses Verfahren wurde jedoch auch von einigen anderen, oft nicht genannten Verlegern tibernommen ${ }^{3} 35$.

Bei den Kupferstichen ist die Möglichkeit der Balkung wahrscheinlich nur ein Nebeneffekt. Ein Hauptgrund für die Veröffentlichung im Stich war sicher die Tatsache, daß Tabulaturen ${ }^{336}$ im Typendruck nicht befriedigend zu drucken sind ${ }^{337}$ und daruber hinaus der Druck einer italienischen Klaviertabulatur im Typendruck ausgesprochen aufwendig ist338.

332. Lücken wurden zur Gliederung auch in verschiedenen Tabulaturarten verwendet, dort jedoch als Ersatz der Takt- bzw. Gliederungsstriche.

333. Vg1. hierzu auch: Erig, Diminutionen, S.44. Dort findet sich auch (S.43) ein Faksimile einer Seite aus Dalla Casas vero modo.

334. Vg1. zu Verovio Casimiri, Verovio; dort auch eine Liste aller bekannten Veröffentlichungen Verovios (S.193 ff).

335. Einige Werke G. G. Kapsbergers erschienen im Stich ohne Nennung eines Verlegers (Verovio ist vom Druckbild her auszuschließen), darunter auch das hochvirtuose Libro primo di arie passeggiate sowie das Libro primo di mottetti passeggiate (beide 1612).

336. Fast alle der frühen Notenstiche enthalten auch Tabulaturen, sofern es nicht gar reine Tabulaturdrucke sind.

337. Es gibt dennoch eine ganze Reihe von Tabulaturen im Typendruck, darunter u.a. die Ricercar-Drucke Andrea Gabrielis.

338. Man benötigt Typen für jede mögliche Verbindung von bis zu drei Stimmen in einem System, nicht nur von den verschiedenen harmonischen, sondern 
Ein Typendruck mit Balken - ihn kennt man z.B. in dieser Zeit in Frankreich ${ }^{39} 9$ oder im 18 . Jahrhundert in Deutschland 340 - wurde wahrscheinlich auch aufgrund der großen Zahl der dabei benötigten Typen vermieden ${ }^{341}$. Sicher auch in Anbetracht der kleinen Auflagen zogen es die Drucker vor, Balken - sofern die Komponisten oder Herausgeber darauf bestanden ${ }^{342}$ - von Hand zu zeichnern ${ }^{343}$.

Solche handgezeichneten Balken finden sich in Musikdrucken Girolamo Frescobaldis ${ }^{344}$, Bartolomeo Montalbanos ${ }^{345}$, Giovanni Maria Trabacis346 und vor

auch von den rhythmischen Varianten.

339. Ein französicher Typendruck mit Balkungen von 1597 ist bei François Lesure, Art. Caignet, Denis in MGG, Bd.2, Sp.640 abgebildet.

340. So sind z.B. die Notenbeispiele in Georg August Griesingers Biographische Notizen über, Joseph Haydn, Leipzig 1810 bei Breitkopf und Härtel (Reprint: Leipzig 1979) im Typendruck erstellt worden.

341. Von jeder Croma-Note benötigt man statt einer Type mit einem Fähnchen je eine Type mit beginnenden Balken aufwärtsweitergehend, mit beginnendem Balken abwärtsweitergehend, mit Balken von unten kommend und nach oben geherid, mit Balken von oben kommend und nach unten gehend, mit Balken von oben kommend und nach oben gehend, mit Balken von unten kommend und nach unten gehend, mit endendem Balken von oben kommend und mit endendem Balken von unten kommend - und auch damit kann man nur Läufe ohne Sprünge darstellen.

342. Es ist jedoch nicht immer gesagt, daß es die Komponisten oder Herausgeber waren, die nach der Balkung verlangten. Auch der gröBere Platzbedarf einzelner Biscromen - zumal wenn man sie mit Luicken gliedern will - gegen uber von Semiminimen, die nachträglich mit Balken zu versehen sind, kann einen Verleger veranlassen, handgemalte Balken zu verwenden. Die "Canzon Seconda" aus B. de Selma e Salaverdes Primo libro de canzoni (1638) hätte nicht auf eine Seite gepaßt, wenn der Drucker nicht zu dem Mittel der handgeschriebenen Balken gegriffen hätte - und auf praxisgerechte Seitenaufteilung wurde bei den italienischen Drucken großer Wert gelegt. Daß die Balkung bei anderen Drucken aber auf Wunsch des Komponisten oder Herausgebers vorgenommen wurde, ist sehr wahrscheinlich (s.u.).

343. Eine AbschluBredaktion der Drucke per Hand wird man ohnehin annehmen könrien. Oft finden sich dieselben Korrekturen in mehreren Exemplaren eines Druckes auf die gleiche Art ausgefuhrt, oder aber Korrekturen sind in einem Exemplar handschriftlich ausgefihrt, in einem anderen - später gedrucktem Exemplar derselben Ausgabe aber bereits berichtigt gedruckt (so beobachtet bei verschiedenen Exemplaren von der Partiturausgabe von Frescobaldis Primo libro de canzoni (1628)). Dariber hinaus gibt es Drucke, in denen bestimmte Zeichen (z.B. Semicromen-Pausen), da eine entsprechende Type feh1te, immer per Hand nachgetragen wurden.

344. In Partitura il primo libro delle canzoni (1628).

345. Sinfonie (1629).

346. Ricercare ... libro primo (1603). In Trabacis zweitem Ricercare-Buch (1615) finden sich zwar keine Balkungen, jedoch ist auch hier von Hand nach- 
allem Giovanni Girolamo Kapsbergers ${ }^{347}$. Mit Ausnahme der Drucke Kapsbergers geschieht die Balkung ausschlieBlich an virtuosen Stellen. Die Gliederung der Musik soll erleichtert - oft aber auch beeinflußt - werden ${ }^{348}$.

In den Drucken Giovanni Girolamo Kapsbergers wird die Balkung nicht nur benutzt, um besonders virtuose Stellen uberschaubarer zu machen, sondern auch, um eine Anzahl von kurzen Noten zusammenzufassen, ganz entgegen einer rein schematischen Gliederung ${ }^{49}$. Besonders interessant ist aber die Verwendung von Balkung, wie sie in dem Libro quarto di villanelle (1623) zu finden ist. Nur einige wenige Stücke dieses Druckes enthalten virtuose Stellen. In der Regel wird die Balkung hier verwendet, um zwei einzelne Cromen miteinander zu verbinden, sofern sie zu einer Textsilbe gehören ${ }^{350}$.

Eine Zusammenfassung von Cromen mit einer Textsilbe unter einem Balken ist in Handschriften und auch in Kupferstichen zu dieser Zeit durchaus iblich; in einem Typendruck erscheint der Aufwand jedoch recht hoch, da keine wesentliche Leseerleichterung von dieser Druckart ausgeht. Daf dennoch bei Kapsberger - und soweit bekannt nur bei ihm - versucht wurde, das Aussehen der Typendrucke dem der Kupferstiche seiner Werke anzupassen, spricht für das hohe Ansehen, das Kapsberger genoß. Offenbar drängten die Herausgeber der Kapsbergerschen Werke - fast bei jedem Druck Kapsbergers ist ein "Sammler" genannt351 -, wenn nicht der Komponist selbst, die Drucker dazu, das Notenbild der handschriftlichen Vorlagen weitgehend beizubehalten.

\section{DiE UNGERADEN TAKTARTEN}

Die Diskussion uber das Tempo der ungeraden Takte in der neueren Literatur wird beherrscht vom Begriff der Proportion. Es ist daher notwendig, sich zunächst eingehender mit den Proportionen und ihrer Verwendung im späten 16. und fruhen 17. Jahrhundert zu befassen, ehe im einzelnen auf die verschiedenen Arten der ungeraden Takte eingegangen werden kann.

getragen worden: Alle Notenwerte, die kürzer als die Semicroma sind, waren als Semiminimen gedruckt und sind von Hand mit Fähnchen versehen worden offenbar fehlten hierfür die Typen.

347. Libro secondo d'arie (1623), Libro quarto di villanelle (1623), Cantiones sacrae... volumen primum (1628) und Modulatus sacri ... volumen secundum (1630).

348. So bei Frescobaldi, vgl. Notenbeispiel I,5 im zweiten Band dieser Arbeit.

349. So insbesondere im Libro secondo d'arie (1623), vgl. Notenbeispiel I,6.

350. Vg1. Notenbeispie1 I,7.

351. Untertitel, wie "Raccolte da1 Signor Marce1lo Pannocchieschi De Conte d'Esci" (Libro quarto di villanelle), "Raccolto dal Sig. Caual. fra. Jacomo Christoforo AD Andlaw del Ordine di S.Gio: Battista" (Libro primo di arie) oder "Raccolte dal Sig. Pietro Contarini nobile venetiano" (Libro secondo d'arie). 


\subsection{DiE PROPORTIONEN ${ }^{352}$}

Die Kunstauffassung der Renaisance wird in vielen Beziehungen von den Proportionen beherrscht. Zurückgehend auf die Antike wurde die Harmonie, die musikalische Intervalle mit einfachen Schwingungs- bzw. Seitenlängenverhältnissen hervorrufen, auf Architektur und Malerei ubertragen. Im einzelnen ausgeführt ist diese Theorie - auf Pythagoras zurückgreifend - z.B. in den Architekturtraktaten Leon Battista Albertis und Palladios 353.

Die Theorie der Proportionen beherrscht auch die Musiktheorie der Renaissance. Neben den Proportionen der Intervalle komnt den Proportionen der Mensuralnotation in den Musiktraktaten des 16. Jahrhunderts große Bedeutung zu. Kaum einer der Traktate, die die Mensuralnotation behandeln, enthält nicht auch Rechentabellen, mit deren Hilfe man die Dauer eines Notenwertes unter den verschiedenen Proportionszeichen - gemessen in der Einheit der Semibrevis, des "integer valor" - ablesen kann. Besonders ausfühı ${ }_{1}$ ich ist dies im 16. Jahrhundert in der Musiktheorie Deutschlands betrieben worden ${ }^{354}$. Die Musikstiicke, die diese Möglichkeit auszuschöpfen suchen, haben große Beruihmtheit erlangt - nicht wegen ihrer musikalischen Qualitäten, sondern weil sie häufig in diesen Traktaten als Beispiele erwähnt, oder auch ausfihrlich behandelt werden ${ }^{35}$.

352. Nicht zur Sprache kommen werden in den folgenden Abschnitten Proportionsangaben in Kanonanweisungen. Diese haben zumindest seit der Wende zum 17. Jahrhundert eine eigene Geschichte, die bekanntlich noch weit über das Ende des 17.Jahrhunderts hinaus reicht. Bereits in dem hier zu behandelnden Zeitraum haben sich die Kanonanweisungen als eine solche Sondertradition etabliert. Ein vereinzeltes Auftreten von Kanonanweisungen, wie es etwa zur Vervielfältigung der Stimmenzahl im 16. Jahrhundert beliebt war, gibt es fast nicht mehr. Zumeist stehen solche Arweisungen nun in besonderen KanonDrucken (z.B. der Musica vaga et artificosa continente motetti con oblighi, et canoni diversi (1615) von Romano Michele). Kanonanweisungen außerhalb solcher Drucke findet man nur noch sehr selten in Drucken mit Kirchenmusik aus der sogenannten römischen Schule, wie z.B. in den Messen Paolo Agostinis (hier im 3. Buch z.B. ein auf 14 verschiedene Arten aufzulösendes Agmus Dei).

353. Vg1. hierzu u.d. Wittkower, Proportion; Braunfels, Kunstgeschichte, S.39 f und S.372 sowie Heinrich Hischen, Art. Harmonie, in MGG, Bd.5, Sp. $1602 \mathrm{ff}$.

354. Vgl. z.B. M. Agricolas Von den Proportionibus (ohne Jahr). Hier folgen auf drei einfuhrende Seiten 23 Seiten mit Rechentabellen und zwei Musikbeispielen - eines davon mit "Resolucio". Ein besonders schönes Beispiel einer solchen Rechentabelle enthält Ambrosius Wilfflingseders Erotemata musices praticae, Nürnberg 1563. Dort sind die Mensurzeichen unter einer drehbaren Scheibe mit Fenstern angeordnet (vg1. Martin Ruhnke, Art. Wilfflingseder, Ambrosius in MGG, Bd.14, Sp.652). Vereinzelt gibt es solche Rechentabellen auch schon im 15.Jahrhundert (freundlicher Hinweis von Prof. Dr. Martin Staehelin, Göttingen). 
Einige Stellen, bei denen eine triolische Bildung einer Stimme mittels eines Wechsels nur dieser Stimme in einen Dreier notiert wurde, oder aber ein $\mathrm{Ab}$ schnitt im Dreier nicht in allen Stimmen zugleich beginnt, belegen, daß zumindest fü solche recht einfachen Bildungen, Taktwechsel auch außerhalb besonderer Proportionensticke - die als solche ja immer sofort $\mathrm{zu}$ erkennen sind - proportional verstanden wurden ${ }^{356}$.

Schon im späteren 16. Jahrhundert sind echte Proportionsstücke äußerst se1ten. Das Paradebeispiel jener Zeit ist Palestrinas Missa L'homme armé, erstmals gedruckt 1570357. Einige weitere, an Kompliziertheit358 an die Palestrina-Messe heranreichende Beispiele, finden sich bei Don Fernando de las Infantes ${ }^{359}$ und Romano Micheli 360 . Andere "Proportionsspielereien" aus dem späten 16. wie frühen 17.Jahrhundert beschränken sich auf die Verwendung einfacher Diminution oder Augmentation ${ }^{361}$ oder anderer einfacher Bildungen362. Diese gehören in den Bereich der Madrigalismen und dienen einer -

355. Vg1. hierzu Dahlhaus, Proportionenlehre, S.336.

356. Im zweiten Band dieser Arbeit ist als Notenbeispiel I, 8 die ubertragung eines Ausschnittes des Madrigales "Vogi'1 tuo corso" aus Cipriano de Rore, Il quatro libro d'i madrigali a 5 (1557) abgedruckt. In diesem Madrigal findet sich sowohl ein versetzter Anfang des ungeraden Taktes als auch ein einzelner Abschnitt im Dreier nur im Tenor. In einer Neuausgabe muß so eine Stelle als Triole wiedergegeben werden - vgl. Rore-GA, Bd. IV, S.90 ff.

357. Missarum liber tertius. Vg1, hierzu Monterosso, Palestrina (mit ubertragung und Faksimile der beiden Ausgaben von 1570 und 1599, sowie Casimiri, Dibattio und ders., Polyphonia.

358. Auch Palestrinas $L$ 'homme armé ist, verglichen mit frïheren Beispielen, nicht sonderlich kompliziert.

359. Einmal in Sacrorum varii styli cantionum ... liber III. sowie zah1reich in Plura modulationum genera (beide 1579).

360. Musica vaga et artificiosa (1615); hier in zahlrejchen Kanonkompositionen.

361. Zu finden in folgenden Drucken: P. Zallamella, Musica (1582), R. Giovannelli, Gli sdruccioli ... libro secondo (1589), G. Metallo, Messe commodissime... libro sesto (1602) und Motetti per tutte le solennita (1610), P. M. Marsolo, Mottecta ... liber primus (1608), G. Montesardo, I lieti giorni (1612), P. Agostini, Spartitura delle messe et motetto (1627), G. Ferrari, Il primo libro de madrigali (1628). In der Regel werden die Proportionen nur in einem Stiick verwendet und betreffen nur eine Stimme. Es handelt sich immer um eine gerade Augmentation oder Diminution (meist 1:2, bei Ferrari 1:12).

362. Hier werden gerade und ungerade Takte miteinander verbunden, doch in der Regel so, daß die Noten des geraden Taktes der Länge eines ganzen ungeraden Taktes entsprechen (i.d.R.: Semibreve im geraden Takt). Auch hier ist also nur das Aussehen kompliziert. In den folgenden der ausgewerteten Drucke ist so etwas anzutreffen: A. Striggio, Il secondo libro de madrigali a 6 
allerdings weder hörbaren, noch im einzelnen Stimmbuch immer sichtbaren Ausdeutung des Textes. Eindeutige Proportionen außerhalb solcher sofort erkennbarer Proportionenstuicke gibt es schon im späten 16.Jahrhundert kaum noch 363 .

Waren die Proportianen in der Kunsttheorie der Renaissance fest verwurzelt, so stehen sie den baracken Idealen eher entgegen. Den Gegensatz RenaissanceBarock beschrieb Heinrich Wölfflin plastisch mit den Worten "Die Renaissance ist die Kunst des schönen ruhigen Seins. (...) Der Barock beabsichtigt eine andere Wirkung. Er will packen mit der Gewalt des Affects, unmittelbar, überwältigend. Was er giebt ist nicht gleichmässige Belebung, sondern Aufregung, Ekstase, Berauschung" 964 . Wölfflin behandelt in erster Linie den Sti1wandel innerhalb der Architektur, aber seine Aussage ist ebensogut fuir die anderen Künste giiltig365. Die "Gewalt des Affects" ist es auch, die ein

(1571), G. B. Cali, Ricercare a 2 (1605), P. M. Marsolo, Mottecta ... 1iber primus (1608) und S. Musotti, L'anima sospirante (1621). Einen Satz mit einer solchen Parallelität von geradem Takt und Dreier findet sich auch noch in der Oper Chi soffre speri von M. Marazeoli (1639; nach 0sthoff, Spätwerk, S.216 und Witzenmann, Mazzocchi, S.207). Das Beispiel von Striggio ist das vielseitigste dieser Stücke. Es ist bereits im 16. als Proportionsbeispiel in die Musiktheorie eingegangen (so in L. Zacconis Prattica musica, (1596), fol. 138. Zu Erwähmungen im 17. Jahrhundert vgl. Ray J. Tadlock, Art Striggio, Alessandro (I) in MGG, Bd.12, Sp.1607 f). Die entsprechende Stelle ist im zweiten Band dieser Arbeit als Notenbeispiel I,9 wiedergegeben.

363. In gewisser Weise mit der Rore-Stelle in Notenbeispiel I, 8 verwandt sind die folgenden späteren Fä1le: In Monteverdis erstem Madrigalbuch (1587) sowie in Montes La fiammetta (1599) gibt es je einen Dreier, währenddessen eine Stimme schweigt. In dieser Stimme ist der Taktwechsel nicht vermerkt. In der "Fantasia seconda sopra vn soggietto solo" in G. Frescobaldis Fantasie (1608) beginnt ein Teil im ungeraden Takt nicht gleichzeitig in den verschiedenen Stimmen (Notenbeispiel I,10 im zweiten Band dieser Arbeit). Große Probleme gibt es hier jedoch nicht. Zum einen verhelfen zahlreiche (perfekte) Semibreven zu einem meist klar iberschaubaren Notenbild (in der Neuausgabe von Pierre Pidoux, Girolamo Frescobaldi: Orge1- und Klavierwerke, Bd.I, S.8, konnte der Herausgeber die Taktwechsel an vielen Stelien einfach ubergehen), zum anderen klärt die Notation in Partitur vieles von selbst. Mit den klassischen Proportionen wäre dieses Beispiel nicht zu 1ösen. Bei Frescobaldi verhält sich der 3/2-Takt zum semicircolo semplice genauso, wie zum semicircolo tagliato, der der Stelle mit der "Proportionsspielerei" voransteht (sonst verwendet Frescobaldi nur den semicircolo semplice). Vie1leicht gaben hier die altertimlichen Proportionen für Frescobaldi auch den Anlaß, den ebenfalls altertümlichen semicircolo tagliato zu verwenden.

364. Wölfflin, Renaissance, S.38.

365. Wölfflins Thesen sind auch in der Musikwissenschaft reichlich rezipiert worden und waren Grundlage fur die Ubernahme des Barockbegriffes in die Musikgeschichte. Während die übertragbarkeit von eher allgemein den 
Theaterpublikum in Tränen versetzt, wie z.B. Marco da Gagliano im Vorwort zu seiner Dafne (1608) von der Uraufführung von Monteverdis Arianna zu berichten weiß366.

Die Ausgeglichenheit von Proportionen steht einer "Affektgeladenheit" direkt entgegen. In der Architektur wurden Proportionen, nach denen vorher mit aller Kraft gestrebt wurde, wie etwa der "Goldene Schnitt", nun gemieden36?.

Eine Beibehaltung des proportionalen Verständnisses der Dreiertakte ist auch in der Musik kaum denkbar ${ }^{368}$. Die Wandlungen, denen die Verwendung der Dreiertakte in den Jahren um 1600 unterworfen waren, haben den bis dahin zumindest theoretisch vorhandenen Zusammenhang zwischen ungeradem und geradem Takt in der bisher gelehrten Form unmöglich gemacht ${ }^{369}$. Schon die Anzahl der zur Verfugung stehenden Dreiertakte 370 veränderte sich stark: Bis gegen Ende des 16.Jahrhunderts fanden - abgesehen von den hemiole (s.u.) - nur der Dreihalbe- sowie der Dreiganzetakt Verwendung; um 1630 findet man in den Que11en dariber hinaus den Dreivierteltakt, den Sechsvierte1-, Sechsachte1und Zwö1fachteltakt, und sogar einen Vierundzwanzigachteltakt ${ }^{371}$.

Zeitgeist betreffenden Aussagen sicher ohne Frage möglich ist (vgl. hierzu auch Friedrich Blume, Art. Barock in MGG, Bd.1, Sp.1286 ff), birgt die (jbertragung der Wölfflinschen Gegensatzpaare ("1inear - malerisch", "F1äche Tiefe" etc.) Gefahren in sich, da Begriffe wie "malerisch" oder "Fläche" nicht direkt auf Musik zu übertragen sind, sondern erst einer Interpretation bedürfen und diese recht unterschiedlich ausfallen kann (vgl. die zum Teil gegensätzlichen Ergebnisse solcher übertragungen bei Sachs, Barockmusik; Wellesz, Renaissance sowi.e Kroyer, Renaissance).

366. Vgl. die Faksimile-Ausgabe sowie bei Vogel, Gagliano, S.433 und Fenlon, Stage Works, S.275. Vg1. hierzu auch das im zweiten Band, S.8 ff abgedruckte Vorwort zu E. De' Cavalieres Rappresentatione (1600) von A. Guidotti.

367. Wölfflin, S.43.

368. Dazu Sachs, Rtythm, S.269: "The end of polyphonic leadership around 1600 emfailed the final collapse of the proportions".

369. Es sei an dieser Stelle auf ein interessantes Zitat aus dern musiktheoretischem Opus Joachim Burmeisters verwiesen. In seiner Chorudioecesis sagt Burmeister nicht nur, daB der Ausdruck "tactus proportionatus" falsch sei, da dieser Takt in keinem wirklichen zahlenmäßigem Verhältnis zum geraden Takt stehe, sondern Burmeister führt zugleich auch eine Autorität zur Bestätigung seiner Meinung an: Henricus Glarean "in Musica sua Mensurali" (Ruhnke, Burmeister, S.77). Ein Werk Glareans mit diesem Titel ist allerdings nicht bekannt. In den entsprechenden Abschnitten des Dodekachordon (1547) Glarean findet sich eine solche Aussage nicht (Liber III, S. $195 \mathrm{ff}$, besonders Caput XII: De Proportionibus musicis, S. $227 \mathrm{ff}$ ). Die Isagoge in musicen Glareans (1517) enthält keine Kapitel zur Mensuralnotation, kann also nicht gemeint sein.

370. Miteinbezogen sind hier auch die Sechser- und Zwölfertakte (zur Berechtigung dieser Bezeichnungen siehe weiter unten). 
Die Wahl zwischen den beiden im späten 16.Jahrhundert zur Verfügung stehenden Dreiern war zunächst abhängig von dem Hauptmensurzeichen, also dem semicircolo tagliato oder dem semicircolo semplice.

\subsection{ABHÄNGIgKEIt DER UngERAdEN TAKTE VON DER MENSUR DES GERADEN TAKTES}

Im späteren 16. Jahrhundert war die Sesquialtera die meistbenutzte Art des Dreiertaktes. Sesquialtera meint in dieser Zeit noch das (theoretische) Verhältnis 3:2, unabhängig von den verwendeten Notenwerten ${ }^{372}$. Für die beiden Signa des geraden Taktes, den semicircolo semplice und den semicircolo tagliato, waren dies verschiedene Taktarten, denn der Theorie nach bestand eine Batutta unter dem semicircolo tagliato aus zwei Semibreven, unter dem semicircolo semplice aber aus zwei Minimen; folglich bestand auch die Sesquialtera unter dem einen Zeichen aus drei Semibreven, unter dem anderen aus drei Minimen.

Diese Verknüpfung der beiden geraden Taktarten mit den beiden Sesquialtere ist, wenn auch nicht konsequent, so doch in immerhin $85 \%$ der ausgewerteten Musikdrucke der Jahre 1571-1600 anzutreffen. Es gibt daneben aber auch Komponisten, die unter dem semicircolo semplice den großen Dreier-373 oder umgekehrt 374 verwendeten. In etwa $7 \%$ der Drucke aus dieser Zeit sind beide Dreiertakte unter einem Signum fiir den geraden Takt anzutreffen (s.u.).

Durch die Bindung der beiden Sesquialtere an die beiden geraden Takte ergab sich zwangsläufig auch eine - zunächst indirekte - Bindung der beiden Dreiertakte an die Gattungen. Sofern aber die Mensurzeichen der geraden Takte verwendet wurden, um einzelne Stücke in Bezug auf das Tempo von

371. Die Fundstellen für die einzelnen Taktarten werden weiter unten im Abschnitt I.2.5. im einzelnen geboten.

372. Im 17. Jahrhundert wurde Sesquialtera auch zu einer Bezeichnung des kleinen Dreiertaktes (Dreihalbetakt). Indem aus dem Proportionszeichen 3/2 das Taktzeichen für den Dreihalbetakt wurde, wurde auch der Name - nun sinnentleert - beibehalten - vgl. dazu weiter unten.

373. So R. Giovannelli (nur im ersten Madrigalbuch zu 5 Stimmen von 1586) und V. Nerito in allen Canzonettenbuichern (1593-1599).

374. Dieser Fall ist sehr viel seltener. Dies hat sicher seine Ursache darin, daß der semicircolo semplice neu hinzugekommen war (s.o.) und somit auch der kleine Dreier. Es leuchtet ein, daß es eher Komponisten gab, die den neueren geraden Takt gebrauchten und dabei den vertrauten Dreier beibehielten, als umgekehrt. Der einzige dem Verfasser bekannte Druck mit dem kleinen Dreier unter dem semicircolo tagliato aus dem späten 16.Jahrhundert ist das zweite Buch der fünfstimmigen Motetten von Orfeo Vecchi (1598) (im ersten Buch benutzt er nur den großen Dreier). Interessanterweise entsprechen die Notenwerte im zweiten Buch (genau wie der verwendete Dreier) im Gegensatz zum ersten Buch eher den in den Madrigalen - und das heißt den unter dem semicircolo semplice - iblichen. 
anderen zu differenzieren, wurde diesen dann in der Regel auch der dem jeweiligen semicircolo entsprechende Dreier zugeordnet.

Um 1600 stieg die Zah1 der Drucke, in denen beide Dreier unter einem Signum für den geraden Takt anzutreffen sind von etwa 7\% (1591-1600) auf zunächst 17\% (1601-1610) und schließlich gar auf fast 44\% (1621-1630) an. Nicht nur diese Verwendung mehrerer Dreiertakte unter einer Grundmensur, sondern auch Abweichungen von der Norm der Sesquialtera bei Drucken mit nur einem Dreiertakt sind ab etwa 1600 vermehrt zu beobachten. Der Grund hierfü ist in der oben beschriebenen Wandlung der Gattungsmensuren in diesen Jahren zu suchen.

\subsection{DiE ABHÄngIgKeIt DER UNGERADEN TAKTE VON DER GATTUNG}

Eine Abhängigkeit der Dreiertakte nicht nur von dem Hauptmensurzeichen, sondern auch von der Gattung tritt dort zu Tage, wo es zu Differenzen zwischen der Gattung und der ihr eigenen Mensur kommt. Solange die Madrigale unter dem semicircolo semplice und die Motetten unter dem semicircolo tagliato geschrieben wurden, entsprechen die Dreier der jeweils zugehörigen Sesquialtera. Beginnend um 1600 wurden, wie oben dargestellt, zunehmend auch Motetten unter dem semicircolo semplice statt unter dem semicircolo tagliato notiert. Als Gründe hierfür konnte eine Angleichung der Notenwerte der Motetten an die der Madrigale sowie ein allgemeiner Bedeutungsverlust der Mensurzeichen für den geraden Takt beobachtet werden.

Schon im 16. Jahrhundert hatten sich insbesondere in den Motetten einige Standardstellen für den Einsatz von Dreiertakten herausgebildet; die wohl häufigste Stelle für die Verwendung eines Dreiers ist das Alleluia375. Die Dreier haben hier Ritornellcharakter. Die Notenwerte, in denen diese Abschnitte im Dreier notiert wurden, waren um 1570 dieselben wie um 1600 (und auch noch um 1630), während in den geraden Takten in diesem Zeitraum eine Halbierung bis Viertelung der iblichen Notenwerte zu verzeichnen ist. Als die Komponisten gegen Ende des 16.Jahrhunderts vermehrt dazu tibergingen, auch Motetten unter dem semicircolo semplice zu schreiben, wurden diese Dreier - längst zu Typen mit eigenem Tempo geworden ${ }^{370}$ - unverändert ubernommen und weiter beibehalten.

Die Abhängigkeit der Dreiertakte ging damit um 1600 von dem Mensurzeichen des geraden Taktes auf die Gattung uber: Sowohl Motetten als auch Madrigale wurden iberwiegend unter dem semicircolo semplice notiert, in den Motetten wurde der grobe Dreier, in den Madrigalen der kleine Dreier vorgezogen. Diese Gattungsabhängigkeit der Dreier ihrerseits wurde jedoch recht bald durch die vermehrte Verwendung von mehreren Dreiertakten innerhalb der Gattungen wieder verdrängt 377 .

375. Vg1, auch Braun, Handbuch, S.188.

376. So auch Bank, Tactus, S.230.

377. Die Tabelle 3 im zweiten Band dieser Arbeit verdeutlicht diese Entwicklung. 
Dieser Bruch in der Zeit um 1600 ist bei den Motetten kaum zu übersehen: Der Anteil des Standards "semicircolo tagliato + großer Dreier" sinkt von $82 \%$ im letzten Jahrzehnt des 16 . Jahrhunderts auf nur noch $22 \%$ im ersten Jahrzehnt des neuen Jahrhunderts. In demselben Zeitraum nimmt die Anzahl der Drucke mit dem gewissermaßen neuen Standard "semicircolo semplice + großer Dreier" sowie die Anzahl der Drucke mit mehreren Dreiern stark zu. Die Bedeutung dieser drei beschriebenen Gruppen (alter Standard, neuer Standard, mehrere Dreier) zeigt sich auch in ihrer Summe: In den Jahren 1591-1620 gehören über 90\% der ausgewerteten Motetten mit Dreiertakten einer dieser Gruppen an. In den Jahren ab 1621 beginnt die Tradition der Verwendung des großen Dreiers in Motetten in Vergessenheit zu geraten, und es erscheinen nun auch vermehrt Motetten mit nur dem kleinen Dreier ${ }^{378}$.

Die Madrigale waren in der Verwendung der Mensurzeichen nie in dem Maße standardisiert wie die Motetten. Bis in die ersten Jahre des 17. Jahrhunderts hinein erschienen auch Madrigaldrucke unter dem semicircolo tagliato und auch vereinzelt Drucke mit dem großen Dreier unter dem semicircolo semplice. Im Laufe des frühen 17. Jahrhunderts setzte sich jedoch auch hier der Standard "semicircolo semplice mit kleinem oder mehreren Dreiern" durch.

Zu den Veränderungen in den Jahren um 1600 gehört auch die viel größere Verbreitung der Dreiertakte iberhaupt. Waren Dreiertakte im 16. Jahrhundert in manchen Gattungen nur selten anzutreffen (so z.B. im Madrigal und in der Carizonette), so nimmt die Zahl der Drucke mit Dreiertakten auch in diesen Gattungen im fribhen 17. Jahrhundert schne11 $\mathrm{zu}^{379}$.

\subsection{DAS TEMPO DER ERSTARRTEN DREIERTAKTE}

Nach wie vor wird im frühen 17. Jahrhundert etwa in der Hälfte aller Musikdrucke jedoch nur eine Art der Dreiertakte verwendet. Die Verwendung verschiedener Dreiertakte zur Tempodifferenzierung (s.u.) wurde im 17.Jahrhundert als Neuerung empfunden. Sie erfuhr auch eine theoretische Behandlung. Diese Tempoabstufungen lassen sich jedoch nicht auf die Drucke ubertragen, in derien nur eine Art des Dreiertaktes Verwendung fand. Hier erfolgte die Wahl des jeweiligen Dreiers in Abhängigkeit von der Gattung (s.o.). Diese Dreiertakte behielten ihr in etwa nach den alten Regeln gewonnenes Tempo bei, während sich das Tempo der geraden Takte verlangsamte. Die Veränderung des Tempos der geraden Takte ist von der Musiktheorie durchaus bemerkt worden 380 , wenngleich ihr wenig Aufmerksamkeit geschenkt wurde. Von der sich

378. Vgl. im einzelnen zu den Prozentverteilungen die Tabelle 3 im zweiten Band dieser Arbeit.

379. Im späten 16. Jahrhundert liegt der Anteil der ausgewerteten Musikdrucke ohne einen einzigen Dreiertakt (nicht hinzugerechnet sind die hemiole - vg1. weiter unter1) bei gut 30\% (genau: 1571-1580: 33\%; 1581-1590: 37\%; 1591-1600: 35\% der ausgewerteten Drucke). In den ersten drei Jahrzehnten des 17. Jahrhunderts sinkt dieser Anteil auf unter 10\% (1601-1610: 17\%; 16111620: 11\% und 1621-1630: 6\%). 
daraus ergebenden "Abkoppelung" der ungeraden Takte wird jedoch nichts berichtet. Die Musiktheorie lehrt weiterhin ein System von Proportionen, von dem, wie bereits dargelegt, keine Hilfe zur Lösung der Tempofrage zu erwarten ist; einige modernere Autoren enthalten sich ganz einer genauen Tempoangabe für diese Takte ${ }^{381}$. Moderne Außerungen zum Tempo dieser zu einem selbständigen Tempotypus gewordenen Dreiertakte fehlen aber fast gänzlich.

Eine konkrete Tempoangabe zu einem solchen Dreiertyp findet sich aus der ersten Hälfte des 16.Jahrhunderts in der Handschrift Venedig, Bibliotheca Marciana, IV, $1227^{382}$. Hier hat ein Musiker offenbar frei von jeder musiktheoretischen Bildung ${ }^{383}$ seinen Tempoeindruck niedergeschrieben. Gegeniber

380. Dies zeigt sich zum Beispiel in einer Bemerkung in S. Cerretos Prattica musica (1601), S.191. Cerreto teilt mit, daß Orazio Tigrini in seinem Compendio di musica (1588) behauptet hätte, daß die Semicroma-Pause nicht verwendet würde. Dem hält Cerreto entgegen, daß nicht verleugnet werden könne, daß die "nuovi" sie benötigen.

381. Es muB als eine solche Enthaltung angesehen werden, wenn, nach einer gesonderten Behandlung der Proportionen (mit dem Hinweis, sie seien nicht mehr in Gebrauch), zu den Dreiertakten nur gesagt wird, dab hier drei Semibreven bzw. Minimen "auf eine Battuta" kommen (so z.B. bei A. Banchieri, Conclusio (1609), S.36 bzw. Cartella musicale (1614), S.30). Der gängige Verweis auf eine immer gleiche Battuta ist auch für das 16.Jahrhundert bereits bezweifelt worden (z.B. Dahlhaus, Tempo, S.768 oder ders., Tactus, S.27 ff). Bei Banchieri kann sie nicht mehr greifen, benutzt er doch bereits Tempowörter - sowohl in seinen musikpraktischen Werken als auch in seinen Traktaten. (s.o.).

382. Vg1. hierzu Jeppesen, Tanzbuch, S.247 f. Die diesem "Tanzbuch" voranstehende Tempoanweisung lautet (die zahlreichen Abbreviaturen wurden aufge1öst): "Nota che tuti $i$ balli che a la zeta imprincipio del ballo che tute le notte bianche con la coda val una nota negra con la Coda. Et una notta biancha tonda senza coda val tanto Come una biancha con la coda. Et una negra con la coda val una Croma Come sta qui notato: VZ:

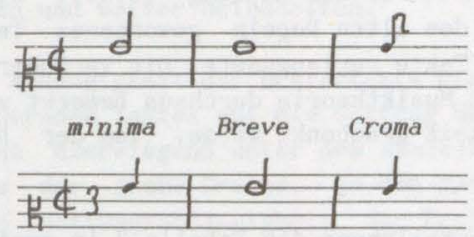

In questo segno tanto val quelle de sopra." Jeppensen datiert die Handschrift auf "um 1520" (S.245).

383. Wie aus dem Text hervorgeht, hält der Schreiber das Zeichen für den Dreiertakt, die "3" für ein "z" ("zeta"), die sich in seiner Schrift tatsächlich ähnlich sehen. Auch bei der Benennung der Noten hat er offensichtlich Schwierigkeiten ("Breve" statt "Semibreve"). 
der Theorie ergibt sich ein schnelleres Tempo: Nicht drei Minimen im Dreier entsprechen zwei Minimen im geraden Takt, sondern eine Minima im Dreier entspricht einer Semiminima im geraden Takt (Verhältnis also 2:4 statt 2:3).

Jeppesen sah in der Tempoanweisung dieses Manuskriptes lediglich eine vereinfachte Auffassung der Temporelationen ${ }^{384}$. Sicher ist ein Verhältnis $1: 2$ einfacher als ein triolisches, aber auch der Beschleunigung gegenúber dem triolischen Verhältnis kommt hier Bedeutung zu. Sie entspricht der - freilich in der ersten Hälfte des 16. Jahrhunderts noch nicht so ausgeprägten Verlangsamung der geraden Takte385. Das Streben nach einfachen Zahlenverhältnissen zur genauen Bestimmung des Tempoverhältnisses verdient allerdings ebenso Beachtung. Sicher ist auch den Musikern des 16.Jahrhunderts bewußt, daß ein solches klares Verhältnis (2:1) - insbesondere beim Ensemblespiel eine große Hilfe ist. Mit den klassischen Proportionen hat dies jedoch nichts zu tun ${ }^{386}$.

Ähnliche Tempoangaben sind leider aus dem späteren 16. und fruhen 17.Jahrhundert nicht bekannt. Die Verwendung eines Dreiertaktes mit losgelöstem, selbständigem Tempo ist dennoch im späteren 16. und fruihen 17.Jahrhundert die häufigste Form; besonders für ritornellartige Einschübe. Der Wahl des Dreiertaktes kormt hierbei keine Bedeutung zu; es wird in der Regel der dem Gattungstyp entsprechende sein (also der Dreihalbetakt in madrigalischer Musik, der Dreiganzetakt in motettischer). Die verwendeten Notenwerte im Dreier bleiben in vokaler Musik nahezu unverändert die, die auch im 16.Jahrhundert für Dreiertakte benutzt wurden: Der taktangebende (also die Semibrevis im Dreiganzetakt), der nächst größere und der nächst kleinere Notenwert - selten auch der ibernächst kleinere Wert 38 ?.

Da die Beibehaltung der alten proportionalen Regeln für die Musik aus der Zeit un und nach 1600 offensichtlich nicht möglich ist - entweder sind die geraden Takte so schnell, daß sie nicht sing- bzw. spielbar sind, oder die Dreier fallen viel zu langsam aus - wurde in der Forschung nach neuen ein-

384. Tanzbuch, S. 248

385. In dem besagten Manuskript werden im geraden Takt (immer semicircolo tagliato) Cromen in Ketten verwendet, im Dreiertakt (meist semicircolo tagliato + "3", aber auch semicircolo semplice + "3/2") Semiminimen in Ketten, so daß hier bei einem Tempoverhältnis von $1: 2$ eine Tempogleichheit der kürzesten Notenwerte bestünde. Außerdem findet auch die hemiola maggiore Verwendung.

386. Auch heute haben sich als Temporelation für Dreiertakte in der Musik dieser Zeit klare Verhältnisse durchgesetzt (6:1 oder 4:1 zwischen dem Dreiganze- und dem Zweihalbetakt) - z.T., weil man sich den Proportionen verpflichtet fiihlt und nur diese Proportion sinnvolle Ergebnisse ermöglicht, z.T. jedoch auch aus praktischen uberlegungen heraus.

387. Nur in der Instrumentalmusik wurden auch kleinere Notenwerte in den Dreiertakten verwendet - hier sind allerdings auch die Notenwerte im geraden Takt z.T. erheblich kürzer als in der Vokalmusik (s.o.). 
deutigen Temporelationen gesucht. Dabei wurde der Versuch unternommen, die Temporelationen zwischen den geraden Takten und den Dreiern mit Hilfe thematischer überlappungen zu definieren ${ }^{388}$. Gemeint sind hiermit Stellen, bei denen entweder ein Motiv sowohl in einem geraden als auch in einem Dreiertakt vorkommt oder aber ein Motiv zunächst in der einen Taktart vollständig erklingt und dann - bei seiner Wiederholung - in der einen Taktart beginnt und erst nach einem Taktwechsel endet. Wenn man nun will, daß das Motiv immer gleich erklingt, kann man davon ein Tempoverhältnis zwischen beiden Taktarten ableiten. Die Temporückschluisse aus solchen thematischen uberlappungen können selbstverständlich nur eine Art des Verhältnisses zur Folge haben: Nicht Proportionen im "klassischen" Sinn (als $3: 2$ oder 3:1), sondern Proportionen mit geraden Werten, also $2: 1,4: 1,8: 1$ etc. Andere Verhältnisse kann es bei ubberlappungen nicht geben, denn die Notation bietet keine Möglichkeiten hierfür ${ }^{389}$. Es ist aber fraglich, ob wirklich Rïckschlüsse auf Tempoverhältnisse aus diesen Fällen gezogen werden können. Bei der Mehrzahl der hierfür genannten Beispiele handelt es sich um einen ubergang von einem Dreier zuruck in den geraden Takt. Ein solcher ubergang ist in der Regel (und so auch bei den meisten der genannten Beispiele) mit einer Kadenz verbunden. Ein "Abfangen" eines Dreiers mit einer Kadenz im geraden Takt kann als ein Stilmerkmal dieser Zeit bezeichnet werden. Häufig stehen einzelne Takte im geraden Takt mit einer Kadenz zwischen zwei Abschnitten im Dreier 390 . Es ist hier gut vorstellbar, daß eine Art ausgeschriebenes Ritardando gemeint ist - die Ungleichheit der Motivwiederholung also bewust eingesetzt wurde. Auch die meisten der Adagio-Einschübe Frescobaldis sind an solchen Stellen zu beobachten (s.o.).

Scheiden die thematischen uberlappungen als Hilfsmittel, die exakte Temporelation zu bestimmen aus, so geben sie dennoch Hinweise auf die ungefähren Relationen; denn auch wenn von einer thematischen uberlappung nicht auf ein gleiches Tempo zu schließen ist, so kann die Differenz der Tempi, sofern es sich nicht nur um Schlußtöne oder Auftakte handelt ${ }^{391}$, auch nicht beliebig groß sein. Eine allgemeinverbindliche Regel kann aber für diese "Ritorne11-

388. So Machatius, Tempi, S.108 ff (u.a.), Osthoff, Spätwerk, S.211 ff und Witzenmann, Mazzocchi, S.206 f.

389. Die Problematik dieser Relationen wird bei Witzenmann, Mazzocchi, besonders deutlich. Obwohl er die Sonderstellung des Sechsvierteltaktes als Triolenschreibweise (hier haben sechs Semiminimen tatsächlich den Wert von vier Semiminimen im geraden Takt) kennt (s.u.) und für allgemein zutreffend hält ( $.207 \mathrm{f}$ ) zwingt ihn die Methode der Tempobestimmung aus Uberlappungen dazu, an einer Stelle die Gleichsetzung der Semiminima und $6 / 4$ mit der Croma im geraden Takt anzunehmen (S.206).

390. Ein solcher einzelner gerader Takt findet sich auch unter den Beispielen 0sthoffs (S.212, aus dem 6.Madrigalbuch Monteverdis). Auch hier ist sicher ein freies Tempo gemeint und dies steht ohnehin jeder Proportion entgegen.

391. Ein Großteil der Beispiele Osthoffs (S.212 ff) weist nur solche Uberlappungen auf. Sie haben kaum Beweiskraft. 
drejer" nicht gegeben werden. Sicher verlangen sie nach einem als schnell zu empfindenden Tempo.

\subsection{DIE NEUEN TAKTARTEN UND DEREN SIGNEN}

Wie bereits erwähnt, nahm nicht nur die Verbreitung der Dreiertakte uberhaupt, sondern auch die Zahl der zur Verfügung stehenden Dreier zu. Standen den Komponisten im 16. Jahrhundert nur der Dreiganzetakt und der Dreihalbetakt zur Verfügung, so kamen in den ersten Jahren des 17. Jahrhunderts der Dreivierteltakt392, der Sechsvierteltakt393 und, seltener, der Zwölfachteltakt 394 sowie der Dreiachteltakt395 hinzu; in einem einzelnen Fall wurde sogar ein Vierundzwanzigsechzehnteltakt396 verwendet.

Auf den ersten Blick scheint es sich auch bei einigen der Zeichen für die neuen Taktarten, nämlich "6/4", "12/8" und "24/16", um Proportionen zu handeln. Sie treten in einzelnen Stimmen alleine auf (in einer Stimme steht "6/4", in einer anderen behält der semicircolo seine Guiligkeit397); das Ende eines Abschnittes in einem dieser Takte kann durch eine Inversion der Signen ("4/6", "8/12" etc.) bezeichnet werden"398. In den alten Proportions-

392. Dreivierteltakte sind in den Werken von D. Caste11o, C. Gesualdo, S. d'India, G. G. Kapsberger, M. Pesenti, E. Radesca di Foggia, R. Rontani, C. Saracini und G. M. Trabaci zu finden. Ein Dreivierteltakt steht auch in der Handschrift Mag. XIX. 115 der Biblioteca Nazionale Centrale, Florenz. Nach Becherini, Catalogo, S.50, stammt diese Handschrift bereits aus dem Ende des 16. Jahrhunderts.

393. In den Werken von V. Calestani, G. Frescobaldi, M. da Gagliano, S. d'India, G. G. Kapsberger, B. Marini, G. Priuli und R. Rontani.

394. Der Zwölfachteltakt findet sich nur in einigen Werken G. Frescobaldis sowie in den Salmi passeggiati (1615) von F. Severo.

395. Dem Verfasser ist nur folgendes Beispiel für einen Dreiachteltakt aus dieser Zeit bekannt: In Le musiche... libro quinto (1623) benutzt S. d'India einen Dreiertakt mit der Vorzeichnung "3" und Taktstrichen nach jeweils sechs Cromen. An der Melodieführung ist deutlich zu erkennen, daß es sich um einen Dreiachteltakt, und nicht um einen Dreivierteltakt handelt.

396. Dem Verfasser aus dieser Zeit nur bekannt aus den Salmi passeggiati (1615) von F. Severo.

397. So z.B. in großer Fülle bei Frescobaldi ( $I 1$ primo libro de capricci (1626), Il secondo libro di toccate (1627), Il primo libro delle canzoni (1628) und In partitura il primo libro delle canzoni (1628)). Auch Severos Vierundzwanzigsechzehnteltakt steht nur in einer Stimme.

398. Solche Inversionen finden sich in folgenden Drucken: F. Anerio, Canzonette ... libro primo (1586; hier " $3 / 2$ " ... "2/3". Eine Inversion von " $3 / 2$ " findet sich auch in der Kasseler Handschrift von C. Cornets Canzon \& 8), F. Severo, Salmi passeggiati (1615); R. Rontani, zweite Auflage von Le varie musiche ... libro primo (1623); G. Frescobaldi, Il primo libro de capricci (1626), Il secondo libro di toccate (1627), Primo libro d'arie (1630). Auch nach 1630 sind Inversionen noch anzutreffen (Vgl. hierzu Geoffrey Chew, Art. 
lehrbichern und in Proportionenstücken wird man solche Proportionszahlen jedoch nicht finden. Als Proportion hat nämlich ein kürzbarer Bruch wenig Sinn.

Die Sechser-, Zwölfer- und Vierundzwanzigertakte dienen im fruhen 17.Jahrhundert als eine Art der Triolenschreibung. Ein Wechsel von einem geraden Takt zu einem dieser Takte bedeutet keinen Wechsel im Taktschlag. Diese Sechser-, Zwölfer- und Vierundzwanzigertakte gehören nicht zu den ungeraden Takten, denn auch hier dauern die beiden Teile der Battuta gleich lang, nur statt zwei Semiminimen missen nun drei unter jedem Teil der Battuta musiziert werden ${ }^{399}$. Die Geschwindigkeit der Battuta bleibt dabei unverändert nur so ist das häufige Auftreten dieser Taktarten nur in einzelnen Stimmen zu erklären.

Der Unterschied zwischen den mathematisch gleichen Sechsviertel-, Zwölfachte1- oder Vierundzwanzigsechzehnteltakten $(6 / 4,12 / 8$ und 24/16 sind der Proportion nach alles Sesquialtere, denn das Verhältnis 1 äßt sich auf $3 / 2$ kürzen) liegt in der Gruppierung der Notenwerte: In einem Sechsvierteltakt kann man zwei Gruppen zu je drei Semiminimen, in einem Zwölfachteltakt vier Gruppen zu je drei Cromen ausmachen. Trotz der, aus der Besonderheit dieser Taktarten herrihrenden, proportionalen Bindung an den geraden Takt handelt es sich bei diesen Zeichen nämlich bereits um Taktzeichen im modernen Sinn.

Der Begriff der "viertel Note", der der heutigen deutschen Bezeichnung des Sechsvierteltaktes ihren Sinn gibt, war freilich damals noch nicht bekannt400. "6/4" bedeutete für einen Zeitgenossen Frescobaldis nichts anderes, als "ein Takt enthält sechs mal den vierten Teil eines geraden Taktes". Eine solche Bezeichnung war möglich geworden, da ein gerader Takt in dieser Zeit immer ein Takt alla Semibreve war ${ }^{401}$. Auch unsere Bezeichnurig "viertel Note" hat dort ihren Ursprung.

Notation in NG, Bd.9, S.381 f und Barthe, Tempo, S.9 ff).

399. In einer von Michel'Angelo Grancino iberarbeiteten Auflage von Orazio Scalettas Scala di musica (Mailand 1657) wird dies wie folgt beschrieben: "Et in questi tempi moderni vi è ancora la Sesquialtera la quale si canta sotto la battuta ordinaria, cioè tre nel battere, \& tre nel levare, \& le Pause giuste à detta battuta." (zitiert nach Aldrich, Rhythm, S.35, Anm, 16. In der dem Verfasser vorliegenden Ausgabe von 1623 fehlt eine Aussage zu den Sechsvierteltakten). Die ubereinstimmung zwischen Triole und Sechsvierteltakt wird deutlich in einem Brief G. B. Buonamentes vom 24.2.1627 ausgedrickt: "An Stellen wo eine 3 steht, bedeutet das drei Semiminimen auf die Hebung, drei auf die Senkung. Die Ciastrupola, die das Zeichen 6/4 trägt, hat dasselbe Zeitmaß. Ich setze dies nicht für die Ausübenden, die es schon wissen, sondern um Dem gefällig zu sein, der es lernen will." (zitiert nach Nett1, Buonamente, S.542).

400. Auch heute ist die Terminologie "viertel Note" etc. keineswegs international iblich, dennoch versteht man auch in den Sprachgebieten, in denen nicht dieser Terminologie gefolgt wird, die Taktzeichen heute nicht anders 
Neben diesem modernen Verständnis der Taktsignen ist offenbar aber auch die a1te proportionale Bedeutung der Zeichen den Komponisten noch bewubt, denn sonst wären die Inversionen der Taktzeichen nicht zu verstehen ${ }^{402}$. Es ist dennoch kaum möglich von dem besonderen proportionalen Verständnis der Sechser- und Zwölfertakte auf die Temporelationen zwischen den geraden Takten und den Dreiertakten zu schließen, da diese eine vollständig eigene Qualität haben.

Auch die Signen der Dreiertakte wurden im fruhen 17. Jahrhundert of fenbar zunehmend als Taktzeichen im heutigen Sim verstanden. Die ubereinstimmung der Taktarten mit den Taktzeichen im modernen Sinn ergab sich fast wie von selbst: Wie oben beschrieben, wurden die großen Dreiertakte beibehalten, als man um 1600 vermehrt auch in Motetten den semicircolo semplice vorschrieb. Diesen Dreiertakten wurde nun in der Regel " $3 / 1$ " vorgesetzt 403 - ob dies geschah, um ein schnelleres Tempo der Dreier vorzuschreiben, oder ob hier schon ein Taktbewußtsein ausschlaggebend war, läßt sich nicht ermitteln 404 .

als im deutschen Sprachraum.

401. Vg1. hierzu weiter oben im Abschnitt I.1.3. Dieser Standard im geraden Takt machte die Entstehung der modernen Taktzeichen erst möglich.

402. Beide Interpretationen finden sich - nur drei Seiten voneinander entfernt - in W. C. Printzens, Compendium musicae (1689), S.22 und S.25. Auf S.22 hat die untere Zahl einer "Proportion" (die Verwendung dieses Wortes hier, zeigt, dak es zu einem sinnentleerten Begriff geworden ist) die Aufgabe, das Tempo anzugeben: "Je kleiner die untere Zahl einer Proportion ist/ ie langsamer soll der Tact geschlagen werden; und ie grösser dieselbe Zahl ist, ie geschwinder soll der Tact geschlagen werden." ( $\mathrm{Zu}$ dieser Tempoangabe vgl. weiter unten). Auf Seite 25 folgt dann, mit einigen Abwandlungen, die klassische Deutung: "Die Zeichen/ so eine Proportion andeuten/ seyn zween Numeri oder Zahlen/ so denen Notis secundae impositionis vorgesetzt werden/ deren oberste ist die Zahl der Noten secundae impositionis, die in einem Tact sollen gesungen werden/ die untere aber die Zahl der Noten primae impositionis, welche in gemeiner Zeit=Maßß=Art auff einen Tact gesungen werden." Beide Interpretationen der Taktzeichen schließen sich gegenseitig aus.

403. Auch die gegenüber $3 / 2$ stärker diminuierende Proportion $3 / 1$ gleicht die entstandene Differenz der Notenwerte zwischen Dreiertakt und geradem Takt nicht annähernd aus.

404. Eine interessante Mischung aus konservativem Verständnis der Proportionszahlen sowie bereits taktmäßigem Empfinden ist sehr schön an den Proportionsbeispielen aus S. Cerretos Prattica musica (1601) zu beobachten. Die Proportionen beziehen sich hier nicht mehr auf irgendwelche Großeinheiten, sondern ganz gezielt auf den jeweiligen Takt. So komnt jeweils nach einem Dreiertakt die Proportion "8/3" und "5/3" vor, unter einem geraden Takt aber die Proportion "5/2" (S.239 f). Ausgefuhrt sind diese Proportionen durch Ersetzung eines oder mehrerer Takte im geraden bzw. ungeraden Takt durch Fiinfer- bzw. Achtertakte. Es kam Cerreto nicht mehr nur darauf an, daß das mathematische Endergebnis stimmte, sondern jeweils auch die Takte muBten in sich aufgehen. 
Mit dem allmählichen Verschwinden des semicircolo tagliato als Mensur der Motetten verschwand auch die im 16.Jahrhundert ubliche Vorzeichnung " $3 / 2$ " fuir den großen Dreier. Beginnend mit den zwanziger Jahren des 17.Jahrhunderts erschien zudem auch der Dreivierteltakt auber mit der für alle Dreier verwendeten Vorzeichnung " 3 " unter dem Signum "3/4"405, das als Proportionsangabe keinen Sinn hat 406 .

Auch weiterhin bleiben dennoch Vorbehalte der Komponisten (Drucker, Herausgeber) gegenuber nach mensuralem Verständnis falschen Taktzeichensetzungen erhalten. So wird es z.B. vermieden, zwei verschiedene Dreiertakte direkt aufeinander folgen zu lassen. Die oben bereits erwähnten eingeschobenen einzelnen Takte unter dem semicircolo können in solchen Fällen das Entstehen von - nach der alten Lehre - ganz und gar widersinnigen Signen vermeiden 407 . Aber auch solche Folgen von Taktzeichen kommen bei einigen Komponisten vor 408 .

\section{2,6. DRUCKE MIT VERSCHIEDENEN DREIERTAKTEN}

Wie bereits erwähnt, stieg nach der Jahrhundertwende die Anzahl der Drucke mit verschiedenen Dreiertakten unter ein und demselben Signum für den geraden Takt stark an. Betrachtet man nur diejenigen der ausgewerteten Musikdrucke, in denen überhaupt Dreier vorhanden sind, so finden sich in rund $12 \%$ dieser Drucke aus dem letzten Jahrzehnt des 16.Jahrhunderts verschiedene Dreier unter einem Signum für den geraden Takt. Dieser Anteil stieg auf iber 40\% der Drucke aus dem dritten Jahrzehnt des 17. Jahrhunderts an. Auch diese Entwicklung verlief in den verschiedenen Gattungen nicht parallel 409 .

405. Die Vorzeichnung $3 / 4$ vor einem Dreivierteltakt findet sich in G. B. Buonamentes Quarto libro del varie sonate (1626) und G. Bruschis Terzo libro delli concerti (1629). Ein Vorzeichnung von $3 / 4$ vor einen Dreivierteltakt beabsichtigte wahrscheinlich auch $\mathrm{R}$. Rontani in der zweiten Auflage seines ersten Buches der varie musiche (1623) - es wurde im Druck dann allerdings 4/3 daraus.

406. Bei einer Proportionsangabe 3/4 wäre der Dreiertakt langsamer als der gerade Takt, obwohl er von zahlreichen Zeitgenossen als besonders schneller Dreier beschrieben wird (s.u.).

407. Etwa ein 6/4-Takt direkt nach einem 3/2-Takt bedeutet nach der alten Lehre: (der Einfachheit halber auf Grund der Einheit vier dargestellt): 4 Semibreven im geraden Takt $=6$ Semibreven nach der Proportion $3 / 2=9$ Semibreven nach der folgenden Proportion 6/4. Dies hat dann mit dem Gemeinten nichts mehr zu tun.

408. Z.B. bei Kapsberger.

409. In den reinen Instrumentalmusikdrucken aus dem zweiten Jahrzehnt des 17. Jahrhunderts liegt dieser Anteil bereits bei uber $50 \%$, in den Madrigaldrucken (hier wurden Dreiertakte insgesamt weniger verwendet) liegt er immer leicht unter dem Durchschnitt, im dritten Jahrzehnt jedoch nur noch sehr gering, in Drucken motettischer Musik wurden verschiedene Dreier in den 
Wie oben beschrieben, wurden seit dem späten 16. Jahrhundert die beiden Zeichen für den geraden Takt, der semicircolo semplice und der semicircolo tagliato, zur Tempodifferenzierung benutzt. Es liegt nahe, auch in der Verwendung verschiedener Dreiertakte Tempodifferenzierungen zu vermuten. Tatsächlich findet sich diese Vermutung auch in Theoretikeräußerungen und Vorworten vom frühen 17 . bis zum 18. Jahrhundert bestätigt 410 .

Es fällt auf, daß mit Ausnahme des Capriccen-Vorwortes von Frescobaldi sowie der kurzen Notiz von Pesenti keine der Quellen, die die Verwendung der Dreiertakte zur Tempodifferenzierung schildern, aus Italien stammt41. Diese Tatsache bedeutet nicht, daß diese Praxis in Italien nicht verbreitet gewesen wäre, sondern bestätigt nur die Praxisferne insbesondere der italienischen Musiktheorie.

Für die Relationen zwischen den verschiedenen Dreiertakten wurden in der Forschung, in der Rege1 unter Berufung auf Frescobaldi 412 , insgesamt vier

Jahren 1591-1600 noch sehr selten verwendet, in den darauffolgenden Jahrzehnten jedoch uberdurchschnittlich viel.

410. Dem Verfasser sind folgende Quellen bekannt (chronologische Ordnung): G. Frescobaldi, Vorwort zu den Capricci (1624); M. Pesenti, Il terzo libro de madrigali (1628), Tempoiberschrift zu "Più non $t$ 'amero" (s.o.); J. A. Ban, Briefe an G. B. Doni, Rom, 1.Januar 1639, sowie Florenz, 12.Apri1 1639 (Mitgeteilt in Frescobaldi, Opere complete, Bd.IV, S.XXXI); ders., Vorrede zu Zangh-Bloemzel, Amsterdam 1642 (He11, Rhythmus, S.125 f); M. Mersenne, Harmonicorum libri XII, Paris 1648 (Heckmann, Takt, S.116 f); D. Friderici, Musica figuralis, Rostock 1649 (Eberlein, Proportionsangaben, S.241); C. Simpson, Compendium of practical musick, London $3 / 1678$, S. 23 ff; J. A. Reinken, Compositionslehre, ms 1670, S.56 der Ed.; W. C. Printz, Compendium musicae, Dresden 1689, S.22; G. Carissimi (?), Ars cantandi, Augsburg 1692, S.93 der Ed., S. de Brossard, Dictionnaire de Musique, Paris 1703 (HerrmannBengen, Tempobezeichnungen, S.49 ff, bes.S.51. Vgl, auch die Faksimiles bei Elisabeth Lebeau, Art. Brossard, Sebastien de, in MGG, Bd.2, Sp.335 f); P. Prelleuer, The Newest Method, London 1731, S.11 f.

In gewisser Weise gehört hierzu auch eine Gruppe deutscher Theoretiker, deren bekanntester Vertreter Michael Praetorius ist. Diese Theoretiker gehen zwar nicht ausdrücklich auf das Tempo der Dreiertakte ein, ordnen jedoch die beiden Dreiertakte in das System "tactus tardior - tactus celerior" ein (vg1. hierzu die Tabelle Praetorius' auf S.78 des Syntagmatis musici ... tomus tertius (1619)); weiter zu nennen ist J. Magirus, Artis musicae, Frankfurt 1611 (No1te, Magirus, S.103 ff).

Ausführlich eingegangen wird auf einen Teil dieser Stellen auber in den bereits zitierten Titeln Darbellays, Heckmanns, Hells und Herrmann-Bengens bei Barthe, Tempo, S.8 ff; Dahlhaus, Proportionenlehre, S.345 ff; Darbellay, Tempo; Murata, Valentini, S.335 ff; Paulsmeier, Temporelationen und Nastasi, Tempofrage. Auf den Sachverhalt geht außerdem Apel, Violinmusik, S.35, ein.

411. Ob die Ars cantandi wirklich eine aus dem Italienischen ubersetzte Lehre Carissimis ist, wie es das Vorwort behauptet, bleibt dahingestellt. 
verschiedene Lösungen angeboten. Diese sollen nun vorgestellt und kritisch hinterfragt werden. Um diese vier Lösungsvorschläge besser in Beziehung zueinander stellen zu können, werden sie jeweils mit einem - plakativen Namen versehen.

\subsection{1. "VerdopplungstheORIE" NACH WILli ApEL}

Willi Ape1413 geht zunächst davon aus, daß der Dreihalbetakt weiterhin in proportionalem Verhältnis (Sesquialtera) zum geraden Takt steht. Die Tempi der anderen Takte erhä1t Apel, indem er die Dauer der Notenwerte in allen Dreiertakten beibehält; eine Minima soll also im Dreiganzetakt genauso lange dauern, wie im Dreihalbetakt oder im Dreivierteltakt; anders ausgedrückt: Ein Dreiganzetakt entspricht in der Zeitdauer zwei Dreihalbetakten oder vier Dreivierte1takten.

\subsection{2. "Hemiolentheorie" nach Karin Paulsmeier}

Ausgangspunkt auch für die Theorie Paulsmeiers ${ }^{414}$ ist die Annahme, daf man dem Dreihalbetakt als dem häufigsten Dreier Frescobaldis seine proportionale Beziehung zum geraden Takt (also 2 Minimen $=3$ Minimen bzw. Takt $=$ Takt) beibelieb. Da die Taktzeichen $6 / 4$ und $12 / 8$ oft nicht in allen Stimmen gleichzeitig erscheinen, kann an dem Tempo dieser Taktarten kein Zweifel bestehen. In ihnen ist eine Art der Triolenschreibung zu sehen (s.o.). Werden nun die drei Taktarten mit - nach Paulsmeier - eindeutiger Beziehung zum geraden Takt $(3 / 2,6 / 4$ und 12/8) miteinander verglichen, so ergibt sich jeweils ein hemiolisches Verhältnis (Abbildung 1). Dieses hemiolische Verhältnis uberträgt Paulsmeier nun auch auf die Beziehung des Dreihalbetakteszum Dreiganzetakt. Daraus ergibt sich, daß eine Minima des Dreiganzetaktes einer Minima des 3/2-Taktes entspricht, (Abbildung 2). Auf den bei Frescobaldi vorkommenden 3/4-Takt wendet Paulsmeier kein hemiolisches Verhältnis an; sie setzt diesen der hemiola minore gleich (vgl. dazu weiter unten bei der Behandlung der hemiola). In den hemiole sieht Paulsmeier Tempozwischenstufen. Sie erhält diese durch Gleichsetzung der colorierten Semibrevis mit der Minima im geraden Takt bzw. der colorierten Brevis mit. der Semibrevis. Für den Dreivierteltakt ergibt sich also eine Gleichsetzung der Minima mit der des geraden Taktes.

412. Zur Erinnerung sei die entsprechende Stelle aus Frescobaldis Vorwort von 1624 hier nochmals zitiert: "... e nelle trippole, o sesquialtere, se saranno maggiori, si portino adagio, se minori alquato piu allegre, se di tre semiminime, più allegre se saranno sei per quattro si dia illor tempo con far caminare la battuta allegra." (zitiert nach Sartori, Bibliografia I, S. 295 f $(1624$ b)).

413. Violinmusik, S.35.

414. Temporelationen. 
Abbildung 1

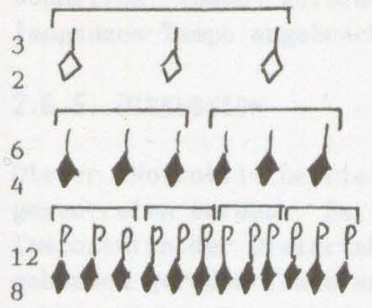

Abbildung 2
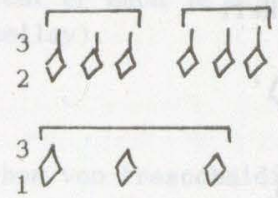

\subsection{3. "MischtheORIE" NACH HELMUT HELL}

In dem Lösungsvorschlag von Helmut He11415 kann eine Verbindung der Theorie Apels mit derjenigen Paulsmeiers gesehen werden. Ausgangspunkt sind zunächst auch für Hell die angenommene Beibehaltung der Proportion für den Dreihalbetakt (Sesquialtera) sowie das gesicherte Verhältnis zwischen geradem Takt und dem Sechsvierteltakt. In der hemiola minore416 sieht Hell eine weitere Tempostufe in der Mitte zwischen Dreihalbe- und Sechsvierteltakt, wobei er den Sechsvierteltakt als (doppelten) Dreiertakt auf der Semiminimenebene interpretiert: Drei (taktgebende) Minimen im Dreihalbetakt entsprechen dann einer Semibrevis im geraden Takt, drei Semiminimen im Sechsvierteltakt einer Minima im geraden Takt, drei schwarze Minimen (als Zwischenstufe) einer punktierten Minima im geraden Takt. Nun überträgt Hell die Temporelation zwischen den beiden Tempostufen Dreihalbetakt und Sechsvierteltakt (nämlich gleiche Dauer der Notenwerte, aber andere metrische Qualität - Höreindruck: doppeltes Tempo, vergleichbar der Theorie Apels) auf die Beziehung der nun als Tempostufe definierten hemiola minore zur verbleibenden Tempostufe, nämlich dem Dreiganzetakt. Eine (taktangebende) schwarze Minima der hemiola minore soll doppelt so schnell sein wie eine (taktangebende) Semibrevis des Dreiganzetaktes. Ein Takt mit drei Semibreven dauert also so lange, wie eine punktierte Semibrevis im geraden Takt (vgl. Abbildung 3) Im Temposystem Hells gibt es also zwei Gruppen zu je zwei Tempostufen. Innerhalb jeder Gruppe verhalten sich die Tempi im Verhältnis $1: 2$ (wie bei Ape1). Das Verhältnis der beiden Tempogruppen zueinander wurde durch Einfuhrung einer Zwischenstufe (hemiola minore) zwischen die ersten beiden gewonnen (auch Paulsmeier gewarn das Tempo der hemiole auf diese Art). Den Dreivierteltakt hält Hell wie auch Paulsmeier für eine notationstechnische Variante der hemiala minore.

\subsection{4. "Notenbildtheorie" nach Etienne Darbellay und Margaret Murata}

Der Lösungsvorschlag von Etienne Darbellay ${ }^{417}$ und Margaret Murata ${ }^{418}$ geht gänzlich andere Wege. Apel, Paulsmeier und Hell versuchten, den Widerspruch

415. Rhythmus, S. $108 \mathrm{ff}$.

416. Die hemiola maiore erwähnt. Hell nicht.

417. Tempo sowie in Vorwort zu Frescobaldi, Opera complete IV. 
Abbildung 3

Tempotabelle nach Helmut He11

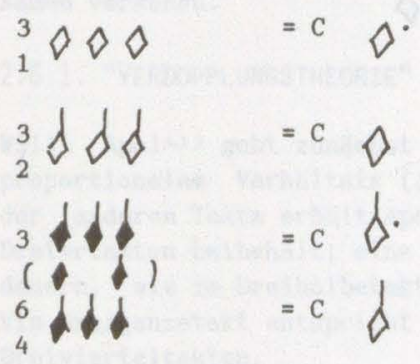

zwischen der Proportionenlehre, deren Guiltigkeit von keinem der Autoren grundsätzlich in Frage geste11t wird, und den damit eigentlich nicht ibereinzubringenden Tempoabstufungen durch die Aufstellung eines neuen (Proportionen-) Systems zu uberwinden. Dabei gingen alle drei von nur einem geraden Takt aus, zu dem sie die verschiedenen Dreiertempostufen auf unterschiedlichen Wegen in Beziehung setzten. Darbellay und Murata erreichen die unterschiedlichen Tempostufen durch Veränderung des Grundtempos (gerader Takt) bei völliger (Murata) bzw. weitgehender (Darbellay) Beibehaltung der Proportionen. Ein langsamer bzw. schneller Dreier ergibt sich also nicht durch ein verändertes Verhältnis zwischen Dreier und geradem Takt, sondern durch ein verlangsamtes oder beschleunigtes Grundtempo. Ein Dreiganzetakt ist also langsam, weil der Abschnitt im geraden Takt davor langsam ist. Das Tempo des geraden Taktes muß am Notenbild (Darbellay) erkannt werden: je größer die Notenwerte, je schneller das Tempo. Als Quelle dienen Darbellay hierfür die Abhandlungen der Theoretiker zum Unterschied zwischen dem Tempo unter dem semicircolo semplice und dem semicircolo tagliato; wurde doch dort darauf hingewiesen, daß das Tempo unter dem semicircolo tagliato aufgrund der vielen weiben Notenwerte schnell sein müsse bzw. umgekehrt. Bei Frescobaldi sei das jeweilige Tempo der geraden Takte zusätzlich an der Taktstrich-

418. Valentini. P. F. Valentinis Traktate dienen Murata hier nur als Beleg für die Gültigkeit der Proportionen auch bei einem Theoretiker der Generation Frescobaldis, der seine "observations and comments clearly derive from firsthand, practical experiance" (Murata, S.327). In der Tat teilt sie eine Reihe interessanter Informationen aus Valentinis Traktaten mit - an den entsprechenden Stellen dieser Arbeit wird darauf verwiesen. Dennoch kann seine Praxisnähe kaum auf die Proportionenlehre ubertragen werden. Wie andere Theoretiker auch, bezieht Valentini seine Beispiele für die Verwendung der Proportionen von Josquin, Compère, Palestrina und Metallo (Murata, S.334). $\mathrm{Zu}$ den vom ihm genannten Proportionen gehören auch 5/4, 7/4 und 9/8 (Murata, S.348, Anm.8), dem 6/4-Takt und dem 12/8-Takt fügt er einen 10/8-Takt hinzu (Murata, S.350, Anm.24). Die Praxisferne - selbst von der Praxis des 16. Jahrhunderts - dürte damit genügend belegt sein. 
setzung zu erkennen: Steht ein Taktstrich nach je einer Brevis, so ist ein schnelles Tempo gefordert, steht er nach je einer Semibrevis so ist ein langsames Tempo angebracht (Darbellay).

\section{$2,6,5$, DISKUSSION}

Dieser "Notenbildtheorie" muß schon von Frescobaldis Formulierungen her entgegentreten werden. Es ist nicht einzusehen, warum Frescobaldi, wenn die Tempostufen der Dreiertakte sich aus den unterschiedlichen Tempi der vorhergehenden geraden Takte ergeben, in seinem Vorwort dem Notenbild der Dreiertakte solchen Raum gewährt, jedoch in Bezug auf die geraden Takt nichts in dieser Richtung schreibt419. Auch muß auf den sich ergebenden Widerspruch zwischen dem Zusammenhang von Notenbild und Tempo im geraden und ungeraden Takt hingewisen werden. Im geraden Takt deuten bei Frescobaldi nach Darbellay große Notenwerte auf ein schnelles Tempo, im Dreiertakt aber auf ein langsames Tempo hin! Die von Darbellay als Erkennungszeichen für ein langsameres Tempo herangefuhrte engere Taktstrichsetzung bei Abschnitten mit vielen kurzen Notenwerten verwundert nach dem oben uber die Taktstrichsetzung Gesagten nicht. Auch in Frescobaldis Partitur dienen die engeren Taktstriche an diesen Stellen einer größeren übersichlichkeit 420 .

Der "Hemiolentheorie" Paulsmeiers wird man ebenfalls schon vom Ansatz her widersprechen müssen. Es ist aufgrund der grundsätzlichen Verschiedenheit von Dreiertakten und dem Sechsvierte1- bzw. Zwölfachteltakten nicht möglich, aus dem Verhältnis der letzteren zum geraden Takt Riickschluisse auf die Beziehungen der Dreiertakte zum geraden Takt zu ziehen. Auf die Besonderheit des Sechsviertel- bzw. Zwölfachteltaktes wurde oben bereits eingegangen 421 . Der gleiche Kritikpunkt gilt auch gegenuiber der Theorie Helmut Hells.

Die Idee von Ape1s "Verdoppelungstheorie" ist zunächst naheliegend: Geht man von einem Fortbestand eines proportionalen Systemes aus, kann "schneller" oder "langsamer" nur als proportionale Beschleunigung oder Verzögerung gesehen werden $(1: 2)$. Allerdings bedeutete $3 / 1$ nach der klassischen Lehre aber

419. Frescobaldis Vorworte in Bezug auf die Tempi im geraden Takt sind weiter oben ausfihrlich dargestellt.

420. Es ist hier nicht möglich, auf alle Unstimmigkeiten insbesondere in den Arbeiten Etienne Darbellays einzugehen. Es wird an vielen Stellen deutlich, daß Darbellay auch mit den klassischen Proportionen-Lehren nicht vertraut ist. So sieht Darbellay bei direktem Aufeinandertreffen zweier Dreiertakte, bzw. eines Dreier- und eines Sechser-Taktes nur das Problem, daß sie - in seiner Theorie - zu unterschiedlichen Arten von geraden Takten gehören, nicht jedoch, daß Proportionszahlen sich nicht unbedingt auf den geraden Takt, sondern auf den jeweils vorangehenden Takt beziehen (Tempo, S.319). Auf solche Probleme wurde weiter oben hingewiesen. Seine Ausgabe der Capriccen ist hingegen vorzüglich, da in dem Partiturteil der Ausgabe, Notenwerte, wie auch Signen und Schwärzung unverändert wiedergegeben sind.

421. Im Abschnitt I.2.5. 
schneller als $3 / 2$ und nicht langsamer. Es ist auch nicht einzusehen, warum eine Proportion (3/2) als solche gedeutet wird, die anderen Dreiertakte aber ihr Tempo unabhängig von deren Signum von dieser einen Proportion her beziehen.

Letztendlich fuhrt Apels Theorie aber auch zu ganz und gar unbrauchbaren Tempi. Setzt man - im Gegensatz zu Apel - ein Tempo von ungefähr Minima = MM 70 als (theoretisches) Tempo voraus, so ergäbe sich unter Anwendung von Apels Theorie für einen Dreiganzetakt eine Dauer von ungefähr 3,5 Sekunden, oder uber einer Sekunde für eine Semibrevis422. Schon ein solcher Dreier ist kaum noch als solcher zu erkennen. Apel nimmt jedoch als Tempo für den geraden Takt Minima = MM 42423 an; dann dauert ein Dreiganzetakt schon 6 Sekunden, eine Semibrevis also 2 Sekunden 424 - das ist sicherlich völlig undenkbar.

\section{$2,6,6$, VERSUCH EINER NEUEN UNPROPORTIONALEN DARSTELLUNG DER TEMPORELATIONEN}

Frescobaldis Wortlaut enthält keinerlei genaue Tempoanweisung. Er beschreibt lediglich recht vage die Tempounterschiede der drei Dreiertakte: Dreiganzetakt = "adagio", Dreihalbetakt = "alquanto più allegre", Dreivierteltakt = "più allegre". Zunächst stellt sich die Frage nach der Bedeutung des Wortes "adagio" in diesem Zusammenhang. Ist dieses "adagio" absolut zu sehen (also vergleichbar einem "adagio" in einem geraden Takt), oder ist es relativ, bezogen auf das sonst ubliche Tempo eines Dreiertaktes zu verstehen? Eher ist hier an ein solches relatives Verständnis zu denken. Auch an anderen Stellen erscheinen die Tempoworte als Zusatz; sie geben nicht ein Tempo an, sondern sie modifizieren ein sich z.B. aus Gattung und Mensurzeichen ergebendes Tempo 425 .

Vergleicht man die verwendeten Notenwerte im Dreiganzetakt mit denen im geraden Takt, so ergibt sich, daß die Semiminima im Dreiganzetakt seltener (und nur in Zweierpaaren) erscheint, als die Semicroma des geraden Taktes. Es ergibt sich - unter der Voraussetzung, dak die Bewegung in den beiden Taktarten ähnlich sein soll - ungefähr das Verhältnis: halber Takt unter dem semicircolo semplice entspricht einem Dreiganzetakt420. Bedenkt man das

422. Minima $=$ MM $70 \rightarrow$ Semibrevis $=$ MM 35. Unter $3 / 2$ also perfekte Semibrevis $=$ MM35. Bei einer Gleichsetzung der Semibrevis im Dreihalbetakt mit der des Dreiganzetaktes ergibt sich für den Dreiganzetakt ein Tempo von etwa MM 17 für eine perfekte Brevis. $60 / 17$ = etwa 3,5.

423. Ape1, Violinmusik, S. 35

424. Minima $=$ MM $42 \rightarrow$ Semibrevis bzw. perfekte Semibrevis $=$ MM $21 \rightarrow$ perfekte Brevis etwa MM 10, also 6 Sekunden.

425. Vgl, oben im Abschnitt I.1.2.4.3.

426. Wenn die Semiminima des Dreiganzetaktes seltener (also wohl schneller) als die Semicroma des geraden Taktes ist, könnte die perfekte Brevis etwa der Minima des geraden Taktes entsprechen (bei diesem Verhältnis wäre die Semiminima des Dreiers so schnel1 wie Cromen-Triolen im geraden Takt). 
"adagio", kann man wahrscheinlich ein langsameres Tempo für die Dreiganzetakte annehmen (also ein Dreiganzetakt dauert länger als ein halber gerader Takt).

Zum Dreihalbetakt besagt Frescobaldis Vorwort, daß er "alquanto più allegre" sei, also "etwas heiterer". Schon das Wort "alquanto" schließt ein doppeltes Tempo aus, beinhaltet es doch die Bedeutungen "wenig" und "unbestimmt". Auch die verwendeten Notenwerte sprechen für ein Tempo, nicht allzuweit von dem des Dreiganzetaktes entfernt: Sie sind - bezogen auf den Wert des tactus, also die perfekte Brevis im Dreiganzetakt bzw. die perfekte Semibrevis im Dreihalbetakt - dieselben ${ }^{427}$; die küzeren Notenwerte sind in Frescobaldis Dreihalbetakten sogar eher häufiger ${ }^{428}$. Nur beim Dreivierteltakt läßt sich die Beschleunigung gegenuber den anderen Dreiern auch an den Notenwerten ablesen: Der Wert, der den kïrzesten Notenwerten der anderen Dreier entspräche (also die Semicroma ${ }^{49}$ ), feh1t hier. Die Tatsache, daß die Notenwerte des Dreivierteltaktes mit denen des Sechsvierteltaktes ubereinstimmen, läßt vielleicht an eine Tempogleichheit der beiden Taktarten denken ${ }^{30}$. Da jedoch in den Zwölfachteltakten - sie kommen im Tempo mit den Sechsvierte1takten ubberein - die Cromen sehr viel häufiger sind als in den Dreivierteltakten, wird man für die Semiminima im Dreivierteltakt doch eher ein schnelleres Tempo annehmen, als für die der Sechsvierte1- und Zwölfachteltakte. Die unterschiedlichen Häufigkeiten der kleinen Notenwerte in den im Tempo gleichen Sechsvierte1- und Zwölfachteltakt beweisen zugleich, wie wenig Endgiiltigkeit solche aus Notenwertsvergleichen gezogenen Rückschluisse auf das Tempo haben.

In Beziehung zueinander gebracht, könnte sich etwa folgendes Bild ergeben:

Dreiganzetakt: etwas langsamer als ein halber gerader Takt, also ungefähr zwei Semibreven $=$ Minima .

Dreihalbetakt: ungefähr Takt $=$ halber Takt, also perfekte Semibrevis = Minima.

Dreivierteltakt: etwas schneller als ein halber Takt, also ungefähr Minima = Semiminima.

oder anders dargestellt:

427. Im Dreiganzetakt wurde selten die Semiminima als kleinster Notenwert verlangt, im Dreihalbetakt selten die Croma.

428. Sicher, da dem Dreihalbetakt kein Adagio-Charakter mehr anhaftet. Wie oben gezeigt waren auch bei Frescobaldis Adagio-Teilen im geraden Takt eher längere Notenwerte notiert.

429. Sie entspricht der Semiminima des Dreiganzetaktes bzw. der Croma des Dreihalbetaktes ( $=$ jeweils ein Sechstel des Taktes).

430. Eine Tempogleicheit zwischen Dreivierteltakt und Sechsvierteltakt würde jedoch bedeuten, daß der Taktschlag im Dreivierteltakt doppelt so schnell wäre (im Dreivierteltakt kommen drei Semiminimen auf einen ungleichen - Taktschlag, im Sechsvierteltakt aber sechs Semiminimen auf einen - gleichen - Taktschlag. 


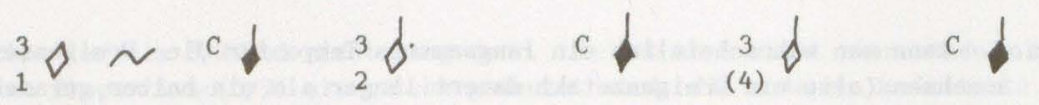

Diese Werte sollen dazu dienen, eine Vorstellung der möglichen Relationen zu vermitteln. Sie verstehen sich nicht als Proportionen. Diese Relationen sind, versucht man sie exakt in die Praxis umzusetzen, freilich sehr schwierig zu treffen. Geht man jedoch grundsätzlich von dem Verhältnis zwei Dreiertakte $=$ ein gerader Takt aus, und verzögert dann den Dreiganzetakt ein wenig, bzw. nimmt den Dreivierteltakt etwas schneller, stellen sich diese Werte fast von selbst in etwa ein.

Diesen Temporelationen - hergeleitet anhand des Vorwortes zu den Capriccen sowie der Werke Girolamo Frescobaldis - steht ein Vorwort Sigismondo d'Indias entgegen431. D'India empfiehlt dort, die "sestupla" (gemeint ist der Sechsvierteltakt) als Dreier mit je drei Semiminimen "a battuta presta" zu singen, da die Geschwindigkeit der "sestupla" den Sängern Schwierigkeiten bereite. Sofern auch d'India den proportionalen Sechvierteltakt (triolische Aufteilung der Minima wie bei Frescobaldi) voraussetzt, ist es unverständlich, warum dessen Schnelligkeit den Sängern Schwierigkeiten bereiten soll. Wahrscheinlich ist der Sechsvierteltakt für d'India ein selbständiger, schneller Takt. Will man statt eines Sechsvierteltaktes (mit damals iblichem zweiteiligen Taktschlag) zwei Dreiertakte dirigieren, so sind nur halbsoviele Noten in einem Takt. Selbst wenn man besonders schnell dirigiert - "a battuta presta" -, ist es gut vorstellbar, daß das sich ergebende Tempo langsamer ist.

Sigismondo d'India ist nicht der einzige, dessen Verwendung der unterschiedlichen Dreiertakte von derjenigen Frescobáldis abweicht. Bei den Werken einiger anderer Komponisten hat man den Eindruck, daß sie die beiden Hauptdreier - den Dreihalbe- und den Dreiganzetakt - genau in umgekehrter Weise verwenden. Für sie ist der Dreiganzetakt der schnellere und der Dreihalbetakt der langsamere Dreier 432 .

Ein einheitliches Notationssystem, wie es im 16.Jahrhundert vielleicht wenn auch eingeschränkt - bestanden hat, gab es im friihen 17.Jahrhundert

431. Le musiche e balli (1621), vg1. Abdruck des Vorwortes im zweiten Band dieser Arbeit auf S. 14. Auch M. Praetorius erwähnt im Vorwort zu seiner Trepsichore (1612) die Möglichkeit, einen Sechsertakt als zwei Dreiertakte zu taktieren (Heckmann, Tact, S.119).

432. Vg1. dazu im nächsten Abschnitt. Zu diesen Komponisten gehören, außer den im nächsten Abschnitt Genannten, B. Strozzi und F. Vitali (ein Beispiel Vitalis wird weiter unten im Abschnitt. I.3.2.2.1. eingehender zu besprechen sein - vg1. auch Notenbeispie1 I,13). Auf diese Uneinheitlichkeit in Bezug auf den Dreiganzetakt machte auch J. A. Reinken in seiner Compositionslehre (ms 1670) aufmerksam: "NB. Dieser Tripla Major erfordert einen langsamen, gravitetischen tact oder Battuta seiner ahrt und Natur nach, wiewohl er aber nebst anderen auch etwa gar geschwinde gebraucht wirdt". (S.56). 
nicht. Eine solche Aufsplitterung in mehrere verschiedene - gattungsabhängige, personenabhängige, ortsabhängige und vor allem vom Entstehungsjahr abhängige - Notationsarten ist nicht nur in Bezug auf die Dreiertakte zu beobachten. Auf diese Tatsache wurde bereits oben im Zusammenhang mit den beiden Zeichen für den geraden Takt hingewiesen. Die Uneinheitlichkeit der Notation tritt noch sehr viel deutlicher hervor, wenn man bedenkt, das im frihen 17. Jahrhundert auch noch Musik aus der Mitte des 16. Jahrhunderts unverändert nachgedruckt wurde - also unter Umständen denselben Ausführenden Madrigale Arcadelts und Monteverdis vorlagen.

Auch bei den anderen Kapiteln dieser Arbeit wird immer wieder auf die Uneinheitlichkeit der Notation dieser Zeit zu verweisen sein. Eine der Konsequenzen daraus ist es, daß man annehmen kann, daß ein Werk schon zu seiner Zeit unterschied1ich aufgefuhrt wurde. Zwar versuchen zahlreiche Komponisten mit Vorworten auf die eine oder andere Besonderheit in ihren Drucken hinzuweisen, doch können auch sie nur ihren Umkreis im Auge gehabt haben. Sie erklären Besonderheiten, die ihnen als solche bewubt sind; sie können nicht wissen, wie ihre Noten in anderen Städten oder Ländern (z.B. in Deutschland!) verstanden wurden.

\subsection{DREIERTAKTE MIT TEMPOWORTEN}

Sowohl die Tatsache, daß die uberwiegende Mehrheit der Dreiertakte Ritornellcharakter hat - die affekthaltigen Abschnitte einer Komposition stehen zumeist im geraden Takt -, als auch das Vorhandensein einer ganzen Reihe von verschiedenen Tempostufen für die Dreiertakte, hat sicher dazu geführt, daß Tempoworte zunächst hauptsächlich für geradtaktige Teile benutzt wurden. Das einzige dem Verfasser bekannte Beispiel für die Verwendung eines Tempowortes fiir einen Dreier aus den ersten zwei Jahrzehnten des 17. Jahrhunderts entstammt der bereits mehrmals zitierten Battaglia Adriano Banchieris. Die Tempoworte dieser Battaglia sind weiter oben bereits ausfuhrlich besprochen worden 433 .

Ab der Mitte des dritten Jahrzehntes des 17 .Jahrhunderts nahm die Zahl der Tempoworte bei Dreiertakten stark $\mathrm{zu}^{434}$. Die Tempoworte finden sich zumeist

433. Im Abschnitt I.1.2.4.3.; vgl. die übertragung dieses Stückes im zweiten Band dieser Arbeit als Notenbeispiel I,2. Ebenfalls bei einem Dreiertakt stehen zwei der oben angesprochenen Tempo- und Registerangaben in Satzform des "Magnificat à versi \& intiero" aus A. Banchiers Salmi festivi (1613). Die beiden Verse im Dreiertakt ("Quia fecit" und "Fecit potentiam") tragen jeweils den Hinweis "Allegro \& pieno".

434. Folgende Drucke mit Tempoworten für Dreiertakte sind dem Verfasser aus dieser Zeit bekannt: N. Corradini, Motetti (1624), A. Grandi, Motetti a 1, 2 et 4 (1625) , ders., Motetti a 1 e $2 \ldots$ libro secondo (1625, nach Seelkopf, Grandi, Bd.II, S.188), ders., Motetti a 1, et 2 ... 1ibro terzo (1629), G. B. Buonamente, Il quarto libro de varie sonate (1626), D. Castello, Sonate concertate ... libro primo (secondo) (1629), B. Montalbano, Sinfonie (1629), 
in Drucken, in denen auch mehrere verschiedene Dreiertakte verwendet wurden435; es sind dies in der Regel der kleine und der große Dreier436. Die Tempoworte wurden hier allerdings nicht dazu benutzt, den Kontrast zwischen den beiden Dreiertypen zu verschärfen oder uberhaupt durch zusätzliche Tempoworte zu verdeutlichen, sondern in der Mehrzahl der genannten Drucke bleibt eine Art des Dreiers gänzlich frei von Tempoworten (es ist in der Regel der Dreihalbetakt), während der andere Dreier mit sowohl verlangsamenden als auch beschleunigenden Tempoworten versehen ist437. Wie man auch an anderen Stellen beobachten kann, ist der Dreihalbetakt zum mittleren, wenn nicht gar langsamen Dreier geworden. Der große Dreier wird als schneller Dreier sowie als variabler Dreier benutzt; vielleicht aufgrund des Fehlens einer theoretischen Bindung an den semicircolo semplice. Dario Castello schließlich beschränkt sich in seinem zweiten Buch auf den Dreihalbetakt, den er in allen Tempostufen benutzt. Hier ist die Entfernung zum späteren System, in dem jede Taktart praktisch jedes Tempo erhalten kann, nicht mehr sehr groß438.

\subsection{Die Mensurzeichen DER DReiertakte}

Den Mensurzeichen der Dreiertakte wurde in dieser Arbeit bisher kaum Beachtung geschenkt. In der Tat waren zumindest im 17.Jahrhundert allein die Notenwerte ausschlaggebend. Frescobaldi spricht nur allgemein von "sesquialtere o trippole", die Taktarten unterscheidet er dann aber nach den Notenwerten439. Wie bereits dargelegt, entwickeln sich die alten Proportionszahlen ( $3 / 2$ etc.) im 17.Jahrhundert immer mehr zu modernen Taktzeichen 440.

G. Rovetta, Madrigali concertati (1629), G: Scarani, Sonate concertate (1630).

435. Lediglich im zweiten Buch von Castello findet sich nur der kleine Dreier, in den Sonate von Scarani nur der kleine Dreier und die hemiola minore.

436. Ausnahmen sind das erste Buch Castellos (Dreihalbe- und Dreivierteltakt) sowie das quarto libro de varie sonate von Buonamente (Dreihalbe-, Dreiviertel- und Sechsvierteltakt).

437. Nur bei Montalbano ist es umgekehrt: Hier ist nur der Dreihalbetakt einmal mit einem Tempowort ("Tardo") versehen.

438. Für die These von Dahlhaus, Proportionenlehre, S.359, daß die Tempoworte in den Dreiertakten darüber unterrichten sollten, ob die Ziffern $(3 / 2$ etc.) als Brüche (= Taktzeichen) oder Gleichungen (= Proportionen) zu lesen seien, 1äßt sich keine Bestätigung finden.

439. Vorwort zu den Capriccen von 1624 - vgl. dazu ausführlich weiter oben. 440. Die Bedeutungslosigkeit der alten Proportionszahlen fuhrte auch zu z.T. unsinnigen Verwendungen. So bezeichnet P. F. Valentini einen geschlossenen Kreis mit Punkt in der Mitte und den Zahlen $3 / 1$ als Zeichen für den Takt mit drei Minimen (Murata, Valentini, S.340 f). Solche Zeichen können bei einem anderen Komponisten oder Theoretiker wieder ganz anders verstanden werden. Die ubertragung dieser Deutung auf einen Ausschnitt aus Frescobaldis Missa sopra l'aria de11a Monica (Murata, S.341, Bsp. 5d) fiihrt zu einem 
Es stellt sich nun auch die Frage, welche Bedeutung den Zeichen der Perfektion und Imperfektion in dieser Zeit zukam.

Bereits im 16.Jahrhundert nahm die Bedeutung dieser Zeichen stark ab. Mit der Festigung des Dreiertaktes als eigenständigem Takttypus neben dem geraden Takt wurde aus der zunächst immer angezeigten Perfektion eine Selbstverständlichkeit.

In den italienischen Drucken der hier zu behandelnden Jahre ist der Kreis als Zeichen für das tempus perfectum, also das Perfektionszeichen für den großen Dreier, noch recht häufig anzutreffen: Nimmt man auch jene Drucke hinzu, die in der Setzung dieses Zeichens nicht ganz konsequent sind, so ist dieses Zeichen während des gesamten Zeitraumes in rund $60 \%$ der ausgewerteten Drucke mit Dreiganzetakten anzutreffen. Die Verwendung des Prolationspunktes für den kleinen Dreier (Dreihalbetakt) hingegen ist stets nur eine Ausnahme gewesen. Auch das liegt sicher in den unterschiedlichen Traditionen begründet: Der Dreiganzetakt war der Standard des 16.Jahrhunderts, der Dreihalbetakt trat erst zusammen mit dem wieder aufkommenden semicircolo semplice häufiger auf. Als - zumindest in dieser Weise - neue Taktart war der Dreihalbetakt hier frei von mehr oder weniger uberkommener Tradition. Daruber hinaus mag die Verwendung der prolatio perfecta als Augmentationszeichen im 16. Jahrhundert 441 manche Komponisten und Drucker zunächst davon abgehalten haben, dasselbe Zeichen nun einem Dreiertakt voranzustellen. In den Drucken des frihen 17.Jahrhunderts ist das Perfektionszeichen für den kleinen Dreier zwar etwas häufiger, aber immer noch recht selten anzutreffen 442 .

Diese Zeichen konnten so oft fehlen, da ihnen kaum noch Bedeutung zukam. Vergeblich ermahnten einige Theoretiker zu Beginn des 17. Jahrhunderts die Komponisten, nur nach den Zeichen für das tempus perfectum (Kreis) oder die prolatio perfecta (Punkt im Kreis oder Halbkreis) perfekte Noten zu schreiben ${ }^{43}$. Giovanni Battista Olifante beschäftigt sich gar in einem ganzen Traktat fast ausschließlich mit dem Problem der Perfektion und Imperfektion 444 - nicht ohne Beispiele für den verbreiteten MiBbrauch zu nennen.

völlig unsinnigen Ergebnis, denn Frescobaldi bezeichnete mit dem beschriebenen Zeichen einen Dreiganzetakt.

441. Eine ausfuhrliche Abhandlung der verschiedensten Arten der prolatio perfecta findet sich bei L. Zacconi, Prattica di musica (1596), fol.107' $124^{\prime}$.

442. In gut 3\% der ausgewerteten Drucke aus dem letzten Jahrzehnt des 16. Jahrhunderts ist das Zeichen für die prolatio perfecta konsequent verwendet worden (tempus perfectum: gut $55 \%$ ) und in etwa $7 \%$ der Drucke gelegentlich (tempus perfectum: rund 17\%); im dritten Jahrzehnt des 17. Jahrhunderts konsequent in gut $6 \%(42 \%)$, gelegentlich in knapp $15 \%$ (28\%).

443. Z.B. S. Cerreto, Prattica musica (1601), fünftes und sechstes Avertimenta (S. $260 \mathrm{ff}$ ).

444. Trattato brevissimo intorno allle (!) proportioni cantabile (1611). Die Proportionen im eigentlichen Sinn sind allerdings nicht Gegenstand 
Adriano Banchieri - als modern orientierter Theoretiker - weist hingegen darauf hin, da日 die Perfektionszeichen auch weggelassen werden können ${ }^{45}$.

Wie fest die Möglichkeit der Perfektion 446 mit einem jeden Dreiertakt verbunden war - unabhängig davon, ob sie durch ein Mensurzeichen angezeigt wurde oder nicht - zeigt sich im fruhen 17. Jahrhundert besonders deutlich in den neu hinzugekommenen Taktarten. Gegen jede Theorie wird nun auch die Minima (im Dreiviertel- und Sechsvierteltakt) als perfekte Note behandelt447; ja sogar geschwärzte Noten in den hemiole sind mitunter perfekt448. Die Art des Perfektionszeichengebrauches zeigt dariber hinaus, daß die Bedeutung der Zeichen auch dort, wo sie notiert sind, oft nicht mehr bekannt war bzw. ignoriert wurde: So wird der geschlossene Kreis - wohl als ein zu einem Dreiertakt gehörendes Zeichen empfunden - nicht selten auch einem Dreihalbetakt vorgesetzt, obwohl er hier keine Funktion erfül1t 449 .

Einige Beispiele durchaus bewußter, wenn auch eher untraditioneller Verwendung der Mensurzeichen lassen sich dennoch ausmachen. So legt eine Anzahl von Komponisten Wert darauf, daß zu Anfang ihrer Stücke immer das Hauptmensurzeichen - der semicircolo semplice oder semicircolo tagliato - steht, auch wenn ein Stück mit einem Dreiertakt beginnt. In vielen Drucken, die sonst den Dreiertakten nur Zahlen vorsetzen, erscheinen die Zahlen also, sofern ein Stlick mit einem Dreiertakt beginnt, zusammen mit einem semicir-

dieses Traktates, sondern lediglich die verschiedenen Dreiertakte mit den dazugehörigen Imperfektionsregeln. Mit den "proportioni cantabili" meint 0lifante offensichtlich nur allgemein die Dreiertakte.

445. Cartella musicale (1614), S.168: "Soggiungendo appresso che tra gli moderni conpositori alcuni vsano auanti gli numeri della proportione il Tempo chiuso semplice ouero tagliato \& altri pongono gli numeri assolutamente tutti pero a mio giuditio fanno bene, ...".

446. Und damit natürlich auch die der Alteration, wenngleich diese kaum noch benutzt wird.

447. So bei S. d'India, Le musiche ... libro quinto (1623), G. Frescobaldi, I1 secondo libro di toccate (1627) und B. Marini, Sonate (1629).

448. G. Grabbe, Il primo libro de madrigali (1609), G. Puliti, Baci ardenti (1609), P. Quagliati, Carro di fedelità (1611) und B. Marini, Scherzi (1622). Der Theorie nach ist eine schwarze Note immer imperfekt. Einen aus diesem Grund uberflüssigen Divisionspunkt verwendete P. A. Giacobetti in Motectorum ... liber primus (1589) in einer hemiola.

449. Im tempus perfectum ist die Brevis perfekt; sie kommt in einem Dreihalbetakt jedoch nicht vor. Die Semibrevis hingegen, die als perfekte Note behande1t wird, ist beim einfachen Kreis eigentlich imperfekt. Diese Anwendung findet sich z.B. regelmäBig im Werk M. da Gaglianos. Ebenso sinnlos ist es, einem geschwärzten Dreiertakt (s.u.) außer einer " 3 " auch ein Perfektionszeichen vorzusetzen, wie es bei A. Feliciani, Brevis ... psalmodia (1590) und G. Carrone, Il primo libro delli motetti (1629) zu beobachten ist, oder die Vorzeichnung einer perfekten prolatio vor einem Dreivierte1takt (G. Frescobaldi, Toccate... libro primo (1615, 4/1628)). 
colo450. In einigen Drucken, in denen jedem Dreiertakt auch ein Perfektionszeichen vorgesetzt ist, steht, sofern ein Stück mit einem Dreier beginnt, zusätzlich ein semicircolo noch vor dem Perfektionszeichen ${ }^{451}$.

Gelegentlich wurde in Drucken mit dem semicircolo semplice als Hauptmensurzeichen großen Dreiertakten ein semicircolo tagliato vorgezeichnet. Hier war dem Komponisten (oder Drucker bzw. Herausgeber) offenbar bewust, das der Dreiganzetakt eigentlich zum semicircolo tagliato gehört452.

Die häufigste gezielte Verwendung eines Mensurzeichens ist die Notation des Zeichens der prolatio perfecta ohne Zahlen. Diese Art der prolatio perfecta ist im 16.Jahrhundert als Zeichen einer Augmentation verwendet worden (s.o.). Beginnend im späten 16.Jahrhundert erscheint die prolatio perfecta bei einer Anzahl von Komponisten ${ }^{453}$ in Verbindung mit weißen Semiminimen 454 als eine Möglichkeit, einen Dreihalbetakt zu notieren. Diese Dreihalbetakte unterscheiden sich - außer in der Notation - nicht von den anderen, wahrscheinlich also auch nicht im Tempo. Adriano Banchieri sieht in dieser pro-

450. Die Zahl dieser Drucke ist nicht gering: In uber 10\% (im zweiten Jahrzehnt des 17. Jahrhunderts sogar in uber 20\%) der ausgewerteten Drucke konnte dies beobachtet werden. Es hat den Anschein, daß der semicircolo zu einem bedeutungslosen Zeichen geworden ist, das eben immer zu Anfang eines Stückes zu stehen hat. Die Dreiertakte nach diesem semicircolo (= Zeichen für das tempus imperfectum) können dennoch perfekte Noten aufweisen.

451. So findet sich das Anfangssignum " $\mathrm{C} \emptyset 3 / 2$ " bei E. Trabattone, Concerti (1629), G. Carrone, Il primo libro delli motetti (1629) und ubrigens auch bei Heinrich Schüz, z.B. vor dem "Cantate Domino" aus den Cantiones sacrae (Freiberg 1624, Vgl. Schütz-GA, Bd.IV, S.101), das Anfangssignum "C 03" bei A. Diruta, Sacri motetti (1630).

452. F. de Soto, I1 primo libro delle laudi (1583); F. Guerrero, Canciones (1589); V. Calestani, Madrigali et arie (1617).

453. Banchieri nennt als Beispiele für diese prolatio Kompositionen von G. Croce (Conclusio (1609), S.35) und L. Marenzio (Cartella musicale (1614), S.32). Außer in den Werken Marenzios und vor allem Croces sind dem Verfasser Werke folgender Komponisten mit solchen Dreiern bekannt: A. Gabrieli, M. A. Ingegneri, P. Masnel1i, M. Chiaula, F. Stivori, L. Bati, P. Sartorius, P. M. Marsolo, A. Mortaro, V. Pozzo, S. Bernardi, G. P. Nodari und G. Frescobaldi. 454. Bei M. A. Ingegneri, Sacrarum cantionum ... liber primus (1586) und M. Delipari, I baci (1630) werden sogar weiße Cromen notiert. Die Notation der weißen Semiminimen ist nicht immer konsequent durchgefuhrt; gelegentlich findet man auch weiße und schwarze Semiminimen gemischt vor. Weiße Semiminimen wurden auch außerhalb der prolatio perfecta in gewöhnlichen Dreihalbetakten oder gar Dreiganzetakten verwendet. S. d'India war offensichtlich die Bedeutung der weiBen Semiminima nicht bekannt, denn er verwendet sie in Le musiche e balli (1621) als Cromen. Auch die Terminologie dieser Noten war uneinheitlich. So bezeichnet $S$. Cerreto sie als "Semicroma bianca nella Prolatione Perfetta... la quale vale quanto una Semiminima" (Prattica musica (1601), S.184). 
latio eine besondere Art der hemiola455. Selten kommt im späten 16.Jahrhundert auch der Kreis ohne Zahlen vor, meist jedoch als - z.T. wohl augmentierendes - Mensurzeichen für gerade Takte ${ }^{456}$.

Ungeachtet der schwindenden Bedeutung dieser Mensurzeichen in der Praxis, werden diese von vielen Theoretikern auch weiterhin in aller Breite behandelt; man gewinnt sogar den Eindruck, daß die Praxisferne den Spekulationen erst richtig "Tor und Tür" geöffnet hat. Als ein extremes aber keinesfalls vereinzeltes Beispiel darf Silvestro Picerli gelten. In seinem Specchio primo di musica (1630) findet sich auf S.28-32 eine "Tavola prima de' diuersi segn' inditiali, de' segni contrasegni, e del valor delle note sotto d'esi". Diese enthält 50 verschiedene Signen, die nur die verschiedenen Perfektionen regeln und verschiedene Klassen der Augmentation und Diminution beinhalten. Es finden sich so aberwitzige Mensurzeichen, wie etwa drei konzentrische Kreise mit Prolationspunkt in der Mitte 457 , oder ein dreimal durchstrichener Halbkreis458. Auch der Verweis Picerlis auf die "antichi" trägt nicht dazu bei, dieser Tabelle einen musikpraktischen Sinn zu geben; die Komposition eines Freundes, die Picerli als Beispiel für die Verwendung fast aller dieser Zeichen erwähnt, ist nicht bekannt - aber deren von Picerli mitgeteilte uberschrift vielsagend: "Pro cappriciosis, \& nemo, nisi Theoreticus" 459 .

455. In beiden oben genannten Quellen. Vg1. hierzu auch weiter unten bei der Behandlung der hemiole.

456. Als Augmentationszeichen wurde der Kreis wahrscheinlich bei S. Felis, Liber secundus motectorum (1585), sowie in den Werken des Don F. de las Infantes - dort besonders in den Plura modulationum genera (1579) benutzt. Als Zeichen für einen äuBerlich nicht von anderen zu unterscheidenden Dreiganzetakt findet es sich bei F. Guerreri in den Motettendrucken von 1589 und 1597.

457. Dieses Zeichen soll anzeigen, daß prolatio, tempus, modus und maximodus perfekt sind. Da die perfekte prolatio bei Picerli zugleich eine Augmentation beinhaltet, bedeutet dies Zeichen z.B. daß eine Maxima (sie ist bei allen Beispielen Picerlis mit aufgefuhrt) 81 Semibreventakte ausgehalten werden muß (!!). Dieses System der Perfektion von modus und maximodus durch konzentrische Kreise findet sich bereits im theoretischen Werk des Johannes Hothby (gest. 1487) (Vgl. Gilbert Reaney, Art. Hothby, Johannes in MGG, Bd. 6, Sp. $771 \mathrm{ff}$, bes. 780), hat jedoch im 16. und 17. Jahrhundert kaum Verbreitung gefunden. Ǎnnliche Mensurzeichen sind dem Verfasser sonst nur aus L. Zacconis Prattica di musica, seconda parte (1622) bekannt. Dort gibt es sogar ein Dreieck (!) als Mensurzeichen (S.16).

458. Hier dauert die Maxima nur einen Schlag.

459. Vollständig lautet diese Stelle bei Picerli: "I musici moderni non si son seruiti de tutt' $i$ sudetti segn' inditiali, ma solo d'alcuni de' primi della prima graticola; si come si può vedere nell' opere di diuersi Autori: se ne possono non dimeno seruire; essendosene seruiti molti (benche antichi) graui Autori; come nell' opere appare; almeno per far qualch' opera capricciosa (è fatta da altri, saperla ben cantare) com' hà fatto vn' amico mio in 
Die Selbstverständlichkeit der Perfektion in den Dreiertakten um 1600 weicht im fruhen 17.Jahrhundert bereits a11mählich einer eindeutigeren Notationsweise. Perfektion wird - auch an offensichtlichen Stellen - durch einen Augmentationspunkt ersetzt 460 . Bei einigen Komponisten sind bereits im frühen 17. Jahrhundert die Noten auch in den Dreiertakten grundsätzlich imperfekt461. Die Taktstriche schließlich bereiten jeder Unklarheit ein Ende ${ }^{462}$.

In der Forschung wurde oft auf Dreiertakte ganz ohne jede Kennzeichnung, also ohne ein Perfektionszeichen und ohne ein Taktzeichen, hingewiesen 463 . Meist handelt es sich dabei um einzelne Abschnitte unter dem Signum des geraden Taktes, deren Rhythmik eher an einen Dreiertakt erinnert. Solche rhythmischen Verschiebungen sind in der Tat in dieser Zeit häufig zu beobachten. Es ist jedoch fraglich, ob es wirklich sinnvoll ist in Editionen jede rhythmische Verschiebung mit einem Taktwechsel zu ibertragen 464 . Ganz unmöglich gemacht wird dieses Verfahren durch - ebenfalls nicht selten zu beobachtende - unterschiedliche Rhythmen in den verschiedenen Stimmen.

In einigen Ausnahmefällen finden sich auch ganze, offensichtich als Dreiertakt gemeinte Sätze unter einem Signum des geraden Taktes notiert. Das bekannteste Beispiel hierfür dürfte die abschließende Moresca aus Claudio Monteverdis L'Orfeo (1609 und 2/1615)465 sein. Hierbei kann es sich durchaus auch un einen Druckfehler hardeln 466 . Auch in Sigismondo d'Indias Le musiche

va suo operetta co' 1 motto sopra, Pro capricciosis, \& nemo, nisi Theoreticus, dou' hà posto quasi tutt' $i$ sudetti segni: dalla cognitione de' quali si può vernir' in cognitione d'ogn' altro, simil' ad essi." (S.42, Abschnitt A).

460. Recht fruhe Beispiele für Augmentationspunkte an Stellen offensichtlicher Perfektion finden sich zum Beispiel in der Barca (1605) von A. Banchieri oder in den Canzonette et arie (1606) von 0. Bartolini.

461. So notierte A. Brunelli, Scherzi ... libro secondo (1614) im Ritornello (in Chorbuchnotation) $z u$ "O soave dolore" ( $\mathrm{S} .7 \mathrm{f}$ ) im Baß in einem Dreihalbetakt eine Folge nicht besonders gekennzeichneter imperfekter Semibreven, aber dennoch in allen Stimmen eine perfekte SchluBsemibrevis (eine ubertragung dieses Ritornelles findet sich im zweiten Band dieser Arbeit als Notenbeispiel I,12). Von einer grundsätzlichen Imperfektion der Noten im Dreiertakt ging S. Rossi in den ersten beiden Büchern delle sinfonie et gagliarde $(1607 / 08)$ aus.

462. Vg1. hierzu auch Wolf, Barbarino, S.37.

463. Vgl. hierzu etwa die 1925 erschienen Aufsätze von Cahn-Speyer, Taktstrich; Wiehmayer, Taktstrichfrage und Keller, Takt sowie vor allem Aldrich, Rhythm.

464. So z.B. im dem Notenteil bei Aldrich.

465. In beiden Auflagen steht nur der semicircolo semplice, vgl. das Verzeichnis der Abweichungen zwischen den beiden Orfeo-Auflagen bei Reichhart, L'Orfeo, S.35 ff.

466. Auch zahlreiche andere Druckfehler befinden sich in beiden Auflagen (vgl. Reichhart, S.35 ff). 
(1609) findet sich ein solcher Dreiertakt. Dort wird aber in den Errori am Ende des Werkes das versehentliche Fehlen dieses Signums festgeste11t467. Am Rande sei hier auch auf die von Michael Praetorius beschriebene Austauschbarkeit von geradem und ungeradem Takt bei der Branle gay 468 hingewiesen. Es ist nicht mehr zu entscheiden, ab Praetorius davon ausging, daß man, da diese Art Tanz ja bekannt war, unabhängig von Notierung und Taktschlag469 die Betonungen an der richtigen Stelle anbringt, oder aber ob er verschiedene Alternativmöglichkeiten für einen ohnehin nicht zu tanzenden Tanz geben wollte 470 .

\section{COLOR UND HEMIOLA}

In den Quellen aus der Zeit um 1600 lassen sich eine ganze Reihe unterschiedlicher, aber doch miteinander zusammenhängender Aufgaben der Schwärzung, in der Musiktheorie auch als color bezeichnet, feststellen. Geschwärzte Notation wurde verwendet:

1. im Dreiertakt, um Imperfektion zu erreichen, Alteration zu vermeiden, aber auch um ungewöhnliche Rhythmen deutlich zu machen,

2. zur Notation der hemiola, einer Art Dreiertakt, bei der statt eines Signums die Schwärze die Dreizeitigkeit anzeigt,

3. in der "unechten" hemiola; gemeint ist ein Dreiertakt mit Signum und dennoch geschwärzt,

4. in den Triolen, in denen zu einem Teil eine Art der hemiola zu sehen ist,

5. im geraden Takt als Schreibweise für die Folgen "punktierte Semibrevis - Minima" und "punktierte Brevis - Semibrevis", insbesondere im Zusammenhang mit Ligaturen (sog. minor color),

6. sowohl in Dreiertakten als auch im geraden Takt zur Notation der sog. "Augenmusik",

7. als Nachahmung der Choralnotation, zumeist in Intonationen, aber auch innerhalb mensuralnotierter Stiicke.

Die Schwärzung hat der Theorie nach zwei Grundbedeutungen: 1. die Imperfektion sonst perfekter Noten sowie 2. die Verkürzung des Notenwertes um ein Dritte1471. Im späten 15. und vor allem im 16. Jahrhundert nahm die Bedeutung des color in der Notation ständig ab. Oft wurde durch den color eine Note

467. Ein weiteres Beispiel für solch einen Dreiertakt steht in C. Saracinis Le terze musiche (1620). Alle die genannten Beipiele stehen in Partitur und sind daher auch mit Taktstrichen versehen. Das problemlose Erkennen des Dreiertaktes ist also gewährleistet.

468. Vg1. Heckmann, Takt, S.119 f.

469. Praetorius geht ausdrücklich auch von verschiedenem Taktschlag aus.

470. Ǎnnliche Fälle führt auch Apel, Notation, S.70 ff an. Schmitz, Continuo-Madrigal, S.520, nennt ebenfalls eine sowohl drei- als auch zweizeitige Mensurierung anbietende Satzuberschrift aus G. Sabbantinis Madrigali à $5(1627,2 / 1634)$.

471. Vg1. Gilbert Reaney, Art. Color in MGG Bd.2, Sp.1567. 
nun nicht mehr verändert, sondern lediglich ihr wert verdeutlicht472. Wie bereits Riemann feststellte, bereitete es den Musiktheoretikern des 16. Jahrhunderts Schwierigkeiten, die Wirkungen des color zu erk1ären ${ }^{473}$; offensichtlich wurde der color bereits im 16.Jahrhundert nicht mehr recht verstanden.

\subsection{COLOR ALS ZEICHEN FÜR IMPERFEKTION SOWIE ALS WARNZEICHEN IM DREIERTAKT}

Die Grundbedeutung "Imperfektion" kann selbstverständlich nur innerhalb perfekter Mensuren Verwendung finden. Da es in der Zeit um 1600 in jedem Dreier-, Sechser-, etc. -Takt zur Pefektion kommen konnte, ist auch überall dort mit Schwärzung zu rechnen, unabhängig vom Mensurzeichen.

Am häufigsten fand die Schwärzung zur Imperfektion in dieser Zeit in den Kadenzen Verwendung. In einem Dreiertakt beinhaltete eine Kadenz zumeist eine Hemiole (im modernen Sinn, also einen Dreiertakt in doppelten Notenwerten). Diese Hemiole hatte in der Regel folgende rhythmische Gestalt:

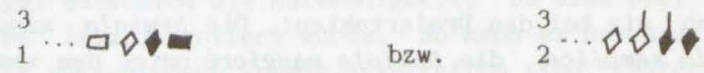

Wenn hier die zweite Semibrevis im Dreiganzetakt bzw. die zweite Minima im Dreihalbetakt nicht geschwärzt wäre, müßte es zur Alteration kommen; entsprechend wäre dann die folgende Note perfekt. Im späten 16.Jahrhundert wurde die Alteration kaum noch verwendet und der Schwärzung kam hier hauptsächlich eine Signalfunktion zu. Sie informiert über die rhythmische Verschiebung und hilft, die Taktschwerpunkte zu erkennen. Zahlreiche sonst unsinnige Anwendungen der Schwärzung an solchen Stellen belegen dies. So wird in einigen Drucken nur eine der beiden Noten geschwärzt474 oder die Schwärzung erscheint an solchen Stellen in allen Stimmen, ungeachtet des jeweiligen Rhythmus475. Wie wichtig diese Schwärzung sein kann, spürt man, wenn man versucht, nach einem der Drucke aus jener Zeit zu musizieren, die diese Hilfe nicht geber, wie z.B. einige Drucke Salomone Rossis.

Besteht eine solche Hcmiole aber aus drei gleichen Noten, z.B. drei imperfekten Breven hintereinander in einem Dreiganzetakt, dient die Schwärzung nicht nur als Signal, sondern ist unbedingt notwendig. Im Gegensatz zur

472. Reaney, Sp. 1572 .

473. Riemann, Color, S.143.

474. Z.B. in den Sacrae cantiones... liber primus von R. Aleotta (1593), den Concenti musicali von G. G. Gastoldi (1604) und den Motetti von A. Bianchi (1612).

475. So in den Motetti... libro secondo von G. Croce, den Sacrae symphoniae von G. Gabrieli (1597), den Vesperae von A. Cifra (1610) und der Scala Iacob von B. Borlasca (1616). In den Responsoria von G. F. Anerio (1614) hat diese Art der Schwärzung ein besonderes Aussehen. Anerio verwendete hier einen Dreihalbetakt mit weiBen Semiminimen (s.o.). Wohl in Anlehnung an das Aussehen der weiken Semiminimen notiert er die schwarze Minima wie eine Croma . 
Alteration wird die Perfektion - insbesondere im Falle aufeinander folgender gleicher Noten - noch häufig vorausgesetzt. Während man die Perfektion auch durch einen Augmentationspunkt ersetzen kann, ist es nur mittels der Schwärzung möglich, eindeutig auf die Imperfektion hinzuweisen.

\subsection{HEMIOLA UND TRIOLE}

\section{2 .1 , VORKOMMEN UND NOTIERUNG}

Die zweite Grundbedeutung der Schwärzung, die Verkiirzung des Notenwertes um ein Drittel, führt zu einer Art Triolierung: Drei um je ein Drittel verkürzte Noten dauern ebenso lange wie zwei nicht verkürzte Noten desselben Wertes. Von diesem mathematischen Verhältnis hat die hemiola ihren Namen: "hemiola" ist das griechische Wort für "eineinhalb"476. Die hemiole werden in allen Traktaten der Zeit mehr oder weniger ausfuhrlich als eine Methode, einen Dreiertakt ohne Mensurzeichen zu schreiben, behandelt. Man unterschied zwischen der hemiola minore mit drei schwarzen Minimen auf einen Takt und der hemiola maggiore mit drei schwarzen Semibreven. Die Theorie kennt hier dieselben Zuordnungen wie bei den Dreiertakten: Die hemiola minore soll unter dem semicircolo semplice, die hemiola maggiore unter dem semicircolo tagliato verwendet werden 477 .

In der Tat ist im späten 16.Jahrhundert eine weitgehende Bindung der hemiola maggiore an den semicircolo tagliato zu beobachten. Im 17. Jahrhundert wurde jedoch aufgrund der größeren Bedeutung des semicircolo semplice in der Regel hierauf keine Rücksicht mehr genommen ${ }^{48}$. In einigen Drucke mit dem semicircolo semplice als Hauptmensurzeichen steht der hemiola maggiore jedoch ein semicircolo tagliato voran 479 .

Während die hemiola maggiore mehrheitlich in ihrer reinen Gestalt - also nur an der Schwärzung zu erkennen - auftritt, ist diese bei der hemiola minore

476. Ape1, Notation, S.140.

477. So z.B. in S. Cerretos Prattica musica, 1601, S.238, und in vielen anderen Traktaten, darunter auch fortschrittlichen, wie A. Banchieris Conclusioni (1609), S.35.

478. In den ausgewerteten Drucken der Jahre 1571-1590 ist die hemiola maggiore zu fast $80 \%$ nur unter dem semicircolo tagliato zu finden. Schon in den Drucken des letzten Jahrzehntes des 16.Jahrhunderts war diese Bindung nur noch in ca. $60 \%$ der Drucke mit der großen hemiola zu beobachten. In den drei Jahrzehnten des 17.Jahrhunderts hingegen findet man die hemiola maggiore zu ca. $75 \%$ unter dem semicircolo semplice. Dies entspricht der Entwicklung bei den weißen Dreiertakten (s.o.).

479. So bei E. Marotta, Aminta musicale (1600), G. Ceresini, Il primo libro de' madrigali (1607), G. B. Nanino, Motecta...liber tertius (1612). Ein weiteres Beispiel bei S. Molinaro ("Domine, ne in fuore tuo" aus dem Samme1druck Sacrarum symphoniarum continuatio, Nürnberg 1600) erwähnt Hell, Rhythmus, S.116. Auch hierzu gibt es Parallelen bei den Dreiertakten (s.o.). 
eher selten anzutreffen. Der Grund hierfür liegt auf der Hand: Eine schwarze Minima ist von einer Semiminima nicht zu unterscheiden. Zur Verdeutlichung der hemiola minore wurde die als Proportionszeichen oft verwendete Zahl "3" wohl zumindest in ganzen 16. Jahrhundert benutzt. Diese Dreien wurden auf verschiedene Weise notiert: Sie standen entweder vor jeder Dreiergruppe, innerhalb jeder Dreiergruppe oder auch nur zu Beginn des Abschnittes in der hemiola minore.

Insgesamt lassen sich also drei Arten der hemiola unterscheiden:

1. die "reine" hemiola, also ohne Zahlen oder Mensurzeichen480,

2. die triolische hemiola, also eine hemiola, bei der jede Dreiergruppe eine Drei beinhaltet, oder jeder Gruppe eine Drei vorangeht (tritt fast ausschließlich in der hemiola minore 481 auf) und

3. die Notation als schwarzer Dreiertakt, also mit einer Drei zu Beginn und einem semicircolo am Ende482.

In der Regel entschied die Notwendigkeit, ob eine Drei verwendet, oder aber die "reine" hemiola notiert wurde. So kann es durchaus vorkommen, daß in einem Druck verschiedene Formen anzutreffen sind - unter Umständen sogar in verschiedenen Stimmen an ein und derselben Stelle $e^{43}$.

Am verbreitetsten ist die Triolennotierung. Im Unterschied zur heutigen Notationsweise ist die Triolendrei zunächst nicht über bzw. unter die Notengruppe, sondern in sie hinein gedruckt. Dies konnte mit dergleichen Type geschehen mit der auch die als Taktzeichen häufige Drei gedruckt wurde. Es gibt keinen festen Platz für diese Dreien innerhalb der Gruppen; sie können vor der Gruppe sowie an jeder Stelle innerhalb der Gruppen stehen - dies kann auch innerhalb einer hemiola wechse $\ln ^{484}$. ijber bzw. unter die Noten

480. Gelegentlich ist eine solche hemiola - wohl um wirklich deutlich zu machen, daß etwas Neues beginnt - von Trennungsstrichen umgeben, so bei C. Antegnati, Salmi (1592) und bei G. Fattorini, I sacri concerti (1600, $2 / 1608)$; vgl, auch weiter unten im Abschnitt IV.2.2.

481. Zwei Beispiele fuir eine hemiola maggiore in Triolennotation gibt $\mathrm{L}$. Zacconi, Prattica di musica (1596), fol. 42'; eines aus einer Motette von "Felice de la Iyolle" und ein anderes aus dem "Liber generationes" von Josquin. Dem Verfasser ist sonst kein Beispiel für eine hemiola maggiore in Triolennotation bekannt. Lediglich die Pausen einer hemiola maggiore versieht A. Ferrabosco in seinem secondo libro de madrigali (1587) mit Dreien (s.u.). A. Banchieri, Cartella musicale (1614), S.31, erwähnt zwar die hemiola maggiore mit einer Drei, bezeichnet sie aber als "impraticate".

482. Gelegentlich steht sogar einem solchen schwarzen Dreier ein - natürlich unsinniges - Perfektionszeichen voran, so bei A. Feliciani, Brevis ... psalmodia (1590), S. Bernardi, Motecta (1610) und F. Costanzo da Cosena, I1 primo libro delli mottetti (1621).

483. Einige Beispiele hierzu weiter unten.

484. Bevorzugt wurde neben der Plazierung am Anfang der Gruppe, die Notierung der Drei nach der Semibrevis bei dem Rhythmus Semimbrevis-Minima, 
gedruckte Dreien (in Handschriften war die heutige Notierung längst ub1 lich 485 ) sind zunächst selten ${ }^{486}$.

Triolennotierung ist nur notwendig, wenn die hemiola minore sonst nicht zu erkennen ist, also zumindest zu Anfang der hemiola-Passage keine schwarze Semibrevis vorkommt (sie kann man am Fehlen der Cauda immer erkennen). Aus diesem Grund kann es sich ergeben, daß eine hemiola in den bewegteren Vokalstimmen mit Triolendreien gekennzeichnet wurde, während in der ruhigeren Basso continuo-Stimme eine "reine" hemiola notiert ist487. Auch Pausen am Anfang einer hemiola veranlassen zu besonderer Kennzeichnung. In solch einem Fall kann in dieser Stimme die hemiola als schwarzer Dreier notiert sein, um den Beginn der hemiola anzuzeigen488. Auch eigentlich unsinnige Triolenpausen gibt es: In einer hemiola in Triolennotation werden Triolengruppen verwendet, die nur jeweils eine (Semibreven-) Pause beinhalten; eigentlich könnten die Dreien hier entfallen ${ }^{489}$.

Ganz ähnlich verhält es sich mit der Kenrzeichnung des Endes einer hemiola. In der Regel ist diese beendet, sobald wieder weiße Noten erscheinen, gleich

sowie nach der ersten Minima bei Gruppen von drei Minimen.

485. Abbildungen von Handschriften aus dem 16.Jahrhundert mit unter den Noten stehenden Triolendreien finden sich bei Franz Krautwurst, Art. Pernner-Kodex in MGG, Bd.10, Tafel 72 sowie Walter Senn, Art. Flori, Franz (I) in MGG, Bd.4, Sp. 420.

486. Dem Verfasser sind solche über die Noten gedruckte Triolendreien aus den Musikdrucken Ottaviano Petruccis (hier möglich aufgrund des besonderen Druckverfahrens; vgl. die Abbildung bei Walter Rubsamen, Art. Frottola in MGG, Bd.4, Sp.1021) sowie aus Diego Ortiz' Trattado de glosas (1553) und aus dem von Clemens Stephani herausgegebenen Sammeldruck Cantiones triginta selectissimae (1568; hier sind die Dreien außerhalb des Notensystemes gedruckt, vgl. die Abbildung bei Dragan Plamenac, Art. Ockeghem in MGG, Bd.9, Sp.1835) bekannt und dann erst wieder aus G. M. Trabaci Ricercare ... libro primo (1603) und P. Quagliatis Carro di fedelita (1611). Ab dieser Zeit mehren sich dann die Drucke mit Triolendreien iber den Noten.

487. Dem Verfasser bekannt aus G. B. Naninos Motecta ... liber tertius (1612).

488. In G. Conversi, Il primo libro de madrigali (2/1584), P. Tristabocca, Missarum... liber primus (1590), V. Liberti, Il primo de madrigali (1608, bei Liberti gibt es aber auch Pausentrialen - s.u.), A. Savetta, Motectorum... liber secundus (1608), A. Scialla, Primo libro de' madrigali (1610).

489. Dies findet sich in dem terzo libro de madrigali von G. B. Moscaglia (1585), in dem primo libro de madrigali v. Libertis (1608) und im primo libro delli mottetti von F. Costanzo da Cosena. Triolenpausen innerhalb einer weißnotierten Triole (s.u.) finden sich bei G. Bruschi, Il terzo libro delli concerti (1629; vgl. zu dieser Stelle auch weiter unten). Eine Besonderheit in dieser Beziehung ist in A. Ferraboscos secondo libro de madrigali (1587) zu finden. Hier werden innerhalb einer "reinen" hemiola nur die Pausen mit Dreien versehen. 
wie sie notiert ist (ob als "reine" hemiola, Triole oder schwarzer Dreiertakt). Bei allen Arten der hemiola kommt es aber ebenso vor, daß am Ende der hemiola ein semicircolo steht. Gründe hierfür können kurze Notenwerte nach der hemiola oder aber auch Pauser am Ende des hemiola-Abschnittes490 sein. Ganz oft wird aber auch nur eine Notationsgewohnheit den Ausschlag für eine Notierung gegeben haben. Wie immer gibt es hier in der Notation dieser Zeit keine Verbindlichkeit. So gibt es durchaus auch komplizierte ubergänge zwischen hemiola und geradem Takt, bei denen die Notation keine Hilfe gibt, und ein "vom Blatt" Musizieren sehr erschwert wird491.

Im beginnenden 17. Jahrhundert wurde die "reine" hemiola minore kaum noch verwendet. Aus den beiden Arten zur Kenntlichmachung der hemiola entwicke1ten sich zwei eigenständige Notierungsformen: Die als eigenständige Taktarten benutzten schwarzen Dreiertakte, die offensicht1ich (insbesondere von Frescobaldi) als weitere Tempostufen der Dreiertakte verwendet wurden (s.u.), sowie die Triolen als unabhängige rhythmische Möglichkeit. Insgesamt ist ein Rückgang der hemiola-Notationen zu verzeichnen, wenngleich sie erst im 18. Jahrhundert endguiltig "aussterben"492.

\section{2,2. DIE VERSCHIEDENEN ANWENDUNGEN DER HEMIOLANOTIERUNGEN}

Ehe nun auf die weitere Entwicklung der Triole eingegangen werden soll, ist zunächst die Frage nach dem Sinn der hemiola-Notierungen ganz allgemein zu stellen. Man kann die hemiole je nach ihrer Anwendung in funf Gruppen unterteilen:

1. als weitere Dreier-Tempostufe,

2. zur Notierung von Dreiern nur in einer Stimme oder mit uberlappungen,

3. zur eindeutigen Notation komplizierter Dreierrhythmen,

4. zur Notation von Neunertakten

5. als Alternative zu einem weißen Dreiertakt,

6. in der sog. Augenmusik (hieruber wird im Abschnitt 3.3. berichtet).

\section{$3,2,2,1$. HEMIOLE ALS TEMPOSTUFEN}

Frescobaldi beschreibt in seinem Vorwort zu den Capriccen von 1624 eine Tempoabstufung der drei Dreiertakte (Dreiganze-, Dreihalbe- und Dreivierte1takt). Außer diesen Taktarter verwendete er neben den bereits angesprochenen Sechsviertel- und Zwölfachteltakten auch die beiden hemiole in der Form von schwarzen Dreiertakten (also mit einen Signum zu Anfang und einem semicir-

490. So in dem secondo libro de madrigali von S. Dentice (1596), dem secondo libro de madrigali von A. Agazzari (1608) und dem primo libro de madrigali von M. Nantermi (1609).

491. Vgl. hierzu das Notenbeispiel I, 15, Takt $44 \mathrm{f}$ im zweiten Band dieser Arbeit (im Original ohne Taktstriche notiert!).

492. In fast jedem zweiten der ausgewerteten Musikdrucke aus den Jahren 1571-1580 fanden hemiole Verwendung, in rund 40\% aus den Jahren um 1600 (1591-1610) und in noch gut 15\% aus Drucke aus den Jahren 1611-1630. 
colo am Ende). Es liegt nahe, auch die hemiole als Tempostufen zu verstehen493. $\mathrm{Zu}$ den hemiole wird allerdings in dem Vorwort von 1624 nichts gesagt494; vielleicht konnte eine entsprechende Anweisung entfallen, da Frescobaldi sich hierin auf eine bereits bestehende Tradition stitzen konnte495. Sicher soll das Tempo der hemiole etwas schneller als das des gleich notierten Dreiertaktes $\operatorname{sein}^{496}$.

Die eine Tempostufe repräsentierenden hemiole können jedoch nicht nur unabhängig von anderen Dreiertakten auftreten, sondern sich auch direkt an diese anschließen und damit eine Beschleunigung des Tempos innerhalb eines Dreiertaktes herbeifuhren. In den Sonaten "E tanto tempo horma $i$ " und "Il Corisino" von Francesco Turini497 wird nach einem langen Abschnitt im Dreihalbetakt nach einer Kadenz ohne Mensurwechsel in der hemiola minore mit Triolendreien weiternotiert498. Dies bedeutet theoretisch, daß eine der Triolengruppen den Wert einer imperfekten Semibrevis des Dreihalbetaktes einimunt, oder daß drei Triolengruppen solange dauern wie vorher zwei Dreiertakte. Dieser Wechsel in die hemiola findet in allen Stimmen gleichzeitig stat,, die Dreien stehen

493. So auch Paulsmeier, Temporelationen, S.199 ff und Hell, Rhythmus, S. $105 \mathrm{ff}$.

494. Zu den uberschneidungen von hemiola minore und Dreivierteltakt s.u.

495. Leider sind nicht viele musiktheoretische Belege für ein gegenüber den weib notierten Dreiertakten schnelleres Tempo der hemiole zu finden. Dem Verfasser sind folgende Belegstellen bekannt: F. Beuerhaus, Erotematum musicae libri duo (1573, 2/1580), S.67: "Hemiola similis est Triplae vel sesquialterae, nisi quod celerius \& subtilius canatur." und A. Gumpelzhaimer, Compendium musicae $(1591,5 / 1611)$ "Hemiola ist gleich der Triplae proportion / allein das sie umb der schwertze willen mehr behendigkeit hat in der Mensur dann die Tripla". (nach Bank, Tactus, S.247, ist diese Aussage bereits in der ersten Auflage von 1591 enthalten). Nach Bank bestatigt auch L. Zacconi, Prattica di musica (1592), daß die hemiola von den "Modernen" schneller gesungen wird, als die Tripla (S.247). In der dem Verfasser vorliegenden zweiten Auflage von 1596 ist eine entsprechende Aussage nicht zu finden.

496. Verschiedene Tempotheorien entwickelten He11, Rhythmus, S.105 if, hes. S.108, sowie Paulsmeier, Temporelationen, S.199 ff. Vgl. dazu auch weiter oben in den Abschnitten I.2.6. - 2.6.6.

497. Aus Madrigali ... con alcune sonate... libro primo (1621, 3/1624). Diese Sonaten finden sich auch in der Neuausgabe, hrsg. von Gustav Leonhard, $\mathrm{S} .20 \mathrm{ff}$ und S.30 ff. Die Sonata "E tanto tempo hormai" ist dariber hinaus in dem Band Die italienische Triosonate von Erich Schenk abgedruckt (S.23 ff).

498. In der Ausgabe Leonhards wird statt dessen mit halben Werten (also im Dreivierteltakt) weiter notiert. Die Triolendreien werden dort, wo sie im Original stehen, ohne Erklärung wiedergegeben (sie sind in dieser ubertragung allerdings vällig unsinnig). Als Fußnote findet sich der Kommentar "Das Original notiert unsere Halben als Viertel ohne Caudae" (S.27)! Schenk notiert ebenfalls einen Taktwechsel vom Dreihalbe- zun Dreivierteltakt, allerdings ohne einen Hinweis auf die originale Notation. 
nur in den ersten Takten499. Die hemiola dauert bis zur Rückkehr in den geraden Takt zur Schlußkadenz an. Es ist durchaus wahrscheinlich, daß hier nicht unbedingt an das mathematische Verhältnis gedacht ist, sondern Turini mit dieser Notation eine Beschleunigung ohne meBbare Proportion herbeifuhren wollte.

Besonders interessant ist ein ähnlicher Sachverhalt in der abschließenden Sinfonia aus Filippo Vitalis Intermedi fatti per la commedia (1623)500. Das Stuick beginnt mit acht Takten unter dem semicircolo semplice. In Takt 9 folgt ein Dreihalbetakt mit der Taktvorzeichnung "3/2". Dieser Dreier wird ab Takt 28 schwarz notiert501. Sicher soll auch hier die Schwärze eine Beschleunigung bewirken. Diese hemiola wird in Takt 59 wiederum von einem Dreiganzetakt (Vorzeichnung ebenfalls "3/2") gefolgt. Es wurde oben dargelegt, daß der Dreiganzetakt, etwa bei Frescobaldi, als langsamster Dreiertakt verwendet wurde. Die recht kurzen Notenwerte in dem Schlußteil dieses Dreiganzetaktes ab Takt 71 deuten auch hier zunächst auf ein langsames Tempo hin 502 . Ein besonders langsamer Dreier nach einer kontinuierlichen Temposteigerung (gerader Takt - Dreihalbetakt - hemiola minore) erscheint jedoch nicht uberzeugend. Vielmehr würde eine weitere Temposteigerung sinnvoll erscheinen; den Schlußteil ab Takt 71 kann man sich gut als virtuoses "Finale" vorstellen. Auch satztechnische Eigenheiten dieses Teiles (z.B. Takt 76) verlangen nach einem schnellen Tempo. Es ist also wahrscheinlich ein schne1ler Dreiganzetakt gemeint (s.o.). Die später iblich gewordene Notation schneller Sätze in großen und langsamer in kleinen Notenwerten 503 , ist zu dieser Zeit auch in anderen Quellen anzutreffen (s.o. zu den Sonaten Dario Castellos) 504 .

499. Sie dienen hier nur dazu, das Erkennen der veränderten Notation sicherzustellen.

500. Die Sinfonia ist im zweiten Band dieser Arbeit als Notenbeispiel I,13 ibertragen.

501. Die schwarze Semibrevis vor dem wiederholungszeichen ist sicher beim ersten Mal als punktierte weiße Semibrevis zu lesen. Sie muß jedoch an dieser Stelle schwarznotiert sein, da sonst der Beginn der hemiola nicht deutlich zu erkennen ist. Sie ersetzt hier gewissermaßen die Dreien Turinis.

502. Es gibt kein weiteres Beispiel für die Verwendung des Dreiganzetaktes in diesem Druck. Auch in Musiche (1617) verwendete Vitali nur den Dreihalbetakt und die hemiola minore.

503. Vgl. Adler, Stil, S.133.

504. Ein Wechsel von weißer in schwarze Notation inmitten eines Dreiertaktes zur Beschleunigung des Tempos findet man daruber hinaus in A. Banchieris Battaglia ( $L$ 'orgnao suonarino 2/1611, dort zusammen mit dem Tempowort "Presto", vgl. Notenbeispiel I,2) sowie im Kyrie der ersten Messe der posthumen Raccolat terza... de messa et salmi del sig. Alessandro Grandi (1630, den Hinweis auf dieses Beispiel verdanke ich Roland Wilson, Brüh1). Dieses Kyrie ist auch bei Seelkopf, Grandi, Bd.2, S.323 ff, abgedruckt. Seelkopf uberträgt die schwarze Semibrevis als Minima (Takt 58 ff). Auf ähnliche Fälle, bei denen jedoch andere Grïnde den Ausschlag zur Schwärzung 


\section{EXKURS: HEMIOLA MINORE UND DREIVIERTELTAKT}

Bereits im 17. Jahrhundert bereitete die Unterscheidung von hemiola minore und Dreivierteltakt Schwierigkeiten - ist doch das optische Erscheinungsbild der in diesen Takten häufigsten Noten, der Semiminima bzw. der schwarzen Minima, gleich. Schon 1593 bezeichnete Giovanni Luca Conforto505 die Noten der hemiola minore als Semiminime506. In englischen Quellen wird bis weit in das 17. Jahrhundert hinein darauf hingewiesen, daß hemiola und Dreivierte1takt austauschbar sind507. Gelegentlich finden sich uneinheitliche Notierungen: Eine Stimme ist in einer hemiola minore notiert, eine andere im Dreivierteltakt oder einzelne schwarze Semibreven treten in Dreiviertetakten bzw. weiße Minimen in hemiole auf. Das allmähliche Aufgreifen des Dreivierteltaktes lä日t sich an einer Canzone Frescobaldis nachvollziehen 508 . In dieser Canzone notierte Frescobaldi einen Abschnitt in einer hemiola minore - vielleicht zu einer Zeit, in der Frescobaldi den Dreivierteltakt noch nicht benutzte. Zur Drucklegung wurde die hemiola minore dann durch einen Dreivierteltakt ersetzt. In in allen drei Ausgaben des primo libro delle

gaben, wird unten noch einzugehen sein.

505. Breve et facile maniera.

506. "Il segno del tre, doue ci sono tre semiminime, che ne segue poi sei crome, \& dodici semicrome alla battuta, è chiamata generalmente emiola minore, \& portaleco il valore delle triple, conforme al loro genere, di tre note alla battuta." (fol.2 der "Dichiaratione"). Dies bezieht sich auf eine Drei vor den Noten zu Anfang eines Beispieles. Eine Drei iber den Noten benutzt Conforto als Verzierungszeichen (s.u.).

507. C. Simpson, A Compendium of practical musick (1668, 3/1678), S.25/26: "There are divers Tripla's of a shorter Measure, which by reason of their quick movement, are usually measured by compting three down, and three up, with the Hand; so that of them it may be said that two Measures make but one. And those quick Tripla's are prickt somtimes with Minims and Crochets; and sometimes with black Semibreves insteaed of Minims; and black Minims, which in appearance are Crochets. (...) Take notice that the balck Semibreves, as also the Minims with stand over them (gemeint: im dazugehörigen Notenbeispie1), are sung or play'd as fast in these quick Tripla's, as Crochets in Common Time; and the black Minims or Crochests (call them which you please) as fast as Quavers. "Auch in J. Playfords Introduction to the Skill of Musick wird auf die Austauschbarkeit von hemiola und Dreivierte1takt aufmerksam gemacht (vgl. Hel1, Rhythmus, S.129, sowie Collins, Coloration, S.167). Aus dem niederländischen Bereich teilt Hell ebenfalls ein Beispiel für diese Austauschbarkeit mit. Auf S.125 zitiert er aus J. A. Bans Vorrede zu Zangh-Bloemzel, in der die hemiola minore unter dem Signum $3 / 4$ notiert ist. Interessant ist in diesem Zusammenhang auch die Vorzeichnung $6 / 4$ vor einer hemiola minore bei G. F. Sances ("Come viver pos.5' io" in der Handschrift Ms.Mus. $2 \circ 57$ e der Murharschen Bibliothek und Landesbibliothek, Kasse1).

508. "Canzona Vigesima, detta la Lipparella" aus Il primo libro delle canzoni , 1628, 1628, 1634 (Titel der Canzone nach der Partiturausgabe). 
canzonisen sind aber Fehler passiert, die die ursprüngliche hemiola deutlich erkernen lassen 510 .

Trotz dieser uberschneidungen ist es kaum zu rechtfertigen, von einer grundsätzlichen Gleichstellung von hemiola minore und Dreivierteltakt auszugehen ${ }^{511}$. Solange beide Notationsformen in denselben Drucken ohne uberschneidungen nebeneinander vorkommen und die hemiola maggiore als Gegenstück zur hemiola minore noch bekannt ist, muß man in den hemiole eine unabhängige Möglichkeit der Notation sehen.

\section{2 .2 .2 , HEMIOLE NICHT IN ALLEN STIMMEN}

Während die hemiole mit vorgesetzten Taktzeichen (schwarze Dreiertakte) den anderen Dreiertakten gleichgestellt waren und damit nicht mehr in ihrem ursprünglichen proportionalen Verhältnis zu den geraden Takten standen, behielten die "reinen" hemiole, also die hemiole ohne Taktzeichen, ihr triolisches Verhältnis zum geraden Takt bei; Triolendreien traten hier je nach Bedarf (s.o.) hinzu. Diese hemiole gingen später ganz in den Triolen auf (s.u.). Aufgrund ihres klaren triolischen Verhältnisses zum geraden Takt eigneten sich die hemiole - ebenso wie die Triolen -, um ternäre Rhythmen nur in einigen Stimmen zu notieren512. Dieses eindeutig triolische Verhältnis veranlaßte sicher auch Claudio Monteverdi eine solche hemiola in seiner "Sonata sopra Santa Maria" zu verwenden513. Die hemiola (in Triolennotation) in Takt $68 \mathrm{ff}^{514}$ der Sonata karn keine Beschleunigung des Grundschlages be-

509. Stimmbuchausgaben Rom 1628 und Venedig 1634, Partiturausgabe Rom 1628. Eine gelegentlich zitierte Stimmbuchausgabe von 1623 beruht vermutlich auf eirem Lesefehler des Titelblattes.

510. In der Partitur von 1628 und in der Stimmbuchausgabe von 1634 ist als zweiter Dreiertakt ein Dreivierteltakt notiert, ebenso wie in der Generalbaßstimme der Stimmbuchausgabe von 1628. Die Canto- und die Basso-Stimme der Stimmbuchausgabe von 1628 hingegen sind in der hemiola notiert. Bis hin zur Musgabe von 1634 findet man aber am Schluß des Dreiers noch Reste der hemiola minore: Die beiden letzten Takte dieses Dreiers sind in der Partitur von 1628 und der Cantostimme von 1634 geschwärzt notiert. In dem als Druckvorlage dienenden Manuskript wird dieser Dreiertakt vermutlich in der hemiola notiert gewesen sein mit einem zusätzlichen Vermerk fü den Drucker, die hemiola als Dreivierteltakt zu drucken. (Vg1. zur Situation in den Que1len zu dieser Canzone Paulsmeier, Temporelationen, S.199 ff mit zahlreichen Faksimilia). Ảhnliches findet man auch in dem secondo libro de ricercate von G. M. Trabaci (1615). In einem Dreivierteltakt (S.80) ist einmal statt einer purktierten Minima eine schwarze punktierte Semibrevis notiert.

511. Von einer Gleichsetzung der hemiola minore mit dem Dreivierteltakt gehen Paulsmeier, Temporelationen, S. 199 ff, und Hel1, Rhythmus, S.119 ff, aus.

512. Noch un 1550 konnte dies auch mit weißnotierten Dreiertakten geschehen (s.o.).

513. Aus: Sanctissimae Virgini missa (1610, "Marienvesper"). 
wirken, denn der Canto ist hier weiterhin weiß notiert (alle wechsel in einen weißen Dreiertakt hingegen stehen auch im Canto). Für eine Gleichzeitigkeit von geradem Metrum (c.f.) und dreizeitiger Bewegung gab es für Monteverdi keine andere Notationsmöglichkeit.

Vergleichbare Stellen sind dem Verfasser in italienischen Quellen dieser Zeit nicht bekannt515. Noch in Quellen aus dem späten 16.Jahrhundert ist es jedoch recht häufig zu beobachten, daß Abschnitte in hemiole nicht gleichzeitig beginnen oder enden 516 oder hemiole nicht in allen Stimmen auftre-

514. Taktzählung nach Malipiero, Monteverdi-GA, Bd.14, 2. Halbband, S. $250 \mathrm{ft}$ - er folgt der Takteinteilung der Bc.-Stimme des Originales. Fine ijbertragung dieser Takte ist im zweiten Band dieser Arbeit als Notenbeispiel I,14 abgedruckt. Auch hier notieren fast alle Neuausgaben die hemiola in halben Werten, obwohl es hier an der Notation in einer hemiola keine Zweifel geben kann: Nicht nur die zahlreichen schwarzen Semibreven sind leicht zu erkennen, sondern auch die Pausenwerte sind eindeutig (sie entsprechen den Werten der ubertragung). Daruber hinaus mußten die Notenwerte des nicht geschwärzt notierten Sopranes in den Ausgaben verkürzt werden. Malipiero weist in einer Anmerkung auf die Verkürzung der Notenwerte des Sopranes hin: "Nell' originale qui valori sono doppi ma conservandoli il basso dovrebbe essere il doppio il che non corrisponderebbe certamente all'intenzione dell'autore." (GA, Bd.14, zweiter Halbband, S.263). Aus der Anmerkung ist zu entriehmen, daß er die hemiola-Notierung nicht erkannt hat, oder nicht zu deuten wußte. Das sich aus der triolischen ubertragung ergebende Tempo wäre erheblich langsamer als das heute ubliche. Man wird vielleicht gegen das sich nun ergebende langsame Tempo dieses Abschnittes der Sonata einwenden, es sei musikalisch weniger iberzeugend. Dieses Empfinden wird jedoch sicher auch von der Gewohnheit geprägt; diese Gewohntheit grindet sich jedoch auf eine fehlerhafte ubertragung. Gewisse Freiheiten im Tempo sind auch hier gegeben, da sowohl vor als auch nach dem Abschnitt in der hemiola nicht ein gerader Takt, sondern ein Dreiertakt folgt; die Bezugseinheit ist also nicht direkt vorhanden.

515. An dieser Stelle sei auf eine recht unverständliche Notation in F, Turinis Motetti a voce sola (1629, S.60 der Partitur) verwiesen. Hier ist die Singstimme in einer hemiola minore in der Form eines schwarzen Dreiertaktes notiert, der Baß aber, solange er in Haltetönen verharrt, als weißer Dreihalbetakt mit perfekten Semibreven bzw. Semibreven mit Augmentationspunkt. Ist der BaB aber bewegter, sind auch hier die Noten geschwärzt.

516. Z.B. in folgenden Drucken: A. Gabrieli, Sacrae cantiones... liber primus (1565, 3/1584), G. Ferretti, Il secondo libro delle canzone (1569, 4/1578) oder in dem Sammeldruck Il secondo libro delle fiamme (1567, $2 / 1570)$. Solche Stellen können hier nicht vollständig genannt werden, da sie am Notenbild selbst nicht ohne weiteres zu erkennen sind. Man mus entweder die Breven auszählen oder den entsprechenden Satz spartieren; beides konnte freilich nur stichprobenweise erfolgen. 
ten ${ }^{517}$. Sehr häufig hingegen sind auch im 17. Jahrhundert einzelne Triolen nur in einer Stimme 518 .

\section{$3,2,2,3$, EINDEUTIGE NOTIERUNGEN KOMPLIZIERTER RHYTHMEN IN HEMIOLE}

Auch im beginnenden 17. Jahrhundert waren die Notenwerte in Dreiertakten noch nicht eindeutig; weiterhin gab es die Möglichkeit der Alteration sowie der Perfektion. Eine eindeutige Notation war nur mit geschwärzten Noten gewährleistet. Gab es in einem Dreiertakt nur gelegentlich uneindeutige Stellen, so wurden nur an diesen die Noten geschwärzt. Dreiertakte, die jedoch uber lange Strecken von der üblichen rhythmischen Gestalt abwichen, wurden z.T. auch ganz als hemiola notiert. Lodovico Zacconi unterschied in der Prattica musica, seconda parte (1622) zwischen weiß notierten "proportioni perfetti" und den schwarznotierten "proportioni imperfetti"519.

Eine solche hemiola liegt in Biagio Marinis "Symphonia La Gambera"520 vor. Zunächst hat man den Eindruck, bei der Schwärze in Takt 27 handele es sich nicht um eine hemiola, sondern um Imperfektionsschwärze521. Auch die Tat-

517. Eine hemiola nur in einer Stimme findet sich in der posthumen RoreSammlung Sacrae cantiones von 1595 (also 30 Jahre nach Rores Tod). Hemiole in allen Stimmen bis auf eine wurden in dem primo libro de madrigali (1604) von F. Spongia Usper, sowie in Pastor fido (1617) von G. N. Mezzogorri notiert.

518. An dieser Stelle soll auch auf eine besondere Notationsform in englischen Quellen hingewiesen werden. Recht häufig findet man hier hemiole, die in den Stimmen nicht gleichzeitig einsetzen, oder uberhaupt nur in einigen Stimmem vertreten sind (interessanterweise nicht selten in den c.f.-gebundenen "In Nomine"-Stiicken, wobei auch hier der c.f. in weißen Noten weiterläuft, vergleichbar Claudio Monteverdis "Sonata sopra Santa Maria"). Bei diesen Stellen hat eine punktierte schwarze Semibrevis den Wert einer weiken Minima (! - es sei an die Gleichsetzung von Dreivierteltakt und hemiola minore in englischen Quellen - s.o. - erinnert!), unabhängig vom Mensurzeichen (sehr oft $6 / 1$ in der Form " $6 i "$ - vgl. Faksinile in der Notenausgabe Englische Fantasien des 16.Jahrhunderts, hrsg. von Uwe Wolf, S.5 sowie die Edition dieses Stiickes auf S.8 f; eine Anzahl ähnlicher Stellen sind in der Handschrift 1113 der Christ Church Library, Oxford, zu finden). Anstelle des Mensurzeichen "6/1" kann auch eine " 3 " stehen, ohne daß sich am Verhältnis zwischen dem geraden Takt und der hemiola etwas ändert. Eine hemiola mit einer Sechservorzeichnung ist dem Verfasser nur aus einem italienischen Musikdruck, nämlich den L'allegre notti von G. Montesardo bekannt (1608). Er verwendete die Vorzeichnung " $6 / 2$ " für eine hemiola minore. Ausfuihrlich behandelt M. Praetorius verschiedenste Arten der hemiola mit Sechservorzeichnungen in Syntagmatis musici... tomus tertius (1619), S.73 ff).

519. S. 22 .

520. Aus Affetti musicali (1617). Eine Ubertragung dieser Symphonia unter Beibehaltung der Schwärze ist im zweiten Band dieser Arbeit als Notenbeispiel I,15 abgedruckt. 
sache, daß der ganze Abschnitt danach geschwärzt ist, ist nicht unbedingt ein Zeichen für eine hemiola 522 . Eindeutig aber ist der ubergang in Takt 45: Der gerade Takt beginnt ohne einen semicircolo - nur herbeigefuhrt durch die weißen Noten 523 .

Der Rhythmus war sicher auch für die schwarze Notation des "In questo prato adorno" aus Claudio Monteverdis L'Orfeo 524 ausschlaggebend. Der ungewöhnliche Rhythmus diese Satzes hätte auch bei weiBer Notierung viele schwarze Noten erfordert. Die Abbildung 4 zeigt den Rhythmus der ersten Strophe in der originalen Notation sowie, darunter, mit der nach damaligem Brauch notwendigen Schwärze. Auch hierbei ist mehr als die Hälfte der Noten geschwärzt.

Monteverdi notierte hier zu Anfang einen schwarzen Dreier (Vorzeichnung in den beiden Violinen " $\mathrm{C} 3 / 2$ ", im $\mathrm{BaB}$ nur "3/2"). Im weiteren Verlauf des Stuickes ist nur noch ein semicircolo notiert (es entspricht dem in der Zeit liblichen, bei einem Wechsel der Stimme im gleichen System die Mensurzeichen zu wiederholen).

Das berihmteste Beispiel für eine Anwendung der hemiola aufgrund eines ungewöhnlichen Rhythmus ist das "Vi ricorda", ebenfalls aus Claudio Monteverdis $L^{\prime}$ Orfeo525. Die Notation dieses Stückes und insbesondere des dazugehörigen Ritornelles hat zahlreiche, oft recht abenteuerliche Deutungen erfahren 526 . Es ist erstaunlich, daß es erst 1975 Helmut Hell gelang, den Befund der Notation mit der seit langem bekannten musikalischen Lösung in Einklang zu bringen 527 .

In der Tat ist die hemiola im Ritornel1 nicht leicht zu erkermen: Erst in den Strophen finden sich geschwärzte Semibreven, die die hemiola zweifels-

521. Diese Schwärze ist allerdings der Theorie nach uberflüsig. In Takt 27 kann es nicht zur Alteration kommen, da eine zweite Minima fehlt. Perfektion ist ebenfalls ausgeschlossen, denn nirgens treffen zwei Semibreven direkt aufeinander. Es entspricht aber dem allgemeinen Gebrauch dieser Zeit, den unublichen Dreierrhythmus mit der kürzeren Note vor der längeren coloriert zu notieren.

522. So wurde z.B. im primo libro delli motetti von G. Carrone (1629) ein längerer Abschnitt mit ungewöhnlichen Rhythmen ganz coloriert, ohne daß man deswegen von einer hemiola sprechen kann.

523. In den taktstrichlosen Stimmbiichern ist dieser Wechsel nicht leicht zu erkennen.

524. S. 28-30 der Ausgabe von 1609.

525. S.32-35 in der Ausgabe von 1609.

526. Ape1, Ritornello, faßt insgesamt 9 Interpretationsvorschläge aus der Zeit bis 1951 zusammen und fügt einen weiteren hinzu. Einige weitere Interpretaionsversuche sind dargestel1t bei He11, Rhythmus, S.87 ff.

527. He11, Rhythmus. Eine musikalisch richtige ibertragung lieferte bereits Epstein, Rhythmisierung (1926). 


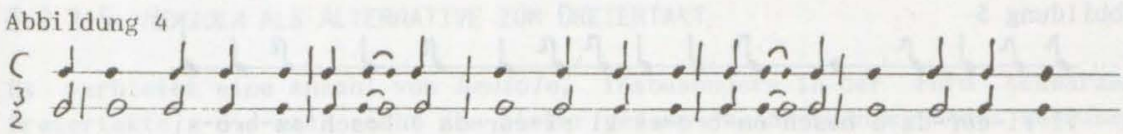

In que-sto pra-to a-dor-no_o-gni sel vag-gio Nu-me So vent'hà per $\mathrm{co}^{-}$

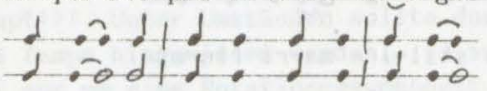

-stu-me Di far lie-to so-gior-no

frei bezeugen. Aus diesem Grunde versah Monteverdi das Ritorne11 bei seinem ersten Auftreten mit den Triolendreien, die in der Forschung soviel Verwirrung stifteten. Daß die Dreien nur zu Anfang notiert sind und nicht immer an derselben Stelle innerhalb der Triolengruppe stehen, ist nichts Besonderes, sondern nur zeitgemäß $(s . o)$. Bei einer jbertragung muß natürlich auch die Schwärze beriicksichtigt werden ${ }^{528}$.

Auch bei dem "Vi ricorda" muißten bei weißer Notation viele Noten geschwärzt werden. Die sich ergebende Notation muß jedoch hierfür nicht, wie für das "In questo prato adorno", rekonstruiert werden, da auch die weibe Notation dieses rhytmischen Modelis sich aus dem späten 16.Jahrhundert erhalten hat. Die Abbildung 5 zeigt die rhythmische Notation des Anfangs der "Vi ricorda"Strophen sowie der Frihfassung von Caccinis "Non ha'1 ciel"529. (Im Gegensatz zu Monteverdi notierte Caccini mit den jeweils nächstgrößeren Werten).

528. Alle dem Verfasser bekannten ubertragungen ubertragen weik, d.h. sie ibertragen die schwarze Minima als viertel Note. Nicht ganz eindeutig ist die Notation des Uberganges vom vorangehenden Chor "Dunque fa degno" zum "Vi ricorda": Dieser unter dem semicircolo semplice notierte Chor endet mit einer einzelnen Minima; zu Beginn des "Vi ricorda"-Ritornelles hingegen fehlt eine Minima. Da aber eine Minima unter dem semicircolo semplice nicht die erste Minima einer Trialengruppe ersetzen kann, erscheint zunächst die Lösung Hells, nämlich die ersten Noten des Ritornelles als ungeschwärzt zu behandeln, befriedigend. Unter musikalischen Gesichtpunkten, insbesondere auch im Hinblick auf die späteren Parallelstellen ist es jedoch besser, den Auftakt in die Hemiola mit einzubeziehen, zumal der Strich am Ende des "Dunque fa degno" einer Fermate gleichkommt (s.u.).

529. Bibliotheque du Conservatoire, Brisse1, ms 704, S.79. Ein weiterer anonymer Satz mit demselben Rhythmus und derselben Notierung ist in dieser Handschrift auf S.75 enthalten ("Amoroso pastore11o"). Eine andere Variante, den Wechsel zwischen schwarzer und weißer Notation zu vermeiden, bot bei diesem Rhythmus die Notation als gerader Takt, wie sie in A. Brunellis "Ballo grave" aus Scherzi... libro terzo (1616) vorzufinden ist (vgl. die Neuausgabe in Baletto delle Ninfe, hrgs. v. Silke Leopold) Zu diesem Wechselrhythmus allgemein vgl. Hell, Rhythmus, S.131 ff. Solche Wechselrhythmen sind noch häufiger anzutreffen als Hell annahm und sind insbesondere auch in der Instrumentalmusik sehr verbreitet. 
Abbildung 5

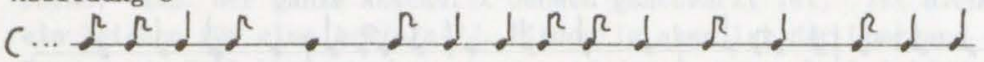

Vi ri-cor-da o bosch'om-bro-si Vi ri-cor-da_o bosch'om-bro-si

$(3+$

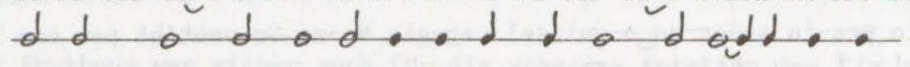

Non ha'l ciel con-tan-ti lu-mi tan-te sti1-1'e ma-ri fiu-mi

\section{$3,2,2,4$, HEMIOLE ZUR NOTATION VON NEUNERTAKTEN}

Die Notation des frïhen 17.Jahrhunderts kannte die Möglichkeit der Perfektion auf zwei Stufen (z.B. tempus perfectum cum prolatione perfecta) nicht mehr. Um solche neungliedrigen Takte zu notieren wurde daher wiederum auf die hemiole zurückgegriffen. Je ein Beispiel für diese Notationsmanier findet sich in Claudio Monteverdis Concerto (1619) und in Dario Castellos Sonate concertate... libro secondo (1629).

Monteverdis Notation in der Schlußsinfonia von "Tempro la cetra" aus dem Concerto (1619) folgt dabei ganz den Gewohnheiten der Zeit. Um einen solchen Neuner darstellen zu können, verwendet er statt des sonst in Marrigalen üblichen kleinen Dreiers den großen, den Dreiganzetakt; nur bei diesem kann er jedes Taktdrittel durch die verbreitete hemiola minore ersetzen. Durch den Vorsatz des semicircolo tagliato (mit "3/2") weißt Monteverdi daraufhin, daß nicht etwa ein langsames Tempo gemeint sei. Der $\mathrm{Ba} \beta$ verläuft weitgehend in Semibreven im normalen Dreiganzetakt, die Oberstimmen aber teilen die Semibreven in je drei geschwärzte Minimen. Deutlich erkennen kann man diese Notation in den Oberstimmen an einigen geschwärzten Semibreven ${ }^{530}$.

Weniger konventione11 ist die Notation des Neunertaktes in der "Sonata Terza d 2" aus Dario Castellos Sonate concertate... libro secondo (1629). Der erste Dreiertakt dieser Sonate, ein Dreihalbetakt mit dem Tempowort "adasio", geht nach 10 Takten in einen Neunvierteltakt (Tempowort "Alegra") uber. Dieser Neunvierteltakt ist nur durch das nochmalige Auftreten einer "3" gekennzeichnet. Die Minimen werden weiterhin notiert wie bisher, die Semiminimen sind jedoch geschwärzt, gleichen also den Cromen des Dreihalbetaktes 531. Der ubergang zurick in den geraden Takt erfolgt durch einen semicircolo semplice.

530. Malipiero überträgt diesen Teil in der Monteverdi-GA, Bd.7, S.6 f, als Neunvierteltakt. Hierbei sind die Viertel als geschwärzte Minimen, die Halben als geschwärzte Semibreven und die punktierten Halben als weiße Semibreven zu lesen.

531. Vg1. das Faksimile im zweiten Band dieser Arbeit als Notenbeispiel $\mathrm{I}, 16$. 


\subsubsection{HEMIOLA ALS ALTERNATIVE ZUM DREIERTAKT}

Es verbleibt eine Anzahl von hemiole, insbesondere in der Form schwarzer Dreiertakte, die sich nicht den bisher genannten Anwendungsgrüinden zuordnen lassen. Oft sind diese hemiole die einzigen Dreiertakte eines Druckes iberhaupt 532 . Unter Umständen sollte durch die schwarze Notation auf ein schne1les Tempo hingewiesen werden; es ist jedoch auch denkbar, daß es sich hierbei nur um eine Notationsgewohnheit handelt.

Ebenfalls nicht selten begegnen uns hemiole in Drucken vornehmlich motettischer Musik des späten 16.Jahrhunderts mit beiden semicircoli: oft steht nämlich hier der semicircolo tagliato mit seiner Sesqiualtera (dem Dreiganzetakt), der semicircolo semplice hingegen mit einer hemiola. Hier ist es sehr unwahrscheinlich, daß der hemiola eine beschleunigende Funktion zukommt. Der Grund für diese hemiola-Anwendung ist eher in dem noch uneinheitlichen Gebrauch des semicircolo semplice und des kleinen Dreiers zu sehen.

\subsection{3, DIE TRIOLEN}

Wie oben beschrieben, ist die Entstehung der Triolen sicherlich auf die schlechte Erkennbarkeit der hemiola minore zurickzufiuhren. Schon recht frïh wurden jedoch nicht mehr alle Triolennotierungen als hemiole verstanden. Die Noten innerhalb einer Triolengruppe konnten auch als weiße Noten angesehen werden533. Das optische Erscheinungsbild ist gleich: Drei schwarze caudierte Noten mit einer Drei zu Anfang oder in der Mitte. Eine solche Triolengruppe kann also sowohl den Wert einer Semibrevis als auch den Wert einer Minima haben534. Oft ermöglicht es eine vorkommende weiße Minima bzw. schwarze

532. Sehr oft insbesondere in Canzonetten-Drucken.

533. Vgl. zu diesen unterschiedlichen Arten der Triolenverwendung auch Collins, Coloration, S.169 ff.

534. Folgende Drucke, in denen nur in Semiminimentriolen notiert wurde, sind dem Verfasser bekannt: D. Ortiz, Trattado de glosas (1553), I. Camatero, Il quarto libro delli madrigali (1569), F. A. Baseo, Il primo libro delle canzoni (1573), J. Peetrinus, liber primus motectorum (1591), J. Peri, Euredice (1600), S. d'India, Le musiche e balli (1621), G. Frescobaldi, Cappricci (1626), G. Bruschi, Il terzo libro delle concerti (1629). Bruschi verwendete die Triole allerdings als Notationsform für den Dreivierteltakt. In der Singstimme sind Triolen (mit einem semicircolo semplice am Ende der Triolenstelle), im Bc. hingegen ein Dreivierteltakt mit der Vorzeichnung $3 / 4$ notiert. Eine verwandte Notationsform findet sich auch in den Concerti accademici... libro primo von S. Bernardi (1616). Hier sind an einer Stelle der Canto, Sesto und Bc. in Triolen, der Alt, Quinto und Basso aber im Dreiertakt notiert. Da ausschließlich der Notenwert der Semiminima (bzw. Schwarzen Minima) verwendet wurde, ist nicht zu entscheiden, ob eine weiße oder schwarze Notation gemeint ist. Semiminentriolen finden sich auch in den Madrigalen G. B. Mostos in dem Sammeldruck Corona de madrigali 


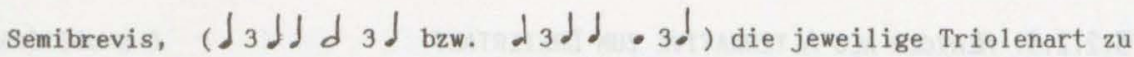
erkennen. Bei einem beträchtlichen Teil der Quellen enthalten die Triolen aber weder eine schwarze Semibrevis noch eine weiße Minima. Hier ist die richtige Ubertragung der Triolengruppen nur durch Ausprobieren zu ermitteln535. Gänzlich kompliziert wird der Sachverhalt, wenn in einem Druck beide Möglichkeiten benutzt wurden. Girolamo Dalla Casa versuchte, die beiden Arten der Triole durch unterschiedliche Setzung der Dreien zu kennzeichnen: Steht die Drei vor der Triolengruppe sind drei schwarze Minimen gemeint, steht sie aber in der Triolengruppe drei Semiminimen536. Ein solches Verfahren ist sonst in Drucken, in denen beide Arten der Triolenschreibung vorkommen, nicht zu beobachten537.

Im Gegensatz zu den hemiole sind die Triolen (und auch die hemiole in Triolennotierung) in der Musiktheorie nicht oft behandelt worden. Verschiedene Triolenarten beschreibt Lodovico Zacconi in der Prattica di musica (1596) unter der uberschrift "Di alcune stravaganze che si trouano nella Musica"538. Dieser Abschnitt ist recht widersprüchlich ausgefallen. Zunächst stellt er die Triolen (diesen Begriff kennt Zacconi freilich noch nicht) "con tre Semiminime" und "con tre Minime" vor. Er weist die beiden Triolenarten den beiden semicircoli zu: In drei Semiminimen wird unter dem semicircolo semplice notiert, in drei Minimen unter dem semicircolo tagliato539. Alle Triolenarten dauern seiner Meinung nach einen "tatto", also die Triole mit drei Minimen eine Brevis, diejenige mit drei Semiminimen eine Semibrevis (!) 540 . Als weitere, seiner Meinung nach seltene Verwendungen dieser Notationsart fügt er je ein Beispiel mit einer schwarzen Brevis und zwei schwarzen Mimimen 541 , sowie mit einer schwarzen Semibrevis und zwei Semiminimen 542 (!!) an. Auf der nächsten Seite versucht er alle erwähnten Arten in ein System zu bringen. Die Triolen mit weißen Minimen sollen nun unter dem semi-

(1579). Die aufgezählten Drucke umfassen nur einen kleinen Teil aller Drucke mit Triolen. Insgesammt wurde in ca. $16 \%$ aller ausgewerteten Musikdrucke der Jahre 1571-1630 Triolen verwendet. Die Tendez ist dabei abnehmend: 15711580: ca. 25\%, 1621-1630: ca. 5\%.

535. Einige der oben genannten Drucke sind durch Ausprobieren als solche ermittelt worden. Bei vielen waren solche Untersuchungen freilich nicht möglich.

536. Il vero modo di diminuir... libro secondo (1584). Es ist möglich, daß die unterschiedliche Behandlung der Triole hier auf die Vorlagen mit den hier bearbeiteten Stücken zurïkgeht.

537. Die Verwendung beider Arten der Triole ist dem Verfasser außerdem bekannt aus Don F. de las Infantes' Sacrarum varii styli cantionum... liber II (1578) sowie M. Nantermis primo libro de madrigali (1609).

538. fol. $42 \mathrm{ff}$.

539. fol. 42.

540. Eine solche Auflösung der Triolen ist aus praktischen Quellen nicht bekannt.

541. $3 \mathrm{~d} d$

542. 3 J 
circolo semplice notiert werden. Für den semicircolo tagliato ergänzt er hier Triolen mit drei schwarzen und mit drei weißen Semibreven ${ }^{543}$. Auch hier weist er jeder Triolengruppe die Dauer eines Taktes zu. Zu den Triolen mit drei Semiminimen bemerkt er jetzt, er glaube, es handele sich in Wirklichkeit um schwarze Minimen ${ }^{54}$. Mit Recht bemerkt er, die Triolen unter dem semicircolo tagliato seien selten $\$ 45$.

Ursprung der Mißverständnisse Zacconis könnten die Formulierungen in dem die Triolen behandelnden Kapitel "Di alcuni altri modi musicali" aus Giovanni Battista Rossis Organo de cantori ${ }^{540}$ gewesen sein. Die Beispiele Rossis entsprechen im wesentlichen denen Zacconis. Rossis Ausführungen legen einige der Mißverständnisse Zacconis geradezu nahe547. Auch der Begriff der "stravaganze" fällt hier.

Dieses Beispiel zeigt deutlich, wie wenig die Triolen in das Denken der konservativen Theoretiker passen. Auch die Triolen sollen sich nach Zacconi auf die beiden semicircoli und die dazugehörigen Taktarten beziehen (wenngleich Zacconi die Bedeutungslosigkeit des Breventaktes wohl erkannt hat - s.o.). Tatsächlich aber beziehen sie sich nicht auf das Taktzeichen oder den Taktschlag, sondern sie unterteilen die Einzelnote: Eine Note wird in drei Noten des nächstkleineren Wertes zerlegt, unabhängig von Taktschlag und Mensur.

Eine theoretische Darstellung der mensurunabhängigen Triole findet sich in den Regole utilissime (1606) von Antonio Brunelli. Er fuhrt dort für die Triole mit drei schwarzen Minimen den neuen Begriff der "meliola" ein548.

543. Aufstellung auf fol. 42' unten.

544. "... \& sappia che $i$ primi essempii delle figure oscure stanno per Minime denegrite, \& non per Semiminime naturali" (fol. 42').

545. Eine Triole mit weißen Semibreven ist dem Verfasser nur aus I. Camateros quarto libro delli madrigali (1569) bekannt; für die Triole mit schwarzen Semibrever nur die Beispiele bei Zacconi selbst (fol 42', vgl. dazu auch weiter oben). Aber auch die Triole mit drei weiBen Minimen ist recht selten. Dem Verfasser ist sie nur bekannt aus Don F. de las Infantes' Sacrarum varii styli... liber II (1588) und liber III (1589).

546. 1585, gedruckt 1618 (s.0.), S.18. Dieser kurze Abschnitt ist im zweiten Band dieser Arbeit auf S.24 f wiedergegeben.

547. So ist es gut möglich, daß die wiedersprüchliche Zuordnung zu den semicircoli bei Zacconi der undeutlichen Benennungsweise Rossis entspringt: Er gibt für jede Art der Triole ("maniere") zwei Beispiele, nämlich einmal mit Punktierungen, und einmal ohne; bei Zacconi wird jedes dieser Beispiele zu einer Art für sich. Rossis Aussage "die ersten beiden Beispiele" gehören zum semicircolo semplice mußte bei einer anderen Zählung der Beispiele zu den Verwirrungen bei Zacconi führen.

548. "La Meliola si puó segnare sotto qual si voglia Tempo, \& e quella che manda tre Minime nere a Battuta due in terra \& una in aria, \& s'usa di farla come dimostrera l'essempio, \& ogni volta che seguira il tre uscira fuori di Meliola, la quale pou uscire tanto in nere, quanto in bianche" (S.29). Vgl. 
Sie kann nach Brunelli unter jeder Mensur benutzt werden. Hier wird also die Triole in ihrer Abhängigkeit nur von dem zu triolierenden Notenwert definiert 549 .

Wie bereits erwähnt, nahm die Zah1 der verschiedenen zur Verfügung stehenden Triolen im frihen 17.Jahrhundert beständig $\mathrm{zu}$. $\mathrm{Zu}$ den bereits erwähnten Formen - praktische Bedeutung hatten im späten 16. Jahrhundert nur die Triolen mit drei schwarzen Minimen und (selten) mit drei Seminminimen - kam sehr bald eine ganze Reihe neuer Möglichkeiten hinzu. Schon 1603 benutzte Ascanio Mayone550 Triolen mit drei Cromen, drei Semicromen und sogar drei Biscromen; alle sind weiB aufzulösen (also drei Triolencromen = eine Semiminima) 551 .

Auch die Notation dieser kurzen Triolen ist noch nicht einheitlich. Neben den oben erwähnten Notationsformen, die bis heute so geblieben sind5s2, gibt. es unter den Triolen mit kürzeren Notenwerten auch solche, die als geschwärzt aufzulösen sind553. Cromen- bzw. Semicromen-Triolen kounten auch in der Form der bereits lange bekannten Semiminimen-Triolen notiert werden.

dazu auch Hel1, Rhythmus, S.123. Der Begriff "meliola" ist dem Verfasser sonst. nur noch bekannt aus den Traktaten Pier Francesco Valentinis (nach Murata, Valentini, S.335).

549. Die meisten der anderen Traktate behandeln nur die hemiole, meist nur in reiner Ausprägung. S. Cerreto erwähnt die Triolen erstmals in seinem Dialogo harmonico von 1631 (Ottava 73), dort unter der iberschrift "Delle Triple" (!). Während ar dort die beiden '(reinen) hemiole den beiden semicircoli zuordnet, notiert er beide Beispiele für die "triple" (entsprechend den hemiole: drei schwarze Minimen und drei schwarze Semibreven) unter den semicircolo tagliato (!).

550. Il primo libro di diversi capricci (1603).

551. Die kürzesten Noten in den geraden Takten in diesem Druck Mayones entsprechen unseren vierundsechszigstel Noten (die zeitgenössische Terminologie hat keinen Namen für diesen Notenwert!).

552. Solche Triolen mit kurzen Notenwerten finden sich bereits in folgenden Drucken: G. M. Trabaci, Ricercate... libro primo (1603) und II secondo libro de ricercate (1615) G. Frescobaldi Toccate... libro primo (1615, 4/1628), S. d'India, Le musiche (1615), F. Rognoni, Selva de varii passaggi (1620) und D. Castello Sonate concertate... libro secondo (1629). CromenTriolen sind auch in A. Virgilianos $I 1$ dolcimelo (ms um 1600) zu finden.

553. Triolen mit drei geschwärzten Semiminimen $(\mathrm{im}$ Aussehen $=$ Cromen) - sie haben zusammen den Wert einer Minima - finden sich z.B. in G. Cavaccios sesto libro de madrigali (1599) und in F. Turinis Motetti a voce sola (1629). Geschwärzte Triolennotierung ist auch noch in Drucken des späteren 17. Jahrhunderts anzutreffen. Noch 1675 notierte J. M. Gletle in seiner Musica genialis latino-germanica (Augsburg: Andrea Erffurt 1675) Triolen mit geschwärzten Semiminimentriolen (11. Trompeter Stückle, vgl. die Neuausgabe von Christian Blümel, S.6, sowie dort im Vorwort. Die ubertragung erfolgte natirlich in Vierteltriolen). 
Diese Notationsweise unterscheidet sich von den anderen nur in der Häufigkeit der Triolendreien. Während Ascanio Mayone schon in jeder Gruppe zu drei Noten eine Drei notierte, stehen in anderen Drucken die Dreien innerhalb oder vor jeder Gruppe von Noten, die zusammen den Wert von drei Semiminimen haben, also nach sechs Cromen bzw. zwölf Semicromen, unabhängig von der rhythmischen Ausprägung der Figuren554. Bereits 1615 notierte Sigismondo d'India Gruppen von je zwölf Cromen, über denen je eine kleine Zwölf steht555. Diese zwö1f Cromen haben den Wert einer Minima. Sextolen finden sich in der, allerdings wohl nach 1630 entstandenen Handschrift Chigi $Q$ IV 28 der Biblioteca Apostolica Vaticana, Rom.

Einige "Sonderlösungen" zur Triolennotation verdienen ebenfalls kurz erwähnt zu werden. Nur aus der Theorie ist die Möglichkeit der Triolierung mittels weißer Semiminimen (Darstellung als "hohle" Cromen) bekannt556. Ebenfalls eine besondere Notenform benutzte Girolamo Dalla Casa zur Notation von Semicromensextolen. Er erklärt im Vorwort seines vero modo di diminuir 557 neben der wohl noch nicht sehr verbreiteten Biscroma (bei Dalla Casa "quadruplicate, che sono 32. per battuta") die besondere Notenform der "treplicate, che sono 24. per batutta" - diese Notierung findet sich allerdings nur bei Dalla Casa558. Einen dritten Sonderweg geht Giovanni Girolamo Kapsbeger. Er benutzt die von ihm grundsätzlich sehr bewußt eingesetzte Balkung559 auch zur Triolierung: Drei Biscromen unter einem Balken haben bei ihm zusammen immer den Wert einer Croma, sechs unter einem Balken immer den einer Semimi-

554. So notierten G. Priuli, Sacrarum concentuum... pars altera (1619) sowie Delicie musicali (1625), F. Rognoni, Selva di varii passaggi (1620, nur die Triole zu drei Semicromen), B. Montalbano, Sinfonie (1629), D. Castello, Sonate concertate... libro primo (1629), G. Scarani, Sonate concertate (1630). Diese Notationsart erimert an die rein quantitativen Relationen der alten Proportionen.

555. Le musiche (1615). Die Verwendung der Zwö1f bei d'India erinnert an die hemiola-Notationen, denn sie steht zwar iber den Noten, jedoch nur beim ersten Auftreten der Figur.

556. A. Banchieri erwähnt diesen Notenwert in seinen Conclusioni (1609) als dritte Art der Hemiola (S.35), L. Zacconi führt gar in seiner Auflistung der verfügbaren Notenwerte (Massima bis Semicroma, Prattica di musica, seconda parte (1622)) die weiße Semiminima als "Figura di prolatione che ne vaño sei alla battuta" an (S.12). Offenbar meinen beide die oftmals in der perfekten prolatio verwendeten weißen Semiminimen und tibersehen, daß das Zeichen der prolatio perfecta, und nicht der Notenwert allein dazu führt, daß "sechs in einen Takt gehen".

557. I1 vero modo di diminuir... libro primo (1584), "Ai lettori".

558. Fs ist oben bereits darauf hingewiesen worden, daß auch A. Virgiliano, 11 dolcimelo (ms um 1600) den Begriff der treplicate kennt. Es ist seinem unvollständig gebliebenen werk jedoch nicht zu entnehmen, was er darunter verstand (vgl. weiter oben).

559. Vg1. oben. Die Balkung erfolgte entweder im Stich oder aber im Typendruck von Hand. 
nima; es liegt hier also wiederum eine Variante der Triolierung durch Schwärze vor. Statt einer Drei wird die Balkung zur Kenntlichmachung der Triolierung benutzt 560 .

Die Tatsache, daß vereinzelt gleichzeitig ternäre (triolische) und binäre (duolische) Rhythmen auftreten, hat immer wieder die Frage aufgeworfen, ob tatsächlich so musiziert wurde, wie es notiert ist, oder ob nicht vielmehr eine der beiden Stimmen angeglichen werden sollte. Georg Schünemann hat darauf hingewiesen, daß es solche Angleichungen wahrscheinlich schon bei den Proportionenstücken des 16.Jahrhunderts gab561. Hinweise auf eine binäre Auflösung der Triolen finden sich auch in einigen musiktheoretischen Quellen des späten 16. und vor allem 17.Jahrhunderts562. Dies hat Michael Bruce Collins zu der Annahme verleitet, daß hemiola und sesquialtera grundsätzlich in binäre Rhythmen abzuwandeln seien 563 . Die Anwendungen der Thesen Collins' auf Monteverdis "Vi ricorda" durch Wolf Frobenius 564 hat jedoch hinlänglich bewiesen, daß dies nicht der Fall sein kann ${ }^{565}$. Zusammenfassend kann festgestellt werden, daß eine solche Angleichung bei gleichzeitigem Auftreten von ternärem und binärem Rhythmus wohl auch in dieser Zeit eine unter mehreren Möglichkeiten darstellte. Treten aber hemiole, Triolen oder sesquialtere in allen Stimmen zugleich auf, verbietet sich eine Umwandlung in binäre Rhythmen.

560. Angewendet in Libro primo di mottetti passeggiate (1612), Libro primo di arie passeggiate (1612), Libro secondo di arie passeggiate (162.3) sowie Cantiones sacrae (1628).

561. Dirigieren, S.58 f. Schünemann erwähnt hier einen Hinweis Fabers (1550), zunächst zum Erlernen der Stücke die ungeraden Takte als gerade zu singen. Er fürt desweiteren verschiedene zeitgenössische ubertragungen eines Proportionenstückes mit Umwandlungen von ungeradem in geraden Takt an.

562. So bei M. Beringer, Musicae ... Erster und Anderer Theil (1610): "Wenn in Dimensione Binaria bißweilen ein schlag oder etlich mit 3 unten verzeichnet/ in einer Stimm gefunden werden." (Notenbeispiel) "Als denn werden die Noten durch drey gezehlt/ aber doch nur durch zwen theil abgemessen. Daher die ersten zwo einen halben Schlag gelten/ und die dritt allein einen halben Schlag. "Ganz ähnlich ist auch die achte Regel aus M. Vulpius' Musica Compendium $(1610,6 / 1636)$, S.52/53 formuliert. In der neunten Regel heiBt es aber dann bei Vulpius: "Wann aber die Ziffer 3. in allen Stimmen unter einer schwarzen Semibrevi und Minima oder unter dreven Minimis erscheint und gesetzt/ als dann werden drey Minimae auff proportiontact gesungen" (S. 52/53). Diese Ausfihrung hat sicher auch A. Brunelli mit der Anweisung "\& e quella che manda tre Minime nere a Battuta due in terra \& una in aria" (Regole utilissime (1606),S.29) gemeint. Weitere z.T. recht fragwiurdige Belege finden sich aufgelistet bei Collins, Coloration, S.298 ff.

563. Coloration, Sesquialtera und Triplets.

564. Ritornel1.

565. Die Unzulänglichkeit dieser Ubertragung hat He11, Rhythmus, S. $90 \mathrm{ff}$, anschaulich nachgewiesen. 


\subsection{COLOR UND "AUgENMUSIK"}

Vielfältige Verwerdung fand die Colorierung bei der sogenannten "Augenmusik"506. Mit "Augenmusik" wird eine Art der Textvertonung bezeichnet, die nicht hörbar, sondern nur für den Ausführenden sichtbar ist567. Bekannte Beispiele hierfür sind z.B. die Vertonung des Textes "di cinque perle" mit fünf aufeinanderfolgenden Semibreven (Marenzio568) oder Semibreven bei dem Wort "bianca" (ebenfalls bei Marenzi(0569). Es liegt auf der Hand, daß sich geschwärzte Noten hierfür geradezu anbieten. In der Tat ist die Augenmusik meist mit geschwärzten Noten ausgefuihrt.

Die Bliitezeit der Augenmusik war das späte 16. Jahrhundert. In etwa jedem fünften der ausgewerteten Musikdrucke aus dem letzten Jahrzehnt des Jahrhunderts sind auch Beispiele für Augenmusik durch Schwärzung zu finden; und dies, obwohl die Zahl der Madrigaldrucke mit Augenmusik in dieser Zeit bereits rückläufig war 570 . In den anderen Gattungen hingegen wird der Höhepunkt in diesem Jahrzehnt erreicht571. Mit Beginn des 17.Jahrhunderts nimmt die Zahl der Drucke mit Augenmusik insgesamt $a b^{572}$. Bemerkenswert ist, daß

566. Zur Kritik an diesem Begriff vgl. Hans Engel, Art. Madrigal in MGG, Bd.8, Sp.1430. Engel schlägt stattdessen den Begriff "Notenbild-Malerei" vor. Da sich jedoch der Begriff "Augenmusik" allgemein durchgesetzt hat, soll er auch hier beibehalten werden.

567. Vg1. hierzu Einstein, Augenmusik oder auch Edward A. Lippmann, Art. Symbolik in MGG, Bd.12, Sp.1789 ff. Die Grenzen zwischen Augenmusik und anderen Arten der Textvertonung sind nicht immer scharf zu zeichnen, da vieles sicht- und hörbar ist. So ist eine aufsteigende Tonleiter bei einem Wort wie "ascendens" natiirlich auch im Notenbild sichtbar. Augenmusik im engeren Sinne wäre dies aber nicht (Einstein rechnet auch solche Beispiele mit hinzu (S.12 f)).

568. Abgedruckt bei Einstein, S.14, sowie Lippmann, Notenbeispiel 3.

569. Abgedruckt bei Ejnstein, S.9.

570. In fast jedem dritten der ausgewerteten Madrigaldrucke aus den Jahren 1571-1580 findet sich diese Art der Augenmusik, jedoch nur noch in etwas mehr als 20\% der Madrigaldrucke aus dem letzten Jahrzehnt des Jahrhunderts. Augenmusik paste in der Tat wenig zu den Idealen der neuen Msuik dieser Zeit.

571. Als andere Gattungen tür Augenmusik kommen nur die verschiedenen Arten der Motette sowie die Canzonetten und Villanelen in Frage. In den Jahren 1571-1580 war solche Augenmusik in etwa $15 \%$ der ausgewerteten Motettendrucke zu finden, im letzten Jahrzehnt des Jahrhunderts hingegen in knapp 20\%; in den Canzonetten und Villanellen aus der Zeit 1571-1580 fehlen Beispiele für solche Augenmusik, im letzten Jahrzehnt des Jahrhunderts sind sie in etwas weriiger als $15 \%$ der Drucke zu finden. (Einstein glaubte, in Canzonetten und Villanellen uberhaupt keine Augenmusik finden zu können, S.11).

572. Madrigale: 1601-1610 noch etwa in 15\% der ausgewerteten Drucke, 16111620 in etwa $7 \%$ und im dritten Jahrzehnt des Jahrhunderts nur noch in gut $2 \%$ (hier nur: Augenmusik durch Schwärzung). 
die Zahl der Motettendrucke mit Augenmusik durch Schwärzung im dritten Jahrzehnt wieder leicht zunimmt573.

Geschwärzte Noten werden als Augenmusik in erster Linie bei Worten mit den Bedeutungen "schwarz", "dunkel", "Nacht" und "blind" verwendet, aber auch Worte die fur "Farbe"574, "Nebe1"575, "Wolke"576, "Schmerz"577, "Trauer"578 und "Tod"579, ja sogar für "schlecht"580 stehen, können zur Notation von schwarzen Noten im Sinn von Augenmusik führen. Vereinzelt wurde Augenmusik bei der bloßen Umschreibung eines dieser Worte angewendet, so z.B. bei dem Text "Vanum est vobis ante lucem surgere" ("es ist vergeblich, daß ihr vor Tag aufsteht", Ps. 127, 2) 581.

Die Verwendung der schwarzen Noten kann unterschiedliche rhythmische Konsequenzen haben. Wird diese Art der Schwärzung in einem sonst weiB notierten Dreiertakt verwendet, hat sie keinen Einflub auf den Rhythmus. Die schwarze Notation einiger Takte innerhalb eines Dreiers besagt der Theorie nach lediglich, daß alle diese Noten imperfekt und nicht alterierbar sind. Der Basso continuo kann in so einem Fall auch ohne die Schwärzung gedruckt. werden 582 .

Stehen jedoch schwarze Noten in einem geraden Takt, kommt es immer zu einem Wechsel von binärem zu ternärem Rhythmus ${ }^{53}$. Hier führt die Augenmusik also

573. 1601-1610: gut 8\%, 1611-1620: gut 3\%, 1621-1630 aber fast $9 \%$.

574. Besonders interessant bei B. Pallavincino, L'ottava libro de madrigali, (1612): Der Text "di piu varii'colori" wird zuerst im geraden Takt, dann im Dreihalbetakt mit weißen und schließlich ein drittes Mal wieder im Dreihalbetakt, aber mit geschwärzten Noten vertont:

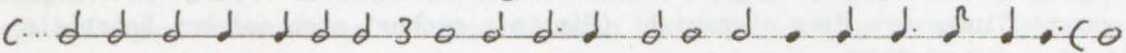

di piu va-rii co-1o-ri, di piu va-rii co-1o-ri, di piu va-rii co-1o--ri 575. Z.B. S. Bernardi, Psalmi integri (1613, 5/1627), G. Ganassi, Vespertina psalmodia (1625) und G. Bruschi, Missa, et psalmi (1627), jeweils bei der Textstelle "nebulam sicut cinerem spargit" ("er streut Nebel wie Asche", Ps. 147, 16).

576. Z.B. "in nubidus coe1i" in A. Savettas Motectorum... liber secundus (1608).

577. Bei G. Ferretti, Il secondo libro de canzone (1569, 4/1578): "Hor doglioso, hor allegre". Bei "Hor doglioso" ist schwarz notiert.

578. F. Spongia Usper, Il primo libro de madrigali (1604): "s'abbruciate". 579. S. Felis, Liber secundus motectorum (1585): "et hora mortis suscipe", bei "mortis suscipe" ist schwarz notiert.

580. 0. Bartolini, Canzonette... libro primo (1606): "Malo fa il mio core" .

581. C. Antegnati, Salmi (1592).

582. So bei G. Bruschi, Missa, et psalmi (1627).

583. Lediglich der "minor color" (s.u.) böte sich als Augenmusik ohne einen solchen Wechsel an. Dem Verfasser ist aber kein Beispiel für so eine Verwen- 
zu einer Veränderung des Rhythmus. Die Schwärzung wird in den geraden Takten selten für nur ein Wort verwendet. Meist sind es längere Abschnitte, die aufgrund entsprechender Textworte in einer hemiola oder in Triolennotation vertont werden 584 .

\subsection{MINOR COLOR UND VERWANDTE NOTATIONSFORMEN}

Schon im 15. Jahrhundert gab es die Möglichkeit, daß eine geschwärzte Semibrevis mit einer folgenden schwarzen Minima, der sogenannte "minor color", statt in triolischer Art als punktierte Minima mit Semiminima zu lesen war. Im 16. Jahrhundert ist diese Lesart die Rege1585. Bereits in den Quellen des späteren 16. Jahrhunderts erscheint der "minor color" fast nur noch in Verbindung mit Ligaturen; hier gab es keine alternative Notationsweise, wollte man die Ligatur beibehalten586. Während Willi Apel die Auflösung von Schwärze als Punktierung auf den "minor color" beschränkt wissen wil1587, ist sie zumindest für das ganze 16. Jahrhundert auch für die Notengruppe schwarze Brevis mit einer folgenden schwarzen Semibrevis belegt 588 .

Entstanden ist diese Notationsform sicherlich aus der rhythmischen Nähe von triolischer und punktierter Auflösung des Rhythmus589. Schon im 16.Jahrhundert bot die Musiktheorie aber auch eine direkte Erklärung dieser Auflösung: Galt bis dahin, daß die Schwärze den Notenwert um ein Drittel verkürze, so kam nun auch die Möglichkeit der Verkürzung um ein Viertel hinzu590. Eine um

dung des "minor color" bekannt.

584. Die große Fillle der Beispiele verbietet hier eine Auflistung.

585. Apel, Notation, S.49 und 137.

586. Bei einer Semibrevenligatur ("cum opposita proprietate") hat die zweite Semibrevis, wenn sie in der Art des "minor color" geschwärzt ist, nur den Wert einer punktierten Minima; dieser Wert ist nicht anders in einer Ligatur zu notieren.

587. Notation, S.138.

588. Z.B. L. Zacconi, Prattica di musica (1596), fol.28' ff und fol.31' ff.

589. Apel, Notation, S.137.

590. Die Theorie der Verkürzung einer geschwärzten Note um ein Viertel findet sich bezogen auf den "minor color" bereits bei Glarean (Riemann, Color, S.150). Auf alle Notenwerte bezogen lautet diese Regel bei $L$. Zacconi, Prattica di musica (1596), fol. 31': "Et si come il punto di agumentatione (!) agumenta (!) le figure d'una terza parte cosi l'oscurità gline scema quanto sia per il vallore di vna quarta parte, dimodo che la massima di otto tatti ne ualeria sei, la Longa tre, la Breve uno e mezzo, et la Semib. tre Semimin. ...". Auch hier wird wieder die Systematisierungsbestrebung Zacconis deutlich: Er beschreibt diese Art der Schwärzung als eine allgemeingiiltige, auf alle Notenwerte anwendbare, Notationsform, obgleich in der Praxis nur die Semibrevis und die Brevis auf diese Art verkürzt werden können. Wieder sehr viel praxisnäher sind auch hier einige deutsche Traktate. So schreibt M. Vulpius, Musica compendium, S.49: "Die vierte Regel. 
ein Viertel verküzte Note hat freilich denselben Wert, wie eine um die Hälfte verlängerte Note des nächstkleineren Notenwertes. Diese Erklärung läßt sich jedoch nur auf den "minor color" selbst anwenden, indem man nämlich in der schwarzen Minima eine Semiminima sieht und die Schwärzung somit auf die Semibrevis beschränkt. Eine solche Lösung ist bei der Form schwarze Brevis mit schwarzer Semibrevis nicht möglich, da die schwarze Semibrevis immer als solche erkannt werden kann. Während Maternus Beringer dieses Problem durch Einführung einer nur für diesen Fall geltenden Regel pragmatisch umgeht591, versuchte Lodovico Zacconi eine Erk1ärung für diesen Fall zu konstruieren 592 ,

Eine schwarze Nota ausser der proportion wird ihres vierdten Theils beraubt / dann die Schwertzung nimpt den vierden Theil hinweg." Als Beispiele werden Brevis und Semibrevis angegeben. Ganz der Anwendung in der Praxis entspricht die Erklärung von M. Beringer, Musicae ... Erster und Anderer Theil (1610) Er schreibt: "Wie werden die Noten gemehret? Durch einen Punct. Wenn derselbige hinter einer Noten stehet/so gilt sie noch halb so viel. Wie werden sie geschmälert? Durch die schwärze. Dieselbe nimbt entweder den vierdten/ oder halben theil darvon. Wo nimbt die schwärtz don vierdten theil? In Ligaturis Brevium und Semibrevium von der letzten Noten" (Notenbeispiele) (...) "Wo nimbt die Schwärtz den halben theil? Inn einer schwartzen Semibrevi, wenn ein schwartze Brevis darvor stehet" (fol. 7 f). Diese Erklärung des "minor color" als Verkürzung um ein Viertel war auch in die moderne Notationskunde eingegangen, wird aber von Ape1, Notation, S.137, Anm.1, bestritten.

591. Vgl. das Beringer Zitat in der vorhergehenden Anmerkung.

592. Schon auf fol.29 spricht Zacconi kurz die Möglichkeit an, daß eine schwarze Semibrevis sowohl für eine punktierte, als auch für eine einfache Minima stehen kann. Fol. 32 unten geht er dann näher darauf ein: "Ma è d'auertire che non sempre stando la Semibr. in questa forma cosi sciolta è di quel vallore che dimostrano gl'adutti essempii, perche se li precede vna Breue oscura non rittiene piu il vallore di tre Semimin. ma entra sotto il vallor di Minime come qui si vede

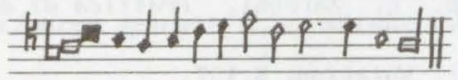

\& chi cerca la cagione, sappia che non per altro ha questo variatio valore che per accompagnamento di figure oscure; per distinguere $i$ Tempi bianchi da gli oscurati, à $i$ quali $i$ Cantori sempre debbano riguardare; accioche 1'vso non gl'ingannino: perche se bene il piu delle volte la Semibreve cosi oscurate et denegrita sta per il vallore d'una Minima puntata che è questa; nondimeno perche nel superiore essempio essa Semibreve oscura si ritroua haver dinanzi vna Breue del istesso collore, volendo equiparar $i$ Tempi non si possano con altra figura che con la Semibreue oscurata equiparare, e percio valendo io che si vegga quanta differenza sia in vna istessa figura gli ho poste le Semiminime a canto; perche se le stessero semplicemente ogni uno s'auederia che la non puó valer piu che per Minima: Ma stando cosi accompagnata molti la torrano per una Minima puntata: \& nondimeno la non è 
Außerhalb von Ligaturen wird der "minor color" in verschiedenen Diminutionstraktaten verwendet, um die Gesangsmanier des "principar sotto le note" zu notieren593. Hier ist aber wahrscheinlich an eine triolische - oder eher noch freie - Auflösung zu denken. Sicher wollte man durch die Schwärzung die Schärfe einer Punktierung vermeiden.

\subsection{SONDERFUNKTIONEN DER SCHWÄRZUNG \\ 3.5.1. SCHWARZE NOTEN ALS CHORALNOTATION}

Intonationen und Antiphone wurden, soweit sie in Drucken figuraler Musik erschienen, in schwarzen Noten, meist Breven, gedruckt. Hiermit soll das Außere der römischen Choralnotation imitiert werden. Sie stehen in der Regel nur im Tenor 594 . In einigen Drucken findet sich in jedem Stimmbuch zu Anfang der figuralen Musik - oft noch vor die Schluissel gedruckt - eine einzelne schwarze Brevis. Sie gibt den Schlubton der Intonation an und soll das Finden des Anfangstones erleichtern595. In Li dilettevoli introiti Valerio Bonas (1611) stehen die Anfangstöne der Intonationen in der Orgelstimme. Der Organist sol1 sie zu Beginn spielen, danit Sänger und Orgel die gleiche Tonhöhe haben 596 .

altro se non quello che qui si vede.

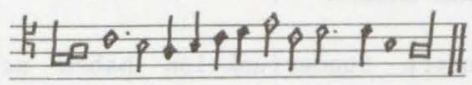

(...)".

593. Vgl. die im zweiten Band dieser Arbeit als Notenbeispiel III,6 wiedergegebene Verzierungstabelle aus F. Rognonis Selva de varii passaggi (1620). Ebenso wird das "principar sotto le note" bei G. B. Bovicelli, Regole passaggi di musica (1594), S.9, 11 etc. notiert. Bovicelli benutzt den color auch für Verzierungen auf einer Schlußnote (vgl. S.13). In deutschen Diminutions- bzw. Gesangslehren wird ebenfalls so notiert, z.B. bei M. Praetorius, Syntagmatis muisci ... tomus tertius (1619), S.232 f (ein Faksimile dieser Stelle ist bei Jacques Handschin, Art, Akzentschriften in MGG, Bd.1, Sp.266 abgedruckt). Hier wird diese Figur als eine Art des "Accentus" behandelt (dieser Abschnitt von Praetorius findet sich wiederum fast wörtlich in der Musica pratica (1642) des J. A. Herbst, S.3 f).

594. Selten auch im Basso continuo, so bei T. Cecchino, Psalmi (1619).

595. Z.B. bei A. Banchieri, Messa solenne (1599) und G. G. Gastoldi, Integra onnium solemnitatum vespertina psalmodia (1600).

596. So mitgeteilt im Vorwort zu Valerio Bonas Li dilettevoli introiti:

"ALI.I HONORATI ORGANISTI. Essendo l'Organo nelle Musiche il principali, di esso si deve far capitale, come quello, dal quale dipendono tutte le voci, \& chi non fa stima dell' Istromento ben spesso resta confusio, per il di sordine che nasce, per la differenza che e tra l'Organo \& l'Intonatori, restono in voce semitonante. Per ouviare dunque à questo di sordine, auanti l'Introite ho posto due note negre, che altro non intendono, che la voce dell'intonatione. Però dovrà l'esperte Organista dar la voce al Maestro di Capella, o Cantore, con quel tasto assegnato con le due note negre, o con la 
Insbesondere in deutschen Quellen des 15. und 16.Jahrhunderts wurden gelegentlich ganze Tenorstimmen mit einem choralen cantus prius factus in Choralnotation, z.B. in Hufnagelnotation 597 geschrieben. In ähnlicher Weise benutzte auch Adriano Banchieri schwarze Breven, um den canto fermo in seiner "Messa domenicale"598 zu kennzeichen. Eine solche schwarze Brevis hat, wie Banchieri in dem Vorwort "alli virtuosi proffessori" ausfihrt599, den Wert einer Semibrevis 600 .

\subsubsection{ANDERE SONDERFUNKTIONEN DER SCHWÄRZE}

Pandolpho Zallamela verwendete in seiner Weihnachtsmotette "Multifariam multisque modis"601 die Schwärze, um die Zweiteiligkeit des Textes in besonderer Art zu vertonen: uber der Motette findet sich die Aufführungsanweisung "asperas nigro demisse concinat unus Ast albo, cuncti uocibus altisonis"0.02; der geschwärzte Teil soll also in solistischer Besetzung und "tiefer" gesungen werden, der weiB notierte Teil hingegen mit vereinten Stimmen, also chorisch und "höher". Die Schwärzung hat hier nur die Funktion, diese beiden Teile zu kennzeichnen, sie hat keine rhythmische Bedeutung. Bei so einer Transpositionsanweisung wird man zunächst an eine Oktave oder allenfalls an eine Quarte bzw. Quinte denken. Der Umfang der Stimmen603 schließt dies jedoch aus. Man wird hier von einem kleineren Intervall - wohl einer Sekunde - ausgehen mússen 604 .

Eine andere Sonderfunktion haben schwarze Noten bei Enrico Radesca di Foggia. In seinem terzo libro delle canzonette (2/1616) notiert er eine Falsobordone-Longa schwarz605, im quarto libro delle canzonette (1610) wurde

stelli intonatione, che alle volte euui poste. Et queste sia can la bana gratia loro, alla quale mi raccomando."

597. Ein Beispiel für die Hufnagelnotation in figuraler Musik ist im Faksimile abgedruckt bei Paul Kast, Art. Messe in MGG, Bd.9, Tafel 12 (aus der Handschrift Cod.542 der Stiftsbibliothek St.Gallen). Ähnliches ist selten auch in italienischen Handschriften des 16. Jahrhunderts anzutreffen - vg1. hierzu Staehelin, Chorbücher, S.666.

598. Aus der Sacra armonica (1619).

599. Vgl. den Abdruck dieses Vorwortes im zweiten Band dieser Arbeit auf S.7 f.

600. Zwei Sätze von I. Camatero (aus $L i$ introiti fondati sopra il canto fermo (1574)), in denen ebenfalls die Choralstimme in schwarzen Breven (Wert: eine Semibreve) notiert ist, sind bei Schneider, Basso continuo, S.163 ff wiedergegeben.

601. Aus Musica (1582). Ein Faksimile aus einem Stimmbuch ist im zweiten Band dieser Arbeit als Notenbeispiel I,17 abgedruckt.

602. Es sei hier auf die Ahnlichkeit dieser Anweisung mit oft ähnlich verschliusselten Kanonanweisungen hingewiesen.

603. Cantus, 1.Tei1 d'-d' ', 2.Teil c'-d''; Baß, 1.Teil G-b, 2.Teil F-c'.

604. Vgl. hierzu auch weiter unten im Abschnitt III.6.

605. Vgl. hierzu im einzelnen weiter unten im Abschnitt IV.1. 
in "Ch'io non fami cor mio"606 eine schwarze Longa im Baß unter einem wohl rezitativisch freiem Canto notiert607. Wahrscheinlich wurde in beiden Beispielen diese ungewöhnliche Notenform gewählt, um die Unbestimmtheit des Notenwertes zu verdeut1ichen.

606. Vgl, die ubertragung des Anfanges dieses Satzes im zweiten Band dieser Arbeit als Notenbeispiel I,18.

607. Auch das Original ist in Partitur gedruckt. 


\section{TEIL: INTAVOLATUR, PARTITUR UND BASSO CONTINUO}

$\mathrm{Zu}$ den entscheidenden Neuerungen der letzten Jahre des 16. Jahrhunderts gehört unbestritten der Basso continuo. Zu dieser Zeit war aber der Basso continuo nur eine unter mehreren Möglichkeiten der Begleitung. Von den unterschiedlichen Arten der Begleitstimmen berichtet Adriano Banchieri bezogen auf die Kirchenmusik - in seinen Conclusioni nel suono dell'organo (1609) unter der Uberschrift "Quattro maniere cō le quali viene praticato il suono dell'Orga."1. Er nennt "Fantasia" (freie Begleitung einer bekannten Komposition), "Intavolatura", also das collaparte-Spiel des in Tabulatur abgesetzten Werkes, "Spartitura", womit sowohl das collaparte-Spiel als auch das Generalbasspiel nach der Partitur gemeint ist, und schlieblich den "Basso seguente"2. Für den Druck von "Basso seguente"-Stimmen weiB er wiederum von drei verschiedenen "maniere" zu berichten. Neben dem "Basso seguente" mit Vorzeichen und dem "Basso seguente" ohne Vorzeichen erwähnt er hier auch den "Basso seguente con il soprano sopra", also eine Baß-SopranPartitur ${ }^{3}$. Dies sei auch die Art, die er, Banchieri, bevorzuge.

Die Wah1 der jeweiligen Art der Begleitstimmen kann von der Art der Komposition bestimmt sein (s.u.). Oft liegt jedoch lediglich eine persönliche Entscheidung des Komponisten vor. Die Befürworter der verschiedenen Arten der Begleitstimme äußern sich nicht selten auch polemisch für oder gegen bestimmte Arten der Begleitung. Vor diesem Hintergrund sind alle theoretischen Außerungen zur Generalbaß- bzw. Begleitproblematik der Jahre um 1600 zu sehen.

Im folgenden werden diese unterschiedlichen Arten der Begleitstimmen im einzelnen anhand der erhaltenen Quellen vorgestellt. Der Blick soll hierbei nicht nur auf die Art der Notation, sondern auch auf die Auswirkungen der jeweiligen Notation auf den Begleitsatz gelenkt werden. Eine Behandlung der ersten von Banchieri genannten Art der Begleitung, nämlich der "Fantasia", entfällt natïrlich.

1. S.24 f. Dieser Abschnitt ist im zweiten Band dieser Arbeit auf S.18 f abgedruckt.

2. Zwischen "Basso seguente" und "Basso continuo" unterscheidet Banchieri noch nicht.

3. Diese Art der Partitur wird im folgenden als Randstimmerpartitur bezeichnet. 


\section{BEgLEITSTIMMEN IN TABULATUR}

\section{1,1, LAUTENTABULATUREN}

In begleiteten Sologesang zur Laute kann ein Vorläufer der Monodie gesehen werden. Lange bevor die "eigentliche Monodie" in Florenz entwickelt wurde, gab es Drucke mit Musik für eine Singstimme und Laute ${ }^{4}$, wenngleich es sich hier meist nicht um für diese Besetzung komponierte Musik, sondern um Bearbeitungen handelts. Diese Lautenlieder wurden in der Regel als Lautentabulatur mit dariber gedruckter bzw. geschriebener Singstimme notierto.

Die Bedeutung der Laute als Begleitinstrument nahm beginnend mit dem Ende des 16.Jahrhunderts in Italien $a^{7}$. In den frihen handschriftlichen Quellen der Florentiner Monodie aus dem späten 16. Jahrhundert sind Monodien noch mit Lautentabulatur bzw. mit Archiliuto-Tabulatur uberliefert ${ }^{8}$. Die intavolierten Begleitsätze entsprechen im wesent1ichen bereits den Chitarronenbegleitsätzen des 17.Jahrhunderts (s.u.).

Seltener erschienen Lautentabulaturen auch zusammen mit mehrstimmiger Vokalmusik, meist Canzonetten?. Es ist hierbei freilich fraglich, ob die Tabu-

4. Zu nennen sind als frihes Beispiel die von Franciscus Bossinensis eingerichteten "Tenori e contrabassi intabulati col soprano in canto figurato per cantar e sonar col lauto" (Ottaviano Petrucci, ab 1509). Ein Faksimile aus dem "libro primo" bietet Gianluigi Dardo, Art. Bossinensis, Franciscus, in MGG, Bd.15, Tafel 35.

5. So bei Petrucci und vielen der nachfolgenden Drucke. Sofern es sich nicht um Intavolierungen handelt, sind die Begleitsätze doch den mehrstimmigen Gattungen nachempfunden, so z.B. in dem "primo libro di napolitane" von G. Gorzanis (1570).

6. Es sind auch Beispiele bekannt, bei denen Singstimme und Intavolatur nebeneinander stehen (vgl, die Abbildung bei Claude V.Palisca, Art. Galilei, Vinvcenzo, in MGG, Bd.4, Tafe1 54) oder iberhaupt nur die Lautenstimme in einer Quelle vorhanden ist und die Singstimme aus einer anderen Quelle zu ergänzen ist (so z.B. in einer Handschrift der Sammlung Thibault - vg1. Thibault, Manuscript, bes. die Notenbeispiele S.67 ff).

7. Fortune, Instruments, S.11.

8. So im sogenannten "Bottegari-Lautenbuch" (vg1. McClintock, Songbook, bzw. seinen Artikel Bottegari, Cosimo in MGG, Bd.15, Sp.999 f) und in großer Zahl in der Handschrift 704 der Bibliotheque du Conservatoire, Brüssel, vgl. Hil1, Accompaniments; Porter, Early Monody und Carter, Caccini, S.211 ff. Auf eine Lautenbegleitung zu Caccinis berihmtem "Amarilli mia bella" von Robert Dowland machte Myers, Caccini-Dowland, aufmerksam. Diese ist jedoch weit mehr einer kontrapunktischen Tradition verpflichtet als die rein akkordischen Begleitungen der italienischen Tabulaturen (vg1. auch Hil1, S.196 ff), Ergänzend sei noch auf eine ebenfalls englische Aussetzung des "Amarilli mia bella" für "Lyra viol" hingewiesen (vg1. hierzu Cyr, Accompaniments, S.45 f mit einer ubertragung des Anfanges dieser in der Vokalstimme uberreich colorierten Fassung). 
laturen immer zusammen mit allen Singstimmen zu spielen sind oder ob durch das Mitspielen der Laute fehlende Singstimmen ersetzt werden sollten 10 oder gar an eine rein instrumentale Aufführung gedacht wurde ${ }^{11}$. Das Zusammenwirken von Singstimmen und Laute ist zumindest bei Orazio Vecchi bezeugt: Die Canzonetten, denen eine Lautentabulatur beigefügt ist, sind uberschrieben mit "Per sonare e cantare insieme"12. Auch in den anderen Quellen ist zumindest auf eine gleichzeitige Lesbarkeit aller Vokalstimmen und der Tabulatur geachtet worden ${ }^{13}$.

\subsection{ChITARRONENTABULATUR}

Der Chitarrone - sowie die ihm verwandte Theorbe und der Archiliuto14 - ist eines der beliebtesten Begleitinstrumente des frühen 17. Jahrhunderts iber-

9. So in Orazio Vecchis Selva de varie ricreatione (1590) und G. G. Gastoldis Balletti (1594). Gedacht sei hier aber auch an die Sammlungen Simone Verovios mit Lauten- und Klavierintavolatur. Casimiri, Verovio, S.193 ff, nennt insgesamt vier Drucke mit Lauten- und Klavierintavolatur. Hinzuzufügen ist noch G. F. Anerios Dialogo pastorale (1600). Ebenfalls mit lautenintavolaturen erschienen die drei Canzonetten-Bücher Canzonette per cantar et sonar di liuto ... libro primo (secondo, terzo) (1591; es handelt sich hierbei um Nachdrucke Vincentis der Ghirlanda Verovios; vgl. Einstein, Bibliography 3, S.65), A. Orologio, Canzonette... intavolate per sonar di liuto (1596; zitiert nach RISM 0 125) und A. Ferrari, Canzonette... con 1 'intavolatura per sonar di liuto ... libro secondo, (zitiert nach RISM F 264).

10. Vg1. Carter, Caccini, S.209.

11. Gelegentlich anzutreffende Diminutionen in den Intavolaturen verovios könnten darauf hinweisen (z.B. in Diletto spirituale (1585) $\mathrm{Nr}$.16). Da es sich aber um sehr sparsame Verzierungen handelt, ist hier auch eine Begleitfunktion denkbar. Sicherlich nicht als Hinweis auf eine rein instrumentale Ausfuihrung kann die Transposition der Intavolaturen gelten. Sie ist gelegentlich auch bei Bc.-Stimmen zu beobachten (vgl. weiter unten).

12. Selva de varii ricreatione. Die Lautentabulatur ist bei vecchi in allen Stimmbüchern abgedruckt. Ein Zusammenwirken von mehreren Singstimmen mit einer Laute bezeugen auch einige außeritalienische Drucke in auffuhrungsorientierter Druckdarstellung (Vokalstimmen kopfstehend gedruckt; vgl. hierzu Besseler, Schriftbild, S.146 f, Wolfgang Boetticher, Art. Denss, Adrian, in MGG, Bd.3, Sp. $194 \mathrm{f}$, sowie Willi Kahl, Art. Köln, in MGG, Bd.7, Sp.1337 f).

13. So sind in Verovios Diletto spirituale die drei bzw. vier Vokalstimmen jeweils auf die linke Seite, die Klavier- und Lautentabulatur auf die rechte Seite gedruckt, auch wenn, wie verschiedentlich deutlich zu erkennen ist, der Platz recht knapp war (z.B. bei $\mathrm{Nr}, 14$ oder $\mathrm{Nr}, 21$ ).

14. Zum Unterschied zwischen Chitarrone und Theorbe bzw. Archiliuto vgl. Buetens, Accompaniment, S.37, Wolfgang Boetticher, Art. Laute in MGG, Bd.8, Sp. $365 \mathrm{f}$ und Ian Harwood, Robert Spencer, Art. Chitarrone in NG Instruments, Bd. I, S.359 f. Vg1. zur Archiliuto auch Hill, Accompaniments, 
haupt 15. In der Regel wurde die Begleitung fiir den Chitarrone nicht in Tabulatur, sondern als Generalbaßstimme notiert. Es haben sich aber auch einige intavolierte Begleitsätze für Chitarrone erhalten.

Wie der Chitarrone bzw. die Theorbe eine Weiterentwicklung der Laute ist, so beruht auch deren Tabulatur auf der Lautentabulatur ${ }^{16}$. Die zusätzlichen, diatonisch gestimmten Chöre des Chitarrone werden einfach durchnumeriert, nur der siebte Chor wird mittels einer "Hilfsline" oberhalb der ersten Linie der Tabulatur notiert (in der Regel mit einer Nul1)17. Rhythmische Zeichen entfallen bei den meisten Begleitintavolaturen, da die Tabulaturen in Partitur direkt unter die Continuo-Stimme gedruckt sind, und der Rhythmus von dort übernommen werden kann ${ }^{18}$. Lediglich bei Rossi19 ist die Begleitintavolatur in einem Stimmbuch neben die Sopranstimme gedruckt.

Der friheste bekannte Druck einer Chitarronentabulatur ist zugleich der älteste Druck fünfstimmiger Madrigale mit vorgeschriebener Instrumentalbegleitung iberhaupt. Im Jahr 1600 erschienen in Salomone Rossis primo libro de madrigali a cinque voci20 sechs fünstimmige Madrigale "Per il Chitarrone"21. Die Chitarronentabulatur ist bei diesen Madrigalen jeweils in dem Canto-Stimmbuch abgedruckt. Es hande1t sich hierbei nicht um eine Intavolatur des Madrigales, sondern um eine weitgehend akkordische Begleitung ${ }^{2}$.

Bei allen weiteren bekannten Publikationen mit Begleitintavolaturen für Chitarrone bzw. Theorbe handelt es sich um Partiturdrucke geringstimmiger

\section{S.202 f.}

15. Fortune, Instruments, S.11 sowie Neemann, Laute. Auf die Frage nach der Besetzung des Continuo wird weiter unten noch ausfuhrlich eingegangen werden (im Abschnitt II.3.3.).

16. Der oder die beiden Diskantchöre sind bei dem Chitarrone um eine Oktave nach unten gestimmt.

17. Chromatische Erhöhung dieser tiefen Chöre werden auf unterschiedliche Art und Weise notiert. Z.T. wird der Zahl für den entsprechenden Chor eine Eins vor oder nachgestellt (gegriffen werden können freilich allenfalls noch der siebte und achte (hor), z.T. wird jedoch auch von umgestimmten bzw. zusätzlichen chromatischen Chören ausgegangen. Die entsprechende Auflösung ist meist einfach, da die tiefen Chöre in der Regel nur für die Baßstimme benötigt werden.

18. Nur selten sind rhythmische Zeichen bei abweichendem Rhythmus notwendig.

19. Il primo Libro de madrigali (1600).

20. Rossis zweites Madrigalbuch (1602) ist das erste Buch mit fünfstimmigen Madrigalen und Generalbaß (s.u.).

21. Jedes dieser sechs Madrigale ist so iberschrieben. Auch im Titel wird bereits auf diese Madrigale hingewiesen (vgl. Quellenliste).

22. Der Anfang eines dieser Madrigale ist im zweiten Band dieser Arbeit als Notenbeispiel II,1 abgedruckt. Auch hier ist die Tabulatur gegenuber den Voka1stimmen transponiert. 
Musik. Es sind dies drei Drucke mit Musik von Giovanni Giaccomo Kapsberger 23 sowie je ein Druck von Flamminio Corradi24 und Belofronte Castaldi25. Die Intavolaturen ersetzen eine Continuo-Stimme in der Regel nicht, sondern ergänzen diese. Sie können Auskunft über die Art des Continuospieles mit Theorben bzw. Chitarronen geben26. Bedingt können jedoch auch Rückschluisse auf eine Aussetzung für Tasteninstrumente gezogen werden. In Anlehnung an Stanley Buetens mögen die Beobachtungen an diesen Begleitsätzen in folgenden zehn Punkten zusammengefaßt werden 27.

1. Die Begleitsätze stehen, bedingt durch die Lage des Instrumentes, in Tenorlage, die Singstimme(n) werden nicht uberschritten.

2. Die Baßstimme wird vollständig gespielt.

3. Baßnoten können oktaviert werden, insbesondere bei Repetitionen. Selten kann in solch einem Fall der Baßton auch durch eine Quinte ersetzt werden 28 .

23. Libro primo di villanelle (1610), Libro primo di arie passeggiate (1612) und Libro terzo di villanelle (1619). Eine der Arien aus dem Buch von 1612 ist im zweiten Band dieser Arbeit als Notenbeispiel II,2 ubbertragen; ein weiteres Beispiel aus den Arie von 1612 ist bei Goldschmidt, Begleitung, S.59 ff abgedruckt. Villanellen aus dem Buch von 1610 sind bei Buetens, Accompaniments, S.43 f (mit Herunteroktavierung nur des höchsten Chores), bei Wolf, Handbuch, Bd.II, S.194 (mit Faksimile S.195; Wolf uberträgt als Lautentabulatur ohne heruntergestimmte Chöre) sowie im zweiten Band dieser Arbeit als Notenbeispiel II, 4 wiedergegeben. Die bei Kapsberger mit dem Zeichen "./." versehenen Akkorde (vgl. die ubertragungen) sollen arpeggiert werden (vgl. hierzu weiter unten im Abschnitt III.4.2.).

24. Le stravaganze d'amore, Venedig 1616, zitiert nach Buetens, Accompaniments, S.38; dort Seite 41 f die Ubertragung eines Satzes daraus.

25. Cappricci a due stromenti cioe Tiorba e Tiorbina, Modena 1622, zitiert nach Buetens, Accompaniments, S.39. Dieser Druck enthält im wesentlichen Instrumentalstuicke, aber auch einige Lieder; eines davon ist bei Buetens auf S. 45 ubertragen. Gelegentlich wird eine Begleitstimme in Chitarronentabulatur auch einem Werk Domenico Bellis, gedruckt in Venedig 1616 (ohne weitere Angaben) zugeschrieben (Riemann Lexikon, Sachteil, S.162 s.v. "Chitarrone" sowie Honegger, Lexikon, Bd.2, S.126 f s.v. "Chitarrone"). Hierbei handelt es sich offensichtlich um einen Irrtum; wahrscheinlich herruhrend vom Titel des primo libro dell'arie (es sind nur drei Musikdrucke Domenico Bellis bekannt und keiner davon enthält eine Tabulatur).

26. North, Continuo, S.161, ist der Meinung, die Aussetzungen seien in diesen Quellen besonders einfach gehalten, da es sich um Amateurmusik handele; Rückschlüsse auf das sonstige Continuospiel seien also nicht möglich. Dem ist jedoch entgegenzuhalten, daß ein Teil der mit Tabulatur gedruckten Werke Kapsbergers sich aufgrund ihrer Virtuosität in der Singstimme wohl doch eher an ausgebildete Musiker richteten. Ausschlaggebend für den Druck mit Tabulatur war hier sicher die Tatsache, daß der Theorbist Kapsberger auf die Ausführung der Theorbenstimme mehr Wert legte, als ein Sänger-Komponist.

27. Die zehn Punkte geben im wesentlichen die zehn Punkte aus Buetens Accompaniment, S.39 ff wieder. Sie wurden an einigen Stellen durch Beobach- 
4. Eine 4-3-Kadenz wird sooft als möglich angebracht.

5. Die Singstimme wird häufig verdoppe1t (auch in Oktaven). Ein Teil dieser Verdoppelungen kam zwangsläufig zustande ${ }^{29}$. Aus den Verdoppelungen kann geschlossen werden, daß man diese nicht mied, nicht aber, daß man danach strebte. Beispiele für Verdoppelungen besonders schwieriger Passagen (so Buetens) sind dem Verfasser nicht bekannt.

6. In den Kadenzen werden kleine Septimen ergänzt ${ }^{30}$, seltener auch Durchgangsnoten an anderen Stellen.

7. Akkorde werden wiederholt, auch wenn der Baßton nicht repetiert wird.

8. Die Begleitung ist durchgängig sehr einfach; es lassen sich keine Parallelen zum solistischen Lautenspiel erkennen ${ }^{31}$.

9. Die Stimmenzahl der Begleitung variiert zwischen zwei und füf.

10. Ein unbezifferter Akkord kann auch als Sextakkord gedeutetet werden. Maßgebend sind die Vokalstimmen, nicht die Bezifferung der Baßstimme.

\subsection{Begleitsätze im "Alfabeto per la chitarRa sPagnola"}

Die "spanische Gitarre", die "chitarra spagnola" war ein beliebtes Modeinstrument etwa ab den zwanziger Jahren des 17.Jahrhunderts 32 . Sie wurde nach Nigel Fortune vor allem in "light and frivolus canzonetts" 3 s vorgeschrieben. Es sind aber auch Solomotetten mit einem "alfabeto per $1 a$ chitarra spagnola" überliefert ${ }^{34}$.

Die Notation der Begleitsätze für die "chitarra spagnola" ist sehr einfach zu erlernen. Wie auch in der heute üblichen Gitarrenakkordschrift wurden uber die Singstimme Buchstaben gedruckt ${ }^{35}$, die jeweils einen Akkord reprä-

tungen des Verfassers erläutert oder ergänzt.

28. So in dem bei Buetens, S. 45 mitgeteilten Satz B. Castaldis. Hier sind im Baß zu Anfang vier Minimen "g" notiert; in der Theorbentabulatur stehen vier Mirimen mit den Tönen "G", "d", "c'" / und "g".

29. Will man z.B. bei der Akkordfolge F-Dur - f-Moll (Notenbeispiel II,2, Takt 13) beide Akkorde vollständig spielen, so muß man die Chromatik der Singstimme verdoppeln, soll es nicht zu unerwünschten Querständen kommen.

30. Notenbeispiel II, 2, Takt 12, 14, 20, 27, 29.

31. Verfehlt ist daher sicher die von Dür/Siegele, Vortrag, geforderte Generalbaßaussetzung auf der Grundlage des Stiles instrumentaler Gattungen.

32. Vgl. zur Häufigkeit des Instrumentes Fortune, Instruments, S.12.

33. Ebenda.

34. G. Marinoni, Il primo libro de motetti a una voce ... pasti in musica per alfabeto, Venedig 1614 (zitiert nach RISM M 699); vg1. Denis Arnold, Art. Marinoni, Giralamo in MGG, Bd.8, Sp.1657, sowie Wolfgang Boetticher, Art. Gitarre in MGG, Bd.5, Sp.187. Eine "chitarina alla spagnuola" wird auch im Vorwort zu E. de' Cavalieres Rappresentatione (1600) verlangt; vgl. den Wortlaut des Vorwortes im zweiten Band dieser Arbeit auf S.8 ff.

35. Getrennt von der Singstimme gedruckte Begleitsätze im alfabeto, wie der von Wolfgang Boetticher, Art. Gitarre in MGG Bd.8, Sp.191 f mitgeteilte (Pietro Milloni, Nuova corona d'intavolatura (Rom 2/1676)), sind aus dem 
sentieren. Im Gegensatz zu der heute ublichen Akkordschrift hatten die damals verwendeten Buchstaben keinen Bezug zu dem Akkord (C-Dur Grundste1lung heute " $\mathrm{C}$ ", im alfabeto "B"). Auch waren keine Modifikationen eines Akkordsymboles möglich (wie $\mathrm{C}^{7}$ o.ä.). Die verschiedenen Akkorde wurden beziehungslos durchalphabetisiert ${ }^{36}$. Ahnlich wie in den Lautentabulaturen des 16. Jahrhunderts, enthalten viele der Drucke, in denen das alfabeto verwendet wird, eine Einweisung in diese Akkordschrift. Die zu den Buchstaben gehörenden Akkorde sind nicht in allen Quellen dieselben. Während es bei den ersten Buchstaben kaum zu Differenzen kommt ${ }^{37}$, weichen die Akkorde zu den letzten Buchstaben des Alphabetes oft recht stark voneinander ab. Unter Umständen sollte damit unterschiedlichen Kompositionen Rechnung getragen werden.

Um die recht eingeschränkten Möglichkeiten des alfabetos zu erweitern, wurden verschiedene Sonderzeichen mit hinzugenommen, z.B. ein Kreuz oder ein Sternchen ${ }^{38}$. Auch die Bedeutung dieser Zeichen weicht von Druck zu Druck voneinander ab. Um der Vorliebe für Quartvorhalte ${ }^{99}$ Rechnung zu tragen, erweiterte Marini das System um Symbole für besondere Kadenzfolgen 40 . DaB trotz allem keine kontrapunktischen Anspruichen genügenden Begleitungen zustande kommen konnten, versteht sich von selbst: Parallelen sind ebenso häufig, wie regelwidrige Quartsextakkorde; passende Akkorde fehlen zum Teil auch ganz ${ }^{41}$. Sicher ist es nicht möglich, von diesen Intavolaturen auf das Continuospiel mit anderen Instrumenten zu schließen ${ }^{42}$.

frihen 17. Jahrhundert nicht bekannt.

36. Vg1. zu dieser Akkordschrift Wolf, Handbuch, Bd.II, S.191 ff, sowie Wolfgang Boetticher, Art. Gitarre, in MGG, Bd.8, Sp.186 ff. Als "Erfinder" dieser Akkordschrift gilt Girolamo Montesardo (1606, vgl. aber Boetticher, Sp.183.).

37. Vg1. die Gegenuiberstellung der alfabetos aus G. G. Kapsbergers Libro secondo di villanelle (1619) und aus B. Marinis Scherzi (1622) im zweiten Band dieser Arbeit als Notenbeispiel II,3a. Daß einige dieser Akkorde selten - wenn uberhaupt je - Anwendung fanden, liegt nahe (besonders Q und S). Die Akkordtabellen sind im Original nach dem Prinzip einer Lautentabulatur gedruckt. Das alfabeto Girolamo Montesardos findet sich bei Ragossnig, Handbuch, S. $64 \mathrm{f}$ mit ubertragung wiedergegeben.

38. Beides bei Marini, aber auch in anderen Quellen. Marini hält das Kreuz, wie auch das " $\$$ " und die Silben "con", "ron" und "bus" offenbar für fest zum alfabeto gehörig, obwohl er die vier letzteren nicht verwendet. Montesardo verwendete außer dem Kreuz und dem " $\&$ " die Sonderzeichen " 9 " und " $R$ " (vgl. Ragossnig, Handbuch, S. 64).

39. Vgl. oben den Punkt 4 der Beobachtungen an den Chitarronentabulaturen, 40. Vg1. die "aggiunte" Marinis (Notenbeispiel II,3b im zweiten Band dieser Arbeit).

41. Marini weist in einem Vorwort zu den Scherzi auf die Unvollkommenheit des alfabetos und die Notwendigkeit vom Baß abweichender Begleitung hin: "Ai Lettori. AVertino, che se non troueranno in qualche loco di quest' opera l'Alfabeto concorde con il Basso, l'animo dell'Autore é d'accompagnar la voce piu che sij possibile, non curandosi in questo d'obligarsi à quello, 


\subsection{INTAVOLIERTE BEgleitsätZe Für TASTENINSTRUMENTE}

Es wurde oben bereits auf einige mit Lauten- und Klaviertabulatur erschienenen Drucke Simone Verovios hingewiesen. Diese Tabulaturen konnten sicher sowohl dem rein instrumentalen Spiel als auch der verdoppelnden Begleitung dienen. Der Vortrag von Canzonetten u.ä. zum Tasteninstrument ist auch anderweitig belegt ${ }^{43}$.

Eine Sonderstellung innerhalb der Drucke Verovios kommt den Madrigali per cantare et sonare a uno, doi, et tre soprani von Luzzasco Luzzaschi (1601) zu. Hier handelt es sich nicht um schlichte Canzonetten, sondern um umfangreiche Madrigale mit hochvirtuosen Singstimmen; der Druck erfolgte statt in Chorbuchmanier (wie dies bei den anderen Drucken Verovios der Fall ist) in Partitur mit einer Klaviertabulatur ${ }^{44}$. Eine Lautentabulatur ist den Madrigalen nicht beigegeben. Obwoh1 1601 gedruckt, dürtten diese Madrigale deutlich älter sein ${ }^{45}$. Das Zusamnenwirken von Cembalo (gespielt von Luzzaschi) und Solosopran ist aus Ferarra bereits von 1571 bezeugt 46 ; das berihmte Vokaltrio "le tre Dame di Ferrara" bestand seit 157947; ein Ende fand die Musikausübung am Hofe von Ferarra vorübergehend mit dem Tode Herzog Alfonsos II. (1597) ${ }^{48}$. Mit diesen Daten dirfte auch die Entstehungszeit der Madrigale Luzzaschis umrissen sein.

essendo la Chitariglia priua di molte bone consonanze."

42. Im zweiten Band dieser Arbeit ist der Anfang einer Villanelle Kapsbergers aus dem ersten Buch der Villanellen von 1610 mit Chitarronentabulatur und alfabeto übertragen (Notenbeispiel II,4).

43. So in der Dedikation (von L. Conforto) zu P. Quagliatis Canzonette ... a tre voci per sonare et cantare ... libro secondo (1588). Sie beginnt mit den Worten: "Mi sapere 'auuto adoperato, che al fine ho hauto nelle mani queste altre canzonette del Sig. Paolo Quagliati; ch'egli ha composte à richesta di varie gentildonne Romane per sonare, \& cantare su'l cembalo ...".

44. Die senkrechte ubereinstimmung von Singstimme(n) und Tabulatur ist allerdings - hervorgerufeñ durch die unterschiedliche Häufung der kurzen Notenwerte - z.T. unbefriedigend; vgl. das im zweiten Band dieser Arbeit als Notenbeispiel II,5 abgedruckte Faksimile.

45. Vgl. Kinkeldey, Luzzaschi, sowie Denis Arnold, Art. Luzzaschi, Luzzasco, in MGG, Bd.8, Sp.1356.

46. Von einem Fest in Ferrara von 1571 wird berichtet, daß "dietro un gravicembalo toccato dal Luzzasco cantarono la signora Lucrezia e 1a signora Isabella Bendidio a solo a solo, e tutt'e due, si bene e cosi gentilmente, che io non credo si possi sentir meglio" (zitiert nach Anna Amalie Abert, Art. Ferrara in MGG, Bd.4, Sp.65. Diese Stelle findet sich auch bei Kinkeldey, Luzzaschi, S.538).

47. Kinkeldey, S.540.

48. Ebenda; vgl. auch Abert, Art. Ferrara in MGG, Bd.4, Sp.66. 
Die Kompositionstechnik dieser virtuosen Musik erinnert stark an die Bearbeitungstechnik der Diminutionstraktate von Ortiz"9 bis Francesco Rognoniso. Diese oft als Improvisationslehren verstandenen - und damit zu einem Teil wohl mißverstandenen - Traktate lehren in erster Linie eine Bearbeitungstechnik. Bereits viel frïher setzten Organisten Vokalmusik in Tabulatur ab und machten diese durch Hinzufuigung von Diminution intrumentengerecht sicher auch, um ihre Fähigkeiten zur Schau stellen zu können. Das Fehlen von solistischen Gattungen hat schlie日lich auch die Virtuosen der Melodieinstrumente und die Sänger dazu bewegt, Anleihen in der vokalen Mehrstimmigkeit zu machen ${ }^{51}$. Sowohl ein Melodieinstrument als auch ein Sänger benötigt hierbei freilich die Unterstützung eines Harmonieinstrumentess2 - es spielt den vollständigen ${ }^{53}$ Satz, während die Solostimme bzw. das Soloinstrument eine der Stimmen reich diminuiert wiedergibt 54 .

Auch der Begleitsatz Luzzaschis gleicht einem intavolierten Vokalsatz55; auch bei ihm sind die Singstimmen in unverzierter Form in der Intavolatur enthalten 56 . Die Besonderheit der Madrigale Luzzaschis liegt darin, dab, so weit man weiß, diese Begleitsätze von vornherein als solche komponiert worden sind.

Weitere italienische Begleitintavolaturen für Tasteninstrumente sind aus dem hier behandelten Zeitraum nicht bekannt. Interessant für die Begleitung vielstimmiger Musik in dieser Zeit ist eine wohl im zweiten Jahrzehnt des 17.Jahrhunderts am Kasseler Hof entstandenene Begleitintavolatur zu einer Canzone Giovanni Priulis57. Diese Canzone ist auch in Priulis Sacrarum con-

49. Trattado de glosas (1553).

50. Selva de varie passaggi (1620). Zu der großen Verbreitung von Quellen dieser Art im späten 16. Jahrhundert vgl. Arnold Geering, Art. Gesangspädagogik in MGG, Bd.4, Sp.1915 ff und Ernest T. Ferrand, Art. Improvisation in MGG, Bd.6, Sp.1101 ff.

51. Dies hat bereits Goldschmidt, Begleitung, S.55, dargelegt.

52. In der Regel enthalten diese Quellen die zugrundeliegenden Sätze nicht. Dort ist nur die jeweils diminuierte Stimme abgedruckt (und natirlich Anweisungen, wie eine solche Bearbeitung selber herzustellen ist). Lediglich Ortiz läßt auch die vollständige Vorlage abdrucken (in Chorbuch-Notation vg1. das Faksimile in der Neuausgabe von Max Schneider, S.XXI und XXII), allerdings getrennt von den Diminutionen.

53. Zur Frage des Mitspielens der Diskantstimme, wenn diese diminuiert wird, vgl. weiter unten. Auch hier kann aber schon gesagt werden, daß sie sowohl gespielt als auch weggelassen werden konnte.

54. Diese Stimme kann auch improvisiert worden sein. Die schriftliche Fixierung war aber zumindest eine gleichwertige Möglichkeit (vgl. z.B. bei Ortiz (Schneiders Ubersetzung des Vorwortes S.XXXI)).

55. Vg1. Denis Arnold, Art. Luzzaschi in MGG, Bd.8, Sp. 1356 sowie Maniates, Manerism, S.413.

56. Vg1. das Notenbeispiel II,5 im zweiten Band dieser Arbeit.

57. Ein Faksimile der Begleitintavolatur ist in der Neuausgabe, Giovanni 
centuum ... pars prima (1618) enthalten ${ }^{58}$, dort allerdings mit einem Basso seguente. Die Seguente-Stimme bildet auch die unterste Stimme der Intavolatur. Bei den anderen Stimmen handelt es sich jedoch weder um eine Generalbaßaussetzung noch um eine vollständige Intavolierung des Stückes. Vielmehr wurde hier versucht, nur das Wesentliche der Komposition zu intavolieren; eine siebenstimmige Komposition ist ja nicht ohne weiteres vollständig auf ein Tasteninstrument zu libertragen ${ }^{59}$. Daß diese Intavolatur nur zu Begleitzwecken zu gebrauchen ist, steht außer Frage. Es ist denkbar, daß auch die Begleitung in dieser Intavolatur nicht vollständig notiert ist. Insbesondere die nur zweistimmige Notierung in den Kadenzen (Takt 13 oder $17 \mathrm{f}$ ) sowie die geringstimmige Notierung bei akkordischen Stellen (Takt $19 \mathrm{ff}$ ) deutet darauf hin, daß auch diese Intavolatur noch zu ergänzen ist. Sie repräsentiert das Gerist der Komposition und teilt dem Continuo-Spieler weit mehr iber deren Aufbau mit als eine Seguente-Stimme. Ahnliches findet man auch in italienischen Musikdrucken, dort dann allerdings in Partiturnotation (s.u.).

Ein Verweis auf englische Quellen, in denen noch aus dem späteren 17. Jahrhundert ausgeschriebene Begleitungen in Klaviertabulatur überliefert sind, sol1 hier nicht fehlen. Dort findet man sowoh1 den Typ des eher exzerptähnlichen Begleitsatzes 60 , wie etwa in der Kasseler Priuli-Handschrift, als auch vollständig ausnotierte Begleitstimmen in Klaviertabulatur ${ }^{61}$. Mit Ausnahme einer Fantasia von William Lawes62 - hier kann man fast von einer konzertierenden Orgelstimme sprechen - werden in allen dem Verfasser bekannten Begleitsätzen die zu begleitenden Stimmen zumindest zum Teil verdoppelt $6{ }^{3}$.

Priuli: Instrumentalkanzonen, 2.Teil, auf S. IX abgedruckt. Zu Datierung und Provinienz vgl. Wolf, Barbarino, S.48.

58. Vgl. die vollständige Edition dieses Druckes, hrsg. von Albert Biales.

59. Vgl. den Anfang dieser Kouposition (in der Fassung der Kasseler Handschrift) in Kollation mit der Intavolatura im zweiten Band dieser Arbeit als Notenbeispiel II, 6 .

60. Vg1. das Faksimile einer solchen Orgelstimme in der Edition John Jenkins, Consort Musik of four Parts, auf S.XIX sowie die Edition dieses Stückes auf S.1 ff. Erfreulicherweise wurde die Orgelstimme in der in der Quelle vorgefundenen Form ibertragen.

61. Solche vollständig ausgearbeiteten orgelstimmen finden sich in der Handschrift Oxford, Bodleian Library, D205-211 (D211 ist das Orgelstimmbuch) mit Werken von John Hingston (gest. 1683). Vgl. auch die Neuausgabe der beiden Fantasien mit Zinken von Robert Paul Block. Zahlreiche Faksimiles von ausgearbeiteten Orgelstimmen sind bei Clark, Transposing, S.74 ff abgebildet; vgl. dort auch S.39 ff.

62. Dieser Satz ist bei Meyer, Kammermusik, S.318 ff abgedruckt; vgl. dazu auch Meyer, S.218.

63. Vg1. ebenda. Bei der von Meyer, S.218, erwähnten ausgeschriebenen Bc.Stimme zu einem Werk Frescobaldis (Mus.Ms.Add. 34003 der British Library, London) handelt es sich um eine Abschrift der Partiturausgabe von Frescobaldis primo libro delle canzoni. Meyer bezieht sich wahrscheinlich auf die Toccata per spinetta, è violino. Die Spinett-Partie ist hier auf zwei Syste- 
Nur schwer abzuschätzen ist die Bedeutung der zah1reichen deutschen Orgeltabulaturen mit vorwiegend geistlicher Vokalmusik für die Begleitung ${ }^{64}$. Aller Wahrscheinlichkeit nach dürften diese Tabulaturen zum größten Teil für den rein instrumentalen Vortrag bestimmt gewesen sein - zahlreiche Diminutionen machen viele dieser Tabulaturen für die Begleitung unbrauchbar65. An Direktions- oder Begleitfunktionen darf jedoch bei undiminuierten Intavolaturen gedacht werden. Dies gilt ganz besonders für Exzerptintavolaturen oder Intavolaturen, die in einer Quelle in mehreren Tonhöhen vorhanden sind 66 .

\section{PARTITUREN}

\subsection{DER BEgRIfF "PARTITUR" UND SEINE BEDEUTUNGEN IM SPÄTEN 16, UND FRÜHEN} 17. JAHRHUNDERT

"Partitura" bzw. "spartitura" hatte in dieser Zeit noch nicht die festumrissene Bedeutung, die der Begriff "Partitur" heute hat. Es lassen sich zwei Grundbedeutungen erkennen, die sicherlich auch im Zusammenhang miteinander stehen.

1. Mit dem Begriff "partitura" oder "spartitura" wurden Partituranordnungen bezeichnet, bei denen jede Stimme auf einem eigenen Notensystem notiert ist. Dies können sowohl Vollpartituren als auch Teilpartituren, wie Baß-Sopran-Partituren (Randstimmenpartituren) oder Baßpartituren doppelchöriger Werke sein, nicht aber Tabulaturen67.

Auch Bc.-Stimmbücher mit mindestens einem Stück in Partitur können mit "partitura" betitelt sein.

2. "Bartitura" kann aber auch für einfache Generalbaßstimmen stehen, ohne Unterschied, ob diese mit Taktstrichen versehen sind, oder ob sie beziffert sind oder nicht 68 .

Der Begriff "Partitur" selbst leitet sich von den in jeder Partitur unerläBlichen Taktstrichen $a^{69}$. Wahrscheinlich gab die Tätigkeit des Abteilens

men ( $\mathrm{BaB}$ und Sopran) notiert. Es handelt sich um eine konzertierende stimme und nicht um einen Basso continuo (eine Continuo-Stimme ist zusätzlich notiert).

64. Vgl. hierzu Schneider, Basso continuo, S.17 sowie Kinkeldey, Orgel, S. $190 \mathrm{ff}$.

65. Schneider, Basso continuo, S.17.

66. Vgl. Kinkeldey, Orgel, S.191 f.

67. Die Grenzen zwischen Partitur und Tabulatur sind unscharf. Auch die italienische Klaviertabulatur ist keine Griffschrift im engeren Sinn und die deutsche Orgeltabulatur in Buchstabennotation wurde auch zur partiturartigen Aufzeichnung von Vokal- und Orchesterstimmen (zur Direktion?) benutzt (vgl. als Beispiel die Abbildung einer in Tabulatur notierten Kantatenpartitur von Balthasar Erben bei Hans Engel, Art. Danzig in MGG, Bd.2, Sp. 1907). Schneider, Basso continuo, S.17, erwähnt Orgeltabulaturen des 16.Jahrhunderts, die ohne Rücksicht auf Spielbarkeit angelegt worden sind, und daher ebenfalls nur Partiturfunktion haben können.

68. Vgl. Arnold, Accompaniment, S.6. 
des Notenblattes in "Taktfächer" der Partitur urspruinglich ihren Namen: "partire in caselle"70.

Die Tatsache, daß auch Generalbaßstimmen mit "partitura" bezeichnet wurden, läßt sich hiermit jedoch nicht erklären. Die These von Frank T. Arnold, "partitura" bedeute eigentlich "Stimme mit Taktstrichen" und sei im Laufe der Zeit zu einem Synonym für "Generalbaßstimme" geworden, da diese immer mit Taktstrichen versehen gewesen seien ${ }^{11}$, kann nicht befriedigen, da im frühen 17.Jahrhundert nur ein kleiner Teil der Generalbaßstimmen mit Taktstrichen ausgestattet war $^{72}$.

Daß auch Generalbaßstimmen als Partituren bezeichnet wurden, lä日t sich eher damit erk1ären, daß in diesen Generalbaßstimmen ein vollwertiger Ersatz der Partitur (und dieser Begriff hatte schon bald seine spätere Bedeutung, losgelöst von den Taktstrichen), gesehen wurde: "vn sicuro modello rapresentante la partitura di tutto il Concerto"73. In ähnlicher Weise 1 äßt sich auch die Verwendung des Wortes "partitura" bei Viadana deuten. Viadana bedient sich dieses Begriffes insgesamt dreimal in seinem beruhmten Vorwort zu den Cento concerti ecclesiastici (1602) 74 . Lediglich einmal ist eine ubersetzung des Wortes "partitura" mit "Generalbaßstimme" möglich75. Aus den anderen Stellen ergibt sich jedoch zweifelsfrei, daß Viadana den vom Organisten zu spielenden Satz, und nicht nur den Baß meint ${ }^{76}$.

69. Zu den sogenannten "pseudo Partituren" ohne Taktstriche vg1. Lowinsky, Early Scores, S.164 f.

70. So erstmals Kinkeldey, Orgel, S.193 f. Dies spiegelt sich auch im Titel eines der ersten Partiturdrucke wieder: Musica de diversi autori ... partite in caselle per sonar d'instromento perfetto (1577).

71. Accompaniment, S.6 f.

72. Von den für diese Arbeit ausgewerteten Continuo-Stimmen aus der Zeit bis 1600 war keine mit Taktstrichen versehen, aus dem ersten Jahrzehnt des 17. Jahrhunderts etwa 27\%, aus dem zweiten Jahrzehnt etwa $35 \%$ und aus dem dritten schließlich 49\%. Dabei ist zu beachten, daß diese Prozentzahlen auch die Continuostimmen umfassen, die nur zum Teil mit Taktstrichen versehen sind. Im dritten Jahrzelunt des 17. Jahrhunderts waren Taktstriche natürlich bereits auch in anderen Stimmen einigermaßen verbreitet (vgl. weiter oben, Abschitt I.1.3. - dort auch zur Frage, warum iberhaupt Continuo-Stimmen besonders häufig mit Taktstrichen versehen sind).

73. A. Banchieri, 1'organo suonarino (2/1611), S.5 der Teilfaksimileausgabe.

74. In Punkt 2, 6 und 9. Dieses Vorwort ist an zah1reichen Stellen abgedruckt, z.B. in Sartoris Bibliografia $I$, S.112 f (1602 a).

75. Punkt 6. Eggebrecht, Generalbaß, S.73, ubersetzt so.

76. "Suonar semplicemente 1a Partitura, \& in particulare con la man di sotto" (Punkt zwei) belegt bereits, daß nicht nur der Baß gemeint ist - die Ergänzung "\& in particulare..." wäre dann schließlich uberfluissig. Bestätigt wird dies durch Punkt neun, in dem Viadana Quinten und Oktaven in der "partitura" erlaubt. 


\subsection{FORM UND ZWECK DER PARTITUREN}

\section{$2,2,1$, KOMPOSITIONSPARTITUREN}

Die Frage nach der Kompositionstechnik im 16.Jahrhundert hat das Interesse der Musikwissenschaft immer wieder auf die spärliche Partituruberlieferung dieses Jahrhunderts gelenkt77. Diesem Interesse verdanken wir heute die Kenntnis einer ganzen Reihe von Partituren, die bereits vor 1600 angelegt wurden 78 .

Die Zahl der erhaltenen Partituren, die tatsächlich von einem Kompositionsproze日 zeugen, ist gering - nach der Anfertigung einer Reinschrift gab es keinen Grund mehr, diese aufzubewahren. Bekannt ist aus dieser Zeit bisher nur ein Skizzenheft Constanzo Portas ${ }^{79}$. Solche Partituren lassen sich aufgrund ihres skizzenhaften Außeren leicht von anderen unterscheiden.

\subsubsection{ZWISCHENPARTITUREN BEIM VORGANG DES INTAVOLIERENS}

Um eine Intavolatur anzufertigen, benötigt man zunächst eine aus den Stimmbüchern herzustellende Partitur. Auch diese Partituren haben ein skizzenhaftes Aussehen. Da sie nach Anfertigung der Intavolatur nicht mehr benötigt wurden, haben sich, sofern sie nicht didaktischen Zwecken dienten 80 , ebenso wie auch Kompositionspartituren nur zufällig erhalten, so z.B. wenn die Rückseite der Partitur für eine Intavolatur (eines anderen Stückes) verwendet wurde ${ }^{81}$. Auch diese Partituren lassen sich leicht erkennen ${ }^{82}$.

\subsubsection{REPRÄSENTATIONS- UND STUDIENPARTITUREN}

Ein Teil der Partituren des frihen 17.Jahrhunderts ${ }^{83}$ erschien unabhängig von irgendwelchen musikpraktischen Absichten. Sie dienten zur Dokumentation

77. Literaturubersicht bei Lowinsky, Use of Scores, S.17 ff.

78. Vg1. insbesondere Lowinsky, Early Scores, sowie Gö1lner, Intabulation, S. 85 ff.

79. Bologna, Civico Museo Bibliografico-Musicale, MS B 140, Faksimiles bei Lowinsky, Early Scores, Abbildung 20-22.

80. Natïrlich beinhalten auch Intavolationsmethoden Partituren; vgl. z.B. M. Carrara, Inatvolatura di liuto (1585).

81. Vg1. Göllner, Intabulation, S.85 ff sowie die dort gebotenen Faksimiles.

82. Einen Spezialfall einer Zwischenpartitur stellt die Handschrift Ant.di Galileo 9 der Biblioteca Nazionale Centrale, Florenz, dar. Hier wurde die Anordnung der Partitur entsprechend der Lautentabulatur umgedreht ( $\mathrm{Ba} \beta$ oben, Sopran unten); vgl. hierzu Lowinsky, Early Scores, S.146 f sowie Abbildung 19 , sowie Göllner, Intabulation, S.84 f.

83. Schon bei jedem fünften der ausgewerteten Drucke aus dem ersten Jahrzehnt des 17. Jahrhunderts handelt es sich um eine Partitur bzw. um einen Druck mit einer Partitur. Bei den Drucken aus dem dritten Jahrzehnt des Jahrhunderts liegt dieser Anteil bereits bei rund $30 \%$ - dazu im einzelnen 
eines Festes bzw. einer Auffuihrung 84 oder hatten musiktheoretische Aufgaben ${ }^{85}$, z.B. in der Kompositionskritik86. Während eine Zuweisung in diese Gruppe bei einigen Partiturdrucken recht leicht fällt, ist bei manchen Partituren ein solcher Zweck nur wahrscheinlich oder möglich. Wahrscheinlich nur zu Studienzwecken erschien die Gesamtausgabe der sechs Madrigalbuicher Gesualdos87. Schon aufgrund des Umfanges ist diese Partitur als Klavierpartitur nur schlecht geeignet88. Möglich ist eine nicht-musikpraktische Intention auch bei anderen Partituren vokaler Musik sofern sie ohne Stimmensatz erschienen (bzw. erhalten sind) ${ }^{89}$ sowie bei Drucken instrumentaler Musik, die aufgrund ihrer Stimmenzahl nicht als Musik für Tasteninstrumente geeignet ist 90 . Diese Gruppe der Partiturdrucke umfabt - rechnet man die Zweifelsfälle mit hinzu, deutlich weniger als $10 \%$ aller Partiturdrucke des ersten Drittels des 17. Jahrhunderts 91.

weiter unten mehr.

84. Hierzu zählen Partituren von Opern und Intermedien.

85. Die Partituren in R. Michelis Musica vaga (1615) z.B. dienen nur dem Studium der auch in verschliusselter - einstimmiger - Form wiedergegebenen Kanons.

86. Dies können einzelne Partiturausschnitte in musiktheoretischen Schriften, z.B. in der berïhmten Streitschrift L'Artusi overo 1'imperfetioni della musica moderna (1600) von G. M. Artusi, fol. 39' f., oder aber auch umfangreiche Partiturdrucke sein, z.B. M. Effrems Censure (1623) mit einer fast vollständigen Partitur des sechsten Madrigalbuches von Marco da Gagliano oder Alcuni salmi et motetti ... posti in spartitura da Filippo Kasperle von Vincenzo de Grandis, gedruckt 1625 bei Vincenti (vgl. Fischer, Aspekte, S.23 ff).

87. Partitura delli sei libri de' madrigali a cinque voci (1613). Auf die Nitzlichkeit der Partitur zum Kontrapunktstudium wird auch in der - wohl in erster Linie zum Spiel auf Tasteninstrumenten gedachten - Partitur Tutti $i$ madrigali di Cipriano di Rore (1577) hingewiesen: "spartiti et accomodati per sonar d'ogni sorte d'Instrumento perfetto, \& per qualunque studioso di contrapunt $i^{\prime \prime}$.

88. Er umfaßt rund 500 Seiten in Folio-Format. Wollte man dennoch die Madrigale auf einem Tasteninstrument spielen, so konnten freilich einzelne Sätze abgeschrieben werden. Auch als Vorlage zum Anfertigen von Einzelstimmen ist eine solche Partitur freilich geeignet.

89. Zu nennen sind hier der Sammeldruck Partitura della nova metarmorfosi de diversi autori (1605), G. F. Biumi, Magnificat, (1612, zitiert nach RISM B 2761) und G. B. Strata, Arie di musica ... aggiuntovi nel fine le letanie... a due chori seperati ... per concertare con voci, e stromenti (1610, zitiert nach RISM S 6929). Es ist freilich nicht auszuschließen, daß Stimmbücher hierzu erhältlich waren und heute verloren sind (vgl. weiter unten).

90. F. Rovigio und R. Trofeo, Partitura delle canzoni da suonare (1613 oder später) und G. F. Biumi, Partito delle canzoni alla francese (1627). Zu beiden Drucken sind keine Stimmen erhalten; bei beiden Drucken verbieten achtstimmige Canzonen die Ausfürung auf einem Tasteninstrument. 


\section{$2,2,4$, PARTITUREN MONODISCHER MUSIK}

Monodische Musik und später auch andere geringstimmige Musik 92 erschien fast ausnahmslos in Partitur. Dieser Publikationsform liegt eine lange, sowohl notationstechnische als auch auffuhrungspraktische Tradition zugrunde. Die Vorläufer dieser Partituren sind die Lautentabulaturen mit einer Singstimme in mensuraler Notation. Auch hier waren Singstimme und Tabulatur in Partituranordnung ibereinander notiert, meist mit Takt- oder Orientierungsstrichen sowoh1 in der Tabulatur als auch in der Singstimme93. Der Grund für diese Anordnung war bei den Lautenbuichern und bei den Partituren monodischer Musik derselbe: Ein einzelner Musiker sollte in die Lage versetzt werden, sich selbst mit der Laute bźw. mit dem Chitarrone zu begleiten. Bei monodischer Musik waren Partituren darüber hinaus auch dann uiblich, wenn nicht von einer Personalunion von Solist und Begleiter ausgegangen werden konnte, wie z.B. in der Kirchenmusik und in der Instrumentalmusik. Hier erschienen die Partituren nicht allein, sondern als Part für den Begleiter zusätzlich zur Einzelstimme für den Solisten. Nur eine Partitur gab die Möglichkeit so frei zu musizieren, wie es in dieser Musik verlangt wurde. Auf diese zusätzlichen Begleitpartituren wird weiter unten noch ausführlich eingegangen werden. Die monodischen Partituren (also die Partituren ohne zusätzliche Einzelstimme) und die Begleitpartituren machen den größten Teil aller gedruckten Partituren aus den ersten dreißig Jahren des 17.Jahrhunderts aus. uber $80 \%$ aller ausgewerteten Partiturdrucke gehören in eine dieser beiden Gruppen.

Eine Sonderform der monodischen Partitur ist Bestandteil einiger Drucke Alessandro Grandis 94 sowie eines Druckes von Bartolomeo Barbarino 95 . Es handelt sich hierbei um Musik für ein bis drei Singstimmen und Bc., z.T. mit zusätzlichen Instrumentalsinfonien. Die Stimmbicher der Singstimmen entha1ten jeweils eine Singstimme sowie den vollständigen Basso continuo in Partitur; den Drucken Grandis ist darüber hinaus eine zusätzliche Orgelstimue (teils nur Basso continuo, teils auch mit einer Randstimmenpartitur) beigegeben. Der Grund für diese Veröffentlichungsform ist dem Titel der Madrigali von Bartolomeo Barbarino zu entnehmen: "Con il Basso continuo a ciasuna parte per poter sonare uolendosi piu di un chittarone". Wahrscheinlich ist

91. Kaum möglich ist es, den ursprünglichen Verwendungszweck bei einigen Partiturhandschriften aus dieser Zeit zu bestimmen (vgl. die Liste bei Lowinsky, Early Scores, S.172f). Die dort genannten Partituren mit vokaler Musik können sowohl zum Studium als auch zur Direktion und Begleitung verwendet worden sein (eine Verwendung als Orgeltabulatur zum reinem orge1vortrag ist aufgrund der großen Stimmenzahl oft nicht denkbar; vgl. dazu auch weiter unten).

92. In der Regel mit bis zu drei Stimmen.

93. Vg1. oben in den Abschnitten I.1.3. und II.1.1.

94. Madrigali concertati $(1615,2 / 1616)$, Motetti a 1 , et 2 (1621) und Motetti a 1,2 et 4 ... libro secondo $(2 / 1625,1$. Auf1. nicht nachweisbar).

95. Madrigali a 3 (1617); vgl. hierzu Wolf, Barbarino, S.42, Anm.41. 
daran gedacht, daß jeder der Sänger auch einen Chitarrone spielt. Eine ähn1iche Begründung der Veröffentlichungsform geben zwei der Titel Grandis 96.

\section{$2,2,5$, KLAVIERPARTITUREN}

Die ersten gedruckten Partituren im eigentlichen Sinn 97 waren Partituren zum Spiel auf Tasteninstrumenten. Die Titel der beiden bei Gardano 1577 erschienenen Partituren fuihren dies aus: Musica de diversi autori, 1a battaglia francese et canzon delli ucelli, insieme alcune canzoni francese, partite in caselle per sonar d'instromento perfetto bzw. Tutti $i$ madrigali di Cipriano di Rore a quattro voci, spartiti et accomodati per sonar d'ogni sorte d'instrumento perfetto \& per qualunque studioso di contrapunti98. Daß es sich bei beiden Partituren um eigentlich vokale Musik handelt, verwundert kaum, wenn man bedenkt, daß eigene instrumentale Formen gerade erst im Entstehen waren. Weitere Klavierpartiturdrucke aus dieser Zeit sind der libro primo di ricerare a quattro voci con alcune fantasie von Roco Rodio, erschienen 1575 bei Cacchio in Neapel (der älteste bekannte Partiturdruck iberhaupt) 99 und die Versi spirituali sopra tutte le note, con diversi canoni spartiti per sonar ne gli organi von Antonio Valente, erschienen 1580 bei Cancer ebenfalls in Neape1100. Eine Anzahl handschriftlicher Orgelpartituren stanmt wahrscheinlich ebenfalls noch aus dem 16.Jahrhundert101.

Im fribhen 17. Jahrhundert wurde Musik fuir Tasteninstrumente bereits recht häufig in Partitur gedruckt102. Eine eindeutige Abhängigheit der uberlieferungsform von der Art der Klaviermusik (polyphone Gattungen, wie Ricercar $\rightarrow$ Partitur, nicht-polyphone Gattungen, wie Toccata $\rightarrow$ Tabulatur) läßt sich noch nicht ausmachen, wenngleich auch um 1600 bereits die polyphonen Gattun-

96. Bei den beiden Motettenbüchern steht im Titel "partiti per cantar, \& sonar $\mathrm{co}^{\prime} \mathrm{l}$ chitarrone".

97. Im Gegensatz zur bloßen Partituranordnung etwa einer Tabulatur und einer Singstimme.

98. Zitiert nach Einstein, Bibliography 2, S.374. Bei dem Rore-Druck handelt es sich - dem Titel nach - bereits um eine zweite Auflage. Eine frühere Auflage ist nicht bekannt.

99. Vgl. Apel, Klaviermusik, S.119.

100. Apel, S.119 sowie Oscar Mischiati, Art. Valente, Antonio in MGG, Bd.13, Sp. 1224 f.

101. Vgl. Lowinsky, Early Scores, ubersicht S.172, sowie die Seiten $134 \mathrm{ff}$ und John Caldwel1, Art. Sources of keyboard music to 1660, NG, Bd.17, S.719 f. Als Orgelpartituren kann auch ein Teil der bei Warwick Edwards, Art. Sources of instrumental ensemble music to 1630, in NG, Bd.17, S.704 ff, genannten Partituren bezeichnet werden. Unklar ist die Trennung zwischen Begleitpartituren und Orge1- bzw. Klavierpartitur auch bei Haller, Partituranordnung, S.92 ff.

102. Dem Verfasser sind aus den Jahren 1601-1630 insgesamt 18 eindeutig zum Spiel auf Tasteninstrumenten bestimmte Partituren italienischer Drucker bekannt. 
gen in den Partituren iberwiegen 103. Es ist denkbar, daß die Partituren als eine normierte uberlieferungsform von Musik für Tasteninstrumente angesehen wurden. Die Musiker konnten diese bei Bedarf in das ihnen geläufige Tabulatursystem ibertragen 104 .

Der Bedeutungsverlust der Mittelstimmen führte in der Klaviermusik zu einer Sonderform der Klavierpartitur, nämlich einer verkürzten Partiturnotation in nur zwei Systemen. Die Mittelstimmen waren hierbei von dem Ausführenden selbständig zu ergänzen. Vorreiter dieser Randstimmennotation - sowohl für reine Klaviermusik als auch als Begleitstimme - war Adriano Banchieri. Die friheste bekannte Randstimmenpartitur ist die Spartitura per sonar nel organo accommodata al primo choro nei concerti di D.Adriano Banchieri (1595). In der Musik für Tasteninstrumente begegnet uns diese Notationsform zuerst in Banchieris L'organo suonarino (1605). Auf den Seiten 106-109 befinden sich vier kurze Capricci in Randstimmennotation. Auf Seite 105 unten steht hierzu "Gli sequenti Capricci seruono per suonare doppó il Magnificat, spartito sopra due parti con gli riempimenti a beneplacito de gl'Organisti" - die Ausfüllung ist also dem Organisten anheimgestellt10s. Die Randstimmenpartitur folgt hierbei dem Seguente-Prinzip: Das untere System enthält die jeweils tiefste, das obere die jeweils höchste Stimme des Satzes106. Die Notation von Klavierwerken in Randstimmenpartitur hat auBer bei Banchieri in den Klaviertanzbüchern Verbreitung gefunden ${ }^{107}$.

103. Deutlich ausgeprägt ist die Zuordnung der einzelnen Stile zu den Veröffentlichungsformen jedoch schon bei Frescobaldi, dessen Ricercare, Capriccen und Canzonen in Partitur, die Toccaten aber in Tabulatur erschienen. Es gibt jedoch aus dieser Zeit auch in Partitur gedruckte Toccaten, z.B. in den beiden Büchern G. M. Trabacis von 1603 und 1615 .

104. A. Banchieri weist im L'organo suonarino (1605) darauf hin, daß man aus der Partitur nicht nur spielen kann, sondern daß diese auch einfaches Intavolieren ermöglicht: "quali Sonate sono commodo per sonare in spartitura; \& ancora facili alle mani per intauolarsi." (S.21). Ahnliches steht auch auf S.58; dort mit einem Hinweis auf die Anleitung zum Intavolieren in Dirutas Tansilvano.

105. Dieses ist ebenfalls in dem Vorwort zu Banchieris Moderna armonica (1612, ebenfalls weitgehend eine Randstimmenpartitur) ausgefuihrt: "Prima, queste possonsi suonare semplicemente nell'Organo, doue con ordine musicale entrano tutte le parti con gli riempimenti a giusto orecchio \& giuditio de11'Organista", zitiert nach Sartori, Bibliografia I, S.184 (1612 a).

106. Pausiert also die Sopranstimme, ist im oberen System eine Altstimme notiert etc.

107. Zu nennen sind vor allem das libro primo $(2 / 1631$, 1 . Auf 1 . nicht nachweisbar) und libro secondo delle correnti francese per sonar nel clavicembalo (1630) von M. Pesenti. Zwei ganz ähnliche Correnten in Randstimmenaufzeichnung befinden sich in E. Radesca di Foggias secondo libro delle canzonette $(2 / 1616,1$. Auf1. nicht nachweisbar); eine von Radesca selbst sowie eine von G. B. Muti. Es handelt sich bei diesen Stücken - wie auch bei den meisten anderen gedruckten Tänzen des 16. und frühen 17.Jahrhunderts - 


\section{$2,2,6$, BEGLEITPARTITUREN}

Ein beträchtlicher Teil der Partituren des späten 16. und frühen 17.Jahrhunderts erschienen als Teil eines Stimmensatzes. Diese Partituren sind entweder ein Teil der Continuo-Stimme (einzelne Sätze sind in der Continuo-Stimme in Partitur notiert) oder aber sie ersetzen die Basso continuo-Stimme insgesamt. Diese Begleitpartituren sind sowohl den Partituren monodischer Musik als auch den Klavierpartituren verwandt: auch die Partituren monodischer Musik dienen der Begleitung und auch nach Begleitpartituren wird in der Regel am Tasteninstrument gespielt. Im Gegensatz zu diesen beiden anderen Partiturarten erschienen sie aber zusätzlich zu Einzelstimmem. Nur bei diesen Partituren ist mit Sicherheit davon auszugehen, daß diese nicht zum reinen Klavierspiel bestimmt sind, und im Gegensatz zu den Partituren monodischer Musik ist die Partiturüberlieferung hier nicht auf die Personalunion von Sänger und Begleiter zurïckzuführen. Die Veranlassung, eine Partitur drucken zu lassen - und dies ist ja erheblich aufwendiger als der Druck einer Continuo-Stimme -, gab die Unzufriedenheit einiger Komponisten mit den Möglichkeiten einer Continuo-Stimme.

Das Erkennen dieser Begleitpartituren wird dadurch erschwert, daß nicht immer auch die Einzelstimmen erhalten geblieben sind. In einzelnen Fällen ermöglichen es aber Hinweise innerhalb der Partituren dennoch ihre Bestimmung zur Begleitung zu belegen 108 .

Die Frihgeschichte der Begleitpartituren liegt ebenso im dunkeln, wie die der Continuo-Stimmen. Beide treten etwa gleichzeitig im letzten Jahrzehnt des 16. Jahrhunderts in den italienischen Musikdrucken auf ${ }^{109}$; beide werden

wohl kaum um zu tarzende Musik, ja oft nicht einmal um tanzbare Musik. Die Tanzform diente hier nur als Kompositionsmodell.

108. So ist G. Guamis Partitura per sonare delle canzonette (1601) lange fur eine Klavierpartitur gehalten worden, da die Einzelstimmen nicht erhalten sind und erst spät ein Antwerpener Nachdruck der Stimmen gefunden wurde (vg1. Denis Arnold, Art. Guami, Gioseffo in MGG, Bd.5, Sp. 999 f). Von den sechs Biichern mit Messen P. Agostinis haben sich nur vom zweiten Buch zwei unvollständige Stimmensätze erhalten, von allen nur eine Partitur z.T. mit zusätzlicher Continuo-Stimme. Die Bestimmung der Partituren zur Begleitung ist aber durch ein Vorwort verbürgt (s.u.). In G. Arnones Partitura del secondo libro delli motetti (1599) bezeugt die Angabe der Stimmbuchseitenzahlen in der Partitur, daß auch diese Partitur urspringlich Bestandteil eines Stimmendruckes war.

109. 1594 erschien mit der Baßpartitur zu G. Croces Motetti a 8 die friheste erhaltene gedruckte Continuo-Stimme. Die ersten einzelnen ContinuoStimuen erschienen spätestens 1598 zu Werken Orfeo Vecchis (vgl. hierzu die Liste der gedruckten Continuo-Stimmen aus den Jahren bis 1605 in zweiten Band dieser Arbeit). Die fruheste bekannte Begleitteilpartitur ist die "Spartitura" zu Banchieris Concerti ecclesiastici von 1595; die erste Begleitvollpartitur erschien 1598 zu Josephus Gallus' Sacri operis ... 1iber 
jedoch bereits vorher bekannt gewesen sein110. Die Begleitpartituren gelten als die ä1tere Form. Es wird davon ausgegangen, daß die Generalbaßstimmen entstanden, weil das Anlegen von Partituren vielstimmiger Stucke zu aufwendig war111. Die Entwicklung beider Formen der Begleitstimme soll hier anhand der gedruckten Quellen, d.h. ab 1594 weiter verfolgt werden.

Zunächst muß die begriffliche Abgrenzung zwischen Begleitpartitur und Continuo-Stimme geklärt werden. Adriano Banchieri bezeichnet in seiner oben zitierten Unterteilung der verschiedenen Arten der Begleitung nur die Vol1partituren als Partituren und sieht in der Randstimmenpartitur eine Art der Basso Continuo-Stimme112; Imogene Horsley hingegen faßt jede Form partiturartiger Stimmenanordnung unter den Begriff "Partitur" zusammen, also auch Partituren der beiden Bässe bei doppelchöriger Musik113. Im folgenden soll die sich aus der jeweiligen überlieferungsform ergebende Methode der Begleitung den Ausschlag für die Zuordnung zu Partitur oder Continuo-Stimme geben. Die Baßpartituren werden hierbei den Continuo-Stimmen zugerechnet, da die Tatsache, daß die Bässe in Partitur angeordnet sind, zwar die Möglichkeit des Begleitens mit einer oder zwei Orgeln bzw. anderen Continuoinstrumenten eröffnet, das Begleiten im einzelnen aber ebenso geschieht wie nach einer Continuo- bzw. Seguente-Stimme. Eine Randstimmen- oder eine andere Art der Teilpartitur hingegen ersetzt zumindest zum Teil die Bezifferung und ermöglicht es daruber hinaus, den Stimnenverlauf mitzuverfolgen und dabei z.B. auf Temposchwankungen zu reagieren. Selbst ein Mitspielen der in der Partitur wiedergegebenen Stimmen ist möglich. Die Teilpartituren werden daher den Partituren zugerechnet.

Da die Besonderheiten einze1ner Partiturformen, aber auch einzelner Partiturdrucke eng mit der Frage verknüptt sind, wie aus der jeweiligen Partitur begleitet werden soll bzw. begleitet werden karm, soll in dem sich anschließenden Kapitel anhand der verschiedenen Quellen den unterschiedlichen Möglichkeiten der Begleitung nach einer Partitur nachgegangen werden.

primus (vgl. die Liste der Begleitpartituren im zweiten Band dieser Arbeit). 110. Die älteste bekannte Generalbaßstimme ist der auf 1587 datierte "Bassone" zu A. Striggios "Ecce beatam lucem" (vg1. Schmeider, Basso contimo, S.66 ff). Wahrscheinlich handelt es sich hierbei um eine Abschrift des Aufführungsmateriales. für die belegte Auffuhrung dieses Werkes in Jahr 1568 (vg1. dazu weiter unten). Auf die Schwierigkeit, den Zweck erhaltener Partiturhandschriften aus dem 16. Jahrhundert zu bestimmen, wurde oben bereits hingewiesen.

111. So Chrysander, Begleitung, Sp.81, Kinkeldey, Orgel, S.195 ff, Schneider, Basso continuo, S.14 ff sowie S.66 ff, Blume, Generalbaßpraxis, S.77, Fritz Oberdörffer, Art. Gerneralbaß in MGG, Bd.4, Sp.1713 f, Eggebrecht, Generalbaß, S.66 und Kirchner, Generalbaß. S.14.

112. Vgl. den Abdruck dieser Stelle im zweiten Band dieser Arbeit auf $\mathrm{S} .18 \mathrm{f}$.

113. Scores. 


\subsection{DiE Begleitung anHAND EINER BEgLeItPARTItUR}

Die Tatsache, daß anstelle von Basso continuo-Stimmen auch Partituren erschienen, hat der Forschung bereits Anlaß zu verschiedenen uberlegungen gegeben. Diese konzentrierten sich vor allem auf die Frage, ob der Organist init Hilfe der Partitur den zu begleitenden Satz notengetreu mitspielen sollte, oder ob es die Aufgabe der Partitur war, ihm nur als Anhaltspunkt für seine Begleitung zu dienen und eine Direktion der Aufführung zu ermöglichen.

Als Hauptargument fiur das Mitspielen nach der Partitur wird auf die Entstehung des Generalbasses verwiesen: Sowohl die Tatsache, daß ein direktes Mitspielen eines vielstimmigen Stückes kaum möglich ist, als auch der große Arbeitsaufwand des Spartierens bzw. Intavolierens führten nach Meinung zah1reicher Autoren zur "Erfindung" des Generalbasses114; eine These die zwar nicht im einzelnen belegbar, aber dennoch wahrscheinlich ist. Wurde nun einem Musikdruck eine Partitur anstelle einer Continuo-Stimme beigegeben, so zeige dies, daß auch hier ein Mitspielen erwinscht sei115. Dies fände eine Bestätigung in einigen Drucken mit doppelchöriger Musik, bei denen alle doppelchörigen Sätze in der Continuo-Stimme als Basso seguente oder Baßpartitur, einchörige Abschnitte hingegen in Vollpartitur wiedergegeben sind116.

Während sich die verschiedenen Autoren daruber einig sind, daß vier- oder fïnfstimige Sätze aus Vollpartituren notengetreu mitzuspielen sind117, gibt es erhebliche Meinungsverschiedenheiten iber die Begleitung der solistischen Musik sowie iber die Begleitung nach Randstimmen- bzw. Teilpartituren: Dienen die Partituren auch hier zum Mitspielen, oder soll durch diese Partituren nur die Orientierung erleichtert werden?

Diese Meinungsverschiedenheit geht auf die Quellen selbst zurück. Auch im späten 16. und fruhen 17. Jahrhundert gab es hieruber keine einhellige Meinung. Dem heutigen Forscher wird daher im besten Fall möglich sein, die Intention eines Komponisten (bzw. in einigen Fällen eines Herausgebers) aufzuspiiren. Auch für die Entstehungszeit der Musik wird man jedoch die Möglichkeit ganz unterschiedlicher Arten der Begleitung nach ein und derselben Begleitstimme annehmen missen. Die unterschiedlichen Auffassungen zur Partitur allein in Norditalien (s.u.) zeigen, wie uneinheitlich die Musikpraxis

114. So Chrysander, Begleitung, Sp.81, Kinkeldey, Orge1, S.195 ff, Schneider, Basso continuo, S.14 ff sowie S. 66 ff, Blume, Generalbaßpraxis, S.77, Fritz Oberdörffer, Art. Gerneralbaß in MGG, Bd.4, Sp.1713 f, Eggebrecht, Generalbaß, S.66 und Kirchner, Generalbaß, S.14.

115. So insbesondere Horsley, Scores, S.466.

116. Auf diese Tatsache verweisen insbesondere Kinkeldey, S.203, Schneider, S. 66 ff sowie Haack, Generalbaß, S.198.

117. Lediglich Peter Williams, Art Continuo in NG, Bd.4, S.685, geht davon aus, daß auch nach Partituren immer in der Art eines Basso continuo gespielt worden sei. Verdoppelnde Begleitung schließt Williams aus. 
in dieser Zeit war; die schnelle Entwicklung zu einer Vereinheitlichung (s.u.) hingegen, wie stark auch der Austausch zwischen den Städten und Regionen florierte. Insgesamt vier verschiedene Aufgaben der Partituren beim Begleiten - und zwar für Teil-wie für Vollpartituren - lassen sich erkennen:

1. Nach den Partituren sollen die Stimmen notengetreu mitgespie1t werden. Hier dient die Partitur als Intavolatur, das heißt als eine ubertragung des zu begleitenden Tonsatzes in eine den Organisten (Cembalisten etc.) geläufige Form, nämlich in Klavierpartitur.

2. Die Partitur soll den Begleiter genau über den Verlauf der zu begleitenden Stimmen und die sich ergebenden Harmonien informieren. Die Partitur ersetzt oder ergänzt hiermit eine Bezifferung.

3. Die Partitur ermöglicht es dem Begleiter, die anderen Stimmen mitzuverfolgen und damit auf die Temposchwankungen eines treien Vortrages zu reagieren.

4. Der Begleiter - oft zugleich auch Leiter der Aufführungen - kann in der Partitur die anderen Stimmen verfolgen, Einsätze und an schwierigen Stellen auch Hilfen geben. Er begleitet aber anhand der zusätzlich abgedruckten Continuo-Stimme. Die Partitur dient nur zur Direktion.

\section{$2,3,1$, PARTITUR ALS INTAVOLATUR}

\section{$2,3,1,1$, VOLLPARTITUREN}

Die eingangs zitierte Aufzählung der verschiedenen Arten der Begleitstimmen durch Adriano Banchieri118 zeigt bereits verschiedene Möglichkeiten der Begleitung nach einer Vollpartitur. Die Begleitung "alla spartitura" erfordert nach Banchieri u,a. die Kenntris aller Schluissel und ein gutes optisches Wahrnehmungsvermögen. Denen, deren Gesicht nicht so gut ist, empfiehlt er daher, sich nur nach der tiefsten Stimme zu richten, und in der Art eines Basso continuo zu begleiten. Diese Einschränkung macht deutlich, daß Banchieri sonst nicht vom Begleiten nach dem Baß ausging, sondern an eiı Mitspielen, sei es aller oder auch nur einiger stimmen, dachte.

Bis in die zwanziger Jahre des 17. Jahrhunderts hinein lassen sich Vorhehalte dem Basso continuo gegenüber verfolgen. Oft sind es Vorreden za Basso continuo-Stimmen, in denen zum Ausdruck gebracht wird, das man, um der allgemeinen Vorliebe zu entsprechen, zwar einen Continuopart in Druck gegeben habe, jedoch nachdrücklich rät, sich lieber eine Partitur oder gar Intavolatur anzufertigen ${ }^{19}$. Selbst die beiden beriihmten Generalbaßtraktate des

118. Vgl. den Abdruck dieser Stelle im zweiten Band dieser Arbeit auf $S$. $18 \mathrm{f}$.

119. Bereits L. Viadana weist 1602 im Vorwort zu den Cento concerti ecclesiastici darauf hin, daß eine Intavolatur einem Baßs vorzuziehen sei (vgl. das Vorwort bei Sartori, Bibliagrafia I, S.112 f (1602 a) unter Punkt 7).

Fünf Jahre später schreibt T. Massaino zur Randstimmenpartitur seines Sacrarum cantinonum ... liber primus (1607), daß er lieber eine Intavolatur in Druck gegeben hätte. Diese hätte doch den Umfang des Werkes allzusthr 
Jahres 1607120 räumen ein, daß für manche Musik eine Partitur unerläßlich sei121.

vergrößert ("Io haveva pensato di stampar seco l'Intavolatura per maggior commodità di semplici suonatori, \& Monache, ma ho mutato pensiero per non accrescere tanto il Volume, che peró hò posti appresso al Basso, una parte che sempre canti"). Auf ähnliche Weise raten auch G. Diruta (Seconda parte del Transilvano (1609), S.16, vgl. die Wiedergabe dieser Stelle im zweiten Band dieser Arbeit auf S.24), G. Picconi (Concerti ecclesiastici (1610): "... Finalmente sarà bene, che quelli Organisti, che non sono pratichi a sonar sopra il Basso seguito, e che non possendono l'Arte della Musica, volendo haver sodisfattione di questa sorte di conceri, $1 i$ spartino, e I'intavolino.", zitiert nach Kinkeldey, Orgel, S.225) und T. Merula (II primo libro delle Canzoni (1615), Vorwort zur Bc.-Stimme: "Benche per maggior facilità di tutti il Signori Organista vi sia posto il Basso continuo alle presente Canzoni, laudo nondimeno partirle.") zum Anfertigen einer Partitur. Noch 1620 bedauert die "Stampa del Gardano" (C. Zoilo, Madrigali a cinque voci), daß eine Intavolatur für den Druck zu aufwendig gewesen sei, und man sich daher mit einer Continuo-Stimme habe begnuigen muissen (abgedruckt bei Kinkeldey, Orgel, S.226, sowie Vogel, Bibliothek, Bd.II, S.357). G. D. Rognoni Taegio berichtet im Vorwort der Partitur zu seinen Canzoni (1605), daß er urspringlich nicht beabsichtigt habe, eine Partitur drucken zu lassen, da man gerade beim Spartieren so viel lernen könne. Auf Drängen seiner Freunde habe er sich dann doch entschlossen, eine Partitur in Druck zu geben, da sein Werk ja der Praxis dienen solle; und dafür sei eine Partitur viel besser als ein Basso continuo ("Alli virtuosi Organisti. Haueuo pensato di non dar alle stampe questo Partito, si perche alcuni non pensassero ch'io lo dassi fuori, accioche con questa commodità 1 'opera hauesse maggior ricapito; si perche anco gli studiosi di questa professione diuengono con questa commodità tepidi, oue nel partire si fanno prattici, et ne cauano molto frutto: Mà alcuni amici m'hanno detto che questa opera ordinariamente sarà suonata, et che vi fá bisogno del partito, onde per compiacerli l'ho dato fuori, conoscendo che in ogni caso meglio è il Partito, che il Basso continuato. Et se quest'opera mia sarà da voi gradita, mi darete animo d'affaticarmi ogn'hora più in case maggiori; et spero che la gradirete se non per bontà sua, almeno per bontà vostra. Viuete felici.").

120. A. Agazzari, Del sonare sopra'l basso con tutti li stromenti, und F. Bianciardi, Breve regola per imparar' a sonar il basso.

121. Während Bianciardi einräumt, daß es auch Stiucke gibt, nämlich ältere fugierte, die sich nicht gut zum Spielen über dem Baß eignen (S.53 der Edition), sagt Agazzari, daß die alten Gesänge, die sich nicht zum Spiel nach dem Baß eignen wegen "der Konfusion und der Suppe der Wörter, die von den langen und verschlungenen Fugen herrihrt" nicht mehr in Gebrauch seien (S.11: "Ma se alcuno mi dicesse, che à suonar l'opere antiche piene di fughe, e contrapunti, non è bastevuole il basso, à ciò rispondo, non esser in uso piu simil cantilene, per la confusione, e zuppa delle parole, che dalle fughe lunghe ed intrecriate nascano"). 
Die Bevorzugung einer Partitur allein bedeutet jedoch noch nicht, daß die Stimmen collaparte begleitet werden sollen. Auch andere Gründe können für eine Partitur sprechen (s.u.). Doch auch das Mitspielen selbst ist uber die bereits mehrfach zitierte Außerung Banchieris hinaus bezeugt. So finden sich Hinweise auf das Mitspielen der in der Partitur notierten Stimmen in der Partitur zu Simone Molinaros Concerti ecclesiastici (1605)122. Hier sind Pausen der Singstimmen durch andere, im Vokalsatz nicht vorhandene Stimmen ausgefuil1t123. Dies ist nur sinnvoll, wenn die notierten Stimmen auch gespielt werden 124 .

Ausfürliche Anweisungen zum Mitspielen nach der Partitur gibt Orazio Scaletta in dem Vorwort zu seiner ebenfalls 1605 erschienenen Partitura della certa spirituale125. Er fordert die Organisten ausdrücklich auf, die "partitura realmente come stà zu spielen. Auch bei Sätzen à due - sie sind zusätzlich mit Mittelstimmen zu begleiten - sollen die Randstimmen nach Möglichkeit unverändert mitgespielt werden ${ }^{126}$. Hier wird für die geringer besetzten Stücke eine Kombination aus Mitspielen und Vervollständigen eines Satzes verlangt, vergleichbar dem Spiel von Klaviermusik nach einer Randstimmenpartitur. Ebenfalls zum wörtlichen Mitspielen rät Giovanni Diruta in der Seconda parte del Transilvano (1609)127.

1610 erschien mit Giovanni Paolo Cimas Concerti ecclesiatici eine weitere wesentliche Quelle für das collaparte-Spiel. Diesen Druck ist eine Begleitpartitur beigegeben, die sowohl Vol1- als auch Teilpartituren beinhaltet128.

122. Die Partitur ist zweistimmig wie auch fast alle Stiicke dieses Druckes. Die Sätze à quattro sind in der Orgel-Stimme nur als Continuo notiert.

123. Ahnlich wie auch bei einigen in Randstimmenpartitur notierten Klavierwerken Banchieris handelt es sich an diesen Stellen um eine "Soprano seguente"-Stimme eines nicht real existierenden Satzes.

124. Vgl. das Notenbeispiel II, 8 im zweiten Band dieser Arbeit.

125. Dieses Vorwort ist im zweiten Band dieser Arbeit auf S.15 abgedruckt. Außer den hier zur Sprache kommenden Anweisungen enthält dieses Vorwort noch eine Reihe detaillierter Regeln zum Spiel geringstimniger Musik nach einer Begleitpartitur.

126. Dies ist mit den Worten "facendo che le parti di mezzo anchor loro accompagnino le due parte estreme più regolatamente sia possibile" gemeint.

127. Vgl. diesen Abschnitt aus Dirutas Transilvano im zweiten Band dieser Arbeit auf S.24.

128. Mit Ausnahme zweier Echo-Stücke (s.u.) sind alle Sätze d̀ uno, à due, da tre und à cinque in Vollpartitur notiert, wobei nur die Sätze ohne gesungenen Baß eine selbständige Continuo-Stimme haben. Die Sätze à quattro sind teils in Vollpartitur und teils in Randstimmenpartitur in der Art eines Basso-Seguente mit Soprano-Seguente wiedergegeben. Bei den in Randstimmenpartitur notierten Sätzen handelt es sich um Concertato-Sätze mit vielen solistischen Passagen. Es erscheint eher fraglich, ob hier wirklich eine Differenzierung in "prima prattica" (Vollpartitur) und "secondo prattica" (Randstimmenpartitur) vorliegt (so Horsley, Scores, S.477). Vielmehr wird 
Ein Nachwort zu dieser Partitur ${ }^{129}$ enthält folgende Feststellungen zur $\mathrm{Be}-$ gleitung:

1. Die Stuicke nur mit Baß und Sopran (gemeint sind sicher sowohl die Sticke "per canto e basso" als auch die nur in Randstimmenpartitur notierten vierstimmigen Sätze) soll der Organist mit Mittelstimmen begleiten ("accompagnarli con le parti di mezzo"). Diese Begleitung soll schlicht sein.

2. Obwohl in der Partitur an vielen Stellen die Stimmen in ihrer ausgezierten Form stehen, soll der Organist in der Regel eine einfache, dekolorierte Fassung spielen. Nur um dem Sänger zu helfen, kann er hin und wieder auch die Verzierung mitspielen ${ }^{130}$.

Unter "accompagnare" versteht Cima offenbar nicht die Tätigkeit des Begleitens selbst, sondern lediglich das Hinzufügen neuer Stimmen. Dies ist nur gefordert bei den in der Partitur zweistimmig notierten Sätzen. Die in der Partitur in allen Stimmen notierten Werke sind collaparte - allerdings in vereinfachter Form - mitzuspielen. Cima gibt keine Anweisungen tiber das Mitspielen der notierten Sopranstimme der in Randstimmenpartitur notierten Sätze. Dies kann jedoch angenommen werden und findet seine Bestätigung auch im Notentext dieser Stiucke.

Bereits in der Nummer 1, einem Stuick für Solosopran und Orge1, ist die Sopranstimme in der Partitur an vielen Stellen in vereinfachter Form notiert. Diese Vereinfachungen sind nur sinnvo11, wenn diese Stimme nach der Partitur mitzuspielen ist, denn das Mitspielen soll ja in vereinfachter Form erfolgen. Noch deutlicher ist an der Nummer $11^{131} \mathrm{zu}$ erkennen, das die in der Partitur notierte Supranstimme zu verdoppeln ist. Obwohl dieses Stück fur "doi Soprani in Ecco" geschrieben ist, handelt es sich dabei eigentlich um ein Solostiuck; die beiden Sopranstimmen singen nur in den letzten Takten gemeinsam. Der erste Sopran steht, in vereinfachter Form, vollständig in der Partitur, statt des Echo-Sopranes sind jedoch immer Pausen notiert. Es wäre denkbar, daß diese Tatsache auf eine erwünschte Tasto solo-Begleitung hindeuten soll. Dagegen spricht jedoch ein Kreuz uber dem Baß in Takt 34. Der Echo-Effekt soll hier durch die fehlende Verdoppelung der Singstimme wahrscheinlich ergänzt durch ein geringstimmigere Begleitung - verstärkt werden 132 .

die Möglichkeit, die Concertato-Sätze in Randstimmenpartitur mit erheblich geringerem Aufwand fast vollständig wiedergeben zu können hier den Ausschlag gegeben haben (vgl. das Notenbeispiel II,7 im zweiten Band dieser Arbeit).

129. Dieses Nachwort ist im zweiten Band dieser Arbeit auf S.12 abgedruckt. 130. Diese schlichte, verzierungslose Fassung bezeichnet Cima als "fermo". Es kann hier kein "canto fermo" gemeint sein (Haller, Partituranordnung, S.76, ging davon aus), da dieser Druck keine echten c.f.-Sätze enthält. Canti fermi werden hier allenfalls in abgewandelter Gestalt und jeweils nur einen kurzen Abschnitt lang zitiert (z.B. in den Magnificat).

131. Dieser Satz ist in Uhertragung im zweiten Band dieser Arbeit als Notenbeispiel II, 9 wiedergegeben. 
Es muß also davon ausgegangen werden, daß fïr Cima verdoppelnde Begleitung auch bei solistiscken Stucken die Regel war.133. Die dabei auftretenden Reibungen zwischen der undiminuierten Orgeloberstimme und der ausgezierteren Solostimme sprechen nicht gegen diese Art der Begleitung. Zum einen sind diese Dissonanzen regelgerecht durchgefihrt und zum anderen können solche Dissonanzen auch an anderer, sicher verbiirgter Stelle beobachtet werden ${ }^{1.4}$.

Die lange währende Tradition, geringstimmige Sätze in Messkompositionen meist Crucifixus, gelegentlich auch Benedictus - in der Bc.-Stimme in Vollpartitur zu drucken, während die anderen Sätze als Continuo-Stimme oder Baßpartitur wiedergegeben sind ${ }^{135}$, geht sicherlich ebenfalls auf das colla-

132. Geht man von einer versteckten Aufstellung des Echo-Sopranes aus (vg1. hierzu weiter unten im Abschnitt III.1.3.1.), wäre eine collaparte Verdoppelung der Echo-Stimme nicht nur unsirmig (sie soll ja nur aus der Ferne kommen), sondern auch mangels Sichtkontakt sehr schwierig. Die These der verdoppelnden Begleitung wird darüber hinaus durch die Abweichung von Sopranstimme und Orgeloberstimme in Takt 12 bestätigt.

133. Zu diesem Schluß konmt - bezogen auf die Irstrumentalwerke - auch Ape1, Violinmusik, S.15 f. Apel fuhrt als Beleg die Tatsache an, daß Pausen in der Solostimme im "Cappriccio" von Andrea Cima in der Partitur ausgefiillt sind (im Gegensatz zu den Ausfuillungen bei Simone Molinaro (s.a.) stehen diese Ausfüllungen in einem anderen Schluissel als die Solostimme, sind also gut zu erkennen). Gänzlich verfehlt ist sicher Klaus Winklers Deutung der Partitur Cimas, hier in Bezug auf die "Sonata per il Cornetta \& Trombone, ouero Violino ò Violone" (Instrumentalwerke, S.66). Winkler weist auf die uberschriften der Sonata im Canto- und Basso-Stimmbuch hin: Dort wird jeweils nur das in dieser Stimme stehende Instrument genarnt, also "Sonata per Cornetto, over Violino" bzw. "Sonata per Trombone over Violone". Winkler schließt daraus, die Sonata könne auch nur mit zink bzw. Violine und orgel oder mit Posaune bzw. Violone und Orgel gespielt werden könmen. Die fehlende Stimme würde dann von der Orgel übernommen; dort sei aus diesem Grund eine Partitur notiert. Ein Hinweis auf eine solche Ausfihrungsmöglichkeit hätte jedoch in der Orgelstimme und nicht in den Solostimmen stehen muissen, Daruber hinaus ist es nicht möglich, die Partituruberlieferung eines einzelnen Stückes aus einem umfangreichen Partiturdruck herausgelöst zu erklären. Die Erklärung mißste auch auf alle anderen Stiicke anwendbar sein. Dafür aber fehlt nicht nur jeder Hinweis, sondern dem stehen dje Ausführungen in Cimas Vorwort direkt entgegen.

134. Z.B. in den Madrigali von L. Luzzasco (1601, vgl, das Notenbeispiel II, 5 im zweiten Band dieser Arbeit).

135. Dies ist erstmals zu beobachten in der 1598 erschienenen Partitur zu J. Gallus' Sacri operis ... liber primus. Es folgen: L. Quintiani, Missa tres (1598, vgl. Horsley, Scores, S.469), G. G. Gastoldi, Messe et motetti (1607), C. Monteverdi Sanctissimae Virgini Missa (1610), A. Bianchi Motetti, e messe (1611, vgl. hierzu unten) sowie G. Valentini, Missae quatuor (1621). Eine Randstimmenpartitur für vierstimmige Sätze gibt die Baßpartitur zu C. Gussagos Psalmi ad vesperas (1610) sowie, in einem Fall, die Continuo-Stimme 
parte-Spiel solcher Sätze zurïck136. Eine ausdrückliche Aufforderung zum Mitspielen befindet sich in der Orgelstimme des Crucifixus der "Missa sexti toni" aus Andrea Bianchis Motetti, e messe a otto voci (1611). Nur das Crucifixus dieser Messe ist teils in Baßpartitur, teils in Vollpartitur ${ }^{137}$ notiert (sonst findet sich in diesem Druck nur eine Continuo-Stimme). Diese Partitur ist mit den Worten "Crucifixus a 2 si suona le Due Parti" bezeichnet - der Organist soll also die beiden notierten Stimmen spielen. Abgesehen von der füfstimmig vertonten Textstelle "passus \& sepultus est" bedeutet dies ein collaparte-Spiel des zweistimmigen Satzes. Die fünfstimmige (und doppelchörige) Stelle "passus \& sepultus est" ist in einer Baßpartitur notiert, jedoch wohl nur, um beide Systeme auszunutzen. Von allen anderen doppelchörigen Stellen enthält die Bc.-Stimme nur einen einfachen Baß.

Einen Beleg für die verdoppelnde Begleitung auch in der solistische virtuosen Musik sieht Willi Ape1139 in der "Sonata prima" aus Biagio Marinis Sonate (1629). In diesem Druck stehen - wie auch in Marinis Affetti musicali (1617) - die vier Solosonaten in Partitur. Apel stüzt seine These vom Mitspielen der Sopranstimme auf eine in der Tat eigenartige Erscheinung in der "Sonata prima" (es ist die einzige der Sonaten mit der Alternativbesetzungsangabe "Cornetto $\delta$ Violino"). In der Partitur sind zwei Pausen der Solostimme mit Noten ausgefiillt. Diese Ausfuillung ist in der Partitur nicht als solche zu erkennen (kein Schliisselwechsel oder ähnlicher Hinweis). Die ergänzten Töne gehen nahtlos in die der Solastimme uber ${ }^{39}$. Da sich die vier in Partitur gedruckten Sonaten (insbesondere die drei nur für Violine bestimmten) dieses Druckes durch ihre z.T. außerordentlich hohe Virtuosität auszeichnen ${ }^{140}$, erscheint es unwahrscheinlich, daß ausgerechnet bei diesen

zu G. Priulis Missae ... octo, novemq. vocibus (1624). Als Beleg für die duplizierende Begleitung wurden einige der fruheren Beispiele von Kinkeldey, Orgel, S.203, Schneider, Basso continuo, S.66 ff sowie Haack, Generalbaß, S. 198 herangefihrt.

136. Es muß aber darauf hingewiesen werden, daß die friheste dieser Quellen, bämlich die Partitur des Druckes von Josephus Gallus, zugleich eine Quelle für das Seguente-Spiel nach einer Partitur ist (s.u.). Diese Partituren geringstimmiger Sätze sind auch keineswegs so verbreitet, wie dies behauptet wurde (Schneider, Basso continuo, S.66 sowie Haack, Generalbaß, S.198). In den achtstimmigen Messen von G. Croce soll die Orgel sogar bei diesen geringstimmigen Sätzen schweigen. (So steht in der Baßpartitur zu den Messe a 8 (1596) bei der zweiten Messe "Crucifixus non est hic" und bei der dritten Messe "Crucifixus tacet". Diese beiden Teile sind im Gegensatz zu den anderen Sätzen nur vierstimmig. Auch in der fünften Auflage von 1612 (die Baspartitur ist hier einer Continuo-Stimme gewichen) findet sich an den entsprechenden Stellen ein (acetvermerk).

137. Das Crucifixus ist als Notenbeispiel II,10 im zweiten Band dieser Arbeit abgedruckt.

138. Violinmusik, S. $37 \mathrm{f}$.

139. Diese Stelle ist im zweiten Band dieser Arbeit als Notenbeispiel II,11 abgedruckt. 
Stücken an eine verdoppelnde Begleitung gedacht wurde. Die hohe Virtuosität lä日t eine duplizierende Begleitung sogar unmöglich erscheinen, ein Mitlesen der Solostimme durch den Begleiter hingegen erscheint unverzichtbar (nur in diesen vier in Partitur gedruckten Sonaten finden sich zahlreiche Tempoworte!).

Es bleibt die Frage nach den Ausfullungen in der Partitur der "Sonata prima". Eine letztendlich gesicherte Erklärung wird man kaum liefern können. Wahrscheinlich sind diese Ausfullungen von der Orgel zu spielen. Dies muibte den Musikern dann in einer Probe auffallen. Vielleicht war ursprïnglich die Violin-/Zink-Stimme so abgefaßt, wie sie in der Partitur uberliefert ist. Aufgrund der größeren Unbeweglichkeit des Zinken und des Luftbedarfes des Zinkinisten ist das Stück dann in die vorliegende Form geändert worden. Auf die spezielle Auffuhrungsart hinzuweisen wurde versäumt ${ }^{141}$.

\subsubsection{TEILPARTITUREN}

Grundsätzlich verschieden von den Vollpartituren sind in Bezug auf die Begleitung die Randstimmen- oder andere Teilpartituren. Ähnlich wie auch Partituren solistischer Musik enthalten diese Partituren keinen vollständigen Satz. Selbst wenn ein Mitspielen vorgesehen ist, bedarf es der Hinzufügung weiterer Stimmen.

Ein Teil dieser Partituren dient sicher in erster Linie dem Organisten zur Orientierung ${ }^{142}$, aber auch das Mitspielen der in der Partitur notierten Oberstimme ist bezeugt. Weiter oben wurde bereits auf die Aussagen Orazio Scalettas ${ }^{143}$ und Giovanni Paolo Cimas ${ }^{144}$ zur Begleitung der Sätze à due hingewiesen. Dort wurde gefordert, die notierten Stimmen auch zu spielen.

140. Vg1. die Ubertragung einer Sonate bei Iselin, Marini, Notenteil, S.11 ff; vgl. dort auch zur wesentlich einfacheren Faktur der anderen Sätze aus diesem Druck (Op.8) S.6 ff und S. 10.

141. Nicht in Betracht als Beleg für eine verdoppelnde Begleitung kommt ein weiteres von Apel genanntes Beispiel, nämlich die partitura zur "Sonata Decima Settima A 4. In Ecco" aus D. Castellos Sonate concertate .. libro secondo (1629, Apel S.24). Es handelt sich bei dieser partitura um eine Art Randstimmenpartitur. Da es bei diesem Stück keine obere Randstimme gibt, wird eine Exzerpt-Stimme aus den beiden Oberstimmen geboten; sie unterrichtet den Organisten über die Harmonie und vermittelt ihm zugleich einen Einblick in den Satzaufbau und die Imitationsfolge (vgl. den Abdruck dieser Sonate mit der originalen partitura in der Neuausgabe Dario Castello, Selected Ensemble Sonatas, Tei1 2, S.81 ff). Das Fehlen einer Bezifferung - von Apel weiter als Argument für seine Mitspielthese angefuihrt - ist in einer Teilpartitur nichts Ungewöhnliches, denn die partitura kann eine Bezifferung ersetzen.

142. Dazu mehr in den folgenden Abschnitten. Grundsätzlich gegen eine Verdoppelung der Oberstimme einer Randstimmenpartitur wendet sich Haack, Generalbaß, S.198 ff. 
Das Mitspielen der in der zweistinmigen Partitur notierten Stimmen ist besonders eindrucksvoll belegt in Domenico Brunettis Unica voce, binis, ternis, quaternis, \& pluribus ad usam ecclesiae ... cum gravi, \& acuti ad organum (1609)145. Hier sind die in der Orgelpartitur notierten Stimmen oftmals unabhängig von den Vokalstimmen. Dies ist besonders auffällig in den Sätzen für Basso Solo. Der Baß der Partitur verläuft hier teils zusammen mit der Solostimme (allerdings ohne Verzierungen), teils aber auch von der Solostimme gelöst als selbständiger Basso continuo unter dem Vokalbaß. Bei der Oberstimme handelt es sich hier um eine selbständige Begleitstimme ${ }^{146}$. Es liegt hier also keine Partitur des Werkes, sondern eine auskomponierte Orgelstimme vor; sie ist als Randstimmenpartitur notiert - vergleichbar den Randstiramenpartituren in der Klaviermusik (s.o.). Auch bei den Sätzen für Sopran Solo sind die Pausen des Sopranes in der Partitur mit Begleitstimmen ausgefiillt: 147 .

Aut ein Mitspielen der in einer Randstimmenpartitur notierten Stimmen deutet auch die "Partitione per l'organo" zu Giovanni Battista Mechis Motecta quinque et acto vocum ... liber primus (1611) hin. Es handelt sich bei dieser "Partitione" um eine Randstimmenpartitur in Seguente-Manier, d.h., wenn die höchste Stirme pausiert, dann steht in der Partitur die zweithöchste Stimme und so fort. In den meisten Partituren dieser Art finden sich an Stimmwechseln unschöne Intervalle; ähnlich wie auch in den Basso Seguente-Stimmen. Mechi vermeidet dies, indem er solche Stellen drei- und einmal sogar vierstimmig notiert. Durch die mehrstimmige Wiedergabe der Stimmwechsel erscheiut der Anfangston der neuhinzutretenden Stimme nicht als (falsche) Fortsetzung der anderen Stimme, sondern als tatsächlicher Neueinsatz ${ }^{148}$. Eine solche Sorgfalt der Stimmführung ist jedoch nur sinnvoll, wenn man davon ausgeht, daß dieser notierte Satz auch tatsächlich erklingen soll.

Eine der interessantesten Randstimmenpartituren ist der Basso principale co'l soprano del quarto libro delle messe a quattro, e cinque voci dell'eccellentiss. Gio. Pietro Aluigi Palestina von Alessandro Nuvalino, erschienen 1610 bei Filippo Lomazzo ${ }^{49}$. In der Dedicatio schreibt Lomazzo, die

143. Partitura della certa spirituale (1605); vg1. auch den Wortlaut des Vorwortes im zweiten Band dieser Arbeit auf S.15.

144. Concerti ecclesiastici (1610); vgl. das Nachwort auf S.12 des zweiten Bandes dieser Arbeit.

145. Vgl. Horsley, Scores, S.483 ff mit einigen Notenbeispielen sowie Oscar Mischiati, Art. Brunetti, Domenico in MGG, Sp.1147 f.

146. Vgl. den Anfang eines Satzes von Brunetti im zweiten Band dieser Arbeit (Notenbeispiel II,12) sowie die Beispiele bei Horsley.

147. Mischiati, Sp.1147.

148. Vgl. das Notenbeispiel II,13 im zweiten Band dieser Arbeit. Aus demselben Grund finden sich zweistimmige Stellen in der Continuo-Stimme zu B. Marimis Sonate (1629).

149. Vgl. hierzu Horsley, Scores, S.490 ff, mit einem Notenbeispiel. 
Musik Palestrinas werde nicht mehr musiziert, da kein Interesse an Musik ohne Basso continuo bestehe. Mit dieser Orgelstimme wolle er der Musik Palestrinas zu neuen Auffürungen verhelfen ${ }^{150}$. Um die eigentlich einem Basso continuo entgegenstehende Musik Palestrinas ${ }^{151}$ den Organisten zugänglich zu machen, wählte Nuvalino die in der überlieferung singuläre Form eines bezifferten Basses mit einer bezifferten Sopranstimme; die Ziffern iber der Sopranstimme bezeichnen eine Stimme unter dieser Stimme (i.d.R. einen Alt), die Ziffern über der Baßstimme - wie ublich - eine Stimme iiber der Baßstimme in Tenorlage (die Zahlen bezeichnen genau die Tonhöhe, also auch ihre Oktavlage). Auf diese Weise waren in der Randstimmenpartitur bis zu vier Stimmen darstellbar. Dies verbindet die Vorteile der schnellen uberschaubarkeit einer Continuo-Stimme mit der Genauigkeit einer Partitur ${ }^{15}$ ?. Sicher sind auch die beiden notierten Stimmen notengetreu mitzuspielen.

Bei den gedruckten Exzerptpartituren, also jenen Partituren, die einen Satz zu sechs oder mehr Stimmen zu drei Stimmen zusammenfassen, ist hingegen kaum von einem Mitspielen der Stimmen auszugehen. Bei den in Exzerptpartitur gedruckten Sätzen in der Bassus generalis-Stimme zu Claudio Monteverdis Sanctissimae Virgini Missa (1610)153 handelt es sich um motettische Sätze zu sechs bzw. acht Stimmen mit - meist zweistimmigen - virtuosen Abschnitten. Die auf drei Systemen zusammengefaßte Partitur stellt somit bei den geringstimmigen konzertanten Abschnitten einen gleichwertigen Ersatz einer Vollpartitur dar und ermöglicht hier insbesondere auch einen im Tempo freien Vortrag, ist jedoch mit erheblich weniger Aufwand herzustellen'154.

150. Horsley, S.490, Anm.59.

151. Horsley, S.493.

152. Bereits im Jahr 1600 erschien in Madrid eine Teilpartitur zu den Missae, Magnificat, Motecta, Psalmi et alia T. L. de Victorias (vgl. Calo, Generalbaß, S.202 sowie Fritz Oberdörffer, Art. Generalbaß in MGG, Bd.4, Sp.1713). Ein Großteil dieser Stücke war bereits vorher ohne Orgel erschienen (vg1. Robert Stevens, Art. Victoria, Tomàs Luis de in MGG, Bd.13, Sp.1592).

153. Es handelt sich um die Sätze "Laudate pueri a 8 voci" und "Laetus sum a 6 voci". Malipiero $1 \mathrm{ie} \beta$ in der Monteverdi-GA, Bd. 14, erster Halbhand, die in den Teilpartituren notierten Stimmen erfreulicherweise in das GeneralbaBsystem drucken, so daß die Faktur dieser Teilpartituren leicht zu studieren ist.

154. Eine dreistimmige Teilpartitur eines sechs-bzw. siebenstimmigen Satzes findet sich auch in A. Mortaros Sacrae cantiones (1598) und T. Massainos Sacrarum cantionum ... liber primus (1607, vgl. Horsley, Scores, $\mathrm{S} .474 \mathrm{f}$ ). Beide Partituren sind jedoch mit den genannten Exzerptpartituren Monteverdis nur bedingt $z u$ vergleichen. Bei Mortaros sonst nur dreistimmigem Druck lag es nahe, auch den einzigen siebenstimmigen Satz auf eine dreistimmige Partitur zu reduzieren; für Massaino gab die Faktur des Satzes den $A n l a ß$ zu dieser Veröffentlichungsform. Als einziger Satz dieses Druckes (die anderen Sätze sind in der Orgelstimme als Randstimmenpartitur wiedergegeben) handelt es sich hierbei weitgehend um einen Satz à tre. Die anderen vier 


\subsubsection{PARTITUR ANSTELLE EINER BEzIfFERUNG ODER ZU DEREN ERGäNZUNG}

Wie bereits mehrfach erwähnt, beschrieb Adriano Banchieri in den Conclusioni nicht nur das collaparte-Spiel nach einer Partitur, sondern auch das Seguente-Spiel nach dem $\mathrm{Ba}$ der Partitur. Die Möglichkeit des Seguente-Spieles nach der tiefsten Stimme einer Partitur hatte bereits elf Jahre friuher Aurelius Ribrochus bedacht. In der Vorrede zu der von ihm herausgegebenen Partitur des Sacri operis... liber primus von Josephus Gallus (1598)15s beklagt Ribrochus, daß die Organisten die Mühe scheuten, eine Partitur anzulegen, und daher "unlieblich und unpassend" spielten. Aus diesem Grund habe er sich entschlossen, diese Partitur herauszugeben. Er dachte dabei jedoch nicht an ein Mitspielen, sondern an ein Seguente-Spiel nach dem Baß. Um dies zu erleichtern, kennzeichnete er an Stellen, an denen der Baß pausiert bzw. nicht die tiefste Stimme ist, die jeweils tiefste Stimme mit einem Kreuz'156. Die Partitur soll also dem Organisten nicht als Intavolatur des zu spielenden Satzes dienen, sondern ihn uber die jeweiligen Harmonien unterrichten.

Die Möglichkeit des Mitverfolgens des Harmonieverlaufes beschrieb Adriano Banchieri als den entscheidenden Vorteil der von ihm bevorzugten Randstimmenpartitur ${ }^{157}$. Auch andere Quellen bezeugen als Veranlassung zur Veröffentlichung einer Randstimmen- oder Vollpartitur die Möglichkeit, die Stimmen mitzuverfolgen; dadurch werde nicht nur eine Bezifferung ersetzt, sondern auch der Organist in die Lage versetzt, den Sängern Hilfestellungen zu geben. Dieser Gedanke findet sich bei Girolamo Giacobbi158 und in besonders

Stimmen treten nur bei den Alleluia-Ritornellen hinzu. Somit erscheint auch die Wiedergabe beider konzertierenden Oberstimmen in der Partitur angebracht. Eine Verwandtschaft mit den Monteverdischen Exzerptpartituren auf drei Systemen haben jedoch die - ebenfalls in dem genannten Monteverdi-Druck zu findenden - Exzerptpartituren auf nur zwei Systemen, wie sie durchgängig für den Introitus (leider keine Wiedergabe der Partitur bei Malipiero) sowie für die solistisch-virtuosen Teile der Sonata (vgl. Monteverdi-GA, Bd.14, 2. Halbband, S. 258 ff) notiert sind. Ähnliche Exzerptpartituren auf zwei Systemen finden sich in A. Grandis Madigali concertati (1626, "O donna troppa cruda") und in D. Castellos Sonate concertate... libro secondo (1629, "Sonata Decima Settima").

155. Das lateinische Vorwort ist vollständig abgedruckt bei Kinkeldey, Orge1, S.199, Anm.1. Das Wichtigste wird von Kinkeldey auf S.199 f paraphrasiert.

156. Vgl. dazu das Vorwort bei Kinkeldey unter "primum". Faksimiles aus dieser Partitur bietet Kunze, Gabrieli, S.226 ff. Solche Kreuze sind in dem Beispiel auf S.227 im drittletzten Takt im zweiten Chor (ein zeitgenössischer Organist hat die entsprechende Stimme auch in den Baß ubertragen!) und auf S.228 im vierten Takt im ersten Chor sowie in den beiden letzten Takten im zweiten Chor zu sehen.

157. Vgl. Banchieris Aufstellung der verschiedenen Arten der Begleitstimmen im zweiten Band dieser Arbeit auf S.18.

158. Vorwort zur Prima parte de Salmi concertati a due e piu chori (1609): 
eindrucksvoller Weise bei Paolo Agostini. Er weist im Nachwort zur Spartitura del primo libro delle messe (1627) darauf hin, daß man Messen und anderes ohne eine Partitur auf den Dörfern nicht musizieren könne, da ja nur wenige Stimmen zur Verfügung ständen. Weil die Orgel in der Lage ist, diese fehlenden Stimmen zu ersetzen, sei es sein Wunsch gewesen, eine Partitur drucken zu lassen, und zwar mit allen Stimmen. Dies sei sowohl für den Organisten als auch für die Sänger gut, um nicht zu viele Fehler zu machen. Mit der Möglichkeit alle Stimmen zu sehen, werde es für diejenigen, die die Partitur aufmerksam beobachten, einfach sein, mit einzuspringen, wenn jemand einen Fehler macht, und dies werde passieren, da alle unvollkommen sind in dieser We1t und die Vollkommenheit dem anderen Leben vorbehalten ist ${ }^{159}$.

\section{3 .3 . PARTITUREN ZUR ERMÖGLICHUNG EINES FREIEN VORTRAGES}

Selbstverständlich ermöglicht jede Art der Begleitpartitur einen freieren Vortrag als eine Basso continuo-Stimme. Der Begleiter kann die andere( $n$ ) Stimme(n) mitverfolgen und auf Temposchwankungen sofort reagieren. Bereits bei der Behandlung der Partituren monodischer Musik wurde auf die Bedeutung der Partitur auch für die Freiheit des Tempos hingewiesen 160 , wenngleich hier nicht mit GewiBheit gesagt werden konnte, daß dies der Grund für die Partiturveröffentlichung war. Die Partitur war hier notwendig, damit der Sänger sich selbst auf der Laute oder dem Chitarrone begleiten konnte

"... con la Partitura poi per 1'Organo, appresso il' Basso continuo, con gli accidenti soliti segnati, si è posto la Parte piu accuta; non perche l'organista l'habbi a rappresentare continuatamente, mà si bene à fine, che havendola innanzi a gli occhi posso \& aiutare \& discretamente accompagnare il cantante, massime quando resta solo, accio gli sia per mezzo di tal discretezza \& accentare \& con passaggi di suo gusto dar quella perfettione che gli parera esser conveniente a tal concerto" (zitiert nach Kinkeldey, Orgel, S.224).

159. "ALLI BENIGNI LETTORI. SI conosce chiaramente, che stampar Messe, $b$ altra cosa, che sia, se non v'è Spartitura non si cantano principamente nelli paesi ch'anno poche voci; perche suplisca l'Organo, hò voluto mettervi in spartitura con tutte le parti tutte stese, per quelli, che non vogliano sonare sul Basso steso, come gia si sonava, \& è molto profitto a quelli, che voglino far professsione di sonare, \& anco per non far tropp'errori nel cătarli, che vscendo qualche parte per quelli che non sono troppo sperimentati nel saper rimettere, con dett' occasione di vedere tutte le parte, saró facilissimo agiustarli, \& per curiosi che le vorranno veder spartite, hauer occassione chi far poca fatica in vederle, \& copartire a quei mancamenti, che vi fussero, essendo noi in questa mondo imperfetti, perche la perfettione e nel'altra vita". Wohl um allen Organisten gerecht zu werden, wurde den folgenden Messpartiturbüchern (nicht dem ersten, aus dem das Nachwort stammt) zusätzlich eine bezifferte Bc.-Stimme beigegeben (auf den letzten Seiten der Partitur).

160. Auf die Zusammenhänge von Partituriberlieferung und Tempo wurde auch in dem Abschnitt I.1.2.5. eingegangen. 
(s.o.). Anders ist dies jedoch, wenn eine Begleitpartitur zusätzlich zu einer Einzelstimme bzw. einem Stimmensatz erschien. Hier kann von einer Personalunion von Sänger und Begleiter nicht mehr ausgegangen werden.

Der Zusammenhang von Partituriberlieferung und Freiheit des Tempos wird in einigen dieser Drucke durch die Tatsache unterstrichen, daß die in der Continuo-Stimme in Partitur notierten Sätze einen Hinweis wie "senza battuta" tragen'61. Bestätigt wird dieser Zusammenhang von freiem Vortrag und Partiturnotation in der Continuo-Stimme auch durch eine Anzahl von Drucken mit Musik unterschiedlicher Besetzungen, in deren Basso continuo-Stimmen nur die solistischen Sätze in Partitur uberliefert sind ${ }^{162}$. 1638 begrindet Ignatio Donati den Abdruck der Singstimme in der Orgelstimme seines secondo libro de motetti an voce sola ausdrücklich mit der bei solistischer Musik gebotenen Freiheit163. Diese Partituren solistischer Musik unterscheiden sich von anderen Begleitpartituren auch darin, daß die Bässe dieser Partituren oft beziffert sind ${ }^{164}$. Schon dies zeigt, daß die Partitur hier nicht in erster Linie der Aussetzung dienen soll.

\subsubsection{DIREKTIONSPARTITUREN}

Bereits die oben erwähnten Partituren solistischer Musik dienen nicht mehr der Generalbaßaussetzung, sondern der Orientierung. Der Generalbaß ist in der Regel auch ohne die notierte Oberstimme voll funktionsfähig, das heißt in dem zu dieser Zeit ublichen Umfang beziffert. Vergleichbar mit diesen Partituren solistischer Musik sind die Direktionspartituren der Ensemble-

161. So in C. Monteverdis Concerto (1619) und G. Valentinis Musiche da camera libro quarto (1621; vgl. Schmitz, Continuo-Madrigal, S.517 f).

162. C. Monteverdi, Sanctissimae Virgini Missa (1610; vg1. im Abschnitt I.1.2.5.), ders., Il sesto libro de madrigali (1614; vgl. Schmitz, ContinuoMadrigal, S.510), ders. Concerto (1619), B. Marini, Affetti musicali (1617), ders., Sonate (1629), F. Turini, Madrigali ... libro secondo (1624), A. Banchieri, Il virtuoso ritrovo (1626), G. B. da Gagliano, Mottetti per concertare (1626), T. Cecchino, Cinque messe a 2 (1628), D. Bellante, Concerti accademici (1629), D. Castello, Sonate concertate... libro secondo (1629) und G. Rovetta, Madrigali concertati (1629). Nur solistische Musik enthalten - ebenfalls mit Einzelstimme und Partitur - Il primo libro de motetti (1620) von I. Vivarino sowie die Motetti a voce sola (1629) von F. Turini.

163. "In questa sorte di Cantilena di voce sola non si deve mai Batter la battuta, ma solo attendere a Cantare con misura Larghissima posatamente, con far quelli affetti; esclamazioni di crescere e mancar la voce a tutte suo potere, con rinforzare la voce a suo luogho, e tempo, e non cantar con ansietà e Timore. Perche il Signor Organista vede nel suo libro tutto quello, che dice il Cantante, e se anco aggiungessere altri passaggi, sempre aspetterà e darà tempo con il suo sonare" (zitiert nach Goldschmidt, Gesangsmethode, S.77).

164. Die Behauptung von Kirchner, Generalbaß, S.40, "Partitur und Bezifferung schließen sich dem Wesen nach aus" ist nicht zutreffend. 
musik. Der Schritt zur ausgesprochenen Direktionspartitur ist mit Bartolomeo Grassis Partitur des primo libro delle canzoni Girolamo Frescobaldis (1628) vollzogen. Der Nutzen der Partitur zur Direktion wird zwar auch in Vorworten fruherer Partituren angesprochen10s, erst bei Grassis Partitur ist jedoch eine Trennung der Partitur von den Generalbaßfunktionen erreicht. Die Geueralbaßstimme ist hier immer einem eigenen System zugewiesen - auch wenn ein BaBinstrument an der Komposition beteiligt ist und dessen Stimme der Continuo-Stimme mehr oder weniger entspricht. Der Generalbaßspieler benötigt für seine Begleitung nur diese Stimme. Die Partitur hingegen dient der ubersicht uber das Werk - gleichzeitig alle Stimmen zu sehen, ist laut Grassi eine "cosa necessarissima a chi desidera sonar bene"106.

Es ist sicher kein Zufall, daß der iberwiegende Teil der Begleitpartituren solistischer Musik und dieser erste Vertreter des neuen Types der Direktionspartitur $z u$ derselben Zeit erschienen ${ }^{10}$ ?. Im dritten Jahrzehut des 17. Jahrhunderts ist die Zeit des "Ausprobierens" auch im Hinblick auf die Begleitung vorbei. Das Spiel nach dem Basso continuo ist fest etabliertic.8. Der Partitur kam damit nicht mehr die Aufgabe zı, den Generalbaßspieler die für seinen Begleitsatz wichtigen Informationen zu geben - diese Aufgabe nahm nun die Bezifferung allein wahr -, sondern ihn als Begleiter konzertant solistischer Musik oder als Leiter eines Ensembles uber den Verlaut der anderen Stimmen $z u$ informieren ${ }^{169}$.

165. Vgl. etwa die oben zitierten Vor- bzw. Nachworte G. Giacobbis und P. Agostinis .

166. Aus dem Nachwort Grassis (vgl. den vollständigen Abdruck dieses Vorwortes bei Sartori, Bibliografia I, S.328 (1628 i). Die Aufgaben der Direktion kommen freilich immer noch dem Generalbaßspieler zu (vgl. weiter unten im Abschnitt II.3.2.).

167. Von den genannten Partituren solistischer Musik entstammen zwei den Jahren 1610 - 1614 (zu Drucken Monteverdis), zwei den Jahren 1615 - 1619, vier den Jahren 1620 - 1624 und neun den Jahren 1625-1629.

168. Die Partituren P. Agostinis stellen einen Sonderfall in der Entwicklung dar. Dies ist auch Agostini bewußt, denu ab dem zweiten Buch gibt er den Partituren zusätzlich auf den letzten Seiten eine bezifferte continuoStimme bei - sicher um der allgemeinen Vorliebe Rechnung zu tragen.

169. An dieser Stelle soll auch auf die beiden Partiturhandschriften Adam Gumpelzhaimers aus der Zeit um 1600 hingewiesen werden (vgl. Lowinsky, Early Scores, S.144 ff). Die beiden umfangreichen Handschriften beinhalten Spartierungen meist vielstimmiger Musik; sie wurden wohl mehrheitlich nach Drucken vorgenommen. Gelegentlich finden sich Hinweise auf die zu benutzenden Stimmen oder auch dynamischen Angaben (Lowinsky, S.145). Ob die Handschriften wirklich der Direktion gedient haben, wie Lowinsky vermutet, ist jedoch zweifelhaft, u.a. da die Bindung der Handschriften schon durch Gumpelzhaimer (Lowinsky, S.145) veranlaßt wurde, und die Handschriften damit ein eher unpraktisches Format besaßen. Durchaus denkbar ist jedoch außer einem reinen Studienzweck auch die Verwendung zur Vorbereitung einer dann nach der Generalbaßstimme geleiteten Aufführung. 


\section{BASSO CONTINUO-STIMMEN}

Trotz der Vorbehalte gegeniber den Basso continuo-Stimmen erleben diese bereits in den ersten Jahren des 17.Jahrhunderts eine grobe Verbreitung. Bereits 12 Continuo-Stimmen ${ }^{170}$ aus den letzten sechs Jahren des 16.Jahrhunderts und 50 Continuo-Stimmen aus den Jahren 1600-1605 sind von italienischen Verlegern bekannt171. Diese Generalbaßstimmen entstammen fast ausschließlich dem Bereich der Kirchenmusik; die Monodie erschien in Partitur, im polyphonen Madrigal wurde der Generalbaß nur zögernd aufgegriffen 172 .

\subsection{DIE AUSBREITUNG DER BEZIFFERUNG ${ }^{173}$ \\ $3,1,1$. MONODIE UND OPER}

Von Anfang an gab es zwei grundsätzlich verschiedene Arten des Generalbasses174, nämlich die zunächst immer in einer Partitur notierten ContinuoStimmen der Oper und der Monodie und die in Einzelstimmen uberlieferten Generalbässe der meist vielstimmigen geistlichen Musik. Es wurde oben bereits darauf hingewiesen, daß die Partituriberlieferung der Monodie in erster Linie in der Personalunion von Sänger und Begleiter begründet $1 \mathrm{ag}$ und

170. Hierzu werden auch die Baßpartituren gerechnet, da sie, wie oben dargelegt, eine größere Verwandtschaft zur Continuo-Stimme denn zur Partitur haben.

171. Vg1. die Liste der nachweisbaren bis 1605 in Italien gedruckten Continuo-Stimmen im zweiten Band dieser Arbeit.

172. Aus den Jahren bis 1605 sind lediglich vier Stimmbuchdrucke weltlicher Vokalmusik mit Basso continuo erhalten, nämlich S. Rossis secondo libro de madrigali (1602), Rossis Terzo libro de madrigali (1603), A. Franzonis nuovi fioretti (1605) und C. Monteverdis quinto libro de madrigali (1605). Nach Schmidts, Continuo-Madrigal, S.509, erschien auch zu P. Singnoruccis Madrigali a cinque voci ... libro primo (1602) eine Continuo-Stimme. Diese ist heute nicht mehr nachzuweisen. In der Liste der Continuo-Stimmen im zweiten Band dieser Arbeit sind die weltlichen Werke durch Fettdruck hervorgehoben.

173. Die folgenden Ausführungen sollen sich im wesentlichen auf die notationstechnischen Probleme und Fragestellungen beschränken und aus dieser Sicht die bisherigen Forschungsergebnisse zum Thema Generalbaß ergänzen.

174. Eggebrecht, Generalbaß, unterschied noch eine dritte Art des Generalbasses, närlich den motettisch-solistischen Generalbaß der Viadana-Schule. Sicher handelt es sich hierbei um eine für begrenzte Zeit eigenständige Erscheinung; sie kommt jedoch den beiden grundsätzlich verschiedenen Hauptgruppen in ihrer Bedeutung nicht gleich. Auch sind die Abgrenzungen zu diesen Hauptgruppen - insbesondere zur Monodie - bei weitem nicht so ausgeprägt, wie Eggebrecht dies darstel1t. Sicher angeregt von der Monodie und den Continuo-Stimmen der vielstimmigen Musik (beides ist bedeutend älter als die motettisch-solistischen Generalbässe) als auch der älteren Praxis, einen Teil der Singstimmen nur der Orgel zuzuweisen, bot Viadana den Organisten und Sängern speziell ihren Bedürfnissen entsprechende Musik. 
damit an die Lautenlieder des 16.Jahrhunderts anknüpfte. Nur im Hinblick auf diese Tradition ist es zu verstehen, daB die Bezifferung zuerst in der Monodie und der Oper auftritt, obwohl die Partiturnotation hier auch ein Begleiten ohne Bezifferung viel eher durchfuhrbar erscheinen läßt als eine Continuo-Einzelstimme. Die Bezifferung ersetzt hier die Tabulatur'175.

Eine Bezifferung tritt, soweit bekannt, erstmals in den Florentiner Opern des Jahres 1600176 sowie in der Rappresentazione di Anima e di Corpo Emilio De' Cavalieris (ebenfalls 1600) auf. Alles deutet darauf hin, daß die Bezifferung ihren Ursprung in der Florentiner Camerata177 hat. Sowohl De' Cavalieri als auch die Komponisten der beiden Opern des Jahres 1600, Jacobo Peri und Giulio Caccini, gehörten dieser Vereinigung zumindest zeitweilig an 178 . Die Bezifferung entstand jedoch nicht zusammen mit der Monodie ${ }^{179}$, sondern kam erst später hinzu180.

Die Verwandtschaft der Bezifferung mit der Tabulatur zeigt sich in diesen Drucken nicht nur in der uberlieferungsform. Ganz ähnlich wie im 16.Jahrhundert vielen Lautentabulaturbüchern kurze Einführungen in die Tabulaturschrift vorausgeschickt waren, findet sich nun in diesen Drucken, wie auch in den früheren Drucken der von der Oper unabhängigen Monodie, eine knappe Einführung in diese neue Akkordschrift181.

175. Die Vermutung, daß die Ziffern eine Art Tabulaturersatz sind, äußerte schon Schneider, Basso continuo, S.79. Auch die Ziffern über 8 sind hierauf zurückzufuhren. Die Bezifferung sollte nicht die Form eines Akkordes bzw. einer Harmonie angeben, sondern die zu spielenden Noten (vgl. auch weiter unten). Wenn in den Ziffern ein (Lauten-)Tabulaturersatz $z u$ sehen ist, erklärt sich damit auch deren Fehlen in Viadanas Cento concerti ecclesiastici (1602) von selbst. Eggebrechts Vermutung, die Partitur in der Monodie sei aus harmonischen Gründen notwendig gewesen (GeneralbaB, S.76) läBt sich ebensowenig halten, wie seine Behauptung, die Bezifferung sei in der Monodie wieder aufgegeben worden (ebenda). Es stimmt zwar, daß in den genannten Beispielen Monteverdis auf eine Bezifferung verzichtet wurde. Dies ist jedoch ausgesprochen untypisch (s.u.).

176. G. Caccini, L'Euredice composta in musica sowie J. Peri, Le musiche ... sopra L'Euredice.

177. Vg1. als uberblick den Artikel Camerata von August Buck in MGG, Bd.2, Sp.719 ff.

178. E. De' Cavalieri war wahrscheinlich kein ständiges Mitglied der Camerata (vgl. Adelmo Damerini, Art Cavalieri, Emilio De' in MGG, Bd.2, Sp. $923 \mathrm{ff}$ ).

179. Eggebrecht, Generalbaß, S.76, geht von einer gleichzeitigen Entstehung von Monodie und Bezifferung aus.

180. Sie fehlt z.B. in der noch aus dem 16. Jahrhundert stammenden Handschrift 704 der Bibliotheque du Conservatoire, Brüssel.

181. Eine solche Einfuihrung enthält außer den Drucken des Jahres 1600 auch G. Caccinis Le nuove musiche (1602) und S. d'Indias Le musiche (1609). Mit der ubernahme der Bezifferung in die geistliche Musik wurden vereinzelt auch 
In Bereich der Monodie gab es immer auch Drucke mit unbezifferten Bässen. Eröffnet wird die Gruppe dieser monodischen Partituren ohne Bezifferung mit dem ersten monodischen Druck außerhalb der Bühnenmusik überhaupt, nämlich mit den Musiche composte sopra alcuni madrigali di diversi von Domenico Maria Megli182. In der Folgezeit erschienen immer wieder einzelne Drucke ohne Bezifferung183 und es gab Komponisten, die grundsätzlich ihre Bässe monodischer Musik unbeziffert ließen ${ }^{184}$. Die Tatsache, daß Monodien in Partitur gedruckt wurden, könnte diese Komponisten zu einer Zeit, in der ein bezifferter $B a ß$ zum Standard auch in anderen Gattungen geworden war, veranlaßt haben, hier auf eine Bezifferung zu verzichten ${ }^{185}$. Diese Drucke blieben aber Ausnahmen; von einer Abkehr von der Bezifferung kann auch bei den Monodisten keine Rede sein.

Zur rhythmischen Notation der Oberstimmen wurde in dieser "neuen Tabulatur", nämlich den bezifferten Bässen, die Teilung der Baßnoten benutzt: Durch die Spaltung von Baßnoten in mehrere kïzere mit einem Bindebogen verbundene Noten konnten bei einem Harmoniewechsel der Oberstimmen die Ziffern genau plaziert werden ${ }^{186}$. In unbezifferten Generalbässen, selten sogar in bezifferten, können mehrere gleiche Baßnoten mit Bindebögen auch allein bereits ein Hinweis auf eine wechselnde Harmonie sein. Auch hier ist allerdings nicht mit einer konsequenten Handhabung zu rechnen. Solche geteilten Baßnoten deuten zwar oft auf einen Harmoniewechsel hin ${ }^{187}$, ein ungeteilter 1 iegender Baßton ist jedoch kein sicheres Anzeichen für eine gleichbleibende Harmonie,

hier kurze Einführungen in die Bezifferung den Generalbaßstimmen vorangestellt, so z.B. in R. Michelis Psalmi ad officium (1610).

182. Vg1. zur Datierung dieses Druckes vor Caccinis Le nuove musiche Nigel Fortune, Art.Melli (Megli), Domenico Maria in MGG, Bd.9, Sp.17 f.

183. G. G. Kapsberger, Libro primo di arie, und Libro primo di mottetti (beide 1612), G. Romano, Fuggilatia (1613), B. Barbarino, Il quarto libro de madrigali (1614) und C. Monteverdi, Lamento d'Arianna (1623); die anderen Drucke monodischer Musik dieser Komponisten hingegen sind beziffert.

184. G. Frescobaldi, B. Marini und C. Saracini (soweit die ausgewerteten Drucke diesen Schluß zulassen).

185. Dies ist ausgefuihrt in dem Nachwort zu C. Milanuzzis Secondo scherzo delle ariose vaghezze (1625). Dort heiBt es: "Non si son posti ne meno nel Basso Continuo $i$ nummeri, $i$ diesis, ne $i$ b molli per dar gli accompagnamenti ... per esser facile à chi sonara l'haver l'occhio all'una, e l'altra Parte." (zitiert nach Vogel, Bibliothek, Bd.I, S.464).

186. Vg1. hierzu Schneider, Basso continuo, S.76 f. Dieses Verfahren wurde erstmals von G. Caccini im Vorwort zu Le nuove musiche (1602) beschrieben.

187. Mit solchen geteilten Baßnoten konnte jedoch auch noch ein anderer Zweck verfolgt werden, so z.B. in C. Monteverdis $L^{\prime}$ Orfeo (1609). Hier sollen sie wahrscheinlich ein Wiederanschlagen des Akkordes anzeigen (vgl. u.a. das Vorwort zur Ausgabe des Orfeo von Edward H.Tarr, S.IV). Die Form des Bindebogens hat dabei sicherlich keine Bedeutung (sie sind in den Drucken dieser Zeit sowohl in der heute ublichen runden Form $\frown$, als auch eckig $\square$ oder 


\subsubsection{DiE BEzIfFERUNG IN DEN GENERALBÄSSEN DER GEISTLICHEN MUSIK}

Die fruheste erhaltene Quelle mit einer Generalbaßeinzelstimme ist, soweit bekannt, der "Bassone" zu Alessandro Striggios "Ecce beatam Iucem à 40"188. Dieses Werk wurde nach Massimo Troiano 1568 bei der Münchner Hochzeitsfeier des bayerischen Erzherzoges Wilhelm V. mit Renate von Lothringen aufgeführt ${ }^{9}$. Das Auffuhrungsmaterial von 1568 ist verloren; erhalten hat sich aber eine auf 1587 datierte Abschrift in der Ratsschulbibliothek Zwickau190. Ganz sicher handelt es sich auch bei der Seguente-Stimme, dem "Bassone", nicht erst um eine Zutat aus dem Jahr 1587. Der Titel dieser Stimme ist so verstiumelt, daß es sich nur um eine Abschrift eines nicht der italienischen Sprache Kundigen handeln kann191. In Anbetracht der Tatsache, dab eine vier-

in Form einer Klammer $\longrightarrow$ anzutreffen). In der Regel wurde ein runder Bindebogen verwendet, um eine geringe Distanz zu iiberbriicken, ein eckiger um längere Phrasen (oder weiter auseinandergedruckte Noten) zu verbinden. Eine besondere Bedeutung will John Eliot Gardiner diesem Unterschied zwischen runden und eckigen Bindebögen in Monteverdis L'Orfeo (1609) zumessen. Er schreibt (Ecco Orfeo, S.17): "I believe that this is the first time an edition and a recording have observed Monteverdi's meticulaus distinction between tied and bracketed notes. In the original printed editions one finds two kinds of slur: bracketed (- linking basso continuo notes of the same pitch; and conventional ties ( $>$ ). The use of shorter note values than was grammatically necessary, and their linking by these bracketed slurs, has always seemed to me too persistent to be ignored or dismissed as just a printer's whim. Rather, their use is fundamental in interpreting Monteverdi's monodic style in L'Orfeo. Accordingly, we treated each new note value, indecated by the bracket, as an occasion for the reiteration of the same chord (...)". Tatsächlich aber findet man die eckigen Bindebögen auch außerhalb der Continuostimme, wenn die zu bindenden Noten weit auseinander stehen (z.B. S.2), sowie runde in der Continuostimme (S.13). Die Gardinet aufgefallene Häufigkeit der eckigen Bindebögen in der Continuostimme ist leicht zu erklären: In den rezitativischen Monodien des L'Orfeo ist die Oberstimme erheblich bewegter als die Baßstimne, so daß die Baßnoten weit auseinanderrücken und daher vom Drucker die eckige (beliebig verlängerbare) Form des Bindebogens verwendet wurde. Ein Neuanschlagen der Akkorde erscheint sinnvoll (s.o.), ist aber unabhängig von der Form des Bindebogens.

188. Schneider, Basso continuo, S.66 ff.

189. M. Troiano, Dialoghi (2/1569), fol. 146'.

190. Einige Faksimiles bei Schneider, S.66 ff. Die Datierung erfolgte auf der Continuo-Stimme als Chronogramm: "Anno DeVs aVXILIVM fer qVoqVe ferre potest". Sie wird bestätigt durch das Wasserzeichen: Es ist auf einer anderen datierten Quelle von 1588 nachweisbar (Wisso Weiß, Historische Wasserzeichen, Leipzig 2/1988, S.86 f). Das Wasserzeichen zeigt das Wappen von Anhalt und stammt aus der Papiermihle JeBnitz (heute im Kreis Bitterfeld).

191. Der Titel lautet "Bassone canato dalla parte piu basce del. 40 Per=/ sona nimero delcircolato con un bronbone No.41. I per sostentameno della 
zigstimmige Komposition keine "Alltagsmusik" sein kann, ist nicht damit zu rechnen, daß dieses Werk eine allzugroße Verbreitung gefunden hatte. Es liegt daher nahe, in der Vorlage für diese Abschrift tatsächlich das Münchner Aufführungsmaterial von 1568 zu vermuten.

Diese früheste bekannte Generalbaßeinzelstimme bestätigt die These, daß die Vielstimmigkeit außerhalb der Monodie den Anlaß zur Verwendung einer Fundamentbaßstimme gab192. Auch die ab 1594 gedruckten Continuo-Stimmen gehören zunächst überwiegend zu Drucken mit Musik zu acht oder mehr Stimmen ${ }^{93}$. Eine Bezifferung ist freilich in dem "Bassone" Striggios nicht vorhanden; sie ist jedoch auch kaum notwendig. Hans Heinrich Eggebrecht hat zu Recht darauf verwiesen, daß die Vielstimmigkeit zu einer derart einfachen Setzweise führte, daß eine Intavolierung - aber auch eine Bezifferung - sich erübrigte194. Der "Bassone" des auf $G$ stehenden Satzes enthält fast nur die Töne "G", "c" und " $d$ ", seltener auch " $A$ " und " $f$ " und zwar stets in klaren Kadenzfolgen. Sextakkorde kommen - soweit dies ohne Spartierung zu beurteilen ist - nicht vor.

Auf Generalbaßvorzeichen verzichteten bereits die Baßpartituren Giovanni Croces aus den Jahren ab 1594 nicht mehr ${ }^{195}$. In diesen Drucken finden sich Generalbaßvorzeichen in der später allgemein ubblichen Form über den Baßsystemen ${ }^{196}$. In dieser Art verwendet auch Adriano Banchieri diese Vorzeichen in seiner Spartitura per sonar nell'organo accomodate als primo choro nei concerti (1595)197. In den Continuo-Stimmen des beginnenden 17.Jahrhunderts

armonica persona / ricon Orgono Liulo \& cimboli ouiole.". Schneider, Basso continuo, S.67, hat dies als "Bassone cavato dalla parte più basse del 40, per sonar in mezzo del circola con un trombone per sostentamento della armonia per sonarsi con Organo, Liuto \& cimbali o viole." entschliisselt. Die "No.41" ist die Zahl der Stimme (alle Stimmen sind durchnumeriert), die der Schreiber der Zwickauer Handschrift versehentlich in den Text integrierte.

192. Oben ist auf die Verbreitung dieser Anschauung bereits verwiesen worden.

193. Vg1. die Liste der ermittelbaren Continuo-Stimmen bis 1605 im zweiten Band dieser Arbeit.

194. Generalbaß, S.66 f.

195. Motetti a 8 (1594), Matetti a $8 \ldots$ libro secondo (1595), Messe a 8 (1596).

196. Generalbaßvorzeichen stehen in den Baßpartituren uber jeder der beiden Baßstimmen.

197. Die Vorzeichen stehen hier zwischen den beiden Randstimmen, in seltenen Fällen jedoch auch iber der Diskantstimme. (Ein Teil der Vorzeichen uber der Diskantstimme bezieht sich aber auf die notierte Note selbst. Hăufig wurden in Musikdrucken dieser Zeit Vorzeichen nicht vor der entsprechenden Note, sondern dariber oder darunter notiert. Der Grund dafür ist zumeist Platzmange1. Ein System sollte nicht mitten im Takt enden. War zu wenig Platz vorhanden, konnte durch Dariber- oder auch Daruntersetzen der Vorzeichen Platz gewonnen werden. Die jeweilige Bedeutung eines Vorzeichen ist 
ist diese Form der Generalbaßvorzeichen nur noch in bezifferten Drucken anzutreffen. In den unbezifferten Bässen wurden dann ausschließlich Vorzeichen verwendet, die durch Notierung der Vorzeichen in das Notensystem hinein, nämlich an der Stelle, an der die entsprechende Note unter dem jeweiligen Schlüssel stände, die zu erhöhende bzw. zu erniedrigende Note direkt bezeichneten. Dies erfolgte unabhängig von der zu spielenden Lage, z.T. sogar unter der Baßnote198. In der Regel bezeichneten diese Vorzeichen eine Terz; gelegentlich aber auch eine Sext oder gar eine Quinte ${ }^{199}$. Bei dieser Art der Vorzeichensetzung sind Fehler sehr häufig anzutreffen. Die Vorzeichen stehen oft an den falschen Stellen innerhalb des Systemes, z.B. vor der Baßnote statt eine Terz höher200. Aufgrund der in dieser Zeit meist gut zu durchschauenden Setzweise ist die Verwechslungsgefahr allerdings nicht sehr grob201.

Obwoh1 Generalbaßvorzeichen bereits in den ersten gedruckten Continuo-Stimmen vorhanden waren, erschienen noch bis in das dritte Jahrzehnt des 17. Jahrhunderts hinein Generalbaßstimmen ohne Bezifferung und ohne Genera1baßvorzeichen. Die Tabelle 4 im zweiten Band dieser Arbeit gibt einen uberblick uber die Verbreitung der Bezifferung und Ausstattung mit Generalbaßvorzeichen in den ersten dreiBig Jahren des 17. Jahrhunderts. Es fällt auf, daß in den ersten 10 Jahren die Mehrzahl der Generalbaßstimmen entweder beziffert oder aber ohne irgendwelche Aussetzungshilfen erschienen. Erst zu einer Zeit, in der der Anteil der bezifferten Continuo-Stimmen bereits bei rund $90 \%$ liegt, ist die Mehrheit der nicht bezifferten Continuo-Stimmen mit Generalbaßvorzeichen versehen. Es hat den Anschein, daß es sich hier weniger um verschiedene Entwicklungsstufen des Generalbasses als vielmehr um gegensätzliche Auffassungen von einer Continuo-Stimme handelt202. Auch druck-

aus dem Zusammenhang heraus dennoch gut zu erkennen).

198. Unter einer Baßnote wurden gelegent1ich Sexten angezeigt. Eine Zusammenstellung der verschiedenen Möglichkeiten der Vorzeichensetzung gibt A. Banchieri in seinem $L$ 'organo suonarino (1605 u.a.), S.2 ("Avertimenti vtile alle guide del Basso"). Sie ist im zweiten Band dieser Arbeit als Notenbeispiel II,14 wiedergegeben. Vg1, hierzu auch Schneider, Basso continuo, S.70.

199. Eine Quintvorzeichnung notierte L. Leoni in Sacri fiori (1612). Hier wird ein H-Dur in der Basso per 1'organo-Stimme durch zwei Kreuze iber einem "H" angezeigt: eines an der Stelle des "dis" und eines an der des " $f$ is".

200. So steht z.B. in G. Montesardos I lieti giorni (1612) kein einziges der Generalbaßvorzeichen an der richtigen Stelle. offenbar war dem Setzer die Bedeutung dieser Zeichen nicht klar und er hat alle "korrigiert" und vor die jeweilige Baßnote gesetzt.

201 Der Tonvorat ist, von Ausnahmen abgesehen, in dieser Zeit doch noch recht uberschaubar. So ist ein "dis" im Baß außerhalb eines chromatischen Ganges eher unwahrscheinlich, und man wird sofort an ein "d" mit vorgezeichnetem "fis" denken.

202. Ein Zeugnis hiervon gibt A. Franzoni im Vorwort zur Basso continuoStimme seinen Concerti ecclesiastici ... L Iibro primo (1611): "A' BENIGNI LETTORI. HAurei potuto nel presente Basso notare alcune Consonanze, e disso- 
technische Schwierigkeiten werden zum Verzicht auf Aussetzungshilfen gefuhrt haben 203 .

Gegen Ende des ersten Jahrzehntes des 17. Jahrhunderts begann man, die von monodischen Partituren her bekannte Bezifferung auf die Generalbaßstimmen zu ubertragen. $\mathrm{Zu}$ den Komponisten, die als erste eine Bezifferung in der Kirchenmusik verwendeten 204 , gehört neben Giulio Bel1i205 und Antonio Cifra206 auch Francesco Bianciardi207, nicht aber Antonio Agazzari208. Agazzari trat in seinem Generalbaßtraktat für eine Bezifferung ein209, Bianciardi erwähnt sie in seiner Generalbaßschrift nicht210. Offenbar war Agazzari nicht in der Lage, auch seine Verleger 211 von deren Notwendigkeit zu iberzeugen.

War die Bezifferung in die polyphone Musik ubernommen, gab es keinerlei Unterschiede zwischen der Bezifferung in der Monodie und in der polyphonen Musik. Die Anwendung der Bezifferung innerhalb bezifferter Bässe vermehrte sich bereits im zweiten Jahrzehnt des 17. Jahrhunderts deutlich. Doppe1stöckige Ziffern waren bereits allgemein iblich und Sonderzeichen wie die durchstrichene Sechs wurden eingeführt212.

nanze perrendere più facile il sonare la presente opera, ma à bello studio l'hò tralasciato per non far torto à signori Organisti, quali col loro purgato orecchio sapranno secondare la compositione co' $i$ loro leggiadri mouimenti. Tanto piu hauendone à bastanza, \& gratiosamente di ciò discorso Agostino Agazaro, nel Secondo Libro de suoi Concerti, alquale io mi riporto: \& viuete felici."

203. Auf drucktechnische Probleme beim Setzen von Generalbaßvorzeichen sowie einer Bezifferung verweist A. Agazzari in Vorwort zur Basso continuoStimme zu Sacrae laudes de Iesu ... liber secundus (1603) hin (vgl. den Abdruck dieses Vorwortes im zweiten Band dieser Arbeit auf S.4).

204. Die früheste dem Verfasser bekannte Begleitstimme mit Bezifferung in der polyphonen welt.lichen Musik ist die Randstimmenpartitur zu P. Quagliatis Madrigali a quattro voci (1608).

205. Missae sacrae (1608).

206. Motecta ... liber primus (secundus, tertius) und Psalmi septem (alle 1609).

207. Sacrarum modulationum (1608).

208. In Agazzaris Drucken erscheint eine Bezifferung erst ab 1611 (vg1. Quellenliste).

209. De1 sonare sopra '1 basso (1607), S.4 ff.

210. Breve regola per imparar' a sonare sopra il basso (1607).

211. Agazzaris geistliche Werke erschienen in Rom bei Zanetti, in Venedig bei Amadino und in Mailand bei Tini \& Lomazzo. Die oben erwähnten vor 1610 erschienen Generalbaßstimmen mit Bezifferung erschienen in Venedig bei Gardano (Belli, Bianciardi) und in Rom bei Robletti (Cifra).

212. Soweit dem Verfasser bekannt, tritt dieses Zeichen erstmals in G. Caccinis Nuove musiche e nuova maniera di scriverle (1614) auf. 
Trotz der großen Verbreitung der Bezifferung bereits im zweiten Jahrzehnt des 17.Jahrhunderts ${ }^{213}$ gab es noch während des ganzen hier zu behandelnden Zeitraumes verschiedene Versuche mit Ergänzungen oder Alternativen zum standardisierten System, die eine Erwähnung verdienen.

Eine Alternative zu den Generalbaßvorzeichen ist in zwei neapolitanischen Drucken zu beobachten 214 . Francesco Costanzo de Cosena215 verwendete den Buchstaben " $M$ " ("maggiore") als Erhöhungs- und die Silbe "mi" ("minore") als Erniedrigungszeichen innerhalb der Bezifferung. Diese Buchstaben stehen jeweils nach der Ziffer "3". In ähnlicher Weise verfuhr Scipione Dentice. Er notiert in seinen Madrigali spirituali (1629)216 die Abkürzungen "Min." und "Mag.". Hier stehen die Abkürzungen jedoch unter den Ziffern21?.

In je einem Satz Grammatico Metallos218 und Giovanni Battista Riccios219 sollen Solmisationssilben über dem Baß eine Bezifferung ersetzen. Während bei Metallo die Situation auch ohne jede Erläuterung eindeutig ist220, hätte bei Riccio eine Bezifferung denselben Zweck besser und eindeutiger erfüllen können221. In dem Druck Riccios sind die anderen Sätze reichlich beziffert.

213. Vgl. die Tabelle 4 im zweiten Band dieser Arbeit.

214. Dem Verfasser sind aus dieser Zeit nur sehr wenige neapolitanische Musikdrucke mit Basso continuo bekannt. Die Blüe der weltlichen Musik Neapels um 1600 (vgl. Anna Mondolfi, Art. Neapel in MGG, Bd.9, Sp.1307 ff, bes.1316 ff.) hatte zur Folge, daß auch die neapolitanischen Drucker hauptsächlich solche Musik (ohne Basso continuo) veröffentlichten. Für diese Arbeit wurden insgesamt funf neapolitanische Drucke mit Basso continuo ausgewertet: S. Anagnino, Nova sacra cantica in Dei (Vitale 1617), F. Costanzo da Cosena, Il primo libro delli mottetti (Vitale 1621), G. M. Sabino, Secondo libro delli mottetti (Magnetta 1626), ders., Psalmi de vespere (Magnetta 1627) und schließlich S. Dentice, Madrigali spirituali (Scoriggio 1629). Lediglich der Continuo Anagninos entspricht denen im ibrigen Italien. Bei den beiden Drucken Sabinos sind die Generalbaßvorzeichen, wahrscheinlich mangels einer entsprechenden Type, immer auf die oberste Notenlinie des Basses gedruckt (statt dariber); zu den verbleibenden Drucken vgl. im folgenden.

215. I1 primo libro delli mottetti (1621).

216. Zu diesem Druck gibt es kein Continuo-Stimmbuch. Lediglich zu dem Madrigal Mentre poposa A 4. steht im Quinto ein Basso, che suona.

217. Die Bezifferung ist ausgesprochen reichhaltig.

218. In Motetti, Magnificat, et madrigali spirituali (1613) auf S.21 des Bassus per sonar 1 'organo.

219. In $I 1$ terzo libro delle divine lodi musicali (1620) auf $\$ .45$ der partitura (hier $=$ Continuo-Stimme) .

220. Hier ist im Baß die Folge "fis" - "d" mit den Silben "mi" - "ut" unterlegt. Daß es sich bei dem "fis" um die Terz eines D-Dur-Akkordes handelt, wäre selbst ohne jede Bezifferung selbstverständlich.

221. Hier werden Kadenzen iber Haltetönen statt mit einer Bezifferung mit Solmisationssilben bezeichnet (zweimal "la sol fa mi" und einmal "la fa 
Sowohl die Minor-Maior-Bezeichnungen als auch die Solmisationssilben sind nur in sehr wenigen Drucken nachzuweisen. Eine größere Verbreitung erfuhr eine dritte Art, die Bezifferung zu ergänzen, nämlich die mehrstimmige Notierung einzelner Stellen innerhalb einer Generalbaßstimme222. Dieses Verfahren fand in der Regel bei geringstimmigen - meist zweistimmigen Passagen nur der tiefen Stimmen Verwendung (z.B. oft am Anfang fugiert beginnender Kompositionen). Sicherlich soll vermieden werden, daß die $\mathrm{Be}^{-}$ gleitung hier die anderen Stimmen zudeckt oder im Ambitus überschreitet. Diese zusätzlichen Stimmen verringern dariber hinaus den Unterschied zwischen Generalbaß- und collaparte-Spiel - er wäre an diesen geringbesetzten Stellen besonders deutlich zu hören. Ohne Zweifel sind diese Stimmen so wie sie notiert sind auch zu spielen'223.

\subsection{Die DiRekTionsfunKtion DER CONTINUO-STIMMEN}

Nach den Generalbaßstimmbichern erfolgte auch die Direktion der Musik 224 . Continuo-Stimmen erschienen zunächst fast uberwiegend zu Sammlungen mit Kirchenmusik. Sicher hat man sich hier den Organisten zugleich auch als Leiter der Aufführungen vorzustellen. In einem Großteil der Generalbaßstimmen stehen ganz konkrete Hilfen für die Direktion der Musik225. Neben Text-

re".

222. L. Balbi, Messe et motetti (1605, nach Kinkeldey, Orgel, S.210), L. Leoni, Primo libro de motetti (1608, nach Kinkeldey, S.210, dort auch ein Notenbeispiel eines dreistimmigen Anfanges), A. Agazzari, Psalmorum ac Magnificat (1611), ders., Psalmi ac Magnificat (1611, 2/1615), 0. Bartolini, Compieta con le lettanie (1613), S. d'India, Il terzo libro de madrigali (1615), T. Merula, Il primo libro delle canzoni (1615), A. Cifra, Motecta, et psalmi (1629) und B. Montalbano, Sinfonie (1629). Auch in Handschriften sind solche zweistimuigen Bässe zu finden, zumal hier keine drucktechnischen Probleme iberwunden werden muissen. Unter den Kasseler Gabrieli-Handschriften sind mehrstimmige Stellen in den Continuo-bzw. Orgelstimmen der Sätze "Surexit Christo" (Landesbibliothek und Murhardsche Bibliothek 20 Ms.Mus. $51^{\mathrm{b}}$ ), "Dulcis Jesu" (20 Ms.Mus. 53०), "Audite principes" (20 Ms.Mus. 62 ${ }^{\mathrm{f}}$ ) sowie "Canzona in Echo" (4० Mus. 147d) zu finden. Die Bc.-Stimme zu "Audite principes" ist teilweise sogar auf zwei Systemen mit bis zu 3 Stimmen notiert.

223. Ebenfalls mitzuspielen sind sicher einzelne Terzen oder ganze Akkorde, wie sie - unabhängig von einer Stimmfuhrung - in der Continuo-Stimme zu G. M. Cesares Concerti ecclesiatici (1614) zu finden sind. Hier wird vor allem die gewiinschte Lage des Akkordes vermittelt.

224. Vg1. Fritz Oberdörffer, Art. Generalbaß in MGG, Sp. 1710. A. Banchieri nennt in der Cartella musicale (1614), S.215, als besonderen Vorzug der "Basso continui spartiti" (Continuo-Stimmen mit Taktstrichen) die größere Sicherheit des Organisten beim Führen des Taktes (vgl. den Abdruck des entsprechenden Kapitels im zweiten Band dieser Arbeit auf S.21 f).

225. Direktionshilfen sind in etwa der Hälfte aller Generalbaßstimmen zu finden, wobei eine steigende Tendenz beobachtet werden kann (1.Jahrzehnt des 
marken sind häufig Angaben tiber Besetzungswechse1, wie "A 3 "2?6, "A 8 ", "Basso (Canto, Tenore, etc.) solo" oder nur "solo" und "tutti"227, in den Continuo-Stimmen enthalten. Abkirzungen wie "C.P." ("Canto Primo") oder "B.S." ("Basso secondo") fanden ebenfalls Verwendung. Auch zu einer Zeit, in der Continuo-Stimmen bereits zu Musik aller Gattungen erschienen, sind Direktionshilfen vor allem in der Kirchenmusik anzutreffen 228 . Dies verwundert nicht, ist doch das Madrigal in erster Linie eine Musik für ein Solistenensemble, während man in der Kirchmusik mit Chören zu rechnen hat. Auf den nicht immer hohen Qualitätsstandard dieser Chöre weist das oben zitierte Nachwort von Paolo Agostini229 recht eindrucksvol1 hin.

Auch wenn die Direktion nicht dem Organisten oblag - und dies ist vor allem bei grobbesetzten Werken wahrscheinlich -, erfolgte sie in der Regel nach einer Continuo-Stimme. Lodovico Viadana empfiehlt dem "Maestro", in die Stimme des Organisten mit hinein zu schauen230, Michael Praetorius sagt, daß aan auch für den "Director" eine Continuo-Stimme abschreiben so11291. In

17.Jahrhunderts: ca. 42\%, 2.Jahrzehnt: ca. 47\%, 3.Jahrzehnt: ca. 62\%).

226. Es können hier aufgrund der Menge dieser Quellen keine einzelnen Belege gegeben werden.

227. Neemann, Laute, S.530, vermutete, solche Angaben bezögen sich auf die Generalbaßbesetzung (vg1. weiter unten). Bei allen ausgewerteten Quellen mit solchen Angaben war der Bezug auf die anderen Stimmen deutlich. Daß diese Angaben jedoch auch Einfluß auf die Ausführung des Continuo haben, liegt nahe ("tutti" $\rightarrow$ zahlreiche Register bzw. Stimmen, "solo" $\rightarrow$ wenig Register bzw. Stimmen; vg1, weiter unten im Abschnitt III.2.2.).

228. In den Drucken geistlicher Musik lag der Anteil der Continuo-Stimmen mit Direktionshilfen bei ca. $60 \%$ (1.Jahrzehnt des 17.Jahrhunderts: $50 \%$, 2.Jahrzehnt: ca. 52\%, 3.Jahrzehnt: ca. 70\%). Verständlicherweise handelt es sich bei der Mehrzahl der Continuo-Stimmen ohne Direktionsangaben um Generalbaß-Stimmen geringbesetzer Musik. Auch persönliche Neigungen bzw. Abneigungen einzelner Komponisten konnten dazu führen, daß die Generalbaßstimmen besonders reichlich oder auch gar nicht mit solchen Hilfen versehen sind. So sind zum Beispiel in keiner der zahlreichen ausgewerteten ContinuoStimmen Antonio Cifras Direktionshilfen zu finden.

229. Spartitura delle messe del primo libro (1627). Das Nachwort ist weiter oben im Abschnitt II.2.3.2 wiedergegeben.

230. In der Vorrede zu den Salmi a quattro chori (1612) heißt es, daß der "Maestro" neben dem Favoritchor stehen und in die Continuo-Stimne des Organisten hineinschauen soll. Er möge den Gang der Musik genau verfolgen und angeben, wann einer, wann zwei, drei, vier oder fuinf Stimmen singen. An Tutti-Stellen soll er alle Chöre anschauen, beide Hände heben und damit anzeigen, daß alle zugleich singen (vgl. den vollständigen Wortlaut dieses Vorwortes im zweiten Band dieser Arbeit auf S.17 f).

231. Syntagmatis musici ... tomus tertius (1619), S. 145. Von den Generalbaßstimmen möge man nach Praetorius mehrere Abschreiben, damit man sie an die verschiedenen Generalbaßspieler verteilen (s.u.) "und der Director auch einen davon vor sich selbsten behalten könne: Damit er nicht allein des 
einigen handschriftlichen Stimmensätzen mit mehreren Continuo-Stimmen ist je eine mit "Guida" bezeichnet232.

\subsection{Die Besetzung DER GENERALBABStIMME/GENERALBABgRUPPE}

Zu den vieldiskutierten Fragen des Generalbaßkomplexes gehört die Frage nach der Besetzung des Continuos. Fast von Anfang an sind auf den Titeln der Generalbaßstimmbicher bzw. der monodischen Partituren häufig Angaben zur Besetzung des Continuos enthalten. Hierin ist nur z.T. eine Parallele zu den ebenfalls um 1600 aufkommenden Besetzungsangaben in den anderen Instrumentalstimmen zu sehen. Die Besetzungsvorschriften in den Continuo-Stimmen knuipfen auch an die bereits seit langem bestehende Tradition an, bei Drucken in instrumentenspezifischer Notation, d.h. in der Regel in Tabulatur, im Titelblatt auf das vorgesehene Instrument hinzuweisen 233.

Schon aus praktischen Grinden ist die Besetzung des Generalbasses von der Art der Musik abhängig: In Kirchen konnte vom Vorhandensein einer Orgel ausgegangen werden, an anderen Orten nicht unbedingt. Ferner spielt die Tradition der Lautenlieder und des sich-selbst-Begleitens bei der Besetzung des Continuos in der Monodie eine bedeutende Rolle. Aus diesen Gründen soll die Behandlung dieser Fragen nach den verschiedenen Musikarten getrennt erfolgen. Zusätzlich sollen in einem abschließenden Kapitel die Ergebnisse zu der Frage nach der Verdoppelung der Baßstimme durch ein Melodieinstrument zusammengetragen und durch weitere Informationen ergänzt werden.

\section{3,1. Die Besetzung des BAsSo CONTINUO IN DER KiRChENMUSIK}

In der Kirchenmusik ist zunächst immer an die Orgel als Continuo-Instrument zu denken. Dieses Instrument war in den Kirchen in aller Regel vorhanden. Es wurde wahrscheinlich schon im 16.Jahrhundert benutzt, um den Chor collaparte zu begleiten, zu stiitzen oder auch fehlende Stimmen zu ersetzen (s.o.). Bei dem iberwiegenden Teil der Continuo-Stimmen zu Drucken geistlicher Musik wird bereits in Stimmbuchtiteln wie "Basso per 1'organo", "Bassus ad organum" oder auch nur "Organo" auf die Orgel hingewiesen. Fehlt eine solche Betitelung gibt es häufig andere eindeutige Hinweise auf die Orgel als Continuo-Instrument. Sie wird in Vor-bzw. Nachworten genannt234, die nicht

Tacts halben / wenn sich derselbe in Tripeln und sonsten verendert / sondern auch einen unnd dem anderen Chor einzuhelffen / und den ganzen Gesang vor sich haben möge." (vgl. den vollständigen Abdruck dieser Stelle im zweiten Band dieser Arbeit auf S.24).

232. Murhardsche und Landesbibliothek, Kasse1, 2॰ Mus. 51b ("Surexit Christo" von. G. Gabrieli), 20 Mus. 49b ("Veni Sancte Spiritus" von H. Schitz) und $2^{\circ}$ Mus. $49 \mathrm{~h}$ ("Liebster sagt in süssen Schmerzen" ebenfalls von H. Schutz).

233. Dazu mehr im Abschnitt III.2.1.1.

234. So bei A. Banchieri, Sacra armonica (1619, wiedergegeben auf S.7 f im zweiten Band dieser Arbeit), V. Bona, Li dilettevoli introiti (1611), G. P. 
selten schon ausdrücklich "alli honorati organisti" (o.ä.) gerichtet sind235. Bei doppelchöriger Musik ist gelegentlich vorgeschrieben, welcher der beiden Chöre "cum Organo" zu musizieren ist236. Die Angabe "solo ne11'organo" wird verwendet, um eine solistische Stelle zu bezeichnen237. $\mathrm{Da}$ eine Orgelbegleitung selbstverständlich war, läßt sich auch daran erkennen, daß die Orgel in manchen Drucken nur erwähnt wird, wenn sie nicht spielen so11238. Registerangaben in Continuo-Stimmen weisen ebenfalls zweifelsfrei auf die Orgel als Generalbaßinstrument hin 239 .

Freilich gibt es auch Ausnahmefälle, bei denen ein anderes Instrument an die Stelle der Orgel tritt. So war die Mitwirkung der Orgel bei Trauerfeiern und Totenmessen vielerorts nicht erlaubt. Es wurde dann entweder "a cappe11a" gesungen oder auf ein anderes Continuo-Instrument zurückgegriffen 240 .

Cima, Concerti ecclesiastici (1610, abgedruckt im zweiten Band dieser Arbeit auf S.12), R. Micheli, Psalmi ad officium (1610), G. P. Nodari, Harmonicum concentum (1620) und E. Porta, Sacro convitio musicale (1620, vgl. im zweiten Band dieser Arbeit auf S.14).

235. Es sol1 hier jedoch auch auf den Umstand verwiesen werden, daß das Vorwort zu G. Marinis secondo libro de madrigali (1618) Alli Organisti gerichtet ist, obwohl der Titel ein Cenbalo als Continuo-Instrument verlangt ("con $i l$ basso continuo per il clavicembalo").

236. In der Baßpartitur zu G. G. Gastoldis Messe et motetti a 8 (1607) steht "secundus Chorus cum Organo".

237. In der Motette "Cantate Domino" aus G. B. Biondis quarto libro delli concerti (1611) - diese Motette zeichnet sich durch wechselnde Besetzung aus - sind die solistischen Teile mit einem Zusatz wie "Tenore solo Con 1'Organo" oder "Un tenore solo Canterà ne11'Organo" kenntlich gemacht.

238. In D. Massentios Motecta ... liber secundus (1614) tragen einzelne Sätze den Hinweis "Con Organo, \& senza" bzw. "Si possono cantare con Organo, \& senza"; bei G. Finetti, Corona Mariae ... liber quintus (1622), sind drei Stiicke mit "sine Organo si placet" iberschrieben.

239. Meist sind Registerangaben nur in auch im Titel als Orgelstimme bezeichneten Stimmen vorhanden. Eine Ausnahme ist hier der "Bassus generalis" zu C. Monteverdis Sanctissimae Virgini missa (1610). Hier belegen nur die Registerangaben, daß die Orgel das gewünschte Continuo-Instrument ist. In A. Banchieris Terzo libro di nuovi pensieri (1613) - hier werden im Titel mehrere Harmonieinstrumente neben der Orgel genannt - sind die Registerangaben ebenfalls eine Hilfe für die Zuweisung an die Orgel.

240. Vg1. Peter Williams, Art. Continuo in NG, Bd.4, S.690 mit einem Hinweis auf die Pompe funebri nel mortoria di Christo. Responsorii delli Matutini la sera nella Settimana Santa ... in concerto co'l basso continuo per lo clavicembalo, tiorba, o simil' istromento von F. Milleville (1624). Weitere Beispiele: "Missa pro defunctis" aus Orfeo Vecchis Missarum quinque vocum ... liber primus (1598, "Basso principale sequente"-Stimme: "Missa pro defunctis in hoc libro non continentur"), V. Bona, Lamentationi della Settimana Santa ... et un motetto del S.Sepolcro ... con il basso continuo per il clavicembalo (1616, zitiert nach RISM B 3434)) und A. Gregori, Cantiones ac 
Auf die Vorzuge von anderen Continuo-Instrumenten auch in der geistlichen Musik - insbesondere bei kleinen Besetzungen - weist Adriano Banchieri hin. Sein Terzo libro di nuovi pensieri ecclesiastici (1613) enthält ein "Concerto alla lettione di Compieta. per cantarsi nell'Organo; ma molto meglio nell'Arpicordo, d Arpichitarone". In der Cartelle musicale (1614) empfieh1t er statt der Orgel auch die "Clauicembali di dui registri" für das Continuo-Spie1241. Die Besetzungsangabe "Organo o Chitarrone" schließlich findet sich in der Bc.-Stimme zu seinen Dialoghi, concerti, sinfonie $e$ canzoni (1625). Sicher ist davon auszugehen, daß die Möglichkeit bestand, konzertante Kirchenmusik, auch wenn es nicht ausdrücklich vorgeschrieben ist, ähnlich zu besetzen wie vergleichbare weltliche Musik. Dies belegen nicht zuletzt die Aufzählungen der verschiedenen "Fundamentinstrumente" von Antonio Agazzari242. Agazzari weist hier auch auf die abweichende Besetzung bei geringstimmiger Musik hin.

Bei den Kirchenmusikdrucken, bei denen mit Sicherheit von der orgel als Continuo-Instrument ausgegangen werden kann, stellt sich weiter die Frage, ob die Orgel das einzige Continuo-Instrument war. Grundlage zahlreicher Stellungnahmen $\mathrm{zu}$ dieser Frage ist Antonio Agazzaris Schrift Del sonare sopra ' 1 basso con tutti li stromenti (1607)243 - ein Traktat der ausschließlich die Kirchenmusik behande1t244. Agazzari nimmt hier eine Unterteilung in Fundament- und Ornamentinstrumente vor. Die Fundamentinstrumente, es sind zunächst die Orgel und das Cembalo, bei geringbesetzten Stücken auch Laute, Harfe, Theorbe etc. 245 stutzen "tutto il corpo delle voci", die Ornamentinstrumente, er nennt Laute, Theorbe, Harfe, Spinett, Violine und

sacrae lamentationes singulis vocibus concinendae cum basso continuo praesertim ad clavicymbalum (1620, zitiert nach RISM G 3811). Auf ein Verbot der Orgel bei Totenmessen weist auch A. Banchieri, Conclusioni, S.17 hin (mit Ausnahmeregelungen für Totenmessen kirch1icher Würdenträger). In A. Brunellis Missae tres pro defunctis können laut Titel jedoch nur die ersten beiden ohne Orgel musiziert werden ("que omnia, excepta prima, et seunda missa, sine organo non modulantur", zitiert nach RISM B 4650).

241. In seinen "Osservationi ... in componere gli Bassi continui sotto le voci" (S.214; dieser Abschnitt ist im zweiten Band dieser Arbeit auf S.21 f abgedruckt). Auch das Vorhandensein einer "Regola in accordare arpicordi" in der zweiten Auflage des L'organo suonarino (1611), S.44 der Faksimile-Teilausgabe, belegt, daß Banchieri an die Möglichkeit der Verwendung anderer Harmonieinstrumente neben der Orgel in der Kirchenmusik dachte.

242. Del sonare sopra' 'l basso con tutti 1 i stromenti (1607). Hierauf wird im nächsten Absatz ausführlich eingegangen werden.

243. Vg1. Arnold, Accompaniment, S.68, Beat, Monteverdi, S.280, Donington, Monteverdi, S.259, Leichtentritt, Vortragspraxis, S.149, Fritz Oberdörffer, Art. Generalbaß in MGG, Bd.44, Sp.1710, ders., Generalbaß, S.195 ff, Rose, Agazzari und Schneider, Basso continuo, S.14. 244. Vg1. z.B. daselbst S.12. 1609 erschien der Traktat auch als Vorwort zu Sacrarum cantionum ... liber II. Vgl. auch Williams, Basso Continuo, S. 137. 245. De1 sonare sopra' ' 1 basso, S.3. 
Pandora246, sollen "sopra $i 1$ medessimo basso compor nuove parti"247. Die woh1 weitgehendste Auslegung haben diese Ausfuhrungen Agazzaris durch Gloria Rose erfahren ${ }^{48}$. Sie sieht hier ein "improvisierendes Orchester" gefordert. Bestätigungen für diese These glaubt sie in Abbildungen 249 , Aufführungsbeschreibungen 250, Opern-Partituren251 und Titeln von Madrigalbüchern (die sie ganz offensichtlich ohne Kenntnis des Inhaltes heranzieht) 252 zu finden.

246. S.3.

247. S. 8 .

248. Agazzari.

249. S.382 (Titelbild zu F. Bianciardis Breve regola per imparar' a sonare sopra il basso, 1607, abgebildet bei Bernhard Billeter, Art. Bianciardi, Francesco in MGG, Bd.15, Tafe1 25).

250. S.384 (Malvezzis Vorwort zu dem Intermediendruck von 1591, wiedergegeben bei Vogel Bibliothek, Bd.I, S.382 ff. Vgl. dazu weiter unten).

251. Rose erwähnt eine ganze Reihe von Opernpartituren, die entweder keine Instrumentalstimmen außer dem Bc. haben oder bei denen die Besetzung der Instrumentalstimmen in Ritornellen nicht oder auch nur im Vorwort genannt wird. Sie ist der Meinung, daß diese ausgeschriebenen Ritornelle Ausnahmen darstellen und die Instrumente sonst (also zu den Vokalteilen) ihre Stimmen nach dem Baß improvisierten (S.384 ff). Sie berücksichtigt nicht, daß alle diese Opernpartituren nicht Aufführungsmaterial, sondern Archivmaterial waren (sofern handschriftlich) bzw. der Dokumentation oder Repräsentation dienten. Auch das Vorhandensein von einer oder zwei zusätzlichen Stimmen bei einem Ritornell belegt nicht unbedingt das Mitwirken von weiteren Instrumenten außer der Continuo-Gruppe. Daß auch partiturartig notierte Ritornelle von dem Continuo-Instrument ubernommen werden können, belegen die Vorworte zu A. Brunellis Scherzi ... libro secondo (terzo) (1614 bzw. 1616, abgedruckt bei Sartori, Bibliografia I, S.200 (1614 f) und S.220 f (1616 a)) sowie die Chitarronentabulatur in G. G. Kapsbergers Libro primo di arie (1612). Zu dem Ritornello zu "Interotte speranze" enthält die Tabulatur keinen Begleitsatz, sondern eine Intavolatur.

252. Die von Rose (in englischer ubersetzung) zitierten Titel lauten im Original: G. Priuli, Il terzo libro de madrigali a cinque voci. Di due maniere, l'una per voci sole, l'altra per voci, \& instrumenti (1612), P. Pace, Madrigali a quattro et a cinque voci, parte con sinfonia se piace, $\theta$ parte senza, avertendo che quelli delle Sinfonie non si possano cantare senza sonarli ma gli altri si (1617) und G. Valentini, Il quinto libro de madrigali divisio in tre parti. Nella prima si contengono madrigali à tre concertati con l'istromento. Nella seconda scherzi à sei concertati con l'istromento. Nella terza madrigali à sei per cantarsi senza istromento (1625). Es kann jedoch kein Zweifel daran bestehen, daß Pace und Valentini nur das Harmonieinstrument bzw. den Basso continuo iberhaupt meinten. Es wird in diesen Titeln zwischen konzertanten Generalbaßmadrigalen und "klassischen" Madrigalen ohne Generalbaß unterschieden (vg1. hierzu auch Hellmut Federhofer, Art. Valentini, Giovanni, in MGG, Bd.13, Sp.1230). Bei Priuli handelt es sich bei den "due maniere" nicht, wie Rose vermutet, um verschiedene Auffuhrungsmöglichkeiten, sondern um Madrigale mit und ohne 
Die These von einem "improvisierenden Orchester" wird, in abgemilderter Form, auch von Hugo Leichtentritt253, Frank T. Arnold254 und Robert Donington 255 vertreten. Lediglich Fritz Oberdörffer ist ihr bisher entschieden entgegengetreten256. Er sieht in Agazzaris Ausfuhrungen zu den Ornamentinstrumenten Anweisungen an den Dirigenten, bei Bedarf anhand der Baßstimme neue Stimmen hinzuzukomponieren 257 , sowie eine Beschreibung der Verzierungspraxis der Instrumentalisten 258 . Das Hauptanliegen Agazzaris ist nach Oberdörffer, "den neuen konzertanten Stil gegen dessen Verächter" zu verteidigen 259 .

In Anbetracht des Wortlautes einiger Stellen in Agazzaris Del sonare stellt sich aber die Frage, inwieweit dieser Traktat bzw. Teile von ihm uberhaupt als Anweisung gedacht waren. Man gewinnt stellenweise den Eindruck, es handele sich hier um eine Streitschrift für den Generalbaß260. Statt um die Vermittlung einer vorhandenen Praxis geht es Agazzari allem Anschein nach vielmehr um eine Darstellung der denkbaren Möglichkeiten des von ihm so gelobten Spielens uber dem Baß. Wird in anderen Quellen auf Agazzaris Schrift hingewiesen, so geschieht dies nur als Anleitung, den Begleitsatz nach den Ziffern einzurichten261. Lediglich Michael Praetorius zitiert auch

obligate (freilich ausgeschriebene) Instrumentalstimmen.

253. Vortragspraxis, S.149.

254. Accompaniment, S. 68 .

255. Monteverdi, S.259.

256. Art. Generalbas in MGG, Bd.4, Sp.1710 und GeneralbaB, S.195 ff.

257. Art. GeneralbaA in MGG, Bd.4, Sp. 1710.

258. Generalbaß, S.195.

259. Art. Generalbaß in MGG, Bd.4, Sp. 1710.

260. Insbesondere S.10 f. Hier nennt Agazzari vol1 Polemik drei Argumente, die für den Generalbaß und gegen die Partitur oder Intavolatur sprächen: 1. der neue Stil, 2. die Bequemlichkeit und 3. die Vielzahl der Werke, die man "al conserto" benötigt (S.10). Im einzelnen führt er zu Punkt 2 (Bequemlichkeit - "commodită" die Schwierigkeit für Augen und Gedächtnis beim collaparte-Spiel an (S.12: "oltre che chi desidera imparare a sonare, e sciolto dalla intavolatura, cosa à molti difficile e noiosa; anzi molto soggetta à gli errori perche l'occhio, e la mente è tutta occupata in guardar tante parti massime venendo occasione di consertar all'improuisio. "). Zu Punkt 3 wird gesagt, daß ein Organist unzählige Werke zu spartieren oder intavolieren hätte, um seinen Dienst zu erfullen, so daß seine Bibliothek schon nach einem Jahr größer wäre als die eines Doktors der Rechte (S.12: "se si havessero ad intavolare, o spartire tutte 1 'opere, che si cantano fra 1 'anno in una sola Chiesa di Roma; doue si fa professione di consertare, bisognarebbe all'Organista che hauesse maggior libraria, che qual si voglia Dottor di legge"). Letztendlich werden freilich diese Grüde auch zum endgültigen Siegeszug des Basso continuo beigetragen haben.

261. So in den Vorworten zu A. Banchieris Ecclesiastiche sinfonie (1607) und zu A. Franzonis Concerti ecclesiastici (1611). Banchieri verweist auch in seinen "Osservationi ... in componere gli Bassi continui sotto le voci" 
Agazzaris Ausfuhrungen zu den Ornamentinstrumenten. Er bezieht diese Ausführungen jedoch auf das Auszieren der Baßstimme262.

Auf jeden Fall bleibt festzustellen, daß sich für eine Praxis eines nach Generalbaßstimmen improvisierenden Orchesters keine weiteren theoretischen Belege finden und auch in den praktischen Quellen Anhaltspunkte für eine solche Verfahrensweise fehlen 263.

Dennoch gibt es eine ganze Reihe von Hinweisen auf die Beteiligung von anderen Instrumenten neben einer Orgel als Generalbaßinstrumente auch in der Kirchenmusik. Dies ist sicher nicht zufällig vor allem für mehrchörige Musik belegt. Mehrchörige Musik war nicht al1täglich. Sie wurde an Festtagen aufgefuhrt, die einigen Aufwand rechtfertigten. Die Mitwirkung von Instrumenten bei solch großbesetzter Musik ist auch aus dem 16.Jahrhundert belegt 264 . Die

aus der Cartella musicale auf Agazzari (1614, der Abschnitt ist im zweiten Band dieser Arbeit auf S.21 f wiedergegeben).

262. Syntagmatis musici ... tomus tertius (1619), S.139-152. Praetorius lehnt sich stark an Agazzari an und ubersetzt dort Teile des Del sonare. Aus Agazzaris "poiche deue sopra il medesimo basso compor nuoue parti sopra, e nuoui, e variati passaggi, e contrapunti. Onde chi suona leuto ..." (S.8) wird bei Praetorius "die weil man alda ober demselben BaB/ newer Passaggien, Contrapunct; und also $f$ a $s t$ ganz newe Parteien oder Stimmen Componieren muB: We 1 ches in den Fundament Instrumenten $n i c h t$ so groB von nöten $\mathrm{i} s t$. Soll der wegen der Lautinist ..." (S.146, 2.Zählung. Hervorhebungen nicht original). Nicht neue Stimmen, sondern fast neue Stimmen álso. Der von Praetorius eingefügte Hinweis, dies sei bei den Fundamentinstrumenten nicht so nötig, erklärt vollends, was Praetorius meint. Es geht nicht um das Hinzufigen ganz neuer Stimmen - dies ist bei den Fundamentinstrumenten freilich nötig (Aussetzung) -, sondern um das Ersetzen der notierten durch eine neue, ausgezierte Stimme. Das Verzieren des Basses ist jedoch "nicht so groß von nöten".

263. Auf ein einziges Zeugnis eines solchen "improvisierenden Orchesters" kann dennoch verwiesen werden, wenngleich hier der Zusammenhang die Sonderstellung dieser Stelle deutlich macht. In den Intermedi fatti per la commedia F. Vitalis (1623, Partiurdruck) endet die Einleitungssinfonia mit fünf Breven " $d$ " im $\mathrm{Ba}$ mit einem "非" darüber. In den anderen Systemen ist nichts mehr eingetragen. Stattdessen findet sich der Text "Sopra questa corda si sonava con $i$ medesimi strumenti della sinfonia a imitatione di trombe a beneplacito de recitanti". Auf einen ähnlichen Fall in einem Musikdruck des späteren 17. Jahrhunderts weißt Mangsen, Ad libitum, S.37, hin (A. Guerrieri, Sonate di violino, Venedig 1673).

264. Einige archivalische Belege von festlichen Aufführungen unter Beteiligung von Orgeln und anderen Instrumenten in Padua aus der Zeit um 1520 hat Ravizza, Ruffino, S.333 und 335 mitgeteilt. Ahnliche Hinweise finden sich bei Dahlhaus, Aufführungspraxis, S.247 f. DaB mehrchörige Musik in erster Linie bei festlichen Anlässen zur Aufführung kam, kommt teilweise bereits in den Titeln der Sammlungen durch Zusätze wie "nelle gran Solenità di tutto 
Instrumente vermehren die Klangpracht dieser Musik. Die Beteiligung von mehreren Continuo-Instrumenten kann dariber hinaus durch die bei räumlich getrennter Aufstellung entstehenden Entfernungen sinnvoll werden.

Bereits die Continuo-Stimme zu Alessandro Striggios vierzigstimmigen "Ecce beatam Iucem"265, die älteste bekannte Continuo-Stimme uberhaupt, nennt eine große Continuogruppe (Posaune, Orgel, Laute, Cembalo und Viola266). Die große Zahl der Instrumente wurde benötigt, um den 40 Stimmen (nach den Ausführungen Massimo Troianos waren darunter bei der Auffuhrung 1568 in Müchen auch acht Posaunen, acht Gamben und acht F1öten ${ }^{267}$ ) ein vernehmbares Fundament zu geben. Hinweise auf eine räumlich getrennte Aufstellung sind hier nicht vorhanden; nach der Besetzungsangabe in der Continuo-Stimme soll die Generalbaßgruppe zusammen in der Mitte des Ensembles, "in mezzo de1 circolo"268, stehen.

Ganz konkrekte Angaben zur Besetzung vielchöriger Musik enthält der "Modo di concertare" zu Lodovico Viadanas "Salmi a quattro chori per cantare, e concertare nelle gran Solenità di tutto 1 'anno" (1612)269. Zur räumlichen Aufstellung wird wenig gesagt; nur, daß der Favoritchor bei der Hauptorgel stehen sol1. Die Besetzung ist aber bis ins Detail aufgelistet: Der Favoritchor soll mit fünf Solisten besetzt werden, dazu die Hauptorgel und ein Chitarrone "à chi piace", der zweite Chor, die Capella, soll mindestens aus 16, besser 20-30 Sängern und Instrumentalisten bestehen. Ein Continuo-Instrument ist nicht vorgesehen. Der dritte Chor, ein Hochchor, ist vokalinstrumental gemischt besetzt mit deutlicher Betonung der tiefsten Stimme: "cantato da più voci, con Tromboni, e Violone. e Organo all'Ottaua alta"27o. Ahnlich ist auch die Besetzung des vierten (Tief-) Chores. Wieder wird fur den $\mathrm{Ba}$ B eine besonders starke Besetzung gefordert: "cantato da profondi Bassi, con Tromboni, e Violoni doppi, e fagotti, con Organo all'ottaua bassa". Für alle erwähnten Orgeln ist dem Druck jeweils eine Stimme beigegeben: Neben dem "Basso Generale" (für die Hauptorgel) ein "Basso per 1'Organo All'Ottaua Alta del Terzo Choro" und ein "Basso per l'Organo All'ottaua Bassa del Quarto Choro Graue"271.

I'anno" (Viadana, Salmi a quattro chori, 1612) zum Ausdruck.

265. 1587, Auffihrung wahrscheinlich bereits 1568 ; zu dieser Stimme s.o.

266. In der Beschreibung der Aufführung von 1568 (M. Troiano, Dialoghi, 2/1569), fol. 146', wird keine Orgel genannt.

267. fol. 146": "otto tromboni, otto viole da arco, otto flauti grossi, uno istromento da penna, \& un liuto grosso, e tutto il restante supplirono le uoci".

268. Vgl. die vollständige Wiedergabe dieser Besetzungsangabe weiter oben.

269. Wiedergegeben im zweiten Band dieser Arbeit auf S.17 f.

270. Die Besetzung der ibrigen Stimmen wird weiter unten zur Sprache kommen.

271. Zwei verschiedene Continuo-Stimmen erschienen auch zu L. Leonis Prima parte dell'aurea corona ingemmata a d'armonici concerti, a dieci con quattro vaci, \& sei istromenti... et a due chori divisi, adoprando $1 i$ bassi 
Eine solche Verteilung verschiedener Continuo-Instrumente auf die einzelnen Chöre wird man bei räunlich getrennter Aufstellung sicher auch dort annehmen können, wo dies nicht ausdrücklich gefordert ist272. Von Abschreiben verschiedener Continuo-Stimmen für mehrchörige Aufführungen berichtet Michael Praetorius 273.

Auch für mehrchörige Musik ist aber die räumlich getrennte Aufstellung mit mehreren Basso continuo-Instrumenten nur eine der Auffuhrungsmöglichkeiten. Die Aufstellung mehrerer Continuo-Instrumente zu einer Generalbaßgruppe, wie in der Striggio-Handschrift beschrieben (s.o.)274, und die Aufführung mit nur einer Orgel ohne große räumliche Trennung wird es ebenfalls gegeben haben; wahrscheinlich ist die letztgenannte Auffuihrungsart sogar die häufigste gewesen.

Es 1 äßt sich ein Bemühen der Verleger und Komponisten erkennen, die mehrchörige Musik in einer Form in Druck zu geben, die ohne große Muihe verschiedene Aufführungsarten zuläßt. Giacomo Vincenti teilt in einem kurzen Nachwort in der nur den ersten Chor umfassenden Randstimnenpartitur ("Spartitura per sonar nel Organo") zu Adriano Banchieris Conceri ecclesiastici (1595) mit, daß der Autor an ein "concertarla à Chori seperati" gedacht habe. Ist dies nicht gewuinscht, solle man an den entsprechenden Stellen die Stimmen des zweiten Chores ebenfalls in die "Spartitura" eintragen275. Bei nicht

dell'uno e l'altro choro con organi, chitaroni, o simili (1615, zitiert nach RISM L 2010, erhalten sind jedoch nur die Stimmbiicher Baß I und "Baritonus" II) und zu P. Quagliatis Motetti, e dialoghi a 8 (1627).

272. So nennt A. Banchieri in seiner Beschreibung einer Aufführung einer vierchörigen Messe in den Conclusioni (1609), S.50 f, zwar eine Reihe von Continuo-Instrumenten ("1'organo grosso dolce, \& soaue di quella Chiesa suonata da Gio. Pietro Negri, appresso dui Violoni continoi in contrabasso, dui Clauicembali tre Liuti, \& dui Chittarroni"), erwähnt jedoch nichts von einer Zuordnung $z u$ den einzelnen Chören (vgl. zu dieser Beschreibung Banchieris Schneider, Basso continuo, S.12, Williams, Basso Continuo, S.139 und Materassi, Teoria e pratica, S.26). Hinweise auf mehrchörige Aufführungen mit mehreren Continuo-Instrumenten finden sich auch bei Wellesz, Musikpraxis, S.134 (von 1603 und 1618) und Paul Kast, Art. Rom in MGG, Bd.11, Sp.718 (von 1626). Jeweils ist im ubrigen auch von einem besonderen Festtag die Rede.

273. Abdruck dieser Stelle aus Syntagmatis musici ... tomus tertius (1619) im zweiten Band dieser Arbeit auf S.24.

274. Auch in den Kasseler Gabrieli-Handschriften mit mehreren ContinuoStimmen (Ms.Mus. 2० $51 \mathrm{a}, \mathrm{b})$ fehlt jeder Hinweis (wie etwa "tace"-Vermerke) auf eine Zuordnung bestimmter Continuo-Stimmen zu einzelnen Chören.

275. "A GLI SIG ORGANISTI. Volendo la Spartitura di tutti due Chori, sarà facil cosa accommodarla prestissimo, pigliando la parte acuta \& graue del Secondo Choro, \& dove in questa dice à 8 . lasciarlo, \& aggiungendo quella d questa, vi saranno tutti due: ma 1'Autore non 1' ha fatta, atteso che l'intenione sua è per concertarla à Chori seperati. In tanto viuete felici." 
getrennter Aufstellung soll der Organist also beide Chöre begleiten, bei einer Aufführung "à Chori seperati" wird nur der erste Chor von der Orgel gestutzt276, eine Praxis die auch anderweitig belegt ist277.

Auch die zu Anfang verbreitetste Form der Continuo-Stimme mehrchöriger Werke, nämlich die Baßpartitur, bot verschiedene Möglichkeiten. Es war leicht möglich, auf einer Orgel beide Chöre zu begleiten, da der Organist beide BaBstimmen vor Augen hatte278. Ebensogut konnte er aber auch nur bei einem Chor mitspielen. Für den anderen Chor konnte dann, bei Bedarf, leicht eine Continuo-Stimme abgeschrieben werden 279 . Baßpartituren wurden zwar bis in das dritte Jahrzehnt des 17 . Jahrhunderts hinein gedruckt 280 , ihre Häufigkeit nimmt jedoch $a^{281}$. Auch konnte bei einer Neuauflage eine ContinuoStimme eine Baßpartitur ersetzen282. Valerio Bona preist 1611 im Titelblatt zu Messa e vespero a quattro chori eine "partito delli bassi ridotti in un solo basso generale" an283. Die Zuwendung auch der mehrchörigen Musik zum einzelnen Generalbaß ist sicherlich auf einen stilistischen Wandel in einem Teil der Drucke mehrchöriger Musik zuruickzuführen. Hier lä日t sich eine Abkehr vom blockhaften Monumentalstil und eine Hinwendung zu einem zumindest mit konzertanten Abschnitten durchwobenen Stil erkennen. Dies schlie日t freilich eine zu große räumliche Distanz aus und läßt auch einen zu groß besetzten Continuo kaum wiinschenwert erscheinen.

Den Belegen für mehrere Generalbaßinstrumente in der mehrchörigen Musik stehen, sieht man von den sehr allgemein gehaltenen Angaben bei Agazzari ab $\left(s .0^{\prime}\right)$, nur sehr wenige Hinweise auf mehrere Continuo-Instrumente in der.

276. Eine Anweisung zur Herstellung einer "Spartitura" für den zweiten Chor hätte sonst sicherlich nicht gefeh1t.

277. In der Baßpartitur zu G. G. Gastoldis Messe et motetti a 8 (1607) steht "secundus Chorus cum Organo", in den Stimmbüchern des "Primo Choro" der Canzoni da sonare von P. Lappi (1616) steht "In Organo" bei der Canzona "La Monteverde".

278. Zu den Möglichkeiten des Begleitens nach einer Baßpartitur vgl. auch Horsley, Scores, S.471.

279. Ein Beleg für die Besetzung jeder Stimme einer Baßpartitur mit einer Orgel findet sich in der Kasseler Handschrift des "Christ ist erstanden à $11^{\prime \prime}$ von H. Schütz (Ms. Mus. $2052^{\mathrm{b}}$ ). Dort trägt die obere Baßstimme die Anweisung "Organo piccolo", vor der unteren steht "Organo grande".

280. So noch zu G. Priuli, Missae ... octo, novemq. vocibus (1624), M. A. Grancino, Messe, motetti et canzoni a 8 (1627), F. Bellazzo, Messe, Magnificat, et motetti ... a 8 (1628).

281. Fast alle BaBpartituren entstammen der Zeit vor 1612.

282. Dem Verfasser ist dieser Fall bekannt von Giovanni Croces Messe a $\boldsymbol{8}$ (1596, BaApartitur, 5/1612 Bc.-Stimme).

283. Leider ist diese Bc.-Stimme nicht erhalten. Ausdrücklich eine Continuo-Stimme für mehrere Chöre erwähnt auch das Titelblatt zu L. Viadanas Salmi campognoli... \& il Basso continuo per l'organo che serve l'un e l'altro choro (1612). 
geringer besetzten geistlichen Musik gegenüber. Weitere Continuo-Instrumente neben der Orgel werden in den Concerti sacri scielti, \& trasportati dal secondo, \& terzo libro de madrigali von Steffano Bernardi (1621)284 sowie in einigen Drucken Alessandro Grandis verlangt, allerdings auch dort nur sehr selten 285 .

An eine Ubertragbarkeit der Besetzungsangaben etwa der Opern (s.u.) auf die geistliche Musik ist keinesfalls zu denken. Die monumentalen Generalba日gruppen, wie sie z.B. für heutige Auffuhrungen von Claudio Monteverdis "Marienvesper" (1610)286 herangezogen werden - nicht selten mit einem Hinweis auf die Besetzungsliste des Orfeo - lassen sich historisch nicht rechtfertigen, zumal Monteverdi die Instrumentation auch in der "Marienvesper" sehr weitgehend (bis hin zur Orgelregistrierung) festgelegt hat28?.

\subsubsection{Die BESETZUng DES GENERALbAsSES IN DER WELTLICHEN MUSIK $3.3,2,1$. DIE GENERALBABINSTRUMENTE IN DER MONODIE}

Auch in der weltlichen Musik ist die Besetzung des Generalbasses abhängig von dem Raum, der Gelegenheit und der Besetzung überhaupt. Eine Untersuchung uber die Generalba日besetzung in der Monodie liegt bereits vor 288 und kann im wesentlichen bestätigt werden. Fast immer werden auf den Titelblättern die gewuinschten (oder auch die möglichen) Instrumente genannt289. Die wichtigsten Continuo-Instrumente sind hier die Zupfinstrumente - zunächst vor allem der Chitarrone und ab dem dritten Jahrzehnt des 17.Jahrhunderts zunehmend auch die "Chitarra spagnola"290 - und, seltener, die Kielklaviere291. Es

284. Bei den Sätzen "Con gli strumenti" wird als Baßinstrument (zusätzlich zum Bc.) mal eine "Thiorba o Chittarrone", mal eine "Trombone d Liutto" verlangt. Diese werden hier auch als Melodieinstrumente verwendet.

285. Ein Chitarrone ist in den Motetten "Date Domini" aus dem secondo libro de motetti (1613) und "Factum est silentium" aus dem quarto libro de motetti (1616) vorgesehen, eine Theorbe in der Motette "Viri diligite" aus dem Sammeldruck Symbolae diversorum musicorum (Venedig 1621) (Seelkopf, Grandi, S.21, 24 und 34). Ein, z.T. wohl auch zwei, zusätzliche Chitarronen werden in den Matetti a 1 , et 2 ... libro primo (secondo) $(1621,1625)$ vorausgesetzt. Hier sind die Singstimmen jeweils in Partitur mit dem Baß gedruckt. Der Titel erklärt dies: "partiti per cantar, \& sonar co'l chitarrone". Eine "Parte per l'organo" ist zusätzlich iberliefert (auf den Sachverhalt ist weiter oben im Abschnitt II 2.2.4. näher eingegangen worden).

286. Aus Sanctissima Virgini missa.

287. S.u. Der Verfasser hatte als Zinkenist Gelegenheit, an zahlreichen Aufführungen der "Marienvesper" mitzuwirken. Generalbaßbesetzungen mit allein vier ( 2 Orgeln, Cembalo, Theorbe) und mehr Harmonieinstrumenten sind nicht. selten. Auch fernliegende Instrumente wie Harfe oder Regal werden herangezogen.

288. Fortune, Instruments.

289. Vgl. die entsprechenden Titel im Quellenverzeichnis zu dieser Arbeit. 
gibt darüber hinaus Belege für die Verwendung auch der Orge1292 und der Kombination von Orgel und Chitarrone293.

\subsubsection{DIE CONTINUOGRUPPE IN DER FRUHEN OPER}

Mit großen Besetzungen der Continuo-Gruppe wartete man in der Bühnenmusik auf. Bereits an den Aufführungen der Intermedien des 16.Jahrhunderts waren mehrere Harmonieinstrumente beteiligt294. Robert L. Weaver nennt bis zu 4 Cembali295 und bis zu 12 Zupfinstrumente296. Die Instrumentationen zu den Florentiner Intermedien von 1589297 beinhalten fast immer große Gruppen von Harmonieinstrumenten.

$\mathrm{Daß}$ die Klangfarbenwechsel bereits im 16. Jahrhundert nicht zufällig, sondern bestimmten Szenentypen und Handlungen entsprechend erfolgten, hat Robert L. Weaver dargelegt298. Noch differenzierter ist der Umgang mit der Instrumentation und ganz besonders der Besetzung der Continuo-Gruppe in der Buihnenmusik des frihen 17. Jahrhunderts. Der Textaffekt soll mit allen Möglichkeiten, und dazu gehört auch die Continuobesetzung299, verstärkt werden.

Wenig bekarnt ist uber die Instrumentation der Opern des Jahres 1600. Da das ganze Interesse bei diesen Opern der neuen Art des Singens galt, waren die Möglichkeiten der Instrumentation offenbar in den Hintergrund gerückt. Lediglich in der Auflistung der Mitwirkenden am Ende des Vorwortes zu Jacobo Peris Le Musiche sopra l'Euredice (1600)300 werden Cembalo, Chitarrone,

290. Fortune, S.10 und S.12.

291. Fortune, S.10. Vg1. auch Arnold, Performing, S.322.

292. So bei J. Peri, Le varie musiche ... per cantare nel clavicembalo, il chitarrone, et ancora la maggior parte di essi per sonare semplicementi nel organo (1609, zitiert nach RISM P 1433).

293. Dies wird in einem Brief C. Monteverdis vom 21.1 .1622 erwähnt (Stefens, Monteverdi: Briefe, S.97 ff). Vgl. auch Peter Williams, Art. Continuo in NG, Bd.4, S.686 und 689.

294. Eine tabellarische Zusammenfassung der aus Festbeschreibungen geschlossenen Besetzungen verschiedener Intermedien des 16.Jahrhunderts bietet Weaver, Instrumentation, S.374 ff .

29.5. S. 374 , Florenz 1539.

296. S.375, F1orenz 1589. Genannt werden 4 Lauten, 2 Harfen, 2 Lire, 1 Chitarrone, 1 Mandola, 1 Psalterium und 1 Zitter.

297. Die in dem Druck von 1591 enthaltenen Besetzungsangaben sind aufgelistet bei Schnejder, Basso continuo, S. $56 \mathrm{ff}$. Zur Vollständigkeit und Glaubwürdigkeit dieser Besetzungsangaben vg1. unten im Abschnitt III.2.1.1.

298. Instrumentation.

299. Bereits in dem Vorwort zu E. De' Cavalieris Rappresentatione (1600) wird auf die Notwendigkeit einer textgerechten Instrumentation des Continuos hingewiesen ("Et il signor' Emilio laudarebbe mutare stromenti conforme all'affetto del recitante", vgl, den gesamten Wortlaut im zweiten Band dieser Arbeit auf S.8 ff). Dazu auch Beat, Monteverdi, S.281. 
"Lira grande" und "Liuto grosso" mit ihren Spielern genannt. Keine Angaben zur Instrumentation sind in Giulio Caccinis L'Euredice composta in musica (1600) zu finden. Auch die Ritornelle sind hier nur als Basso continuo notiert301. Etwas genauere Angaben gibt das Vorwort zu der, den Opern verwandten Rappresentatione di anmima, et di corpo Emilio De' Cavalieris (1600, wiedergegeben im zweiten Band dieser Arbeit auf S. 8 ff). Genannt werden eine "Lira doppia", ein Cembalo, ein Chitarrone oder eine Theorbe und, zusammen mit dem Chitarrone zu spielen, eine Holzorgel ("Organo soave"). Auf der Buihne sollen von dem "Piacere con $1 i$ due compagni" ein Chitarrone, eine "Chitarina alla Spagnuola" und ein "Cembaletto" gespielt werden. Auf diesen Instrumenten sollen auch die Ritornelle (meist vierstimmig notiert!) erklingen.

Uberaus detaillierte Besetzungsangaben auch der Generalbaßgruppe finden sich in Claudio Monteverdis L'Orfeo (uraufgeführt 1607, gedruckt erstmals 1609). Die Partitur enthält insgesamt 21 Angaben uber die jeweilige Continuobesetzung ${ }^{302}$, teilweise für ganze Stücke, teilweise aber auch innerhalb einzelner Nummern wechselnd. Von besonderem Interesse sind nicht so sehr die Besetzungen des Continuos bei den Ritornellen oder anderen grobbesetzten Stücken, als vielmehr die Angaben zu den rezitativischen Stellen, da hier ein Text-Besetzungs-Bezug besonders deutlich hervortritt. So wird beim Auftritt der Messaggierasos der Stimmungskontrast zwischen Messaggiera und Pastore durch unterschiedliche Besetzungen verstärkt (Messagiera: Holzorgel und Chitarrone, Pastore: Cembalo, Chitarrone und Viola). Noch deutlicher wird in den Unterweltszenen die Verschiedenheit von Caronte (Regal) und Orfeo (Holzorgel, z.T. mit Chitarrone) unterstrichen 304.

Besonders interessant sind die Wechsel in der Generalbaßbesetzung am Höhepunkt der Handlung: Orfeo (keine Angabe uber die Begleitung, vermutlich Holzorge1305) zweifelt, ob Euredice ihm folgt. Er kämpft mit seinem Verlangen, sich umzuschauen. Plötzlich hört er Lärm ${ }^{306}$ und befürchtet, daßs die Furien ihm Euredice rauben. An dieser Stelle wechselt die Besetzung. Orfeo singt nun begleitet von Cembalo, Viola und Chitarrone ${ }^{307}$. Der Stimmungsumschwung, hervorgerufen durch den Lärm, wird deutlich, der Einschub "Md che

300. Abgedruckt bei Voge1, Bibliothek, Bd.II, S.65.

301. Nur als Basso continuo notierte Ritornelle sind dem Verfasser außerdem aus C. Monteverdis Sanctissimae Virgini missa (1610), A. Banchieris Salmi festivi (1613), G. Caccinis Le nuove musiche e mova maniera di scriverle (1614) und G. F. Anerios La bella clori (1619) bekannt.

302. Auf den Seiten $10,27,28,32,36,39,42,47,50,52,64,67,68,70$, $80,81,88$ und 89 .

303. Atto secondo, S.36 der Partitur von 1609.

304. Atto terzo, S.50 der Partitur von 1609.

305. Die letzten Besetzungsangaben für die Begleitung Orfeos verlangen nur die Holzorgel (S.67 und 68 der Partitur von 1609).

306. S.80: "Qui si fa strepito dietro la tela".

307. S.80. 
odo ..." wird auf eine andere Ebene gehoben. Dann dreht er sich um. Wieder lyrisch singt er zur Holzorgel "O dolcissimi lumi, io pur vi veggio, io pur", doch damn wird ihm klar, daß er damit Euredice verwirkt hat. Wieder zu Cembalo, Chitarrone und Viola singt er "ma qual Eclissi, oimè, V'oscura?" 308 .

Monteverdi verwendete die verschiedenen Generalbaßbesetzungen hier, um unterschiedliche Handlungsebenen zu verdeutlichen, sowohl räumlich (aus der Ferne heraneilende Messaggiera und bereits in der Handlung gegenwärtiger Pastore) als auch inhaltlich (1yrisch entrückter und in die Wirklichkeit zurückgerissener Orfeo), oder um Personen zu charakterisieren (Caronte $\rightarrow$ Rega1). Die Angaben zur Besetzung können in Monteverdis L'Orfeo so detailliert sein, da es sich nicht um eine Aufführungsvorschrift, sondern um eine dokumentierende "Nachschrift" hande1t. Auf abweichende Besetzungen an anderen Höfen etwa brauchte keine Riicksicht genommen zu werden ${ }^{309}$.

Große Continuo-Gruppen sind auch von anderen Opernaufführungen oder Opernpartituren bekannt, so von Monteverdis Arianna (1608) 310 und Filippo Vitalis L'Aretusa (1620)311. Etwas kleinere Continuo-Gruppen werden in den Partituren zu den "azione" Paolo Quagliatis (1611 und 1623) ${ }^{912}$, in den Szenen aus Monteverdis Concerto $(1619)^{313}$ und in den Hochzeitsmusiken Sigismondo d'Indias aus dem Jahr $1621^{314}$ vorausgesetzt.

Diese großen Continuo-Besetzungen haben zu der verallgemeinernden Annahme gefuihrt, "daß man sich in der Generalbaßzeit für die Ausführung des Conti-

308. S.81.

309. Es soll jedoch auch darauf hingewiesen werden, daß es zwischen der Auffuhrung von 1607 und dem Druck Differenzen gibt. Das woh1 urspringlich geplante Ende (so wie es auch in der Partitur steht) war - unter Umständen aus technischen Grinden - 1607 nicht realisierbar (vgl. Fenlon, Stage Works, S.264). Auch an Wiederauffuhrungen zumindest von Teilen hatte man sicher bei der Drucklegung gedacht. Nur so ist es zu erklären, daß von der Arie "Possente spirito" zwei Fassungen - eine schwierige und eine "entschärfte" gedruckt wurden (S.52 ff der Partitur von 1609).

310. Arnold, Performing, S.326, nach Federico Follino, Compendio delle sontuose feste fatto l'anno M.DC.VIII nella città di Mantova (1608). Genannt werden Holzorgel, Chitarrone, Cembalo und Regal.

311. Leichtentritt, Vortragspraxis, S.149. Besetzt sind hier laut Vorwort zwei Cembali, zwei Theorben, zwei Violinen, eine Laute und eine Viola da gamba.

312. Carro di fedeltà (1611) mit Cembalo, Laute und Theorbe sowie La sfera armoniosa (1623), in der Vermerke, wie "tiorba tace" oder "tiorba sonate" die Beteiligung mehrerer Continuo-Irstrumente bezeugen (Leichtentritt, Vortragspraxis, S.150).

313. Vg1. hierzu Bianconi, 17th Century, S.27.

314. Le musiche e balli, vgl. das Vorwort im zweiten Band dieser Arbeit auf S. 14 . 
nuoparts eines Instrumentalkörpers bediente, der Cembalo, Spinett, Lauten und Theorben (wie auch Chitarronen), Harfe, Baßviola, Violoncello und Kontrabaß - gelegentlich auch Orgel - zu einem klangprächtigen Ensemble vereinte"915. Tatsächlich blieben diese großen Besetzungen (zumindest in Italien) jedoch auf die Bühnenmusik und großbesetzte Kirchenmusik beschränkt.

\section{$3,3,2,3$, CONTINUO-INSTRUMENTE IM POLYPHONEN MADRIGAL 316}

In der geistlichen Musik, der Monodie und der frühen Oper wurden häufig, ja fast immer, Angaben uber die gewiinschten Continuo-Instrumente gemacht, sei es in den Titelblättern, in Vorworten, Generalbaßstimmbuchtiteln oder auch durch eine Durchinstrumentierung. Im polyphonen Generalbaßmadrigal sind solche Angaben eher selten. Im Madrigal kam, wie bereits erwähnt, der Generalbaß erst verhältnismäßig spät zu breiter Anwendung. Noch im zweiten Jahrzehnt des 17.Jahrhunderts erschienen zahlreiche Madrigaldrucke ohne Continuostimmen. Sofern ein Generalbaß vorgesehen war (auch "se piace".317), wird er ohne Nennung eines Instrumentes als "Basso per sonare" o.ä. bezeichnet. Diese ungenauere Bezeichnung des Basso continuo mag darin begrindet liegen, daß zum einen die meisten polyphonen Generalbaßmadrigale erst zu einer Zeit erschienen, in der der Generalbaß bereits weithin bekannt war - jeder wußte, wie er zu besetzen ist -, und zum anderen der Generalbaß hier nicht eine so wesentliche Funktion hatte wie in der Monodie. Dies zeigt sich bereits in den Titeln: Der Generalbaß wird bei den polyphonen Madrigalen meist gegen Ende des Titels als eine Zutat erwähnt ("... con il basso per sonare"), während er bei monodischen Drucken direkt zur Beschreibung des Inhaltes gehört ("Arie per cantar a voce sola ne11' chitarrone" o.ä. 318).

Eine Durchsicht der Titel in Emil Vogels Bibliothek der weltlichen Vokalmusik ergab, daß bei 17 Drucken polyphoner Generalbaßmadrigale ${ }^{319}$ Angaben uber die Continuo-Besetzung in den Titeln enthalten waren. Alle diese Drucke erschienen vor dem Anfang des dritten Jahrzehntes des 17.Jahrhunderts, also vor Beginn der Bluitezeit des Concertato-Madrigales mit Generalbaß320. Neum dieser 17 Titel verlangen ein Kielklavier ${ }^{321}$ (in der Regel Cembalo, manchmal

315. Neemann, Laute, S.527.

316. Der Begriff "polyphones Madrigal" wird hier zur Abgrenzung von der Monodie benutzt.

317. Z.B. bei G. A. Fabri, Il primo libro de madrigali (1620).

318. Vgl. die entsprechenden Titel im Quellenverzeichnis dieser Arbeit.

319. Als Druck polyphoner Madrigale galt hier nicht ein Druck, der auBer polyphonen Madrigalen auch Monodien und Duette enthielt, da sich hier die Titel ganz deutlich an diejenigen der Monodien anlehnen. Nicht mit einbezogen wurden aufgrund ihrer Nähe zur Oper auch die Madrigalkomödien A. Banchieris.

320. L. Bianconi, 17th Century, S.2.

321. Freilich findet sich auch hier oft der Zusatz "ò simil altro stromento" (o.ä). Hierin ist jedoch in erster Linie ein Entgegenkommen der 
auch Cembalo oder Spinett) als GeneralbaBinstrument322, fünf weitere ein Cembalo oder einen Chitarrone ${ }^{323}$. Nur in einem Titelblatt wird ausschlie日lich der Chitarrone 324 vorgeschrieben. Die Orgel wird nur selten genannt: einmal im weltlichen Madrigal325 und einmal für Madrigali spirituali ${ }^{326}$.

Man kann hier von einer deutlichen Tendenz zum Kielklavier sprechen. Das einzige Madrigalbuch, im dem nur der Chitarrone verlangt wurde, Salomone Rossis primo libro de madrigali, erschien außerordentlich frih (1600) und steht noch vor der Durchsetzung des Generalbasses ${ }^{327}$. Ein Teil der Madrigalbïcher, die die Alternative Cembalo oder Chitarrone vorschlagen, gehört ebenfalls zu den frühen Vertretern des Continuo-Madrigales 328 .

Auch Kombinationen von mehreren Continuo-Instrumenten sind, wenngleich se1ten, für späte Concertato-Madrigale belegt. Eine eigene Stimme für den Chitarrone gehört zu den Stimmensätzen von Giovanni Valentinis Musiche di camera libro quarto (1621) ${ }^{329}$ und Francesco Turinis Madrigali a cinque cioe

Drucker, wohl in der Hoffrung auf eine Steigerung der Verkaufsaussichten, zu sehen.

322. F. Comanedo, Secondo libro de madrigali (1615, Vogel, Bd.I, S.178), A. Freddi, Il secondo libro de madrigali (1614, Vogel, Bd.I, S.249), G. Ghizzolo, Secondo libro de madrigali (1614, Vogel, Bd.I, S.291), G. B. Leonetti, Il primo libro de madrigali (1617, Vogel, Bd.I, S.365), G. Marini, Il secondo libro de madrigali (1618, Voge1, Bd.I, S.419), C. Monteverdi, II sesto libro de madrigali (1614, Voge1, Bd.I, S.509. Vgl. hierzu auch Schmitz, Continuo-Madriga1, S.510: Auch innerhalb der Continuo-Stimme steht "concertato nel clavicembalo".), P. Pace, Il quarto libro de madrigali (1614, Vogel, Bd.II, S.36), H. Petrolini, Hore armoniche (1613, Vogel, Bd.II, S.77), B. Tomasi, Il primo libro de madrigali (1611, Vogel, Bd.II, S.244). Weitere Belege für Kielklaviere als Continuo-Instrumente finden sich in den bei Vogel i.d.R. nicht genannten Titeln der Continuo-Stimmbücher, wie z.B. in G. Priulis, terzo libro de madrigali (1612): "Basso grave per sonar nel Clavicembalo, Spinetta \& altro simile instromento".

323. A. Anglerio, II primo libro de madrigali (1617, Vogel, Bd.I, S.23), G. N. Mezzogorri, Il pastor fido armonico (1617, Vogel, Bd.I, S.460), C. Monteverdi, Il quinto libro madrigali (1605, Vogel, Bd.I, S.506), M. Nantermi, I1 primi libro de madrigali (1609, Vogel, Bd.II, S.5), N. Rubini, Madrigali (1615, Voge1, Bd.II, 170).

324. S. Rossi, Il primo libro de madrigali (1600, Vogel, Bd.II, S.161. Ab der 4.Auflage (1618) tritt anstelle des Chitarrone ein "Basso continuo").

325. A. Antone11i, Madrigali (1614, Vogel, Bd.I, S.26).

326. G. F. Anerio, Teatro armonico spirituali di madrigali (1619, Vogel, Bd.I, S.18),

327. Hier handelt es sich auch noch nicht um eine Continuo-Stimme, sondern um eine Tabulatur (s.o.).

328. C. Monteverdis funftes Madrigalbuch (1605) sowie das erste Madrigalbuch M. Nantermis (1609).

329. Voge1, Bd.II, S.264; vg1, auch Schmitz, Continuo-Madrigal, S.517 f. 
tre voce e due violini ... libro terzo (1629)3э0. In einem Vorwort zu Turinis "Basso per il Chitarrone" wird darauf hingewiesen, daß man auch mit dem Chitarrone oder dem Tasteninstrument allein musizieren könne, aber der Chitarrone den Violinen mehr "spirito" gäbe als ein Tasteninstrument, ein Chitarrone alleine jedoch zu leer klänge, um damit alle Stimmen zu beglei$\operatorname{ten}^{331}$.

Ahnliche Continuo-Besetzungen wie im Madrigal wurden auch für die - recht seltenen - Canzonetten und Villanellen (u.ä.) mit Generalbaß verlangt. Von Auffuhrungen mit Cembalo wird bereits 1588 in der Dedikation Luca Confortos zu Paolo Quagliatis Canzonette... a tre voci per sonare et cantare... libro secondo berichtet 332 . Die geistlichen Canzonetten in den Publikationen Simone Verovios erschienen mit je einer Tabulatur für Laute und Cembalo ${ }^{333}$. Ausdrücklich für Cembalo bestimmte Continuo-Stimmen gehören zu den Stimmensätzen von Giovanni Pietro Flaccomios Madrigali a tre voci (1611) 334 und Giulio Medicis Amorosi concetti musicali a tre voci (1619) ${ }^{395}$. Die Mitwirkung eines "Instrumento da tasti" wird in den Vorworten zu Antonio Brunellis Scherzi ... libro secondo (terzo) (1614 bzw. 1616) vorausgesetzt ${ }^{336}$.

Die Besetzung Cembalo oder Chitarrone wird wiederum für die fruhesten Generalbaßstimmen in diesen Gattungen, nämlich in den ersten beiden Büchern der

330. Voge1, Bd.II, S.260.

331. "Avertimento. Anchor che $i$ presenti Madrigali possino esser concertati con l'instromento solo da tasto senza Chitarrone; overo un Chitarrone, ò altra simil istromento senza quello da tasto; nulladimeno faranno assai miglior riuscita l'uno, \& con l'altro: poiche l'istromento da tasto non dà quel spirito a $i$ Violini cha da il Chitarrone, \& il Chitarrone solo senza l'istromento da tasto riesce troppo vuoto ne li accompagnamenti de li parti di mezzo, \& massime nelle ligature ..." (zitiert nach Vogel, Bibliothek, Bd.II, S. 260; vgl. auch Materassi, Teoria e pratica, S.30). Hinweise auf mehrere Harmonieinstrumente finden sich auch bei A. Banchieri, Il virtuoso ritrovo (1626). Genannt werden "Arpicordo" und Spinett.

332. Beginn der Dedikation: "Mi sapere 'auto adoperato, che al fine ho hauuto nelle mani queste altre canzonette del Sig. Paolo Quagliati; ch'egli ha composte d richesta di varie gentildonne Romane per sonare, \& cantare su'l cembalo ...". Auf die Mitwirkung eines Harmonieinstrumentes wird auch in den Titeln der amorosi pensieri. Canzonette... libro secondo sowie dem terzo libro dell'amorose canzonette (beide 1607) von G. S. P. del Negro verwiesen ("da sonare, \& cantare su'l chitarrone, clauicembalo, \& altri stroment $\left.i^{\prime \prime}\right)$, ohne dab jedoch eine Stimme hierfuir vorhanden ist.

333. Vg1. zu den anderen Titeln Verovios Casimiri, Verovio, S.193 ff.

334. Voge1, Bibliothek, Bd.I, S.241.

335. Vogel, Bd.I, S.443.

336. Brunelli führt aus, daß man die Ritornelle, sofern keine Melodieinstrumente vorhanden sind, auch allein auf dem Tasteninstrument ausführen kann (vgl. die Vorworte bei Sartori, Bibliografia I, S.200 (1614 f) und S. $220(1616$ a)). 
nuovi fioretti musicali von Amante Franzoni (1605 und 1607) verlangt ${ }^{337}$. Die ganze Vielfalt der Continuo-Instrumente steht in zwei Canzonettendrucken zur Auswahl. Im Titel von Bernardino Borlascas Canzonette a tre voci (1611) werden "chitarrone, lira doppia, cembalo, arpone, chitariglia alla spagnuola; ò altro simile strumento da concerto" genannt 338 ; Marcello Albano erwähnt in dem Vorwort zu seinem primo libro di canzoni e madrigaletti a tre, \& à quattro voci (1616) Laute, Cembalo und Harfe als mögliche ContinuoInstrumente ${ }^{339}$.

Die Angaben zur Besetzung des Basso continuo in der Vokalmusik bestätigen nochmals die Wurzeln des Basso continuo, nämlich das collaparte-Spiel auf der Orgel in der geistlichen Musik sowie die Lautenlieder in der weltlichen Musik. Während in der Monodie wie auch in der Kirchenmusik an den bereits aus dem 16. Jahrhundert bekannten Begleitinstrumenten im wesentlichen festgehalten wurde, haben sich bei der polyphonen weltlichen Musik mit Generalbaß schon fribh die Kielklaviere durchgesetzt.

\subsubsection{Die BESETZUNG DER GENERALBÄSSE IN DER INSTRUMENTALMUSIK}

Auch bei der Instrumentalmusik ist der Anlaß bzw. der Ort für den sie bestimmt ist, entscheidend für die Besetzung des Continuos. Ein grober Teil der Instrumentalmusik ist der Kirchenmusik zuzurechnen. Mehr als zwei Drittel der Drucke mit vokaler und instrumentaler Musik aus den ersten 30 Jahren des 17.Jahrhunderts enthalten außer den meist wenigen Instrumentalwerken geistliche Musik ${ }^{340}$. Die Hauptgattung der in Sammlungen geistlicher Musik iberlieferten Instrumentalwerke ist die Canzone; Sinfonien und Sonaten erschienen zunächst auch in erster Linie in Drucken sonst geistlicher Musik, später aber auch vermehrt zusammen mit Madrigalen ${ }^{341}$.

337. Voge 1, Bd.I, S.248.

338. Vogel, Bd.I, S.111. Verwiesen sei auf das Vorwort zu Borlascas Scala Jacob (1616, vgl. den Abdruck im zweiten Band dieser Arbeit auf S.8). Dort wird ein ähnliches Instrumentarium (u.a. auch "Arpone" und "Lirone", mit Zusatz "e simil come hoggi si costuma; e massime nella Corte di Bauaria" genannt).

339. Vgl. den Abdruck dieses Vorwortes im zweiten Band dieser Arbeit auf S.6.

340. Eine Durchsicht von Sartoris Bibliografia ergab, daB fast $70 \%$ der Drucke aus dieser Zeit mit Instrumentalmusik neben vokaler Musik der Kirchenmusik zuzurechnen sind. Dieser Anteil ist jedoch innerhalb dieser Zeitspanne rückläufig (1601-1610: fast $80 \%, 1621-1630$ etwa 55\%).

341. In den Jahren 1621-1630 gehören bereits fast 70\% aller Drucke mit vokaler Musik und als "Sonata" betitelten Instrumentalstücken sowie rund 50\% derjenigen mit vokaler Musik und als "Sinfonia" betitelten Instrumentalstiicken der weltlichen Musik an. Interessant sind in diesem Zusammenhang die Beobachtungen von Eleanor Selfridge-Field zum Zusammenhang von Stellung des Komponisten (Organist, maestro di cappella, Instrumentalist etc.) und bevorzugter Gattung (Instrumentation, S.61 f). 
Auch für die Instrumentalwerke in Drucken geistlicher Musik wird man eine Verwendung im Rahmen der Gottesdienste annehmen können. Einige Hinweise auf den liturgischen ort bei instrumentalen Kompositionen belegen dies ${ }^{342}$. Als Continuo-Instrument kommt hier, nicht anders als bei der vokalen Kirchenmusik auch, vor allem die Orgel in Frage. Auch bei reinen Canzonendrucken sind "Basso per 1'organo"-Stimmbücher häufig 343 bzw, ist die Orgelbegleitung auf andere Weise belegt ${ }^{344}$. Seltener sind Hinweise auf die Orgel als Continuo-Instrument in den anderen instrumentalen Gattungen ${ }^{345}$.

Die Orgel wird in Instrumentaldrucken auch als eines von mehreren alternativ vorgeschlagenen Continuo-Instrumenten genannt ${ }^{346}$. Dies mag zum Teil ebenfalls im Hinblick auf mögliche Auffuhrungen im gottesdienstlichen Rahmen geschehen sein ${ }^{347}$. An eine kammermusikalische Aufführung mit einer $\mathrm{Holz}^{-}$ orge $1^{348}$ ist freilich auch zu denken.

342. Vgl. hierzu vor allen Bonta, Sonata, S.54 ff sowie S.72 ff (Tabelle). Bonta nennt insgesamt fünf italienische Drucke aus der Zeit bis 1630, in denen solche Angaben die Verwendung der instrumentalen Sätze innerhalb der Messe belegen; zwei weitere Drucke fiihrt Schnoebelen, Violin, S.538 f, an. Hinzuzufugen sind A. Burlinis, Salmi intieri che si cantano al vespero (1613; sie enthalten eine "Sinfonia avanti il Magnificat"). Belegt wird die Aufführung von Instrumentalmusik im Gottesdienst auch durch A. Agazzaris Angabe "Prima si fa vna Sinfonia di stromenti" in den Psalmi sex (1609).

343. Eine "Basso per l'organo"-Stimme (o.ä.) gehört zu folgenden Canzonendrucken: A. Troilo, Il primo libro delle canzoni da sonare (1606), Samme1druck Canzoni da sonare (1608), V. Bona, Sei canzoni italianae da sonare (1614, nach Sartori, Bibliografia II, S.63 (1614 j)), G. Gabrieli, Canzoni e sonate (1615), G. Mussi, Il primo libro delle canzoni da sonare (1620, nach Sartorio, Bibliografia I, S.269 (1620 i)).

344. Ein Vorwort an die Organisten enthalten die Partitur zu G. D. Rognoni Taeggios Canzoni a 4. \& 8 . voci ... libro primo (1605) und die ContinuoStimme zu T. Merulas primo libro delle canzoni (1615); in Pietro Lappis Canzoni da suonare ... libro primo (1616) ist bei der Canzona "La Monteverde" der erste Chor mit "in Organo" gekennzeichnet; in der "Basso continuo"-Stimme zu G. Picchis Canzoni da sonare (1625) steht bei allen Stücken außer der "Sonata Nona. A 3" zu Beginn jedes Stückes "ORGANO" (die Continuo-Stimme der "Sonata Nona. A 3" ist init "Basso Continuo" bezeichnet; unter Umständen, um auf eine andere Besetzung hinzuweisen. Gut möglich ist jedoch auch ein Versehen).

345. Eine "Basso per 1'organo"-Stimme (o.ä.) gehört auch zu folgenden Instrumentalmusikdrucken: L. Viadana, Sinfonie musicali (1610, nach Sartori, Bibliografia I, S.172 (1610 a)), B. Montalbano, Sinfonie (1629) und O. M. Grandi, Sonate (1630). Eine Ausfuhrung des Continuos durch eine Orgel kann auch für Kapsbergers Sinfonie (1615) wahrscheinlich gemacht werden (s.u.). 346. So auf den Titelblättern zu D. Castellos Sonate concertate (1629). Als andere Möglichkeit wird das Spinett (Libro primo) bzw. das Cembalo (1ibro secondo) vorgeschlagen.

347. D. Castello bezeichnete sich auf den Titelblättern seiner Drucke als 
Ahnlich wie bei den polyphonen Generalbaßmadrigalen und den generalbaßbegleiteten Canzonetten beobachtet werden konnte, wurde in einigen fruihen Instrumentalmusikdrucken außerhalb der Kirchenmusik der Chitarrone als Begleitinstrument verlangt, und zwar - auch hier eine Parallele zu den Madrigalen - in Werken Salomone Rossis ${ }^{349}$. Diese Besetzung hat Rossi auch fur weitere Bücher beibehalten350. Eine Begleitung nur durch ein Zupfinstrument ist auch in zwei Sätzen aus Biagio Marinis Sonate (1629) vorgesehen ${ }^{351}$.

Dariber hinaus werden Zupfinstrumente innerhalb der Instrumentalmusik gelegentlich als zusätzliche Harmonieinstrumente verlangt. Besonders bemerkenswert sind in dieser Hinsicht die Sinfonie a quattro voci Giovanni Girolamo Kapsbergers (1615). Ein Nachwort besagt, daß die beiden Baßstimmen jeweils mit Harmonieinstrumenten ("strumento che suoni in consonanza") wie Laute, Chitarrone, Cembalo, Harfe oder dergleichen zu besetzen sind. Diese Stimmen sind auch beziffert. Ein Melodieinstrument kann nach Kapsberger nach Belieben hinzugefugt werden ${ }^{52}$. Diese bezifferten Baßstimmen (im Alto- und Basso Stimmbuch) sind jedoch zusätzlich zur (ebenfalls bezifferten) Basso continuo-Stimme vorhanden. Der Basso continuo verdoppelt die jeweils spielende

Chef der Instrumentalmusik an San Marco (vg1. zu seiner Identität bzw. zu den Spekulationen dariber Selfrigde-Field, Castello und Winkler, Instrumentalwerke, S.162 ff), stand demnach also im Dienst einer Kirche.

348. Holzorgeln waren auch im außerkirchlichen Bereich verbreitet (s.o. zu den "Organi da legno" bei Intermedien- oder Opernauffuhrungen).

349. Il primo libro delle sinfonie et gagliarde... per sonar due viole, ouero doi cornetti, \& vn chittarrone o altro istromento da corpa (1607) und Il secondo libro delle sinfonie e gagliarde à tre voci per sonar due viole, \& vn Chittarrone (1609).

350. Il terzo libro de varie sonate ... per sonar due viole da braccio, \& un chittarrone o altro stromento simile (2/1623) und $I l$ quarto libro de varie sonate... per sonar due violini et un chitarrone o altro stromento (1622).

351. Das "Pass'e mezzo Concertato in otto Parti" trägt die Instrumentationsangabe "Doi Violini e Chitarrone ö altro simile istromento", die "Sonata VII A voi do vinto il cor" soll von zwei "violini e Chitarrone o Arpa doppia" ausgeführt werden. Da diese Sätze nicht im Baß-, sondern nur im Continuo-Stimmbuch vorhanden sind, kann mit dem erwähnten Chitarrone nur das Continuo-Instrument gemeint sein (bei anderen Sätzen aus diesem Druck wird der Chitarrone zusätzlich zum Bc. verlangt (s.u.)). Die Instrumentation erfolgte bei den beiden genannten Stücken nur im Titel, nicht aber speziell in den einzelnen Stimmbiichern.

352. "AVVERTIMENTO. PEr Primo, \& secondo Basso s'intende qual si voglia strumento che suoni in consonanza, come sarebbe Lauto, Chitarrone, Cimbalo, Arpa \& suoi simili. Per primo, \& secondo Canto, Violino, Cornetto, \& suoi simili. Per primo, \& secondo Basso primo, \& secondo Choro se piacerà con accrescimento de strumenti." 
BaAstimme353. An Tutti-Stellen gehen alle drei Baßstimmen (die beiden Bässe und der Basso continuo) collaparte. Für den Basso continuo hat Kapsberger die Besetzung mit einer Orgel offenbar als selbstverständlich vorausgesetzt 354 .

Eine ähnliche Besetzung wird in zwei Canzonen Gregorio Allegris verlangt35s. Auch hier handelt es sich um doppelchörige Kompositionen, deren erster Chor mit Violine und Laute, der zweite mit Zink und Theorbe besetzt ist. Dazu gehört ein Basso seguente mit der Instrumentation "Organo".

Zusätzliche Zupfinstrumente - alternativ zu Melodiebaßinstrumenten356 werden auch in den Sonaten aus Steffano Bernardis Madrigaletti... con alcune sonate a tre per due violini, overo cornetti, \& un chitarrone, trombone, overo fagotto ... libro secondo (1621) und in Biagio Marinis Sonate (1629) verlangt. Bernardi nennt im Titel und in der Tavola fur die sieben Canzonen (sie werden nur im Titel des Druckes als Sonaten bezeichnet) eine Besetzung mit zwei Violinen oder Zinken und als Baßinstrument eine Posaune oder ein Fagott oder einen Chitarrone bzw. eine Theorbe ${ }^{357}$; ein Basso continuo ist freilich zusätzlich vorgesehen. Nur bei zwei Sätzen findet sich in der Baßstimme eine auf ein Stück bezogene Instrumentation (einmal "Fagotto" und einmal "Liuto o Fagotto"35s).

Biagio Marinis umfangreiches 0p. 8 , die Sonate von 1629 , enthält neben den bereits erwähnten Sätzen mit Chitarrone als einzigem Harmonieinstrument eine

353. Die meisten der Sinfonien haben uber weite Strecken eine doppelchörige Anlage. Die beiden Chöre (jeweils eine Sopran- und eine Baßstimme) musizieren oft abwechselnd, so daB nur eine der beiden Baßstimmen erklingt.

354. Um jedes der vier Stimmbücher auf 24 Seiten (= 6 Bögen) unterzubringen, sind einige geringerbesetzte Stücke anders auf die Stimmbiicher verteilt worden, als dies nach den Stimmbuchtiteln zu erwarten ist (die Tavola gibt an, welche Stimme in welchen Stimmbuch zu finden ist). Bei den Nummern 4 und 13 (beides Sätze fuir Canto solo und Continuo) steht auch der Continuo nicht im Generalbaßstimmbuch sondern je einmal im Altstimmbuch (Nr.4) und im BaBstimmbuch (Nr.13) - diese Stimmen pausieren ja bei diesen Sätzen. Um k1arzustellen, daß es sich bei diesen Stimmen nicht um eine normale Baßstinme handelt (die ja laut Nachwort mit Zupfinstrumenten oder Kielklavieren zu besetzen sind), sind sie mit "Basso per Organo" uberschrieben - für Kapsberger offenbar ein Synonym für Continuo-Stimme.

355. Vg1. Katalog Proske 3, S.9. Eine dieser Canzonen ist bei Schlecht, Kirchenmusik, S.411 ff abgedruckt.

356. Lauteninstrumente werden z.T. auch als reine Melodieinstrumente verwendet, so z.B. in T. Massainos Canzone für "Quattro viole, e quattro Chiteroni, o Leuti" ("Canzona Trigesimaquarta" aus dem Sammeldruck Canzoni per sonare (1608). Es handelt sich hier um zwei in etwa gleiche Chöre jeweils mit Sopran, Alt, Tenor und Baß.

357. Titel: Chitarrone, Tavola: Theorbe.

358. Mit Laute, Chitarrone und Theorbe ist hier sicher ein und dasselbe 
Anzahl kürzerer Sätze (Sinfonien, Balletti, Correnti, Gagliarde und Ritornelli), bei denen ein Chitarrone oder - alternativ - eine "Arpa doppia" bzw. ein "altro Basso" zusätzlich zum Basso continuo verlangt wird ${ }^{359}$. Für die Zupfinstrumente ist hier eine eigene, mit der Basso continuo-Stimme identische, jedoch ohne Taktstriche notierte und sehr viel spärlicher bezifferte Stimme im Basso-Stimmbuch vorhanden ${ }^{360}$.

Nur z.T. darf man hinter den Besetzungsangaben für den Basso continuo konkrete klangliche Absichten vermuten. Die Betitelung eines Continuo-Stimmbuches mit "Basso per l'organo" (o.ä.) besagt vor allem, daß hier an eine Auffuhrung in einer Kirche gedacht war, wo eine Continuo-Stime ganz selbstverständlich eine Orgel-Stimme ist. Bei einer Auffuhrung außerhalb der Kirche wird man je nach vorhandenem Instrumentarium auch ein anderes Harmonieinstrument wohl bedenkenlos eingesetzt haben. Auch Steffano Bernardis Alternativangaben mit Posaune, Fagott oḍer Chitarrone können kaum einer klanglichen Vorstellung entsprungen sein. Hier wollte man den Auffuhrenden entgegen kommen. Diese Angabe zeigt aber, daß ein Chitarrone als zusätzliches Baßinstrument sicher häufig verfügbar war. An recht konkrete Absichten kann hingegen bei Biagio Marinis Besetzungsangaben gedacht werden. Sowohl die Verwendung eines Zupfinstrumentes anstelle des Standardcontinuoinstrumentes (wohl Orge1361) als auch die genau angegebene zusätzliche Besetzung mit einem Zupfinstrument bei den Tanzsätzen und den anderen kurzen Kompositionen dieses Druckes läßt eine Absicht erkennen.

\subsubsection{VERDOPPELUNG DER BABLINIE DURCH EIN MELODIEINSTRUMENT}

Die Frage, inwieweit die Baßlinie des Continuos auch im frühen 17.Jahrhundert durch ein Melodieinstrument zu verdoppeln ist - dies war im 18.Jahrhun-

Instrument gemeint; Vgl. hierzu auch im nächsten Abschnitt.

359. Die Instrumentation erfolgte in manchen der Stimmbuicher in Form einer Satzuiberschrift (oft in Canto secondo und im Basso continuo), in den Tavole der Stimmbicher und als Instrumentation im Basso. Die Zugehörigkeit der Instrumentation $z u$ allen Sätzen eirier Art wird in den Tavole mittels einer Klammer ausgedriirkt. Sie stehen innerhalb der Stimmbicher nur beim jeweils ersten Satz einer Art.

360. Eine bezifferte Stimme für eine Viola da gamba enthält Marinis 0p.8 fuir die "Sonata sopra la Monica". Hier gleichen sich die Baß- und die Basso continuo-Stimme genau (lediglich die Seitenzahl ist verschieden und ein Lagenvermerk ist nur in der Baßstimme enthalten). Beide Stimmen sind beziffert und tragen die Instrunentation "Basseto o Viola da gamba". Es ist nicht auszuschließen, daß die Viola hier ebenfalls Harmonien spielen soll. Sehr viel wahrscheinlicher ist jedoch, daß beide Stimmen nach derselben - nur wenig veränderten - Druckplatte gedruckt bzw. nach einer Vorlage gesetzt wurden, und die Bezifferung in der Basso-Stimme ebenso wie die Instrumentation in der Basso continuo-Stimme versehentlich stehengeblieben ist.

361. Bei der letzten Komposition dieses Druckes wird die Orgel (hier obligat) ausdriicklich erwähnt ("Sonata per l'organo e violino ò cornetto"). 
dert allgemein ublich - ist in der Literatur bisher recht unterschiedlich beantwortet worden. Das Meinungsspektrum reicht von der Auffassung, es sei von Anfang an grundsätzlich auch ein Melodieinstrument hinzugezogen worden 362 , über differenziertere Darstellungen 363 bis hin zu einer völligen Ablehnung der Baßverdoppelung in dieser Zeit ${ }^{364}$.

Eine Sonderstellung nimmt auch in dieser Beziehung der Chitarrone ein. Entsprechend der Zuweisung Agazzaris365 wird er teils als Harmonieinstrument, teils aber auch als Melodieinstrument vorgeschrieben, etwa bei den oben bereits erwähnten Canzonen Steffano Bernardis 366 . Die Instrunentationsangabe lautet hier "Chitarrone, ó Fagotto o Trombone". Die Baßstimme, für die diese Besetzungangabe bestimmt ist, ist nicht eine Duplette der Continuo-Stimme, sondern eine Melodiebaßstimme, wie sie in Triosonaten dieser Zeit iblich ist367. Im Gegensatz zum Continuo hat diese Baßstimme zahlreiche Pausen. Sie ist an den Imitationsfolgen der anderen beiden Melodiestimmen beteiligt und hat eigene Soloste1len. Diminuierte Stellen dieser Melodiebaßstimme sind im Continuo vereinfacht 368 .

Es ist natürlich denkbar, daß der Spieler des Chitarrone hier auch den einen oder anderen Akkord spielt. Die Hauptaufgabe des Chitarrone ist hier jedoch zusammen mit den beiden anderen (Sopran-)Melodieiristrumenten zu konzertieren, wie dies auch bei einer Besetzung mit Posaune oder Fagott der Fall wäre 369 . Auch in anderen Kompositionen, in denen ein Chitarrone zusätzlich zu einem weiteren Harmonieinstrument (meist Orgel) verlangt wird, ist unter Umständen an eine Funktion als Melodiebaßinstrument zu denken.

Gestrichene oder geblasene Melodiebaßinstrumente zur Verdoppelung der Continuo-Stimme sind weit seltener belegt. Sicher wird man auf solche Fundamentbässe bei großbesetzter Musik nicht verzichtet haben. Die oben beschriebenen großen Besetzungen der Continuo-Gruppe( $n$ ) bei mehrchöriger Musik beinhalten

362. Williams, Basso Continuo, S.138 und S.148.

363. Fortune, Instruments; Mueller, Basso continuo, S.273 ff (die hier genannten Feststellungen lassen sich jedoch nicht bekräftigen) und Mangsen, Ad libitum, S.32 ff. Nur auf das späte 17. Jahrhundert geht Allsop, Stringed Bass ein.

364. Arnold, Performing, S.322.

365. Del sonare sopra' 1 basso, S.3. Vg1. hierzu weiter oben im Abschnitt II.3.3.1.

366. Madrigaletti ... con alcune sonate a tre per due violini, overo cornetti, \& un chitarrone, trombone, overo fagotto ... libro secondo (1621).

367. Die Canzonen Bernardis liegen in einer - allerdings nicht sehr lobenswerten - Neuausgabe vor (Steffano Bernardi, Canzonas from Opus 12, hrsg. von Robert Paul Block).

368. Die Baßstimmen entsprechen damit dem in dieser Zeit in Kompositionen à tre für die Melodiebaßstimme ublichen.

369. Ahnliche Besetzungsangaben finden sich in S. Bernardis Concerti sacri scielti, \& trasportati (1621). 
fast immer auch mehrere Melodiebaßinstrumente ${ }^{370}$. Auch in der Bühnenmusik sind gelegentlich Melodiebaßinstrumente zusätzlich zu Harmonieinstrumenten vorgeschrieben ${ }^{371}$, jedoch nur selten zu solistischen Sätzen ${ }^{372}$. Bei geringbesetzter Musik werden die Melodiebaßinstrumente vor allem zusammen mit Zupfinstrumenten und Kielklavieren, nicht aber mit der z.B. im Orfeo Monteverdis ebenfalls häufigen Holzorgel verlangt. Die Vermutung liegt nahe, daß diese Instrumente das Vorhandensein des Baßtones auch nach Verklingen des Akkordes sicherstellen sollten ${ }^{373}$.

Keine Belege finden sich aber für eine Baßverdoppelung mit einem Streichoder Blasinstrument bei monodischer Musik außerhalb der Oper ${ }^{374}$. Es wäre bei der oft geforderten Freiheit im Vortrag - im Gegensatz zu einem zusätzlichen Zupfinstrument - sicher störend. Vö1lig iberfluissig ist die Verdoppelung der Baßlinie bei Kammermusik vokaler oder instrumentaler Art, bei der bereits ein Melodiebaß besetzt ist. Die Continuo-stimme wird hier ohnehin collaparte mit dieser Stimme gefiihrt.

370. Vg1. aben im Abschnitt II 3.3.1. In der Handschrift von A. Striggios vierzigstimmigen "Ecce beatam lucem" wird eine Baßverstärkung mit Posaune und Viola gefordert, A. Banchieri beschreibt in seinen Conlusioni (1609), $\mathrm{S} .50 \mathrm{f}$, die Auffuhrung einer vierchörigen Messe mit "dui Violoni continoi" in der Generalbaßgruppe, in L. Viadanas Vorwort zu den Salmi a quattro chori (1612) wird die Besetzung des Basses fuir den dritten Chor mit Posaunen und einer Violone, für den vierten Chor mit Posaunen, "Violoni doppi" und Fagotten vorgeschlagen.

371. Neben vielen Zupfinstrumenten werden fast immer in den Besetzungsangaben zu den Florentiner Intermedien von 1589 Baßmelodieinstrumente genannt (sehr oft "Basso di Viola", vgl. Schneider, Basso continuo, S.56 ff). In C. Monteverdis $L^{\prime}$ orfeo (1609) ist häufig vor allem bei größerbesetzten Stücken ein "contrabasso" vorgeschrieben (vgl. S.10, S.32, S.64, S.67, S.70, und S.88). In den Vorbemerkungen zu Monteverdis Combattimento di Tancredi et Clorinda (1624 uraufgefihrt, gedruckt 1638 in den Madrigali guerrieri, et amorosi) wird ein "contrabasso da Gamba, che continuoerà con il Clavicembalo" (zitiert nach der Monteverdi-GA, Bd.8, erster Halbband, S.132) verlangt.

372. Der im Combattimento (vg1. vorherige Anmerkung) vorgesehene "contrabasso" soll sicher das ganze Stïck über spielen ("continuerà con il Clavicemba $\left.10^{\prime \prime}\right)$, also auch bei den solistischen Teilen. In Morteverdis L'Orfeo singt einmal ein Pastore und zweimal Orfeo zu einer aus Cernalo, Chitarrone und "Viola da braccio basso" bestehenden Continuo-Gruppe (S.36, S.80 und S.81. Die Ergänzung "basso" steht nur auf S.81).

373. Eine solche Verdopplung einer durch ein Zupf- oder Kielinstrument ausgefubrten Continuo-Stimme schlägt G. G. Kapsberger im Vorwort zu seinen Sinfonie (1615) ad libitum vor, nicht jedoch für den Orgel continuo (vg1. im Abschnitt 11.3.3.3. dieser Arbeit).

374. Vg1. Fortune, Instruments, S.13 und Arnold, Performing, S.322. 
Bei Kammermusik ohne selbständige Melodiebaßstimme, ist eine den Continuo verdoppelnde BaAstimme soweit gewiinscht, in den Stimmbiichern vorhanden, z.T. mit einem "se piace"-Vermerk ${ }^{375}$. Bei Besetzung des Continuo mit Orgel empfiehlt sich ein Zupfinstrument, bei Besetzung mit einem Kielklavier ein Streichbak.

375. Z.B. in B. Marinis Affetti musicali (1617). Vgl. hierzu Mangsen, Ad libitum, S.31 ff. Mangsen fuihrt hierfür Belege auch noch aus dem späteren 17. Jahrhundert heran. 


\section{TEIL: BESONDERE AUFFÜHRUNGSVORSCHRIFTEN}

Der neue Stil in Komposition und Auf fuhrungspraxis manifestiert sich auch in den in den letzten Jahren des 16 . und den ersten Jahren des 17.Jahrhunderts aufkommenden Auffuhrungsbezeichnungen. Diese Angaben (Besetzungsangaben, Bindebögen, Lautstärkebezeichnungen etc.) erschienen alle innerhalb weniger Jahre erstmals in den italienischen Musikdrucken. Das Notenbild beginnt damit sein bis heute gültiges Aussehen zu gewinnen. Aspekte der Auffuhrung werden allmählich zum notierten Bestandteil einer Komposition. Zu den nicht notierten "Selbstverständlichkeiten" treten beabsichtigte, unter der Herrschaft des Affektes stehende, "Nichtselbstverständlichkeiten" als individuelle Ausdrucksmittel der Komponisten hinzu.

\section{DYNAMISCHE ANGABEN}

Die Tonstärke ist als wesentlicher Parameter eines akkustischen Signales in der Musik immer von Bedeutung ${ }^{1}$. Bis ins 16.Jahrhundert hinein war sie jedoch kein Bestandteil der Komposition selbst. Um die Mitte dieses Jahrhunderts begannen Komponisten und Musiktheoretiker sich für die Tonlautstärke $\mathrm{zu}$ interessieren. In der Musiktheorie wird ab der Jahrhundertmitte gefordert, da日 sich die Lautstärke nach dem Text ${ }^{2}$ und nach dem Ort $^{3}$ zu richten $^{2}$ habe und das die Themen in polyphonen Kompositionen besonderer Hervorhebung bediirfen ${ }^{4}$. Solche dynamischen Differenzierungen dienen der Verdeutlichung einer Komposition ohne jedoch bereits Bestandteil derselben zu sein. Zum unmittelbaren Teil der Komposition (oft sogar zum Kompositionsprinzip) wird die Dynamik in den im späteren 16.Jahrhundert häufig werdenden Echo-Kompositionen.

\subsection{ECHO-KOMPOSITIONEN DES SPÄTEREN 16. JAHRHUNDERTS}

Im 16. Jahrhundert, einer Zeit, in der die Nachahmung der Natur insbesondere im Madrigal große Bedeutung erlangte5, bot sich der Effekt des Echos zur Nachahmung geradezu an. Nach vereinzelten Versuchen bereits um $1500^{\circ}$ er-

1. Vg1, auch Boyden in Symposium, Teil II, S.126.

2. N. Vincentino, L'antica musica ridotta (1555), fol.37. Vg1, hierzu Zenk, Vincentino, S.97 ff, sowie Kunze, Gabrieli, S.177, und Berger, Chromatic, s.37.

3. Vincentino, fol. 88'. Hierzu auch Adler, Stil, S.131, Machatius, Tempi, S.120, und Berger, Chromatic, S.37.

4. Meier, Fink, S.46. Weitere Belege hierfuir aus der deutschen Musiktheorie des späten 16. und frihen 17.Jahrhunderts teilt Bartels, Aspekte, S.146, mit.

5. Vg1. u.a. Carapetyan, Imitazione,

6. Karl Michael Komma, Art. Echo in MGG, Bd.3 erwähnt eine nicht erhaltene Echo-Komposition Heinrich Isaacs (Sp.1076) und weist auf Echo-Wirkungen in den Orgeltabulaturen des Johannes Kotter hin; vgl. dazu auch den Art. Echo Organ von Peter Williams in NG, Bd.5, S.822. Auf Echo-Wirkungen vor allem im 
schienen im 1etzten Drittel des 16.Jahrhunderts Echo-Kompositionen in großer Zah17 . Dynamische Bezeichnungen waren in diesen Kompositionen freilich nicht notwendig. Es genugte, das Kompositionsprinzip im Titel zu nennen ${ }^{8}$. Gelegentlich wurde auch eine einzelne Stimme oder ein Chor mit "Echo", häufig aber auch mit "proposta" bzw. "risposta" bezeichnet?. Das Prinzip des Echos und das des Dialoges gehören hier zusammen. Der Mythos von der Nymphe Echo - sie verliebt sich in NarziB und magert aus Liebeskummer ab bis nur noch ihre bald versteinerten Knochen und ihre Stimme ibrigbleiben 10 - ist im 16. und 17.Jahrhundert gegenwärtig. Auch in zahlreichen Kompositionen gibt das Echo tatsächlich eine Antwort auf eine Frage (z.B. "che è cagion del pianto mio" - "Io") 11 .

\subsection{Die Verwendungen der dynamischen Angaben "PIANo" und "Forte" IN DEN JAHREN KURZ VOR 1600}

Die Echo-Kompositionen konnten in der Regel ohne Lautstärkebezeichnungen auskommen. Es verstand sich von selbst, daB das Echo leiser zu singen oder zu spielen war. Eine Kennzeichnung wurde nötig, wenn Lautstärkeunterschiede außerhalb eines Echos oder aber ein Echo außerhalb einer durchgängigen EchoKomposition verlangt werden sollten. Beides ist noch in Quellen des 16.Jahrhunderts zu beobachten. Die frihesten dem Verfasser bekannten Beispiele für die Verwendung der Lautstärkeangaben "piano" und "forte" bzw. " $p$ " und " $f$ " sind je ein Satz aus Aurelio Bonellis primo libro delle villanelle12 und aus Adriano Banchieris Canzoni all francese a quattro voci per sonare ... libro secondo, beide erschienen 159613. Beide Sätze sind mit "Echo" bzw. "In Echo" betitelt, bei beiden Sätzen ist das Echo jedoch nicht durchgängiges Satzprinzip, sondern tritt nur in einem Teil der Komposition (Banchieri) bzw.

frïhen 16.Jahrhundert geht Sternfeld, Echo Music, ein. Seine Beispiele sind zum Teil jedoch wenig uberzeugend (vgl. insbesondere die Notenbeispiele auf $\mathrm{S} .51 \mathrm{f})$.

7. Vgl. die Auflistung bei Kroyer, Dialog und Echo, S.32 sowie ergänzend die bei Komma, Art. Echo, genannten Beispiele. Diese Listen sind freilich bei weitem nicht vollständig.

8. Beispiele bei Kroyer, S.32.

9. Auch hierfür geben Kroyer und Komma zahlreiche Beispiele. Zum Weiterleben dieser Notationsart im 17.Jahrhundert s.u.

10. Vg1. Ovid, Metamorphosen 3,356 ff. Auf andere Auftritte der Nymphe Echo in antiker Poesie weist Hermann Breitenbach in der von ihm herausgegebenen und ubersetzten Ausgabe (Zürich 1958 (Bibliothek der alten Welt)) der Metamorphosen, S.182, hin.

11. Zahlreiche Beispiele bei Kroyer, S.27.

12. "Sott'il tuo regn'Amore". Ein Faksimile des Canto ist im zweiten Band dieser Arbeit als Notenbeispie1 III,2 wiedergegeben.

13. "L'Organista Bella", vg1. die Neuausgaben der Sammlung von 1596 durch L. Bartholomew, S.31 ff. Ein Faksimile der Canto-Stimme dieser Canzone ist dort auf S.16 abgedruckt. Vgl. weiter Bartholomew, Raverij, S.213 f, und Kämper, Ensemblemusik, S. 222 und S. 228. 
nur an einer Stelle (Bonelli) auf; in beiden Kompositionen gibt es keine besonderen Echo-Stimmen. "Proposta" und "risposta" werden von denselben Stimmen ausgefihrt. Die dynamischen Bezeichnungen tragen hier also zur Erkennbarkeit der Echo-Stellen wesentlich bej.

Die verwendete Terminologie - "piano" und "forte" - war seit langem bekannt. Eine einzelne Anweisung "tocca pian piano" steht schon in dem etwa 1517 angelegten "Capirola-Codex"14. Die Musiktheorie bediente sich dieser Begriffe bereits in der Mitte des 16.Jahrhunderts ${ }^{15}$. Zur Anwendung dieser Lautstärkebezeichnungen in den Musikdrucken in den letzten Jahren des 16. Jahrhunderts hat nicht die Einführung neuer Vokabeln geführt, sondern neue Arten der Verwendung der Dynamik haben die Einfuhrung solcher Zeichen in die Notenschrift notwendig gemacht.

Ein Jahr nach den Echo-Stiicken Banchieris und Bonellis erschienen in Giovanni Gabrielis Sacrae symphoniae (1597) die berihmte "Sonata Pian \& Forte"16. Aus nicht erkennbaren Gründen wird auch diese Sonate in der Literatur als Echo-Stück bezeichnet17, obwohl es hier nicht eine einzige Echo-Stelle gibt. Theodor Kroyer hat bereits auf die dynamische Wirkung verwiesen, die durch unterschiedliche Besetzungsstärke hervorgerufen werden kann18. Auch in Echo-Stuicken wurde dieses Prinzip gerne angewendet (z.B. "proposta": Chor, "risposta": nur eine Stimme)19. Eben dieses Prinzip liegt auch Gabrielis "Sonata Pian \& Forte" zugrunde, nur das der Wechsel von halber Besetzung (ein Chor) zum Tutti (beide Chöre) hier zusätzlich durch dynamische Angaben verstärkt und zum kompositorischen Prinzip erhoben wurde.

Bereits in der ein weiteres Jahr später erschienenen Pazzia senile Adriano Banchieris (1598)20 sind die dynamischen Angaben vollständig verselbstän-

14. Fo1. 49'. Faksimile bei Geoffrey Chew, Art. Notation in NG, Bd.8, S.398.

15. So in den oben angefihrten Außerungen Vincentinos zur Dynamik (fo1.37 und $\left.88^{\prime}\right)$.

16. Zahlreiche Neuausgaben, z.B. bei in IeM 2, S.64 ff.

17. So bei Heuss, Dynamik, S.144, Harding, Expression, S.86, Braun, Sti1wande1, S.85, und Leopold, Handbuch, 2. Teilband, S.587.

18. Dialog und Echo, S.19 ff.

19. Vg1. Kroyer, Dialog und Echo, S.26 f, mit einem Notenbeispiel aus einer Komposition Bertoldo Sperindios. Ein ähnliches Notenbeispiel Palestrinas findet sich bei Schwarz, HaBler, S.33.

20. Bei Komma, Art. Echo in MGG, Bd.3, Sp.1081, wird als Erscheinungsjahr der Pazzia 1585 genannt. Eine Auflage von 1585 ist jedoch nirgends nachzuweisen. Die Angabe "libro secondo a tre voci", die sich bei einigen Auflagen im Titel der Pazzia findet (bei allen italienischen Ausgaben bis einschlieBlich 1607, vgl. Voge1, Bibliothek, Bd.I, S.56 ff), meint nicht, daß es sich hier um ein zweites Buch der Pazzia senile handelt, sondern um Banchieris zweites Buch mit weltlichen Kompositionen "a tre voci". Auch in anderen Titeln Banchieris stehen ährliche Angaben, wie z.B. "terzo libro 
digt. Hier sind einige Sätze mit "Cantate questo forte"21, andere mit "Cantate questo piano"22 uberschrieben. Bei zwei Dialogen zwischen Gratiano und Pantalone wechselt die Dynamik innerhalb eines Satzes. Dies geschieht entweder durch im Verlauf des Satzes wechselnde dynamische Vorschriften ${ }^{23}$ oder aber bei verteilten Rollen - und dies ist in der Madrigalkomödie die Ausnahme - durch unterschiedliche Satzuberschriften in den einzelnen Stimmbuichern"24. In den "Avvertimenti" heißt es hierzu "Per ultimo avertarsi in alcune Scene dove es scritto Piano, \& Forte, che vuol significare si canti con mutatione, o per meglio dire alteratione di voce, \& questo per conoscere la diversità de gl'interlocutori"25. Die Dynamik soll also dem Hörer helfen, die jeweils sprechende Person zu erkennen.

\subsection{DYNAMISCHE ANWEISUNGEN IM FRÜHEN 17. JAHRHUNDERT $1.3,1$, ECHO-DYNAMIK}

Der größte Teil der dynamischen Vorschriften des frühen 17.Jahrhunderts steht bei Echo-Stellen oder dem Echo nachempfundenen dynamischen Effekten.

madrigalesco" beim Festino nella sera (1608, vg1. Quellenliste) oder "terzo libro de gli suoi conceri" bei den Messe solenne (1599, vgl. Quellenliste).

21. In der dem Verfasser vorliegenden Auflage von 1599: der "Intermedio di Solfanari" ("Strazz'e ciabatte") und der "Ragionamento secondo" des ersten Teiles ("Burattin, Burattin").

22. In der Auflage von 1599 der "Raginamento primo" des ersten Teiles ("Povero Pantalone").

23. So in dem "Ragionamento quarto" des ersten Teiles ("Sassa d'al ben"). Dieser Satz ist mit "piano \& forte" uberschrieben. In der Auflage von 1599 ist der Wechsel der Person zusätzlich durch einen Wechsel in der Besetzung angedeutet (jeweils $\mathrm{Ba}$ und nur einer der beiden Tenöre). In der auch der Neuausgabe von Bonaventura Somma zugrundeliegenden Ausgabe von 1607 ist der Satz durchgängig dreistimmig. In dieser Neuausgabe ist leider die originale Setzung der dynamischen Bezeichnungen durch eine Vielzahl wenig zeitgemäBer Angaben ersetzt worden. Luigi Torchi hat die originalen Angaben in seiner Ausgabe in L'Arte Musicale in Italia, Bd.4, S.281 ff, beibehalten.

24. So der "Ragionamento primo" des zweiten Teiles ("Tio, Burattin"). In den beiden Tenorstimmbüchern (Burattin) steht "Cantate tutto il canto piano", im Baßstimmbuch (Pantalone) hingegen "Cantate tutto il canto forte". 25. Die "Avvertimenti" zu den verschiedenen Ausgaben der Pazzia senile weichen an dieser Stelle voneinander ab. Die oben wiedergegebene Fassung findet sich nach Vogel, Bibliothek, Bd.I, S.56 f, auch in der ersten Ausgabe von 1598. Die veränderte Fassung von 1599 lautet: "Per ultimo auertarsi in alcuni ragionamenti dove sard̀ $P . \& F$. che vuol dire Piano, \& Forte, cioè cantate con mutatione di voce, \&questo acció si conoschi la diversità de $i$ parlanti." (Vg1. auch Voge1, S.56 f). 1621 erschien nochmals eine leicht geänderte Fassung, die jedoch der von 1599 im wesentlichen gleicht (Vogel gibt zur Ausgabe von 1621 keine Angaben uber die "Avvertimenti" (S.58). Sie sind aber faksimiliert in der Neuausgabe der Pazzia senile von Bonaventura Somma). 
Reine Echostiucke sind jedoch auch im fruhen 17.Jahrhundert in der Regel nicht mit dynamischen Angaben versehen. Hier genügt weiterhin eine Uberschrift "In Ecco"26 und/oder eine "proposta"-"risposta"-Zuweisung 27. Auch bei solchen Kompositionen sind jedoch die Echo-Stellen in den Basso continuo-Stimmen oft mit dynamischen Angaben ausgestattet. Da der Basso continuo in den meisten Stuicken sowoh1 "proposta" als auch "risposta" begleitet, mußten die Wechsel der Dynamik hier angezeigt sein²8.

Einzelne Echos wurden aber auch zunehmend außerhalb durchgängiger Echo-Kompositionen verwendet. Dies ist besonders häufig in der Instrumentalmusik zu beobachten 29 . In Kompositionen, in denen nur vereinzelte Echo-Stellen anzutreffen sind oder etwa jeder der Chöre einer doppelchörigen Komposition sowohl die Rolle der "proposta" als auch der "risposta" ubernimmt, bedurfte es in allen Stimmen einer genaueren Kennzeichnung. Anstelle des "piano" konnte auch "Echo" innerhalb solcher Kompositionen als dynamisches Zeichen verwendet werden ${ }^{30}$. Die unterschiedliche Ausstattung der verschiedenen Arten

26. "In Ecco"-iberschriften (o.ä.) ohne weitere dynamische Angaben im Stiuck (mit Ausnahme der Continuo-Stimme - s.u.) sind dem Verfasser aus folgenden Drucken des frihen 17. Jahrhunderts bekannt: A. Gualtieri, Motecta ... liber primus (1604), L. Spontoni, Motetti ... libro secondo (1609), C. Monteverdi, L'Orfeo (1609), ders., Sanctissimae Virgini missa (1610), G. P. Cima, Concerti ecclesiastici (1610, vgl. Notenbeispiel II, 9 im zweiten Band dieser Arbeit) A. Bianchi, Motetti, e messe (1611), ders., Motetti (1612), G. B. Riccio, Il secondo libro delle divine lodi (1614) P. Lappi, Canzoni da sonare (1616), A. Diruta, Sacrae cantiones (1617), G. B. Grillo, Sacri concentus ac symphoniae (1618).

27. Zu firiden im Sammeldruck Canzoni per sonare (1608) und in folgenden Individualdrucken: G. B. Riccio, Il secondo libro delle divine lodi (1614), A. Borsara, Odorati fiori (1615), G. B. Grillo, Sacri concentus ac symphoniae (1618), D. Castello, Sonate concertate ... libro secondo (1629) und B. Marini Sonate (1629),

28. Eine solche vollständigere Notation der dynamischen Zeichen bei EchoStellen in der Generalbaßstimme ist dem Verfasser aus folgenden Drucken bekannt: C. Monteverdi Sanctissimae Virgini missa (1610), G. B. Riccio I1 secondo libro delle divine lodi (1614), G. Valentini Salmi, hinni, magnificat (1618, hier ist in der Bc.-Stimme die Folge "echo" - "forte" verwendet worden) und G. Mussi IL primo libro delle canzoni (1620).

29. In ungefähr jedem zweiten Instrumentaldruck aus den Jahren 1621-1630 sind solche Echos zu finden. Auch in manchen sowohl Instrumental- als auch Vokalmusik enthaltenden Drucken sind Echo-Stellen und dynamische Angaben liberhaupt auf die instrumentalen Stücke beschränkt (so bereits vor der Jahrhundertwende in 6. Gabrielis Sacrae symphoniae (1597); aus dem frihen 17. Jahrhundert sind neben den drei Büchern delle divine lodi von G. B. Riccio (1612-1620) folgende Drucke zu nennen: G. Priuli Sacrarum concentuum ... pars altera (1619), F. Turini Madrigali ... con alcuni sonate ... libro primo $(1621,2 / 1624)$ sowie S. Bernardi I1 terzo libro de madrigali ... con alcune sonate (1624)). 
der Echo-Kompositionen mit dynamischen Zeichen kann an zwei direkt hintereinander stehenden Canzonen in Giovanni Battista Grillos Sacri concentus ac symphoniae (1618) studiert werden. Grillos achtstimmige "Canzon in Echo" ist ein reines Echo-Stück in "klassischer" Manier. Der erste Chor, betitelt "Proposta", bestreitet die Komposition im wesentlichen allein; nur die ein bis zwei letzten Takte der z.T. recht langen Phrasen des ersten Chores werden vom zweiten Chor, der "Risposta", als Echo wiederholt. Dynamische Angaben konnten hier freilich entfallen. In der ebenfalls achtstimmigen "Canzon pian e forte" hingegen sind die beiden Chöre gleichberechtigt ${ }^{31}$. Jeder der Chöre dieser abwechslungsreichen und vom iblichen Schema der doppelchörigen Canzone entfernten Komposition ist teils "risposta", teils "proposta". Eine genaue dynamische Bezeichnung war hier unumgänglich ${ }^{32}$.

Auch in den genauer bezeichneten Echo-Stuicken ist das Ende des Echos vielfach nicht gekennzeichnet. In vielen Quellen steht der "proposta" ein " $f$ " und der "risposta" ein " $p$ " voraus. Die Riickkehr in die Normallautstärke aber wird nicht angezeigt. Das Ende der "risposta" ist aber, vor allem, wenn "proposta" und "risposta" von der- oder denselben Stimmen ausgeführt werden, ohne weiteres zu erkennen ${ }^{33}$.

30. Dies ist in zah1reichen Musikdrucken des fruihen 17. Jahrhunderts zu finden. Auch Abkürzungen, wie "E.", "Ec." oder "Ecc." wurden verwendet. In einem Satz aus G. B. Riccios primo libro delle divine lodi (1612), der "Canzon Basso \& Sopran", ist "echo" mit "pian" kombiniert worden.

31. Vgl. die vom Verfasser herausgegebene Neuausgabe dieser Canzone.

32. Ein interessantes Beispiel einer Echo-Komposition enthalten B. Marinis Sonate (1629). Es handelt sich bei der Sonata in Ecco aus dieser Sammlung um eine typisches Echo-Stiuck. Die Komposition wird im wesentlichen van der ersten Violine bestritten; ihre Stimme ist mit "Questa e la parte che propone Forte" iberschrieben; sie allein soll für das Publikum sichtbar sein (im Bc. steht "Il Primo Violino deue essere visto, \& gli altri due nó" entsprechende Anweisungen finden sich in der zweiten und dritten Violinstimme). Die beiden anderen Violinstimmen - zu Anfang mit "sempre piano" bezeichnet - treten an einigen Stellen als zweifaches Echo hinzu. In der ersten Violine sind in einer Passage Doppelgriffe vorgesehen, die Marini offenbar den "Echo-Geigern" nicht zumuten möchte. Hier spielen beide EchoStimmen zusammen - die eine die oberen, die andere die unteren Töne der Doppelgriffe. Für das zweite Echo stehen dann aber an diesen Stellen keine weiteren Violinen zur Verfügung, also missen die beiden Echo-Violinen auch das zweite Echo ibernehmen. Dies ist dann mit "più piano" bezeichnet.

33. Hier sei auf die besondere Art der Kennzeichnung des Echos in L. Viadanas "Laude somma A quattro voci" aus den Cento concerti ecclesiastici $(1602,4 / 1605)$ hingewiesen. Die Echo-Stellen sind hier an der Schreibung des Textes in Großbuchstaben zu erkennen. Zur Ausfuhrung dieser Stellen steht uber dem Satz die Vorschrift "Le risposte si dicono piano". Tatsächlich gibt das Echo hier auch eine Antwort (z.в. "... exurge URGE, "... implora PLORA", "... exclama CLAMA" etc.). 
Selbst in den ausgesprochenen Echo-Kompositionen begnigte man sich im frihen 17. Jahrhundert oftmals nicht mehr mit einer schematischen Unterteilung in "proposta" und "risposta"-Stimmen. Giovanni Priulis Canzone (!) in echo a $12^{34}$ z.B. enthält neben verschiedenartigen Echo-Stellen auch zahlreiche Tuttis im forte, die ebenfalls eine Ausstattung mit dynamischen Bezeichnungen notwendig machen.

Auch die eigentlichen Echo-Stellen lösten sich bald von der reinen Naturnachahmung. Das Echo erfolgte nicht mehr immer als wörtliche Wiederholung mit geringerer Lautstärke; es konnte auch stattdessen in einer niedrigeren Tonhöhe notiert sein ${ }^{35}$. Nur in manchen Kompositionen wird ein solches Echo durch eine zusätzliche piano-Vorschrift verstärkt ${ }^{36}$. Die Abstraktion vom Naturphänomen "Echo" wird hier z.T. auch durch die für ein Echo ungewöhnliche Länge der "risposta" unterstrichen.

Weitergehende Abstraktionen vom Naturphänomen "Echo" waren in der Vokalmusik möglich. So verwendet Alessandro Grandi echo-ähnliche Stellen, bei denen nur Text und Rhythmus, nicht aber die Akkorde gleich bleiben (die "proposta" endet als Halbschluß und erst die "risposta" führt zum Ganzschluß)3 ? . Man wird solche Stellen nicht mehr als Echo bezeichnen können, wenngleich aber der Ursprung dieses dynamischen Effektes sicher im Echo zu suchen ist.

Die einfache Nachahmung eines Echos allein fand schon im zweiten und dritten Jahrzehnt des 17. Jahrhunderts nur noch wenig Interesse bei den Komponisten. Zwar wurden noch lange verschiedene Arten des Echos in den Kompositionen verwendet, jedoch nicht mehr als das beherrschende Satzprinzip, sondern nur noch als ein Effekt unter anderen ${ }^{38}$. Sicher hat auch das abnehmende Interes-

34. Aus: Sacrarum concentuum ... pars altera (1619).
35. Vgl. Karl Michael Komma, Art. Echo in MGG, Bd.3, Sp.1080. Besonders
reich mit diesem Effekt sind die Canzonen für Zink oder Violine und General-
baß von G. M. Cesare (aus Musicali melodie, München 1621, vg1. die Neuaus-
gabe von Konrad Ruhland). Beide Möglichkeiten der "risposta" (forte - piano
und hoch - tief) finden sich dicht beieinander in G. Fantinis Modo per
imparare a sonare di tromba (1638, in der "Sonata a due Trombe detta del
Gucciardini", S.68, und vor allem in der "Sonata di Risposte detta la
Saluiati", S.69. In jeder dieser Sonaten werden beide Prinzipien an-
gewendet). 36. So in den Canzonen "La Cornera" und "La Zifara" aus P. Possentis Concentus armonici (1629). Hier wird jeweils eine längere Schlußphrase eine Oktave tiefer und zugleich piano wiederholt (während bei "La Cornera" lediglich ein "piano" notiert ist, steht bei "La Zifara": "piano sino al fine"). 37. Vgl. im zweiten Band dieser Arbeit als Notenbeispiel III,1 abgedruckten Ausschnitt aus dem Madrigal "Non miri il mio bel sole" aus den Madrigali concertati $(1615,2 / 1616)$.

38. Auch in D. Castellos "Sonata Dacima settima A 4 in ecca" sind die beiden Echo-Stimmen nur an einem sehr kleinen Teil der Komposition beteiligt (in der 135 Takte umfassenden Komposition gibt es drei Abschnitte mit zusam- 
se am reinen Echo dazu geführt, daß Ottavio Rinuccinis Opernlibretto Narciso (1608 ?) - ein Libretto uber die Nymphe Echo selbst - keinen Komponisten mehr zu reizen vermochte ${ }^{39}$.

\section{3,2, DYNAMISCHE KONTRASTE DURCH WECHSELNDE BESETZUNGSSTÄRKEN UND DEREN VERDEUTLICHUNG DURCH DYNAMISCHE ANGABEN}

Schon im 16. Jahrhundert wurden mit unterschiedlichen Besetzungsstärken dynamische Effekte erzielt. In einigen Echo-Kompositionen wiederholt nur eine Stimme als Echo das zuvor vom Chor als "proposta" Vorgetragene (s.o.). Auch in Echo-Sätzen des 17. Jahrhunderts ist auf verschiedene Weise eine Verminderung der Besetzung bei Echo-Stellen vorgeschrieben. In der "Sonata Decima settima A 4 in ecco" von Dario Castello wird die Echo-Dynamik unterstiutzt durch Tasto-solo-Anweisungen in der Basso continuo-Stimme" 0 , in der "Canzon La Pichi In Ecco" von Giovanni Battista Riccio schweigt bei längeren EchoStellen das sonst collaparte mit dem Continuo geführte BaBinstrument 41 . Giovanni Paolo Cima ließ in seinen Echo-Motetten die "proposta" vom Organisten verdoppeln, die "rispasta" aber nicht42. In den Motetti a una, due, tre, e quattro voci (1624) von Nicolo Corradini werden Echo-Stellen in den Vokalstimmen mit "P." und "F.", in der der Orgelstimme aber mit "Pieno" und "Vuoto" bzw. mit den Abkiirzungen "P." und "V." gekennzeichnet, womit der Organist angewiesen wird, eine größere bzw. kleinere Zahl von Registern zu ziehen ${ }^{43}$. Adriano Banchieri schlieBlich erklärt den Organisten im Titel zu einer Echo-Fantasie für Orgel allein, daß sie die dynamische Wirkung durch Hinzu- bzw. durch Wegnahme eines Registers erzeugen solle 4. $^{2}$

men 37 Takten, an denen die Echo-Instrumente mitwirken), vgl. die Neuausgabe von Eleanor Selfridge-Field, Part II, S.81 ff.

39. Vg1. Wilhelm Pfannkuch, Art. Rinuccini, Ottavio in MGG, Bd.11, Sp.543 und bes. 545 sowie Leopold, Monteverdi, S.127.

40. Auf S.4 und S.6 der Continuo-Stimme steht zu den Stellen mit Zink und Echo-Zink bzw. Violine und Echo-Violine: "ecco. Va sonato il basso solo sino al duo"; im Schluateil der Sonate (ab S.6 unten), den beide Instrumente mit ihren jeweiligen Echo-Instrumenten zusammen bestreiten, steht abwechselnd "Basso solo" (Echo) und "Insieme" ("proposta").

41. Vg1. in der Neuausgabe G. B. Riccio, Canzoni da sonare a 1-4 voci, hrsg. von Rudolf Ewerhart, Heft II, S.19.

42. Vg1. weiter oben im Abschnitt II.2.3.1. sowie das Notenbeispiel II, 9 im zweiten Band dieser Arbeit.

43. Vg1. hierzu die Erk1ärung ähnlicher Angaben in L. Viadanas Salmi a quattro chori (1612; das Vorwort Viadanas ist im zweilen Band dieser Arbeit auf S.17 f wiedergegeben).

44. "Fantasia sesta in eco movendo un registro" aus Fantasie overo canzoni alla francese (1603). Die Stellen, an denen umregistriert werden soll, sind mit " $f$ " und " $p$ " gekennzeichnet (vgl. die Neuausgabe Adriano Banchieri, Fantasie overo canzoni alla francese von André Vierandeels, S.7 f). Auf änliche Anweisungen in den Continuo-Stimmen der Vokalmusik wird noch einzugehen sein. 
Diese dynamische Wirkung, die durch eine wechselnde Stärke der Besetzung hervorgerufen wird, fand freilich auch außerhalb besonderer Echo-Sätze Anwendung, z.B. in mehrchörigen Kompositionen mit Capellchören, deren Hinzutreten bei Tutti-Stellen immer einen Forte-Effekt hat ${ }^{45}$. Bei solchen Stuicken waren dynamische Angaben in der Regel nicht notwendig, denn die unterschiedliche Besetzungstärke alleine bewirkte die Lautstärkekontraste ${ }^{46}$. Unerläßlich konnte aber auch bei solchen Stücken eine genaue dynamische Bezeichnung in der Continuo-Stimme sein. In den Orgelstimmen ${ }^{47}$ bestimmen diese Angaben zumeist die jeweilige Registrierung, gleich ob sie direkt auf die Registrierung eingehen 48 oder aber in Form der Lautstärkebezeichnung "piano" und "forte" auftreten, dem nur durch eine wechselnde Registrierung können deutliche dynamische Abstufungen auf der orgel realisiert werden. Sehr genaue Anweisungen dieser Art enthält die Orgelstimme zu den Messe, salmi ... concertati a cinque, ò nove voci von Giovanni Ghizzolo (1619). Die nur in der Orgelstimme notierten dynamischen Angaben "FORTE", "PIANO" und als dritte Lautstärkestufe - "F." sollen den Organisten uber die jeweilige Besetzungsstärke informieren ${ }^{49}$. Im Vorwort dieser Sammlung heibt es, der Organist solle die in die Continuo-Stimme hineingeschriebenen Zeichen genau beachten, um zu wissen, wann Register hinzugefügt oder weggenommen werden

45. Noch W. C. Printz rechnet die Angaben "Capella" und "Tutti" zu "denen Zeichen/ so da andeuten Latitudinem Soni oder die Stärcke des Klanges oder Stimme." (Compendium musicae (1689), S.30 f).

46. Daß dynamische Angaben dennoch auch bei solchen durch die wechselnde Besetzung allein hervorgerufenen Lautstärkekontrasten zusätzlich notiert sein können, um die Wirkung noch zu steigern, wurde weiter oben anhand $G$. Gabrielis "Sonata Pian \& Forte" gezeigt.

47. Solche Effekte sind vor allem in großbesetzter Musik - und das heibt i.d.R. Kirchenmusik - zu beobachten. Hier ist eine Continuo-Stimme meist eine Orgelstimme (vgl. oben im Abschnitt II.3.3.1.).

48. Solche registrationsbezogenen dynamischen Angaben können entweder die jeweiligen Register selbst benennen (s.u.), die Zahl der Register angeben (so z.B. in der uberschrift zu A. Banchieris "Undecimo Concerto" aus dem "Terzo libro di nuovi pensieri (1613): "Cantasi piano con vn Registro, \& forte con dua") oder auch nur auf die Menge der Register hindeuten, wie es in der Hauptorgelstimme zu L. Viadanas Salmi a quattro chori (1612) mit den Worten "VOTO" und "PIENO" vorgenommen wurde. Im Vorwort heiBt es hierzu "e quanto trouerà queste parole VOTO, e PIENO, douerà registrare, voto; $e$ pieno" (vgl. das vollständige Vorwort im zweiten Band dieser Arbeit auf S.17 f). Auf eine ähnliche Bezeichnungsweise bei Nicolo Corradini wurde oben bereits hingewiesen.

49. Die dynamischen Angaben "piano" und "forte" wurden auch von G. Gabrieli in der Orgelstimme (Randstimmenpartitur) der Canzoni e sonate (1615, vgl. auch Kunze, Gabrieli, S.179) und Alessandro Grandi in der Orgelstimme zu den Salmi ... brevi (1629; vgl. das Notenbeispiel 102 bei Seelkopf, Grandi, Bd.II, S.255 ff) verwendet, un auf die jeweilige Größe der Besetzung hinzuweisen (in den anderen Stimnen stehen an den entsprechenden Stellen keine dynamischen Angaben). 
muissen. "FORTE" gäbe an, daß der zweite Chor hinzutritt, und zwar zusammen mit den Ripieni, nur "F." allein bedeute, daß der zweite Chor ohne die Ripieni einsetzt. "PIANO" schließlich besage, daß nur der erste Chor alleine fortfahre ${ }^{50}$.

Die Unterscheidung dieser drei unterschiedlich lauten Registrierungen genügte Ghizzolo jedoch noch nicht. Auch innerhalb der mit "PIANO" gekennzeichneten Teile, in denen der erste (der Favorit-) Chor alleine singt, soll der Organist auf die in der Orgelstimme stehenden Buchstaben " $C$.", " $A$. ", "Q.", "T." und "B." (= Canto, A1to, Quinto, Tenore und Basso) achten und dementsprechend mehr oder weniger Register ziehen ${ }^{51}$.

Ein Fulle von dynamischen Effekten, hervorgerufen durch zunehuende oder abnehmende Besetzung, verwendete Claudio Monteverdi in der sogenannten "Marienvesper"52. Besonderes Interesse verdienen die Besetzungsveränderungen in den beiden Magnificat53, da auch hier Registrationsanweisungen in der Continuo-Stimme die beabsichtigte dynamische Wirkung unterstreichen 54 . In eindrucksvoller Weise beginnen diese Kompositionen mit einem crescendo zu einer viermaligen Wiederholung des Wortes "Magnificat". Dies ist in der

50. Der entsprechende Abschnitt in Ghizzolos Vorwort lautet: "Quarto è che 1i Organisti, per hauer più facilità nel mettere, ó levare li registri, secondo il bisogno, potrāno riguardare all'infrascritti segni, e primieramète doue trouerāno questa parola FORTE tutta distesa, sarà inditio, che entri il Secondo Choro, et si facci ripieno: ma quando troueranno la sola lettera F. sarà segno, che entri il Secondo Choro, ma senza ripieni, e qundo si trouerà la parola PIANO, sarà segno che cessi il Secondo Choro, e canti solo il Primo." (zitiert nach Sartori, Bibliogratia I, S.250 (1619 c)). Dem Verfasser ist keine fribhere Verwendung von drei Lautstärkestufen bekannt.

51. Dies geht aus dem nächsten Punkt dieses Vorwortes hervor: "Quinta è che ritrouandosi l'infrascritte lettere C.A.Q.T.B. Sarà segno, che sotto a dette lettere entrino le parti, ò del Canto, Altro, Quinto, si che, secondo la necessità di più o meno Organo per il concerto, potranno l'Organisti porre più ò meno registri conforme al bisogno." (zitiert nach Sartori, Bibliografia I, S.250 (1619 c)).

52. Aus: Sanctissimae Virgini missa (1610).

53. Dieser Druck enthält zwei Fassungen derselben Magnificat-Komposition; eine erste für sieben Stimmen, Instrumente und Orgel und eine zweite für sechs Stimmen und Orgel a cappella. Beide sind abgedruckt in der MonteverdiGA, Bd.14, zweiter Halbband, S.285 ff und $327 \mathrm{ff}$.

54. Besondere Erwähnung verdient außerhalb der beiden Magnificat das auskomponierte diminuendo im Schlußamen des "Laudate pueri A 8 vori sole nel organo" (vgl. Monteverdi-GA, Bd.14, erster Halbband, S.168 f). Dieses letzte Amen wird von sechs Stimmen (nur zwei Tenöre fehlen) in kurzen Abständen (Semiminima oder Minima) imitatorisch begonnen. Bereits nach der Dauer einer Brevis setzt die erste Stimme aus. Ihr folgen nach und nach (innerhalb von zwei Breven) die anderen Stimmen. Ubrig bleiben nur die kurz vor Erde dieses diminuendo einsetzenden Tenöre. 
ersten (vokal-instrumentalen) Fassung folgendermaßen ausgefuhrt: Es beginnt nur eine Singstimme (Sopran) ohne Orgel, nach eineinhalb Takten ubernehmen zwei hohe und eine tiefere Singstimme mit Orgel, einer Violine und einem Zink, wieder eineinhalb Takte später fahren drei tiefe Stimmen mit Orgel fort, bis schließlich nach wiederum eineinhalb Takten ein Tutti (7 Singstimmen, 2 Violinen, 3 Zinken, "Viola da brazzo" (basso) und Orgel) einsetzt55; von Takt 8 ("Anima mea") bis zum Ende dieses Teiles singt nur noch ein Sopran zur Orge1. Dieses crescendo wird in der Orge1stimme durch das Hinzufugen von Registern ausgefihrt: erst "principale solo", dann "principale \& ottaua" und schlieblich "principale ottaua \& quintadecima"56. Zum "Anima mea" ist wieder nur "principale solo" vorgesehen. Ahnliche dynamische Effekte finden im Quia respexit des ersten Magnificat 57 , im Quia respexit des zweiten Magnificat58, im Quia fecit a 6 in Dialogo des zweiten Magnificat59 sowie - besonders eindrucksvoll - im Gloria patri des zweiten Magnificato0 Verwendung.

55. Die Tatsache, daß zwei hohe Stimmen mit collaparte-Instrumenten und einer tieferen Stimme hicr mit einer schwächeren Registrierung begleitet werden als die drei tiefen Stimmen (ohne Instrumente), zeigt, daß Monteverdi das unterschiedliche Klangvolumen der Knaben und der Männerstimmen und auch die akkustisch unterschiedlichen Wirkungen von tiefen und hohen Stimmen mit bedacht hat.

56. Aus Platzgründen ist die Zuordnung der einzelnen Registerangaben zu den jeweiligen Takten nicht vollständig gegliuckt. Sie ist aber wesentlich deutlicher als in der Monteverdi-GA. Malipiero verstand den Sinn dieser Angabe offensichtlich nicht und hat daher die wechselnden Registrierung einfach beziehungslos hintereinander gedruckt.

In der zweiten Fassung ist dieses crescendo noch ibersichtlicher ausgefuihrt. Hier uberschneiden sich die Eirsätze nicht, so daß die Besetzung alle zwei Takte wechselt: erst eine Singstimme ohne orgel, dann eine, vier und schließlich sechs Singstimmen mit Orgel. Leider wurde hier in der Orgelstimme die zweite (mittlere) Registerangabe vergessen.

57. Monteverdi-GA, Bd.14, 2.Halbband, S.289 ff: Ritornelle: "sei instrumenti $1 i$ quali suoneranno con più forza che si puó" und Orgelregistration "Principlae, ottava et quintadecima" (Registrationsanweisung zum zweiten Ritornell fehlt in der GA, nicht aber im Original. Eine Auflistung der in der GA fehlenden Registrationsanweisungen findet sich bei Tagliavini, Registratione, S.367, Anm.7), Mittelteil mit einer Singstimme und je zwei Instrumenten und der Orgelregistration "Principale solo".

58. GA, S.330 f: die Ritornelle werden hier von der Orgel alleine mit der entsprechenden Registrierung ausgeführt.

59. GA, S.331 ff (Registrierung fehlt in der GA): Dialog der Halbchöre mit Registrierung "principale solo", sechsstimmiger Schluß "principale \& ottaua".

60. GA, S. $347 \mathrm{ff}$ : Zunächst sind die sechsstimmigen Teile mit "Principale e ottava", die solistischen aber mit "principale solo" zu registieren. Der letzte sechsstimmige Abschnitt beginnt wie die anderen auch mit der Registrierung "Principlae e ottava". Dies wird jedoch bei der letzten 


\subsubsection{ANDERE DYNAMISCHE EFFEKTE IM DIENSTE DES TEXTAFFEKTES}

Auch die bisher angesprochenen dynamischen Effekte stehen in vokaler Musik in aller Regel im Zusammenhang mit dem Text. Ein Echo antwortet oder erklärt; eine dynamische Steigerung, hervorgerufen durch eine Besetzungsvermehrung, unterstreicht einen Wortsinn ("magnificat"). Bei einigen weiteren dynamischen Effekten ist diese Bindung an den Text noch unmittelbarer. Auch diese Effekte imitieren außermusikalische Erscheinungen. Im Gegensatz zu Echo oder Besetzungsänderung sind deren Vorbilder jedoch nicht sprachunabhängige Naturphänomene, sondern der außermusikalische Umgang mit der Sprache.

Eine eher äußerliche Funktion erfüllt die Dynamik in diesem Sinne in den Madrigalkomödien Adriano Banchieris. Auf ihre Anwendung in der Pazzia senile wurde oben bereits eingegangen61. In der Barca di Venetia per Padova (1605) vermehrte Banchieri die Funktionen der Dynamik. Besonders häufig kommt hier die in einem Dialog in der Pazzia bereits angewendete Bezeichnung der verschiedenen Stimmen eines Satzes mit unterschiedlichen Lautstärkeangaben vor $^{62}$. Dies geschieht in der Barca jedoch auch außerhalb von Dialogen. So preist der Austernverkäufer seine Ware im forte $\mathrm{an}^{63}$, während die uibrigen Stimmen piano Freud und Leid der Fischer besingen 64 . Der als Säufer charakterisierte Deutsche ruft sein "Zum Wohle" natiirlich im forte aus65, die ubrigen Stimmen ubernehmen piano die Rolle des Erzählers66, und Rizzolina singt forte zur von den anderen Sänger piano imitierten laute67. Hier soll die jeweilige akkustische Situation imitiert werden (Markts $\mathrm{c}$ h $\mathrm{r}$ e $\mathrm{i}$ e $\mathrm{r}$, "gröhlender" Säufer, im Verhältnis zum Sänger leise Laute).

Nicht nur durch eine besondere Situation (Marktschreier), sondern auch durch den Textinhalt kann eine Veränderung der Sprechlautstärke hervorgerufen werden. Ein guter Redner unterstreicht mit der Lautstärke seiner Stimme den

Wiederholung der Worte "et Spiritu Sancto" zu "Principale ottava et quintadecima" gesteigert, ohne daß damit eine Vermehrung der Besetzung einhergeht. 61. Im Abschnitt III.1.2.

62. Ein Dialog mit unterschiedlich lauten Partnern wurde von A. Banchieri auch in der Orgelmusik imitiert. An die Stelle von dynamischen Bezeichnungen traten hier Registerangaben ("Secondo Dialogo: Acuto \& Graue" aus der zweiten Auflage des L'organo suonarino (1611), auf S.32 der Teilfaksimileausgabe.

63. "Intermedio de pescatori", Tenore: "Ostreghe da bruazo ...".

64. Canto, Alto und Basso: "Pescatori nù semo ...".

65. "Bell'humore venetiano", Tenore: "Brindis ió iò iò iò iò ...".

66. Canto, Alto und Basso: "Hor suso el fiasco in ronda ..."; nur hei diesem Satz ist die originale Dynamik auch in der Neuausgabe von Elio Piattelli nach der Ausgabe von 1623 beibehalten worden.

67. "Rizzolina canta \& Horatio suona il Lauto", Canto: "Io mi ricordo ...". Alto, Tenore, Basso: "ten ten" bzw. "tronc tronc" bzw. "trinc trinc" ete. 
jeweiligen Textaffekt. Auch dies wurde in der textgebundenen Musik imitiert und konnte mit dynamischen Anweisungen notiert werden 68 .

Ein recht frihes Beispiel für in dieser Weise verwendete dynamische Bezeichnungen findet sich wiederum im Werk Adriano Banchieris. Sein Terzo libro de nuovi pensieri ecclesiastici (1613) enthält ein "Concerto piano \& forte" 69 uber eine Episode aus dem Hohen Lied70. Die hier vorgenommene Zuordnung von Text, Dynamik und Tempo soll der Deutlichkeit halber im folgenden tabe1larisch wiedergegeben werden:

Text

Ego dormio, et cor meun vigilat.

Lautstärke- und Tempoangabe

Aperti mihi, soror, mea sponsa.

PIANO, \& graue

Amica mea, formosa mea, et veni.

FORTE, \& spirituoso

Expoliavi me tunica mea, quomodo induar illa? FORTE

Lavi pedes meos, quomodo inquinabo illos? PIANO

Lavi pedes meos, quomodo inquinabo illos? FORTE

Trotz des Schematismus im zeilenweisen Wechsel der dynamischen Vorschriften ist der Zusammenhang von Text und Dynamik deutlich: piano zu "ich schlief", dann der Ruf "offne mir" natiurlich forte. Die Bitte "Amica mea, formosa mea et veni" leise - flüsternd - aber fröhlich. Der letzte Satz schließlich wird als Frage durch die Wiederholung bekräftigt, zuerst leise, dann laut und aus ladend 71 .

Ebenso deutlich ist dieser Zusammenhang auch zu Anfang der Motette "Plangite mortales" aus Giacomo Finettis Corona Mariae quatuor concinenda ... liber quintus (1622). Auch hier ist die dynamische Unterscheidung mit der Verwendung unterschiedlicher Tempi gekoppelt, wenngleich die Tempodifferenzierung hier nicht durch Tempoworte, sondern durch die Verwendung unterschiedlicher Notenwerte hervorgerufen wird.

Text

Laustärkeangabe und Tempo

Plangite, plangite mortales, plangite piano, lange Notenwerte omnes morimini

forte, kurze Notenwerte

plangite piano, lange Notenwerte

omnes morimini ... forte, kurze Notenwerte

68. Es kann davon ausgegangen werden, daß dynamische Differenzierungen dieser Art bereits im 16. Jahrhundert iblich gewesen sind (s.o.) und auch im 17. Jahrhundert weit häufiger als notiert verwendet wurden.

69. Der Titel "Concerto piano, \& forte" steht nicht iber dem Satz, sondern nur iber der zweiten Seite dieses Stiuckes (S.27): "Residuo a1 XI. Concerto piano, \& forte". iberschrieben ist dieses Concerto mit "Vndecimo Concerto. Cantasi piano con vn Registro, \& forte con dua" (S.26). Dieser Satz ist Notenbeispiel III,3 im zweiten Band dieser Arbeit im Faksimile abgedruckt.

70. Es handelt sich im wesentlichen um Hohes Lied 5, Vers $2+3$. Vers 2 ist jedoch gekürzt und z.T. durch den letzten Satz von Hohes Lied 2, Vers 10 (leicht verändert) ersetzt worden.

71. Die textbedingte Dynamik in diesem Satz von der Echodynamik unterschie- 
In etwas anderer Weise steht die Dynamik in dem an ein Accompagniato erinnernden SchluB des "Possente spirito" aus Claudio Monteverdis L'Orfeo (1609)72 im Zusammenhang mit dem Text. Orfeo singt hier von den "corde soaui" seiner goldenen Leier, mit denen allein er bewaffnet sei ${ }^{73}$. Hier wird nicht die Dynamik eines Redners, sondern die im Text erwähnten "corde soaui" sowoh1 mit einer dynamischen Angabe - "tocchi pian piano" - als auch mit einer Instrumentation (drei "Viole da braccio" und ein "contrabasso de Viola") imitiert 74 .

Besondere Erwähnung verdient die Behandlung der Dynamik in einer Komposition Alessandro Grandis. Während die bisher zitierten Beispiele die bei der jeweiligen Textaussage naheliegende Dynamik vorschrieben, verlangt Grandi in seiner Vertonung des "Lauda Jerusalem" (Ps.147 bzw. 147, Vers 12 - 20) aus der Raccolta terza (von Leonardo Simonetti 1630 bei Magni herausgegeben) 75 eine der Textaussage eigentlich entgegenlaufende Dynamik. Zu erwarten ist eine Vertonung wie die aus Claudio Monteverdis Sanctissimae Virgini missa $(1610)^{76}$, in der klangstark mit Rufintervallen zum Lob herausgefordert wird. In Grandis "Lauda Jerusalem" ist jedoch mit Ausnahme des Schlußverses 77 und des "Gloria patri et filio sancto" "piano" vorgeschrieben; selbst das abschließende "Sicut erat" ist wieder piano zu musizieren. Unterstrichen wird die verhaltene Stimmung durch lange Notenwerte (z.B. "Lauda" mit zwei Semibreven gleicher Tonhöhe) und die tiefe Lage des Satzes78.

Die Dynamik ist hier zu einem frei einsetzbaren Mittel der Komposition geworden. Ein Komponist kann sie verwenden, um seine individuelle sicht eines Textes zu unterstreichen. Hier sind die dynamischen Vorschriften freilich unabkömmlich, da nur mit ihrer Hilfe einer Komposition die zunächst fernliegende Färbung gegeben werden konnte.

den: Die beiden kurzen Echo-Stellen dieses Satzes sind nicht mit piano und forte, sondern lediglich mit "ECCO" bezeichnet.

72. S.65 der Ausgabe von 1609.

73. Der Text lautet: "Sol tu, nobile Dio, puoi darmi aita, ne temer dei, che sopra un'aurea certa sol di corde soavi armo le dita, contra cui rigida alma invan s'impera".

74. Die Anweisung auf S.64 unten lautet: "Furno sonate le altre parte da tre Viole da braccio, \& vn contrabasso de Viola tocchi pian piano".

75. Die Kenntnis dieses Satzes verdanke ich Herrn Prof. Dr. Rudolf Ewerhart, Hochschule für Musik, Köln.

76. Vg1. Monteverdi-GA, Bd.14, 2. Halbband S.237 ff.

77. Vers 20: "Non fecit taliter omni nationi, et iudica sua non manifestavit eis."

78. Die originale Schlüsselung ist dem Verfasser nicht bekannt. Der Sopran des ersten Chores (Hochchor) iberschreitet das $e^{\prime \prime}$ nicht, die erste Stimme des zweiten Chores (Tiefchor) nicht das a'. Die Tiefe wird zusätzlich durch zwei Posaunenstimmen betont. 


\subsection{CRescendo, Diminuendo und "Gesangsdynamik"}

Es konnte bereits auf eine ganze Reihe recht unterschiedlicher Anwendungen der Lautstärkeangaben verwiesen werden. Diese belegen, daß im fríhen 17. Jahrhundert von dynamischen Effekten weit uber das Echo hinaus ${ }^{79}$ bereits reichlich Gebrauch gemacht wurde. Alle erwähnten dynamischen Angaben bezeichnen jedoch nur Lautstärkestufen bzw. Lautstärkeflächen. In der Tat erweckt die Anwendung der dynamischen Bezeichnungen im fruihen 17.Jahrhundert (und noch dariber hinaus ${ }^{80}$ ) den Eindruck einer "Terassendynamik"81. Dabei wird jedoch verkannt, $\mathrm{da}$ auch eine Folge von dynamischen Zeichen ein crescendo oder diminuendo andeuten kann 82 und, daß die notierte Dynamik nur einen Aspekt der Dynamik in der Musik des'Frihbarock (und des Barock iberhaupt) repräsentiert³.

Durch Anweisungen in Vorreden besonders gut dokumentiert ist die nichtnotierte "Feindynamik" in der Monodie. Die in der Monodie geforderte, sich nach der Sprache richtende Art des Gesanges imitierte auch die "Dynamik" eines affektiert sprechenden Rezitators. Zu den besonders augenfälligen Lautstärkeeffekten eines Rezitators gehört der Ausruf; er hat mit Caccinis "esclamatione" seinen Niederschlag in der Gesangslehre gefunden ${ }^{84}$. Auch zahlreiche andere dynamische Effekte werden bei Caccini beschrieben ${ }^{85}$;

79. Insbesondere bei Harding, Expression, S.85 ff sowie S.90 wird dem EchoPrinzip viel zu große Bedeutung zugemessen.

80. Das Crescendo-Zeichen ist erst seit Anfang des 18. Jahrhunderts bekannt (vgl. Boyden, Dynamics, S.187).

81. Siehe Heuss, Dynamik, S.144 f. Heuss räumte aber in der sich an seinen Vortrag anschließenden Diskussion ( $\mathrm{S} .146 \mathrm{f}$ ) ein, daß es in der Vokalmusik darüber hinaus eine "Gefühlsdynamik" (S.146) gab. Diese sei jedoch kein eigentlicher Bestandteil einer Komposition (S.147).

82. Bsp. hierfür bei Boyden, Dynamics, S.186, und Witzenmann, Mazzocchi, S.213. Besonders deutlich hier die von Witzenmann beschriebene Folge "P." "F." auf einem ausgehaltenen Ton. Vgl. bei Witzenmann auch S.139 und $188 \mathrm{ff}$. 83. Hierauf hat bereits mit Nachdruck Kolneder, Dynamik, S.343 ff hingewiesen (S.345: "Es ist doch einfach ummöglich sich vorzustellen, Orfeo hätte um Euridice in Terassendynamik geklagt").

84. "1'esclamatione, che è mezzo più principale per muouere l'affetto: \& esclamatione propriamente altro non è, che nel lassare della voce rinforzarla alquāo" (Le nuove musiche (1601/02), 3.Seite des "Ai lettori").

85. Auf der 3.und 4. Seite des "Ai lettori" ist an zahlreichen Stellen von einem "crescere" oder "scemar della voce" die Rede. Diese Stellen sind der Forschung hinreichend bekannt und brauchen hier nicht wiederholt zu werden. Auf Caccini verweist im besonderen Harding, Expression, S.88 ff. Eine englische ibersetzung von Caccinis Vorwort nach John Playfords Brief introduction of the Skill of Music, erstes Buch (1670) ist dort ab S.88 unten mitgeteilt. Informationen hierzu auch in den Untersuchungen von Goldschmidt, Gesangsmethode, S.67 ff (dort auch Hinweise auf weitere auf Caccini bezugnehmende Aussagen in anderen Vorworten des fribhen 17.Jahrhunderts), Boyden, 
manche von ihnen fuhren bereits über die Dynamik des Redners hinaus, wie das "messa di voce"86. Diese Effekte blieben freilich nicht auf die Monodie beschränkt, sondern werden auch in den anderen expressiven Musikgattungen Anwendung gefunden haben87. Der Sänger sollte "seine ganze Seele in die gesungenen Töne" hineinlegen 8 8.

In Anlehnung an Caccini wurden diese dynamischen Verzierungen bald auch in zahlreiche andere Gesangslehren aufgenommen ${ }^{89}$. Francesco Rognoni Taeggio fordert in einem "Auertimenti à Cantandi" zu seinem Selva de varii passaggi (1620)90 die Sänger auf, bei Worten, die Schmerz, Kummer, Sorge, Qual und ähnliche Dinge ausdruicken anstatt "passaggi" besser "gratie", "accenti" und "esclamationi" zu verwenden und die Stimme bald abnehmen, bald zunehmen zu lassen, mit süßen und lieblichen Bewegungen, mit rührender und schmerzhafter Stimme, ganz dem Sinn der Rede angepaßt91. Auch in der der Selva vorausgehenden Verzierungstabelle spricht er die zu den jeweiligen Verzierungen gehörende Dynamik an ${ }^{92}$.

Der nächste Schritt in der Entwicklung dynamischer Bezeichnungen, nämlich die Einführung eines Zeichens für eine wechselnde Lautstärke, wurde erst 1638 in Domenico Mazzocchis Madrigali a cinque voci vollzogen. Mazzocchi beschreibt im Vorwort zu diesen Madrigali neben den Abkiirzungen " $P, F, E, t$. intesse per Piano, Forte, Echo, e trillo, già sono volgari, e note à tutti" und den Abkürzungen " $X$ " und " $V$ " (diese Zeichen gehören in den eher peripheren Bereich der Experimente mit dem enharmonischen Tongeschlecht) ${ }^{9} 3$ auch ein

Dynamics, S.185 und Robinson, messa di, voce, S.1 und S.6, sowie in den MGGArtikeln Gesangskunst von Hans Joachim Moser (Bd.4, Sp.1898) und Gesangspädagogik von Arnold Geering (Bd.4, Sp.1918 f).

86. Ein An- und Abschwellen auf einem Ton. Den Begriff "messa di voce" verwendet Caccini nicht. Vgl. hierzu Robinson, messa di voce.

87. Z.B. im polyphonen Madrigal. Auch hier finden sich die für die Anwendung der dynamischen "Figuren" typischen Melodiewendungen; vgl. - um ein berümtes Beispiel zu nennen - den Anfang von Monteverdis "Cruda Amarilli" (füntes Madrigalbuch (1605), Nr.1 = Monteverdi-GA, Bd.5, S.1-4) mit den in Caccinis "Deh, deh doue son fuggiti" (7.Seite des "Ai lettori") mit "escla." bezeichneten Stellen.

88. So um 1600 D. P. Cerone (nach Hans Joachim Moser, Art. Gesangskunst in MGG, Bd.4, Sp. 1896).

89. Vg1. Arnold Geering, Art. Gesangspädagogik in MGG, Bd.4, Sp. 1919.

90. Letzte Seite des ersten Teiles.

91. "S'hanno ancora a guadare da passaggi sopra parole significanti doglia, affanni, pene, tormenti. \& simili cose, perche iuvece de passaggi, s'vsano fare gratie, accenti, \& esclamationi, scemando hor la voce, hor accrescendola, con mouimenti dolci, e soaui, \& tal'hora con voce mesta, \& dogliosa, conforme il senso dell'oratione."

92. Unter Punkt 1 ("portar della voce") und Punkt 6 ("esclamationi").

93. Vg1. hierzu Harding, Expression, S.90 ff. Mazzocchis Vorwort ist hier auf S.91, Anm.2, abgedruckt. 
Zeichen (" $C$ ") für die heute als "messa di voce" bekannte dynamische Verzierung 94 . Dieses Zeichen blieb aber zunächst ohne Nachfolge. Weitere Zeichen für dynamische Entwicklungen erschienen erst wieder in der Violinmusik des 18. Jahrhunderts95.

Aus dem Bereich der reinen Instrumentalmusik fehlen aus der Zeit bis 1630 konkrete Hinweise auf eine solche Feindynamik. In dem 1638 gedruckten Combattimento Claudio Monteverdis (Urauffuhrung allerdings bereits 1624) steht in den Instrumentalstimmen eine interessante diminuendo-Anweisung: "questa ultima nota va arcata morendo"96. Eine Anweisung zur Dynamik enthä1t auch der ebenfalls 1638 veröffentlichte Modo per imparare a sonare di tromba von Girolamo Fantini97. Fantini verlangt hier, daß jede längere Note im "modo cantabile" (!) ausgefuhrt werilen soll. Der von Fantini beschriebene "modo cantabile" entspricht einem "messa di voce"98. Wahrscheinlich steht diese Anweisung zum "modo cantabile" ausgerechnet in dieser Trompetenschule (dort wïrde man vielleicht am wenigsten damit rechnen), weil Fantini mit dieser Methode auch der Trompete zum Einzug in die Kunstmusik verhelfen wollte 99 . Bei den anderen Instrumenten wurde der "modo cantabile" wahrscheinlich ohnehin angewendet.

Nur die Instrumente, die in der Lage waren, verschiedene Lautstärkestufen hervorzubringen, werden auch in den Instrumentationsangaben des frïhen 17. Jahrhunderts verlangt100. Die Instrumente mit starrer Dynamik (wie Windkapselinstrumente und viele der Rohrblattinstrumente uberhaupt) sind in diesen Besetzungsangaben nicht zu finden. Der Zink - zunächst das Virtuoseninstrument des späten 16.Jahrhunderts - wird besonders gelobt wegen seiner

94. Vg1 hierzu weiter unten. Mazzocchi bezeichnet in seinem Vorwort abweichend von der späteren Terminologie ein crescendo bei gleichzeitig steigender Tonhöhe als "messa di voce". Dies komme aber nur im enharmonischen Tongeschlecht vor (vgl. hierzu aber auch im Abschnitt III.3.2.2.1. dieser Arbeit).

95. Boyden, Dynamics, S.187.

96. Vg1, hierzu Boyden, Dynamics, S. 186. Weitere Beispiele für - allerdings nicht so ausdrücklich notierte - Schlußdiminuendi bzw. piano-Schlüse bei Witzenmann, Mazzocchi, S.213.

97. Das Titelblatt nennt als Erscheinungsort "Francofort".

98. S.6: "Si deue anco auuertire, che quando si troueranno note di valore, cioe di vna, di dua, e quattro battute, si deuono tenere in modo cantabile, con mettere la voce piano, e poi venir crescendo sino al mezo valore della nota, e con l'altro mezo andar calando sino al fine della battuta, che a pena si senta, che cosi facendo si renderà perfetta armonica.".

99. "HAuendo mandato alle stampe questo mio debil volume per benefizio di chi professa, o volesse professare di sonar di Tromba: non più in aria come gia si soleua, ma co'l vero fondamento come gli altri strumenti perfetti" (Anfang des "L'Autore a $i$ Lettori", S.6).

100. Vgl. hierzu in den folgenden Abschnitten. 
Fähigkeit, die verschiedenen Lautstärkestufen ebenso nutzen zu können wie die menschliche Stimme101.

Uberaus deutlich kommt der Wunsch nach dynamischer Gestaltung auch in der Instrumentalmusik in dem bei Michael Praetorius mitgeteiltem Traktat des Instrumentenbauers Hans Haiden ${ }^{102}$, eines "Instrumentenmachers vnnd Erfinders eigene Wort vnnd gedancken/ welche er in einem kleinen Tractätlein/ Anno 1610. im Druck herfür gegeben" 103 zum Ausdruck. Dort wird bedauert, daß es den Tasteninstrumenten an "der schönsten Zier/ nemlich der Moderation der Stimmen" mangelt. Dies sei ebenso schlimm, wie wenn ein Redner "im außsprechen mit erhebung vnd niederlassung der Stimm/ wie es der Text vnd affectus erfoddern/ keinen decorum helt; Sondern immer im gleichen Thon an einander vnabgesetzt fort redet"104. Auch das Wechseln der Orgelregister sei nur ein Behelf, da ja weiterhin die Moderation fehle105. Als Lösung dieser Probleme empfieh1t dieser Instrumentenmacher das von ihm erfundene Geigenwerk. "Und ob wohl der Text mit Worten sich nicht auBsprechen lest/ so kan doch der Instrumentist seinen sensum zu erkennen geben/ ob trawrige oder fröhliche Gedanken in ihme sind"106.

\section{ANGABEN ZUR KLANGFARBE}

2.1. INSTRUMENTATION

\subsubsection{INSTRUMENTATION IN DER ZWEITEN HÄLFTE DES 16. JAHRHUNDERTS}

Genaue Angaben uber die zu verwendenden Instrumente finden sich bereits im 16.Jahrhundert regelmäßig auf den Titelblättern der Drucke mit Musik für Harmonieinstrumente ${ }^{107}$. Diese Angaben waren hier kein aufführungspraktischer Zusatz, sondern als wesentliche Grundinformation im Titel der Drucke unerläBlich, handelt es sich doch bei diesen Drucken um Musik in instrumenten-

101. G. Dalla Casa, I1 vero modo di diminuir ... libro primo (1584), "Ai lettori", 3.Teil: "Del Cornetto. DE gli Stromenti di fiato il più eccellente e $i 1$ Cornetto per imitar la uoce humana piu de gli altri stromenti. Questo stromento si adopera piano, \& forte, \& in ogni sorte di Tuono, si come fa la voce". Dalla Casa folgend schreibt G. M. Artusi, L'Artusi (1600), fol.5: "Ma notate, che questo Instromento è atto quanto ogni altro ad imitare la voce humana; e'l buono, \& eccellente Sonatore l'adopra forte, cioe lo fa sentire con suono gagliardo, \& con suono più rimesse, alto, basso, \& in ogni sorte di tuono, come più li piace, piu e manco, seconda la sua eccellenza".

102. Vg1. hierzu v. d. Meer, Musikinstrumente, S.94.

103. Syntagmatis musici ... tomus secundus (1618), S.68 ff. Auf diese Stelle weist auch Boyden, Dynamics, S.186, hin.

104. S. 69 .

105. Ebenda.

106. S. 70 .

107. Vgl. die bei Sartori, Bibliografia, mitgeteilten Titel der Drucke 1517, 1540, 1543?, 1549 a), 1551 b), s.a Posteriore al 1555, 1567, 1568 a), 1568-1569, 1576, 1580, 1586 a bis), 1588 c), 1590 b), 1591 b), c), 1592 a), c), d), 1593 b), c), etc. 
spezifischer Notation, nämlich in Tabulatur. Ein Druck in Lautentabulatur mubte bereits im Titel einen Hinweis auf die Laute tragen, denn nur für den Lautenisten war dieser Druck von Wert. Diese Tradition setzte sich im 17. Jahrhundert fort; auf sie ist es auch zurückzuführen, daß in den frihen Drucken mit Basso continuo fast immer ein Continuoinstrument genannt wird, denn auch bei der GeneralbaAschrift handelte es sich (zunächst) um eine solche instrumentenspezifische Notationsform 108 .

Fehlte aber eine solche direkte Bindung der Notation an ein bestimmtes Instrument, so konnte auch eine Intrumentenangabe entfallen ${ }^{109}$. Diese ergab sich aus der Art der Musik110 und vor allem aus dem in Frage kommenden Instrumentarium; und dies war in Italien zumindest im späteren 16.Jahrhundert recht begrenzt. In erster Linie ist hier an die Zinken-Posaunen-Gruppe und an Violenensembles zu denken. Die Violen wie auch die Zinken-Posaunengruppe werden in jeder Intermedienbeschreibung genannt111 und in den theoretischen Schriften des späteren 16.Jahrhunderts beschrieben, sofern diese uberhaupt die Musikinstrumente behandeln ${ }^{112}$. Andere Instrumentenfamilien werden lediglich von Lodivico Zacconi erwähnt (er nennt je drei verschiedene Lagen von den Blockflöten und den "Doppioni")113.

108. Vg1, weiter oben im Abschnitt II.3.3.

109. In den folgenden Ausfuhrungen kann die Frage der Besetzung im späten 16. Jahrhundert freilich nicht erschöpfend behandelt werden. Einige grundsätzliche Bemerkungen hierzu sind hier jedoch unerläßlich, da im späten 16. Jahrhundert Entwicklungen zu beobachten sind, die die Verwendung von Instrumenten und Instrumentationen im frihen 17. Jahrhundert entscheidend bestimnt haben.

110. So kann z.B. angenommen werden, daB die im 16. Jahrhundert verbreiteten Lehrduos entweder gesungen oder aber auf Streichinstrumenten gespielt wurden (vgl die Dedikationen zu Agostino Lincinos secondo libro di duo chromatici (1545), abgedruckt bei Sartori, Bibliographia I, S.13 (1546), sowie zu den Contrapuncti a due voci von Vincenzo Galilei (1584), abgedruckt bei Sartori, $\mathrm{S} .48(1584 \mathrm{c}))$.

111. Vgl. die Tabellen bei Weaver, Instrumenation, $\mathrm{S} .374 \mathrm{ff}$.

112. Es wurden folgende Quellen aus den Jahren um 1600 herangezogen: E. Bottrigari, Il desidero ouero de' concerti di varii strumenti musicali (1594), L. Zacconi, Prattica di musica (1596), G. M. Artusi, L'Artusi overo delle imperfettioni della moderna musica (1600) sowie A. Virgiliano, I1 dolcimelo (um 1600) und, als Vergleichsquelle, M. Praetorius, Syntagmatis musici ... tomus secundus de organographia (1619). Eine besondere Bedeutung kommt hier dem - leider unvollständig gebliebenen - "1ibro terzo, doue si contengono tutti modi da fare qualsiuoglia instrumento; con $i$ loro accordi, tanto in concerto quanto seperati" aus A. Virgilianos I1 dolcimelo (um 1600) zu. Diese "Organographia" Italiens behandelt neben zah1reichen einzelnen Musikinstrumenten auch zwei Ensemblebesetzungen, nämlich das Violenensemble (S. (93), S. (95) und S.(98)) und das Zink-Posaunenensemble (S.(102)).

113. Prattica di musica, fol. 218 f. Zum Vergleich: M. Praetorius, Syntagmatis musici ... tomus secundus de organographia (1619), nennt nicht weniger 
Eine gewisse Verbreitung hatten im späten 16.Jahrhundert dariber hinaus Blockflöten, vornehmlich in Diskantlage114, Traversflöten ${ }^{115}$ sowie einige nicht eindeutig zu definierende Instrumente, nämlich die "Dolzaine"116, "Cornamusen"117 (Windkapselinstrumente?) und "Pifari" bzw. "Fifari"118 (ge-

als 15 mehr oder weniger vollständige Instrumentenfamilien (vg1. die "Tabella Universalis", S.20 ff). Vgl. zu den Aufzählungen von Instrumenten in deutschen Traktaten Bartels, Aspekte, S.66 ff. Bartels bestätigt, daß das aufgelistete Instrumentarium nicht unbedingt dem tatsächlich verwendeten entspricht (z.B. werden Instrumente genannt, die die Verfasser offensicht1ich nicht kannten; Bartels, S.77, Anm. 330). Auch Praetorius setzt nur einen kleinen Teil der von ihm beschriebenen Instrumente in seinem Instrumentationsvorschlägen tatsächlich ein (Bartels, S.95).

114. Virgiliano, dolcimelo, beschreibt nur eine Blockflöte in g (S.(111)), Zacconi, Pratica di musica, nennt daruber hinaus noch eine Tenorblockflöte in $\mathrm{C}$ und eine Baßblockflöte in F (fol.218'). Weaver gibt in seinen tabellarischen Ubersichten (Instrumentation, S.374 ff) zu fast jedem Intermedium "flauti" an, teilweise als "diritti" näher bezeichnet, selten auch "tenori di flauto" (Florenz 1539), "Flauto grande di tenore" (Florenz 1565) oder "Flauti grande" (Florenz 1585). In den anderen oben genannten Quellen wird nur der "Flauto" oder "Flauto diritto" genannt (Bottrigari, Il desidero, Seite 11 und S.40, Artusi, L'Artusi, fol. 1' und fol. 11). Auch in den Instrumentationsvorschriften des frühen 17. Jahrhundert wird die Flöte nur als Diskantinstrument verlangt (s.u.).

115. Die Traversa wird in den meisten Intermedienbeschreibungen und allen herangezogenen theoretischen Quellen genannt. Wahrscheinlich beziehen sich Zacconis Außerungen zum "Fifaro" (Prattica di musica, fol.218) auch auf die Traversflöte (s.u.).

116. Die Dolzaina wird ebenfalls in allen der genannten theoretischen Quellen sowie in den Auffuhrungsbeschreibungen der Intermedien von Florenz 1567, 1569 und 1585 (Weaver, S.376 und 378) erwähnt. Leider ist bei Virgiliano, II dolcimelo, S.(117), von dem Abschnitt uber die Dolzaina nur die Uberschrift fertiggestellt. Zacconi gibt für dieses Instrument einen Tonumfang von 9 Tönen (c-d') bzw. mit Klappen von 11 Tönen (c-f') an (Prattica di musica, fol. 218). Dieser Tonumfang deutet auf ein Windkapselinstrument (so auch $v$. d. Meer, Musikinstrumente, S.81. V. d. Meer glaubt, die Dolzaina sei mit der von Praetorius beschriebenen Cornamuse identisch - s.u.). Praetorius kennt den Namen "Dolzaina" sowohl als Namen für den Fagotto (S.38) als auch für Sordunen (S.39).

117. Eine Cornamuse wird nach Weaver nur in einer italienischen Intermedienbeschreibung genannt, nämlich in derjenigen der Auffuhrung von 1539 in Florenz (Weaver, S.376). Außer bei Bottrigari wird die Cornamuse in allen genannten Quellen erwähnt. Konkrete Angaben zu dem Instrument fehlen allerdings. Der Name ist eine Verballhornung von "Corno muto" (Sachs, Musikinstrumente, S.92). Sachs hält die Cornamuse fiir eine Art Schalmei, kann dirs jedoch nicht belegen. Es ist zumindest zweifelhaft, ob hiermit immer das heute unter diesem Namen bekannte (und ohne historische Vorbilder gebaute) Windkapselinstrument ("gerades Krummhorn") gemeint ist. Sicher nicht gemeint 
meint sind z.T., Schalmeien, iberwiegend aber wohl Traversflöten). Hinzu kommen noch einige Rohrblattinstrumente, die jeweils nur in einer italienischen Quelle genannt werden ${ }^{119}$, und das im späten 16.Jahrhundert ebenfa11s noch sehr seltene Fagott 120 .

Auch wenn im 16. Jahrhundert noch keine Instrumentationsangaben notiert wurden, kann dennoch nicht von einem "Zufallsorchester" gesprochen werden. Das Instrumentarium war übersichtlich. Eine der beiden "Familien" (Blechb1äser ${ }^{121}$ oder Streicher) muBte vorhanden sein. Eine nicht sehr große Anzah1 von vornehmlich Baß- und Sopraninstrumenten konnte hinzugenommen werden. DaB dies nicht willkirlich geschah, sondern zumindest bei den Intermedien dem Typ der Szene entsprechend erfolgte, hat Robert L. Weaver anschaulich gezeigt122. Blasinstrumente etwa (vornemlich Flöten und Rohrblattinstrumente) dominieren bei Hirtenszenen123, tiefe Instrumente - mit Vorliebe Posaunen - bei "infernal or horrendous scenes"124 und so fort.

Direkt ausgefihrte Instrumentationen vor 1597, wie sie als Anweisungen für Laienmusiker in Drucken Attaingnantsi25 oder wenigen mitte1- und nordeuropäischen Handschriften als Merkhilfen ${ }^{126}$ vorkommen, sind dem

ist der Dudelsack (ital.: Cornamusa).

118. Sie werden bei Bottrigari, Il desidero, S.40 ("piffarotti"), Zacconi, Prattica di musica, fol.213 ff ("Fifari") und Virgiliano, I1 dolcimelo, S.(107), erwähnt. Der bei Zacconi, fol. 218 f, angegebene Tonumfang deckt sich mit demjenigen einer Traversflöte (vg1. auch Smith, Renaissancequerflöte, S.18). Praetorius erwähnt "Fiffaro" als italienischen Namen für "Querpteife" (S.35). Verschiedene Instrumentationen des frühen 17.Jahrhunderts deuten ebenfalls auf eine Traversflöte hin (s.u.). Virgiliano hingegen bildet die "Pifari" (S.(17)) als Schalmeien ab.

119. Virgiliano, Il dolcimelo, S.(113) f, gibt Zeichnung der sonst nicht bekannten "Armilloni"; Zacconi, Prattica di musica, fol. 213 ff, erwähnt

"Doppioni" (die schon M. Praetorius nicht zu Gesicht bekommen hatte (S.39)) und Sordunen.

120. Der Fagotto wird ebenfalls bei Zacconi, fol. $213 \mathrm{ff}$ genannt.

121. Es verzerrt das Bild, daß Weaver die Zinken zu den "flutes" rechnet. Auch wenn sie nicht aus Messing sind, gehören die Zinken eindeutig in die bei Weaver so benannte Kathegorie "Brass".

122. Instrumentation.

123. Vg1. die Tabelle 2 bei Weaver (S.376).

124. Vgl. die Tabelle 3 bei Weaver (S.377).

125. Pierre Attaingnant, Chansons musicales a quatre parties desquelles les plus convenables a la fleuste dallemant sont signees en la table cy dessoubz escripte par a. et a la fleuste a neuf trous par b. et pour les deux fleutes sont signes par $a b$, Paris 1533 sowie Pierre Attaingnant, Vingt \& sept chansons musicales a quatres parties desquelles les plus convenables a la fleuste dallemant sont signees en la table cy dessoubz escripte par a. et la fleuste a neuf trous par $b$. et pour les deux par ab, Paris 1533 (zitiert nach Smith, Reanissancequerflöte, $\mathrm{S} .52 \mathrm{ff}$; vgl. dort auch $\mathrm{S} .28 \mathrm{ff}$ ). 
Verfasser aus italienischen Quellen nicht bekannt. Ansätze zu einer Instrumentation finden sich im Vorwort des Intermediendruckes von 1591127. Hier wird zu fast jedem Satz eine Besetzung genannt. Im Vordergrund stand jedoch hier der Wunsch, die beteiligten Personen aufzufuhren ${ }^{128}$. Als Vorstufe einer Instrumentation können auch Hinweise auf eirue der beiden "Grundfamilien" betrachtet werden, wie sie in zwei Drucken des Jahres $1590 \mathrm{zu}$ finden sind. In der Selva di varie ricreatione von Oratio Vecchi steht iber dem "Saltarello Gioite tutti": "Per Sonare con gli Stromenti da corde A 5"; in den Dialoghi musicali de diversi eccelentissimi autori liest man uber den beiden Battaglien129 "Aria della Battaglia per sonar d'Insrumenti da fiato".

\subsubsection{INSTRUMENTATION AB 1597}

\subsubsection{AUFKOMMEN UND VERBREITUNG DER INSTRUMENTATIONSANGABEN}

Soweit bekannt, erschien eine Instrumentation im modernen Sinn, also eine genaue Angabe von Instrumenten bei den Noten selbst, erstmals in den Sacrae symphoniae von Giovanni Gabrieli (1597) im Druck130. Auch hier sind bei weitem nicht alle Instrumentalstimmen mit Instrumentationen versehen: Von insgesamt 16 Instrumentalwerken sind nur vier durchinstrumentiert131, ein

126. Zu nennen sind (aus studentischen Kreisen?) die handschriftlich eingetragenen Instrumentationsvermerke in dem Exmplar des ersten Teiles von Georg Forsters, Frische teutsche Liedlein, 4/1552 der Schermar-Sammlung in Ulm (vg1. Smith, Renaissancequerflöte, S.30), die vereinzelten Instrumentationshinweise in der Kopenhagener Handschrift KB 1872 der Kopenhagener Hofkapelle (Dania sonans IV, hrgs. von Henrik Gluhn, S.19), sowie die sicher von Stadtpfeifern stammenden Handschriften Hs.A.R.775 und 777 der Bischhöflichen Proske-Bibliothek, Regensburg, mit Madrigalen und Motetten von Orlando di Lasso (A.R.775 datiert auf 1579). Hier finden sich zahlreiche Instrumentenangaben, namentlich Zinken, Posaunen und Pommern (vgl. Dickey, Musik fïr Zink, S.287 f).

127. Intermedii et concerti, fatti per la commedia rappresentata in Firenze (1591, aufgefuhrt 1589). Vgl. zu den Angaben Vogel, Bibliothek, Bd.I, S.382 ff und Schneider, Basso continuo, $\$ .56 \mathrm{ff}$. Die hier gegebernen Angaben zur Instrumentation lassen sich allerdings nur mit einigen Schwierigkeiten auf die Musik übertragen.

128. Die Instrumente werden jeweils mit ihren Spielern genannt.

129. Je eine von Annibale Padoano und Andrea Gabrieli.

130. Vg1. hierzu die Neuausgabe der Instrumentalsätze dieses Druckes in IeM 2. Die Besetzungsangaben können sehr ibersichtlich in dem "Indice tematico" dieser Ausgabe studiert werden.

131. "Sonata Pian \& Forte": 1.Chor: Zink und drei Posaunen, 2.Chor: "Violino" und drei Posaunen; "Canzon in echo duodecimi toni": beide Chöre je: 4 Zinken (3 Chorzinken, 1 Alktzink) und eine Posaune; "Canzon sudetta accomodate per concertar con l'organo": wie "Canzon duodecimi toni", aber zusätzlich Orgel (fiir die jedoch keine Stimme vorhanden ist); "Canzon quarti toni a 15": 1.Chor und 3.Chor je: Zink und 4 Posaunen, 2.Chor: "Viol ino" und 4 Posaunen. 
weiteres trägt lediglich im ersten Sopran die Angabe "Cornetto"132. Die drei bei Gabrieli genannten Instrumente, Violine, Zink und Posaune, können als das Standardinstrumentarium auch des frühen 17.Jahrhunderts bezeichnet werden (s.u.) 133 .

Die Besetzung vornehmlich mit Posaunen und Zinken ist bei solcher Musik eindeutig festlichen Charakters eigentlich nicht auffällig. Ein Hinweis, warum Gabrieli dennoch den ungewöhnlichen Schritt tat, diese Stuicke mit einer Instrumentation zu versehen, gibt vielleicht die Auswahl der durchinstrumentierten Sätze. Die "Sonata Pian \& Forte" sowie die beiden Fassungen der "Canzon in echo duodecimi toni" haben gemeinsam, daß sie auch in dynamischer Hinsicht experimenteller Natur sind. Die Festlegung der Instrumentation bestimmte hier nicht nur die Klangfarbe, sondern legte auch die beabsichtigte dynamische Wirkung fest - diese konnte bei einer anderen Besetzung ganz anders ausfallen.

Im 17. Jahrhundert wurde eine genaue Bezeichnung der vorgesehenen Instrumente bald die Regel. Wenn auch gelegentlich noch einzelne Stimmen unbezeichnet blieben (insbesondere Mittelstimmen), so beträgt doch der Anteil der Drucke, in denen grundsätzlich auf eine nähere Bezeichnung der Melodieinstrumente verzichtet wurde, in den Jahren 1621-1630 nur gut 10\% aller ausgewerteten Drucke mit Instrumentalstimmen.

Besonders rasch fanden genaue Angaben zur Besetzung in Vokalwerken mit obligaten Instrumentalstimmen Verbreitung. In solchen, kurz vor 1600 erstmals in Erscheinung getretenen 134 Kompositionen war irgendeine Art der Bezeichnung der Instrumentalstimmen unbedingt notwendig, um diese von den Vokalstimmen zu unterscheiden. Nur in Ausnahmefällen geschah dies mit allgemeinen Angaben, wie "Instromento grave" bzw. "Instrumento acuto"135. In der Regel wurden hierfür die Instrumentennamen selbst verwendet - z.T. freilich mit Alternativangaben.

132. "Canzon duodecimi toni a 10".

133. Der "Violino" Gabrielis ist jedoch ein Altinstrument (im 17.Jahrhundert wird mit "Violino" vereits uberwiegend ein Diskantinstrument gemeint). Vg1. hierzu auch weiter unten im Abschnitt III.6.3.2,

134. Die fruhesten dem Verfasser bekannten Kompositionen mit ausdrücklicher Aufteilung in Vokal- und in Instrumentalstimmen außerhalb der solistischen Musik sind in zwei Musikdrucken des Jahres 1598 enthalten, nämlich in J. Gallus Sacri operis ... libro primo und A. Soderinis Sacrarum cantionum ... liber primus. Es handelt sich hierbei jeweils um doppelchörige Werke, von denen der eine Chor vokal und der andere instrumental auszufuhren ist. Diese Stiucke sind in beiden Drucken mit "Concentus duplex vocum, \& instrumentorum" uberschrieben (vg1. zu dem Gallus-Druck Kunze, Gabrieli, S.223 ff mit zwei Faksimile-Tafeln).

135. Z.B. in A. Burlinis Salmi intieri che si si cantano al vespero (1613, dort nennt der Stimnbuchtitel allerdings eine Violine als Diskantinstrument). Vgl. zu diesen Angaben Bonta, Instruments, S.524 und S.533. 
Das in den Instrumentationen des frühen 17.Jahrhunderts am häufigsten genannte Instrument ist die Violine. Bereits in fast $85 \%$ aller ausgewerteten Musikdrucke mit Instrumentationen iberhaupt 136 aus dem ersten Jahrzehnt des Jahrhunderts wird auch die Violine verlangt. Aus dem dritten Jahrzehnt des 17. Jahrhundert ist dem Verfasser kein Druck mit Instrumentationen bekannt, in dem nicht auch die Violine vorgeschrieben ist. Als Diskantinstrument ist außer der Violine nur noch der Zink einigermaßen häufig anzutreffen (in rund 50\% der ausgewerteten Musikdrucke mit Instrumentationen - allerdings mit deutlich abnehmender Häufigkeit). Flöteninstrumente werden noch nicht eirmal in $10 \%$ dieser Drucke genarnt.

Die Baßstimmen sind insgesamt ungenauer bezeichnet als die Diskantstimmen 137; Angaben wie "per Violino e Basso" sind nicht selten138. Das meist verlangte Baßinstrument ist die Posaune. In etwa der Hälfte der ausgewerteten Drucke mit Instrumentationsangaben wird auch eine Posaune verlangt. Deutlich seltener sind Violone und Fagott vorgeschrieben (jeweils nur in rund 15\% dieser Drucke). Der Anteil der Drucke, in denen die Besetzung mit einem Fagott vorschlagen wird, steigt jedoch innerhalb des behandelten Zeitraumes stark an ${ }^{139}$. Relativ oft findet man daruber hinaus den Sammelbegriff "Viola" (in knapp 30\% der Drucke) 140.

Die genannten Häufigkeiten beziehen sich immer auf die Anzahl der Drucke, nicht aber auf die Zahl der einzelnen Stuicke. Die z.T. unvollständigen Instrumentationen, widersprüchlichen Angaben (im Titel anders lautend als in der Tavola o.ä.141) oder globalen Instrumentationen nur in den Drucktiteln machen eine sinnvolle statistische Auswertung nach einzelnen Stiicken unmöglich. Bei einer solchen näheren Betrachtung der Drucke käme die Beschränkung auf nur wenige Instrumente aber noch deutlicher zum Ausdruck. Die Violine wird nicht nur in fast allen Drucken mit Instrumentationen genannt, sie ist

136. Gemeint sind natürlich nur Instrumentationen für Melodieinstrumente.

137. In einigen wenigen Drucken ist die Bezeichnung der BaBiustrumente genauer, z.B. in den beiden Buichern der Sonate concertate (1629) von D. Castello.

138. Vg1. z.B. B. Marinis Affetti musicali (1617). Mehr als drei Viertel aller Baßstimmen sind unbezeichnet, aber bei keiner Diskantstimme fehlt eine Instrumentation.

139. Während das Fagott in den ersten 10 Jahren des Jahrhunderts noch nicht und im zweiten Jahrzehnt nur in knapp $8 \%$ der ausgewerteten Drucke mit Instrumentationen verlangt wird, ist es in den Jahren 1621-1630 immerhin bereits in rund $25 \%$ der Drucke vorgeschrieben.

140. Zu ganz ähnlichen Ergebnissen kommen - jeweils auf grund unterschied1icher Quellengruppen - Bonta, Instruments, S.524, und Mangsen, Ad libitum, S.31, Tabelle 4. Vg1. hierzu auch Selfridge-Field, Instrumentation, S.66 f.

141. Diese Abweichungen sind natürlich meist nicht grundsätzlicher Art. Oft werden an verschiedenen Stellen unterschiedlich viele Alternativinstrumente genannt oder aber eine Instrumentenangabe durch eine Lagenbezeichnung ersetzt. 
auch innerhalb dieser Drucke bei fast jedem Stück vorgeschrieben. Die F1öteninstrumente hingegen werden auch in den wenigen Drucken, in denen sie überhaupt erwähnt werden, nur jeweils für ein - selten zwei - Sätze verlangt.

\section{$2,1,2,2$. TEXTBEZOGENE INSTRUMENTATION}

Es 1 iegt zunächst nahe, auch die Instrumentation in den Dienst des Textaffektes zu stellen. Diese bereits aus den Intermedien des 16. Jahrhunderts bekannte Praxis ist aber nur in wenigen italienischen Quellen des 17.Jahrhunderts zu beobachten. Der Grund hierfur ist in dem deutlich verkleinerten Instrumentarium zu suchen. Auch in den vokalinstrumentalen Kompositionen wird in erster Linie die Violine, etwas seltener die Posaune und der Zink verlangt ${ }^{142}$. Es 1 äßt sich die Bestrebung erkennen, durch verschiedene Spie1techniken die Violine zu einem jedem Textaffekt gerecht werdenden Instrument zu machen 143 .

Claudio Monteverdis L'Orfeo $(1607 / 09)$ ist mit seiner Anknüpfung an die Intermedientradition und der Vervollkommung der affektgerechten Instrumentation ohne direkte Nachfolge geblieben ${ }^{144}$. Einzelne Beispiele textabhängiger Instrumentation finden sich auch in späteren Werken Claudio Monteverdis145. So ist ein textlicher Bezug auch bei der wechselnden Besetzung im "Quia respexit" des ersten Magnificat aus Sanctissimae Virgini missa (1610) gegeben ${ }^{146}$. In dem Madrigal "A quest'olmo a sei voci concertato" aus dem

142. Von insgesamt 34 Drucken mit vokalinstrumentalen Sätzen aus den ersten 30 Jahren des 17. Jahrhunderts wurde in 30 die Violine, in 15 die Posaune und in 12 der Zink verlangt. Die Violine war schon gegen Ende des zweiten Jahrzehntes des 17. Jahrhunderts so sehr zum Standardinstrument geworden, daß Bernardi in seinen Concerti accademici (1616) in die Generalbaßstimme den Direktionshinweis "doi Violini" eintrug, obwohl die Instrumentalstimmen nicht näher bezeichnet sind und auch sonst nichts auf die Verwendung von Violinen hindeutet. Auch in G. B. Riccios "Canzon in Ecco La Moceniga A 4" aus dem terzo libro delle divine lodi (1620) werden Duett-Abschnitte in allen Stimmbiichern mit "Violini soli" bzw. "Tromboni soli" bezeichnet, obwohl für dieses Stiuck (im Gegensatz zu anderen Instrumentalsätzen dieses Druckes) sonst keine Instrumentation angegeben ist.

143. Das wohl bekannteste Beispiel hierfür ist der von C. Monteverdi sogenannte "stile concitato", angewendet im Combattimento (1624/1638). Ein weiteres schönes Beispiel fiir diese Technik findet sich in dem "Dixit Dominus" aus A. Grandis "Salmi ... brevi" (1629, abgedruckt bei Seelkopf, Grandi, Bd.2, S.255 ff (Notenbeispiel 102)). Hier ist es die Textstelle "conquasabit capita" (Takt $115 \mathrm{ff}$ ), die Grandi mit einer Violinstimme in der Art des "stile concitato" unterstreicht.

144. Vg1. hierzu Wolff, Instrumentation sowie Beat, Monteverdi.

145. Allerdings sind diese Instrumentationen im Vergleich zum L'Orfeo wesentlich sparsamer.

146. Vg1. Monteverdi-GA, Bd.14, 2.Teilband, S. $289 \mathrm{ff}$ : 
Concerto (1619) benutzte Monteverdi einen Instrumentationswechsel zur Hervorhebung der zentralen Textstelle $\mathrm{e}^{147}$ - Tirsi erinnert sich an die Zeit, da Clori ihm sich und ihr Herz zum Geschenk machte148. In diesem umfangreichen Madrigal zu sechs Stimmen mit zwei Violinen und Basso continuo werden nur für diese 19 Takte die beiden Violinen durch zwei Block- oder Querflöten ("Flautino o Fifara") ersetzt. Diese Takte werden dariber hinaus durch die Besetzung ( $\mathrm{BaB}$ solo) und die außergewöhnlich langen Notenwerte hervorgehoben ${ }^{149}$.

In großer Fülle hingegen wurde wechselnde Instrumentation zur Textverdeutlichung in deutscher Musik dieser Zeit verwendet, z.B. besonders häufig bei Vertonungen des zu Instrumentationswechseln geradezu herausfordernden 150 . Psalmes ${ }^{150}$. Solches ist dem Verfasser aus Italien aus dieser Zeit nicht bekannt 151.

\section{$2,1,2.3$. Vokal instrumentale Ausfijhrung von Musik ohne obligate Instrumente}

Die Frage nach der vokalinstrumentalen Besetzung der Vokalmusik ohne obligate, d.h. ausdrücklich als solche bezeichnete Instrumentalstimmen gehört im wesentlichen nicht mehr in den Bereich der Notation. Sie kann daher hier

Ritornel1: 2 Violinen, drei Zinken, Viola da brazo (basso) "con più forza che si pud"

Qui respexit humilitatem

2 Fifare

ancillae suae

2 Tromboni

ecce enim ex hoc beatam me dicent omnes generationes

2 Flauti

wie Ritornell

In der a cappella-Fassung des Magnificat wird die wechselnde Besetzung im Mittelteil teilweise durch eine wechselnde Registration (in der GA unvollständig) imitiert (vgl. dazu weiter unten im Abschnit.t III.2.2.).

147. Dazu Whenham, Later Madrigals, S.219.

148. "... all'hor che la mia Clori, Tutt'in dono se stessa e'l core mi diede".

149. Vg1. Monteverdi-GA, Bd.7, S.26 f.

150. Man denke an die Vertonungen durch H. Schütz in den Psalmen Davids von 1619 ("Alleluja, Lobet den Herren", SWV 38, Schütz-GA, Bd.3, S.34 ff und in dem "Concert Jauchzet dem Herren", SWV 47, Schütz-GA, Bd.3, S.239 ff).

151. Um solche Kompositionen zu erkennen, ist es Notwendig, sie zu spartieren. Dies konnte innerhalb dieser Arbeit nur begrenzt geleistet werden. Einige Sätze mit auffälligen Instrumentationen, z.B. die beiden vokalinstrumentalen Motetten aus G. B. Riccios terzo libro delle divine lodi (1620; "O salutaris hostia" für Sopran, Tenor, Violine, Fagott und Bc. sowie "Iubilent omnes" für Sopran, Violine, Flöte, Fagott und Bc.) wurden auf Verdacht. spartiert; eine direkte Beziehung zwischen Instrumentation und Text konnte nicht festgeste11t werden (lediglich die Erwähnung von Instrumenten (" \& exultantes in himnis \& canticis in cordis \& organo, in cymbalis bene sonantibus") hat Riccio wohl bewegt, den Satz "Iubilent omnes" vokalinstrumental zu besetzen). 
nicht erschöpfend behandelt werden. Erwähnung verdienen jedoch einige notierte Hinweise auf eine vokalinstrumentale Besetzungspraxis in einigen der ausgewerteten Drucke. So sind in einigen Drucken Zeichen $z u$ finden, die angeben sollen, wann ein collaparte mitgefühtes Instrument schweigen oder auch allein ohne die Singstimme fortfahren soll. Bernardino Borlasca notierte in der Scala Jacob (1616) zu diesem Zweck in den Vokalstimmen "V." für "voci", "Sinfonia" für rein instrumentale Teile und "T." für "Tutti"152; Annibale Orgas benutzte die Arweisungen "solus" und "Cum Instrumentum"153. Um solche Besetzungswechsel darzustellen, konnten auch Großbuchstaben verwendet werden 154 .

In einem Vorwort erklärt Borlasca, wie er sich im einzelnen die Besetzung der beiden Chöre in seiner Scala Jacob vorstellt155. Der erste Chor soll aus den vier "voci principali" mit einem Kastrat oder Falsettist als Sopran, verstärkt durch ein Streicherensemble, bestehen. Der zweite Chor möge die gleiche Zusarmensetzung der Singstimmen haben, aber mit Blasinstrumenten, nämlich Zinken und Posaunen, begleitet werden. Ergänzend kann jedem Chor noch ein Diskantinstrunent der jeweils anderen Gruppe (eine Violine zum zweiten Chor und ein Zink zum ersten) beigefügt werden. Diese sollen die Altstimmen um eine Oktave nach oben transponiert spielen.

Solche differenzierten Anweisungen zur Instrumentierung von Vokalmusik sind selten und auch kaum allgemein ubertragbar. Einige weitere Angaben finden sich zur Besetzung von Ripienochören ebenfalls mehrchöriger Musik. Es wird sowohl die collaparte-Verdoppelung 156 als auch die rein instrumentale Aus-

152. Vg1. den Abdruck dieses Vorwortes im zweiten Band dieser Arbeit auf S.8.

153. Sacrarum cantionum ... liber primus (1619).

154. Davon berichtet Anne Schnoebelen in dem Artikel Cortellini, Camilo in NG, Bd.4, S.808. Nach Schnoebelen benutzte Cortellini in den Messdrucken von 1616 und 1621 Großbuchstaben als "a unique notation to indicate the partecipation of Instruments". Es sei an dieser Stelle an die Verwendung der Großbuchstaben in Adriano Banchieris Sacra armonica (1619) erinnert. Dort zeigte die Textierung mit Majuskeln eine "missura larga" an (vg1. im Abschnitt I.1.2.4.2.). Um Choralzitate als solche zu kennzeichnen benutzte $G$. Metallo, Messa, motteti, et un Magnificat (1611), Großbuchstaben. Teile des Textes sind auch in P. Lappis Misarum ... liber secundus (1608) in Majuskeln notiert, hier allerdings ohne Angabe von Gründen.

155. Wiedergegeben im zweiten Band dieser Arbeit auf S.8.

156. Dies wird besonders detailliert von L. Viadana im Vorwort zu den Salmi a quattro chori (1612) besprochen (vgl. den Abdruck des Vorwortes im zweiten Band dieser Arbeit auf S.17 f). Eine instrumentale Verdoppelung von Ripienostimmen wird auch von G. B. Grillo, Sacri concentus (1618) vorgeschlagen (S.3 der Pars ad organum: "Radopiate le parti con voci \& Instrumenti nelli ripieni se vi piace, $₫$ il simile nel Magnificat $\left.d 8^{\prime \prime}\right)$. Ahnliche Anweisungen finden sich in einem Vorwort Amadio Freddis aus dem Jahr 1616 (Messa, vespero et compieta; abgedruckt bei D'Arpa, Freddi, S.293). Es sei an dieser 
fuihrung solcher Stimmen beschrieben157. Hier finden sich ebenfalls Hinweise auf Oktavverdoppelung durch zusätzlich Instrumente158. Viadana weist auch darauf hin, daß eine exponierte Stimme von Instrumenten alleine ausgefuhrt werden kann ${ }^{159}$.

ähnliche Angaben zur Besetzung von geringer besetzter Musik sind dem Verfasser aus italienischen Musikdrucken und Handschriften dieser Zeit nicht bekannt. Sicher wird es auch hier die Möglichkeit der collaparte-Verdoppelung gegeben haben. Es sind jedoch auch einige Werke erhalten, bei denen für einen Teil der Stimmen eine instrumentale statt einer vokalen Besetzung vorgeschrieben ist, ohne daß es sich dabei um instrumental gedachte stimmen handelt160. Diese Instrumentalstimmen wären in aller Regel problemlos textierbar. Zahlreiche Belege für solche instrumentalvokalen Besetzungen ohne collaparte-Verdoppelungen finden sich auch im Werk von Heinrich Schütz

Stelle auch auf die Motette "Jubilate Deo" aus dem postum erschienenen zweiten Teil der Symphoniae sacrae von G. Gabrieli (1615) hingewiesen. Während hier bei den meisten der fünf vokalinstrumentalen Motetten klar zwischen Vokal- und Instrumentalstimmen unterschieden wird (hier tritt auch "voce" als "Instrumentation" auf - vgl. zu diesen Angaben bei Gabrieli Charteris, Gabrieli, S.337), überlä日t es Gabrieli in dieser Motette den Ausführenden, die Instrumentalstimmen (natülich mit Ausnahme der Sinfonie) zusätzlich vokal zu besetzen.

157. Eine Aufteilung in einen Vokal- und einen Instrumentalchor ist bereits in J. Gallus Sacri operis ... liber primus (1598) und A. Soderinis Sacrarum cantionum ... liber primus (1598) fest vorgeschrieben (s.o.). Dies hat im 17. Jahrhundert zahlreiche Nachfolger gefunden (G. M. Radino, Concerti per sonare et cantare (1607), F. Rognoni, Messa, salmi intieri e spezzati (1610), E. Porta, Sacro convitio,(1620), P. Tarditi, Psalmi magnificat (1620) und S. Bernardi, Il terzo libra de madrigali (1624)). Eine Besetzung des zweiten Chores mit Instrumenten statt mit Sängern schlägt auch G. Ghizzolo, Messa salmi, lettanie B.V. (1619, vgl. das Vorwort bei Sartori, Bibliografia I, S.250 (1619 c) unter Punkt 2) vor.

158. L. Viadana im Vorwort zu den Salmi a quattro chori (1612) (vgl. den Abdruck des Vorwortes im zweiten Band dieser Arbeit auf S.17 f).

159. Dies schlägt Viadana fiur den Sopran des Hochchores vor (Vorwort zu Salmi a quattro chori) - sicher auch ein Beleg dafür, daß uber- bzw. Unterschreitungen des vokalen Ambitus nicht zwangsläufig die Notwendigkeit einer Transposition bedeuten.

160. Besonders beliebt war die Mischung von Singstimmen und Posaunen. Sie ist schon in L. Viadanas Cento concerti (1602) zu finden ("O bone Iesu a tre voci" für Tenor, zwei Posaunen und Bc., abgedruckt bei Schneider, Basso continuo, S.185 ff). Weitere Beispiele: "Corda Deus" für Sopran, Alt und drei Posaunen aus Sacro convitio musicale von E. Porta (1620) sowie verschiedene Sätze von A. Grandi aus der Raccolta terza di Leonardo Simonetti (Magni 1630, den Hinweis hierauf verdanke ich Prof. Dr. Rudolf Ewerhart, Hochschule für Musik, Köln). Die Posaunenstimmen sind hier jeweils vokal komponiert und wären ohne weiteres auch singbar. 
- interessanterweise vornehm1ich in Handschriften, und damit einer Auffihrung oft näherstehenden Que11en 161 .

An eine nicht verdoppelnde, sondern ersetzende instrumentale Beteiligung ist wohl auch bei den in 17. Jahrhundert noch erscheinenden Kompositionen "per cantare \& sonare" (o.ä.) zu denken ${ }^{162}$. Diese These wird unterstitzt durch die Tatsache, daß Formulierungen ganz ähnlicher Art auch auf eine Generalbaßbegleitung oder auf obligate Instrumente verweisen können ${ }^{163}$.

\section{$2.1,2,4$. ALTERNATIVBESETZUNGSANGABEN}

In den ersten Jahren des 17. Jahrhunderts war die Nennung von Alternativen bei Besetzungsangaben recht verbreitet164. Am häufigsten anzutreffen sind die Angaben "Violino o Cornetto"1 65 und "Trombone ò Violone" bzw. "Trombone ¿ Viola" und "Trombone d Violetta"160. Mit der zunehmenden Verbreitung des Fagottes 167 tritt auch dieses Instrumerit ab 1620 als Alternative zu Streich-

161. Vgl. die Besetzungsangaben bei Bittinger, SWV, S.105 ff). Solche Besetzungen finden sich in großer Fulle auch in den Psalmen Davids von 1619 (vg1. Schiitz-GA, Bd.3, S.34 ff, $89 \mathrm{ff}, 133 \mathrm{ff}, 142 \mathrm{ff}, 167 \mathrm{ff}, 182 \mathrm{ff}, 217$ $\mathrm{ff}, 239 \mathrm{ff})$. Diese Art der vokalinstrumentalen Besetzungspraxis ist auch Gegenstand der ausführlichen Vorrede zur Geistlichen Chormusik (1648; vgl. die wiedergabe in der Schütz-GA, Bd.8). Die letzten beiden Sätze der Geistlichen Chormusik sind bereits in dieser Art bearbeitet.

162. Eine vorläufige Liste von Drucken mit solchen Angaben auf den Tite1blättern gibt Bonta, Instrunents, S.528 f.

163. Bei L. Luzzaschi, Madrigali (1601), weist die Formulierung "per cantare, et sonare" auf die Begleitung durch das Tasteninstrument hin. Ahnliche Formulierungen sind auch in der Folgezeit nicht selten (z.B. G. S. P. del Negro, Gl'amorosi pensieri ... da sonare, \& cantare su'l chitarone (1607)). Im Titel der otto ordine di litanie della Madonna von V. Bona (1619, RISM B 3435) bezieht sich diese Formulierung offensichtlich auf obligate Instrumente (im Titel werden auch "le parti de gli instrumenti" erwähnt).

164. Vgl. hierzu auch Mangsen, Ad libitum. Mangsen bietet zahlreiche Hinweise auf die verschiedensten Arten von Alternativbesetzungen in instrumentalen Trios bis 1675. Ihre Darstellung geht allerdings wenig tiber das Auflisten verschiedener Alternativbesetzungsangaben hinaus.

165. Die Alternativangabe "Violino ó Cornetto" ist in etwa $90 \%$ der ausgewerteten Drucke mit Alternativangaben iberhaupt zu finden. Auch dieser Anteil liegt jedoch bereits im dritten Jahrzehnt des 17. Jahrhunderts bei nur noch etwas iber $60 \%$.

166. Die Alterrativangaben "Posaune oder Streichinstrument" können zusammen betrachtet werden. Die unterschiedlichen Namen der Streichinstrumente liegen zum Teil in der uneinheitlichen Nomenklatur, zum anderen aber auch in den verschiedenen durch die Posaunen vertretenen Lagen (Alt bis Baß) begrïndet. In etwa $30 \%$ der ausgewerteten Drucke mit Alternativbesetzungsangaben waren auch solche Alternativen zu finden. 
instrumenten oder zur Posaune auf 168 . Andere Nennungen von mehreren Instrumenten sind in italienischen Quellen selten und oft situationsbedingt. So können z.B. eine oder zwei von vier Posaunen durch Violinen in der höheren Oktave ersetzt werden, falls nicht genugend Posaunen verfiigbar sind ${ }^{169}$, oder es stehen zwei klanglich verwandte Instrumente (Block- oder Querflöte) zur Auswah1 170 .

Das Vorhandensein von Alternativbesetzungsangaben - zumal von solchen, die nach heutigem Verständnis völlig unverständlich erscheinen - hat zu der Annahme gefuhrt, die Instrumente seien in dieser zeit grundsätzlich beliebig austauschbar gewesen171. Dem ist jedoch entgegenzuhalten, daß es auch im frihen 17. Jahrhundert zunehmend Besetzungsangaben ohne solche Alternativnennungen gab $^{172}$. Ferner ist auf die ingesamt geringe Anzahl von Instrumenten

167. Das Fagott des frihen 17. Jahrhunderts wird nur heute allgemein als "Dulzian" bezeichnet, in den Quellen dieser Zeit hingegen fast ausnahmslos als "Fagotto" (auch in Deutschland).

168. Im dritten Jahrzehnt des 17. Jahrhunderts erschienen Alternativbesetzungsangaben mit Fagott bereits in über $20 \%$ der ausgewerteten Drucke mit alternativen Besetzungsangaben iberhaupt.

169. In den in Venedig 1629 erschienenen Symphoniae sacrae von H. Schitz lautet die Besetzungsangabe der ersten beiden Instrumentalstimmen der beiden Werke für vier Posaunen, Baß und Bc. ("Fili mi Absalon" und "Atendite popule meus") "Trombone o Violino all'ottava". Nur bei einer stimme steht eine entsprechende Angabe bei der "Canzona terza à 4 Tromboni" aus B. Marinis Sonate (ebenfalls 1629); dort steht im Canto-Stimmbuch "Questa parte puo esser Sonata da vn Corneto, ò Violino al'Octaua" (S.28). Eine ähnliche Besetzungsangabe findet sich auch beim letzten' Stück dieser Samnlung, der "Sonata per 1'Organo, \& Violino o Cornetto". Das Canto secondo-Stimmbuch enthält eine Stimme mit der Uberschrift "Trombone ad Libitum per la Sonata del Organo, \& Violino, o Cornetto. Puo esser anco Sonata, da vn Violino o Cornetto al octaua". Bei dieser Stimme handelt es sich um die obligate Diskantstimme der Orge1, um eine Oktave nach unten transponiert (!!). Uber der Orgelstimme steht "Dovendo l'Organista sonar il Basso \& il secondu soprano con uno registro de' Flauti all'ottava \& Pedale overo si farà sonare quel secondo soprano ad un Violinio ò Trombone all'ottava" (zitiert nach Schering, Solosonate, S.320. Die Orgelstimme zu dieser Sonate ist heute weitgehend zerstört).

170. So in dem oben bereits angesprochenen Madrigal "A quest'olmo" aus C. Monteverdis Concerto (1619).

171. So z.B. Fischer, Instrumentalmusik, S.550.

172. Bereits in den mit Instrumentationen ausgestatteten Musikdrucken der Jahre bis 1610 sind nur in etwa $60 \%$ Alternativangaben vertreten. In dritten Jahrzehnt des 17.Jahrhunderts beträgt dieser Anteil bereits nur noch $40 \%$. Auch bei diesen Angaben ist zu bedenken, daß die Tatsache, daß in einem Druck Alternativangaben benutzt wurden, nicht bedeutet, daß alle Stimmen mit solchen Angaben verseher sind. So enthält z.B. der mit Instrumentationen reich ausgestattete Druck Sanctisssimae Virgini missa (1610) von C. Montever- 
zu verweisen, die in solchen Angaben genannt werden (s.o.). Es ist daraus zu schließen, dab auch dort wo eine Besetzungsangabe fehlte, nicht ein beliebiges Instrumentarium zur Auswahl stand 173 .

Die Besetzungsangaben bezeichneten in dieser Zeit, insbesondere in der solistischen Musik, nicht in erster Linie die Klangfarbe, sondern die musikalischen Möglichkeiten. Nur so ist die verbreitete Angabe "Violino $\delta$ Cornetto" (bzw. umgekehrt) zu verstehen ${ }^{174}$. In Anbetracht der Tatsache, daß die Besetzungsangaben in dieser Zeit als etwas Neues auftraten, erscheint es undenkbar, in der Angabe "Violino d Cornetto" ein Synonym für "irgendein Diskantinstrument" zu sehen; dann hätte die Angabe auch entfallen können. Der Sinn dieser Instrumentationen ist auch in einer ausschließenden Funktion zu suchen. "Geeignet für Violine oder Zink" kann auch "ungeeignet für Blackflöte175, Diskantgambe etc." heißen. Es ist zu bedenken, daß es besonders in einer Zeit eines Umbruches nötig sein kann, auch eine vielleicht für modern eingestellte Musiker selbstverständliche Besetzung trotzdem ausdrücklich vorzuschreiben, um unerwuinschten Fehlbesetzungen durch mit der neuen Praxis - vielleicht durch räumliche Distanz - nicht vertraute Musiker vorzubeugen 176 .

Nicht selten verfolgt die Setzung einer Alternivangabe den Zweck, einzelne Stuicke besonders vielfältig einsetzbar zu machen. Hier stellt die Alternativangabe bereits ein Zugeständris dar; ein eigentlich nur für ein Instrument geschriebenes stück kann zur Not auch auf dem alternativ genannten Instrument ausgefuhrt werden. Dies ist z.B. ganz offensichtlich bei den oben erwähnten Stuicken für Posaunenensemble der Fall. Eine Notlösung stellt eine Alternativangabe auch bei Stuicken dar, die einem bestimmten Instrument "auf den Leib" geschrieben wurden.

Entgegen der weit verbreiteten Meinung von der Austauschbarkeit der Instrumente in dieser Zeit, gibt es schon aus den ersten Jahren nach 1600 Komposi-

di nur eine Alternativangabe, und zwar für eine unbedeutende Stimme ("Sonata sopra Santa Maria", Settimo: "Trombone, ouero Viuola da brazzo").

173. Es wurde oben bereits auf zwei Quellen aufmerksam gemacht, in denen auch dort, wo eine Instrumentation fehlt, im Verlauf der Stücke Hinweise wie "Violini soli" o.ä. stehen (S. Bernardi, Concerti accademici (1616) und G. B. Riccio, Il terzo libro delle divine lodi (1620)).

174. Werner Brauns Deutung dieser Alternativangabe, man solle auch unter bescheidenen Verhältnissen die Bläserpracht imitieren (Handbuch, S.270), kann bestenfalls auf die Ensemblemusik zutreffen. In der solistischen Musik - und dort ist diese Angabe am häufigsten zu finden - kann nicht von einer "Bläserpracht" die Rede sein.

175. Die wenigen für Blockflöte bestimmten Sätze unterscheiden sich deut$1 \mathbf{i} c h$ - in Schlisselung, Ambitus und Virtuosität - von denen für Zink oder Violine.

176. Auf solche "vorbeugenden" Anweisungen wird weiter unten im Abschnitt III.4.1. näher einzugehen sein. 
tionen, in denen spieltechnische Möglichkeiten sowie Charakteristik einze1ner Instrumente gezielt berücksichtigt und eingesetzt werden. Als besonders eindrucksvolle Beispiele sollen im folgenden die beiden Sonaten "à due" aus Giovanni Paolo Cimas Concerti ecclesiastici (1610) betrachtet werden ${ }^{177}$. Die erste der beiden Sonaten trägt A1ternativbesetzungsangaben ("Cornetto \& Trombone, ouero Violino $\delta$ " (muß heißen: "e") "Violone")178, die zweite Sonate hingegen nicht ("Violino \& Violone")179. Die erste der Sonaten kann als durchaus "bläserisch" bezeichnet werden. Sie beginnt mit feierlichen Akkorden und steht insgesamt in einer als feierlich zu bezeichnenden Tonart180, sie enthält genügend Pausen ${ }^{181}$ und typische Bläserfiguren (z.B. Akkordbrechungen)182. Die Baßstimme ist deutlich ruhiger gehalten als die der zweiten Sonate und wird damit der langsameren Posaune gerecht ${ }^{183}$. Die Sonate kann als virtuos bezeichnet werden, ohne aber bis an die Grenzen des bläserisch Machbaren zu gehen.

Die zweite Sonate unterscheidet sich von der ersten in vielerlei Hinsicht. Sie steht in einer eher melancholischen Tonart184, beginnt nicht akkordisch, sondern melodisch und ubertrifft die erste Sonate an Virtuosität bei weitem ${ }^{185}$. Akkordbrechungen und ähnliche Figuren fehlen gänzlich. Sie ist für Zink und Posaune einfach nicht geeignet (was nicht heißt, daß sie auf diesen Instrumenten unspielbar wäre186). Nur die erste Sonate ist den Bläsern "auf den Leib" geschrieben und ideal auf diesen Instrumenten ausfuhrbar. Sie

177. Beide Sonaten sind in der Neuausgabe Giovanni Paolo Cima, Drei Sonaten (1610) für Soloinstrumente und B.C., hrsg.v. Karl Grebe enthalten. Diese Neuausgabe weist erhebliche Mängel auf. Nicht nur die Instrumentation, sondern vor allem der Notentext ist merklich entstellt. Die Concerti ecclesiastici sind dariber hinaus seit 1986 auch als Faksimileausgabe (siehe Quellenverzeichnis) erhältlich.

178. In dem Basso-Stimmbuch steht richtig: "Trombone, ouero Violone" (S.52). In der Neuausgabe (dort heiBt diese Sonate "Sonate in $d^{\prime \prime}$ ) lautet die Instrumentation: Oboe, Baß, Cembalo.

179. In der Neuausgabe heißt diese Sonate "Sonate in $g$ ".

180. Sie steht in d dorisch.

181. Diese Sonate enthält in der Diskantstimme vier "Entspannungspausen" von vier oder mehr Semibreven, die zweite Sonate nur zwei (beide Sonaten sind in etwa gleich lang).

182. Vgl. insbesondere in der Partitur von $1610 \mathrm{~S} .146 / 147$ oben bzw. in der Partitur der Neuausgabe S.11, drittes und viertes System.

183. Die Posaunenstimme der ersten Sonate enthält keine Semicromen, die Violonenstimme der zweiten Sonate fast 100, davon allein fast 50 in einem Lauf (vg1. Basso-Stimmbuch von 1610, S.53, die letzten beiden Systeme bzw. Partitur der Neuausgabe, S.6).

184. Sie steht in $\mathrm{g}$ hypodorisch.

185. Vg1. u.a. S.150/151 oben der Partitur von 1610 bzw. S.6 der Neuausgabe.

186. Unter Verweis auf die "allgemeine Austauschbarkeit" wird sie heute auch von Zinkinisten geblasen. 
trägt die Alternativbesetzungsangaben vielleicht, weil schon zu dieser Zeit die Nachfrage nach Violinliteratur größer war als die nach Bläserstücken. Sicher jedoch ist diese Alternativangabe bereits ein Zugeständnis und keinesfalls ein Indiz für eine beliebige Besetzung. Ebenfalls verkaufstechnische Bedeutung kommt der gelegentlich auftretenden Formulierung " $o$ simil altro stromento" $\mathrm{zu}^{187}$. Auch hier ist ein anderes Instrument eine freilich mögliche - Notlösung.

\subsection{REGISTRIERANWE ISUNGEN}

Auf die Registrieranweisungen wurde im Zusammenhang mit der musikalischen Dynamik bereits mehrfach verwiesen. Die meisten der gedruckten Registrieranweisungen sind in erster Linie als dynamische Anweisungen zu verstehen. Antonio Agazzari188, Lodovico Viadana189 und Giovanni Ghizzolo190 schreiben, daß man die Registration der Orgel der Zah1 der sonstigen Stimmen anpassen solle; Adriano Banchieri weist die Organisten an, die Lautstärkeangaben "piano" und "forte" durch Hinzu- bzw. Wegnahme eines Registers umzusetzen 191 .

Auch die erste Registrationsangabe innerhalb eines Musikstückes, nämlich in der "Terza Sonata in dialogo" aus der ersten Auflage von Adriano Banchieris $L$ 'organo suonarino (1605)192, nennt nicht einzelne Register, sondern gibt lediglich an, daß die letzten Takte im "pieno" (hier: "Ripieno") zu spielen sind. Diese "Sonata in dialogo" besteht aus einem Wechsel (Dialog) zwischen einem Hochchor und einem Tiefchor. Lediglich die letzten zwei Takte sind als Tutti komponiert193. Um mit den zur Vefügung stehenden vier Stimmen wirklich

187. Z.B. bei G. Gabrieli, Canzoni e sonate (1615): "Sonata con tre Violini o altro simile stromento" und I. Vivarino, Il primo libro de motetti ... con otto sonate per il violino ó simil altro stromento (1620).

188. Del sonare sopra'1 basso (1607), S.6: "si deue suonare con molto giudizio, hauendo la mira al corpo delle voci; perche se sono molte, conuien suonar pieno, e raddoppiar registri; ma se sono poche, schemarli, e metter poche consonanze".

189. Im Vorwort zu Salmi a quattro chori (1612, vg1. im zweiten Band dieser Arbeit auf S.17 f).

190. Im Vorwort zu Messa, salmi lettanie B.V. (1619, vg1. das Vorwort bei Sartori, Bibliografia I, S.250 (1619 c) sowie im Abschnitt III.1.3.2.)

191. In den Fantasie overo canzoni alla francese (1603) im Titel der "Fantasia sesta": "in eco movendo un registro" (die Echo-Stellen sind mit " $p$ " und " $f$ " gekennzeichnet, vgl. die Neuausgabe, hrsg. von André Vierandeels) und im Terzo libro di nuovi pensieri (1613), S.26: "Cantasi piano con vn Registro, \& forte con dua."

192. S.64 f. Diese Sonate ist im zweiten Band dieser Arbeit als Notenbeispiel III, 4 im Faksimile wiedergegeben.

193. Die oberen drei Stimmen sind als Hochchor geschlüsselt, der BaB bleibt jedoch in der Tiefchorlage, so daß der gesamte Ambitus beider Chöre abgedeckt ist. 
eine Tutti-Wirkung zu erreichen, ist hier eine stärkere Registration, ausgedrückt mit der "Ripieno"-Anweisung, vorgesehen. In der Auflage von 1611194 kommt ein langsameres Tempo hinzu. Hier steht an der entsprechende Stelle "Pieno \& graue"195.

Registrierangaben mit Nennung der jeweiligen Register enthält die Bassus generalis-Stimme zu Claudio Monteverdis Sanctissimae Virgini missa (1610) zu den beiden Magnificat196. Auf die dynamischen Effekte und deren Unterstreichung durch Regitrierangaben in diesen Stücken wurde weiter oben bereits eingegangen ${ }^{197}$. Auch die meisten der aben nicht erwähnten Registrierangaben dieses Druckes haben lediglich die Aufgabe, die Orgelbegleitung in der Lautstärke den anderen Stimmen anzupassen ${ }^{198}$. Drei dieser Angaben sind jedoch deutlich auf die Klangfarbe ausgerichtet. Die Dynamik wurde in allen Fällen mit den Registern "Principale", "Ottava" und "Quintadecima" bestritten. Bei beiden Magnificat kommt aber im "Fecit potentiam"199 jeweils ein weiteres, nur in diesem Satz auftretendes Register hinzu: im ersten Magnificat "Principale et registro delle zifare o voci humana", im zweiten Magnificat "Principale et Pifara".

Besonderes Interesse verdient aber die dritte eindeutig klangfarbenbezogene Registrieranweisung dieser Generalbaßstimme. Wie oben dargelegt handelt es sich bei dem "Quia respexit" des ersten Magnificat um eines der wenigen Bejspiele für textbezogene Instrumentationswechsel in italienischen Quellen dieser Zeit200. In der zweiten Fassung des Magnificat (ohne Instrumente) wird nun dieser Instrumentationswechsel teilweise mit wechselnden Registern imitiert:

194. S.32 f der Teilfaksimileausgabe. Es handelt sich hier um eine Neufassung der Sonate.

195. Hinzu tritt eine unterschiedliche Registration von Hoch- und Tiefchor (Hochchor: "Principale \& Ottana", Tiefchor: nur "Principale"). Die Auflage von 1611 enthält ein weiteres Stück dieser Art, den "Primo dialogo acuto \& sopra'acuto" (S.31). Auch hier steht beim Schlußtutti "Pieno \& Graue".

196. S.41 ff. Vg1. Monteverdi-GA, Bd.14, 2.Teilband, S.289, 291, 294, 300, $304,310,313,319,327 \mathrm{f}, 330 \mathrm{f}, 336,338,342,344,347 \mathrm{ff}$. Die in der GA fehlenden Angaben sind bei Tagliavini, Registratione, S.367, Arm.7 aufgelistet.

197. Im Abschnitt III.1.3.2.

198. So ist für solistische Sätze in der Regel nur der Prinzipal vorgeschrieben, für chorische hingegen mehrere Register.

199. S.42 und 50, Monteverdi-GA, S. 300 und 336.

200. Vgl, oben im Abschnitt III.2.1.2.2. 
Ritorne11

Qui respexit...

ancillae suae

ecce enim ex hoc...

omnes generationes
Magnificat I

sechs Instrumente "con più "Principale, ottaua et forza che si puó"

2 Fifare

2 Tromboni

2 Flaut $i$

wie Ritornel1
Magnificat II quintadecima" 201

"Principale solo"202

"principale e tremo-

lare" 203

$-204$

wie Ritornell mit dem $\mathrm{Zu}$ satz "et la voce canta forte"

Solche deutlich klangbezogenen Registrieranweisungen sind in den vereinze1ten Anweisungen aus den Jahren bis 1630 nur selten zu finden. Am verbreitetsten sind allgemein gehaltene Vorschriften wie "pieno"205. Werden einzelne Register genannt, so sind dies meist "principale"206 und "ottaua" 207 bzw. "Flauti all'ottaua"208 und "Flauto"209. Tremolo-Register werden auch in Biagio Marinis Affetti musicali (1617)210 und der dritten Auf lage von Adriano Banchieris L'organo suonarino (1622) 211 verlangt. Eine klangliche Absicht ist außer bei den erwähnten Registerangaben Monteverdis

201. Diese Registrierung ist auch im Magnificat I vorgeschrieben. Im zweiten Magnificat ist das Ritorne11 nur als Generalba日 notiert.

202. Diese Registrierung ist im ersten Magnificat für den gesamten Mitte1teil vorgeschrieben.

203. Diese Angabe fehlt in der $G A$.

204. Es ist zumindest denkbar, dab hier ursprünglich ebenfalls eine Registerangabe stand (weiter oben wurde bereits darauf hingewiesen, daß im zweiten Magnificat in dem crescendo gleich zu Anfang eine Registerangabe fehlt (im ersten Magnificat ist sie korrekt notiert).

205. A. Banchieri, L'organo suonarino (2/1611), ders., Terzo libro di nuovi pensieri (1613), ders., Salmi festivi (1619), G. B. Biondi, Il quarto libro delli concerti (1611, dort auch "Pieno sino all fine"), N. Corradini, Motetti (1624)

206. A. Banchieri, L'organo suonarino (2/1611 und $3 / 1622)$, ders., Terzo libro di nuovi pensieri (1613) und S. Anagnino, Nova sacra cantica (1617). 207. A. Banchieri, L'organo suonario (2/1611) und S. Anagnino, Nova sacra cantica (1617).

208. A. Banchieri, L'organo suonarino (3/1622) und B. Marini, Sonate (1629).

209. A. Banchieri, L'organo suonarino (2/1611).

210. "La Foscarina Sonata a 3 con il Tremolo". In den beiden mit "Violino $o$ Cornetto" bezeichneten Stimmen steht "Tremalo con 1'arco" (!!), in der Baßstimme ("Trombone o Fagotto") "Tremolo col strumento" und in der Orgelstimme "Metti il Tremolo"; vgl. hierzu Dickey, Vibrato, S.105 f sowie Carter, Tremolo, S.43 ff. Die Sonata "La Foscarina" ist als Neuausgabe erschienen in Biagio Marini, Sonata à 3 "La Foscarina" and Sinfonia d̀ 3 "La Giustiniana", hrsg. von Robert P.Block und David Stuart.

211. S.130: "Qui tollis peccata mundi Suscipe. con Tremolo". 
und den Tremolo-Stellen nur bei den wenigen Sätzen zu vermuten, deren Registerangaben für einen ganzen Satz gelten, also nicht der Vermehrung oder Verminderung der Lautstärke dienen. Dies sind die mit Registrieranweisungen versehenen Sätze aus Banchieris dritter Auflage des L'organo suonarino $(1622)^{212}$ sowie Biagio Marinis "Sonata per l'organo e Violino o Cornetto"213. Bei dieser Sonate kommt der Registerangabe ein besonderes Gewicht zu, da die entsprechende Orgelstimme obligat ein zweites Soloinstrument ersetzt214.

\section{BINDEBÖGEN}

Auch die Bindebögen zwischen Noten wechselnder Tonhöhe erschienen in den letzten Jahren des 16.Jahrhunderts erstmals in italienischen Musikdrucken215, und auch sie wurden innerhalb von wenigen Jahren zu einem festen Bestandteil der Notation. Im dritten Jahrzehnt des 17.Jahrhunderts erschien bereits fast kein Musikdruck mehr ohne solche Bindebögen. In dieser Zeit wurden die Bindebögen bereits auf recht unterschiedliche Art und Weise verwendet. Ihre auffuhrungspraktische Bedeutung ist hier oftmals kaum zu erkennen216. In den Jahren um 1600 aber waren die Bindebögen zunächst auf sehr wenige Melodiefiguren begrenzt. Von diesen kann z.T. auch die gewuinschte Auffuhrungsart abgeleitet werden.

Neu war aber um 1600 nur die Anwendung des Bindebogens zwischen Noten wechselnder Tonhöhe. Das Zeichen selbst war seit langem bekannt.

212. S.133 ("Principale"), 135 und 136 ("Flauto").

213. "Flauti a11' ottaua" s.o.

214. Diese Registerangabe bezieht sich nur auf den zweiten Sopran ("Dovendo l'Organista sonar il Basso, \& il secondo soprano con uno registro de'Flauti all'ottava \& Pedale overo si farà sonare quel secondo soprano ad un Violino o Trombone all'ottava", zitiert nach Schering, Solosonate, S.320; dort findet sich auch eine Edition dieses Stückes.).

215. Bindebögen sind bereits in dem 1582 in Paris erschienenen Balet comique de la Royne enthalten (vgl. das Faksimilie bei Paul Nettl, Art. Ballett in MGG, Bd.1, Sp.1166). Der Bindebogen dient hier - ganz in Gegensatz zu seiner Verwendung in den italienischen Drucken aus der Zeit um 1600 - nur der Zuordnung von Text und Musik. Uberaus lange Bindebögen finden sich auch in der, in wesentlichen aus den achtziger Jahren des 16.Jahrhunderts stammenden Handschrift Mus.G1 5 der Sächsischen Landesbibliothek, Dresden (vgl. zur Datierung Wolfram Steude, Musiksammelhandschriften, S.53 ff; den Hinweis auf diese Quelle verdanke ich Herrn Prof. Dr. Martin Staehelin, Universität Göttingen). Diese Bindebögen sind vermutlich nachträglich eingetragen worden (die Tintenfarbe unterscheidet sich von der des Notentextes Briefliche Mitteilung von Frau Marina Lang, Sächsische Landesbibliothek, Dresden, vom 22.5.90).

216. Dies ist z.B. bei den relativ häufigen Bindungen von zwei Noten inmitten eines längeren Melismas der Fall (s.u.). 


\subsection{BINDEBÖGEN ZWISCHEN NOTEN DERSELBEN TONHÖHE}

Der Bindebogen wurde bereits im 16.Jahrhundert in allen Notationsformen verwendet, in denen senkrechte Striche (vergleichbar dem Taktstrich) zur Gliederung des Notenbildes benutzt wurden, also in den Tabulaturen und Partituren 217 . Hier waren Bindebögen notwendig, um iber einen solchen Gliederungsstrich hinaus andauernde Notenwerte darzustellen. Punktierte Notenwerte konnten ohne Bindebogen durch Herïbernehmen des Punktes auf die andere Seite des Gliederungstriches notiert werden218, Waren aber beide Teile eines Notenwertes gleich lang, so konnte dieser nur durch zwei kürzere, mit einem Bindebogen verbundene Noten dargeste11t werden. Die Notationsform mit einer langen Note (z.B. Semibrevis) direkt auf einen Taktstrich findet sich zwar in Handschriften219, war aber im Druck nicht darstellbar.

Ein weiterer Grund für den Einsatz eines Bindebogens zwischen Noten gleicher Tonhöhe ergab sich aus der, zunächst im Madrigal zu beobachtenden, Verwendung von Notenwerten sehr unterschiedlicher Dauer innerhalb eines Stückes (oft Brevis bis Semicroma). Hieraus ergaben sich auch zusammengesetzte Notenwerte, die sich nicht mittels eines Augmentationspunktes, sondern nur durch Anbindung darstellen ließen (z.B. Minima mit angebundener Croma) 220 .

Zu Beginn des 17. Jahrhunderts kamen weitere Anwendungsgebiete für Bindebögen zwischen Noten gleicher Tonhöhe hinzu. Zu nennen ist vor allem die weiter oben bereits behandelte Verwendung des Bindebogens in den Basso continuoStimmen221. Hier konnte mittels eines Bindebogen zwischen zwei gleichen Noten ein Akkordwechsel (meist zugleich Bezifferungswechsel) genau plaziert werden. Bindebögen zwischen gleichen Noten fanden weiter bei der schrift1 ichen Fixierung des "tremolo" oder "trillo"222 Verwendung. Hier kennzeichnet der Bindebogen bereits eine besondere Artikulation, allerdings eine Artikulation auf einem Ton. Ebenfalls im Zusammenhang mit Verzierungen wurden Bindebögen in einigen Drucken zur genauen Plazierung eines Verzierungszeichens verwendet. Dieses Zeichen (in der Regel " $t$.") steht hier erst auf der zweiten von zwei, mit einem Bindebogen verbundenen Noten 223.

217. Vgl. oben, Abschnitt I.1.3.

218. Später wurden vereinzelt auch solche Punkte auf der anderen Seite des Taktstriches mittels eines Bindebogen angebunden. Dies ist zu beobachten in den Notenbeispielen zu G. B. Chiodinos Arte pratica latina et volgare (1609) sowie in A. Cifras Scherzi et arie (1614) und in B. Marinis Affetti musicali (1617).

219. Vg1. Lowinsky, Early Scores, S.146.

220. Vg1. hierzu die Beispiele A. Banchieris zu den "legature moderne" in der Cartella musicale (1614), S.48 f (dieser Abschnitt ist im zweiten Band dieser Arbeit auf S.19 ff abgedruckt).

221. Vgl, im Abschnitt II.3.1.1.

222. Das früheste dem Verfasser bekannte Beispiel hierfür findet sich in dem primo libro de madrigali von D. Valla (1605). Zu diesen Begriffen vgl. weiter unten im Abschnitt III. 4.3. 
Zuletzt sei darauf verwiesen, daß in einer Anzahl von Musikdrucken Birdebögen auch zur Verdeutlichung von Synkopen benutzt wurden224. Der synkopierte Notenwert wurde dann so zerteilt, da $\mathrm{zu}$ Beginn der neuen Taktzeit auch eine neue Note steht. Sie ist dann mit einem Bindebogen mit der vorhergehenden verbunden.

\subsection{BINDEBÖGEN ZWISCHEN NOTEN UNTERSCHIEDLICHER TONHÖHE IN DEN JAHREN BIS 1610}

\section{$3,2.1$, BINDEBÖGEN ANSTELLE VON LIGATUREN}

Die Vermutung, die Bindebögen zwischen Noten unterschiedlicher Tonhöhe seien zunächst nur eine andere Notationsform für Ligaturen, liegt nahe225. In Partiturquellen des 16.Jahrhunderts sind Ligaturen, die aufgrund eines Gliederungsstriches in der Partitur nicht notiert werden konnten, tatsächlich durch Bindebögen ersetzt worden226. Auch in Musikdrucken des fruihen 17. Jahrhunderts stehen gelegentlich Bindebögen in Partituren oder in mit Gliederungsstrichen versehenen Generalbaßstimmen, die offensichtilich eine Ligatur vertreten227.

223. Die fruhesten dem Verfasser bekannten Beispiele fïr diese Anwendung stehen in B. Barbarinos secondo libro de madrigali (1607).

224. Solche Bindebogensetzungen sind vereinzelt in folgenden Drucken zu beobachten: C. Monteverdi, L'Orfeo (1609), A. Grandi, Il secondo libro de motetti (1613, 4/1623), C. Orlandi, Arie (1616), G. Fornaci, Amorosi respiri musicali (1617), G. Marini, Il secondo libro de madrigali (1618), P. Pace, I1 nono libro de motetti (1619), C. Saracini, Le quinte musiche (1624), G. Bruschi, Liber secundus sacrarum modulationum (1625), ders., Missa, et psalmi (1627).

In diesem Zusammenhang sei auch die Handschrift II.I.295 (olim Magl. XIX.107) der Biblioteca Nazionale Centrale, Florenz (Becherini, Catalogo, S.41, zweite Hälfte 16. Jahrhundert) erwähnt . In dieser Partiturhandschrift sind nicht nur Punktierungen konsequent durch Anbindungen ersetzt worden, sondern auch fast alle anderen länger als eine Minima andauernden Notenwerte sind als Minimen mit Bindebögen notiert (Zählhilfe?).

225. So Hermann Keller, Art. Artikulation in MGG, Bd.1, Sp.744.

226. Beispiele hierfür finden sich in der Handschrift MS Q 42 des Civico Museo Bibliografico-Musicale, Bologna (um 1575, vgl. Lowinsky, Early Scores, $\mathrm{S.140}$ sowie Abb.15) und gedruckt in G. Arnones Partitura del secondo libro de11i motetti (1599).

227. In: B. Binaghi, Coronae divinarum laudum ... liber primus (1604), 0. Scaletta, Certa spirituale (1605), Sammeldruck Della nuovi metarmorfosi (1605), A. Savetta, Matectorum ... liber secundus (1608), A. Cifra, Motecta ... liber septimus (1614), Psalmorum, sacrorum cancentuum .. liber secundus (1621), A. Franzoni, Messe a 5 (1623), P. Agostini, Spartitura delle messe del primo libro (1627), ders., Spartitura del secondo libro delle messe e motetti (1627), ders., Libro quarto delle messe in spartitura (1627) sowie ders., Spartitura della messa et motetto Benedicam Dominum (1627). Vg1. hierzu auch M. Praetorius, Syntagmatis musici ... tomus tertius 


\subsubsection{BINDEBÖGEN IN VERBINDUNG MIT BESTIMMTEN MELODIEFIGUREN}

\section{$3,2,2,1$, CHROMATIK}

Eine gewisse Năhe zur Ligatur ist auch in den ersten gedruckten Beispielen für die Verwendung des Bindebogen in Stimmbuchnotation ohne Gliederungsstriche zu spüren. In Simone Balsaminos "Canto e piang'" aus den sechsstimmigen Madrigalen von 1594 sind jeweils zwei chromatisch aufeinanderfolgende Semibreven (der auch in Ligaturen dieser Zeit häufigste Notenwert) mit Bindebögen verbunden228. Die Notation einer Ligatur wäre hier nicht möglich gewesen, da die beiden gebundenen Noten stets auf derselben Notenlinie stehen (zunächst ohne und dann mit Vorzeichnung eines Kreuzes bzw. zunächst mit Vorzeichnung eines $\mathrm{b}$ und dann mit Auflösungszeichen). Eine Ligatur kann jedoch nur notiert werden, wenn die beiden Noten an unterschiedlichen Ste1len in das Notensystem zu notieren sind 229 . Obwoh1 hier eine moderne Auffiihrungsmanier notiert wurde, ist die Nähe $\mathrm{zu}$ den alten Ligaturen nicht zu übersehen. Auch in theoretischen Außerungen zu diesen modernen Bindungen bleibt der alte Begriff bestehen230. Ganz ähnlich wie in dem Madrigal Balsaminos, wenn auch nur sehr vereinzelt, wurden Bindebögen in dem 1598 erschienenen achten Madrigalbuch von Luca Marenzio verwendet231.

Diese Nähe zur Ligatur erklärt jedoch das Auftreten der Bindebögen an diesen Stellen nicht. Ligaturen konnten zwar anzeigen, daß zwei Noten zu einer Textsilbe gehören, die Verwendung einer Ligatur an einer solchen Stelle war jedoch keineswegs selbstverständlich. Im Gegenteil: Da im späteren 16.Jahrhundert - insbesondere im Madrigal - der Semibrevis für die Deklamation kaum noch Bedeutung zukam, waren Ligaturen längst eine Seltenheit geworden ${ }^{32}$.

Auch die weitere Entwicklung im 17.Jahrhundert bestätigt, daß die Chromatik den AnlaB zur Setzung dieser Bindebögen gab. In fast der Hälfte der ausgewerteten Musikdrucke mit Bindebögen zwischen Noten unterschiedlicher Tonhöhe aus den Jahren 1601 bis 1610 stehen diese Bindebögen nur, in nochmals knapp 30\% der Drucke auch (und oft iberwiegend) bei Chromatik. Drucke, in denen solche Bindebögen nur bei Chromatik verwendet wurden, erschienen, wenn auch

(1619), S.29.

228. Der chromatische Anfang dieses Madrigales ist im zweiten Band dieser Arbeit als Notenbeispiel III,5 wiedergegeben.

229. Folgerichtig wurden die zwei Semibreven zu "piang'" im zweiten Takt in den beiden Mittelstimmen auch als Ligatur notiert.

230. A. Banchieri nennt die Bindebögen "Legature moderne" (Cartella musicale (1614), S.48 f - dieses Kapitel ist im zweiten Band dieser Arbeit auf S.19 ff abgedruckt); 0. Durante spricht in Bezug auf solche chromatische Bindungen von der "nota ligata" (Vorwort zu den Arie devote (1608) - s.u.).

231. In: "Pur venisti cor mio" und "Perfida in su 1 'estrema".

232. Die Verwendung von Ligaturen parallel zu einer chromatischen Bindung bei Balsamino (vgl. den zweiten Takt im Notenbeispiel III,5) läßt jedoch eine gleiche Artikulation von Bindebogen und Ligatur hier wahrscheinlich erscheinen. 
mit abnehmender Häufigkeit, noch zu Ende des hier zu behandelnden Zeitraumes233. Natürlich wurde im frühen 17.Jahrhundert nicht mehr nur der ligierbare Wert der Semibrevis für solche Bindungen verwendet, und auch längere chromatische Gänge konnten mit Bindebögen versehen werden ${ }^{2}{ }^{4}$.

Die Textworte zu den chromatischen Gängen mit Bindebögen lauten "piango"235, "piangi e sospira"236, "struggi"237, "morte"238, "lagrime"239, "languire"240, "dolore"241, "soavis"242, etc. Sie stehen für eine Außerung des Schmerzes oder aber für "sanft", "süß". Selten tritt eine solche Chromatik auch bei Worten wie "iniquus" 243 auf 244 .

Eine Anweisung zur Ausfuhrung solcher chromatischen Bindungen gibt Ottavio Durante in seinem uberaus informativen Vorwort zu den Arie devate, le quale in se contengono la maniera di cantar con gratia (1608)245. Die hier beschriebene Ausfuhrungsart kommt einem Glissando gleich246. In deutschen

233. Der späteste dem Verfasser bekannte Druck mit Bindungen nur im Zusammenhang mit Chromatik ist P. Quagliatis Motetti, e dialoghi (1627). Dieser konservative Zug Quagliatis könnte auf das Alter des Komponisten zum Zeitpunkt des Erscheinens dieses Druckes zurückzuführen sein. Das genaue Geburtsjahr Quagliatis ist nicht bekannt. Er duirfte 1627 aber uber 70 Jahre alt gewesen sein (vgl. Paul Kast, Art. Quagliati, Paolo in MGG, Bd.10, Sp.1794 ff).

234. Vg1. das Notenbeispiel III,7 aus B. Barbarinos secondo libro de madrigali (1607) im zweiten Band dieser Arbeit. Hier sind funf chromatisch aufeinanderfolgende Minimen durch Bindebögen verbunden.

235. Z.B. in den (Madrigali) a sei vaci von S. Balsamino (1594).

236. Z.B. im vierten Madrigalbuch von C. Monteverdi (1603).

237. Z.B. im achten Madrigalbuch zu fünf Stimmen von L. Marenzio (1598).

238. Ebenda.

239. Z.B. im ersten Madrigalbuch von T. Pecci (1602).

240. Ebenda.

241. Z.B. in B. Barbarinos secondo libro de madrigali (1607).

242. T. Massaino, Musica per cantare (1607).

243. Ebenda.

244. An dieser Ste1le soll auch auf eine ganz andere Verwendung eines solchen gebundenen chromatischen Ganges in einem Credo aus dem Sacro convitio musicale von E. Porta (1620) aufmerksma gemacht werden. Porta vertont die Worte "passus \& sepultus est" mit je zwei chromatisch aufeinanderfolgenden gebundenen Tönen zu den beiden Silben des Wortes "passus", gefolgt von einem Oktavsprung abwärts zwischen " $\$$ " und der ersten Silbe von "sepultus" mit einer anschließenden Kadenz in tiefer Lage zu "sepultus est" (Tenorstimmbuch, S.19).

245. Dieses Vorwort ist vollständig abgedruckt und ibersetzt bei Goldschmidt, Gesangsmethode, S.29 ff.

246. "Per il crescimento della voce dal tuono al semituono si assegna il diesis nella nota ligata per dar ad intendere, che bisogna comminciar a crescere a poco a poco facendo conto che vi siano 4 come, sino che si arrivi 
Quellen des 17.Jahrhunderts spricht man vom "Ziehen"247 oder von der "schleifenden Manier"248, um die Ausführung von Bindebögen zu beschreiben. Hinzu kommt wahrscheinlich noch eine deutlich unterschiedliche Betonung der beiden Noten (schwer-1eicht) ${ }^{249}$. Nur so ist es zu erk1ären, daß in chromatischen Stellen in einer Stimme, in der Chromatik aus harmonischen Gründen nicht möglich ist, zu der entsprechenden Silbe statt dessen zwei gleiche Noten mit einem Bindebogen notiert sind250. Hier soll offenbar die Art, eine solche Figur zu singen, imitiert werden.

\subsubsection{BINDEBöGEN BEI ANDEREN MELODIEFIGUREN}

In den Musikdrucken des frühen 17. Jahrhunderts sind die Bindebögen nicht mehr ausschließlich an die Chromatik gebunden. Auch die anderen Melodiefiguren, die hier mit Bindebögen auftreten, stehen aber bei ähnlichen Textworten wie die chromatischen Gänge, also bei Worten mit den Bedeutungen "Trauer", "Schmerz" oder auch "süß" und "sanft". Nach der Chromatik ist am häufigsten folgende, bei Francesco Rognoni Taegio als "accento" bezeichnete Melodiefigur 251, mit einem Bindebogen anzutreffen252: $d^{R} d$

Dieser "accento" gehört in den Bereich der Gesangsmanieren. Während die Chromatik immer zum unmittelbaren Bestandteil einer Komposition gehört, ist der "accento" eine Zutat, die entweder bereits der Komponist oder auch erst der Ausfürende anbringen konnte. Rognoni fordert in einem Nachwort an die Sänger zum ersten Teil der Selva (1620) dazu auf, bei Worten, die Leid, Trauer etc. bedeuten, nicht "passaggi", sondern "gratie" wie "accenti", "esclamationi" etc. zu benutzen'253. Auch die Ausführung des "accento" gehörte damit freilich in den Bereich der Gesangslehre. Die besondere, durch den Bindebogen angedeutete Ausfuihrungsart des "accento" ist sicher auch dann angebracht, wern der Bindebogen nicht notiert ist254. Offensicht1ich ver-

al perfetto crescimento, il che quando è fatto bene commove assai." (zitiert nach Goldschmidt, Gesangsmethode, S.32).

247. M. Praetorius, Syntagmatis musici ... tomus tertius (1619), S.232.

248. A. Hammerschmidt in der Bc.-Stimme zum dritten Teil der Musicalischen Andachten (1642, vgl. das Faksimile bei Adam Adrio, Art. Hammerschmidt, Andreas in MGG, Bd.5, Sp.1431; die Jahreszah1 1638 bei Carter, Tremo1o, S.44, beruht auf einem Irrtum).

249. Vgl. hierzu auch weiter unten.

250. So in G. Ghizzolos, Madrigali a 5 .. 1ibro primo (1608) und G. Valentini, Missae concertatae (1617).

251. In der Verzierungstabelle aus der Selva de varii passaggi (1620). Diese Tabelle ist im zweiten Band dieser Arbeit als Notenbeispie1 III, 6 wiedergegeben. Die Bedeutung des Wortes "accento" ist in den Quellen dieser Zeit nicht einheitlich.

252. In etwa einem Viertel der ausgewerteten Musikdrucke der Jahre 16011610 ist die Melodiefigur zusammen mit einem Bindebogen zu finden.

253. Auf diese Stelle wurde oben im Abschnitt III.1.4. bereits ausfuhrlicher eingegangen. 
1ießen sich im frihen 17. Jahrhundert - hier war der "accento" noch neu nicht alle Komponisten darauf, daß die Sänger mit seiner Ausfihrung vertraut waren.

Mit bereits notierten Bindebögen erschien häufig ebenfalls folgende, dem "accento" verwandte Melodiefigur:

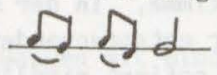

Auch sie entstammt den Gesangsmanieren neuen Stiles. Giulio Caccini führt diese Figur in der tabellarischen Aufstellung verschiedener Melodiefiguren und derer Ausführung in der Nuove musiche (1601/02)255 an und schlägt fuir sie eine lombardische Ausfihrung, also eine Verkirzung der jeweils ersten Note zugunsten der zweiten, vor. In einer sehr ähnlichen Tabelle zu Anfang von Antonio Brunellis Varii esercitii (1614) sind den Ausführungsvorschlägen bereits die Bindebögen beigefügt 250 , mit denen sie auch in zahlreichen Musikdrucken erschienen257.

Selten treten Bindebögen zwischen wechselnden Noten bereits im ersten Jahrzehnt des 17.Jahrhunderts innerhalb einer Quartvorhaltskadenz auf. Hier wird entweder die auflösende Terz an die vorangehende Quart258 oder aber an die nachfolgende U1tima259 angebunden.

Bindungen längerer Gruppen von Noten sind in dieser Zeit seltene Ausnahmen . Bereits 1594 verwendete Giovanni Battista Bovicelli in seinen "Avertimenti intorno alla note" 260 einen Bindebogen uber 7 Noten, um die Ausfihrung des "Groppetto raffrenato" zu erläutern201. Gruppen von vier Noten finden sich in Amante Franzonis nuovi fioretti musicali (1605). Hier werden die vier Noten der oben beschriebenen Melodiefigur unter einem Bogen zusammengefaßt. Weitere frühe Beispiele für längere Bindebögen enthä1t Bartolomeo Barbarinos secondo libro de madrigali (1607). Auf eine Bindung von fuinf chromatisch aufeinanderfolgenden Minimen zum Wort "dolore" ist oben bereits hingewiesen worden ${ }^{262}$. Weitere Gruppenbindungen finden sich in der Basstimme des "Dialogo Ferma, ferma caronte". Diese Bindebögen können bereits als Phrasierungsbögen bezeichnet werden. Innerhalb eines 27 Noten umfassenden Melis-

254. Einige Uberlegungen zur Ausfihrung dieser Bindungen folgen aru Ende dieses Abschnittes.

255. Auf der füften Seite des Ai lettori.

256. Vgl. S.2 der Neuausgabe von Richard Erig.

257. Das fruheste dem Verfasser bekannte Beispiel hierfür steht in A. Franzonis I nuovi fioretti musicali (1605). Dort stehen bereits alle vier Noten der Figur unter einem Bogen.

258. Der früheste dem Verfasser bekannte jtalienische Druck mit einer solchen Bindung ist M. Perdsфns, Madrigali a 5, 1 ibro primo (1608).

259. Bereits bei B. Barbarino, Il secondo libro de madrigali (1607) und S. Bonini, Madrigali, e canzonette spirituali (1607).

260. Regole, passaggi di musica (1594), S.10 ff.

261. S.12.

262. Vgl. das Notenbeispiel III,7 im zweiten Band dieser Arbeit. 
mas sind dreimal vier Noten unter je einem Bindebogen zusammengefaßt worden. Mit diesen Bindebögen war es Barbarino möglich, eine vom ublichen abweichende Gruppierung vorzunehmen 263 .

Ebenfalls noch in den ersten Jahren des 17. Jahrhunderts treten, wenn auch sehr vereinzelt, Zweierbindungen bei einfachen Folgen kurzer Notenwerte (i.d.R. Cromen) außerhalb der erwähnten Melodiefiguren auf. Auch diese Bindebögen stehen allerdings bei Textworten, für die eine der erwähnten Melodiefiguren hätte verwendet werden können. Ein frühes Beispiel hierfür ist in dem Madrigal Troppo ben può aus Claudio Monteverdis füntem Madrigalbuch (1605) enthalten: Obwohl es hier zahlreiche kleine Melismen gibt, stehen die Zweierbindungen nur in Verbindung mit dem Wort "core".

Es ist auszuschließen, daß die Bindebögen im frühen 17. Jahrhundert notiert wurden, um die Textunterlegung zu regeln. Die Bindebögen stehen nur bei einem verschwindend kleinen Teil aller melismatischen Textaufteilungen. Bindebögen, die einzelne Noten innerhalb längerer Melismen verbinden, können mit der Textverteilung nicht in Zusammenhang gebracht werden. Diese Bindebögen haben nur einen Sinn, wenn sie auf eine besondere Auf fuhrungsart der gebundenen Noten hinweisen 264 . Die von Ottavio Durante (s.o.) beschriebene Ausfuhrung der Bindungen bei Chromatik ist freilich nicht auf andere Melodiefiguren ubertragbar. Die einzige weitere dem Verfasser bekannte Anweisung zur Ausführung von Bindebögen aus dem frühen 17. Jahrhundert, nämlich das Kapitel Praticamento delle legature moderne aus Adriano Banchieris Cartella musicale (1614)265, gibt nur für die Ausfihrung von Bindebögen zwischen Noten gleicher Tonhöhe nähere Hinweise - und auch diese können bestenfalls a1s "Eselsbriicken" bezeichnet werden.

Einen Hinweis auf die gewiunschte Ausführung können aber sowohl die Melodiefiguren als auch die mit gebundenen Noten vertonten Textworte geben. Es liegt nahe, hier an eine, dem Seufzen ähnliche Artikulation zu denken. Eine solche Ausfiihrung - die erste Note betont und, sofern möglich, mit einem messa di voce versehen, die zweite unbetont "angeschleift" - ist auch bei der Chromatik zusätzlich zum Glissando gut denkbar266.

263. Diese Stelle ist im zweiten Band dieser Arbeit als Notenbeispiel III, 8 wiedergegeben.

264. Ein Beispiel zu einer solchen Bindung mit chromatischen Noten zeigt das Notenbeispiel III,7 im zweiten Band dieser Arbeit. Ein weiteres Beispiel einer Bindung innerhalb eines Melismas ist als Notenbeispiel III, 9 abgedruckt.

265. Dieses Kapitel ist im zweiten Band dieser Arbeit auf S.19 ff wiedergegeben.

266. Eine solche Ausfiihrung von Bindungen ist - allerdings 150 Jahre später - in der Gründlichen Violinschule (erste Auflage 1756) von Leopold Mozart beschrieben worden. In den Ausfuhrungen zum Akzent heißt es: "Wenn nun dergleichen mehrere Noten nacheinander folgen, iber deren zwo und zwo ein Bogen stehet: so fällt auf die erste der zwoen der Accent, und sie wird nicht nur 


\subsection{Die WEITERE VERBREITUNG DER BINDEBögen IN DEN JAHREN BIS 1630}

In den Jahren von 1611 bis 1630 nahm die Verbreitung der Bindebögen stark zu. In annähernd 75\% der ausgewerteten Drucke aus den Jahren von 1621 bis 1630 sind bereits Bindebögen zu finden (1601-1610: etwa 20\%). Während die Bindebögen in den ersten Jahren des 17. Jahrhunderts vor allem in der Monodie und den Madrigaldrucken zu finden waren ${ }^{267}$, sind sie im dritten Jahrzehnt des Jahrhunderts auch in den Motettendrucken in gleicher Häufigkeit vertre$\operatorname{ten}^{268}$.

Vermehrt hat sich aber nicht nur der Anteil der Drucke mit solchen Bindebögen, sondern auch die Zahl der Bindebögen in einem Druck sowie die Menge ihrer Anwendungsmöglichkeiten. Vor allem die bereits in den ersten Jahren des 17.Jahrhunderts gelegentlich anzutreffenden Bindungen zweier kurzer Noten desselben Wertes verbreiteten sich sehr stark und wurden nun auch innerhalb von Dreiertakten verwendet269. Dort sind jeweils zwei Noten auf der Ebene des Notenwertes unter dem Taktgebenden (also der Minima im Dreiganzetakt) mit einem Bindebogen verbunden - besonders häufig mit dem in Dreiertakten oft vertonten Textwort "alleluia". Diese Art der Zweierbindungen in Dreiertakten gehören in den Drucken aus dem dritten Jahrzehnt des 17. Jahrhunderts zu den häufigsten Anwendungen von Bindebögen überhaupt.

In den geraden Takten wurden auch in dieser Zeit Bindebögen meist mit Textworten eher traurigen Affektes verbunden. Als weitere Melodiefigur kamen hierfür "Seufzerbindungen" uber große Intervalle abwärts hinzu270. Die Zweierbindungen boten sich aber auch an, um bestimmte Geräusche, wie z.B. ein Lachen271 oder das "Tremolieren" der Vöge1272 lautmalerisch zu vertonen. Auch dies ist durchaus geeignet, die oben aufgestellte These uber die Ausfuhrung von Bindungen in dieser Zeit zu bestätigen.

etwas stärker angespielet, sondern auch etwas länger angehalten; die zwote aber wird ganz gelind, und still, auch etwas später daran geschleifet" (zitiert nach der dritten Auflage von 1789, S.261 f). Dies erinnert an den "jeu inégal" des sogenannten französischen Stiles im 18.Jahrhundert.

267. Bindebögen zwischen Noten unterschiedlicher Tonhöhe sind bereits in uber 40\% der ausgewerteten Madrigal- und Monodiedrucke der Jahre 1601 bis 1610 vorhanden, aber nur in etwas $10 \%$ der gleichzeitigen Motettendrucke.

268. In dieser Zeit erschienen nur noch ein recht kleiner Anteil sowohl der Madrigal- als auch der Motettendrucke ohne Bindebögen zwischen Noten wechselnder Tonhöhe (je etwa 15\%).

269. Dem Verfasser erstmals bekannt aus L. Leonis, Sacri fiori (1612).

270. Z.B. in B. Barbarinos quarto libro de madrigali (1614) uiber "moro".

271. In G. Priulis terzo libro de madrigali (1612) und G. Valentinis Secondo libro de madrigali (1616).

272. In G. L. Missionis Tirsi doglioso (1615). Die entsprechende Textstelle lautet: "garrir l'ucceli a tremolar". 
Weiterhin sind häufig Bindungen einzelner Zweiergruppen innerhalb längerer Melismen anzuteffen. Es sind nun freilich nicht mehr nur die oben erwähnten Melodiefiguren, die mit Bindebögen ausgestattet sind. So findet man auch Melismen die aus mehreren Dreierfiguren (z.B. je eine Croma und zwei Semicromen) bestehen, bei denen jeweils eine Dreiergruppe unter einem Bindebogen zusanmengefaßt ist273. Mehrfach sind auch nur die ersten oder die letzten beiden Noten eines längeren Melismas gebunden274.

In der Instrumentalmusik wurden Bindebögen zwischen Noten wechselnder Tonhöhe erst verhältnismäßig spät angewendet. Der früheste dem Verfasser bekannte Irıstrumentalmusikdruck mit solchen Bindebögen ist Biagio Marinis Opus 1 aus dem Jahr 1617 mit dem programmatischen Titel Affetti musicali275. Die Bindebögen treten innerhalb dieses Druckes in vier Stücken auf 276, darunter zwei deutjich in Anlehnung an die vokale Monodie komponierte Solosonaten277. Die Bindebögen stehen hier bei den auch in der Vokalmusik mit Bindebögen versehenen Melodiefiguren. In drei der vier Sätze stehen die Bindebögen nur am Anfang der Stücke278. Hier sollte auf die in der Instrumentalmusik bis dahin wahrscheinlich wenig angewendete Auffuhrungsart dieser Melodiefiguren hingewiesen werden. Sicher sollen diese auch im weiteren Verlauf dieser Sätze ebenso gespielt werden, ohne daß dies jedesmal ausdriicklich notiert worden ist.

273. Z.B. in A. Banchieris Terzo libro di nuovi pensieri (1613), in A. Cifras $L i$ diversi scherzi ... libro primo (1613) und G. Bruschis Liber secundus sacrarum modulationum (1625, dort zweimal drei Noten in einem tiber elf Takte langem Melisma (sonst ohne Bindebögen) uber dem Wort "Amen" (Canto secondo, S.6)).

274. Solche Bildungen sind sehr häufig. Frühe Beispiele sind zu finden in C. Saracini, Le musiche (1614), A. Burlini, Concerti spirituali (1617) und V. Calestani, Madrigali et arie (1617). Zwei Beispiele hierfür (aus Calestanis Madrigali et arie und E. Portas Sacro convitio (1620)) sind im zweiten Band dieser Arbeit als Notenbeispiel III,10 wiedergegeben.

275. Nicht unerwähnt bleiben soll hier eine dem Bindebogen sicher verwandte Notationsart in der Trompetenmethode C. Bendinellis (Volume di tutta l'arte della trombetta, ms. 1614). Er notiert in den Militärsignalen und den Toccaten (fol.3 bis fol.7) gelegentlich zwei mit einem Strich verbundene Noten $\left(\mathrm{C}-\mathrm{g}\right.$ oder $\left.\mathrm{g}-\mathrm{C}^{\prime}\right)$, meist eine schwarze Minima mit folgender schwarzer Semibrevis. Zur Ausfuhrung sagt er in der Vorrede "et doue trouera $1 i$ segnati iui dirà sempre 'dran' toccando apena la prima notta et passando al altra per modo d'accentare".

276. "La Foscarina", "La Orlandina", "La Gardana" und "La Agguzzona".

277. "La Orlandina" und "La Gardana". Sie erschienen in der Basso principale-Stimme in Partitur.

278. Nur bei "La Agguzzona" stehen die Bindebögen etwas vor der Mitte des Satzes. Sie stehen hier zumeist nicht bei den erwähnten Melodiefiguren, sondern verbinden Cromen zu Vierergruppen. Es handelt sich wahrscheinlich bereits um Artikulationsbögen. 
Gut zehn Jahre später werden gerade in der Instrumentalmusik Bindebögen in sehr großer Zahl verwendet279. Insbesondere Bindungen von Gruppen zu vier, acht oder mehr Noten sind in der Instrumentalmusik der späten zwanziger Jahre des 17.Jahrhunderts sehr verbreitet280. Dies sind bereits Artikulationsbögen. Die entsprechenden Gruppen sollen unter einem Bogenstrich gespielt werden (die Streichinstrumente sind in dieser Zeit bereits der unumstrittene Standard)281. Ein Modo di lireggiar ogni stromento di Archo im zweiten Teil der Selva de varii passaggi (1620) Francesco Rognonis zeigt verschiedene gebundene Notengruppen und versieht diese mit Strichbezeichnungen. Es geht daraus hervor, daB die unter einem Bogen notierten Noten auch auf einem Bogenstrich zu spielen sind282. Bindungen sind in dieser zeit als etwas "Streichertyppisches" empfunden worden283. Daß es sich hierbei um Hilfen zur Gruppierung handelt, wie z.B. in dem oben beschriebenen Beispiel aus Bartolomeo Barbarinos zweitem Madrigalbuch284, ist auch unwahrschein1ich, da die in der Instrumentalmusik von den Bindebögen dargesteliten Gruppierungen ohnehin naheliegend $\operatorname{sind}^{285}$.

279. In den Instrumentaldrucken der Jahre 1629 und 1630 von D. Castello, B. Marini, B. Montalbano und G. Scarani (vgl. Quellenliste im zweiten Band dieser Arbeit).

280. Bindungen mit mehr als vier Noten sind in der Vokalmusik auch nach 1610 noch selten. Eine Bindung von 10 Noten, gefolgt von einer zu zwei, einer zu drei und noch einer zu fünf Noten in B. Barbarinos quarto libro de madrigali (1614) soll sicher die komplizierte Textverteilung verdeutlichen (abgedruckt im zweiten Band dieser Arbeit als Notenbeispiel III,11).

281. Auf Blasinstrumenten können solche Bindungen freilich ebenfalls ausgeführt werden (beschrieben wird eine solche Artikulation, soweit dem Verfasser bekannt, allerdings erstmals in B. Bismavontas Compendium musicale (ms 1677; vgl. Dickey, Bismavonta, S.152)).

282. S.5. Die Strichbezeichnungen lauten "T." ( "vuol dire tirar in giü") und "P." ("pontar" (= portar) "in sù 1'arco").

283. S. Scheidt bezeichnet einige Stellen in der Tabulatura nova (1624) mit. Bindebögen über Gruppen zu je vier Noten (wechselnder Tonhöhe) als "Imitatio Violistica" (vg1. Scheidt-GA, Bd.6, S.7, 9, 25, etc.). In einer "N.B." bemerkt er hierzu, "WO die Noten/ wie allhier/ zusammen gezogen seind/ ist solches eine besondere art/ gleich wie die Violinisten mit dem Bogen schleiffen zu machen pflegen." (vg1. das Faksimile diese "N.B." in der Scheidt-GA, Bd.5, S.126). Daß Scheidt in dieser "N.B." - und nur dort - die gebundenen Noten nur auf einer Tonhöhe notiert, hat zu Verwechslungen mit dem "tremolo" gefuhrt, der jedoch hier nicht gemeint ist (vgl. unten im Abschnitt III.4.3.).

F. Rognoni gibt unter dem oben erwähnten Modo di lireggiar ogni stromento di Archo ein Modo di Dar la lingua al Corneto o altro instrumento di fiato. Behandelt der erste die Ausfuihrung gebundener Noten, so zeigt der zweite ausschlieblich wie man Einzelnoten kunstvoll artikuliert.

284. Vg1. das Notenbeispiel III,8 im zweiten Band dieser Arbeit.

285. Vgl. hierzu das Notenbeispiel No.9 bei Iselin, Marini, Notenteil, S.11 ff, bes. S.14. 
Die Plazierung der langen Bindebögen ist teilweise recht ungenau. Insbesondere wenn die Noten das System uber- oder unterschreiten, also auf Hilfslinien stehen, ist oft nicht mehr genügend Platz für den Bogen vorhanden. Im allgemeinen ist aber - auch unter Zuhilfenahme von Parallelstellen - das Gemeinte gut zu erkennen.

Neben den Bindebebögen wurden freilich auch Ligaturen vor allem in der Kirchenmusik in dem gesamten hier zu behandelnden Zeitraum weiter verwendet. Sie sind in immerhin noch fast zwei Drittel aller ausgewerteten Motettendrucke und in fast allen Messdrucken der Jahre 1621 bis 1630 anzutreffen. Die Mehrzahl dieser Ligaturen waren freilich Ligaturen cum opposita proprietate, also Semibrevenligaturen. Die Kenntnis auch der anderen Ligaturen war aber auch für einen Musiker des fruhen 17.Jahrhunderts wichtig286. Noch in vier der ausgewerteten Mottetendrucke287 und etwa einem Drittel der Messdrucke der Jahre 1621 bis 1630 wurden auch solche Ligaturen verwendet.

\section{VERZIERUNGEN UND VERZIERUNGSZEICHEN .}

\subsection{DIE VERZIERUNGSPRAXIS IM SPÄTEN 16, UND BEGINNENDEN 17. JAHRHUNDERT}

Im späten 16. Jahrhundert erschien in Italien eine ganze Reihe von Diminutionstraktaten288. Einem offenbar großen Bedarf an virtuoser Musik standen zumindest im vokalen Bereich, aber auch für Melodieinstrumente - fast keine entsprechenden Kompositionen gegenüber 289 . Die Ausfuhrenden schafften sich ein Repertoire virtuoser Musik, indem sie mehrstimmige Vokalkompositionen 290 für virtuos gefuhrte Solostime und Begleitung bearbeiteten291 - vergleichbar den diminuierten Intavolaturen der Organisten und Lautenisten. Diese

286. A. Banchieri behauptet fälschlicherweise in der Cartella musicale, daB man die Kenntnis dieser Ligaturen nur benötige, um ältere Werke zu spartieren ( $\$ .47$ im Abschnitt "Delle legature o congivntioni antiche"): "Posero gli compositori antichi un profluuio di note straordinarie da loro dette Legature, \& perche parmi discorso superfluo il ragionarne, diro solo che vi sono Libri antichi, $i$ quali pongono tali legature, che a volere conoscere da gli moderni saria necessario spartire le compositioni ...".

287. G. F. Anerio, Litanie deiparae Virginis (1626), A. Cifra, Motecta, et psalmi (1629), A. Signoretti, Vespertinae omnium solemnitatum psalmodiae (1629) und B. Strozzi, Per compagnie musica concertata (1630).

288. Vg1. hierzu Erig, Diminutionen.

289. Fiir Tasteninstrumente ist bereits aus dem 15.Jahrhundert ein virtuoses Repertoire erhalten; für Laute ab dem 16.Jahrhundert. Die anderen Instrumentalisten konnten bis zum Beginn des 17.Jahrhunderts nur auf Diminutionen zurickgreifen.

290. Eine entsprechende Bearbeitung von einer instrumentalen Komposition ist dem Verfasser erst aus dem 17. Jahrhundert bekannt ("Canzon del Mortara detta la Porcia" in F. Rognonis Selva di varii pasaggi parte seconda (1620). Es handelt sich um eine Bearbeitung der Diskantstimme einer Canzone aus Antonio Mortaros Primo libro di canzoni (1600)).

291. Vgl. die verschiedenen Beispiele bei Erig, Diminutionen. 
Technik haben die Diminutionstraktate - neben zah1reichen Beispielen bereits bearbeiteter Stimmen - zum Inhalt. Aber auch beim Musizieren im Ensemble sind vielerorts Verzierungen von den Ausführenden hinzugefügt worden, eine Tatsache die keinesfalls die Zustimmung aller Zeitgenossen fand ${ }^{292}$.

Bereits im späten 16.Jahrhundert wurde auch vereinzelt virtuose Musik komponiert, wenngleich die Technik zunächst den Diminutionen gleicht. Auch die frihen Beispiele komponiert virtuoser Musik - zu nennen sind die Madrigali von Luzzasco Luzzaschi293 oder die Solostücke aus dem Intermediendruck von 1591294 - beruhten auf vier- oder fünstimmigen Kompositionen, aus denen einzelne Stimmen herausgezogen und koloriert wurden - auf dieses Verfahren wurde weiter oben bereits hingewiesen295.

Die entscheidenden Veränderungen geschahen auch im Bezug auf die virtuose Musik in den Jahren um 1600. Zunächst in der Monodie, dann aber auch im konzertanten Madrigal, geistlichen Konzert und in ganz besonderem Maße in der Instrumentalmusik, wurden virtuose Passagen zum Bestandteil der Komposition. Die "antica maniera di passaggi" wird hierbei von Anfang an getadelt. Giulio Caccini schreibt in dem beruhmten Vorwort zu Le nuove musiche (1601/02) lang und breit darüber, daß diese Verzierungen, da sie keine Rücksicht auf den Text nehmen, die Musik nur entstellen können. Sie dienten nur dazu, ein ahnungsloses Publikum in Staunen zu versetzen 296 . Es fehlt auch in

292. Vg1. z.B. Hans Engel, Art. Diminution in MGG, Bd.3, S.493. Bei der Frage nach dem Umfang der angebrachten Verzierungen ist Vorsicht geboten. Zum einen ist es wahrscheinlich, daß die Gegner des willkuirlichen Verzierens bei ihren Darstellungen des "ubels" ubertrieben haben und zum anderen fehlt uns heute für die verwendeten relativen Begrifte der Maßstab. Das die Verzierungen beim Ensemblespiel tatsächlich den solistischen Diminutionen in Virtuosität und Ausmaß ähnelten, ist eher unwahrscheinlich.

293. Gedruckt erst 1601, entstanden aber um 1570 - \$.o.

294. Aufgefuhrt 1589. Einige Beispiele hieraus sind abgedruckt bei Schneider, Basso continuo, S.116 ff.

295. Im Abschnitt II.1.4.

296. Einige Beispiele hierzu aus Caccinis "Ai lettori": 1. zu den virtuosen Bearbeitungen eigentlich polyphoner Musik: "... e che no poteuano farsi per il contrappunto nelle moderne musiche," (modern hier im Gegensatz zu antik, nicht im Sinn von neu) "e particolarmente cantando sopra qualunque strumento di corde, che non se ne intendeua parola per la moltitudine de $i$ passaggi, tanto nelle sillabe breui quato lunghe, \& in ogni qualita di musiche pur che per mezzo di essi fussero dalla plebe esaltati, e gridati per solemi cantori" (1.Seite); 2. zum Verzieren allgemein: "...ma perche di sopra io ho detto essere malamente adoperati quei lunghi giri di voce, e d'amuertire, che $i$ passaggi no sono stati ritrouati per che siano necessarij alla buona maniera di cătare, ma credo io più tosto per una certa titillatione a gli orecchi di quelli, che meno intendono, che cosa sia catare con affetto, che se cio sapessero indubitatamente $i$ passaggi sarebbono abborriti, non essendo cosa più contraria di loro all' affetto; ..." (2.Seite). 
der Folgezeit nicht an virtuosen Stellen und an "passaggi", die Komponisten haben jedoch die Ausstattung der Stücke mit den Diminutionen zu ihrer eigenen Sache gemacht und tiberlassen dies nicht mehr den Ausfiuhrenden allein'297.

In Vorworten zu Musikdrucken aus den ersten Jahren des 17.Jahrhunderts sind nicht selten Mahnungen an die "unverbesserlichen" Sänger enthalten, die Kompositionen doch bitte so zu singen, wie sie notiert sind, ohne sie mit zusätzlichen Diminutionen zu versehen 298 . Diese Mahnungen werden meist als Beleg für die Verbreitung von Verzierungen durch die Ausfürenden in dieser Zeit gedeutet. Dies ist sicher auch z.T. richtig. Es ist jedoch in einer solchen Umbruchszeit auch zu erwarten, daß ein Vorwort etwas für die mit dem neuen Stil vertrauten Sänger Selbstverständliches mitteilt, da noch nicht allgemein mit dieser Vertrautheit gerechnet werden kann. Ebenfalls gegen Diminutionen richten sich einige "come stà"-Anweisungen, die nicht nur in Vorworten, sondern auch bei den Musikstücken selbst auftreten299.

297. Ein besonderes Beispiel für bewußt eingesetzte Virtuosität stellt C. Monteverdis "Possente spirito" aus L'Orfeo (1609) dar (der "Meistersänger" Orfeo bezaubert Caronte auch mit seiner Virtuosität). Erwähnenswert sind auch die Arie passeggiate von G. G. Kapsberger (erstes Buch 1612, zweites 1623). Leopold, Monteverdi, S.83, sieht in Kapsbergers Vertonung des die Kunst einer Sängerin lobenden "Mentre vaga Angioletta" (vollständiger Text mit ubersetzung bei Leopold, S.77 f) das Bemihen, "einen Katalog wortausdeutender Verzierungen zu erstellen". Nicht zutreffend ist die Behauptung von Braun, Handbuch, S.156, Kapsbergers Arienbuch von 1612 enthalte eine Diminution von Caccinis "Amarilli mia bella".

298. Ein ausdrickliches Verbot von "passaggi" findet sich in den Vorworten zu folgenden Drucken: E. De' Cavalieri, Rappresentatione (1600, wiedergegeben im zweiten Band dieser Arbeit, S.8 ff), G. P. Cima Concerti ecclesiastici (1610, abgedruckt im zweiten Band dieser Arbeit, S.12), A. Banchieri, Vezzo di perle musicali (1610, im zweiten Band dieser Arbeit auf S.7 wiedergegeben), E. Radesca di Foggia, Il quinto libro delle canzonette (1617: "HAuendo 1'Autore a richiesta mia composto le presenti Armonie Spirituali, per ció ho uoluto narraui tre cose principali da me richiesteli. La prima $e$ in non farli passaggio acciò che chi dalla natura non è dotato d'hauere la dispositione; non sia affatto priuo del'Opera; tanto più che chiaramente si vede che all'inprouiso, per valente cantante che sia, non fara mai quel passagio giusto come sara scritto al libro. Si che chi non haverà dispositione le canti come sono notate che son sicuro non saranno ingrate, \& che ne hauerá, potra accomodarsi il passaggio a sui gusto conforme il parera (...)"), P. Quagliati, La sfera armoniosa (Rom: Robletti 1623: "Avertimento per il Violino. Ne11' Opere concertate con il Violino, il Sonatore ha da sonare giusto come stà adornandola con trilli, \& senza passaggi.", zitiert nach Voge1, Bibliothek, Bd.II, S.111). Ein maßvolles Diminuieren fordert auch 0 . Scaletta im Vorwort zu der Cetra sprituale (1605, abgedruckt im zweiten Band dieser Arbeit auf S.15 f).

299. Solche "come stä"-Anweisungen stehen außer in dem zitierten Vorwort Quagliatis in einem Vorwort G. Marinis, dort allerdings in Bezug auf den 
Waren die Verzierungen fest vorgeschrieben, muste auch hierbei dem unterschiedlichen Können der verschiedenen Sänger Rechnung getragen werden. Ercole Radesca di Foggia erlaubt in seinem quinto libro delle canzonette (1617) den Sängern, die Verzierungen nötigenfalls zu vereinfachen 300 . Bartolomeo Barbarino beklagt jedoch, daß seine Monodien dabei von überforderten Sängern furchtbar entstellt worden seien ${ }^{301}$. Einen Ausweg bot der Druck von zwei verschiedenen Fassungen - einer einfacheren und einer schweren -, wie er im frihen 17. Jahrhundert vereinzelt zu belegen ist 302 .

Trotz vieler Mahnungen und Verbote gelang es im fruhen 17. Jahrhundert nicht, die Verzierungspraxis aus der Musik zu verdammen. Diese lebte vielmehr noch bis ins 19.Jahrhundert hinein fort. Die Jahre um 1600 stellen dennoch einen

Basso continuo: "REsteranno serviti il Signori Organisti di sonar la Partitura" (= Bc.-Stimme? Zu dem Stimmensatz diese Druckes gehört nur eine Bc.Stimme, aber keine Partitur) "di questi miei Madrigali come stà toccando le consonance senza variare, altrimenti non riuscirebbero, \& si sonarebbero contra la testitura essi Madrigali" ( $I 1$ secondo libro de madrigali, 1618). "Come stà"-Anweisungen uber den Noten selbst können sich auch auf die notierte Tonhöhe beziehen, sofern sie in Continuo-Stimmen stehen (etwa bei einem in einem Chiavetten-Schluissel notierten BaB - vgl. hier weiter unten im Abschnitt III.6.). Als Verbot von Verzierungen sind jedoch sicher die "come stă"-Anweisungen in G. Frescobaldis primo libro delle canzoni (1628, sowohl in der Stimmbuch- als auch in der Partiturausgabe) zu verstehen.

300. Aus dem Vorwort: "Si che chi non hauerà dispositione le canti come sono notate che son sicuro non saranno ingrate, \& che ne hauerà, potra accomodarsi il passaggio a sui gusto conforme il parera" - vgl. weiter oben. 301. Im Vorwort zu seinem secondo libro de madrigali (1607) begründet Barbarino hiermit, daß er seine Madrigale in Druck gegeben hat: ".. la qual' è che molti di essi, hauendogl'io date à ciascheduno che me gli recercaua, indi à poco tempo mi ritornarono cosi trasformati, ch'io non gli riconosceuo per miei, essendo loro stato leuato via il spirito da certi che non hanno voluto hauer' un' poco di patienza, con veder bene alcuni passi ch' in apparenza difficili, $i$ quali poi se gl'hanno accomodati à lor' dosso trasformandoli ...". Ganz ähnlich begründet auch G. Caccini den Druck seiner nuove musiche, allerdings beklagt er, daß seine Stücke zuviel mit "passaggi" versehen wurden: "... Ma ora veggendo andare attorno molto di esse lacere, e guaste, \& in oltre malamente adoperasi qui lunghi giri di voci semplici, e doppi, cioe raddoppiate, intrecciate l'una nell' altra ritrouate da me per isfuggire quella antica maniera di passaggi che gia si costumarono ..." (1.Seite des "Ai Lettori" aus Le nuove musiche (1601/02)).

302. Das beruhmteste Beispiel einer solchen Doppeledition ist wohl das "Possente spirito" aus C. Monteverdis L'Orfeo (1609, vgl. hierzu Leopold, Monteverdi, S.120 ff). Als ganze Sammlung mit zwei unterschiedlich diminuierten Singstimmen erschienen B. Barbarinos Secondo libro de motetti (1614). Er begruindet diese Doppeledition mit einem Hinweis auf viele Sänger, die die Motetten seines ersten Buches zu schwierig getunden hätten (vgl. Jerome Roche, Art. Barbarino, Bartolomeo in NG, Bd.1, S.130). 
wesentlichen Einschnitt in der Entwicklung der Verzierungsweise dar. Zum einen entwickelte sich ein Bewußtsein, daß Verzierungen nicht iberall angebracht werden können ${ }^{30}{ }^{3}$ und zum anderen bilden sich hier bereits Ansätze zu den späteren "wesentlichen Manieren" heraus (s.u.). Auch in Bezug auf die Verzierungen kann jedoch nicht unbedingt von Italien auf andere Länder geschlossen werden. So wurde in Deutschland auch noch um die Mitte des 17. Jahrhunderts die "italiänische Manier zu singen" als Kunst des Verzierens gelehrt - wenn auch nicht ohne Mahnungen zur Zurüickhaltung ${ }^{304}$. Dabei werden z.T. italienische Quellen in entstellter Art zitiert und dadurch erst zu Verzierungslehren gemacht 305 .

303. Bezeichnend ein Abschnitt aus den "Auertimenti à Cantanti" aus F. Rognonis Selva di varii passaggi (1620), S.51, also aus einer Diminutionslehre, die, wie auch im 16.Jahrhundert, zunächst die virtuose Bearbeitung als Ziel verfolgt: "S'hanno ancora a guadare da passaggi sopra parole significanti doglia, affanni, pene, tormenti. \& simili cose, perche iuece de passagi, s'vsano fare gratie, accenti, \& esclamationi, scemardo hor la voce, hor accrescendola, con mouimenti dolci, e soaui, \& tal hora con voce mesta, \& dogliosa, conforme il sento dell'oratione." Interessant ist auch der Versuch von H. Schütz, den virtuosen Sängern einen Raum für zusätzliche Verzierungen zur Verfügung zu stellen, wie dies im "Cantabo Domino" aus den Symphoniae sacrae (1629) beim letzten "Alleluja" geschehen ist. Hier steht iber der, in verhältnismäßig langen Notenwerten notierten Singstimme "passagio" (vgl. Schütz-GA, Bd.5, S.24).

304. So in M. Praetorius' Syntagmatis musici ... tomus tertius (1619), S.229: "Das IX. Capite1. Introductio pro Symphoniacis Wie die Knaben/ so vor andern sonderbare lust und Liebe zum singen tragen/ uff jetzige Italianische Manier zu informieren und zu unterrichten seyn.", dort S.229 unten: "Sintemal die jenigen gar nicht zu loben/ welche von Gott und der Natur/ mit einer sonderbaren lieblichen zitternden und schwebenden oder bebenden Stimme/ auch einen runden Hals unnd Gurgel zum diminuieren begabet/ sich der Musicorum leges nicht binden lassen/ sondern nur fort unnd fort/ mit ihrem allzuviel colorirn, die im Gesang vorgeschriebenen limites uberschreiten/ unnd denselben dermaßen verderben und verdunckeln/daß man nicht weik was sie singen" (etc.).

305. Besonders deutlich ist dies bei J. A. Herbst. Er zitiert in seiner Musica Practica (1642), S.29 ff, A. Banchieris "Cento variati passaggi accentuati alla moderna" aus der Cartella musicale (1614, S.216 ff), verkirzt aber Banchieris "Annotationi" in seiner ubersetzung (S.41) um entscheidende Punkte. Banchieri versteht diese "Cento passaggi" nämlich in erster Linie nicht als Verzierungslehre, sondern als ubungen für die Sänger, bereits vom Komponisten modern ausgezierte Stimmen zu singen. Die verzierten Stimmen hat er aus verschiedenen Kompositionen der "autori moderni" zusammengesucht und - zu ubungszwecken - eine vereinfachte Fassung hinzugefügt: "2 La Memoria non hó trouato scritta mà da me composta sopra il Passaggio, che seruira tal studioso, à gli principanti apprendere il modo far cantare le parte passaggiate \& accentuate all 'uso odierna". Es geht Banchieri hier a1so ausdruicklich um das Singen der "parte passagiate" und nicht um das Ver- 


\subsection{AUFKOMMEN UND STANDARDISIERUNG VON VERZIERUNGSZEICHEN}

Im letzten Jahrzehnt des 16. Jahrhunderts wurde, soweit bekannt, erstmals in der Vokalmusik der Versuch unternommen, immer wiederkehrende Verzierungen durch Zeichen zu ersetzen ${ }^{306}$. Giovanni Luca Conforto notierte in seiner Breve et facile maniera (1593) eine Drei unter die Noten, auf denen ein "trillo" auszufuhren ist307; ein Jahr später benutzte Giovanni Battista Bovicelli in den Regole, passaggi di musica ein nach unten offenes Dreieck als Zeichen für den "tremo10"308.

Breiteren Gebrauch von Verzierungszeichen auch außerhalb eines didaktischen Werkes machte wohl erstmals Emilio De' Cavalieri in der Rappresentatione di anima, et.di corpo (1600). Am Ende des umfangreichen Vorwortes werden gleich vier Buchstaben eingefüht, die jeweils stellvertretend für eine Verzierung stehen: " $g$ " für den "groppolo", "m" für die "monachina", " $t$ " für den "trillo" und schließlich " $z$ " für den "zimbelo"s09. Alle diese Verzierungen gehören nicht zu den "passaggi", sondern es handelt sich um kleine, sich nicht weiter als eine Sekunde von der Ausgangsnote entfernende Figuren. Diese Verzierungszeichen wurden nicht, wie später ublich, uber die entsprechende Note notiert, sondern vor die Note, in das System hineingedruckt.

Ebenfalls mehrere Verzierungszeichen finden sich in Giulio Caccinis Le nuove musiche (1601/1602). Im Gegensatz zu De 'Cavalieri verwendete Caccini nicht einzelne Buchstaben, sondern ganze Worte oder längere Abkürzungen (z.B. "Escla" für "Esclamatione") jeweils über den damit zu verzierenden Noten. Während diese Zeichen in den zum "Ai Lettori" gehörenden Musikbeispielen recht häufig notiert sind, sind sie im weiteren Druck nur selten anzutreffen ${ }^{310}$. Caccini teilt hierzu im Vorwort mit, daß er sie in diesen Beispielen so genau gesetzt habe, um sie im weiteren Verlauf seines Druckes nicht immer notieren $\mathrm{zu}$ müssen ${ }^{311}$. Caccini bezeichnet auf diese Weise die Verzierungen "Esclamatione", "Trilla" und - selten - den "Gruoppo".

zieren der "Memoria", wie dies bei Herbst der Fall ist.

306. Es ist bekannt, daß solche Zeichen in den Tabulaturen bereits sehr viel fruher auftreten, vgl. z.B. Apel, Notation, S.27 f.

307. Dazu heibt es in den "Dichiarationi": "L'altro tre, che si vede sotto duoi crome, ouero nel fine delle cadenze, altro non voglio dire che trillo, che rendendo al doppio il numero, imbellisce il canto \& copre molli difetti." (fol.2).

308. Dieses Zeichen wird - wie auch der Bindebogen (s.o.) - nur in den "Avertimenti per $1 i$ passaggi" verwendet, und auch dort nur auf Seite 13. Zur Bedeutung der Worte "tremolo" und "tri11o" s.u.

309. Vgl. den Abdruck des Vorwortes im zweiten Band dieser Arbeit auf S.8 ff.

310. Ein "Tri11o" wird auf den Seiten 3, 4, 14, 20 und 39 verlangt, eine "Esclamatione" auf S. 20 ("Es") und 39 ("Escl").

311. Caccini bemerkt zu der Notation dieser Verzierungszeichen: "E perche negli vltimi due versi sopra le parole "Ahi dispietato amor" in aria di 
Wenig später erschien in Giovanni. Maria Tarbacis Ricerare ... libro primo (1603) erstmals das in den nächsten dreißig Jahren häufigste Verzierungszeichen, nämlich das " $T$ ". Trabaci schreibt hierzu, daß er sich dieses Zeichens bediene, um den "trillo" nicht jedesmal ausschreiben zu muissen ${ }^{312}$. In die virtuose Vokalmusik wurde das Trillozeichen " $T$ " (o.ä.) ${ }^{13}$ s sätestens 1607 aufgenommen. Es erschien in diesem Jahr in Bartolomeo Barbarinos secondo libro di madrigali 314 und in den Passaggi sopra tutti li salmi von Giovanni Luca Conforto.

Eine erste theoretische Behandlung erfuhr das Zeichen " $t$ " im Vorwort zu den Arie devote von Ottavio Durante $(1608)^{315}$. Dort liest man, daß man immer, wenn man ein " $t$ " findet, einen "trillo" ausführen soll. Falls das " $t$ " aber uber einem (bereits ausgeschriebenen) "trillo" steht, so solle man um so mehr "trillern"316. Diese letzte, zunächst wiedersprüchlich klingende Bemerkung bezieht sich auf die in der Tat häufig anzuteffende Notation des Verzierungszeichens " $t$ " uber einem ausgeschriebenen "trillo"s17.

Eine wirkliche Verbreitung dieses Verzierungszeichens setzt erst im zweiten Jahrzehnt des 17. Jahrhunderts ein ${ }^{318}$. Bereits rund 15\% der ausgewerteten Drucke der Jahre 1611 bis 1620 sind mit diesem Verzierungszeichen versehen.

romanesca, e nel madrigale appresso "Deh doue son fuggiti" sono dentro tutti $i$ migliori affetti, che si possono vsare intorno alla nobilita di questa maniera di canti gli ho voluti per ció scrivere; si per mostrare doue si deue crescere, e scemar la voce: à fare l'esclamazioni, trilli, e gruppi, \& in somma tutti $i$ tesori di quest'arte, come anco per non essere necessitato altra volta à dimostrar cio in tutte le opere, che appresso sequiranno: \& acciache seruano per esempio, in riconoscere, in esse musiche $i$ medesimi luoghi, oue saranno più necessari secondo gli affetti delle parole".

312. S.73: "Per non scrivere il Trillo sempre disteso, doue si ritrouerà questa littera. . . sempre si farà il Trillo".

313. Es gibt verschiedene Schreibweisen für dieses Zeichen: neben " $T$ " auch " $t$ " sowie " $t$.", " $T$.", ".t." etc. Vereinzelt und vor allem gegen Ende des hier zu behandelnden Zeitraumes wurde bereits "tr." notiert.

314. Nur im ersten Madrigalbuch (1606) verwendete Barbarino kein Trillozeichen.

315. Das Vorwort ist vollständig abgedruckt und ubersetzt bei Goldschmidt, Gesangsmethode, S. 29 ff.

316. "Dove sarà notata la lettera " $t$ " si deve trillar sempre con la voce anchor che sia notata sopra il trillo o groppetto stesso e all hora si deve trillar tanto più" (zitiert nach Goldschmidt, S.32).

317. Hierauf wird im nächsten Abschnitt noch näher einzugehen sein.

318. Außer den bereits genannten Drucken G. M. Trabacis, B. Barbarinos und G. L. Confortos ist dem Verfasser aus der Zeit bis 1610 nur ein Druck S. d'Indias mit dem Verzierungszeichen " $t$ " bekannt, nämlich Le musiche ... da cantar solo (1609). Vorhanden war dieses Zeichen freilich auch in Arie devote von Ottavio Durante $(1608, \mathrm{s.o.})$. 
Mit anderen Besonderheiten des monodischen Stiles fand das Verzierungszeichen in diesem Zeitraum auch Aufnahme in geistliche Kompositionen, vor allem in Werken Antonio Cifras ${ }^{319}$, Alessandro Grandis 320 und Seraphino Pattas321. Insgesamt bleibt die Verbreitung des Verzierungszeichens aber gegenuiber den anderer Neuerungen in der Notation zuruick: Während z.B. Bindebögen in etwa drei Viertel der ausgewerteten Musikdrucke aus den Jahren 1621 bis 1630 zu finden sind, tritt das Verzierungszeichen nur in etwa einem Viertel der Drucke dieses Zeitraumes auf. Die Komponisten iberließen das Hinzufügen dieser Verzierung neuer Art noch weitgehend den Ausführenden. In Vorworten findet sich der Hinweis, man möge bitte keine "passaggi", wohl aber "tril1i" und "accenti" anbringen 322.

Andere Verzierungszeichen ${ }^{323}$ blieben eine Seltenheit; oft sind sie nur in einem einzigen Druck iberhaupt anzutreffen. Allgemein bekarnt war offenbar neben dem "trillo" der "groppo" (oder "gruppo", "gruoppo" ete.). Von ihm wird nicht nur in Traktaten und Vorworten ${ }^{324}$ geschrieben, sondern er wurde gelegentlich auch mittels eines Verzierungszeichens verlangt ${ }^{325}$. Das

319. Beginnend mit dem sechsten Motettenbuch von 1613.

320. Es ist dem Verfasser nicht genau bekannt, wann das Verzierungszeichen Aufnahme in die Drucke A. Grandis fand, da dem Verfasser von den ersten vier Motettenbiichern jeweils nicht die ersten Auflagen vorlagen. Die ausgewerteten Nachdrucke aus den 1620er Jahren sind allesant mit Verzierungszeichen ausgestattet. Es ist sehr wahrscheinlich, daß diese auch in den ersten Auflagen von 1610 bis 1616 nicht fehlten.

321. Sacrarum cantionum ... liber secundus (1613) und Motetti et madrigali cavati da le poesie sacre (1614). Letzterer ist ein Druck ausschlieblich geistlicher Monodien. Ein kurzes Beispiel für den virtuosen Kompositionsstil Pattas wie auch für seine Anwendung des Trillozeichens ist bei Goldschmidt, Gesangsmethode, S. 16 des Anhanges, abgedruckt.

322. So in den Vorreden zu G. P. Cimas Concerti ecclesiastici (1610, abgedruckt im zweiten Band dieser Arbeit auf S.12) und P. Quagliatis La stera armoniosa (1623, abgedruckt bei Voge1, Bibliothek, Bd.II, S.111, s.0.). Interessant in diesem Zusammenhang ist auch ein Vorwort A. Piccinis (Intavolatura di liuto, e chitarrone, 1623). Piccini schreibt, er hätte die Stellen, an denen ein "tremolo" angebracht werden soll, nicht bezeichnet, da dies zu viele gewesen seien (Dodge, Ornamention, S.322 f).

323. Nicht mit einbezogen sind hier die Verzierungszeichen in den Tabulaturen; sie haben eine eigenständige Geschichte. Vgl. hierzu vor allem Dodge, Ornamention. Nach Dodge wurde auch in italienischen Tabulaturen für Lauteninstrumente, beginnend mit Pietro Paolo Mellis Intavolatura di liuto attiorbato (1614) der Buchstabe " $T$ " verwendet (S.322).

324. Vg1. hierzu Goldschmidt, Gesangsmethode, Anhang, S.14 ff.

325. Die Verzierungszeichen - darunter auch " $G$." und "T." - in G. Dirutas Seconda parte del Transilvano (1609) sind nicht vorschreibend, sondern nur erklärend gemeint. Sie zeigen innerha1b einer diminuierten Intavolatur an, welche Art der Verzierung angewendet wurde (vg1. Krebs, Diruta, S.351 1, sowie S.379 ff). 
früheste Beispiel hierfür ist, wie oben beschrieben, Emilio De' Cavalieris Rappresentatione (1600). In Le nuove musiche verwendete Caccini das Wort "gruppo" nur in den Beispielstuicken innerhalb seiner Vorrede. In der Nuove musiche e nuova maniera di scriverle (1614) hingegen ist es auch bei den Stiucken selbst zu finden, wenngleich erheblich seltener als das Trillozeichen. Meist steht "grup" oder "gruppo" hier uber einem bereits ausnotierten "groppo". Nur an einer Stelle steht "grup" uber einem unverzierten Quartvorhalt326. An den anderen Stellen weist es wahrscheinlich auf die besondere Ausfuhrungsart hin. Ebenfalls sowohl bei bereits ausgeschriebenen "groppi" als auch bei unverzierten Kadenzformeln ist das Wort "groppo" in Biagio Marinis Sonate (1629) notiert327. Ein "G." als Zeichen für den "groppo" findet sich in Sigismondo D'Indias Le musiche (1609)328.

Mehrere verschiedene "trilli" schrieb Giovanni Maria Trabaci in seiner Bearbeitung des "Ancedetimi pur" von Arcadelt für Harfe329 vor. Außer dem ublichen " $t$." verwendete er ein " $t$." mit einem Kreuz (" $t+$ "). Die damit bezeichnete Verzierung ist zu Anfang des Stückes einmal ausnotiert. Uber dem Satz steht der Hinweis, daß dieses Zeichen immer für diese $z \cdot \mathcal{A}$ Anfang ausnotierte Verzierung steht ${ }^{300}$. Dariber hinaus findet sich in diesem Stiick die Anweisung "trillo doppio", die nicht näher erläutert wird. Im füften Takt steht gar geschrieben: "qui trilla sep finch'è finit'il Bas.". Hier soll in den Oberstimmen während eines aus 16 Biscromen bestehenden Laufes des Basses durchgetrillert werden.

Nicht erklärt wird ein weiteres, sonst nicht bekanntes Verzierungszeichen in Michele Deliparis I baci (1630). Außer dem " $t$." wird hier auch das Zeichen "m.t." (wahrscheinlich "mezzo trillo" = kurzer "trillo") notiert. Die Abkuirzung "m.t." steht ausnahmslos auf dem Wert der Semiminima.

In gewisser Weise gehören auch die arpeggio-Anweisungen, wie sie selten in Basso continuo-Stimmen Adriano Banchieris und reichhaltig in den Tabulaturen Giovanni Girolamo Kapsbergers zu finden sind, zu den Verzierungszeichen.

326. S. 34

327. Bei unverzierten Quartvorhalten steht "groppo" in der "Sonata quarta" (vgl. die Edition bei Iselin, Marini, Notenteil S.11 ff, bes. S.12 und S.14), bei bereits ausgeschriebenen "groppi" in der "Sonata per 1 'organo $e$ Violino ò Cornetto" (vgl. die Edition bei Schering, Solosonate, S.320 ff, bes.S.323).

328. Ein "G." als Groppozeichen steht auch in G. Fantinis Modo per imparare a sonare di tromba (1638) auf S.11.

329. Il secondo libro de ricercate (1615), S.126 ff.

330. "Questo Madrigale particolarmente si sonerà in Battuta larga, ma in principio del Tenor' e Contralto per non scriuere quella sorte di Trillo sempre disteso, doue se ritrouerà questo segno $+v i$ seruirete di detto Trillo." Bei diesem "trillo" handelt es sich um eine Diminution der Melodie- 
Während eine solche Anweisung in den Continuo-Stimmen ausgeschrieben wurde ${ }^{331}$, führte Kapsberger hierfür ein neues Zeichen, nämlich "./." $\operatorname{ein}^{33^{2}}$.

\subsection{Die Bedeutung des Verzierungszeichens " $T$ " UND Der Begriffe "TRILlo" UND "TREMOLO"}

Es verbleibt nun noch, die Ausfuhrung der mit " $t$ " sowie mit den Begriffen "trillo" und "tremolo" bezeichneten Verzierung zu erklären. Dies wird erschwert durch z.T. recht widerspriichliche Erläuterungen hierzu in den Quellen des 16. und 17.Jahrhunderts. Um die folgenden Definitionsversuche nicht gänzlich verwirrend zu machen, bleiben die deutschen Quellen dabei unberuicksichtigt ${ }^{3}{ }^{3}$.

Die Bezeichnung "tremolo" ist die ä1tere. Sie ist bereits in Quellen des friheren 16. Jahrhunderts nachweisbar. Silvestro Ganassi bezeichnet hiermit in seinen Opera intitulata Fontegara (1535) einen Tonwechseltriller ${ }^{334}$. Auch Girolamo Diruta meint im ersten Teil des Transilvano (1593) mit "tremolo" einen solchen Triller335. Bei Giovanni Battista Bovicelli (Regole, passaggi di musica, 1594) ist jedoch zu lesen, der "tremolo" sei nichts anderes, als ein Beben auf ein und derselben Note ${ }^{336}$. Dem schließt sich auch Lodovico Zacconi an ${ }^{337}$.

Erst in den letzten Jahren des 16. Jahrhunderts kam der Begriff "trillo" hinzu. Giovanni Luca Conforto gibt zwar in den "Dichiarationi" zu seiner Breve et facile maniera (1593) an, welches Zeichen er für einen "trillo" verwendet hat (s.o.), nicht aber, wie er ausgefuhrt werden soll. Sowohl die Beschreibung der Vorzuige des "trillo" ("che rendendo al doppio il numero, imbellisce il canto"338) als auch die Notation des Verzierungszeichens mit Vorliebe uber zwei gleichen Noten deuten auch hier auf eine Verzierung ohne Tonwechsel hin ("Bockstriller"). Auch im frühen 17.Jahrhundert steht das Verzierungszeichen " $t$ " sehr häufig uber bereits ausnotierten Tonrepetitionen. Ottavio Durante schrieb hierzu, daß man dann um so mehr trillern soll 337.

331. A. Banchieri, Vivezze di flora e primavera (1622) und L'organo suonarino $(3 / 1622)$.

332. Vg1. Dodge, Ornamentation, S.321, sowie die Notenbeispiele II, 2 und II, 4 im zweiten Band dieser Arbeit.

333. Zu den deutschen Quellen und der dort anzutreffenden Terminologie vgl. Goldschmidt, Gesangsmethode, bes. S.86 ff.

334. Dickey, Vibrato, S.79.

335. Ebenda.

336. S.12: "Il tremolo nondimeno, che non é altro, che un tremar di uoce sopra ad una stessa note".

337. Prattica musica (1596), fol.60: "il tremolo, cioè la voce tremante".

338. Fo1.2.

339. Vgl, weiter oben. 
Innerhalb der Vokalmusik ist im fruhen 17.Jahrhundert mit "tri1lo" - und auch mit dem, fast immer einen "tri11o" vorschreibenden Zeichen " $t$ " 340 wahrscheinlich immer der Tonwiederholungstriller gemeint ${ }^{341}$. In der Musik für Tasteninstrumente ist dies jedoch offenbar nicht immer der Fall. Giovanni Maria Tarbacis Zeichen " $t+$ " ist eindeutig eine Sonderform eines Tonwechseltrillers (s.o.). Der Zusatz "con un tasto solo" ("trillo con un tasto solo") in der Handschrift Chigi Q IV 28 der Biblioteca Apostolica Vaticana, Rom, beweist ebenfalls, da日 diese Ausführung des "tri11o" nicht die einzig mögliche war.

Wie bereits erwähnt, steht das Verzierungszeichen " $t$ " häufig uber einem bereits ausgeschriebenen "trillo". Für einen Teil dieser Notierungen verschafft die Forderung ottavio Durantes, in einem solchen Fall "um so mehr" zu "trillern" (s.o.), Klärung. Die Zahl der Tonwiederholungen soll also vermehrt (d.h. in der Regel wohl verdoppelt) werden. Dies deckt sich auch mit der Formulierung aus Giovanni Luca Confortos Breve et facile maniera ${ }^{42}$, daB der "trillo" (auch hier steht das Verzierungszeichen mit Vorliebe uber einer bereits ausgeschriebenen Tonwiederholung) durch sein "rendere al doppio il nummero" 343 den Gesang verschönt. Sehr oft aber ist eine solche Vermehrung der Tonwiederholungen nicht möglich, da der notierte "trillo" bereits aus sehr kurzen Notenwerten besteht. In einem solchen Fal1 soll die zusätzliche Angabe " $t$ " entweder auf die besondere Artikulation hinweisen, oder aber dem Auffuhrenden anheimstellen, hier den ihm gewohnten "trillo" anzubringen, unabhängig von seiner jeweiligen Ausformulierung.

Auch in Quellen des frïhen 17. Jahrhunderts gibt es aber neben dem "trillo" auch den "tremo1o". Beide Verzierungen bestehen aus einer Tonwiederholung. Während der "trillo" zu den Gesangsmanieren gehört, ist der "tremolo" nun eine weitgehend instrumentale Verzierung ${ }^{344}$. Das "tremolo" ist deutlich

340. Daß das Zeichen " $t$ " den "trillo" meint, ist ausdruicklich erklärt bei E. De' Cavalieri, Rappresentatione (1600, vg1. den Abdruck des Vorwortes im zweiten Band dieser Arbeit auf S.8 ff), G. M. Trabaci, Ricercari ... 1ibro primo (1603, s.o.), ders., Libro secondo de ricercari (1615, s.o.), 0. Durante, Arie devote (1608, s.o.) und F. Severi, Salmi passeggiati (1615, vg1. den Abdruck des Vorwortes im zweiten Band dieser Arbeit auf S.16 f). Nur bei P. P. Melli, Intavolatura di liuto attiorbato (1614) meint das " $T$ " einen "tremolo" (Dodge, Ornamention, S.322).

341. Ausführlich beschrieben wird der Tonwiederholungstriller bei $G$. Caccini, Le nuove musiche (1601/02), G. G. Kapsberger, Libro primo d'intavolatura di chitarone (1604 nach Dodge, Ornamentation, S.321). Lediglich E. De' Cavalieri löst das " $t$ " im letzten Teil des Vorwortes zur Rappresentatione als Tonwechseltriller auf (vgl. im zweiten Band dieser Arbeit auf S.11).

342. 1593. Die vollständige Anweisung zum "trillo" ist weiter oben bereits abgedruckt.

343. Fo1.2.

344. Vgl. zum Tremolo im 17. und auch 18. Jahrhundert Carter, Tremolo, mit 
langsamer als der "trillo"345 und meist auch bedeutend länger (ein "trillo" dauert in der Regel höchsten eine Semibrevis, ein "tremolo" kann sich über mehrere Takte erstrecken) ${ }^{346}$. Es handelt sich beim "tremolo" um eine Imitation des Orgeltremulanten ${ }^{347}$. Hierbei werden lange Noten (Minimen oder Semibreven) in Folgen von Cromen zerteilt. Der "tremolo" konnte nur als Anweisung notiert348 (dann hatte der Ausführende die Zerteilung der Noten selbst vorzunehmen), oder aber ausgeschrieben werden; darn weisen entweder Bindebögen ${ }^{349}$, oder aber auch nur das zu Anfang notierte wort "tremolo"350 auf die gemeinte Ausführung hin.

zahlreichen Notenbeispielen.

345. Der "trillo" wird, soweit ausgeschrieben, häufig in Semi- oder gar Biscromen notiert.

346. Braun, Handbuch, S.153, ist der Meinung, der Unterschied zwischen "trillo" und "tremolo" liege in der gleichbleibenden Geschwindigkeit des "tremolo" im Gegensatz zur stetigen Beschleunigung beim "trillo". Dies ist jedoch nur ein sekundärer Unterschied. Freilich ist es bei einem "trillo" als Gesangsmanier möglich, eine solche Beschleunigung durchzuführen (sie wird auch in einigen Quellen erwähnt, z.B. im Vorwort zu Giulio Caccinis Le nuove musiche (1601/02), sie ist jedoch nicht das eigentliche Erkennungsmerkmal des "trillo").

347. Entsprechende Unterweisungen finden sich in den Vorworten zu C. Farinas Ander Theil nawer Paduanen (Dresden, 1627: "So wird das Tremulieren mit pulsierender Hand/ darinnen man den Bogen hat/ auff Art des Tremulanten in den Orgeln imitiret", zitiert nach Moens-Haenen, Vibrato, S.134) und A. Hammerschmidts dritten Teil der Musicalischen Andachten (Freiberg, 1642: "Es wird derselbe" (gemeint: der Leser) "in den Violinen bißweilen etliche Noten/ nemlich also" (folgt Notenbeispiel mit vier Cromen e', vier Cromen f' ' und acht Cromen e', jeweils vier unter einem Bogen) "finden/ welche so gemennt/ dab man mit dem Bogen ihrer viere auff einen strich (gleichsam wie einen tremulanten in einer Orgel) machet ..." (vgl. das Faksinile bei Adam Adrio, Art. Hammerschmidt, Andreas in MGG, Bd.5, Sp.1431). Brauns Behauptung, Samuel Scheidt würde in der Tabulatura nova (1624) den "tremolo" als eine "Imitatio violistica" bezeichnen (Handbuch, S.272), beruht auf einem Irrtum. Zwar notiert Scheidt in seiner "N.B." die gebundenen Noten der "Imitatione violistica" auf einer Tonhöhe (sie gleichen dann einem "tremolo"), innerhalb der verschiedenen Stuicke jedoch handelt es sich bei der "Imitatione violistica" immer um gebundene Noten wechselnder Tonhöhe (vg1. Scheidt-GA, Bd.6, S.7, 8, 9, 25 etc. sowie S.126 und weiter oben im Abschnitt III.3.3.). Scheidt wählte in seiner "N.B." offenbar die gleichen Noten als verallgemeinernde Form. Ein "tremolo" ist nicht gemeint.

348. So in B. Marinis Affetti musicali (1617). In "La Foscarina Sonata A 3" steht in den Diskantstimmen (Instrumentation: "Violini o Cornetti") "Tremolo con L'arco" (!!), in der Baßstimme (Posaune oder Fagott) "Tremolo cal strumento" und in der Orgelstimme "Metti il Tremolo" (vgl. hierzu Dickey, Vibrato, S.105, sowie die Neuausgabe dieser Sonate, hrsg. von Robert P.Block und David Stuart).

349. In N. Corradinis Motetti (1624), P. Possentis Concentus armonici 
Trotz der allmählichen Verbreitung des Verzierungszeichens " $t$ " wurde im frihen 17. Jahrhundert diese Verzierung oft auch ausgeschrieben. Das wohl bekannteste Beispiel hierfür dürten die zahlreichen atemberaubenden "trilli" in dem "Concerto Duo Seraphim" aus Claudio Monteverdis Sanctissimae Virgini missa (1610, sog. "Marienvesper") sein 351 . Hier ist der "trillo" sowoh1 mit Semicromen als auch Biscromen notiert - voran gehen jeweils Sekundpunktierungen in der Art von De' Cavalieris "Zimbe10"352.

\section{VORZEICHENSETZUNG UND "MUSICA FICTA"}

Die Setzung der Vorzeichen sowie die Anwendung der Regeln einer "musica ficta" wurde im beginnenden 17. Jahrhundert in Italien recht uneinheitlich gehandhabt. Natürlich gibt es eine große Zahl von Musikdrucken aus dieser Zeit, insbesondere im Bereich der traditionsgebundeneren Gattungen ${ }^{35} 3$, deren Vorzeichensetzung weiterhin auf den alten Regeln einer "musica ficta" beruht; obgleich auch dort zu beobachten ist, daß die Stellen, an denen diese Regeln zur Anwendung kommen, seltener werden - die meisten Vorzeichen sind nämlich bereits notiert. Besondere Bedeutung kommt auch im frühen 17. Jahrhundert der Regel "una nota super la semper est canendum $\mathrm{fa}$ " $\mathrm{zu}$, wenngleich das Fortleben dieser Regel sich sehr oft nur noch an Warnakzidentien an den Stellen, an denen diese Regel nicht angewendet werden soll, erkennen 1äbt. In der Musiktheorie werden die Regeln der "musica ficta" bis zum Ende des hier zu behandelnden Zeitabschnittes weiter tradiert354. Als Folge der zunehmenden Chromatisierung in Madrigal und Monodie mußten aber Möglichkeiten gefunden werden, die Gültigkeit der Akzidentien eindeutig festzulegen $^{355}$.

(1628) und D. Castellos Sonate concertate... libro secondo (1629). Die "tremoli" stehen jeweils nur in Instrumentalstimmen. Bei Corradini stehen acht, sonst je vier Noten unter einem Bogen. Castello schreibt zusätzlich "tremolo" in die stimmen.

350. G. B Riccio in der "Canzon In Ecco con il tremolo La Pichi" (zwei Violinen und Posaune) und "Canzon con il Tremolo La Grimaneta" (zwei "Flautini" und Fagott) aus dem terzo libro delle divine lodi (1620, vg1. die von Rudolf Ewerhart herausgegebene Neuausgabe, Heft 2).

351. Monteverdi-GA, Bd.14, zweiter Halbband, S.190 ff.

352. Vgl. das Vorwort zu den Rappresentationi im zweiten Band dieser Arbeit auf S.8 ff, bes. S.11. Diese Sekundpunktierungen gehen den "trilli" häufig voraus.

353. Dazu zählen vor allem die nicht konzertante Motette und die Messe (s.o.).

354. So geht noch S. Picerli im Specchio primo di musica (1630), S. 13 f, auf die Grundregeln der "muscia ficta" ein. Auch A. Banchieri behandelt in seiner als fortschrittlich zu bezeichnenden Cartella musicale (1614) einige dieser Regeln (S.46).

355. Vg1. Kroyer, Akzidentien, S.113, und Wolf, Akzidentien, S.125. 
Ein Teil der Komponisten des frihen 17. Jahrhunderts $1 \mathrm{ieB}$, aufbauend auf der älteren Praxis, ein Akzidens zwar für ein Folge gleicher Noten gelten, vorausgesetzt, daB diese unmittelbar nacheinander stehen und nicht von einer Pause getrennt werden356, Hinzufügung von Vorzeichen aber im Sinne einer "musica ficta" lehnen sie ab. Ausformuliert ist dies bei Manilio Caputi357, Giovanni Battista de Bellis ${ }^{358}$ und Domenico Mazzocchi359.

Andere Komponisten gingen dazu über, die Geltung eines Vorzeichens genau auf die Note zu beschränken, vor der es steht, ohne irgendwelche Ausnahmeregelungen anzuerkennen. Dies hat unter anderem zur Folge, daß, wenn mehrere gleiche Noten aufeinanderfolgen, ein Akzidens bei jeder dieser Noten stehen muß. Diese Regel ist ausformuliert in der Vorrede zu Emilio De' Cavalieris Rappresentatione $(1600)^{360}$, sowie in Scipione Stellas Hymorum ecclesiasticorum liber primus $(1610)^{361}$. Nicht ganz so deutlich sind die Hinweise in

356. Bereits eine Zäsur kann jedoch eine Pause in diesem Sinne sein; vg1. Schwartz, Akzidentienfrage, S.111, sowie Robert Donington, Art. Musica ficta in NG, Bd.6, S.809.

357. Libro primo de' madrigali (1592). Im Baßstimmbuch auf Seite 24 unten sind folgende Anmerkungen zu lesen: "Auvertano $i$ Cantanti, che in tutte 1 'Opere del Caputi non s'ha da cātat mai co'l b, molle la Nota che stà in b fa b mi, ouero in Elami, eccetto quando ui sarà il detto b, molle segnato; per euitar l'inconuiente d'hauerui à ponere altro segno, quando non alzando il canto più d'una Nota sopra della Là, conuenga che quella tal Nota non sia Fà, ma Mi: Et similmente auuertano, che quando si trova segnato il, hà da seruire per quell'vna, ó più Note, che solamente il seguando appresso nel medesimo luogo senza intervallo d'altre Note più acute, ò più graui, $\dot{o}$ di pause."

358. Il primo libro di madrigali a 4 (1619), "A'Lettori": "Facciasi il Semitono tanto alla prima nota, ove sarri egli signato, quanto alle seguenti, \& spettando da me la terza muta di madrigali à cinque con la seconda cose Ecclesiastiche. Le bacio le mani."

359. La catena d'Adone (1626), "Avvertimento": "Che non si faccino ne "k, ne b. se non dove si ritrovano segnati, eccettuando le note, che seguitano immediatamente nel medemo grado vn' altra segnata, le quali s'intendino sempre esser del medemo tenore della prima corda precedente, mentre non saranno disferentiate con segno contrario, che non per altro fine vi sarà posto.".

360. Vg1. die vollständige Wiedergabe dieses Vorwortes im zweiten Band dieser Arbeit auf S.8 ff, sowie Schneider, Basso continuo, S.74 f. Diese Stelle ist sicher im Sinne einer neuen Einschränkung gegenüber der alten Praxis zu deuten und kommt daher keinesfalls als Beleg für zurückhaltenden Gebrauch der "musica ficta" im 16. Jahrhundert in Frage (so Albrecht, Akzidentien, S.128).

361. Dort steht im Canto-Stimmbuch unter dem letzten Stiuck: "SI auertisce, che all'hora solo si canteranno $i$ b molli, $i$ diesis, \& $i$ b quatri quando saranno scritti, cioè faranno due, ò più note in vno stesso virgalo, o spatio, auanti alla prima nota solo sarà scritto vno b molle, ò diesis, $\dot{o} b$ 
den beiden ersten Madrigalbuichern von Crescenzio Salzilli (1607 bzw. $1611)^{362}$ und im siebten Madrigalbuch von P. Nenna (1608)363. Aus der Handhabung der Vorzeichen in den dazugehörigen Drucken ist jedoch ersichtlich, daß sie dasselbe meinen.

Diese Art der Vorzeichenbehandlung findet sich dariber hinaus auch in Musikdrucken zahlreicher anderer Komponisten ${ }^{364}$; meist ist sie jedoch nicht sehr konsequent gehandhabt worden ${ }^{365}$. Nur eine kleine Zah1 der Komponisten, darunter vor allem Carlo Gesualdo und Pomponio Nenna, führen die Anwendung dieser Regel so weit, daß sie bei einem chromatischen Gang kein nach dieser Regel uberfluissiges Akzidens setzen: Ein chromatischer Gang abwärts kann also z.B. mit einer Note mit Kreuz und einer darauffolgenden gleichen Note ohne Vorzeichen notiert werden ${ }^{366}$.

Die Anwendung dieser Regel sowie auch die vielen Ausnahmen die ihr gegenúber selbst in Drucken mit vergleichsweise strenger Einhaltung der Regeln eingeräumt werden, kann sehr gut anhand der Ausgabe der sechs und siebenstimmigen Cantiones von Carlo Gesualdo (1603) studiert werden ${ }^{367}$. Da dieser Druck nur unvollständig uberliefert ist 368 , verzichtete der Herausgeber (Glenn E.

quattro all prima nota solo si canteranno, \& alle altre no."

362. Il primo libro de madrigali a 5 (1607): "S'averte alli Signori Cantanti che non cantino \# $o$ b se non dove sono segnati." sowie Il secondo libro de madrigali a 5 (1611): "Non ch'à da Cantar $\$ \$, \zeta o b$, se ne dove stà segnato." (beide zitiert nach Voge1, Bibliothek, Bd.II, S.188)

363. Il settimo libro de madrigali a 5 (1608), unter der Tavola: "Facciasi il \# in quelle note solamente oue stà notato."

364. Dem Verfasser ist eine solche Vorzeichenbehandlung aus Drucken von G. Aichinger, S. Bernardi, A. Banchieri, A. Bianchi, A. Bonelli, G. Carrone, T. Cecchino, G. P. Cima, G. Croce, G. Del Turco, C. Gesualdo, A. Grandi, T. Graziani, S. D'India, E. Marotta, D. M. Megli, G. Metallo, P. de Monte, M. A. Negri, P. Nenna, G. P. Nodari, S. Patta, J. Peri, G. Piccioni, G. Pingirolo, A. Porto, C. Salzilli, C. Saracini, 0. Scaletta, F. Soto, S. Stella, F. Stivori, A. Tarroni, G. Trofea, G. Turini, S. Venturi di Nibbo, L. Viadana, S. Visconti und F. Vitali bekannt.

365. Insbesondere bei den früheren Komponisten wie P. de Monte oder F. Soto ist die Vorzeichenbehandlung recht inkonsequent. Auch ist die Ausdehnung der Gultigkeit von Vorzeichen bei einzelnen Komponisten von Druck zu Druck verschieden. Als besonders konsequente Vertreter dieser Auffassung können hingegen C. Gesualdo, S. D'India, P. Nenna und S. Venturi di Nibbo gelten.

366. Beispiele hierfür im Madrigal "La mia doglia" in P. Nennas Primo libro de madrigali a 4 (1613) oder in großer Zahl im Madrigal "Se la mia morte brami" des sechsten Madrigalbuches von Gesualdo (vgl. Bd.6 der Gesualdo-GA. Dort ist auf S. $12 / 13$ der Anfang dieses Madrigales im Faksimile des Partiturdruckes von 1613 dem Anfang der ubertragung gegenubergestellt. Die genannte Vorzeichenbehandlung kann sehr gut in den beiden Sopranen in Takt 2 (Vg1. Tenor Takt 4) etc. beobachtet werden).

367. Gesualdo-GA, Bd.9. 
Watson) in diesem Band der Gesamtausgabe auf eine Modernisierung der Vorzeichensetzung. Hier kann die Notation von Chromatik durch Fehlen eines weiteren Akzidens 369 und Chromatik durch Auflösungszeichen ${ }^{370}$, die Geltung eines Vorzeichens für mehrere gleiche Noten ${ }^{371}$ sowie Wiederholungen des Vorzeichens vor jeder dieser Noten ${ }^{372}$, ein Akzidens an Stellen "una nota super 1a"373 sowie ein Akzidens an Stellen, wo diese Regel nicht gelten sol1374 und die mehrfache sowie nur einfache Angabe der Erhöhung in der Kadenzklause1 ${ }^{375}$ beobachtet werden.

Wenn auch diese Beispiele eine uneinheitliche, ja widerspriichliche Praxis belegen, so ist in den meisten Fällen doch zweifelsfrei zu erkennen, was gemeint ist ${ }^{376}$.

Neben solchen doch weitgehend pragmatischen Handhabungen der eng begrenzten Gültigkeit der Vorzeichen, gibt es auch Drucke, in denen die beiden Möglichkeiten der Vorzeichensetzung - Gültigkeit nur für eine Note sowie Gültigkeit nur für eine Folge gleicher Noten - nebeneinander in verschiedenen Stiucken vertreten sind. In Giovanni Paolo Cimas Partitio de ricercari (1606) z.B. steht im Hauptteil des Druckes oft, etwa bei einer Kadenzforme1, das Akzidens nur beim ersten Auftreten der erhöhten Note. Im Anhang diese Druckes, der "breue regola per imparare à far prattica di suonare in qual si voglia luoco" hingegen ist die Geltung der Vorzeichen eindeutig auf die direkt folgende Note beschränkt. Nichtwiederholung des Vorzeichens bedeutet hier Auf1ösung ${ }^{377}$. Der Grund für die unterschiedliche Praxis liegt in der größeren

368. Vgl. das Vorwort sowie den Revisionsbericht $z u$ dieser Ausgabe.

369. In $\mathrm{Nr} .5$ ("O Oriens splendor"), in Takt 58 und Takt 61.

370. Ebenda, Takt 55.

371. Ebenda, Takt 53.

372. Ebenda, Takt $40,48 \mathrm{f}, 53$.

373. In Nr.11 ("Veni sponsa Christi"), Takt 20, 21, 24, 28, etc.

374. Ebenda, Takt 9, 27.

375. In Nr.5, mehrfach: Takt $36 \mathrm{f}$, einfach: Takt 21. Die Beispiele lassen sich aus den anderen Sätzen vermehren und verdeutlichen. Die Beschränkung jm wesentlichen auf die $\mathrm{Nr} .5$ sol1 zeigen, wie eng die unterschiedlichen Geltungen der Vorzeichen zusammenstehen (lediglich für die Regel "una nota super ..." wurde auf die Nr.11 zurückgegriffen, da diese dort aus tonalen Gründen häufiger zur Anwendung kommt).

376. Dennoch verbleiben einige Fälle, in denen eine Entscheidung nicht mit Sicherheit zu treffen ist-vgl. z.B. Takt $37 \mathrm{im}$ Tenor von "O Oriens splendor" von Gesualdo (Gesualdo-GA, Bd.9, S.33).

377. Vg1. hierzu das Notenbeispie1 bei Ape1, Klaviermusik, S.409. Auch bei anderen Komponisten kann diese Verwendung unterschiedlicher Regeln für verschiedene Stücke beobachtet werden. Bei tonal einfachen Sätzen gilt dann ein Versetzungszeichen vor mehreren gleichen Noten für alle diese Töne, bei chromatischen Sätzen betrifft das Vorzeichen nur die erste Note (z.B. A. Banchieri, L'organo suonarino, 1/1605, "Sonata settima concerto enarmonico": Vorzeichen nur für eine Note; "Sonata ottava in aria francese": ein Vor- 
Notwendigkeit einer eindeutigen Vorzeichensetzung bei diesen vorzeichenreichen Sätzen ${ }^{378}$.

Es gehört zu den Selbstverständlichkeiten lebendiger Notation, daß alle diese Regeln nur selten konsequent durchgehalten wurden. Oft fehlen in den Drucken auch weiterhin Vorzeichen. Dies ist zum Teil auf Ungenauigkeit der Drucker zurückzuführen, zum Teil aber sicher auch auf immer noch gegenwärtige Regeln einer "musica ficta". Diese wirkte insbesondere dort weiter, wo Vorzeichen zweifelsfrei zu setzen waren, nämlich in den Kadenzen. Oft fehlen die Vorzeichen hier an unmißverständlichen Stellen. So wurde sehr häufig in einer ausgeschriebenen Kadenzdiminution das Akzidens nur beim ersten Ma1, oder aber erst bei der Penultima notiert. In beiden Fällen ist davon auszugehen, daß immer erhöht wurde. Abweichungen zwischen Parallelstellen sowie zwischen Melodiestimmen und Basso continuo bestätigen dies ${ }^{379}$. Die anderen Regeln der "musica ficta" finden kaum noch Anwendung. Nur in den Randstimmen begegnet noch selten der Fall des "fa super 1a".

Eine eigenständige Art der Vorzeichenbehandlung hat Giovanni Maria Trabaci in seinen beiden Ricercar-Buichern (1603 und 1615) entwickelt. Er schreckte offensichtlich vor einem ausgedehnten Gebrauch des "b-quadratum" (4) 380 ebenso zurück, wie vor der Anwendung der Akzidentien b und \# als Auflösungszeichen. In chromatischen Stiicken verwendet er daher Tonbuchstaben, um die Geltung eines Vorzeichens zu beenden ${ }^{81}$. Ein Buchstabe nach einer alterierten Note besagt, daß die folgende Note nicht mehr alteriert sein so11382.

Weitere Sonderzeichen waren sowohl für die Versuche der Wiederbelebung der antiken "genera" als auch in Kompositionen für das "Cimbalo Cromatico"38 3

zeichen auch für mehrere Noten). Diese Inkonsequenz hat hier jedoch keine praktische Bedeutung, da die Lösung bei den einfacheren Sätzen offensichtlich ist.

378. Hier wird ein Stück auf sämtliche Halbtonstufen transponiert. Vor den Stiicken steht jeweils eine Anweisung zum Umstimmen des Instrumentes (vgl. hierzu Rayner, Cima). Auf einige weitere Transpositionsanweisungen, die ebenfa11s in vorzeichenreichere Tonarten führen, wird im Abschnitt III.6. noch hingewiesen werden.

379. Ein Beispiel hierfür findet sich im Notenbeispiel II,7 im zweiten Band dieser Arbeit im Takt 10.

380. Das " $b$-quadratum" beginnt in dieser Zeit sich als Auflösungszeichen durchzusetzen. Viele Komponisten benutzen es jedoch noch gar nicht oder nur beschränkt auf den Ton " $h$ ". Vgl. zur Verwendung des "b-quadratum" im 17. Jahrhundert auch: Brozska, Notationspraxis.

381. Vg1. das Faksimile im Anhang, Notenbeispiel III,12.

382. Erklärung Trabacis im ersten Buch auf S. 91: "Le lettere che stanno per mezo di questa partita, non vol dir altro che leuar lo Semitono da quella nota che segue dopoi la lettera."

383. Hier ist inbesondere wieder Trabaci zu nennen. Im Secondo libro de ricercare steht auf S.88 ff ein "Ricercar' sopra 11 Cimbalo Cromatico". Auf 
bzw. "Archicembalo" notwendig. Hier wurde eine Anzah1 neuer Vorzeichen geschaffen, die oft schon die Formen der Vierteltonschrift des zwanzigsten Jahrhunderts vorwegnehmen. Da diese Experimente aber kaum musikpraktische Bedeutung haben, sollen sie hier nicht im einzelnen behandelt werden.

Zusammenfassend kann zur Vorzeichensetzung im 17.Jahrhundert - wie auch zur Notation insgesamt - gesagt werden, daß das für die Praxis Notwendige den Ausschlag für die Anwendung der einen oder anderen Regel gab. Bei Kenntnis der möglichen Geltungsausdehnungen der Akzidentien sowie der wenigen noch zu erwartenden Anwendungsmöglichkeiten einer "musica ficta" bereitet die Vorzeichensetzung, von wenigen Ausnahmen abgesehen, keine Probleme. Im wesentlichen kann es hier nur bei Chromatik Schwierigkeiten geben.

\section{Transpositionsangaben - "Chiavettenfrage" \\ 6.1. "Chiavette" als Transpositionsanweisung?}

Die Diskussion un die absolute Tonhöhe der Musik des 16. Jahrhunderts und deren Zusammenhang mit den jeweils vorgesetzten Notenschlisseln erhielt ihre ersten Anstöße bereits 1820 durch Raphael Georg Kiesewetter ${ }^{384}$ und wurde insbesondere in den zwanziger und dreiBiger Jahren des 20. Jahrhunderts durch Theodor Kroyer, Robert Haas auf der einen Seite und Richard Ehrmann auf der anderen gefuhrt385. Es ging um die Frage, ob Sätze, die mit einer hohen Schlüsselkombination ("Chiavette") 386 notiert sind, nach unten zu transponieren seien. Während Ehrmann eine solche Transposition grundsätzlich ablehnte, traten Kroyer und Haas dafuir ein, solche Stücke um eine Terz nach unten zu transponieren. Diese Diskussion ging ins Leere, da zum einen die unterschiedlichen Stimmtonhöhen ${ }^{387}$ ein Festlegen einer absoluten Tonhöhe verbieten und zum anderen die Frage nach der erklingenden Tonlage erst durch

S.87 unten werden in einem "A' LETTORI" die besonderen Notenzeichen erklärt und deren Notwendigkeit begründet. Auch den besonderen Gebrauch des Akzidens " $b$ " in A. Banchieris "Sonata settima concerto enharmonico" (aus L'organo suonarino 1605) fuhrt Apel, Klaviermusik, S.407, auf die Gegebenheiten des Archicembalos zurück. Das " $b$ " bedeutet bei Banchieri nach Apel in diesem Stück ebenfalls eine Erhöhung (vgl. das Notenbeispiel bei Ape1). Apel sieht diese Notation in der Tastenaufteilung des Archicembalo begründet. Seiner Meinung nach bedeutet diese Notation, daß die Taste fuir die enharmonische Erhöhung unterhalb der Taste für den nicht erhöhten Ton gelegen haben muß.

384. Vgl. Hans Enge1, Art. Chiavette in MGG, Bd.2, Sp. 1188.

385. Vgl. Ebenda.

386. Der Terminus "Chiavette" ist nicht zeitgenössisch. Diese hohe Schliisselkombination besteht im vierstimmigen Satz in der Regel aus Violin-, Mezzosopran-, Alt und Baritonschliusse1, die "Normalschliusselung" ("Chiave naturale") aus Sopran-, Alt-, Tenor- und Baßschliissel.

387. Gegen die Einbeziehung solcher musikpraktischer Gesichtspunkte hat sich Hermelink, Chiavettenfrage, bes. S.265, gewendet. Hermelink möchte eine Erklärung der Schlüsselungen "innerhalb der Vokalpolyphonie des 16. Jahrhunderts selbst" finden (S.265). 
den Basso continuo und vor allem durch obligate Instrumente an Bedeutung gewann388. Beim a cappella-Vortrag konnte die Tonhöhe ohnehin frei gewäh1t werden389. Eine Tiefertransposition hochgeschlisselter Sätze bot sich in der Regel an, um den Sängern den Vortrag zu erleichtern. Von den Organisten ist bezeugt, daß sie das Transponieren beherrschen mußten ${ }^{390}$. Wurden weitere Instrumente herangezogen, so konnten entweder die für diese anzufertigenden Stimmen gleich transponiert notiert werden, oder aber die Instrumentalisten transponierten ebenfalls die für sie meist unkomplizierten Vokalstimmen ${ }^{391 .}$ Eine Terz als Regeltranspositionsintervall aber ist, zumindest bei Mitwirkung von Instrumenten, recht unwahrscheinlich ${ }^{32}$. Die sich ergebende Vorzeichenhäufung ist weder auf einem mitteltönig gestimmten Tasteninstrument noch auf den meisten der damaligen Blasinstrumente sinnvoll ausführbar ${ }^{393}$. Eine solche Praxis bestätigendes Auffuhrungsmaterial - sofern diese Tranpositionen uberhaupt schriftlich festgehalten wurden - ist aus dem 16.Jahrhundert, abgesehen von einigen transponierten Orgeltabulaturen ${ }^{394}$, nicht erhalten.

Das Aufkommen des Basso continuo - also schriftlich fixierter Orgelstimmen sowie obligater Instrumentalstimmen in den Jahren um 1600 zwang zunehmend zu genauerer Fixierung der Tonhöhe ${ }^{395}$. Dennoch lebte zunächst auch die Transpositionspraxis des 16.Jahrhunderts im 17. Jahrhundert fort. Hel1mut Federhofer hat bereits auf den Zusammenhang von Hochschluisselung und Transpositionsforderungen in Generalbaßstimmen sowie auf die unterschiedliche notierte Tonhöhe in den Vokalstimmen und der Continuostimme in zwei Messen aus Giovanni Valentinis Missae quatuor (1621) hingewiesen396. Federhofer nennt jedoch auch Quellen, die bezeugen, daß Transposition bei hochgeschlüsselten Werken möglich, aber nicht zwingend bzw. sogar unerwiunscht war ${ }^{397}$ und schließt sich

388. Vgl. hierzu vor allem Federhofer, Schlüsselung, S.111.

389. Man vergleiche entsprechende Zitate bei Federhofer, Chiavetten, S.141 (nach J. Cochlaeus, L. Zacconi, M. Praetorius, W. C. Printz; diese Belege ließen sich vermehren).

390. Vg1. hierzu die Zitate bei Parrott, Transposing, $\mathrm{S} .491 \mathrm{ff}$.

391. Vgl. Parrott, S.505 ff, sowie weiter unten im Abschnitt III.6.3.2.

392. Im 17.Jahrhundert ist die Terz als ein mögliches Transpositionsintervall allerdings selten bezeugt (s.u.).

393. Ein aufwendiges Umstimmen, wie es G. P. Cima in seiner Transpositionslehre (Partito de ricercarii ... et in ultimo una breue regola per imparare à far prattica di suonare in qual si voglia luoco, 1606) beschreibt, ist sicherlich nicht immer möglich gewesen. Vgl. hierzu auch Rayner, Cima.

394. Auf solche Orgeltabulaturen bereits wurde oben im Abschnitt II.1.4. hingewiesen.

395. Dies hat schließlich zur Vermehrung der Vorzeichen im Laufe des 17. Jahrhunderts geführt.

396. Federhofer, Chiavetten, S.148.

397. Chiavetten, S.151, und vor allem Schlüsselung, S.106 f. Der Verfasser schließt sich hier der Deutung des oft zitierten Abschnittes aus Thomas Morleys A plaine and easie introduction (1597), abgedruckt und Ubersetzt bei 
Arthur Mendels Fazit an, daß Hochschlüsselung ein Indiz für Transposition sein kann, aber keineswegs immer ist398.

In jüngerer Zeit wurde der Transpositionsfrage im Zusammenhang mit der z.Zt. zum gängigen Konzertrepertoire gehörenden "Marienvesper" C1audio Monteverdis wieder zunehmendes Interesse gewidmet. Besonders Andrew Parrott vertritt hier erneut die Auffassung, daß bei Hochschliusselung grundsätzlich zu transponieren $\operatorname{sei}^{399}$. Ehe im folgenden auf die "Marienvesper" eingegangen wird, soll allgemein der Befund zu dieser Frage in den für diese Arbeit ausgewerteten Musikdrucken dargestel1t werden. Um eine wichtige Voraussetzung zur Interpretation der Quellen zu schaffen, soll zunächst auf die Erweiterung des zur Verfügung stehenden Tonraumes und die sich dadurch ergebenden Konsequenzen für die Schluisselsetzung eingegangen werden.

\subsection{DiE ERWEITERUNG DES TONRAUMES IM SPÄTEN 16, UND FRÜHEN 17, JAHRHUNDERT}

Bereits gegen Ende des 16.Jahrhunderts vergröberte sich der von den Komponisten eingesetzte Tonraum 400 . Ausgelöst wurde diese Entwicklung sicher durch die mehrchörige Musik. Der Stil der mehrchörigen Werke ist im späten 16.Jahrhundert und vor allem im 17. Jahrhundert zunehmend verfeinert worden. Sowohl eine Zunahme der Stimmenzah1401 als auch eine sehr viel differenziertere Anwendung der Gestaltungsmöglichkeiten in der mehrchörigen Musik ist zu beobachten. Die Chöre sind nicht mehr gleich besetzt, sondern es gibt Hochchöre, Tiefchöre, Favoritchöre und Capellchöre - z.T. sogar "Chöre" mit nur einem Solisten und Generalbaß; unterschiedliche instrumental-vokale Besetzungen kamen hinzu. An die Stelle des bloßen abwechselnden Musizierens der Chöre - freilich wurde dieses wesentliche Element mehrchöriger Musik nicht aufgegeben - traten nun auch zahlreiche Tutti-Blöcke, nicht mehr, wie bisher, nur an den Satzenden. Es entwickelte sich ein ausgesprochener "Prachtsti1"402. Sowohl der Wunsch nach Kontrastierung (Hochchor, Tiefchor)

Hermelink, Chiavettenfrage, S.265 ff, durch Arthur Mendel und Hellmut Federhofer (Schliusselung, S.106 f) an.

398. Federhofer, Chiavetten, S.151.

399. Parrott, Transposing, S.491: "In this article I shall try to show that 'obligatory transposing' is implicit in the notation of much vocal music of the late 16th and early 17 th centuries".

400. Dies zeigt sich in der Musiktheorie sehr schön in der Hinzufügung einer elften Linie zur "scala decemlinealis" bei A. Gumpelzhaimer (Compendium musicae, erste Auflage 1597) - vgl. Federhofer, Schlüsselung, S.108.

401. Eine Ausnahmestellung hatten in der Entwicklung der Mehrchörigkeit sicher die vierzigstimmigen Kompositionen von A. Striggio (1568 - s.o) und, wahrscheinlich etwas fruher, T. Tallis (vgl. Denis Stevens, Art. Tallis, Thomas in MGG, Bd.13, Sp.68). Erst in den Jahren um und nach 1600 erschienen jedoch 12, 14 etc. bis 24 stimmige Stücke in größerer Zahl.

402. Zahlreiche Beispiele für diesen "Prachtstil" finden sich bereits in G. Gabrielis postum erschienen Sacrae symphoniae ... liber secundus (1615; vgl. Giovanni Gabrieli-GA, Bd.5). 
als auch die große Zahl der Stimmen, die in Tutti-Blöcken auf engem Raum ohne allzuviele Einklangsparallelen zusammenzubringen waren, haben zwangsläufig zu einer Ausdehnung des Gesamtambitus gefuhrt. Kam man um die Mitte des 16. Jahrhunderts noch mit einem Ambitus von 19 oder 20 diatonischen Tönen aus403, so wurde dieser bereits in den letzten Jahren des 16.Jahrhunderts in der mehrchörigen Musik auf 27 diatonische Töne erweitert404. In der mehrchörigen Musik der ersten zwei Jahrzehnte des 17. Jahrhunderts kann bereits ein Tonumfang von 28 diatonischen Tönen in vokaler Musik bzw. von 30 Tönen in instrumentaler Musik beobachtet werden405. Ganz selbstverständlich bedeutet dies, daß alle zur Verfügung stehenden Schliussel verwendet werden musten 406 ; oft kommen dabei die bekannten Standardschliusselkombinationen in den verschiedenen Chören solcher Kompositionen gleichzeitig vor.

Eine Transposition aufgrund der Schlüsselungen in Musik mit Hoch- und Tiefchören verbietet sich natürlich von selbst. Das offensichtliche Vorhandensein solcher extremen Lagen schließt den Tonumfang allein als Argument für die Transposition aus. Es war, wie Thomas Morley beschrieb407, dem Komponisten überlassen, für fröhliche Stücke eine höhere Tonlage, für traurige eine tiefere zu wählen 408 .

403. Vg1. Hermelink, Chiavettenfrage, S.269. Geschlossen wird dieser Tonumfang aus der "scala decemlinealis". Ganz sicher ist dies aber auch im 16. Jahrhundert allenfalls ein "Regelumfang", an den sich der Kompositionsschuller zu halten hat. uber- und Unterschreitungen waren freilich möglich (erinnert sei an die mehrfach in der Chiavetten-Debatte angefuhrte Motette "De profundis" von Josquin, deren Transposition nach oben Glarean trotz der tiefen Lage ausdricklich verbot (Federhofer, Schlüsselung, S.107 u.a.)).

404. Bereits die Motette "Deus meus" von G. Gabrieli aus den Concerti di Andrea, et di Gio: Gabrieli (1587, Giovanni Gabrieli-GA, Bd.1, S.18) erstreckt sich uber einen Tonumfang von C - a''.

405. Die Töne Kontra B bis a" verwendete G. Gabrieli in einem Magnificat in Sacrae symphoniae ... liber secundus (1615, Giovanni Gabrieli-GA, Bd. 5, S.56). Eine Ersetzung von Singstimmen in extremer Lage durch Instrumente war freilich möglich (siehe oben). Ebenfalls 1615 erschienen Giovanni Gabrielis Canzoni e sonate. Der Tonumfang der Canzone XV reicht vom D bis zum d', (vgl. Giovanni Gabrieli, Canzoni e sonate, hrsg. von Michel Sanvoisin, S.137 ff). Ein e'"' wird in der Canzone $X$ verlangt, jedoch innerhalb eines hochgeschliusselten Satzes, für den Parrott eine Transposition fordern würde. Einen Tonumfang C bis d'' hat die Canzone prima a 12 aus G. Priulis Sacrorum concentuum ... pars altera (1619).

406. Vgl. die Vorsätze in den Neuausgaben der erwähnten Komposition von G. Gabrieli.

407. Abdruck der entsprechenden Stelle bei Hermelink, Chiavettenfrage,

S.265 ff. Zur Deutung dieser Stellen vgl. Federhofer, Schlüsselung.

408. "the high key be made for more life, the other in the low key with more gravitie and stadiness", zitiert nach Hermelink, Chiavettenfrage, S. 266 . 


\subsection{VORGESCHRIEBENE ODER BEREITS AUSGEFÜHRTE TRANSPOSITION IN BC-STIMMEN}

War eine Transposition im 17.Jahrhundert erwünscht, so konnte sie entweder verbal vorgeschrieben werden oder aber in den auf eine absolute Tonhöhe angewiesenen Instrumentalstimmen bereits ausgeführt sein. Es wurde weiter oben bereits darauf hingewiesen, daß verschiedene Begleittabulaturen gegeniber den Vokalstimmen transponiert sind 409 . Auch in einer ganzen Reihe von Basso continuo-Stimmen aus dem frühen 17. Jahrhundert sind einzelne (in einem Fall sogar alle) Sätze in einer tieferen Tonlage als die Vokalstimmen notiert.

\subsubsection{TRANSPONIERTE ORGELSTIMMEN}

Die ersten dem Verfasser bekannten Beispiele für solche, gegenüber den Vokalstimmen bereits transponierte Orgelstimmen sind in Lodovico Viadanas Cento concerti ecclesiastici (1602) enthalten. Transponiert ist die Orge1stimme immer dann, wenn die Singstimme(n) in den zur "Chiavette" gehörenden Schluisseln notiert sind (Sopran im Violin-, Alt im Mezzosopran-, Tenor im Alt- und Baß im Baritonschlüsse1) 410 . Nur in einem Satz, in dem ein solcher Schluissel verwendet wurde - es handelt sich um ein Stück für BaBsolo im Baritonschlüsse1 - ist der Basso continuo nicht transponiert 411 . Hier wurde der Baritonschliissel offenbar verwendet, um den großen Tonumfang dieser Baßstimme besser darstellen zu können412. Sie unterschreitet den Tonumfang der anderen Stimmen im Baritonschliussel deutlich413. Eine Transposition wäre hier unangebracht und ist von Viadana daher auch nicht vorgenommen worden ${ }^{44}$. Bei einem weiteren Satz für zwei Bässe ist offenbar an eine Transposition des Continuos nach oben (statt der Singstimmen nach unten) gedacht worden: uber der Continuo-Stimme steht: "I Cantori la Cantano Alla quarta Alta" 415 .

Transponierte Orgelstimmen für einzelne Stïcke finden sich auch in einigen Drucken Adriano Banchieris416, Giulio Bellis417 - diese sind mit "trasporta-

409. Vgl. oben im Abschnitt II.1.1. Solche Transpositionen der Tabulaturen sind in den Drucken S. Verovios, im ersten Madrigalbuch S. Rossis (vgl. Vorsatz zum Notenbeispiel II, 1 im zweiten Band dieser Arbeit) und auch in der Handschrift 704 der Bibliothéque du Conservatoire, Briissel enthalten (vg1. zu letzterer auch Carter, Caccini, S.211).

410. Insgesamt sind 13 der hundert Concerti in der Orgelstimme transponiert.

411. "Super flumina".

412. Der Tonumfang der Baßstimme erstreckt sich hier von $F$ bis $d^{\prime}$.

413. Im den anderen in Baritonschliusseln notierten Sätzen wird das c nicht unterschritten.

414. Schon dies beweist, daß die These von der 'obligatory transposing' nicht zutreffend ist.

415. "Muro 1uo".

416. Ecclesiastiche sinfonie (1607). Auf den Sachverhalt in diesem Druck wird weiter unten noch einzugehen sein. 
to alla quarta bassa" bzw. "trasportato alla quinta bassa" gekennzeichnet -, Giovanni Felice Anerios418, Giovanni Ghizzolos419 - hier werden für einige Stïcke sogar zwei verschiedene Transpositionen angeboten ("alla quarta bassa" und "alla quinta bassa")420 - und Giovanni Valentinis421.

In allen genannten Drucken sind die in der Orgelstimme transponierten Stücke in den Vokalstimmen in einer "Chiavette" notiert. Nur in den Missae quatuor Valentinis und dem terzo libro delli concerti Ghizzolos aber sind alle in einer "Chiavette" notierten Sätze auch in den Orgelstimmen transponiert wiedergegeben. Bei Anerio ersetzt bei manchen Stücken eine Transpositionsanweisung die Transposition422, in den Drucken Bellis gibt es ebenfalls Transpositionsanweisungen 423 , aber auch eine Anzahl von hochgeschliusselten Sätzen ohne einen Hinweis auf eine Transposition. Die bereits durchgefuhrten Transpositionen erfolgten (mit Ausnahme der in zwei Transpositionen gegebenen Sätze Ghizzolos) in die Unterquinte, wenn die Vokalstimmen ohne Vorzeichen notiert sind (im Continuo sind sie dann mit einem b versehen), und in die Unterquarte, falls in den Vokalstimmen ein b vorgezeichnet ist (dies entfällt dann in der Continuo-Stimme).

Adriano Banchieri macht die Transposition in seinen Ecclesiastiche sinfonie (1607) von der Besetzung dieser zumeist sowohl für rein vokalen als auch rein instrumentalen Vortrag bestimmten Sätze abhängig. Die nur vokal auszuführenden Stücke sind in "Normalschluisselung" notiert und enthalten keinerlei Transpositionsanweisung 424 . Eine Sinfonie ist nur für Instrumente gedacht 4\%5. Auch für sie gibt es keinen Hinweis auf eine Transposition, obwohl

417. Compieta, falsi bordoni, antifone et litanie (1607), Missae sacrae (1608) und Concerti ecclesiastici (1613).

418. Antiphonae, seu sacrae cantiones (1613, nach Parrott, Transposing, S.496).

419. Il terzo libro delli concerti (1615, 2/1623).

420. Ghizzolo bietet für drei Sätze den Basso continuo in zwei verschiedenen Transpositionen, für vier weitere Sätze nur eine Continuo-Stimme in der Unterquinte und fiir einen nur in der Unterquarte an. Das jeweilige Transpositionsintervall ist auch verbal angegeben ("Trasportato alla ..."). Eine sich durch die Transposition ergebende b-Vorzeichnung steht zu Anfang des Satzes, eine Keuz-Vorzeichnung nur vor den jeweiligen Noten (vgl. hierzu auch weiter unten).

421. Missae quatuor (1621). Hierauf wurde bereits von Federhofer, Chiavetten, S.148 f, hingewiesen. Auf eine ganze Anzahl transponierter Orgelstimmen (zumeist ausgeschrieben) in englischen Quellen geht Clark, Transposing ein. Er sieht den Grund fur diese Transpositionen in den damaligen englischen Orgelstimmungen (10' statt $8^{\prime}$ Pfeifen (S.9)).

422. Parrott, Transposing, S.498.

423. In Bellis Missae sacrae (1608) ist auch eine in "Normalschluisselung" notierte Messe mit der Anweisung "In tuono, Overo vno Tono piu Basso" versehen - vgl. hierzu weiter unten im Abschnitt III.6.3.2.

424. Es sind dies die "Concerti" und die 14. Sinfonia. 
sie in einer "Chiavette" steht. Nicht transponiert werden sollen schließlich auch fünf der insgesamt zwölf für Instrumente oder Stimmen gedachten Werke, gleich wie sie besetzt sind426. Die ubrigen Sinfonien sind jeweils für die Sänger tief und für die Instrumentalisten hoch aufzufuhren. Vier dieser Stücke sind hochgeschlüsselt und bei vokaler Ausfuhrung nach unten zu transponieren 427 - im Seguente sind sie bereits transponiert notiert -, drei weitere stehen in einer "Normalschlüsselung" und sind für instrumentale Aufführungen nach oben zu transponieren ${ }^{428}$. Eine verbale Angabe weist in der Seguente-Stimme auf die jeweils vorzunehmenden Transpositionen hin ${ }^{29}$.

Das späteste dem Verfasser bekannte Beispiel für eine transponierte Continuo-Stimme in einem italienishen Musikdruck ist die Seguente-Stimme der Neuauflage von Adriano Banchieris Barca di Venetia per Padoua von 1623430 . Hierbei handelt es sich um einen nachträglich mit einem Basso continuo versehenen Druck. In der Auflage von 1605 (sie hat dieselbe (Hoch-) Schluisselung wie diejenige von 1623) fehlt sowohl eine Generalbaßstimme als auch irgendein Transpositionshinweis - die Sänger konnten sich bei solcher zweifellos a cappella vorzutragenden Musik eine bequeme Lage aussuchen. Das Hinzufugen eines Generalbasses zwang in der Ausgabe von $1623 \mathrm{zu}$ einer Festlegung auf eine Tonlage. Die Seguente-Stimme steht durchgängig um eine Quarte tiefer als die ubrigen Stimmen. Da die Barca in den Singstimmen ohne Vorzeichen notiert ist, ergibt sich für die Continuo-stimme die bereits bei Ghizzolo zu beobachtende Transposition in eine, die Vorzeichnung eines Kreuzes verlangende Tonlage. Im Gegensatz zu den Beispielen Ghizzolos stehen die Kreuze hier nicht vor den jeweiligen Noten, sondern als Generalvorzeichnung vor den Stuicken ${ }^{41}$. Die Basso continuo-Stimme zu Adriano Banchieris

\section{Sinfonia 13.}

426. Die Sinfonie 5 - 9 .

427. Sinfonie $1-4$.

428. Sinfonie $10-12$.

429. Sie lautet bei den Sinfonie 1 und 4 "Trasportato alle quinta per le voci" bzw. "Trasportato alla quinta per cantare" bei den Sinfonie 2 und 3, bei den Sinfonie 10 - 12 steht hingegen "In tuono per cantare, \& una quarta superiore per gli stromenti" (zitiert nach $\mathrm{Nr} .10$, die anderen Angaben weichen leicht ab (statt "per cantare": "alle voci" bzw. "per voci")). Bei den funf in keinem Fall zu transponierenden Sinfonie steht: "In tuono per voci \& stromenti".

430. Die Ausgabe von 1623 bildete die Grundlage für die Neuausgabe von Elio Piattelli. Alle im folgenden gemachten Angaben zur Ausgabe von 1623 beziehen sich auf die Vorsätze sowie den Editionsbericht dieser Ausgabe.

431. M. Praetorius bezeichnet die Transposition eines ohne Vorzeichen notierten Satzes in die Unterquart im Vergleich zu der ublichen in die Unterquinte als "frischer", räumt jedoch ein, daß die Organisten dies nicht gewohnt seien (vgl. Syntagmatis musici ... tomus tertius (1619), S. $80 \mathrm{ff}$, sowie einen bei Parrott, Transposing, S.507, abgedruckten Ausschnitt aus Praetorius' Vorbemerkung zur Nr.15 der Polyhymnia caduceatrix (1619)). Ebenfalls in eine Tonart mit einem Kreuz ist die Basso continuo-Stimme zu dem 
Barca ist daruber hinaus mit dem Vermerk "alla quarta" versehen. Dieser Transpositionsvermerk ist hier ganz offensichtlich nicht vorschreibend 432 , sondern erklärend gemeint 433 .

\subsubsection{VERBALE TRANSPOSITIONSANWEISUNGEN}

Sehr viel häufiger als bereits transponierte Generalba日stimmen sind verbale Transpositionsanweisungen anzutreffen. Auch sie stehen zumeist in den Continuo-Stimmen. Es muß dabei zunächst zwischen zwei verschiedenen Arten der Transpositionsanweisung unterschieden werden. Ein Teil dieser Transpositionsanweisungen verstehen sich nämlich als zusätzliche Hilfe zur Ausführung von Alternativbesetzungsangaben. Neben dem häufigen "Canto o Tenore a11' ottava" wurden hierbei auch andere Transpositionsintervalle vorgeschlagen, z.B. "Canto, Alto, e Tenore O alla quinta Bassa. Alto T. e B."434, "Soprano solo, o Tenore alla ottaua, \& Alto alla quinta"435 oder "Doi Alti, o Soprani alzato alla 4 over Tenori alle 5"436. Auch für instrumentale und vokale Ausführungen konnten zwei verschiedene Tonlagen angegeben werden 437 .

Psalm "Nun lob mein Seel den Herren" aus den Psalmen Davids von H. Schütz transponiert (1619, SWV 41. Vg1. hierzu Schiitz-GA, Bd.3, S.X). Interessanterweise sind die beiden instrumentalen Cape11-Chöre hier ebenfalls bereits transponiert notiert. Solche transponierten Stimmen für Melodieinstrumente sind dem Verfasser aus dieser Zeit aus italienischen Quellen nicht bekannt. Parrott, Transposing, S.505, erwähnt eine Sammlung G. A. Rigattis (Messe e salmi, 1640) mit gegeniber den Vokalstimmen und den verbleibenden Instrumentalstimmen transponierten Violinstimmen.

432. Es wäre ohne Zweifel sinnlos, den Basso continuo eine Quarte tiefer zu notieren, damit der Generalbaßspieler ihn wieder zurücktransponiert (dies ist in der Neuausgabe geschehen!).

433. Es ist ein "trasportato" zu ergänzen. Solche erklärenden Transpositionsangaben konnten auch in anderen Continuo-Stimmen beobachtet werden (vg1. oben).

434. G. N. Mezzogorri, La celeste sposa (1613).

435. T. Massaino, Musica per cantare (1607).

436. B. Tomasi, Il primo libro de sacri fiori (1611). Xhnliche Angaben finden sich auch in G. B. Riccios primo libro delle divini lodi (1612) und A. Grandis secondo und terzo libro de motetti a 2, 3 et $4(1613,2 / 1617$ bzw. $1614,2 / 1618)$. Eine Alternativangabe "Flautini ouero Cornetti alla quarta" findet sich in B. Marinis Sonate (1629).

437. Auf diese Fälle in A. Banchieris Ecclesiastiche sinfonie (1607) wurde oben bereits eingegangen. Eine ähnliche Alternativangabe steht bei den beiden im Violinschlüssel notierten Motetten für Sopransolo aus L. Viadanas Cento concerti ecclesiastici (1602). Sie sind nach Angabe Viadanas auch auf dem Zink zu spielen, allerdings in der, in der Sopranstimme notierten Tonhöhe. Der Organist muß dann also die gegenuber der Singstimme transponierte Orgelstimme um eine Quarte nach oben transponieren. Um dem Organisten dies $\mathrm{zu}$ erleichtern, ließ Viadana unten auf den entsprechenden Seiten den Anfang des Basses in nach oben transponierter Form abdrucken. Dabei steht die 
Solche Transpositionen je nach Besetzung konnten nicht nur verbal angezeigt werden. Weitere Möglichkeiten sind die Doppelschluisselung (vor den Stimmen stehen jeweils zwei verschiedene Notenschliusse1)438 oder die Doppeledition in der Continuo-Stimme439.

Diese besetzungsabhängigen Transpositionsangaben beruhen freilich nicht auf einer Tradition der "Chiavettentransposition". An einen Zusammenhang mit dieser Tradition ist jedoch bei der zweiten Art der Transpositionsanweisungen zu denken. Diese Transpositionsangaben haben unabhängig von einer Besetzungsänderung Gültigkeit. Sie weisen den Organisten an, ein Musikstück grundsätzlich zu transponieren. Verlangt werden erneut Transpositionen in die Unterquart, Unterquint oder auch alternativ "alla quarta o quinta bassa". In den Singstimmen sind diese Stücke zumeist in einer "Chiavette" notiert. Die frühesten dem Verfasser bekannten Beispiele für solche Transpositionsangaben finden sich bereits in einer der ersten gedruckten Genera1baßstimmen uberhaupt, nämlich in dem Basso principale zu Orfeo Vecchis Psalmi integri (1598). In den folgenden Jahren erschien eine ganze Reihe vor Continuo-Stimmen mit solchen Transpositionsanweisungen.

Diese ausdrücklichen Transpositionsanweisungen belegen bereits, daß es sich hierbei nicht um eine Selbstverständlichkeit handelte. Daß diese Transpositionspraxis bereits im "Aussterben" war, beweist die stark abnehmende Häufigkeit, mit der Transpositionsangaben in den Quellen des frihen 17.Jahrhunderts auftreten. Ihre größte Verbreitung hatten diese Angaben im ersten Jahrzehnt des 17. Jahrhunderts440. In den ersten Continuo-Stimmen aus dem

Transpositionsanweisung "Sonando questo Concerto $\mathrm{co}^{\prime} 1$ Cornetto 1 'Organista Sonara la quarta alto cosi" (vgl. das Faksimile einer dieser Seiten aus dem Basso continuo-Stimmbuch der vierten Auflage von 1605 in der Neuausgabe, hrsg. von Claudio Gallico).

438. Solche Doppelschlisselungen sind dem Verfasser bekannt aus 0 . Scalettas Certa spirituale (1605) und G. P. Cimas Concerti ecclesiastici (1610). In diesen beiden Drucken wird bei den Doppelschlüsselungen auch die sich andernde Vorzeichnung bedacht: Der Schluissel in der mit einem b zu versehenden Tonlage steht (mit dem dazugehörigen b) voran, der andere folgt (vg1. Notenbeispiel III,13 im zweiten Band dieser Arbeit). Eine ähnliche Doppelschlüsselung ist in der Symphonia à doi Fagotti, overo Tromboni alla quinta sopra aus J. E. Kindermanns Deliciae studiosorum ... Dritter Theil (1647) notiert (Hier fehlt das für die transponierte Fassung notwendige Vorzeichen (ein Kreuz) - vgl. die vom Verfasser herausgegebene Neuausgabe. In den Stimmen wurde hier die Notation mit Doppelschliusselung beibehalten). Zwei weitere Beispiele von Doppelschluisselung nennt Federhofer, Chiavetten, S.142 ff. Diese Fälle sind jedoch mit den genannten nur bedingt vergleichbar (vgl. bei Federhofer).

439. Dies ist dem Verfasser nur aus F. Turinis Motetti a voce sola (1629) bekannt. Während in der Solostimme nur die Transpositionsangaben stehen, sind die Transpositionen in der Partitura vollständig ausgeführt und die Baßstimmfuhrung ist hier und da ein wenig der neuen Lage angepaßt. 
späten 16.Jahrhundert konnten solche Angaben vielleicht noch fehlen, da die Möglichkeit der Transposition noch weitgehend bekannt war. Im frihen 17. Jahrhundert erschienen diese Angaben zunächst vermehrt. Entsprechend der insgesamt genauer werdenden Notation 441 konnte nicht mehr davon ausgegangen werden, daß die Organisten von selbst transponieren würden. Aber diese Transpositionsangaben werden im Zuge der weiteren Konkretisierung der Notation nicht mehr, sondern weniger442. Dies kann nur darauf zurlickzufuhren sein, daß die Komponisten vermehrt auch die erklingende Tonhöhe zu einem festen Bestandteil der Notation machten. Wie auch in den anderen Gebieten der Notation ist aber nicht mit einer einheitlichen Praxis insgesamt zu rechnen. Vereinzelt gibt es auch aus späterer Zeit noch Belege fü eine transponierende Auffuihrungsweise ${ }^{443}$. Der Uneinheitlichkeit der Notationspra$\mathrm{xis}$ ist es auch zuzuschreiben, daß gelegentlich Transpositionsverbote notiert wurden 444 .

440. In etwa $12 \%$ der ausgewerteten Motetten- und Messdrucke dieser Jahre sind Transpositionsangaben zu finden.

441. Vgl, oben.

442. In nur noch etwa $9 \%$ der ausgewerteten Motetten- und Messdrucke des zweiten Jahrzehnts und in nur etwa $2 \%$ derjenigen des dritten des 17.Jahrhunderts sind Transpositionsanweisungen vertreten.

443. Vg1. Parrott, Transposing, S.497 und $500 \mathrm{ff}$.

444. Dies ist ganz deutlich bei P. Signorucci, I1 secondo libro de'concerti ecclesiastici (1608). Außer einigen Transpositionsanweisungen enthält die Continuo-Stimme auch einmal die Anweisung "in Tuono". Parrott hat darauf hingewiesen, daß auch "come stà" ein Transpositionsverbot meinen können. Dies ist wahrscheinlich bei G. F. Anerio der Fall (Motectorum ... liber secundus (1611, 2/1612), Responsoria nativitatis Domini (1614, 2/1629) und La bela clori armonica (1619), ein weiteres Beispiel bei Parrott, Transposing, S.498). Hier tritt die "come stä"-Anweisung nur im Basso continuo auf, wern der Continuo in einem höheren Schliussel (Tenor oder Bariton) notiert ist, ohne daß es sich dabei um eine "Chiavette" handelt; dies ist zum Beispiel der Fall bei einem Satz für Sopran und Tenor, bei dem der BaB als Seguente gefuhrt ist. Konsequent sind die Anweisungen allerdings nicht verwendet worden. Ebenfalls gegen fälschliche Transposition richten sich wohl die "come stà"-Anweisungen bei S. Anagnino, Nova sacra cantica Dei ... liber secundus (1619) und G. Allegri, Motecta (1612). Eine besondere Art des Transpositionsverbots ist in S. Rossis primo libro delle sinfonie (1607) zu finden. Hier steht bei drei in einer "Chiavette" notierten Sätzen "Va sonata alla quarta alta". Eine Hochtransposition dieser ohnehin schon hohen Sätze ist kaum denkbar. Wie auch Parrott, Transposing, S.506, vermutete, soll diese Angabe eine Tiefertransposition verhindern. Einen Sinn hat diese Angabe freilich nur in der Continuo-Stimme (notiert im Baritonschluisse1). In den Violin- bzw. Zinkstimmen ist eine Notation im Violinschlissel ohnehin iblich. Sicher ist diese Angabe in diesen Stimmen versehentlich stehengeblieben (ein Hinweis auf eine Partitur als Druckvorlage!). Nur bei einem, in den Diskantstimmen tatsächlich sehr exponiertem Satz (Spitzenton hier d"' - sonst nur b") ist der Baß im Baritonschlüssel geschrieben, ohne daß 
Neben diesen Transpositionsanweisungen in die Unterquarte oder Unterquinte finden sich in den Generalbaßstimmen des fruhen 17. Jahrhunderts aber auch Anweisungen, in ein anderes Intervall zu transponieren, nämlich um eine Sekunde (aufwärts wie abwärts)445, eine Terz abwärts oder aber alternativ eine Terz oder Quart abwärts446. Diese Transpositionsangaben sind jedoch unabhängig von einer bestimmten Schliusselung und verfolgen zumeist den Zweck, Vorzeichenhäufungen zu vermeiden.

Eine solche Transposition um ein kleines Intervall wird daruber hinaus in einigen Continuo-Stimmen nicht mehr vorgeschrieben, sondern nur noch vorgeschlagen (z.B. "In Tono, Ouero uno Tono più basso"447). Auch hier handelt es sich meist um Sekundtranspositionen ${ }^{448}$, in einem Fall steht jedoch auch eine Transposition in die Unterquarte zur Auswahl449. Am Rande sei hier auf die weiter oben ausfühlicher behandelte Transpositionsanweisung mittels Schwärzung in einer Motette Pandolpho Zalamellas hingewiesen. Auch hier ist an eine Sekundtransposition zu denken ${ }^{40}$.

Nur sehr wenige Transpositionsanweisungen sind in den Quellen der Instrunentalmusik enthalten. Eine Freiheit in der Wahl der Tonlage, vergleichbar der Situation in der a cappella-Musik, hat es hier nie geben können. Es wurde oben bereits darauf hingewiesen, daß die Instrumentalisten dennoch in der Lage sein mubten, zu transponieren, um auch im Falle einer transponierenden

die erwähnte Anweisung notiert ist. Hier ist eine Tiefertransposition zumindest möglich.

445. Abwärts: G. Croce, Basso' per sonare nell'organo delli vesperi (1610), S. Bernardi, Motecta (1610), A. Banchieri, Terzo libro di nuovi pensieri (1613, "Un tuon tuono basso per commodità di Choro"); aufwärts: C. Monteverdi, L'Orfeo (1609). Hier steht eine Transpositionsangabe bei der Toccata (sie macht lediglich darauf aufmerksam, daß man bei Verwendung von gedämpften Trompeten (die damaligen Trompetendämpfer erhöhen die Stimmung) einen Ton höher spielen sol1) sowie ein weiteres Mal im Verlauf der Oper (S.76: "Vn spirito del cor. Vn tuono piu alto"). Diese letzte Transpositionsangabe ist tonal sehr interessant. Nach modernem Tonartenverständnis hätte dieser Satz auch einen Ton höher ohne Vorzeichen notiert werden können. Monteverdi umgeht mit dieser Transpositionsangabe die Anerkennung des Aeolischen. Er notiert diesen eigentlich aeolischen Abschnitt in G dorisch mit häufiger Vorzeichnung des "es". Hätte er ihn in dieser Art und Weise in der gemeinten Tönhöhe (auf A) notieren wollen, hätte er ein Kreuz vorzeichnen und dann stets vor den Tönen auflösen missen. Dieses Kreuz jedoch wurde, wie bereits mehrfach festgestellt, nach Möglichkeit umgangen).

446. Beides in G. Croces, Basso per sonare nell'organo delli vesperi (1610).

447. Nach G. Belli, Missae sacrae (1608).

448. P. Signorucci, Il secondo libro de' concerti eclesiastici (1608), G. Belli, Missae sacrae (1608) und E. Porta, Sacro convitio musicale (1620).

449. In G. B. Riccios secondo libro delle divine lodi (1614).

450. Vgl. Abschnitt I.3.5.2. dieser Arbeit. 
Auffuhrung Chorstimmen verdoppeln oder ersetzen zu können. Transpositionsgrifftabellen für Violenensemble sowie für Zink-Posaunenensemble sind in Aurelio Virgilianos drittem Buch des dolcimelo (ms um 1600) enthalten 451. Diesen Transpositionstabellen geht eine Darstellung der "Segni per conoscer tutti $i$. Modi da sonar qualsivoglia Instru:to"452 voraus. Hier nennt Virgiliano für die Sopraninstrumente (für die anderen Instrumente war es sicher geplant, ist jedoch nicht mehr vollendet worden ${ }^{43}$ ) $\mathrm{zu}$ den verschiedenen Schluisseln, mit $b$ und ohne $b$ jeweils eine Transpositionsanweisung ${ }^{454}$. Dies reicht von "In tuono" bis zur Terz tiefer für den Sopranschlüssel und von der Sekunde tiefer bis zur Quint tiefer für den Violinschlüssel. Auch in den erwähnten Transpositionsgrifftabellen wird zwischen hoher und tiefer Schlüsselung unterschieden. Für die tiefe Schlïsselung sieht Virgiliano das Spiel "Vn tūo piu alto"455, "In Tuono", "Vn Tūo piu basso"456 sowie "Alla Terza" vor, für die hohe Schlusselung ("Chiavette") aber "Alla Quarta", "Alla Quinta", "A11a Sesta" und sogar "Alla Settima" (jeweils "bassa")45?. Auch bei seinen Grifftabellen für die einzelnen Instrumente gibt Virgiliano verschiedene Transpositionshilfen 458 . Nicht nur die Vielzahl der Transpositionsmöglichkeiten, sondern auch die unterschiedlichen Transpositionsintervalle für Stlucke mit und ohne Vorzeichnung eines b, belegen, daß es sich hier um ein Angebot von verschiedenen Möglichkeiten handelt. Mit den nur in die Unterquarte- bzw. Unterquinte vorgenommenen Transpositionen in den Orgelstimmen hat dies nicht viel gemein. Auch fehlt eine Bindung an die "Chiavette", denn hier wird aus allen Schlïsselkombinationen transponiert.

Diese Transpositionsgrifftabellen dienen sicher, wie oben bereits angedeutet, mehr der Chorbegleitung, denn der reinen Instrumentalmusik. In den Instrumentalstimmen des frühen 17. Jahrhunderts entsprechen die notierten Tonlagen in der Regel sehr genau der Tonumfängen der Instrumente ${ }^{49}$. Trans-

451. S. (98) f und S. (102) f.

452. S. (97).

453. Zur Unvollständigkeit dieses Werkes vgl. oben im Abschnitt III.2.1.1.

454. Im einzelnen: Sopranschlüssel ohne Vorzeichen: "In Tuono", Sopranschlüssel mit b: "Vn Tuō piu bass. Et Alla 3a: bassa.", Mezzosopranschluissel mit b: "Vn tūo piu alto.", Violinschluissel ohne Vorzeichen: "Alla 5a: bassa." und Violinschlüsel mit b: "Vn tūo piu basso Et Alla 5a: Bassa.".

455. Beim Violenensemble mit "commodissimo" bezeichnet.

456. Beim Violenensemble mit "scommodisso." bezeichnet.

457. Diese Transpositionen lassen sich auf den Blasinstrumenten aber z.T. nicht vollständig ausführen.

458. Beim Zink (S.(105)) für den Sopranschlüssel: eine Terz tiefer, eine Sekunde tiefer, im Ton, ein Ton höher und eine Quint höher, im Violinschlüssel: eine Sept tiefer, eine Sext tiefer, eine Quint tiefer, eine Quart tiefer, im Ton und eine Sekunde höher. Ahnliches nennt Virgoiliano auch zur Traversa (S.(109)) und Blockflöte (S.(111)). Dies bezeichnet jedoch woh1 eher das Denkbare oder Mögliche, als das ständig Verwendete (deut1ich auch in der Hochtransposition aus dem Violinschliusse1). Vg1. hierzu auch Parrott, Transposing, S.505 f. 
positionsvorschriften sind in der reinen Instrumentalmusik mit Ausnahme einer Sonate Giovanni Gabrielis auf die unten noch einzugehen sein wird, nur in Innocentio Vivarinos primo libro de motetti ... con otto sonate (1620) hier handelt es sich um eine Alternativtranspositionsangabe 460 - Biagio Marinis Sonate (1629) - ebenfalls für eine Alternativbesetzung 461 - und in Salomone Rossis drittem Buch der varie sonate (1613 (?), 2/1623) 462 notiert worden. Bei Rossi trägt der einzige in den Diskantstimmen das b' 'uberschreitende Satz, die "Gagliarda Prima detta la Turca", die Transpositionsanweisung "va sonata a la quarta bassa"463. Unklar formuliert ist eine zweite Transpositionsangabe dieses Druckes: Die "Gagliarda Seconda detta l'incognita" ist uberschrieben mit "va sonata una terza piu bassa di quello si sona all'alta". Wahrscheinlich meint Rossi damit eine Terztransposition nach unten ${ }^{464}$ und möchte mit dem Zusatz unterstreichen, daß von der notierten Tonhöhe, und nicht von einer bereits um eine Quarte erniedrigten Lage ausgegangen werden sol1465. Diese beiden Sätze sind als einzige im Baß in einem zur "Chiavette" gehörenden Schlüssel, nämlich im Baritonschliussel geschrieben. Darüber hinaus enthält diese Sammlung auch einen um eine Sekunde nach oben zu transponierenden $\operatorname{Satz}^{466}$.

Keine vollends befriedigende Erklärung vermag der Verfasser zu der Transpositionsangabe uber Giovanni Gabrielis "Sonata Pian \& Forte" (Sacrae symphonie, 1597) zu geben. Auch uber dieser Sonata steht "Alla Quarta Bassa"467. Die Sonate ist bereits in der bei Gabrielis Instrumentalstuicken

459. Daß Violine und Zink fást ausschließlich im Violinschlissel notiert sind, schließt eine Transposition aufgrund einer "Chiavette" ohnehin aus.

460. Hier steht bei der letzten der acht Solosonaten - sie ist als einzige im Sopranschlüssel notiert (alle anderen stehen im Violinschluissel) - die Anweisung: "In Tuono, \& Alla Quinta Alta". In der höheren lage entspräche diese Sonate im Tonumfang den anderen sieben. Es ist denkbar, daß Vivarino bei dieser letzten Sonate an eine Blockflöte gedacht hatte. Auch die anderen Blockflötensätze aus dieser Zeit sind fast ausschließlich im Sopranschiussel notiert.

461. Hier findet sich die Angabe "Flautini ouera Cornetti alla quarta" (Sonata Sesta).

462. Auf die als Transpositionsverbot gedeutete Angabe "Va sonata alla quarta alta" aus dem ersten Buch "delle sinfonie" (1607) wurde oben bereits hingewiesen.

463. Auf die Möglichkeit der Transposition in dem einzigen das b' 'uberschreitenden Satz aus Rossis primo libro delle sinfonie (1607) wurde ebenfalls oben bereits verwiesen.

464. Die transponierte Fassung würde die Vorzeichnung von zwei Kreuzen erfordern. (Ein Satz mit zwei b ist in dieser Sammlung ausnotiert vorhanden ( "Gagliarda Terza")).

465. Diese Angabe ist also mit der "va sonata alla quarta alta"-Angabe aus Rossis erstem Buch der Sinfonien (1607) verwandt.

466. "Corrente Quinta va sonata una voce più alta". Diese Hochtransposition verlangt nach der Vorzeichnung von einem Kreuz. 
eher seltenen tiefen Schluisselung notiert468. Eine weitere Abwärtstransposition um eine Quarte ist zwar tonal gut denkbar (der Sonate ist ein b vorgezeichnet), würde aber in eine für die vorgeschriebenen Instrumente sehr tiefe Lage füren (Zink dann a bis c", "Violino" (wohl eher Bratsche) A bis d' und Baßposaune C bis f). Liest man "alta" statt "bassa" - ein solcher Schreibfehler ist nicht ausgeschlossen - so wäre die mit "Violino" bezeichnete Stimme auf einer Violine ausfuhrbar ${ }^{499}$, es ergäbe sich jedoch die ungewöhnliche Vorzeichnung von zwei $\mathrm{b}^{470}$.

\subsection{TRANSPOSition in MonteVERDis "MarienVesPeR"? - Zusammenfassung}

Die Situation im frühen 17. Jahrhundert ist zunächst sicher noch uneinheitlicher als sie im 16.Jahrhundert bereits war. Ein Komponist konnte, wenn er ein Stück in einer "Chiavette" notierte, nicht davon ausgehen, daß der Generalbaßspieler es selbständig transponiert, andererseits mußte er, schrieb er ein Stück in einer hohen Lage (was zwangsläufig zur Verwendung der hohen Schlüsselung führt), damit rechnen, daß der eine oder andere Organist dieses Stück nach unten transponiert auffuhrt. Sowohl Transpositionsanweisungen als auch Transpositionsverbote bezeugen diese Uneinheitlichkeit.

Gegen eine Transposition der hochgeschlüsselten Sätze aus Monteverdis "Marienvesper" 471 aber spricht vor allem die Tatsache, daß bei den wenigen anderen Beispielen für eine gesicherte Transposition in Satzen mit obligaten Instrumentalstimmen, die Instrumentalstimmen bereits transponiert notiert wurden (s.o.). In der reinen Instrumentalmusik sind Belege für Transpositionen sehr selten (s.o.). Auch der Tonumfang der in "Chiavette" notierten Sätze aus diesem Druck ist keinesfalls singulär. Auf einige Beispiele von ähnlich hohen Instrumentalstimmen (ohne Transpositionsmöglichkeit) wurde oben bereits hingewiesen. Die Lage der Vokalstimmen scheidet ebenfalls als Argument für eine Tiefertransposition aus. Deren Ambitus bleibt im Rahmen des damals ublichen. Der Ambitus der Stücke in Hochschlüsselung uberschreitet auch innerhalb dieses Druckes den der anderen selten und auch nur um eine Sekunde 472 . In anderen Quellen dieser Zeit wird von den Spitzentönen

467. Vgl, in der Ausgabe in IeM 2, S.64,

468. In der Sammlung von 1597 ist nur noch die Sonata Octavi Toni A 12 (IeM 2 , S.270) in einer ähnlich tiefen Schliusselung notiert.

469. Es sei jedoch darauf hingewiesen, daß dies nicht die einzige mit "Violino" bezeichnete Altstimme dieses Druckes ist (vg1. IeM 2, S.290 sowie oben im Abschnitt III.2.1.2.).

470. Eine sich ergebende ungewöhnliche Tonart wäre dann allerdings auch ein Grund für die Anwendung einer Transpositionsanweisung.

471. Sanctissimae Virgini missa (1610).

472. Der höchste Ton der hochnotierten Sätze ist das a' ', der der meisten anderen das $g^{\prime \prime}$. Das a' wird nicht häufig verlangt. Bei Tiefertransposition wïrden diese Sätze allerdings auch die anderen nur wenig unterschreiten: Der tiefste Ton der "normalgeschluisselten" Sätze ist das E, transponiert man die Hochnotierten um eine Quarte nach unten, so wird einige Male ein D verlangt. 
(insbesondere vom a' im Sopran) weitaus ausgedehnter Gebrauch gemach4473. Nicht zuletzt paßt es wenig zu der sonst sehr genauen Notationsweise Monteverdis (auch in diesem Druck genaue Instrumentationsangaben, Registerangaben für die Orgel, ausnotierte Verzierungen und "trilli" etc.), eine solche Transposition einfach ohne Vermerk vorausgesetzt zu haben. Auch musikalisch wäre es verwunderlich, wenn ausgerechnet die beiden Sätze, bei denen eine hohe Lage besonders gerechtfertigt erscheint, nämlich Lauda Jerusalem und das Magnificat, in iberdurchschnittlich tiefer Lage musiziert werden sollten.

Wie oben bereits erwähnt (im Abschnitt III.6.2.) wurde das a' häufig auch in anderen Vokalstücken (ohne Transpositionsmöglichkeit) verlangt, das D sogar mehrfach unterschritten.

473. Vg1. z.B. die oben genannten Kompositionen Giovanni Gabrielis. 


\section{TEIL: WeItere BESONDERHEITEN IN DER NOTATION DIESER ZEIT}

In den ersten drei Teilen dieser Arbeit sind die wesentlichen Wandlungen in der Notation in den Jahren um 1600 beschrieben worden. Darüber hinaus sind einzelne Abweichungen von unserer heutigen Notenschrift und oft auch von der des friberen 16. Jahrhunderts zu beobachten, die keinem der drei bisher behandelten Bereiche zufallen. Es ist dies in erster Linie die recht unterschiedliche Behandlung von Wiederholungszeichen, Fermaten und weiteren auch zur Kennzeichnung von Wiederholungen verwendeten Zeichen, sowie die Behandlung der Longa und damit insbesondere die Falsobordone-Notation.

\section{FALSOBORDONE-NOTATION UND LONGA}

Der reine Falsobordone ist eine im 16.Jahrhundert in Italien uberaus verbreitete Art des mehrstimmigen Psalm-Vortrages ${ }^{1}$. Ein solcher vierstimmiger Falsobordone besteht in der Regel aus einem Rezitationsakkord mit anschließender Kadenz auf einem Nebeninterval1 and einem weiteren Rezitationsakkord mit abschließender Kadenz auf dem Grundton. In Musikdrucken sind solche Falsobordoni (sie stehen in zahlreichen Motettendruclen am Ende der Sammlung) untextiert und für alle (meist acht) Kirchentöne vorhanden. Der Falsobordone wurde nach Francesco Severo in Rom auch als Grundlage des virtuos-solistischen Psalmvortrages verwendet 2 .

In späten 16.Jahrhundert traten in der Art eines Falsobordone notierte Chorrezitationen auch innerhalb von Motetten, insbesondere Psalmmotetten, auf. Frühe Beispiele für solche Falsobordone-Abschnitte finden sich in Drucken von Bartolomeo de Ratti ${ }^{3}$ Giulio Belli ${ }^{4}$, Giuliano Cartari ${ }^{5}$ und Tiburtio Massaino ${ }^{6}$. Im frühen 17. Jahrhundert sind solche Falsobordone-Abschnitte etwa in einem Viertel aller Drucke mit geistlicher Musik vorhanden. Falsobordone-Stellen wurden jedoch auch in weltlicher Musik verwendet, sowohl in Madrigalen und Canzonetten als auch in der Oper. Das wohl beruhmteste Beispiel hierfür ist der Arfang von Claudio Monteverdis Madrigal "Sfogava con le stelle" aus dem vierten Madrigalbuch $(1603)^{7}$. Außer von Monteverdi 8

1. Vg1. hierzu Ludwig Finscher, Art. Psalm in MGG, Bd.10, Sp.1694 ff sowie Murray C.Bradshaw, Art. Falsobordone in NG, Bd.6, S.375 ff.

2. F. Severo, Salmi passeggiati ... nella maniera che si cantano in Roma sopra $i$ falsi bordoni (1615, vgl. den Abdruck des Vorwortes Severis im zweiten Band dieser Arbeit auf S.16 f). Passaggi iber Falsobordoni bietet auch G. B. Bovicelli, Regole passaggi di musica (1594), S.73 ff.

3. Cantiones in laudem Deiparae Virginis Mariae ... liber primus (1594).

4. Psalmi ad vesperas ... acto voc. $(1596,3 / 1615)$, Psalmi ad vesperas... quinque vocibus (2/1598, 1. Auf1. nicht nachweisbar).

5. Beatissimae Virg. integerrimae laudes (1597).

6. Musica super Threnos Ierimie prophete (1599).

7. Vg1. hierzu Fortune, Monteverdi, S.200 ff, sowie Leopold, Monteverdi, S. $157 \mathrm{f}$.

8. Im dritten und funften Madrigalbuch (1603 bzw. 1605; später auch in der 
sind dem Verfasser Falsobordone-Abschnitte auch aus weltlichen Kompositionen von Adriano Banchieri ${ }^{9}$, Domenico Valla10, Amante Franzoni11, Marsilio Casentini12, Giulio Santo Pietro del Negro13, Marc Antonio Negri14, Giovanni Lorenzo Missioni15, Enrico Radesca di Foggia16, Guiseppe Marini17 und Stefano Landi 18 bekannt.

Die Falsobordone-Stellen sind weitgehend einheitlich als eine mit mehreren Textsilben unterlegten Longa ${ }^{19}$ notiert worden; gelegentlich findet man statt einer Longa aber auch eine Brevis20. Nicht selten sind die FalsobordoneAbschnitte zusätzlich mit senkrechten Strichen im Notensystem (Trennungsstrichen, s.u.) vom ubrigen Notentext abgeteilt. Gänzlich anders notierte Falsobordone-Abschnitte finden sich in Werken Orfeo Vecchis ${ }^{21}$, Romano Michelis 22 Gasparo Villanis ${ }^{23}$ und Giacomo Finettis ${ }^{24}$. Orfeo Vecchi notierte für jede Silbe des mit Trennungstrichen von der ubrigen Motette abgetrennten Falsobordone-Abschnittes eine Semibrevis; diese Semibreven sind gewiB ohne Rhythmisierung auszufuhren. Romano Micheli ordnet je drei Silben einer Minima zu. Auch hier ermöglichen es Trennungsstriche Anfang und Ende dieser besonderen Gültigkeit der Minima zu erkennen. Villani und Finetti schließlich schrieben als ersten Falsobordone-Ton eine Semibrevis, gefolgt von einem Custos mit unterlegtem Text 25.

Die Longa hat in diesen Fällen keinen definierten Notenwert. Sie zeigt nur die Tonhöhe an, auf der rezitiert werden soll. Ebenfalls keinen fest defi-

Incoronazione - vg1. Braun, Handbuch, S.102).

9. Il zabaione musicale (1604).

10. Il primo libro e madrigali a tre voci (1605).

11. I nuovi fioretti musicali (1605) und Il secondo libro delli fioretti (1607).

12. Tirsi e Clori (1607).

13. Gl'amorosi pensieri... libro secondo (1607) und Il terzo libro dell'amorose canzonette (1607).

14. Affetti amorosi a tre vaci (1608).

15. Tirsi doglioso (1615).

16. Il terzo libro delle canzonette (2/1616, 1. Auflage nicht nachweisbar).

17. Il primo libro de madrigali (1617) und Il secondo libro ... (1618).

18. La morte d'Orfeo (1619, nach Braun, Handbuch, S.102).

19. Bei E. Radesca di Foggia, Il terzo libro delle canzonette (2/1616, 1. Auflage nicht nachweishar) ist diese Longa schwarz notiert - zu schwarznotierten Longen bei Radesca di Foggia s.o. im Abschnitt 1.3.5.2.

20. So bei C. Monteverdi, Il quarto libro de madrigali (1603), A. Banchieri, Il zabaione musicale (1604), S. Molinaro, Concerti ecclesiastici (1605) und F. Rognoni, Messa salmi intieri (1610).

21. Motectorum quinque vocibus liber secundus (1598).

22. Psalmi ad officium (1610).

23. Ad Deum Opt. Max. ... liber quartus (1611).

24. Cantiones ... liber tertius (1620).

25. Vgl. das Notenbeispiel IV, 1 im zweiten Band dieser Arbeit. 
nierten Notenwert hat die Longa als Schlußnote. Im Gegensatz zur heutigen Praxis mußte die Schlußnote im 16. und 17. Jahrhundert nicht in allen Stimmen gleichzeitig eintreten. Der Schlußton wurde ausgehalten, bis alle Stimmen den Schlußakkord erreicht hatten. Sehr schön zu sehen ist dies in Partiturnotation. Die Schlußnote steht hier oft in einer Stimme bereits einen oder mehrere Takte vor dem Schlußakkord26. Auch in Stimmennotation wurde die SchluBlonga in gleicher Weise verwendet 27 . Innerhalb eines Satzes und außerhalb eines Falsobordone aber hat die allerdings nur noch selten auftretende l.onga ihren vollen Wert.

\section{WIEDERHOLUNGSZEICHEN, TRENNUNGSSTRICH, FERMATE UND KONGRUENZZEICHEN}

Im 16. und 17.Jahrhundert gab es keine einheitlich Methode, Wiederholungen zu notieren. Das häufigste Wiederholungzeichen war auch damals der Doppe1strich mit Punkten (meist zwei) auf jeder Seite. Dieses Zeichen 1ieß jedoch an Eindeutigkeit zu wuinschen übrig; es war z.B. nicht klar, ob nur wiederholt werden soll, was vor dem Zeichen steht, oder auch das danach - Wiederholungszeichen mit Punkten nur auf einer Seite waren noch nicht üblich. Um dennoch die Wiederholungen genau eingrenzen zu können, wurden verschiedene anderen Zeichen hirzugenommen. Da diese Zeichen meist auch noch andere $\mathrm{Be}-$ deutungen haben können (s.u.), sind Verwechslungen nicht auszuschließen. Eine annähernde Genauigkeit in der Notation der Wiederholungen wurde auch mit diesen Zeichen nicht immer erreicht28. Im folgenden sollen diese Zeichen mit ihren verschiedenen Bedeutungen beschrieben werden.

\subsection{ZUR SETZUNG DES WIEDERHOLUNGSZEICHENS}

Abweichend vom heutigen Gebrauch konnte ein Wiederholungszeichen an jeder denkbaren Stelle eines Taktes stehen. Beginnt ein Stück mit einem Auftakt, so mußte der erste Takt mit Pausen aufgefuilt werden. Ein Wiederholungszeichen wurde dann so gesetzt, dak diese Pausen bei der Wiederholung entfallen konnten, d.h. die Noten vor dem Wiederholungszeichen vervollständigen den ersten Takt. Vor allem in Canzonetten, aber auch in anderen Gattungen, wird ein Wiederholungszeichen am Schluß eines Stückes oft von einer SchluBlonga gefolgt. Diese ersetzt in der Regel die Schlußsemibrevis (oder auch eine andere Schlußnote) der "prima volta". Zwischen dem letzten Wiederholungszeichen und der Schlußlonga steht gelegentlich ein Custos oder auch eine Note. Beide bezeichen den Anfangston der Wiederholung ${ }^{29}$. Selten steht

26. Vgl. hierzu das Notenbeispiel IV,2 im zweiten Band dieser Arbeit.

27. Vollkommen unsinnig ist es, die Schlußlongen in einer modernen Edition in ihrem eigentlichen Wert, also als vier mit Bindebögen versehene ganze Noten zu ibertragen (so ist in Eleanor Selfridge-Fields Ausgabe Dario Castello, Selected Ensemble Sonatas verfahren worden).

28. Auf unklare Wiederholungsangaben in Drucken italienischer Klaviermusik aus dieser Zeit hat Judd, Repeat problems, hingewiesen. In vokaler Musik treten Probleme dieser Art allerdings nicht auf.

29. Vgl. das Faksimile aus A. Bonellis primo libro delle villanelle (1596) 
nach dem letzten Wiederholungszeichen statt einer Schlußlonga auch eine Art $\operatorname{Coda}^{30}$.

\subsection{DIE ANWENDUNGEN DES TRENNUNGSSTRICHES \\ 2.2.1. DER TRENNUNGSSTRICH ALS WIEDERHOLUNGSZEICHEN}

Fast zu einer Norm im Bereich der Canzonette und Villanelle war die Verwendung von Trennungsstrichen als vordere Begrenzung einer Wiederholung geworden ${ }^{31}$. Diese Stücke bestehen häufig aus einem zu wiederholenden Anfangsteil, einem nicht $z u$ wiederholenden Mittelteil sowie einem zu wiederholenden Schluß. Am Ende des ersten Teiles sowie am Schluß des Stückes steht ein Wiederholungszeichen, der Mittelteil ist nach hinten mit einem Trennungsstrich abgegrenzt. Die Setzung eines Wiederholungszeichens an dieser Stelle war nicht möglich, da dies auch die Wiederholung des Mittelteiles zur Folge gehabt hätte. Diese zunächst nur in Canzonetten und Villanellen verbreitete Art, die Wiederholung nach vorn zu begrenzen, fand vereinzelt auch in anderen Gattungen Anwendung ${ }^{32}$.

In einer Anzahl von Drucken wurde nur der Trennungsstrich verwendet, um eine Wiederholung anzuzeigen. Daß hier eine Wiederholung gemeint ist, läßt sich entweder an der, nach dem als Wiederholungszeichen gemeinten Trennungsstrich zusätzlich notierten Schlußlonga ${ }^{3}{ }^{3}$ oder aber an einer Textmarke bzw. einem Musikinzipit des zu wiederholenden Teiles - im Notentext meist ebenfalls durch einen Trennungsstrich gekennzeichnet - erkennen ${ }^{34}$.

im zweiten Band dieser Arbeit (Notenbeispiel III,2).

30. Z.B. in T. Merulas primo libro delle canzoni (1615).

31. Diese Verwendung des Trennungsstriches ist auch von der Musiktheorie beschrieben worden, so von L. Zacconi, Prattica di musica (2/1596), wie immer sehr ausfürlich und kompliziert unter der uberschrift "Delle fine che hanno le Villanelle, \& le canzonette. Cap. LXXII" (fol, 80'). 0. Scaletta beschreibt im Abschnitt uber den "Ritornello" (Wiederholungszeichen) in der Scala di musica $(1585,12 / 1626)$, S.5, nicht nur das Wiederholungszeichen selbst, sondern, im Bezug auf die "Canzone", auch diesen Trennungsstrich, den er als "bastone" bezeichnet. D. Hizler, Newe Musica (2/1628) benennt diesen Strich "Signum Inchoandae Repititionis" (S.19). Wieder mit besonderem Bezug auf die Canzonette beschreibt $S$. Picerli im Specchio prima di musica

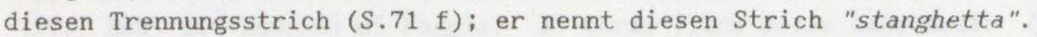

32. Eine Besonderheit stellt G. B. Rossis Verwendung des Trennungsstriches nicht als vordere Wiederholungsbegrenzung, sondern als Fine-Zeichen dar (Organo de cantori, 1618). Zu Verwechslungen kann es allerdings nicht kommen, da ausdrüicklich auf diese Bedeutung hingewiesen wird ("Da Capo, fino all traverso", S.98 f).

33. $\mathrm{Zu}$ beobachten in den Rose fresche von $\mathrm{J}$. de Castro und in D. Peccis Sacri modulatus (1629).

34. Folgende Beispiele sind dem Verfasser bekannt: A. Agazzari, Sacrae luades de Iesu ... liber secundus (1603), ders., Sertum roseum (1611), A. Cifra, Motecta liber primus $(1609,4 / 1611)$, ders., Motecta ... liber quartus 


\subsection{2, DeR TREnNungsstrich ZUR AbSChnittsbildung als "PAusa generalis" - TRENNUNGSSTRICH UND FERMATE}

Die meisten Trennungsstriche in Musikdrucken des 16. und 17.Jahrhunderts sind jedoch nicht im Zusammenhang mit Wiederholungen zu sehen. Sie dienen vielmehr der Gliederung der Musik und sind an musikalischen und textlichen Einschnitten notiert - besonders häufig z.B. in Motetten vor der Doxologie oder dem letzten "Amen". Solche Trennungsstriche kommen in allen musikalischen Gattungen außer der Canzonette und Villanelle vor ${ }^{35}$. Sie sind bereits in den Frottolen-Drucken Petruccis anzutreffen ${ }^{36}$. Gelegentlich sind solche Trennungsstriche auch zusammen mit Fermaten notiert (schon bei Petrucci). Fermaten und Trennungsstriche sind im späten 16. und frühen 17. Jahrhundert häufig bedeutungsgleich ${ }^{37}$. Während die italienische Musiktheorie hierzu nur wenig mitteilt38, werden beide Zeichen in deutschen Musiktraktaten aus dem frihen 17. Jahrhundert genau erk1ärt. Danach sind Fermate und Trennungsstrich alternative Zeichen für die "Pausa generalis"39. Diese "Pausa generalis" "bringt allen stimmen eine gemeine stillschweigung"40. Die Bedeutung der Fermaten unterscheidet sich damit deutlich von der heutigen ${ }^{41}$.

Daß diese in den deutschen Quellen beschriebene Bedeutungsgleichheit von Fermate und Trennungsstrich auch in italienischen Quellen gilt, belegt die Austauschbarkeit der beiden Zeichen in den italienischen Musikdrucken. So können Fermate und Trennungsstrich in verschiedenen Stimmbüchern eines Stückes an den sich entsprechenden Stellen stehen ${ }^{42}$. Eine Fermate konnte den

$(1609,3 / 1610)$, F. Beccari, Il secondo libro de sacri concenti (1611), V. Ugolini, Motecta sive sacrae cantiones ... liber quartus (1619). Eine bevorzugte Verwendung dieser Art der Wiederholungen durch irgendeinen Drucker ist nicht erkennbar (die genannten sechs Drucke erschienen bei fünf verschiedenen Verlegern aus drei Städten).

35. Dort stehen Trennungsstriche immer im Zusammenhang mit Wiederholungen.

36. Vg1. bei Jeppensen, Frottola, die Faksimiles auf den Seiten IX und XX.

37. Auf von der Fermate abweichende Bedeutungen des Trennungsstriches wird in den Abschnitten IV.2.2.1. und 2.2.3. hingewiesen.

38. Lediglich bei L. Zacconi, Prattica di musica (1596), fol. 80, findet sich eine Bemerkung iber die Fermate, die man "per dimostrare che quello non è l'ultimo fine" verwendet.

39. So bei J. Burmeister, Musica poetica (1606), S.10, A. Gumpelzhaimer, Compendium musicae (5/1611), fol. 11' und $14^{\prime}$, D. Hizler, Newe musica (2/1628), S.8 ff, und M. Vulpius, Musica compendium $(1610,6 / 1636)$, S.66 f. 40. Gumpelzhaimer, fol.11'.

41. Nur so ist es auch zu verstehen, daß gelegentlich kurze Schlußnoten der in Madrigalen häufigen "abgebrochenen" Schluisse (z.B. gerne bei "morire") mit einer Fermate versehen sind. Ein Aushalten dieser Schlußnoten wäre widersinnig.

42. So in F. Canales Missae, introitus, ac motecta (1588). 
Trennungsstrich auch in mit Taktstrichen versehenen Basso continuo-Stimmen ersetzen ${ }^{43}$.

Eine interessante Unterscheidung von Fermate und Trennungsstr ch ist in Bernardo Strozzis Musica concertata (1630) zu beobachten. Ein Trennungsstrich wurde hi r verwendet, wenn ein Abschnitt ganztaktig endet, wird eine abschnittsbildende Kadenz aber inmitten eines Taktes erreicht, so ist dieses Abschnittsende mit einer Fermate gekennzeichnet.

Dieselbe Bedeutung kommt sicher auch anderen Methoden der Abschnittsgliederung zu. So kann anstelle eines Trennungsstriches auch eine Wiederholung des Schluissels 44 oder des Taktzeichens 45 stehen (beides kommt auch in Verbindung mit einem Trennungsstrich vor ${ }^{46}$ ).

\subsubsection{WEITERE VERWENDUNGEN DES TRENNUNGSSTRICHES}

Außer in den beiden Hauptfunktionen des Trennungsstriches - Wiederholungszeichen und Abschnittsbildung ("Pausa generalis") - wurden Trennungsstriche gelegentlich auch für eine ganze Anzahl weiterer Abgrenzungen innerhalb des Notentextes verwendet. Es wurde oben bereits darauf hingewiesen, daß rege1mäßig Falsobordone-Abschnitte in Trennungsstriche eingefaßt sind ${ }^{4}$. Trennungsstriche konnten dariber hinaus einen piano-Abschnitt ${ }^{48}$, eine hemiola 49 sowie einen längeren Pausenteil50 einschließen. Immer dienen diese Trennungsstriche der ubersichtlichkeit des Notentextes - ihnen kommt keine weitere auffuhrungspraktische Bedeutung zu, aber sie belegen einmal mehr die Auffuhrungsbezogenheit der Musikdrucke dieser Zeit.

\subsection{DAS KONGRUENZZEICHEN}

Das im 16.Jahrhundert im wesentlichen zum Bezeichnen der Kanon-Einsätze verwendete "signum congruentiae" wurde im späten 16 . und frühen 17.Jahrhundert

43. In L. Leoni, Sacrarum cantionum, liber primus (1608). In anderen Drucken wird der Trennungsstrich in der (mit Taktstrichen versehenen) Bc.Stimme auch als Doppelstrich notiert - dies wäre sicher auch die angemessene moderne Ubertragung sowoh1 von Fermaten, als auch von in diesem Sinne verwendeten Trennungsstrichen.

44. So in S. d'Indias Le musiche... libro quarto (1621).

45. Z.B. in G. Conversis primo libro de canzoni $(1572,2 / 1580)$.

46. Wiederholung des Taktzeichens mit Trennungsstrich: 0. Columbani, Liber secundus sacrarum cantionen (1592); Wiederholung des Schlissels mit Trennungsstrich: S. Bernardi, Psalmi integri (1613) und P. Pace, Il sesto liro de motetti (1618).

47. Vgl. im Abschnitt IV.1.

48. S. Arsilli, Messa e vesperi (1621) und G. Finetti, Corona Mariae ... liber quintus (1622 - dort allerdings nur in der Echo-Motette "Nos autem").

49. C. Antegnati, Salmi (1592) - vg1. oben im Abschnitt I.3.

50. S. Bernardi, Messa $(1615,2 / 1624)$. 
ebenfalls als Wiederholungszeichen verwendet51. In Notierungen mit Taktstrichen (meist Partituren ${ }^{52}$ ) konnte der Trernungsstrich als vordere Wiederholungsbegrenzung nicht verwendet werden. Stattdessen griff man auf das Kongruenzzeichen zuriick (gedruckt als ".S." oder ".5." o.ä.). Die Notation des Kongruenzzeichens auch iber dem Wiederholungszeichen macht auf die Verwendung des Kongruenzzeichens als vordere Begrenzung der Wiederholung aufmerksam ${ }^{53}$.

Ebenso wie der Trennungsstrich wurde auch das Kongruenzzeichen in manchen Quellen als alleiniges Wiéderholungszeichen verwendet. Diese Art der Notierung von Wiederholungen findet sich nur in Quellen monodischer Musik. Bereits in der noch dem 16. Jahrhundert entstammenden Brüsseler Monodienhandschrift sind Wiederholungen auf diese Art gekennzeichnet54. Im frühen 17. Jahrhundert sind solche Wiederholungen bei Bartolomeo Barbarino55, Giulio Caccini ${ }^{56}$, Vincenzo Calestani ${ }^{57}$, Sigismondo d'Indias 58 und Jacobo Peri59 zu finden. Sie notierten lediglich am Ende eines Satzes sowie an der Stelle, von der aus wiederholt werden soll, je ein Kongruenzzeichen. Ein gelegentlich am Ende des Satzes zusätzlich notierter Inzipit belegt, daß die Kongruenzzeichen hier tatsächlich eine Wiederholung anzeigen ${ }^{\circ 0}$. Gelegentlich fehlt sogar das Kongruenzzeichen am Ende des Satzes, so daß die Wiederholung alleinig durch das vordere Zeichen bezeichnet wird.

51. Freilich wird es auch in Quellen des 17.Jahrhunderts ggf. zur Bezeichnung von Kanoneinsätzen benutzt.

52. Dem Verfasser ist nur ein Stimmbuchdruck bekannt, im dem das Kongruenzzeichen als Wiederholungszeichen verwendet wurde, nämlich S. Bernardis Concerti academici ... libro primo (1616) - auch dieser Stimmbuchdruck ist allerdings teilweise mit Taktstrichen versehen. A. Brunelli verwendete das Kongruenzzeichen in seinen zweitem Buch der Scherzi (1614) sowohl bei den in Partitur notierten als auch bei den in Chorbuchnotation gedruckten Sätzen; bei den letzteren allerdings oft zusätzlich zum Trennungsstrich.

53. In dieser Art wird das Kongruenzzeichen z.B. in S. Molinaros Partiturausgabe der sechs Madrigalbucher Gesualdos (1614) sowie in A. Brunellis Scherzi ... libro secondo (1614) verwendet.

54. Bibliotheque du Conservatoire, Brissel 704 (vgl. das Notenbeispiel IV, 3$)$.

55. Madrigali (1606, 2/1609), Il secondo libro de madrigali (1607), Il terzo libro de madrigali (1610), Canzonette (1616) sowie in der Wolfenbuitteler Barbarino-Handschrift (Cod. Guelf. 19 Musica Hdschr.).

56. L'Euridice (1600), Nuove musiche (1602) und Le nuove musiche e nuova maniera (1614). Ein Faksimile einer solchen Stelle aus L'Euredice bietet Besseler, Schriftbild, S.159. Die Punkte zu dem Doppelstrich am Ende sind von Hand ergänzt (vgl. die auch im Bezug auf die Wiederholung fehlerhafte ubertragung auf S. 158 unten).

57. Madrigali et arie (1617).

58. Le musiche (1609) und Le musiche a due voci (1615).

59. Le musiche ... sopra l'Euridice (1600).

60. Vgl. das Notenbeispiel IV, 3 im zweiten Band dieser Arbeit. 
Anste1le des ublichen Kongruenzzeichens konnten auch andere Zeichen dense1ben Zweck erfüllen. Giovanni Maria Tarbaci verwendete in Ricercate ... libro primo (1603), einem Partiturdruck, das Symbol einer Hand, um die Wiederholungen kenntlich zu machen ${ }^{1}$. Diese Hand steht an der Stelle des Trennungsstriches als vordere Begrenzung der Wiederholung; am Satzende steht ebenfalls diese Hand mit dem Vermerk "Replica". Orazio Scaletta notierte in der Partitur der Certa spirituale (1605) statt eines Kongruenzzeichens drei Kreuze. Am Ende des Satzes steht "Tornate al Segno delle tre croci".

In anderer Weise verwendeten Adriano Banchieri und Emilio De' Cavalieri das Kongruenzzeichen in ihren Musikdrucken. Bei Banchieri bezeichnet es nicht eine Begrenzung der Wiederholung, sondern das Fine62 - bei De' Cavalieri werden die Stellen, an denen kurz innegehalten und Atem geschöpft werden so11, mit einem Kongruenzzeichen versehen63. Beide Funktionen kornten in nicht in Partitur notierten Sätzen auch Trennungsstriche wahrnehmen. Dies verdeutlicht abschließend nochmals die enge Verwandtschaft zwischen diesen Zeichen.

\section{HORIZONTALE StRICHE ZUR KENNZEICHNUng INSTRUMENTALER ABSCHNitTE}

In vokaler Musik mit gelegentlichen instrumentalen Einwiirfen war es unangemessen aufwendig, für diese Einwuirfe gesonderte Instrumentalstimmen zu drucken. Längere Ritornelle konnten mittels einer überschrift. ("Ritornello") als solche gekennzeichnet und separat notiert werden. Wollte man aber kurze instrumentale Abschnitte notieren, die inmitten einer Strophe zu musizieren sind, ohne daß der musikalische Ablauf dadurch unterbrochen wird, bot sich ein anderes Verfahren an: Man kennzeichnete die instrumentalen Abschnitte als eine besondere Art der "Textierung". Statt eines Textes steht bei den Instrumentalteilen ein horizontaler Strich bzw. eine gestrichelte linie. Diese Art der Unterscheidung zwischen vokalen und instrumentalen Teilen wurde offensichtlich von Giulio Cesare Monteverdi in den von ihm herausgegebenen Scherzi musicali (1607) seines Bruders Clandio eingefuhrt ${ }^{64}$. In diesem Druck gibt es längere, separat notierte Ritornelli, die jeweils zu Anfang und Ende der Strophen zu musizieren sind, sowie kurze, in der beschriebenen Art notierte Einwirfe. Das Verfahren wurde auch von einigen anderen Komponisten ibernommen 65 .

61. Eine solche Hand als Kongruenzzeichen findet man auch der Handschrift 704 der Bibliotheque du Conservatoire, Brisse1.

62. Vg1. das Vorwort zur Partitura moderna armonica (1612), abgedruckt bei Sartori, Bibliografia I, S.184 (1612 a) unter "Quarta". Zahlreich mit "Da Capo" angezeigte Wiederholungen sind auch in den Auflagen des L'organo suonarino zu finden.

63. Vg1. den Abdruck des Vorwortes im zweiten Band dieser Arbeit auf S. 8 ff (das Kongruenzzeichen entspricht damit einem "Pausa generalis"-Zeichen).

64. In den "Avvertimenti" heißt es hierzu: "Doue si vedranno tirate linee nella sede delle parole, quelle note che sono ad esse linee sopraposte douranno esser sonate, ma non cantate." 


\section{TEXTIERUNGSZEICHEN}

Insgesamt ist in den Drucken des hier behandelten Zeitraumes auch die Textunterlegung sehr sorgfältig vorgenommen worden. Ein Bedarf an Textunterlegungsregeln - sie findet man in theoretischen Quellen des friberen 16. Jahrhunderts 60 - bestand nicht67. Auch in den Textarmen Teilen der Messe (z.B. Kyrie) wird der Text zunehmend genau unterlegt68, Dies konnte z.T. auch geschehen, da man sich oftmals von der liturgisch vorgeschriebenen Anzahl der Textwiederholung (dreimalige Anrufung) 10̈ßte69; dann war auch das Kyrie nicht. mehr ganz so textarm.

Wurde der Text nicht direkt unterlegt, sondern - bei einer Textwiederholung - durch ein Textierungszeichen ersetzt, so geschah dies auch weiterhin mit dem Zeichen " $i j$ ". Dieses Zeichen wurde mit Vorliebe an Stellen syllabischer Textunterlegung 70 oder bei Textwiederholungen bei gleichbleibendem Rhythmus angewendet ${ }^{11}$. Fine hiervon abweichende Art, solche Textwiederholungen zu kennzeichnen, findet sich in einer Anzahl von Drucken mit Musik Grammatico Metallos $\sin ^{72}$. Metallo ersetzte in seiner uberaus genauen Textunterlegung das Zeichen " $i j$ " durch den Anfangsbuchstaben des zu wiederholenden Textes, z.B. Sicut erat $S \quad S \quad S$.

65. Dieses Verfahren ist dem Verfasser dariber hinaus aus folgenden Drucken bekannt: R. Rontani, Le varie musiche ... libro primo $(1614+1623)$, M. da Gagliano, Musiche (1615), V. Calestani, Madrigali et arie... parto primo (1617), G. C. Monteverdi, Delli affetti musici, libro primo (1620) und D. Mazzocchi, La Catena d'Adone (1624). Ein Faksimile einer solchen Stelle aus Monteverdis Scherzi ist in zweiten Band dieser Arbeit als Notenbeispiel IV, 4 wiedergegen.

66. Vgl. hierzu z.B. Harrén, Text Underlay.

67. So enthält L. Zacconis Kapitel "Come, \& in che modo si cantino le parole sotto le figure Musicali" (Prattica di musica (1596), fol. 57) auch nur eine Erklärung der Textwiederholungszeichen und einige wenige allgemein gehaltene Hinweise - z.B. Ausspracheregeln.

68. So ist in den drei erhaltenen Vokalstimmbüchern von A.Banchieris Sacra armonica (1619) in insgesammt 6 kyrie nur ein einziges mal das Textierungszeichen " $i j$ " angewendet worden - und dies an einer Stelle fast syllabischer Textunterlegung. Komplizierte ist die Textunterlegung z.T. in Monteverdis "Missa In illo tempore" aus Sanctissimae Virgini missa (1610).

69. Bei Banchieri wird der Text in den Kyrie zwischen ein- und sechsmal wiederholt; bei Monteverdi drei-bis fünfmal.

70. Vgl. z.B. im zweiten Band dieser Arbeit das Notenbeispiel I, 17, dritte Zeile.

71. Z.B. Notenbeispiel II, 9, Takt 13.

72. Villanelle (1592), Messe comodissime ... libro sesto (1592), Magnificat (1603), Messe a 5 (1610), Motetti a 5 (1610), Motetti per tutte le solennita (1610), Messa, motetti (1611), Motetti, Magnificat (1613). 


\section{DANKSAGUNGEN}

An dieser Stelle soll all denen gedankt werden, ohne deren Unterstiutzung diese Arbeit nicht hätte zustande kommen können. An erster Stelle sei hier mein verehrter Lehrer, Herr Prof. Dr. Martin Staehelin, Universität Göttingen, genannt. Ihm ist es zu verdanken, daß diese Arbeit in dieser Form uiberhaupt zustande kommen konnte, und er hat ihre Ausfertigung mit Interesse und Rat begleitet.

Besonderer Dank gebihrt auch den folgenden Bibliotheken sowie deren Mitarbeitern:

Bayerische Staatsbibliothek, Muinchen

The British Library, London

Christ Church Library, Oxford

Deutsches Musikgeschicht1iches Archiv, Kasse1

Diözesan-Bibliothek, Muinster

Herzog August Bibliothek, Wolfenbittel

Landesbibliothek und Murhardsche Bibliothek, Kassel

osterreichische Nationalbibliothek, Wien

Ratsschulbibliothek, Zwickau

Staats- und Stadtbibliothek, Augsburg

Stadt- und Universitätsbibliothek, Frankfurt a.M.

Dort wurde es mir jeweils ermöglicht, zumeist große Mengen von Quellen einzusehen. Ohne diese Bereitschaft wäre diese Arbeit nicht durchfuhrbar gewesen.

Für die Anfertigung von Mikrofilmen oder Fotokopien bin ich, neben den bereits genannten, folgenden Bibliotheken zu Dank verpflichtet:

Biblioteca Municipale "A. Panizzi", Reggio Enilia

Biblioteca Nazionale Centrale, Firenze

Biblioteca Uniwersytecka, Warszawa

Bibliothéque Royale Albert Ier, Bruxelles

Bodleian Library, Oxford

Civico Museo Bibliografico Musicale, Bologna

Gemeente Museum, Den Haag

The Newberry Library, Chicago

Niedersächsische Staats- und Universitätsbibliothek, Göttingen

Ratsbücherei, Lüneburg

Seminario Arcivescovile, Lucca

Staatsbibliothek Preussischer Kulturbesitz, Berlin

Schließlich möchte ich meiner Frau danken. Sie hat nicht nur die Entstehung dieser Arbeit mit Interesse und Hilfe begleitet, sondern auch zahlreiche Stunden mit Korrekturlesen zugebracht. Ihr möchte ich diese Arbeit widmen. 

Cimband mil blaw Tin't boschmitas

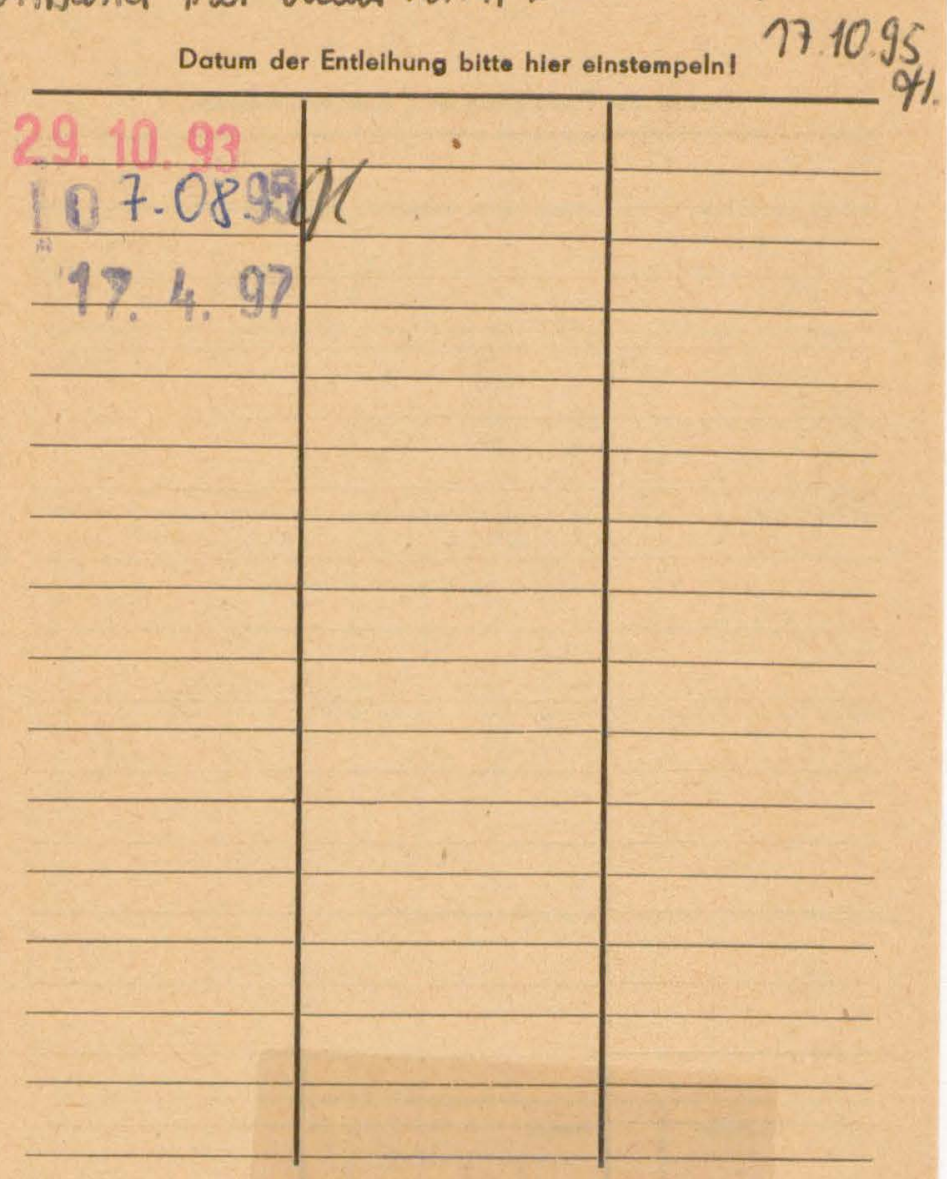




\section{Berliner Studien zur Musikwissenschaft}

\section{Auswahl}

Band 2 Erika Gessner

Samuel Scheidts geistliche Konzerte

Ein Beitrag zur Geschichte der Gattung. 216 Seiten mit 89 Notenbeispielen und 9 Tabellen im Anhang

EM 1452

Band 5 Walter Reckziegel

Das Cantional von Johan Herman Schein

Seine geschichtlichen Grundlagen. 237 Seiten mit 9 Abbildungen und 10 Notenbeispielen

EM 1455

Band 6 Dieter Krickeberg

Das protestantische Kantorat im 17. Jahrhundert

Studien zum Amt des deutschen Kantors. 243 Seiten

EM 1456

Band 10 Friedhelm Krummacher

Die Überlieferung der Choralbearbeitungen

in der frühen evangelischen Kantate

Untersuchungen zum Handschriftenrepertoire evangelischer Figuralmusik im späten 17. und beginnenden 18. Jahrhundert. 591 Seiten mit Abbildungen und Tabellen

EM 1460

Band 11

Ute Meissner

Der Antwerpener Notendrucker Tylman Susato

Eine bibliographische Studie zur niederländischen Chansonpublikation in der ersten Hälfte des 16. Jahrhunderts. 2 Bände mit zusammen 397

Seiten, 9 Abbildungen und 23 Seiten Notenanhang

EM 1461

Band 12 Marianne Henze

Studien zu den Messenkompositionen Johannes Ockeghems

264 Seiten mit Notenbeispielen und Tabellen

EM 1462

Band $13 \quad$ Wolfgang Nitschke

Studien zu den Cantus-firmus-Messen Guillaume Dufays

2 Bände mit zusammen 468 Seiten, 44 Seiten Notenbeispiele und $10 \mathrm{Ta}$ feln mit Notentabellen

EM 1463

\section{Merseburger}

SHCHSISCHE LANDESBIBLIOTHEK

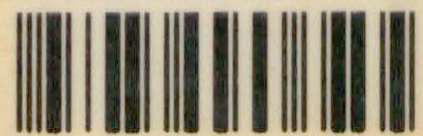

EM 1s

20125608 